
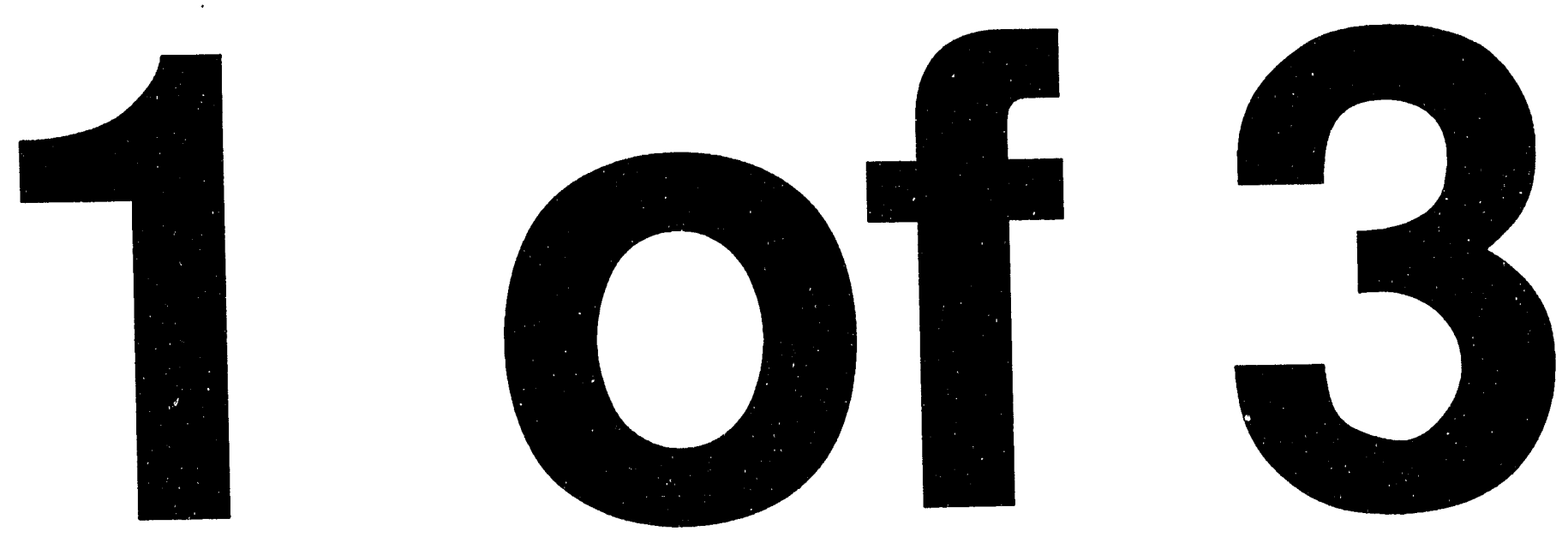
NUREG/CR-6128

BNL-NUREG-52396

\section{Piping Benchmark Problems for the ABB/CE System $80+$ Standardized Plant}

Manuscript Completed: May 1994

I .te Published: July 1994

Prepared by

P. Bezler, G. DeGrassi, J. Braverman, Y. K. Wang

Brookhaven National Laboratory

Upton, NY 11973-5000

Prepared for

Division of Engineering

Office of Nuclear Reactor Regulation

U.S. Nuclear Regulatory Commission

Washington, DC 20555-0001

NRC FIN E2024 


\begin{abstract}
To satisfy the need for verification of the computer programs and modeling techniques that will be used to perform the final piping analyses for the $\mathrm{ABB} / \mathrm{Combustion}$ Engineering System $130+$ Standardized Plant, three benchmark problems were developed. The problems are representative piping systıms subjected to representative dynamic loads with solutions developed using the methods being proposed for analysis for the System $80+$ standard design. It will be required that the combined license licensees demonstrate that their solutions to these problems are in agreement with the benchmark problem set.
\end{abstract}


ABSTRACT $\ldots \ldots \ldots \ldots \ldots \ldots \ldots \ldots \ldots \ldots \ldots \ldots \ldots \ldots \ldots \ldots \ldots \ldots \ldots \ldots \ldots \ldots \ldots$

LIST OF FIGURES AND TABLES $\ldots \ldots \ldots \ldots \ldots \ldots \ldots \ldots \ldots \ldots \ldots \ldots \ldots \ldots \ldots \ldots \ldots \ldots \ldots$

EXECUTTVE SUMMARY $\ldots \ldots \ldots \ldots \ldots \ldots \ldots \ldots \ldots \ldots \ldots \ldots \ldots \ldots \ldots \ldots \ldots \ldots \ldots \ldots \ldots$

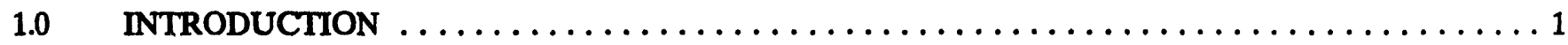

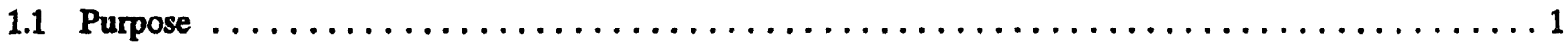

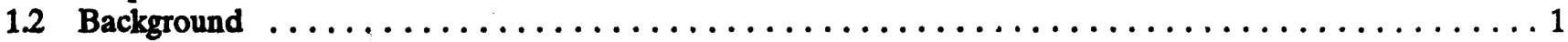

1.3 Benchmark Program Overview $\ldots \ldots \ldots \ldots \ldots \ldots \ldots \ldots \ldots \ldots \ldots \ldots \ldots \ldots \ldots \ldots$

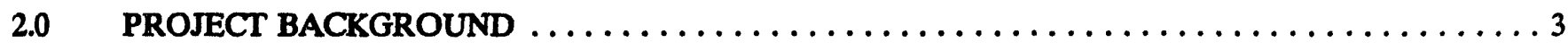

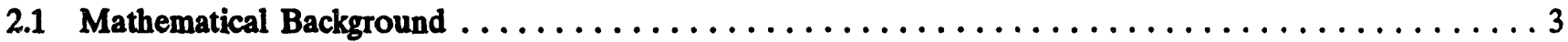

2.1.1 Uniform Support Motion Response Spectrum Analysis $\ldots \ldots \ldots \ldots \ldots \ldots \ldots \ldots \ldots \ldots \ldots$

2.1.2 Modal Superposition Time History Analysis $\ldots \ldots \ldots \ldots \ldots \ldots \ldots \ldots \ldots \ldots \ldots \ldots$

2.1.3 Direct Integration Time History Analysis $\ldots \ldots \ldots \ldots \ldots \ldots \ldots \ldots \ldots \ldots \ldots \ldots$

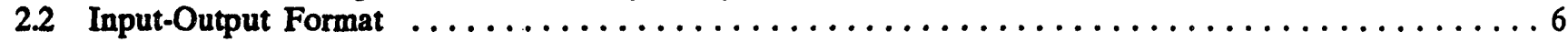

3.0 DESCRIPTION OF PIPING BENCHMARK PROBLEMS $\ldots \ldots \ldots \ldots \ldots \ldots \ldots \ldots \ldots \ldots \ldots$

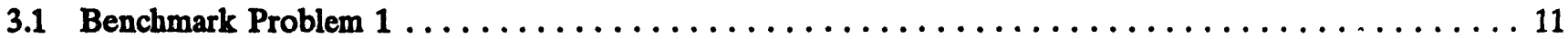

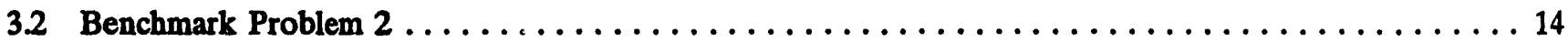

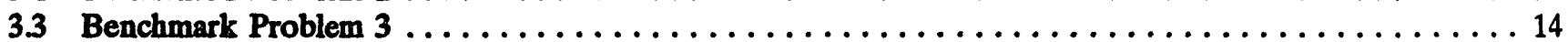

4.0 BENCHMARK ACCEPTANCE CRITERIA $\ldots \ldots \ldots \ldots \ldots \ldots \ldots \ldots \ldots \ldots \ldots \ldots \ldots \ldots$

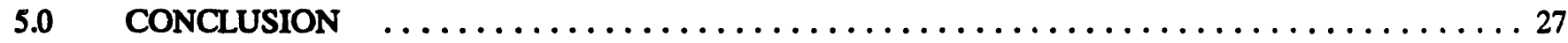

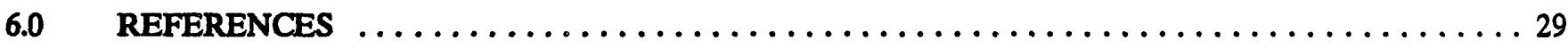

\section{APPENDIX A}

UNITS OF MEASURE $\ldots \ldots \ldots \ldots \ldots \ldots \ldots \ldots \ldots \ldots \ldots \ldots \ldots \ldots \ldots \ldots \ldots \ldots \ldots \ldots \ldots$

BENCHMARK PROBLEM 1 - UNIFORM SUPPORT MOTION RESPONSE SPECTRUM ANALYSIS .. A-1

Benchmark Problem 1 - Lower Frequency Amplified Response . . . . . . . . . . . . . . . . . . A-13

Benchmark Problem 1 - High Frequency Response (Rigid Response) $\ldots \ldots \ldots \ldots \ldots \ldots \ldots \ldots \ldots$. . . . . . . . . .

Benchmark Problem 1 - Total Response $\ldots \ldots \ldots \ldots \ldots \ldots \ldots \ldots \ldots \ldots \ldots \ldots \ldots \ldots \ldots$

BENCHMARK PROBLEM 2 - DIRECT INTEGRATION TIME HISTORY ANALYSIS . . . . . . A A-73

BENCHMARK PROBLEM 3 - MODAL SUPERPOSITION TIME HISTORY ANALYSIS . . . . . . . A-159 
Figure 1 Sign Convention for Forces and Moments $\ldots \ldots \ldots \ldots \ldots \ldots \ldots \ldots \ldots \ldots \ldots \ldots \ldots \ldots$

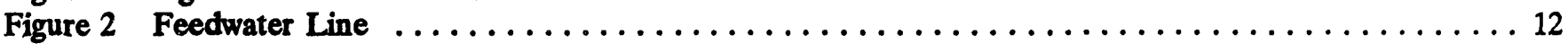

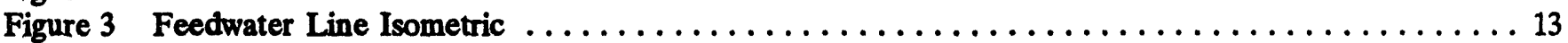

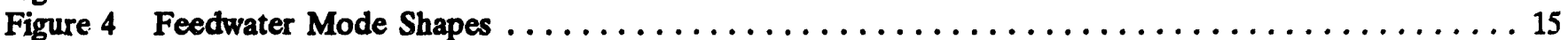

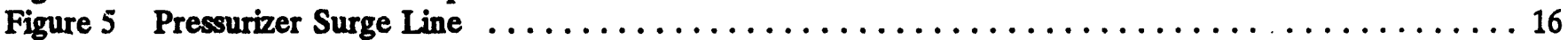

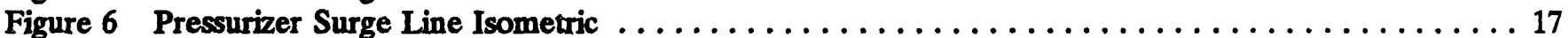

Figure 7 Pressurizer Surge Line Mode Shapes $\ldots \ldots \ldots \ldots \ldots \ldots \ldots \ldots \ldots \ldots \ldots \ldots \ldots \ldots \ldots \ldots$

\section{LIST OF TABLES}

Table $1 \quad$ Feedwater Piping Modal Analysis Results $\ldots \ldots \ldots \ldots \ldots \ldots \ldots \ldots \ldots \ldots \ldots$

Table 2 Pressurizer Surge Line Piping Modal Analysis Results $\ldots \ldots \ldots \ldots \ldots \ldots \ldots \ldots \ldots \ldots \ldots \ldots$

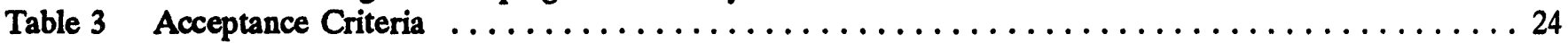

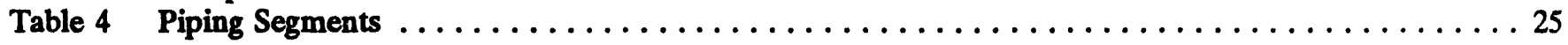




\section{EXECUTIVE SUMMARY}

The NRC staff has identified piping and pipe support design as one technical area where engineering information in sufficient detail can not be provided for the ABB/Combustion Engineering System $80+$ standard design to allow the staff to make a final safety decision under the rules of 10. CFR, Part 52. For this and similar areas the staff will use design acceptance criteria (DAC) verified by inspections, tests, analyses, and acceptance criteria (ITAAC) to enable the final safety determination. One element of the piping DAC requires the combined license (COL) licensee to verify the sufficiency of the computer codes and modeling techniques to be used to complete its piping stress analyses. To provide the basis for this verification, the staff developed a System $80+$ specific piping benchmark program. The COL licensee will be required to develop solutions to the benchmark problems and to demonstrate that those solutions meet the acceptance criteria specified in this benchmark program report.

The benchmark program consists of three piping problems involving two piping systems representative of the System 80+ design. One system represents a portion of the Main Feedwater Line and the other represents the Pressurizer Surge Line. The problems provide analytical benchmarks for three analysis methods, the uniform support motion response spectrum analysis method, the modal superposition time history analysis method, and the direct integration time history analysis method. The response spectrum method and the direct integration time history methods are applied to the Feedwater Line while the modal superposition time history method is applied to the Surge Line. In all analyses the excitation functions are representative for the System $80+$ standard design. This report presents the benchmark problems and includes all the information needed by the COL licensee to perform the analyses and evaluate the results. Acceptable analysis methods will estimate natural frequencies to within $2 \%$, maximum pipe moments to within $5 \%$, and support reactions and maximum displacements to within $10 \%$ of the benchmark results. A COL licensee having demonstrated this level of accuracy may use the benchmarked analysis method without further review. In instances where some deviations from the acceptance criteria occur, the results and justification for such deviations shall be documented and submitted to the staff for review and approval before initiating piping qualification analyses. 
This report describes the NRC benchmark program for the verification of computer programs that will be used by combined license (COL) licensees to complete the jesign and analysis of piping systems in the $A B B / C o m b u s t i o n$ Engineering (ABB/CE) System 80+ Standardized Plant. It provides detailed descriptions of the benchmark problems including geometries, material properties, analytical methods, input loads and solutions. The report also provides the acceptance criteria which must be met to demonstrate that the COL licensee's solutions to the benchmark problems are acceptable to NRC.

In reviewing the design certification application for the ABB/CE System 80+ under the rules of 10 CFR Part 52, Reference 1, the NRC staff identified a number of technical areas in which the applicant did not provide design and engineering information in sufficient detail to make a final safety decision. One of these areas was piping and pipe support design where $A B B / C E$ did not have the as-built or as-procured information to complete the final design. To resolve this issue, the staff developed an alternate approach using design acceptance criteria (DAC). The DAC are a set of prescribed limits, parameters, procedures, and attributes upon which the NRC relies in making a final safety

determination to support a design certification. The DAC are objective (measurable, testable, or subject to analysis using pre-approved methods), and must be verified as a part of the inspections, tests, analyses, and acceptance criteria (ITAAC) used to demonstrate that the as-built facility conforms to the certified design. The combined license (COL) licensee will use the DAC to demonstrate conformance with the System $80+$ standard design during construction. This will enable the NRC staff to make a final safety determination through the review of the COL licensee's satisfactory implementation and verification of the ITAAC.

The NRC staff's evaluation of ABB/CE's proposed DAC approach for the System 80+ piping design was documented in their final safety evaluation report. In that document the staff provided their evaluation of the $A B B / C E$ certified design commitments and corresponding ITAAC for the System $80+$ piping design. One of these commitments was to verify the piping analysis modeling and the computer code to be used by the COL licensee to complete its piping stress analysis. The COL licensee will verify the sufficiency of the computer code and modeling techniques in conjunction with the DAC. The NRC found this commitment acceptable provided that the computer program and the modeling techniques will be evaluated using the NRC benchmark program. The staff concluded that once the COL licensee successfully completes the DAC verifying that the piping benchmark results are within the acceptable range of values specified in the benchmark program, there is reasonable assurance that the computer code and analytical modeling techniques to be used to complete the System $80+$ piping design and analyses are adequate.

\subsection{Benchmark Program Overview}

The benchmark program requires the $\mathrm{COL}$ licensee to construct mathematical models and perform dynamic analyses of specified representative System $80+$ piping systems using his own computer program. When the analyses are completed, the COL licensee will compare his results to those of the benchmark problems given in this report to ensure that the results meet the range of acceptable values. Any deviations from these values, as well as, the justification for such deviations, shall be documented and submitted to the NRC staff for review and approval before initiating final certified piping analyses. This benchmarking will provide assurance that the computer program used to complete the System $80+$ piping design and analyses will produce results that are consistent with results considered acceptable to the NRC staff.

This report presents the benchmark problems and gives all information needed by the COL licensee to perform the analyses and evaluate the results. The Brookhaven National Laboratory (BNL), under contract with the NRC staff, developed these problems based on representative piping system design information provided by $\mathrm{ABB} / \mathrm{CE}$ during the NRC technical review and evaluation of the System $80+$ standard design. The benchmark problems represent a portion of the System $80+$ Main Feedwater Line and the Pressurizer Surge Line. Although the piping benchmark problems are considered representative System 80+ piping configurations and loadings, they are not intended to be final designs. BNL constructed mathematical models of these two piping systems using the PSAFE2 piping analysis program. 


\section{ABB/CE-SYSTEM 80+}

This report provides a complete description of the input parameters for each problem including piping dimensions, material properties, weights, support stiffnesses and locations, load definitions and damping values. The dynamic analysis methods benchmarked include the uniform support motion response spectrum method, the modal superposition time history analysis method, and the direct integration time-history analysis method. The BNL solutions to each problem are presented in this report. Specific guidelines for comparing COL licensee results to these published results and acceptance criteria to be satisfied in order to demonstrate acceptability are also given. 
The PSAFE2 program is a full feature, elastic piping analysis code based on the finite element method. The program, a modified version of the general purpose computer program SAP IV, Reference 2, was developed by BNL to analyze piping systems subjected to both static and dynamic loading. Dynamic analysis capabilities include both response spectrum and time history analysis for systems subjected to either uniform or independent support motions.

The PSAFE2 program and its precursor EPIPE, Reference 3, have been extensively tested and verified against other piping programs and against physical test results. The programs have been applied to develop earlier NRC benchmark problem solutions to confirm the adequacy of programs used by nuclear power plant license applicants (References 4 and 5).

This report presents the solutions to piping benchmark problems with configurations that are representative of $A B B / C E$ System $80+$ piping systems. The solution methods that were applied were not in their entirety included in earlier NRC benchmark studies. They include the uniform response spectrum method with high frequency mode responses, the modal superposition time history analysis method, and the direct integration time history analysis method. The following section provides a brief description of the analytical methods.

\subsection{Mathematical Background}

Since elastic piping analysis is a well established procedure, only a brief outline of the theoretical considerations used in obtaining the dynamic solutions will be presented. A more detailed description of the analysis methods and solution schemes is given in Reference 2.

The analysis of a piping system is carried out by use of the stiffness matrix method, in which the piping is represented by a network of basic elements, straight and curved beams, and one-dimensional elements interconnected at the nodes. The dynamic response of the network is described mathematically by the equation of motion:

$$
[M]\{\ddot{u}\}+[C]\{\dot{u}]+[K]\{u\}=\{R(t)\}
$$

where $[M]$ is the mass matrix, $[C]$ is the damping matrix, and $[K]$ is the stiffness matrix of the element

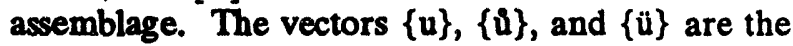
nodal displacements, velocities, and accelerations, respectively. $\{R(t)\}$ can be a vector of time varying loads or of effective loads which result from ground motion. The PSAFE2 program can carry out time history or response spectrum analysis for the solution of this equation. A brief description of the methods used in these benchmark problem solutions is provided below.

\subsubsection{Uniform Support Motion Response Spectrum Analysis}

In the case of ground motion, if it is assumed that the piping system is uniformly subjected to the ground acceleration, $\tilde{u}_{z}$, the equations of motion can be expressed as follows:

$$
[M]\left\{\tilde{u}_{r}\right]+[C]\left(\dot{u}_{r}\right\}+[K]\left\{u_{r}\right\}=-[M]\left\{\tilde{u}_{g}\right\}
$$

where $\left\{u_{r}\right\}$ is the relative displacement of the system with respect to the ground, $\left\{u_{\mathrm{r}}\right\}=\{u\}-\left\{u_{\mathrm{g}}\right\}$.

The equations are then expanded in terms of the system modal matrix and generalized coordinates:

$$
[M][\phi]\{q\}+[C][\phi]\{\dot{q}\}+[K][\phi]\{q\}=-[M]\left\{\tilde{u}_{g}\right\}
$$

where:

$$
\begin{aligned}
& {[\phi]=\text { normalized modal matrix }} \\
& \text { such that }[\phi]^{T}[M][\phi]=[I]
\end{aligned}
$$

$\{q\}=$ generalized coordinates vector.

Multiplication by the transpose of the modal matrix yields:

$$
\{\not{q}\}+[\Delta]\{q\}+\left[\omega^{2}\right]\{q\}=-[\phi]^{T}[M]\left\{\ddot{u}_{g}\right\}
$$

where:

$$
\begin{aligned}
{\left[\omega^{2}\right]=} & \text { diagonal matrix of eigen-values } \\
{[\Delta]=} & \text { diagonal matrix of modal damping } \\
& \text { coefficients, where the damping is } \\
& \text { assumed to satisfy the modal } \\
& \text { orthogonality condition. This results in } n \\
& \text { uncoupled equations for the generalized } \\
& \text { coordinates. }
\end{aligned}
$$


The equation set can be solved for the maximum modal response for each system degree of freedom corresponding to excitation in each spatial direction as follows:

$$
u_{m, N}=\frac{S_{n d}}{\omega_{n}^{2}} \phi_{m, n} L_{n d}
$$

where:

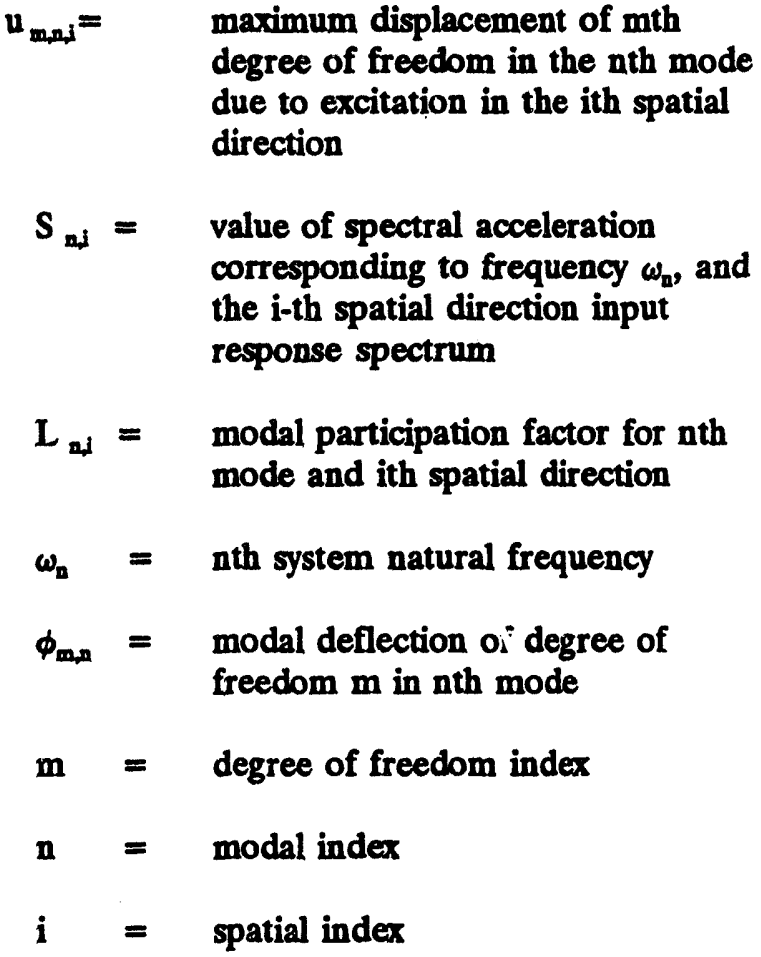

For the final solution, the maximum modal responses are combined over the first n modes up to the cutoff frequency (the lower frequency nodes) at which the spectral acceleration roughly returns to the zero period acceleration (ZPA). For the benchmark problem given in this report, the modal combination is performed in accordance with the grouping method as described in Section 1.2.1 of Regulatory Guide 1.92, Reference 6.

In some piping systems the responses of modes above the cutoff frequency (the high frequency modes) may have a significant effect on the total response, especially on support reactions. In order to ensure that these high frequency mode effects are accounted for, an additional calculational procedure based on the methodology described in Appendix A to Section 3.7.2 of the Standard Review Plan,
Reference 7, was implemented. This procedure is as follows:

For each degree of freedom (DOF) included in the dynamic analysis, the fraction of DOF mass, $d_{\text {, }}$, included in the summation of all of the modes up to the cutoff frequency was calculated for each DOF as follows:

$$
d_{i}=\sum_{n=1}^{N} L_{n} \times \phi_{n}
$$

where:

n is order of the mode under consideration,

$\mathbf{N}$ is the number of modes up to the cutoff frequency,

$\phi_{n, j}$ is the nth natural mode of the system, and

$\mathrm{L}_{\mathrm{n}} \quad$ is the participation factor given by:

$$
L_{n}=\frac{\left\{\phi_{n}\right\}^{T}\{\lambda\}}{\left\{\phi_{n}\right\}^{T}[M]\left\{\phi_{n}\right\}}
$$

The fraction of DOF mass not included in the summation of these modes, $\mathrm{e}_{\mathrm{b}}$, was then calculated as:

$$
e_{i}=d_{i}-\delta_{i j}
$$

where $\delta_{\mathrm{ij}}$ is the Kronecker delta, which is one if DOF $i$ is in the direction of the earthquake motion and zero if DOF $i$ is a rotation or not in the direction of the earthquake input motion.

Since higher modes can be assumed to respond in phase with the ZPA and thus, with each other, these modes should be combined algebraically. This is equivalent to pseudostatic response to the inertial forces from these higher modes excited at the ZPA. These pseudostatic inertial forces for all higher modes for each DOF $i$ are given by:

$$
P_{i}=Z P A \times M_{i} \times e_{i}
$$

where:

$P_{1}$ is the force or moment to be applied to DOF i. 
$M_{4}$ is the mass or mass moment of inertia associated with DOF $\mathbf{i}$.

In order to determine the maximum responses associated with the high frequency modes, a static analysis is performed by applying this set of pseudostatic inertial forces to all of the degrees of freedom in the model. The total high frequency mode responses are then conbined by the squareroot-of-sum-of-squares method with the total combined response from the lower-frequency modes to determine the overall piping system peak responses.

\subsubsection{Modal Superposition Time History Analysis}

The time history response of a piping system subjected to a known forcing function can be obtained by the modal superposition method. This procedure requires the solution of the generalized eigenvalue problem to determine the frequencies and mode shapes of the system. It uses the same modal transformation procedures described above for the response spectrum method. The general equations of motion in matrix form are expressed as follows:

$$
[M]\{\tilde{u}]+[C]\{\dot{u}\}+[K](u)=\{R(t)\}
$$

Applying the transformation:

$$
\{u\}=[\Phi]\{q\}
$$

The equations can be expressed in terms of the generalized modal coordinates:

$$
[M[\phi]\{\dot{q}]+[c][\phi]\{\dot{q}\}+[K][\phi]\{q]=\{R(t)\}
$$

Multiplication by the transpose of the modal matrix and application of the modal orthogonality assumptions yields the following set of equations:

$$
\{q]+[\Delta]\{\phi\}+\left[\omega^{2}\right]\{q\}=[\phi]^{T}\{R(t)\}
$$

This set of decoupled differential equations is solved numerically for a sufficient number of modes needed to adequately characterize the dynamic response of the piping system. In selecting the integration time step and the number of modes, the dynamic characteristics of the system as well as the forcing function must be considered. The PSAFE2 program uses the Wilson $\theta$ numerical integration method which is an unconditionally stable step-bystep integration scheme. Finally, the modal responses are converted back to geometric coordinates $u$ and combined algebraically at each time step to provide the total response of the piping system as a function of time.

\subsubsection{Direct Integration Time History Analysis}

In this solution mode the equation of motion is solved directly by numerical integration. Eigen value evaluations are not made and no simplification associated with eigen vector orthogonality properties is used. As such, no approximation associated with a modal assumption is introduced. The method is a basic approach and is described in any reference on numerical methods. References 2 and 3 are particularly applicable.

In the PSAFE2 program the Wilson $\theta$ method is used to perform the numerical integration. The advantage of this method is that it is unconditionally stable. As a matter of good practice, the time step should be essentially $1 / 10$ the shortest period of interest. In this method, Rayleigh damping is assumed such that the damping matrix is given by:

$$
[C]=\alpha[M]+\beta[K]
$$

Where $\alpha$ and $\beta$ satisfy the following relation:

$$
\xi_{i}=\frac{\alpha}{2 \omega_{i}}=\beta \frac{\omega_{i}}{2}
$$

where $\xi_{1}$ is the modal damping ratio corresponding to natural frequency $\omega_{i}$.

The $\alpha$ and $\beta$ constants may be determined from the above relationship by assigning a prescribed modal damping ratio, $\xi$, to any two frequencies, $\omega_{1}$ and $\omega_{2}$ and solving the following set of simultaneous equations:

$$
\begin{aligned}
& \xi=\frac{\alpha}{2 \omega_{1}}+\beta \frac{\omega_{1}}{2} \\
& \xi=\frac{\alpha}{2 \omega_{2}}+\beta \frac{\omega_{2}}{2}
\end{aligned}
$$

Once the $\alpha$ and $\beta$ constants are established, the modal damping ratio, $\xi_{i}$, for each remaining mode of vibration with natural frequency, $\omega_{i}$, is determined from the equation given above. Thus, the damping ratio is a function of frequency. This differs from 
the modal analysis methods described above in which a constant damping ratio may be assigned to all modes. With Rayleigh damping, a plot of $\xi$ versus $\omega$ shows modal damping to be lower than the prescribed value within the frequency range of interest between $\omega_{1}$ and $\omega_{2}$ (although the curve is relatively flat). Modal damping is higher at frequencies below and above this range.

In an analysis where a constant modal damping is required, (e.g., R.G. 1.61 damping) the analyst must exercise extreme caution and good engineering judgement in determining the $\alpha$ and $\beta$ constants. The prescribed damping ratio should be assigned to the two frequencies that bound the significant frequency range. For example, the prescribed damping ratio may be assigned to the fundamental frequency and the ZPA frequency. This will ensure that all modes within the amplified range of response will have a modal damping value that is close to but does not exceed the prescribed value. Other values of $\omega_{1}$ and $\omega_{2}$ can be selected if appropriate justification is provided to ensure that the prescribed modal damping has been adequately approximated.

\subsection{Input-Output Format}

To facilitate proper use of the enclosed data a brief summary of the PSAFE2 input-output data lists will be presented. For a basic description of element properties, coordinates, etc., reference can be made to the earlier benchmark reports, References 4, 5, and 8.

The first page of each problem shows a table listing the significant parameters for the problem and the analysis options activated for the analysis. The number of element types is the number of boundary-spring element and pipe element groups used to define the model. The number of frequencies is the number of frequencies solved for in the eigen value routine and used in the subsequent response spectrum analyses. The interpretation of the various option flags is obvious, although not all of the options are used in the benchmark problems.

The next pages show the nodal point list. The column labeled old node is the label assigned the nodal point in the finite element model. The column labeled new mode is the sequential label assigned to the node within the program and used in all program algorithms. The boundary condition codes define the constraints imposed at the node, a " 1 " signifying that the degree of freedom is completely restrained while " 0 " or $("-0 ")$ signifies complete freedom. As indicated, each node potentially has six degrees of freedom, " $X$ " signifying displacement in the $\mathrm{X}$ global direction and "XX" signifying rotation about the $X$ global axis. The following nodal coordinates define the location of the node in the global coordinate system and are in inches. The last column designated " $T$ " is the nodal temperature.

The next blocks of input information are the input element data lists. Since the system may be subdivided into groups of elements this data is entered group by group. The possible element types are spring elements, anchor movement elements, snubber elements, and pipe (either tangent or bend) elements. A single group is comprised of all pipe elements or all spring elements as intermixing element types within a group is not allowed.

For a typical pipe element group, the first page lists control information for the group, i.e., type of element, number of elements, etc. This is followed by a material properties table where on each line of this table are specified, the material identification number, the temperature for which the data applies and the corresponding modulus of elasticity, Poisson ratio and coefficient of thermal expansion. For cases where the variation of material properties with temperature are considered, the element temperature is the average of its bounding nodal temperatures and a linear variation of the input material properties data with temperature is assumed. Following this is the section properties table where all data entries are clearly labeled. Next, if appropriate, is a branch point list specifying the nodes where pipe runs intersect, followed on the same page by entries entitled, "Load Case Multipliers." These only apply to static analysis runs and may be ignored herein. The last and largest list in the group data is the element definition and connectivity table. Again, the columns in this table are clearly labeled. For the bend elements. two lines are shown. The pressure indicated on the first line is the value of pressure used in computation of the bend flexibility. The entries on the second lines are the bend radius, third point declarer and the $X, Y$, $Z$ coordinates of the third point. If the third point declarer is "TI" the coordinates of the bend tangent intercept are listed, if "CC," the coordinates of the 
bend center of curvature are listed. The columns entitled direction cosines, wall fraction and input tag may be ignored.

The group data list for boundary/spring elements or snubber elements is somewhat simpler. There is the element control information followed by element load case multipliers which again may be ignored. The element definition and connectivity list follows and is clearly labeled. The interpretation of the pertinent information in this table is as follows: support group number NG designates the excitation group to which the spring belongs; code $\mathrm{KD}$ and $\mathrm{KR}$ are spring type declarers where $\mathrm{KD}=1$ signifies a linear spring while $K R=1$ signifies a rotational spring. The direction cosines listed define the line direction along or about which the spring acts in relation to the global axss. The specified displacement - rotation columns relate to imposed displacements and can be ignored. The last column lists the element spring rate in $\mathrm{lb}$. per inch or inch lb. per radian.

The next table of input is labeled nodal loads. For the dynamic cases the table is a listing of all the concentrated masses, and the nodes at which they are located, acting on the system. A complete absence of this table indicates that there are no concentrated loads. The total mass used in each analysis is the sum of these quantities plus any distributed mass developed in the pipe elements. Distributed mass is developed in the pipe elements only if quantities appear in the columns entitled weight/unit length - mass/unit length in the pipe element section property tables.

The remaining input data describes the input response spectra or the time history forcing function, as appropriate. For a response spectrum case, the input spectra appear immediately after the output list of frequencies and modal participation factors. This data includes the direction weighting factors, a descriptive title and a table of the spectrum values given in " $\mathrm{g}$ 's". If only one spectrum is provided it applies to all three directions as modified by the weighting factors. If two spectra are provided, the first applied only to the $X$ direction while the second applies to the $Y$ and $Z$ directions. Intermediate values between data points are obtained by linear interpolation.

For a modal superposition time history case, the definition of the time history forcing functions follows after the output list of frequencies. This data is input by support groups and includes first a summary of control information specifying forcing function number, type and size, analysis time step, phasing (controlled by arrival times), duration and modal damping information (applies to all modes), and output print interval. This is followed by forcing function type, direction and arrival time declarers followed by the detailed definition of the forcing functions. The forcing function record is digitized and includes a listing of the time and corresponding function value for each point of the function definition and is preceded by scale factor, function number and heading information. The function value for intermeciiate times is derived by linear interpolation. Regarding arrival times, an arrival time of 0.0 connotates forcing function application at time equal to 0.0 . This information is provided sequentially for each support group in the system and acts simultaneously on all supports within a group.

For the time history case, the nodal load table is followed by a page summarizing control information unique to the time history analysis. This includes a definition of the number of solution tir.e steps, time step size and the values of the damping parameters $\alpha$ and $\beta$. The next table provid es the definition of the points of application of the forcing functions, the degree of freedom they act in, function number and arrival (start) time declarers and the function multiplier. Regarding the function multiplier, it is the amount by which the forcing function is amplified for the listed nodal point, the amplification being constant for the duration of the forcing function. Two further listings complete the input. The first is a listing of the arrival times and the second, the listing of forcing functions. The forcing function listing includes the time and corresponding function value for each point of the function definition.

The output provides listings of all the pertinent results. For the response spectrum solutions these include the system natural frequencies, modal participation factors, global displacements/rotations of all nodal points and element forces/moments for all elements based on the sign convention shown in Figure 1. The displacement and force results are provided first for the lower frequency modes (amplified response), then for the higher frequency modes (rigid response), and lastly for the final results corresponding to SRSS combination of the response due to the lower frequency modes with the response due to the higher frequency modes. For the rigid response, the contribution to response for excitation in each of the coordinate directions is, in 


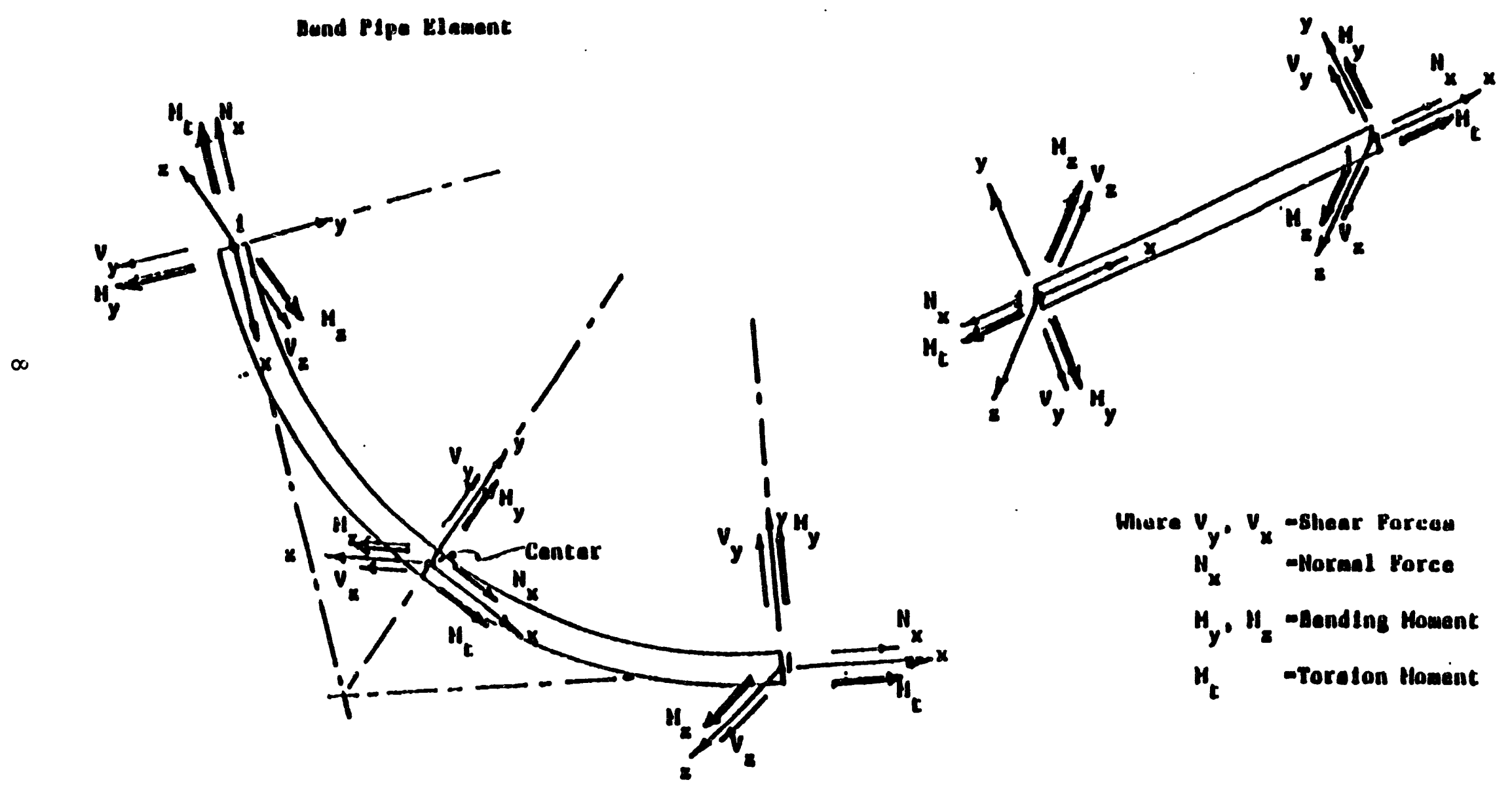

FIGURE 1 - SIGN CONVENTION FOR FORCES AND MOMENTS 
fact, provided. These are labeled load cases 1,2 and 3 corresponding to excitation in the $X, Y$, and $Z$ dire. ions respectively. These results are developed by static analyses and are combined by SRSS before combination with the amplified response. In effect, the rigid response is treated as an additional mode and summed with the other, lower frequency modes.

The time history output is simpler. It consists of three tables of maxima listed with their corresponding time at maxima. The first table is for nodal displacements with six displacement components for each node. The displacement component declarers have the following interpretation: 1,2 , and 3 are the translations of the node in the $X, Y$, and $Z$ directions respectively while 4,5 , and 6 are the rotations of the node about the $X, Y$, and $Z$ axis respectively. The next table provides the spring/boundary element force (labeled stress) maxima. A component declarer equal to one in this table indicates a force while the declarer equal to two irdicates a moment. The last table provides the maxima for pipe element force components (labeled stress). For each element twelve components of force are listed; the first six corresponding to the $i$ and the remaining six to the $j$ end. The component declarers in this table have the following interpretations: 1,2 , and $3 / 7,8$, and 9 are the $i$ end $/ j$ end forces in the element $X, Y$, and $Z$ axes respectively while 4,5 , and $6 / 10,11$, and 12 are the $i$ end / $j$ end moments about the element $X$, $Y$, and $Z$ axis respectively. 

BENCHMARK PROBLEMS

For the System $80+$ standardized design three piping benchmark problems involving two representative piping systems were developed. The first piping system is a section of the feedwater piping, while the second is the pressurizer surge line. These two systems were used by $A B B / C E$ in sample analyses to demonstrate the analysis methodology proposed for design analysis of piping in the System $80+$ design. The solutions developed represent analytical benchmarks for three analysis methods, the uniform support motion response spectrum method, the modal superposition time history analysis method and the direct integration time history analysis method. Only these methods are proposed for use in the design of piping in System $80+$ and were demonstrated in the sample analyses. The COL licensee having demonstrated that the results developed for these problems are in agreement with the benchmark results will be entitled to use these methods in design analysis.

The computational options used in the benchmarks are considered acceptable. For the response spectrum method clustering between closely spaced modes in accordance with the grouping method as described in Section 1.2.1 of Regulatory Guide 1.92 and additions to account for high frequency modes per SRP 3.7.2 Appendix A, must be made. Interspatial combination using the SRSS method and any sequence in performing the combinations is acceptable.

No definition of acceptable combination procedures are required for the time history method. Good practice would require that the time step used be sufficiently small to provide good estimates of system response. One tenth the smallest period of interest is a recognized rule for time step size. In any case, having selected a time step, its adequacy should bu demonstrated by showing that a reduction of time step size by a factor of two does not change the predicted response by more than $10 \%$. This sensitivity to time step check was made in the time history benchmarks. For the modal superposition method a check to assure that a sufficient number of mcdes has been used to ensure participation of all significant modes must be made. The criterion for this check is specified in SRP 3.7.2 and requires that the inclusion of additional modes does not result in more than a $10 \%$ increase in response.
The listing of the output results for each problem is relatively complete. The greatest omission is the deletion of the digitized mode shape information for the response spectrum and modal superposition problems. The inclusion of this data would have resulted in a five fold increase in the size of this report. Further omissions are the calculations for high frequency effects in Problem 1. These are simple static analyses which can be performed in various ways. A full description of the format of the output is provided in the preceding section.

\subsection{Benchmark Problem 1}

The first System 80+ piping Uenchmark is a uniform support motion response spectrum solution for one section of the feedwater piping subjected to safe shutdown seismic loads. The piping system, shown in Figure 2, extends from two nozzles on steam generator 2 to an anchor at the main steam valve house wall (MSVH). It consists of two 20 inch $(50 \mathrm{~cm})$ diameter branch lines converging into a 24 inch $(60 \mathrm{~cm})$ diameter main line which expands into a 28 inch $(70 \mathrm{~cm})$ diameter line at the MSVH. The pipe is schedule 80 , schedule 120 and special walled ASME SA106B carbon steel piping. Both the nozzles on the steam generator and the penetration at the MSVH wall are anchors for the system. The system is dynamically restrained by six rigid restraints in the vertical direction and six snubbers in the horizontal direction. The line includes two 20 inch diameter and five 24 inch diameter motor operated valves and two butt weld reducers. Nominal temperature and pressure for the line are $650^{\circ} \mathrm{F}\left(343^{\circ} \mathrm{C}\right)$ and $1200 \mathrm{psig}(8274 \mathrm{kPa})$ respectively.

The finite element nodes and discretization are shown in Figure 2 while a computer generated isometric of the system is shown in Figure 3. The model consists of 70 pipe elements and 71 nodes. The anchors exist at nodes 1 and 14, the steam generator nozzles and node 71 , the MSVH wall. Vertical restraints act at nodes $10,26,34,44,55$ and 66 and horizontal snubbers restrain nodes $10,25,26$, 34,44 and 55. In the analyses the anchors are modeled as stiff springs $(K=1 E+13 \mathrm{lb} /$ in in translation and $1 E+15$ in-lb/rad in rotation) in the six degree of freedom directions. The snubbers and vertical restraints are modeled as linear springs with spring constants consistent with their properties. The valves in the system are modeled with heavy walled $(t=10$ inches) pipe elements and concentrated masses chosen to simulate valve 


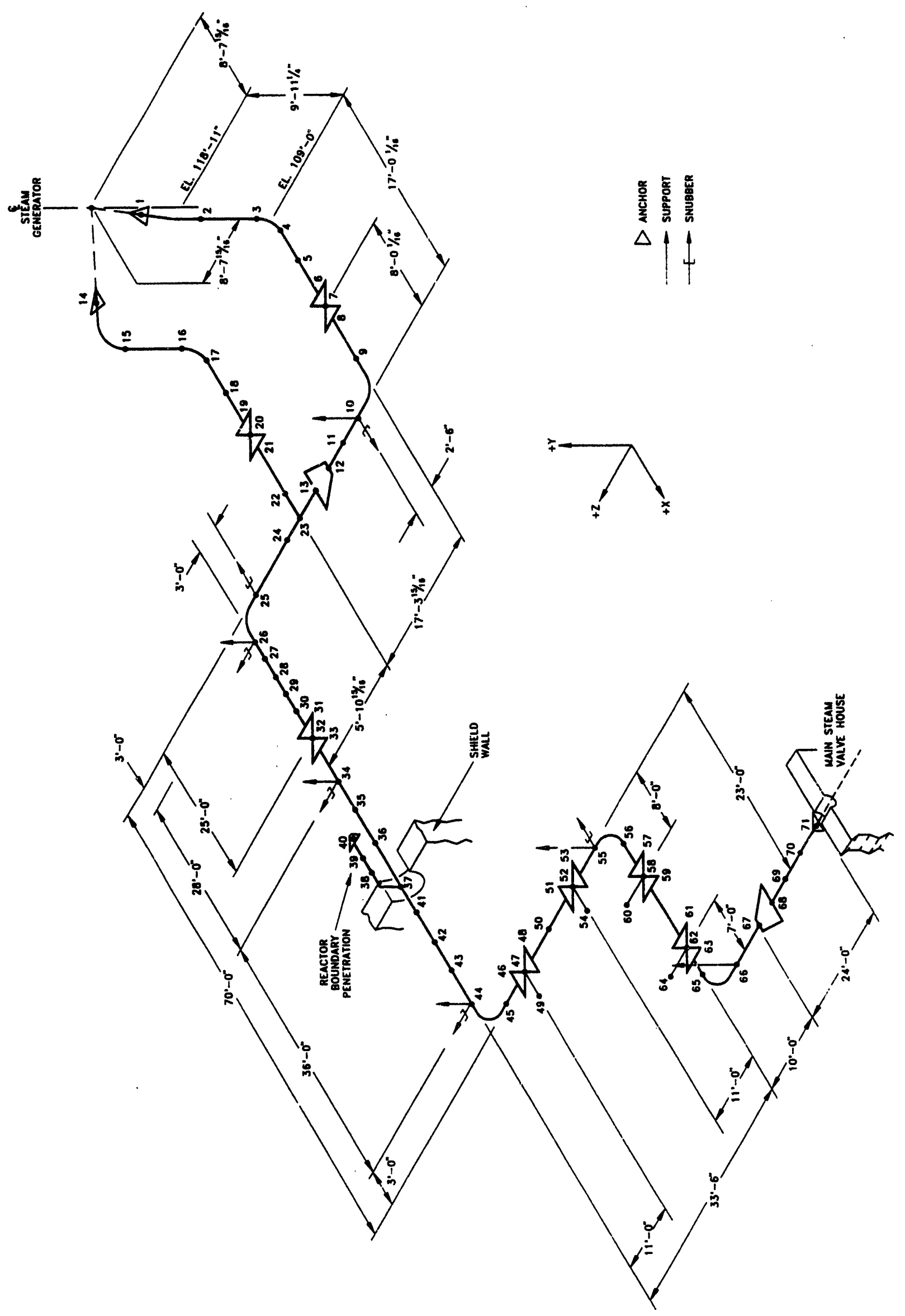

四 
STSTEM 60 + FEEDWATER AMLTSSS

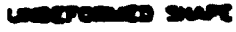

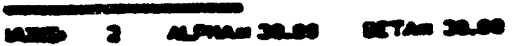
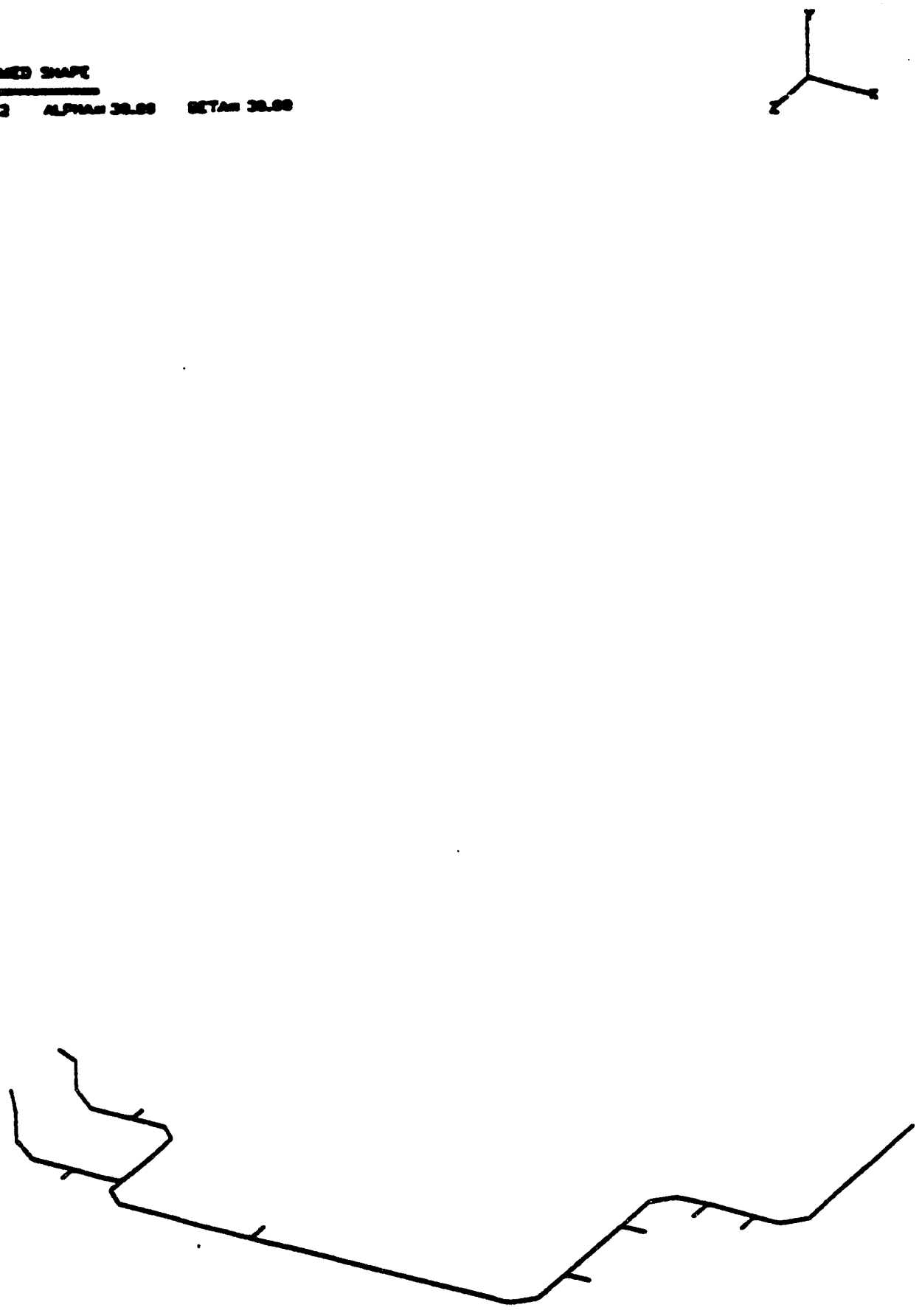

FIGURE 3 - FEEDWATER LINE ISOMETRIC 
properties. All parameters relating to the model are specified in the included computer listing.

In the analysis a cutoff frequency of $40 \mathrm{~Hz}$ was used. $A$ listing of the first 10 natural frequencies is presented in Table 1. Computer generated isometries of the first four modes are shown in Figure 4. As stated, the loading function was the SSE. A digitization of the spectra is included in the input listing. In the calculation, combination between the low frequency (amplified) and high frequency (rigid) responses was made by the SRSS combination method. Listings of the low frequency, high frequency and total responses are presented in the output listings. In the analysis, interspatial combination was performed first using the SRSS method followed by a modal combination with the contribution of the high frequency modes being treated as an additional mode.

\subsection{Benchmark Problem 2}

The second System $80+$ piping benchmark is a time history solution for the feedwater piping subjected to the transient loading induced by a water hammer. The piping and finite element model are identical to those used in Benchmark Problem 1 and the preceding section should be referred to.

In the analysis, the excitation consisted of eleven forcing functions acting on different nodes in the system. The loading essentially reproduces the effects of a water hammer shock wave traveling through the piping from the steam generator nozzles to the MSVH anchor. A full tabulation of the forcing functions digitized at $\mathbf{0 . 0 0 5} \mathrm{sec}$ intervals is provided in the computer listing.

The solution is developed using the direct integration time history method of analysis with, in this case, a BNL version of the SAP V computer code. As stated, the listings of the input forcing functions are provided in the input listing. This list also provides a clear delineation of the nodal points on which the loads act, any weighting or scale factors associated with them, and their line direction of action. The solution time step was $0.001 \mathrm{sec}$. and the solution extended for $\mathbf{4 0 0 0}$ time steps $(4 \mathrm{sec})$. A reduction of time step to $0.0005 \mathrm{sec}$ was found to change results by a factor less than $10 \%$ verifying the adequacy of the chosen size. The Rayleigh damping coefficients were taken as $\alpha=0.0000377$ and $\beta=0.00009549$ to be consistent with the
ABB/CE application. All other pertinent parameters are identical to those used in the preceding problem and are specified in the input list.

The output consists of the maxima and their time of occurrence for each response component. Although a more comprehensive tabulation of results would be desirable, the results provided are adequate for the benchmarking purpose. The tabulated outputs are well labeled and should be readily understood.

\subsection{Benchmark Problem 3}

The third System $80+$ piping benchmark is a time history solution of the pressurizer surge line subjected to the accelerations induced by a main steam line pipe break. The line, Figure 5, extends from a nozzle on the hot leg to a nozzle on the pressurizer. It is restrained in the horizontal directions by two rigid supports and one snubber. It is supported against dead weight and in the vertical direction with three constant force hangers. There are no valves or vertical dynamic restraints in the system. Nominal temperature and pressure for the line are $653^{\circ} \mathrm{F}\left(345^{\circ} \mathrm{C}\right)$ and $2250 \mathrm{psig}(15514 \mathrm{Kpa})$.

The finite element nodes and discretization are shown on Figure 5 while a computer generated isometric of the system is shown in Figure 6. The model consists of 44 pipe elements and 45 nodes. Anchors exist at nodes 1 and 45, the two end points of the system. These were simulated in the analysis with stiff springs $(K=1 E+13 \mathrm{lb} /$ in in translation and $1 E+15 \mathrm{in}-\mathrm{lb} / \mathrm{rad}$ in rotation) in the six degree of freedom directions. The horizontal restraints act at nodes 19 and 23 while the lone snubber acts at node 7. Each of these elements was modeled with a linear spring with a stiffness consistent with their properties. No pipe supported equipment exists in the system. A full definition of all system parameters is provided in the input list.

The solution is developed using the modal superposition time history method of analysis. The loading function is an acceleration in the three coordinate directions imposed on node 1 , the pressurizer nozzle. A full digitized record of the forcing function is provided in the input list. In the analysis a cutoff frequency of $100 \mathrm{~Hz}$ was used resulting in a 27 mode modal approximation. A listing of the first ten natural frequencies is presented in Table 2. Computer generated 


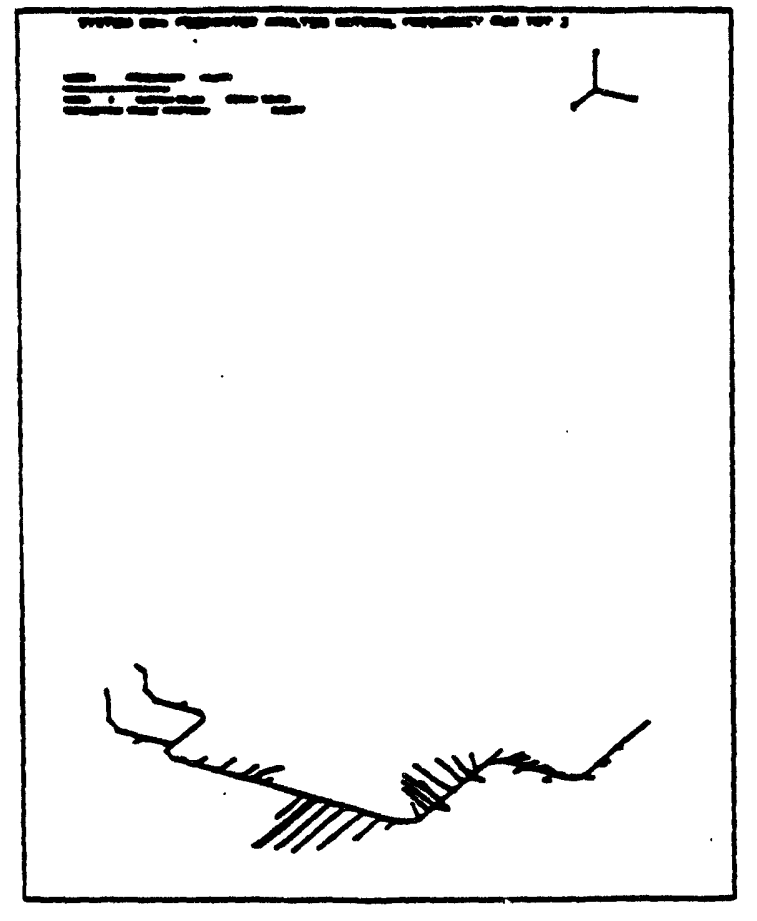

MODE 1

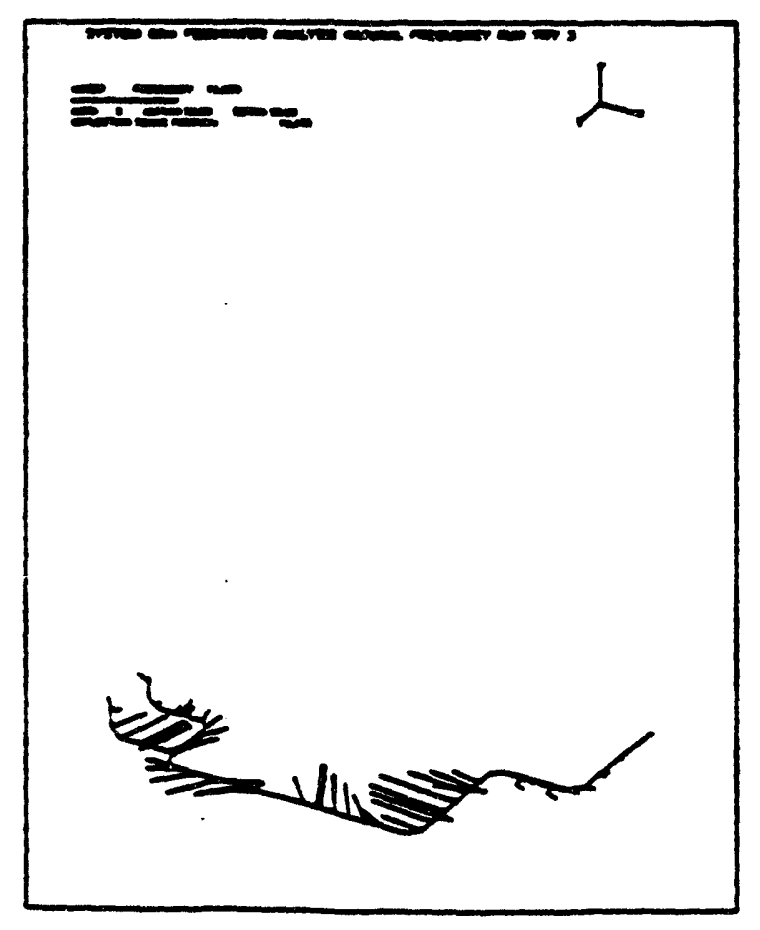

MODE 3

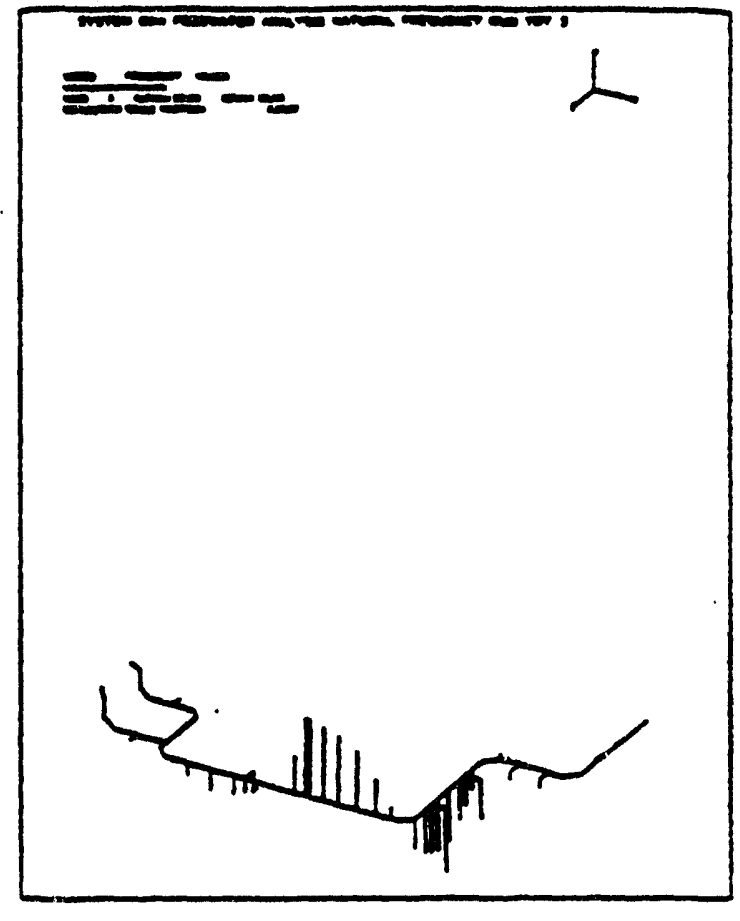

MODE 2

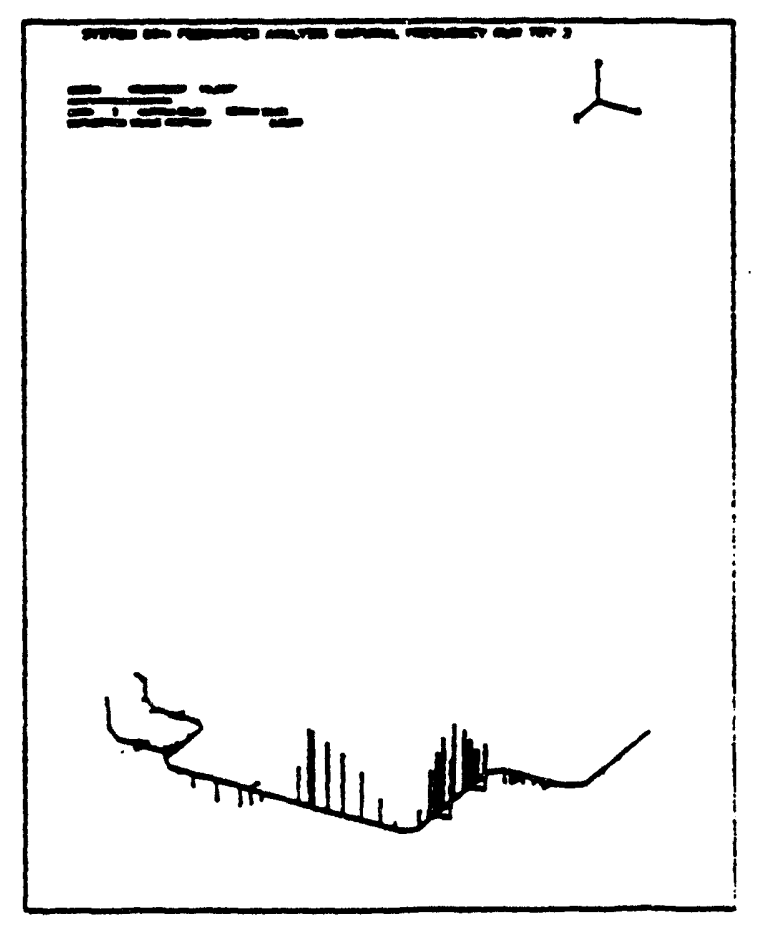

MODE 4

FIGURE 4 - FEEDWATER LINE MODE SHAPES

15 


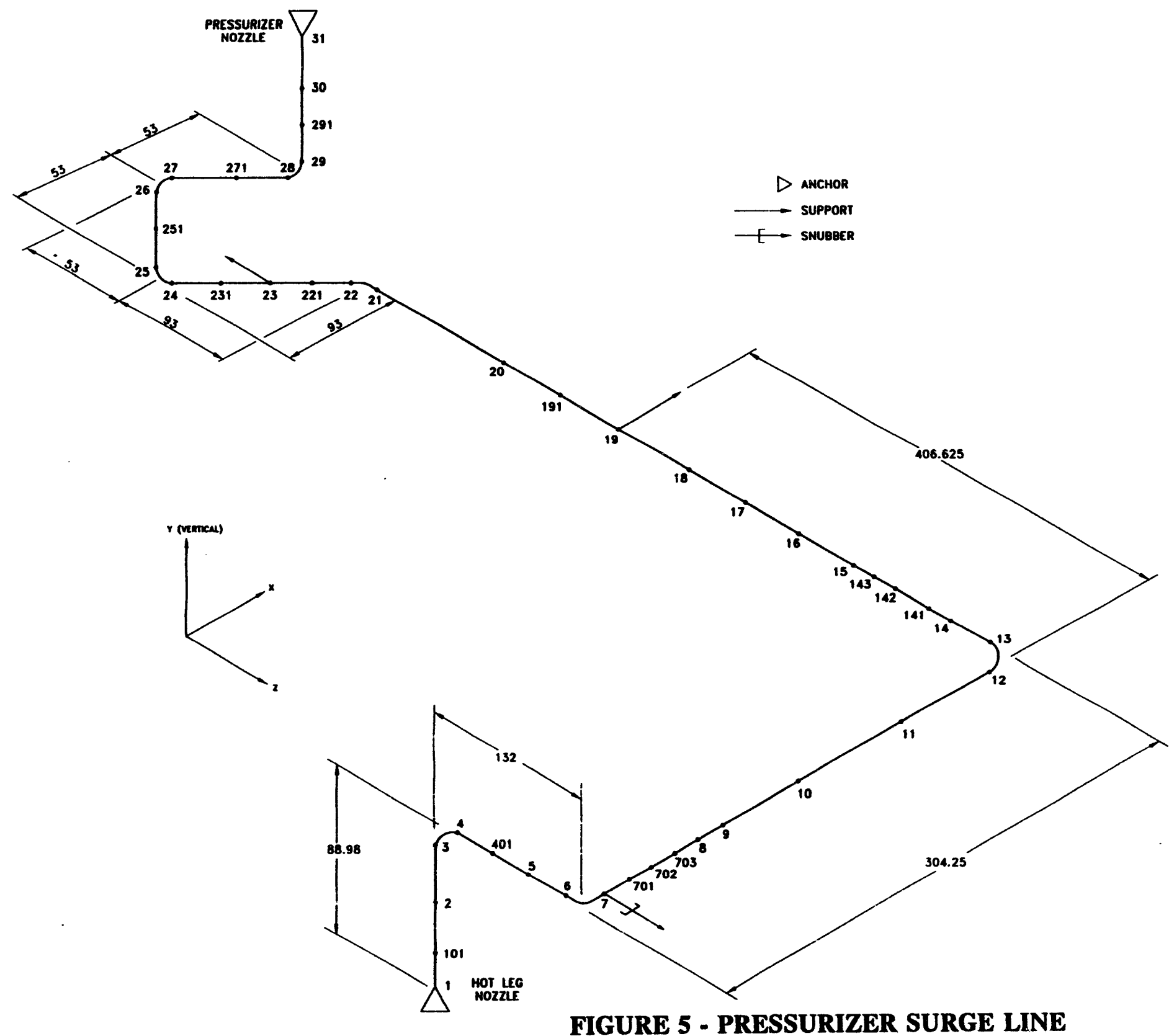




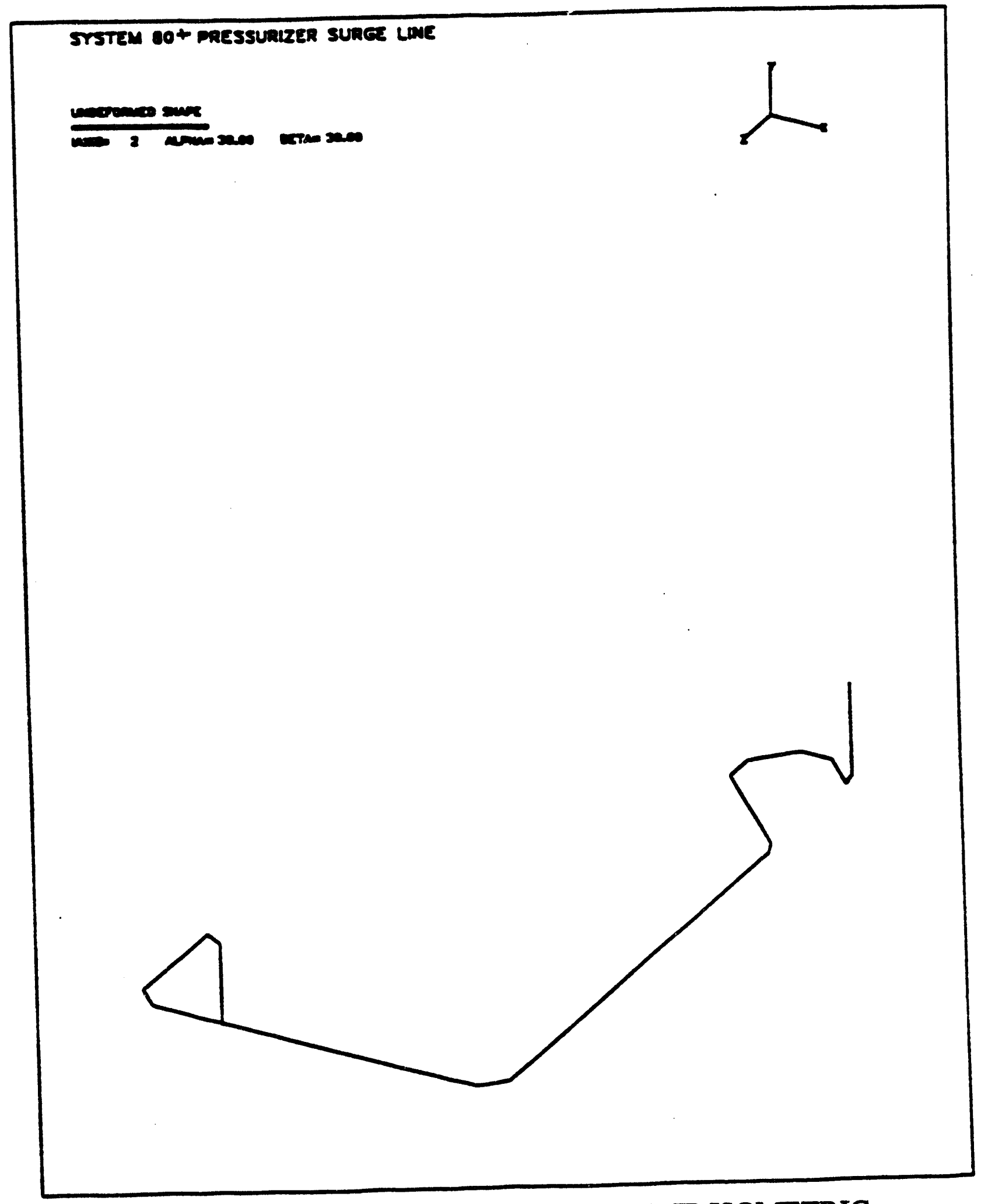

FIGURE 6 - PRESSURIZER SURGE LINE ISOMETRIC

17 


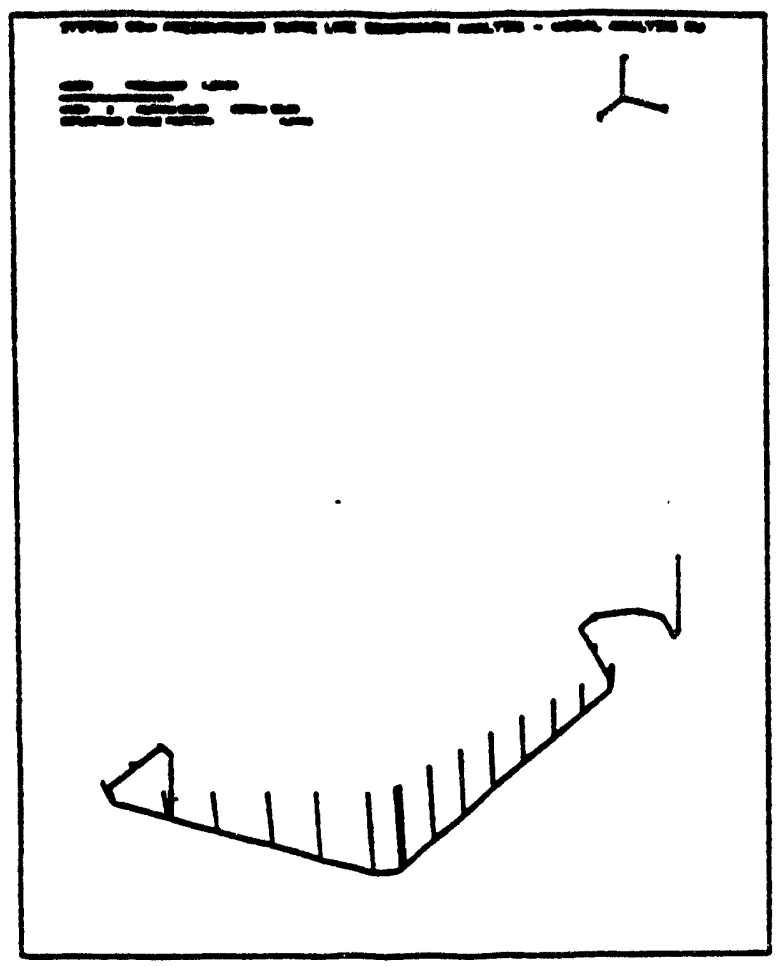

MOUE 1

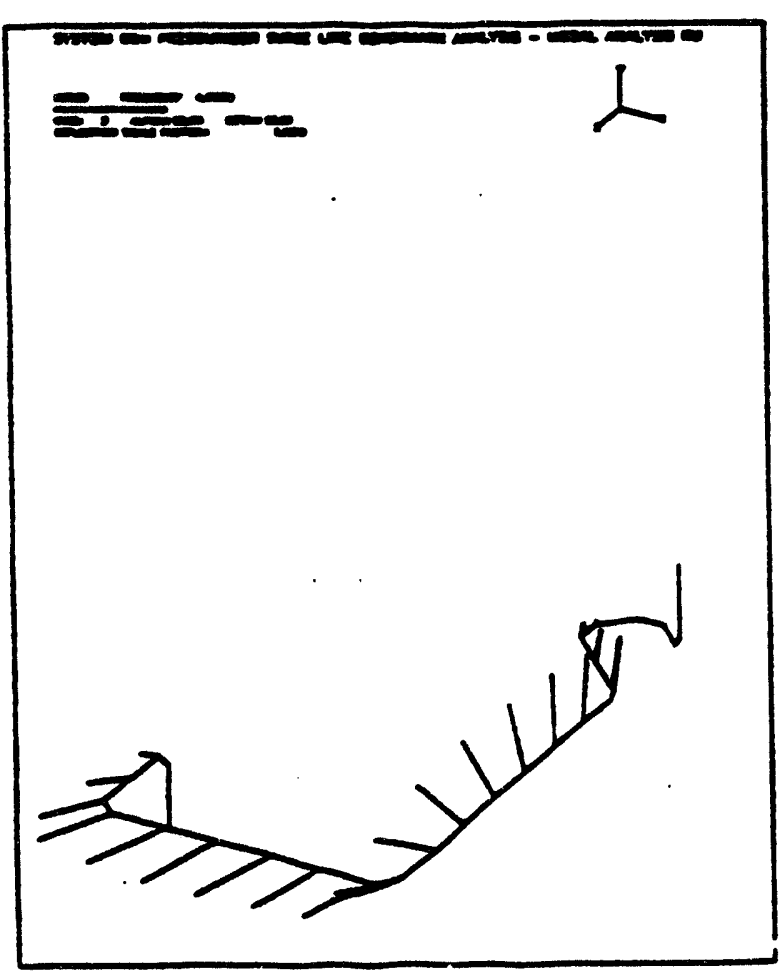

MODE 3

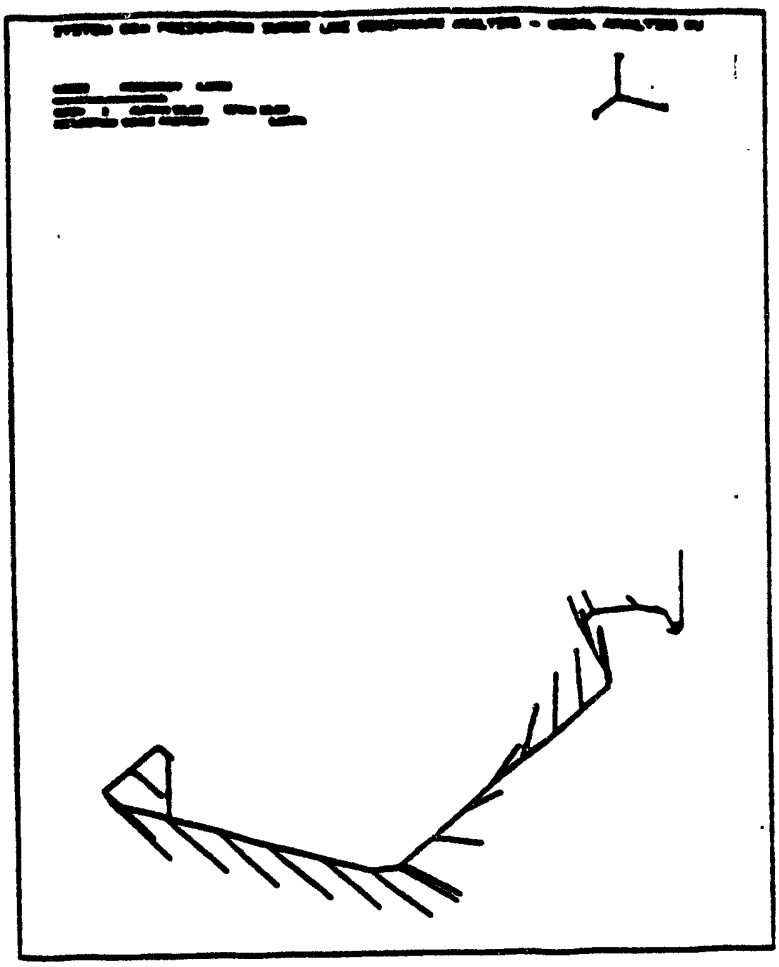

MODE 2

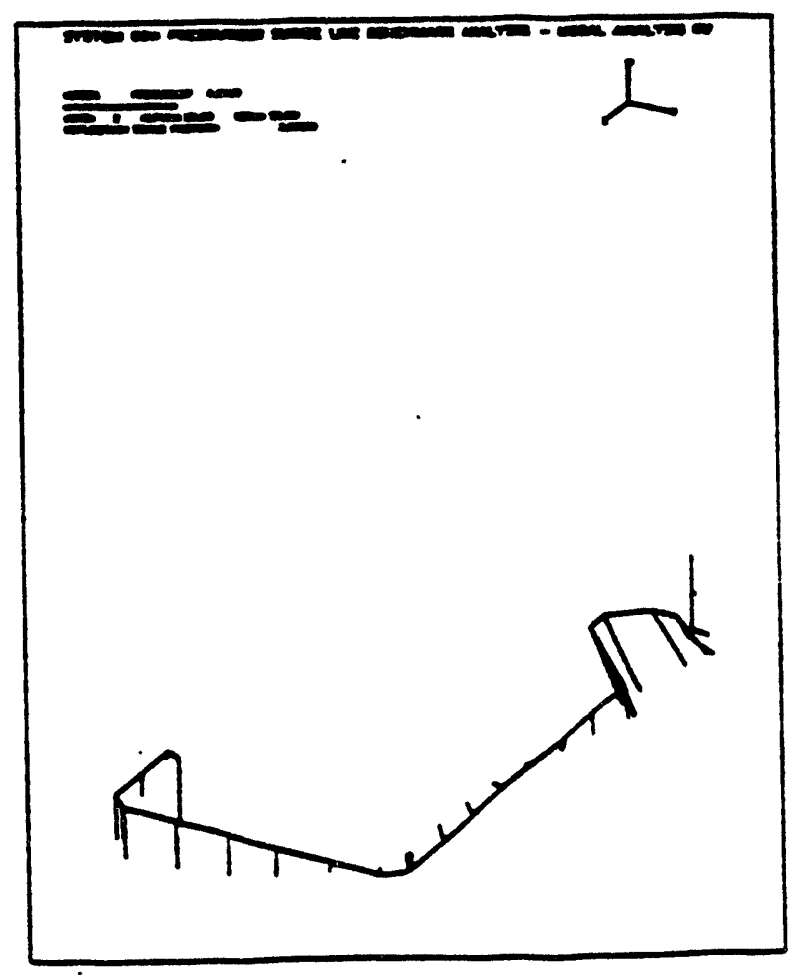

MODE 4

FIGURE 7 - PRESSURIZER SURGE LINE MODE SHAPES 
TABLE 1

FEEDWATER PIPING MODAL ANALYSIS RESULTS

\begin{tabular}{|c|c|c|}
\hline MODE NO. & $\begin{array}{c}\text { FREQUENCY } \\
\text { (CPS) } \\
\end{array}$ & $\begin{array}{l}\text { MODE DOMINANT } \\
\text { DIRECTION }\end{array}$ \\
\hline 1 & 9.158 & $Y$ \\
\hline 2 & 9.429 & $X, Z$ \\
\hline 3 & 10.870 & $X, Z$ \\
\hline 4 & 10.893 & $Y$ \\
\hline 5 & 12.815 & $\mathbf{Z}$ \\
\hline 6 & 14.139 & $X, Z$ \\
\hline 7 & 19.342 & $\mathrm{X}, \mathrm{Z}$ \\
\hline 8 & 19.781 & $\mathbf{Z}$ \\
\hline 9 & 19.814 & $\mathrm{Y}, \mathrm{Z}$ \\
\hline 10 & 20.380 & $X, Y, Z$ \\
\hline
\end{tabular}


TABLE 2

\section{PRESSURIZER SURGE LINE MODAL ANALYSIS RESULTS}

\begin{tabular}{|c|c|c|}
\hline MODE NO. & $\begin{array}{c}\text { FREQUENCY } \\
\text { (CPS) }\end{array}$ & $\begin{array}{c}\text { MODE } \\
\text { DOMINANT } \\
\text { DIRECTION }\end{array}$ \\
\hline 1 & 1.312 & $\mathrm{Y}$ \\
\hline 2 & 4.307 & $\mathrm{X}$ \\
\hline 3 & 4.757 & $\mathrm{X}$ \\
\hline 4 & 6.820 & $\mathrm{X}, \mathrm{Y}, \mathrm{Z}$ \\
\hline 5 & 8.550 & $\mathrm{X}, \mathrm{Y}$ \\
\hline 6 & 12.637 & $\mathrm{Z}$ \\
\hline 7 & 13.883 & $\mathrm{X}, \mathrm{Z}$ \\
\hline 8 & 14.667 & $\mathrm{X}, \mathrm{X}, \mathrm{Y}$ \\
\hline 10 & 15.837 & \\
\hline & & \\
\hline
\end{tabular}


isometrics of the first four modes are shown in Figure 7. A listing of modal participation factors corresponding to uniform support excitations, developed in a different analysis of the system, is provided in the computer list for model verification purposes. Damping was taken as a uniform 2\% of critical for all modes. The solution time step was $0.0001 \mathrm{sec}$ for a total duration of one second ( $*$ 10,000 time steps).

The output results again consist of the response maxima and their times of occurrence for each response component. In this case, however, the maxima were based on a sampling at every tenth time step. That is only the response at every tenth time step or 0.001 secs was compared to determine the maxima. If a finer sampling rate is used, the times of maxima occurrence and in fact the magnitudes of maxima will change. This sampling rate was selected to be consistent with the sampling rate used by $A B B / C E$ in their solution for this problem. For the benchmarking purpose the prediction of comparable results for the same sampling frequency will be adequate.

Further, regarding this benchmark it should be realized that the solution represents only the inertial component of response. For a complete evaluation the response due to the time varying displacements of the anchor/support points must be evaluated and added to the inertial response to obtain total response. 


\subsection{BENCHMARK ACCEPTANCE CRITERIA}

Earlier NRC benchmark reports provided comprehensive descriptions of the benchmark problem inputs and results but did not provide any definition of the acceptance criteria used to judge adequacy. Instead, the results of a benchmarking effort were submitted to the staff for evaluation on a case-by-case basis. This procedure was followed for most of the "work horse" computer codes then used by industry to qualify piping, including PISYS, ADLPIPE, WECAN, PISTAR, NUPIPE, SUPERPIPE, and PIPSYS.

For the System $80+$ standard design benchniarks it was considered to be more appropriate to specify the acceptance criteria to be met. This will allow the COL licensee to assess the adequacy of his methods as the benchmark solutions are developed; precluding the striving for "perfect" results and flagging early on, analysis methods that are deficient. When succescful, the COL licensee will simply have to retain an auditable record of the benchmarking effort to verify the adequacy of the methods to be used to qualify piping.

For the acceptance criteria, maximum allowed differences, expressed as a percent, rather than specific values, are specified and a summary of the criteria is presented in Table 3. As can be seen, the criteria is different for each response parameter, ranging from $2 \%$ for every natural frequency, to $10 \%$ for maximum displacements. Comparisons are required to be made for all natural frequencies and support reaction forces, but for oniy the maximum displacements and pipe moments in the designated pipe segments given in Table 4. The percentage differences are given by 100 (PE-BE)/BE, where PE is the predicted value, and $B E$ is the benchmark value, for a specific response.

Comparisons are required to be made for every natural frequency and every component of support reaction. Regarding natural frequencies, these are fundamental characteristics of a system, and if they are not predicted accurately there is little possibility that correct estimates of system response can be developed. Consequently, the requirement to compare all frequencies to a tight tolerance was specified. Support reactions on the other hand are not fundamental characteristics of a system, but unlike pipe moments and forces, every component of reaction is used explicitly in the design process. That is, supports and anchors are typically designed individually for the specific loads they will carry with only a small level of conservatism. Consequently, it is important to provide reasonably accurate estimates of all reactions and the benchmarking requirement was set accordingly.

For pipe displacements and moments, only comparisons of maximum values are required. This is again consistent with the design/qualification process where only the maximum values impact on design. These comparisons are required to be made in each unique section of pipe for each problem. This was specified to assure that reasonably accurate estimates of all system responses was being made rather than good estimates for only a local region. The accuracy tolerances specified denote the relative importance assigned these parameters.

Again, reflecting design practice, some relaxation of criteria is allowed for pipe moments. Specifically, for these, if the estimates of the maximum components of moment do not meet criteria, comparisons can be made to the resultant moment at each location of maxima, and if these meet criteria, the benchmarking is acceptable. This reflects design practice in that it is only the resultant moments that enter into the qualification evaluations.

Finally, it is recognized that the benchmarking effort may not be clean cut in every application. Candidate analysis methodologies may not meet criteria for every parameter for all comparisons. In these instances, the COL licensee must interact with the st providing additional justifications for acceptance. These applications will be assessed on a case-by-case basis. 
TABLE 3

\section{ACCEPTANCE CRITERIA}

\begin{tabular}{|c|c|c|}
\hline PARAMETER & CRITERIA & COMAENAY 8 \\
\hline $\begin{array}{l}\text { Natural } \\
\text { Frequencies }\end{array}$ & $2 \%$ & $\begin{array}{l}\text { All natural frequencies up to } \\
\text { cutoff frequency. }\end{array}$ \\
\hline $\begin{array}{l}\text { Maximum } \\
\text { Displacements }\end{array}$ & $10 \%$ & $\begin{array}{l}\text { Comparison to be made for } \\
\text { maximum nodal translation* in } \\
\text { each global coordinate } \\
\text { direction for each segment* } * \text { of } \\
\text { piping. }\end{array}$ \\
\hline $\begin{array}{l}\text { Maximum Pipe } \\
\text { Moments }\end{array}$ & $5 \%$ & $\begin{array}{l}\text { Comparison to be made for } \\
\text { maximum element moment* about } \\
\text { each local coordinate axis for } \\
\text { each pipe segment**. Failure } \\
\text { to meet criteria is acceptable } \\
\text { if sRss of three components at } \\
\text { each point of maxima meet the } \\
\text { criteria. }\end{array}$ \\
\hline $\begin{array}{l}\text { Support } \\
\text { Reaction }\end{array}$ & $10 \%$ & $\begin{array}{l}\text { Comparison to be made for each } \\
\text { component of force and moment } \\
\text { for each support and anchor. }\end{array}$ \\
\hline
\end{tabular}

* Maxima for each direction not necessarily at same point. ** Pipe segments defined in Table 4. 
TABLE 4

PIPING SEGMENTS

\begin{tabular}{|c|c|}
\hline \multicolumn{2}{|c|}{ A. FEEDWATER LINE } \\
\hline SEGMENT & NODE NUMBERS \\
\hline 1 & $1-10$ \\
\hline 2 & $10-26$ \\
\hline 3 & $26-34$ \\
\hline 4 & $34-44$ \\
\hline 5 & $44-55$ \\
\hline 6 & $55-66$ \\
\hline 7 & $66-71$ \\
\hline B. PRESSURIZER SURGE LINE \\
\hline SEGMENT & NODE NUMBERS \\
\hline 1 & $1-7$ \\
\hline 2 & $7-19$ \\
\hline 3 & $19-23$ \\
\hline 4 & $23-31$ \\
\hline
\end{tabular}




\subsection{CONCLUSION}

Three benchmark problems have been developed for the purpose of assessing the adequacy of the analysis techniques that will be used by $\mathrm{COL}$ licensees to qualify piping for the System $80+$ standardized plant. If a COL licensee can demonstrate that a candidate analysis methodology provides solutions to the benchmark problems that meet all acceptance criteria, the COL licensee may use that methodology for piping design without further approval. In that instance the COL licensee must submit documentation to the staff of the benchmarking effort in sufficient detail to support the conclusion.

In the event that the benchmarking is essentially, but not wholly successful, the COL licensee may petition for staff approval on a case-bycase basis. In these instances, the documentation of the benchmarking must be augmented with additional justifications of adequacy and the use of the methodology must await staff approval. 
1. 10CFR, Office of Federal Register National Archives and Research Administration, "Code of Federal Regulations," Title 10, Part 52, (54 FR 15386), May 1.989.

2. Bathe, J.K., Wilson, E.L., Peterson, F.E., "SAP IV-A Structural Analysis Program for Static and Dynamic Responses of Linear Systems," Report No. EERC 73-11, University of California, Berkeley, CA, 1973.

3. Subudhi, M., Bezler, P., "EPIPE, Piping Analysis Program User's Manual -Version 1981," BNL NUREG/30784, December 1981.

4. Bezler, P., Hartzman, M., Reich, M., "Piping Benchmark Problems," NUREG/CR-1677, August 1980.

5. Bezler, P., Subudhi, M., Hartzman, M., "Piping Benchmark Problems," NUREG/CR-1677, Vol. II, August 1985.

6. Regulatory Guide 1.92 , "Combining Modal Responses and Spatial Components in Seismic Response Analysis," Revision 1, February 1976.

7. U.S. Nuclear Regulatory Commission, "Standard Review Plan for the Review of Safety Analysis Reports for Nuclear Power Plants," Section 3.7.2, 1989.

8. Bezler, P., Gardner, D., Hartzman, M., "A Dynamic Benchmark Solution for a Hypothetical Reactor System," BNL-NUREG23645, December 1977. 
APPENDIX A 


\section{UNITS OF MEASURE}

The attached computer input and output data is presented in the following units of measure:

Length

Angular

rotation

Force

Moment

Time

Pressure

Acceleration

Frequency

Temperature
- inches (in)

- $\quad$ radians (rad)

- $\quad$ pounds (lb)

- in-lbs.

- $\quad$ seconds (sec)

- $\quad$ lbs./in ${ }^{2}(p s i)$

- $\quad$ g's (in/sec $/$ gravitational constant)

- $\quad$ Hertz (Hz) or cycles per second, cps

- degrees Fahrenheit ( $\left.{ }^{\circ} \mathrm{F}\right)$ 
BENCHMARK PROBLEM 1

UNIFORM SUPPORT MOTION

RESPONSE SPECTRUM ANALYSIS 
SYSTEM 80+ FEEDWATER PIPING, BENCHLARK PROBLEM 1, UNIFORM SUPPORT MOTION

CONTROL INEORMATION

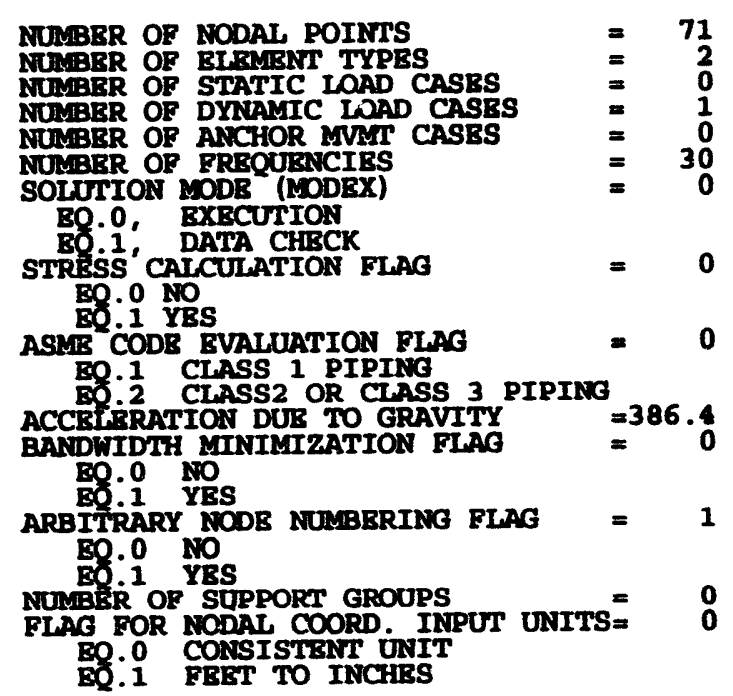

NUMBER OF NODAL POINTS

NUMBRR OF RIKLIRNT TYPES

NULBRR OF STATIC LOAD CAES

NUMBRR OP ANCHOR MVIT CASES

NUMBSR OP ANCHOR MVMT CASBS

MOTI

EO.0, EXECUTION

STRESS' CAICULATION FLAG

EO. 0 NO

ASUB CODR BVALUATION FLAG

EO.1 CLASS 1 PIPING

B. 2 CIASS2 OR CIASS 3 PIPING

ACCEIRRATION DUR TO GRAVITY $=386$.

BO.0 NO

PBITRARY NODE NUMBERING FLAG

BO.0 No

EO.1 YES

TOR NODAL COORD. INPUT UNITS=

EO.0 CONSISTEIN UNIT

LIST OF ANALYSIS TO BE PERFORUIED

UNIFORM RESPONSE SPECTRUM ANAL. 


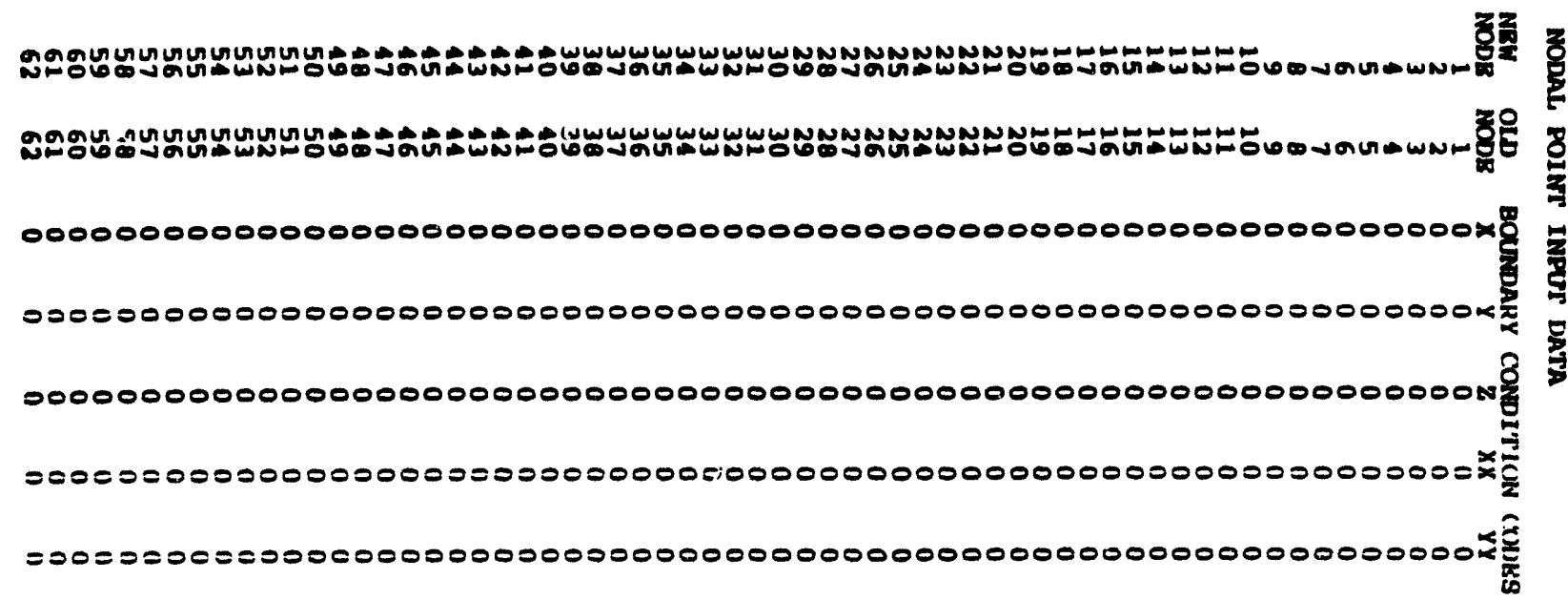

00000000000000000000000000000000000000000000000000000000000000 药

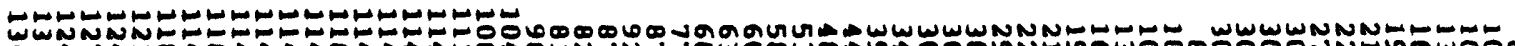

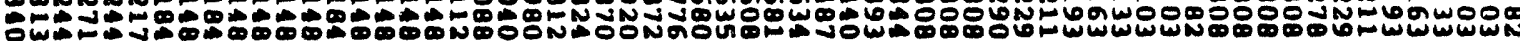

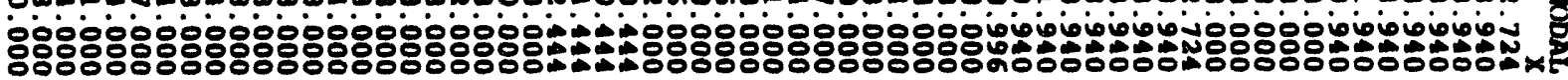

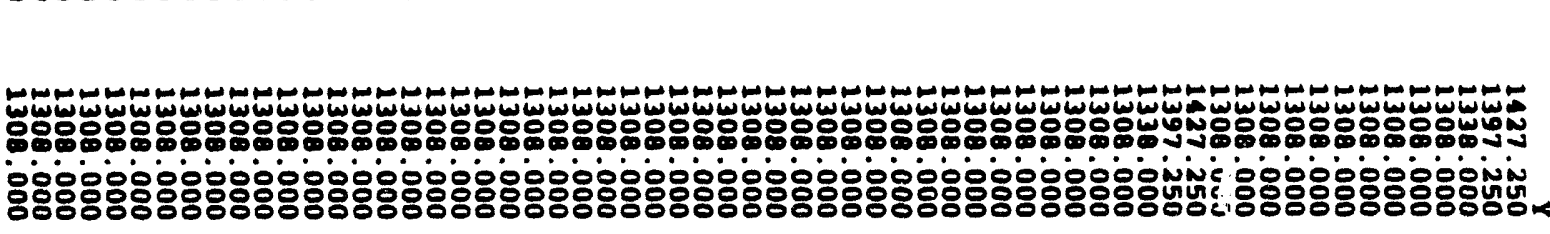

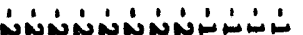

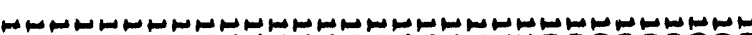

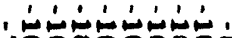

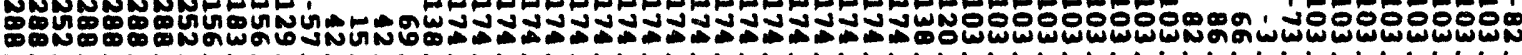

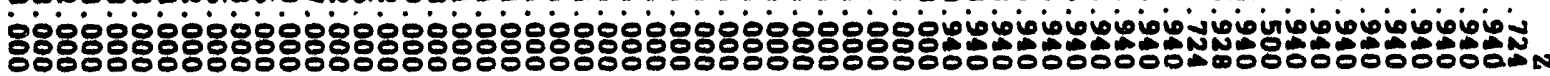

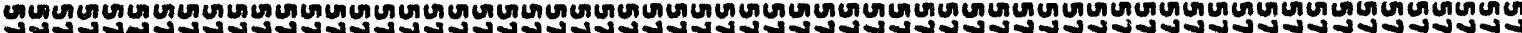

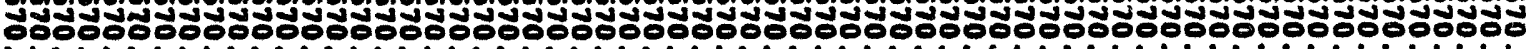

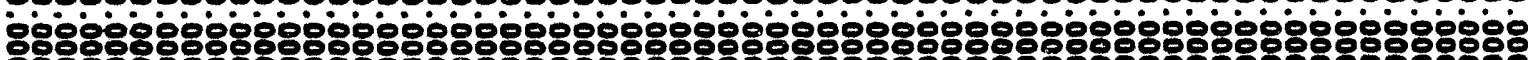

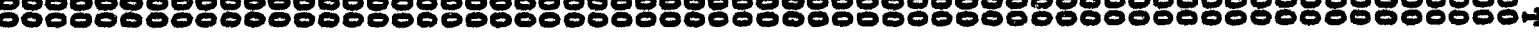




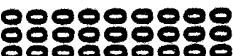 \\ ¿00000000 \\ ñnvinininininis}

\section{ㅇํㅇํㅇㅇㅇㅛ \\ ini-inio \\ ๑ง⿵⺆⿻二丨冂 \\ พสสม}

요요요요용

808888088

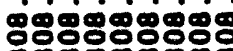

p户pmpmpmpm

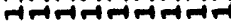

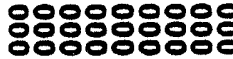

ธำสสำกี่

miñ

000000000

000000000

000000000

000000000

000000000

000000000

ํํำแัธ็ํำ

ํํํํํํํำำ 


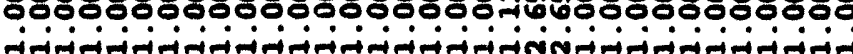

影

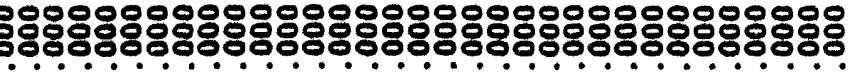

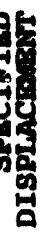

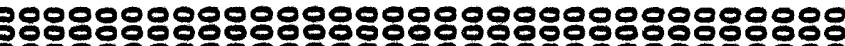

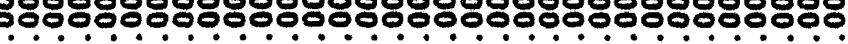

퉁요

.

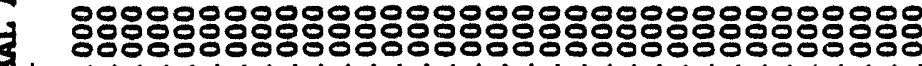

SN

0000000000000000000000000000000

๖ำㅇำ

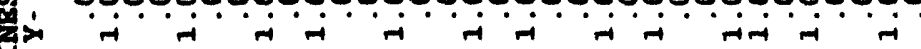

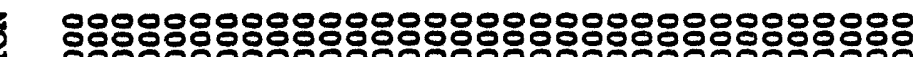

ర

通

营

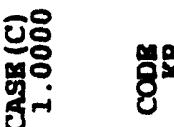

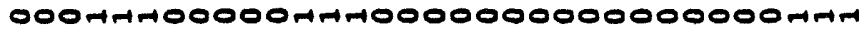

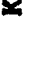

영료

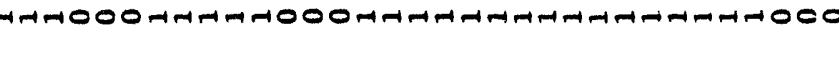

o

" "

商

홍응

At

घ

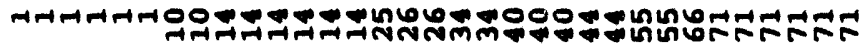

క용

-กM 

P I P $\mathbf{B}$
B L B M N N
I N U T
D A T A

CONTROL INFORMATION
NUMBER OR PIPE BLEMRNIS
$=70$
MLEBRR OP MATERIAL SETS
$=1$
MAXIMY MUABR OF MATBRTA
TEIPRRATURE INPUT POINIS
$=2$
MUABR OP SBCTION PROYEKIYY SETS $=11$
MEARR OP BRANCH POINT NOUES a 2
YAXIMTA NTMBRR OP TANGRN'S
CONON TO A BRANCH POINT
FLAG FOR NEGLECTING AXIAL,
DEPORLATIONS IN BEND BI.BMENTS = 0
(BQ.1, NROLECT) 
T A B L B S

\begin{tabular}{|c|c|c|c|c|}
\hline 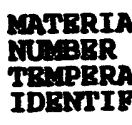 & $\begin{array}{l}\text { MURBRR } \\
\text { PRE POINTS }= \\
\text { CATION }\end{array}$ & $\begin{array}{l}\text { 1) } \\
\text { 2) SA106 B }\end{array}$ & & \\
\hline $\begin{array}{l}\text { POIFT } \\
\text { NUEBRR }\end{array}$ & TRMPPRRATURB & $\begin{array}{l}\text { Young's } \\
\text { MODULUS }\end{array}$ & $\begin{array}{l}\text { POISSON'S } \\
\text { RATIOO }\end{array}$ & $\begin{array}{l}\text { THRRMAL } \\
\text { EXPANSION }\end{array}$ \\
\hline$\frac{1}{2}$ & $\begin{array}{r}70.00 \\
651.00\end{array}$ & $\begin{array}{l}2.950 \mathrm{~B}+07 \\
2.610 \mathrm{E}+07\end{array}$ & $\begin{array}{r}.300 \\
.300\end{array}$ & $\begin{array}{l}5.600 \mathrm{~B}-06 \\
8.700 \mathrm{~B}-06\end{array}$ \\
\hline
\end{tabular}




\begin{tabular}{|c|c|c|c|c|c|c|}
\hline $\begin{array}{l}\text { 3BCTION } \\
\text { NUMBER }\end{array}$ & $\begin{array}{l}\text { OUTSIDB } \\
\text { DIALIBTER }\end{array}$ & $\begin{array}{r}\text { WAI,I, } \\
\text { THICKNESS }\end{array}$ & $\begin{array}{l}\text { SIIAPE FACTOR } \\
\text { FOR SHRAR }\end{array}$ & $\begin{array}{l}\text { WEIGHT/ } \\
\text { UNIT LBMOTH }\end{array}$ & UNI' LBNGTH & D B S C R I P T I O N \\
\hline $\begin{array}{r}1 \\
2 \\
3 \\
4 \\
5 \\
6 \\
7 \\
8 \\
9 \\
10 \\
11\end{array}$ & $\begin{array}{l}20.000 \\
24.000 \\
24.000 \\
28.000 \\
33.000 \\
22.062 \\
26.436 \\
27.624 \\
27.600 \\
22.000 \\
26.000\end{array}$ & $\begin{array}{r}1.0310 \\
1.2180 \\
1.8120 \\
2.0000 \\
1.5000 \\
2.0620 \\
2.4360 \\
3.6240 \\
10.0000 \\
1.1240 \\
1.9060\end{array}$ & $\begin{array}{l}.0000 \\
.0000 \\
.0000 \\
.0000 \\
.0000 \\
.0000 \\
.0000 \\
.0000 \\
.0000 \\
.0000 \\
.0000\end{array}$ & $\begin{array}{l}2.8977 \mathrm{~B}+01 \\
4.0750 \mathrm{~B}+01 \\
5.0428 \mathrm{~B}+01 \\
3.169 \mathrm{~B}+01 \\
1.2053 \mathrm{~B}+01 \\
8.333 \mathrm{~B}+01 \\
7.4070 \mathrm{~B}+01 \\
7.4070 \mathrm{~B}+01 \\
0.0000 \mathrm{~B}+00 \\
4.0751 \mathrm{~B}+01 \\
3.7367 \mathrm{~B}+00\end{array}$ & $\begin{array}{l}7.4994 \mathrm{~B}-02 \\
1.0546 \mathrm{~B}-01 \\
1.3051 \mathrm{~B}-01 \\
8.2024 \mathrm{~B}-02 \\
1.0883 \mathrm{~B}-01 \\
2.1567 \mathrm{~B}-01 \\
1.9169 \mathrm{E}-01 \\
1.9169 \mathrm{~B}-01 \\
0.0000 \mathrm{~B}+00 \\
1.0546 \mathrm{~B}-01 \\
9.6705 \mathrm{~B}-03\end{array}$ & $\begin{array}{l}20880 \\
24980 \\
248120 \\
289 p 8 C \\
301 D \\
20980 \text { VLVR } \\
24980 \text { VIVB } \\
249120 \text { VLVB } \\
\text { VOOP } \\
\text { BRRD 24:20 } \\
\text { BRED 28*24 }\end{array}$ \\
\hline
\end{tabular}

BRANCH POINT NOERTL I S T

$\begin{array}{rr}\text { BRANCH } & \begin{array}{r}\text { NODE } \\ \text { POINT }\end{array} \\ \text { NUMBRR }\end{array}$

B L E M B N T LOA D

C A S E M ULT I P L I E R S

X-DIRRCTION GRAVITY
Y-DIRRCTION GRAVITY

CASE

$\begin{array}{rrrr}\text { SE A } & \text { CASE B } & \text { CASEC } & \text { CASE D } \\ .000 & .000 & .000 & .000 \\ .000 & .000 & .000 & .000 \\ .000 & .000 & .000 & .000 \\ .000 & .000 & .000 & .000 \\ .000 & .000 & .000 & .000\end{array}$

$\begin{array}{lllll}\text { Z-DIRRCTION GRAVITY } & .000 & .000 & .000 & .000 \\ \text { THEREAL DISTORTION } & .000 & .000 & .000 & .000 \\ \text { PRBSGURR DISTORTION } & .000 & .000 & .000 & .000\end{array}$


窟

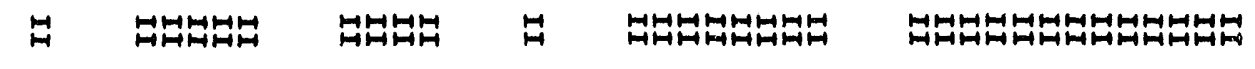

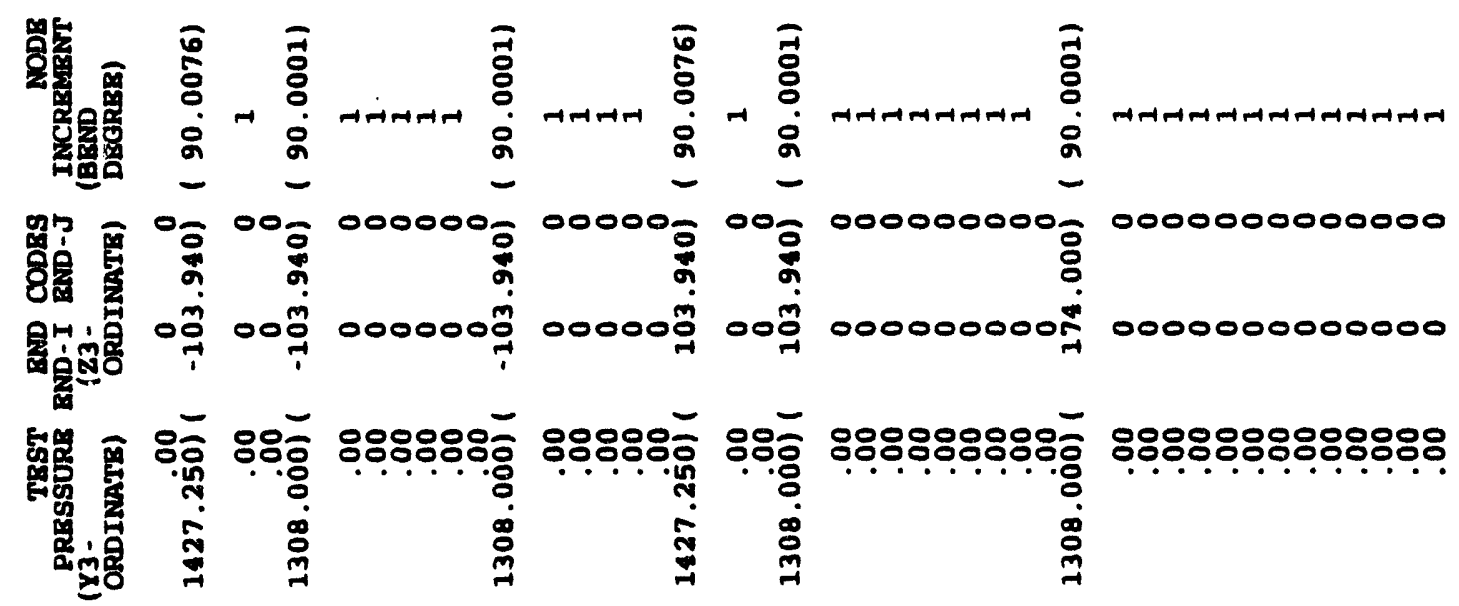

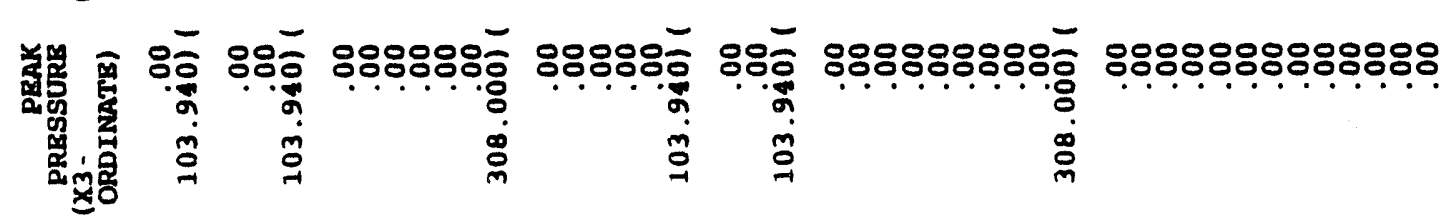

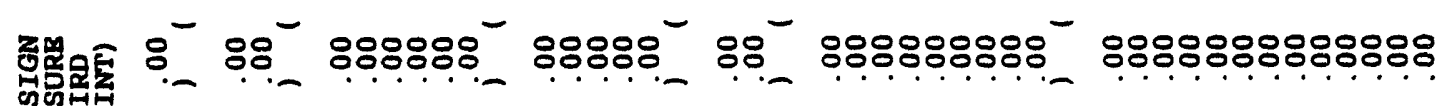

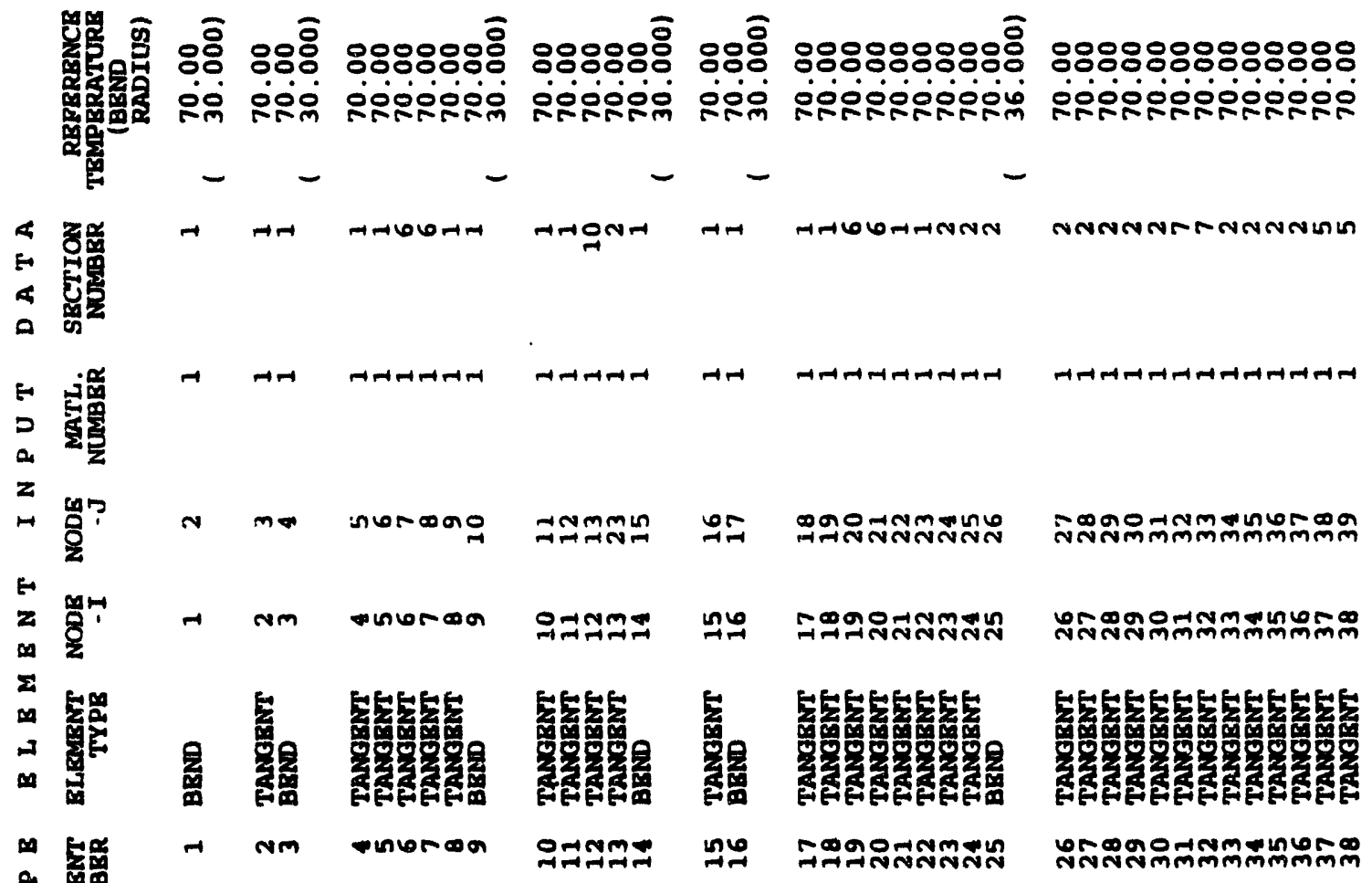




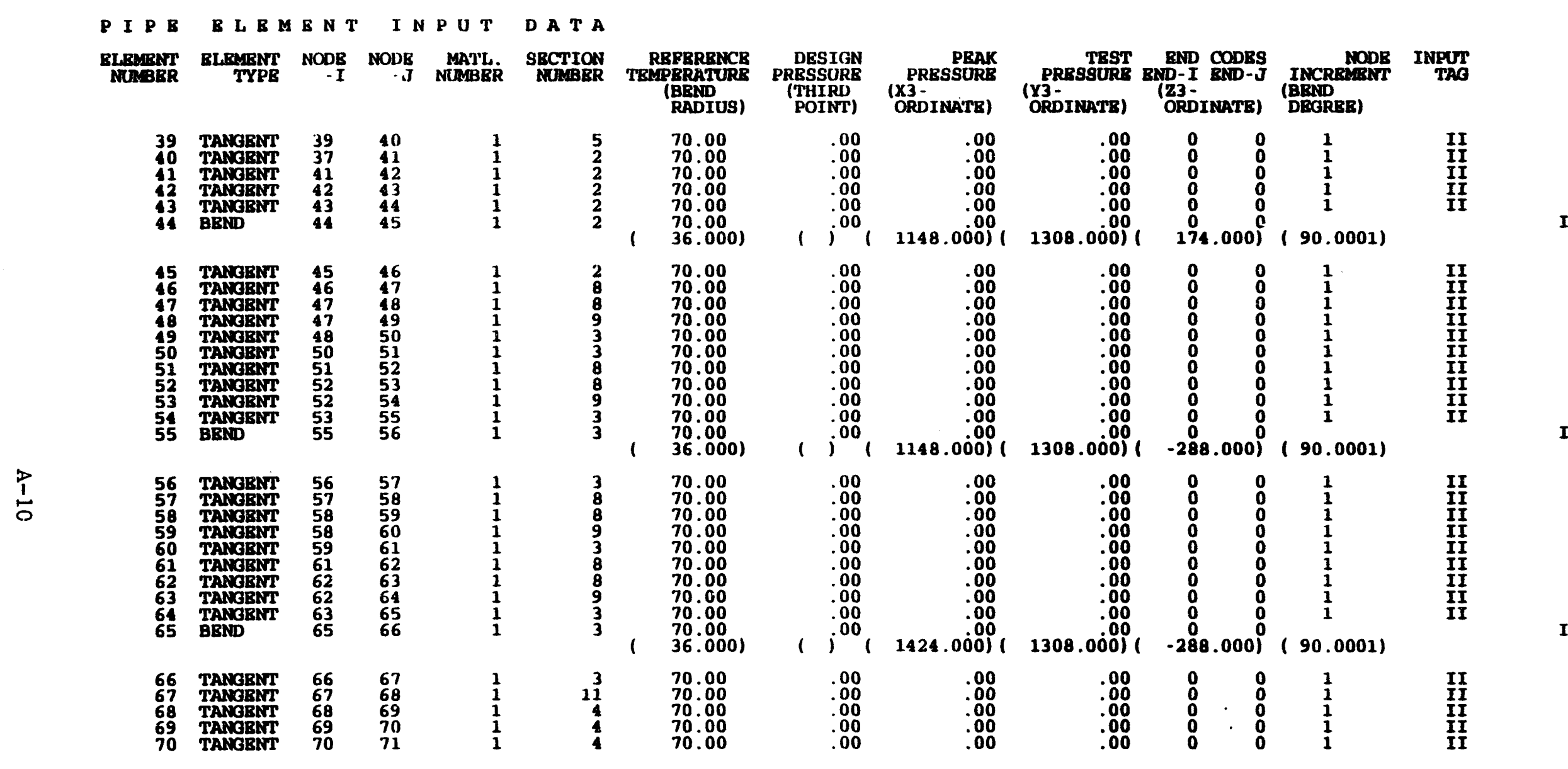


B ANCH POINT DATA

BRAHCH MODR

23 -22AT J

$\begin{array}{ll}-13 A T & J \\ -36 A T & -22 A T \\ J\end{array}$

23AT I

$\underset{1}{2}$ 

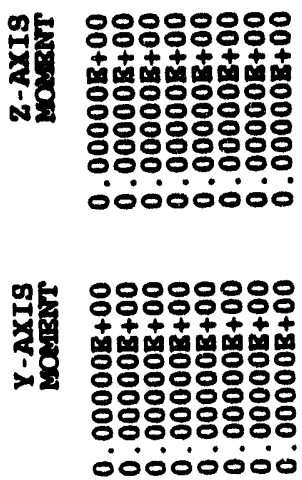

3

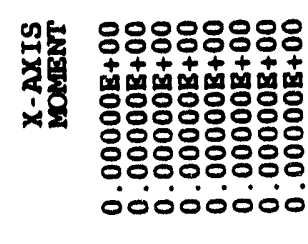

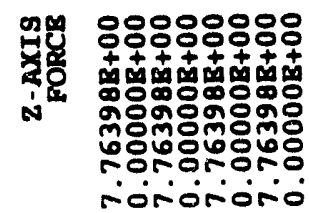

2

$\propto$

무붕 웅웅요웅

연

붕ํํㅇํㅇㅇํ용

m

E

4

on

씹 응응응용응

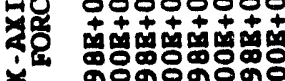

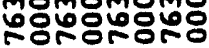

ronoriro

0

0

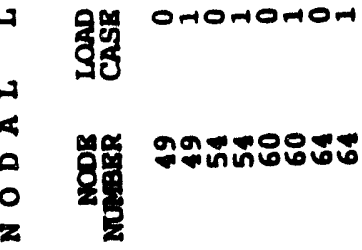




\section{BENCHMARK PROBLEM 1}

\section{LOWER FREQUENCY AMPLIFIED RESPONSE}


PRINT OP RREOUENCIES

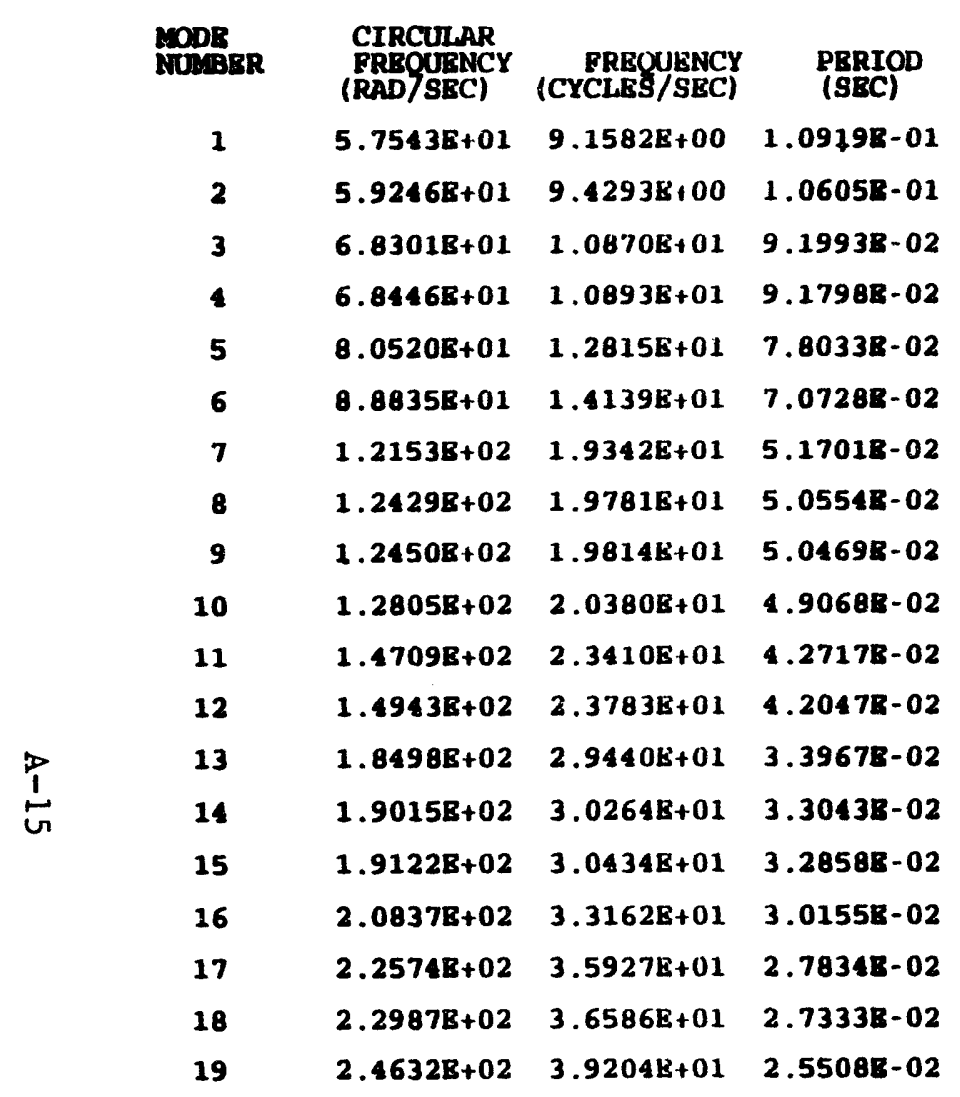


MOAAL PARTICIPATIOA BACTORS IODE PRRO (CPS) X-DIRBCTION

$1 \quad 9.158 \quad-2.9006 \mathrm{~B}-02$

$2 \quad 9.429 \quad 3.0460 \mathrm{z}+00$

$3 \quad 10.870 \quad-7.6548 R+00$

$4 \quad 20.893-1.00968-01$

$5 \quad 12.815 \quad 7.80328-01$

$6 \quad 14.139-1.59298+00$

$7 \quad 29.342 \quad 5.11998+00$

- $19.781 \quad 1.0254 B+00$

$9 \quad 19.014 \quad-6.8280 \mathrm{~B}-01$

$10 \quad 20.380 \quad 1.0290 \mathrm{~B}+00$

$11 \quad 23.410 \quad 5.0000 \mathrm{~B} \cdot 02$

$12 \quad 23.783 \quad 4.80828+00$

$\frac{5}{1}$

29.440

$6.18478+00$

$5.2935 \mathrm{~B}+00$

30.26

1.21838-01

4. $10678-02$

33.162

35.927

6.9973B-02

36.586

$-1.78818+00$

39.204

Y-DIRICTIOA

$2.61025+00$

$-1.0507 \mathrm{~B}-02$

$-6.68708-02$

B. $92278+00$

6. 2645E-01

1. $.89235 \cdot 02$

-1 .01695-01

$-1.72795+00$

$-5.3100 \mathrm{~B}+00$

$-1.51718+00$

$-8.2597 \mathrm{~B}+00$

2.2761B-01

-1. 18508-02

$-3.7780 \mathrm{~B}-02$

2. 18835+00

$6.16903+00$

$5.6306 \mathrm{~B}+00$

$-1.10308-02$

$-5.9229 \mathrm{~B}-01$
Z-DIRRCTIOA

-2 .6883B-02

$-1.00578+00$

$-4.0077 \mathrm{R}+00$

$7.1187 \mathrm{E}-02$

$-3.84628+00$

$-9.1802 \mathrm{E}-01$

$-2.60218+00$

$-9.9896 \mathrm{x}+00$

$3.72928+00$

$-1.99818+00$

-.65548-01

$6.33448+00$

$-2.34268-01$

$-3.4609 \mathrm{~B}+00$

$-1.10338-01$

5.75748-01

$-1.01018-03$

$-6.15198-01$

$-2.00358-03$ 
D Y A I C ANALYSI:S

\section{STRucture}

1 USM RBgPONgR SPECTRA ANALYSIS

\begin{tabular}{|c|c|c|}
\hline $\begin{array}{l}\text { BLEMBNT } \\
\text { A }\end{array}$ & $\mathrm{B}^{\text {LAAD }}$ & MUTIPLIR \\
\hline & & .000 \\
\hline
\end{tabular}

Case...?

DIRnction pactors

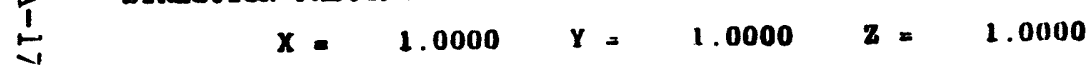

INDICATOR POR DIgPLACBMENT OH ACCLLERATION SPBCIRUA * 2

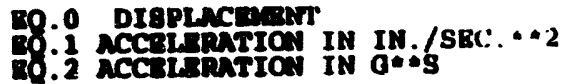

3 gPECTRA ARE ENTERED POR CASB 1. KIND- 2

CLustitr factor, CF $=.10000$

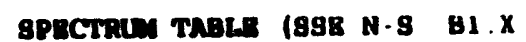

SCAn pheror $=14.000008100$

\begin{tabular}{|c|c|c|}
\hline 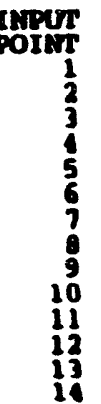 & 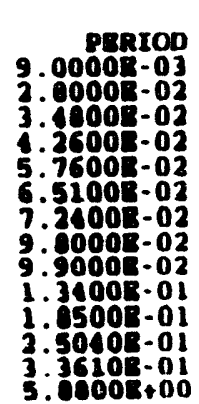 & 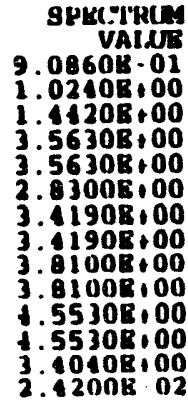 \\
\hline
\end{tabular}


SPBCTROA TABLB ISSE Y BI.Y

MERPR OP POIMTS $=10$
SCAIS BACTOR

\begin{tabular}{|c|c|c|}
\hline $\begin{array}{r}\text { Iupur } \\
\text { Porkin } \\
1 \\
2 \\
3 \\
4 \\
5 \\
6 \\
7 \\
8 \\
9 \\
10\end{array}$ & $\begin{array}{r}\text { PRRIOD } \\
9.0000 \mathrm{~B}-03 \\
2.7600 \mathrm{~B}-02 \\
3.6300 \mathrm{~B}-02 \\
4.6700 \mathrm{~B}-02 \\
6.0500 \mathrm{~B}-02 \\
7.8000 \mathrm{~B}-02 \\
9.9000 \mathrm{~B}-02 \\
1.3400 \mathrm{~B}-01 \\
3.0200 \mathrm{~B}-01 \\
1.1760 \mathrm{~B}+01\end{array}$ & $\begin{array}{r}\text { SPECTRUY } \\
\text { VALUR } \\
9.4240 \mathrm{~B}-01 \\
1.4610 \mathrm{~B}+00 \\
3.0510 \mathrm{~B}+00 \\
1.2772 \mathrm{E}+01 \\
1.2772 \mathrm{~B}+01 \\
3.9410 \mathrm{~B}+00 \\
1.1400 \mathrm{~B}+00 \\
1.1400 \mathrm{~B}+00 \\
1.6490 \mathrm{~B}+00 \\
3.1000 \mathrm{~B}-03\end{array}$ \\
\hline
\end{tabular}

8PRCTRUA TABLB (SSB z B1.z

MCAIBR OP POINTS $=12$
SACTOR

\begin{tabular}{|c|c|c|c|}
\hline & & & \\
\hline & & & 4 \\
\hline & 1 & $9.0000 \mathrm{~B}-03$ & $8.7670 \mathrm{~B}-01$ \\
\hline & $\frac{2}{3}$ & $1000 \mathrm{~B}-02$ & $9424 B+00$ \\
\hline$\infty$ & 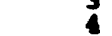 & $3500 \mathrm{~B}-02$ & $0300 \mathrm{~B}+00$ \\
\hline & 5 & $300 \mathrm{~B}-02$ & $100 \mathrm{~B}+00$ \\
\hline & 6 & $1700 \mathrm{~s}-02$ & $270 \mathrm{~B}+00$ \\
\hline & : & $900 \mathrm{~B}-02$ & $3 \mathrm{~B}+00$ \\
\hline & & $3005-02$ & $0 \bar{B}+0$ \\
\hline & $\begin{array}{l}10 \\
11\end{array}$ & $\begin{array}{l}2210 \mathrm{~B}-01 \\
1800 \mathrm{-0}-01\end{array}$ & $0 B+00$ \\
\hline & & $3 \cdot+4=0$ & \\
\hline
\end{tabular}

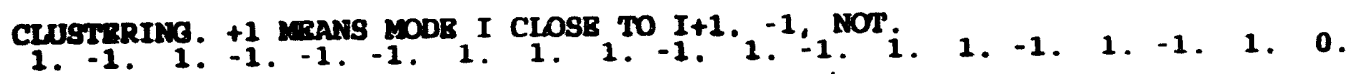




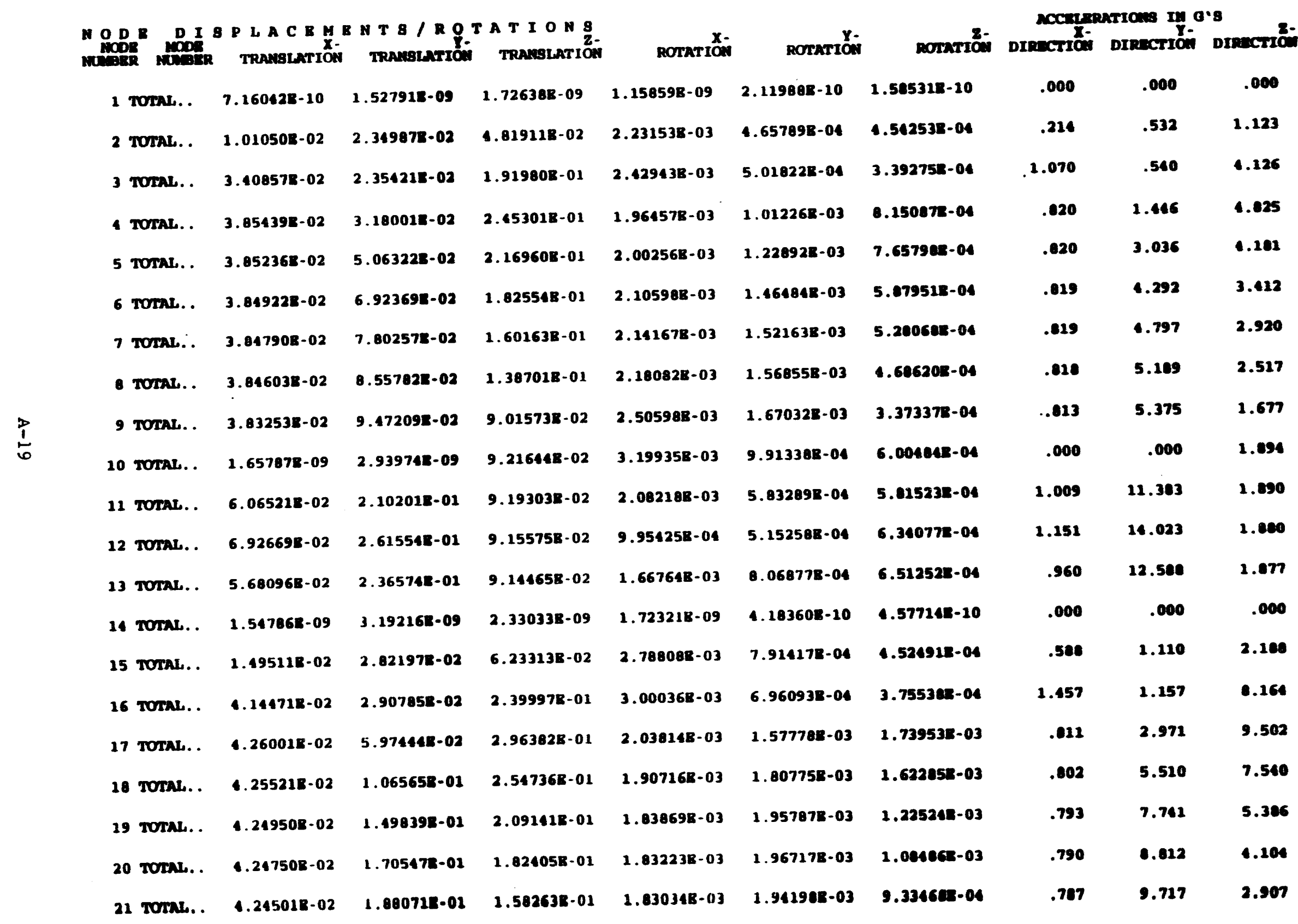




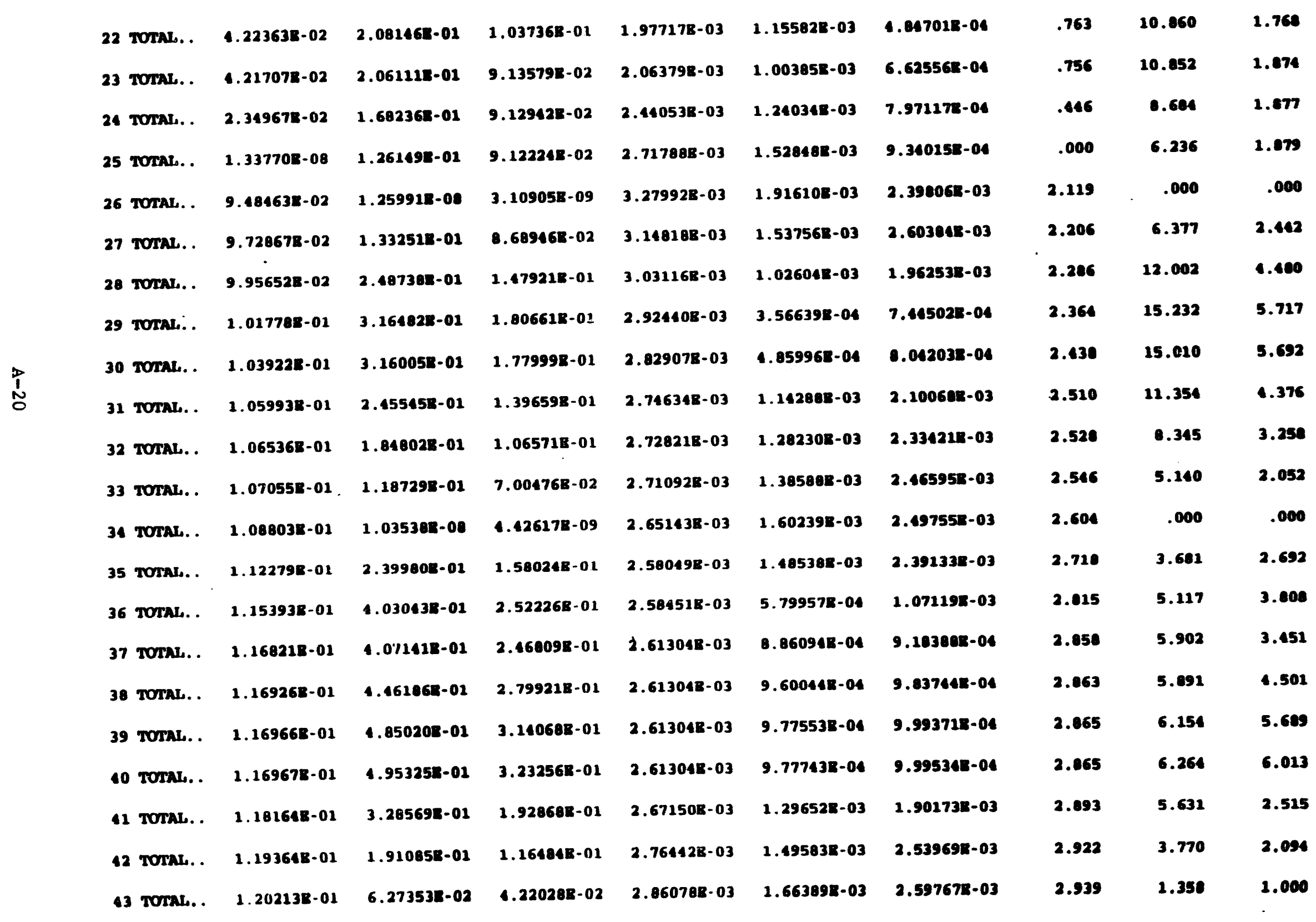




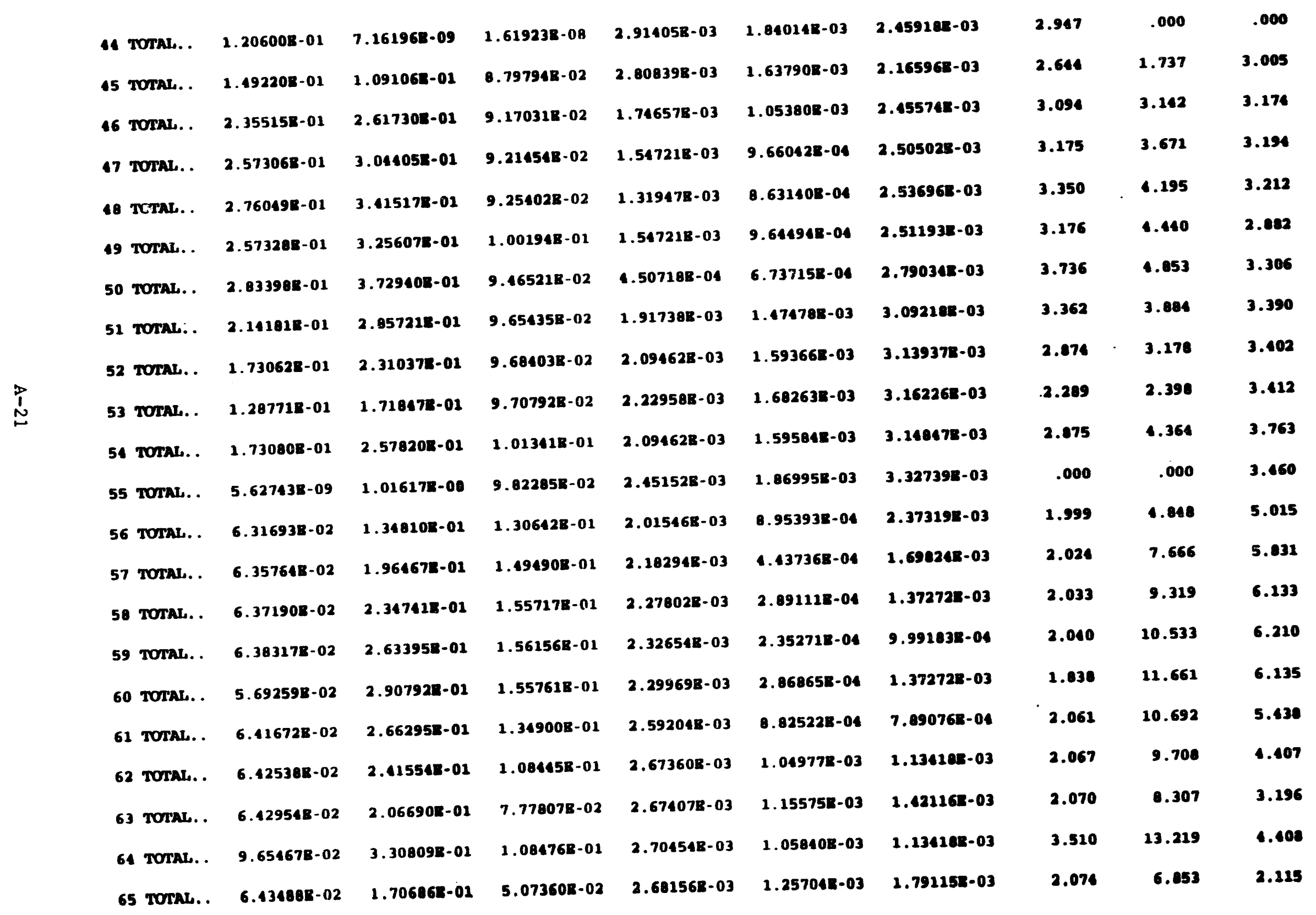




\begin{tabular}{|c|c|c|c|c|c|c|c|c|c|}
\hline 66 TOTAL. . & $4.24091 B-02$ & $1.159808-08$ & $5.04635 B-03$ & $1.77738 \mathrm{~B}-03$ & $6.92097 \mathrm{~B}-04$ & $1.99844 \mathrm{~B}-03$ & 1.370 & .000 & .214 \\
\hline 67 TOTAL. . & $4.54928 B-02$ & 8.933308-02 & $3.687898-03$ & $3.87211 \mathrm{~B}-04$ & $2.194198-04$ & $1.32851 \mathrm{~B}-03$ & 2.470 & 3.849 & .156 \\
\hline 68 TOTAL. . & $4.498928-02$ & $9.492608-02$ & $3.34603 \mathrm{~B}-03$ & $1.225268-04$ & $1.53272 \mathrm{~B}-04$ & $1.18638 \mathrm{~B}-03$ & 2.555 & 4.147 & .142 \\
\hline 69 TOTAL. . & 3.339358-02 & 7.71621B-02 & $2.23503 \mathrm{~B}-03$ & $4.20258 B-04$ & $2.03322 B-04$ & $7.90917 \mathrm{~B}-04$ & 2.065 & 3.529 & .095 \\
\hline 70 TOTAL. . & $1.28057 \mathrm{~B}-02$ & $2.982558-02$ & $1.11882 \mathrm{~B}-03$ & $5.028078-04$ & $2.163458-04$ & 3.954598-04 & .820 & 1.415 & .048 \\
\hline 11 TOMAL. & $1.29526 \mathrm{~B}-09$ & $2.981368-09$ & 5.519318-09 & 3.417798-09 & $1.46764 \mathrm{~B}-09$ & $1.275578-09$ & .000 & .000 & .000 \\
\hline
\end{tabular}




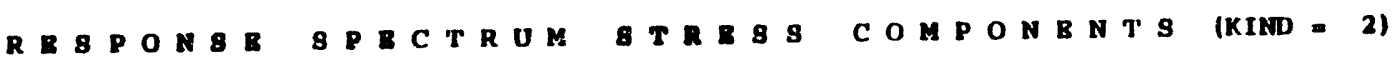

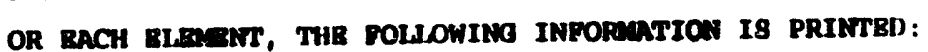

1. POR ENCH BARTH QUAKR DIRBCTIOA, THE MURBRR OP THE MODE WITH THB LARGEgT 9TREgs.

2. TH VAHE OP THT STPBS

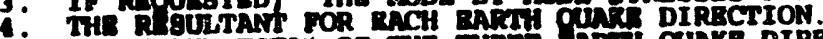

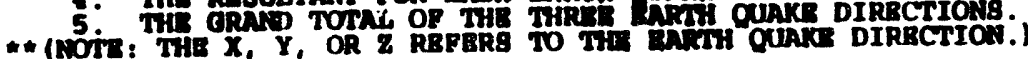

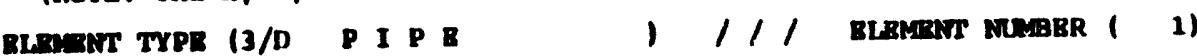

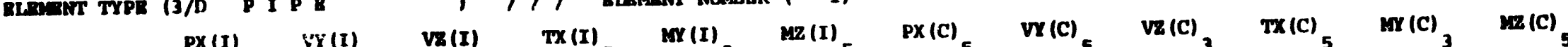

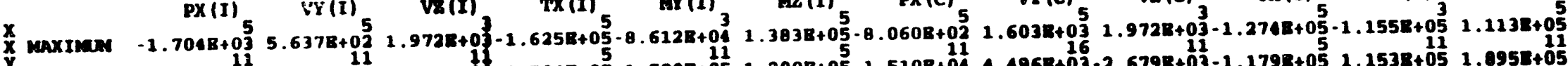

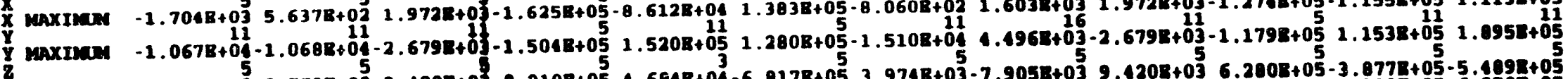
$y$
$z$

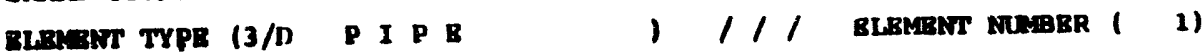

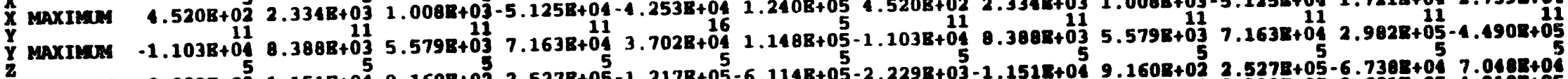

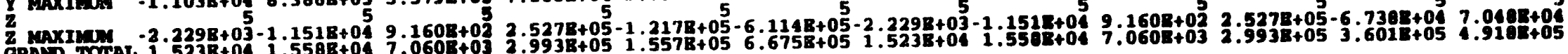
GRAID TOTAL 1.523B+04 $1.558 B+047.060$ +

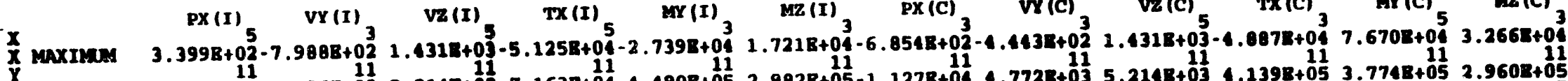

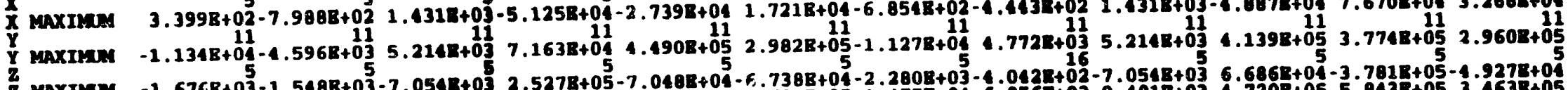

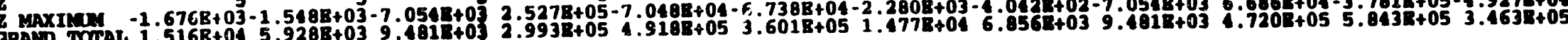

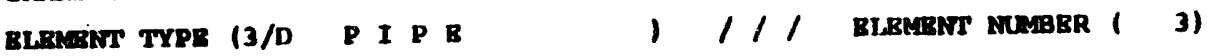

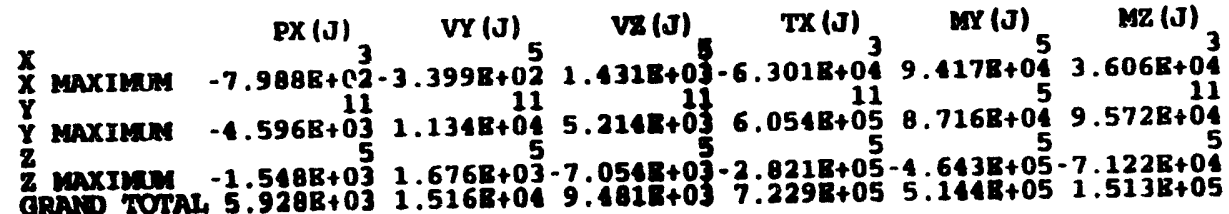




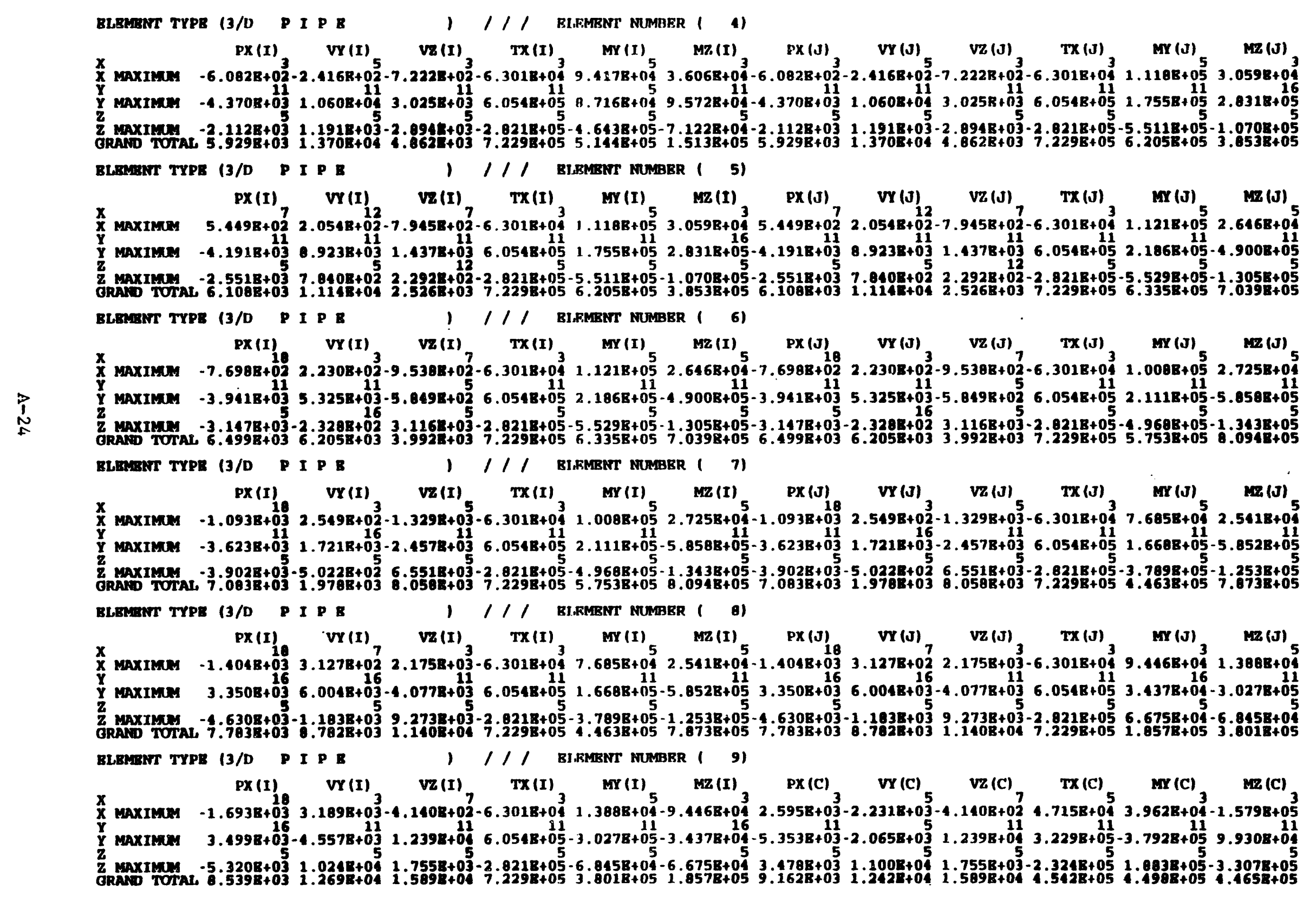




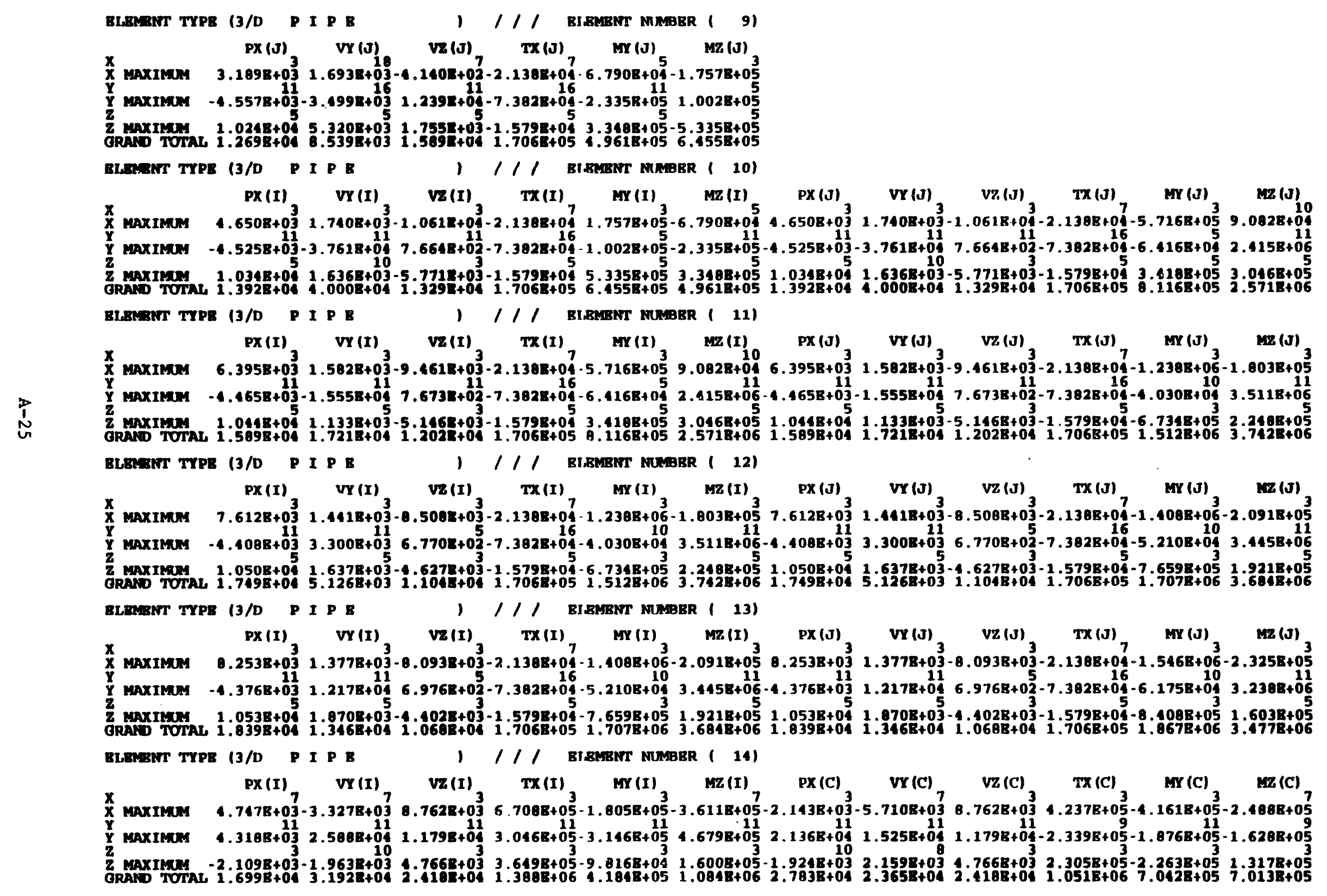




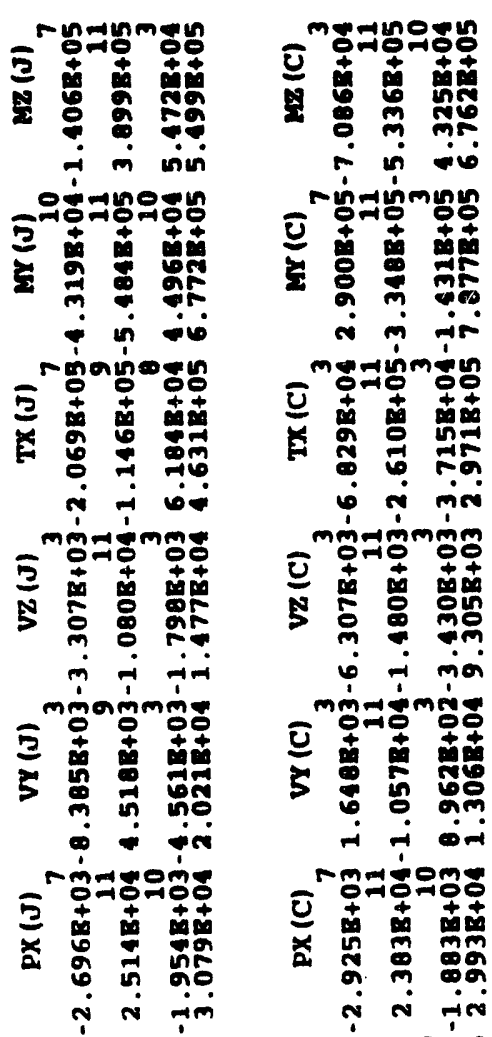

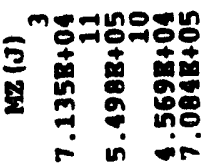

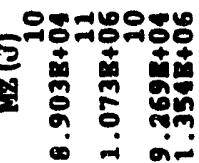

minonymingn

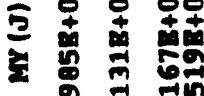

m i viv

minzunming

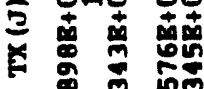

$\therefore$ - ir

mịañming

క

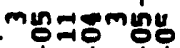

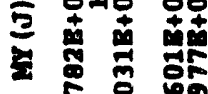

- $\therefore$ ivi

mex่

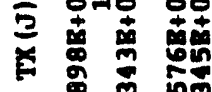

i : ir

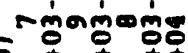

క

- i vir

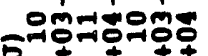

క

$5 \underset{m}{m}$ :

m an

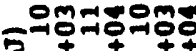

站 融

$\rightarrow$ in

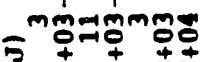

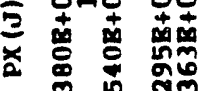

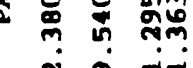

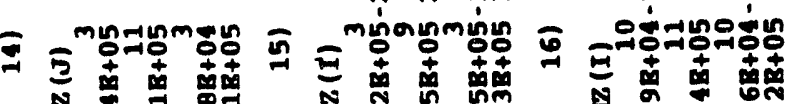

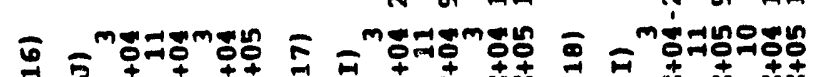

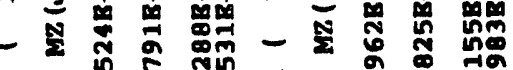

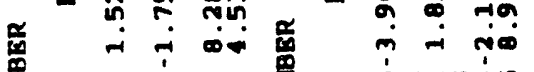

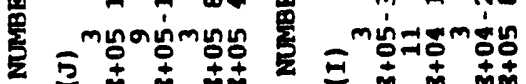

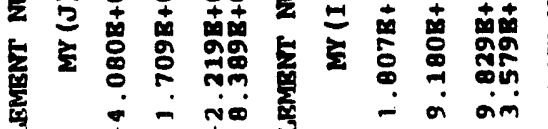

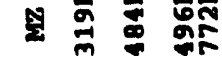

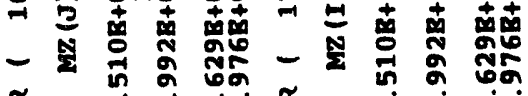

E-

₹

$\therefore \dot{m}$ inis

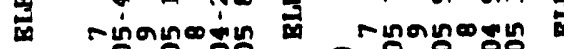

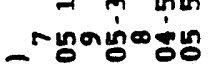

$\therefore-$ -

$\because-10$

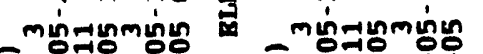

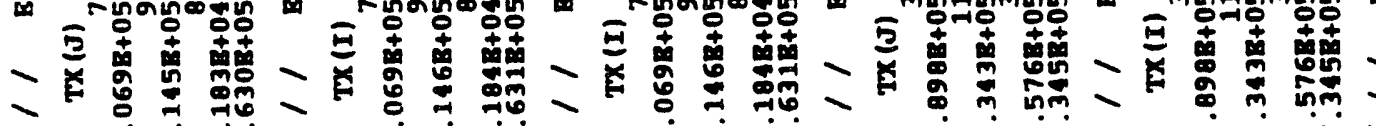

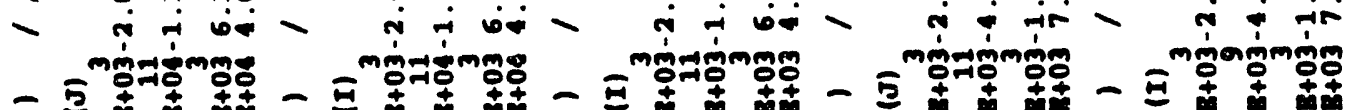

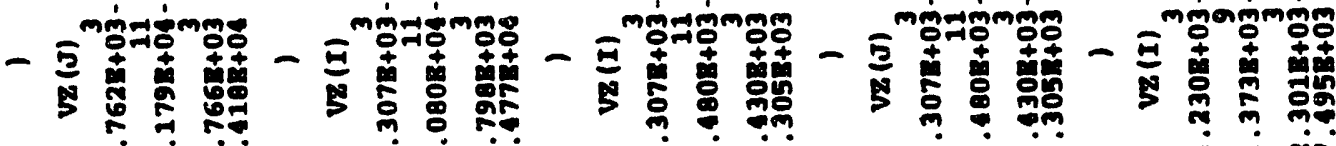

$\circ \dot{-1}$

- i rir

riñ-iom

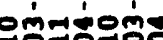

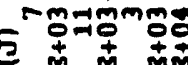

? ? ?

2. 立 离

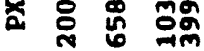
$\therefore$ a -1

논

- 楸

些

mingonming

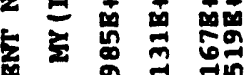

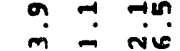
miñ 互 E की - 플 产

mminmming

Nongmm

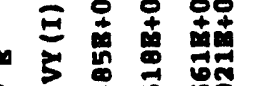

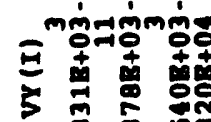

.

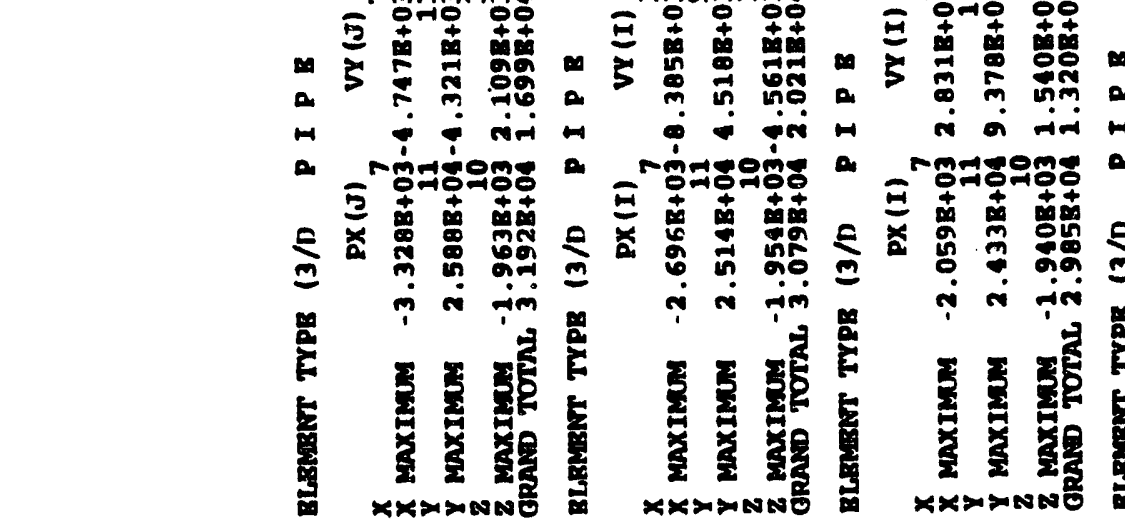

$\infty$

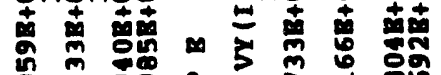
กै รู० i i

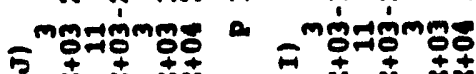

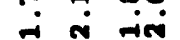
-i त

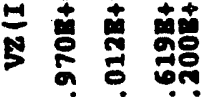
i - 둔

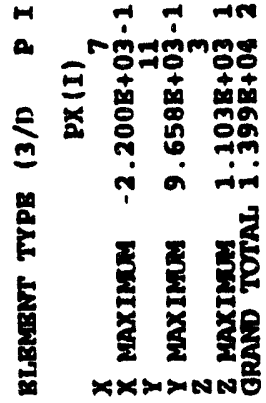




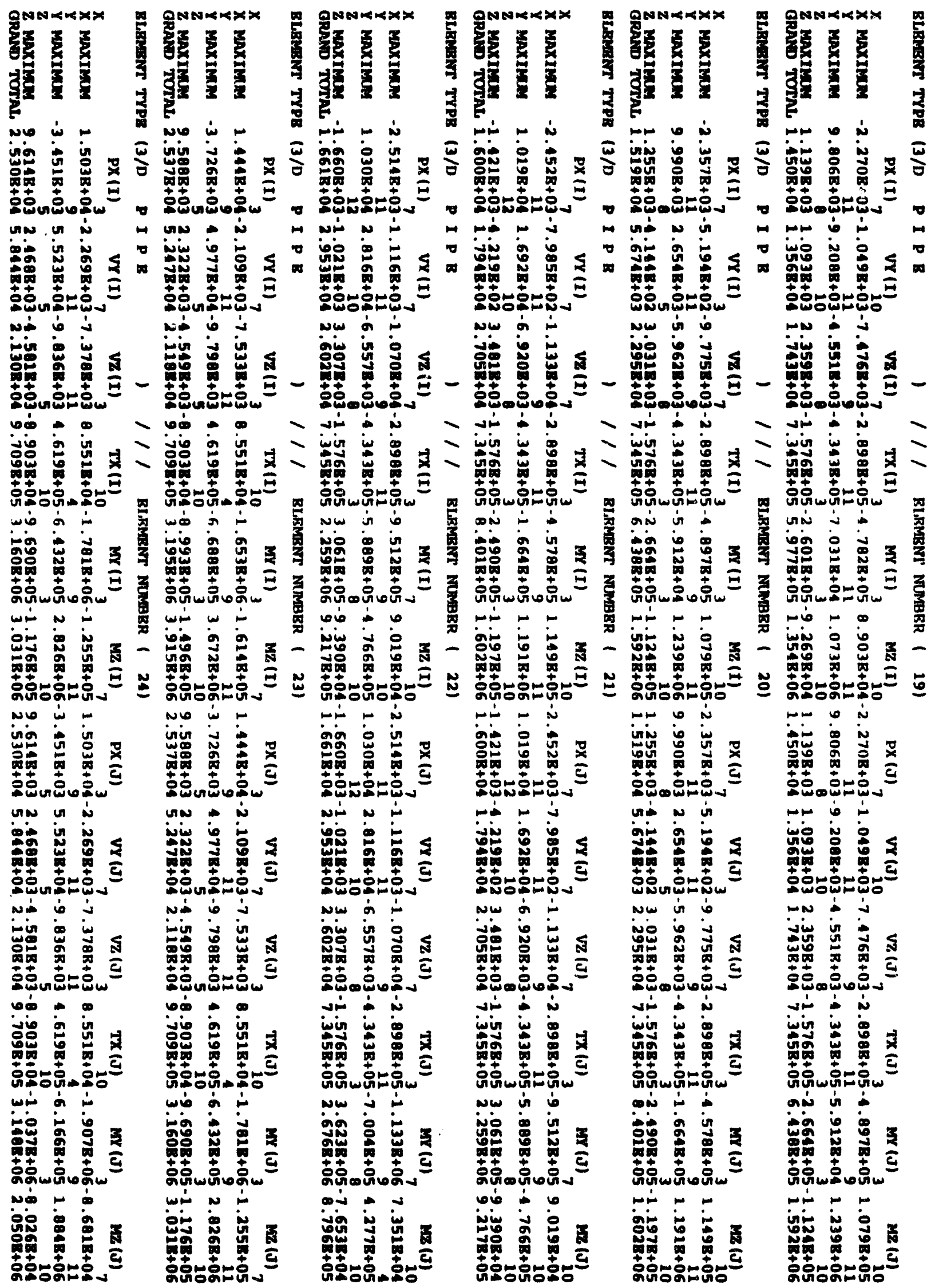




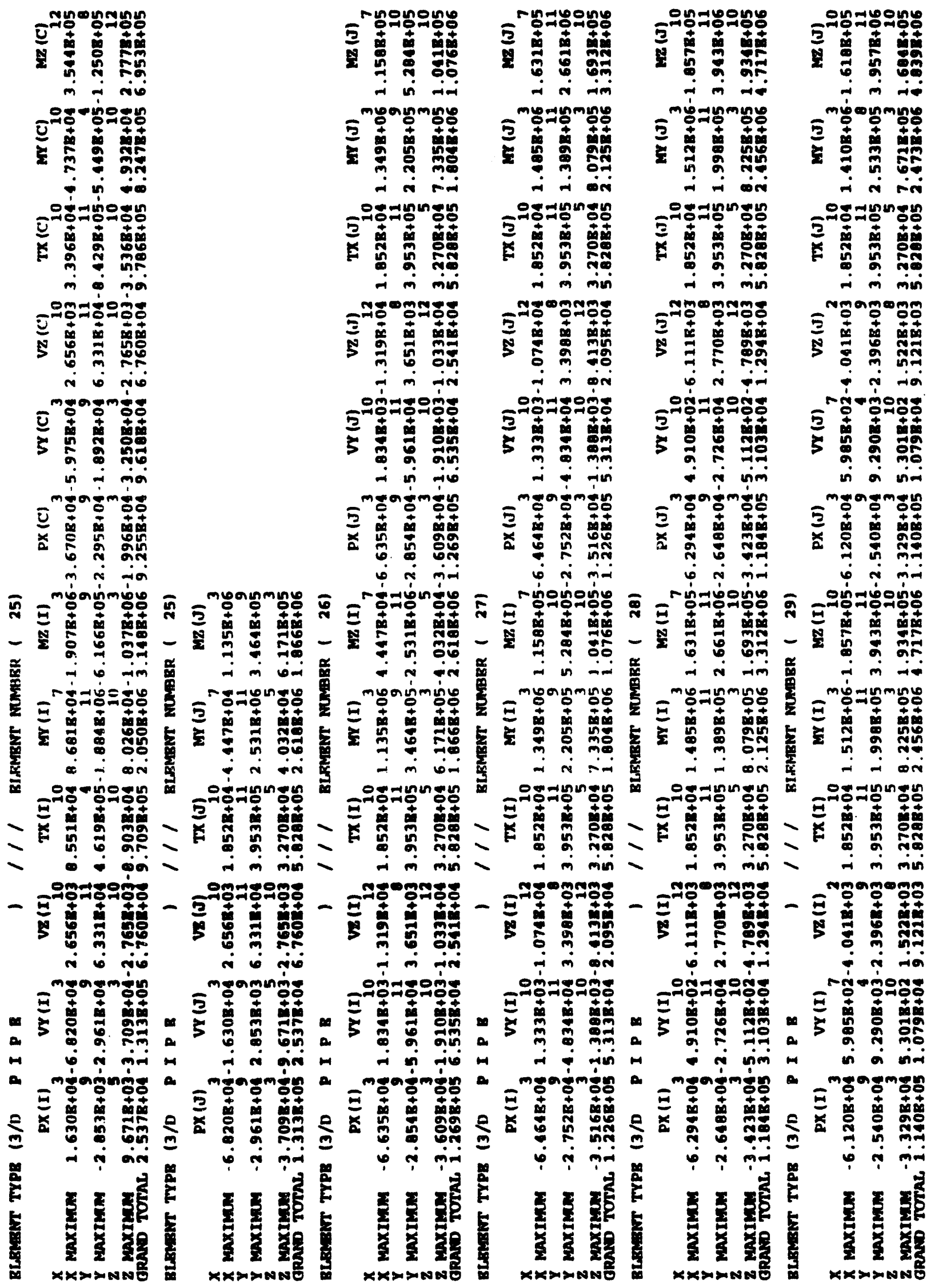


รํำำํำ

2.

or in aim

móøñming

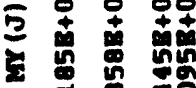

$\therefore$ i in

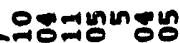

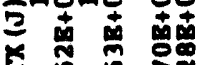

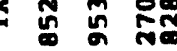

$\rightarrow$ in min

-7mamprọo:

ร

$\approx$ ส

in $-i-$

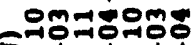

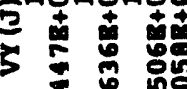

i i im

mjagmgng

范

in ते $\dot{m}-$

- ㅇำ

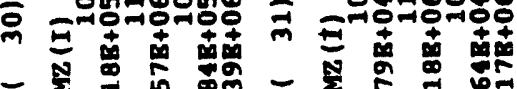

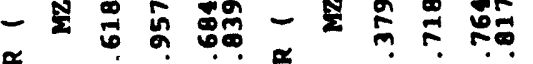

\%

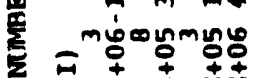

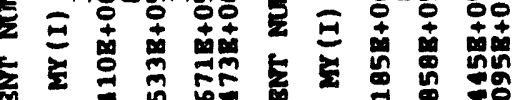

㮍

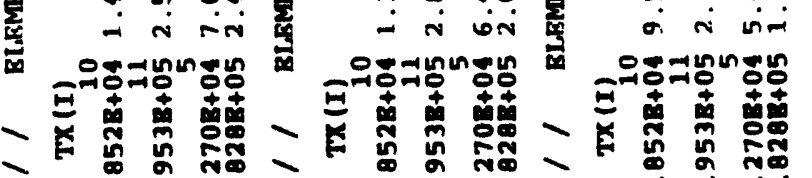

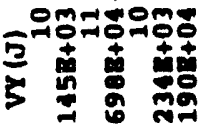

i * in

इ

$\because \bar{m}$ 的

in ति लि-

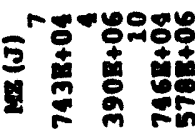

in $\dot{\text { min }}$

minongngnge

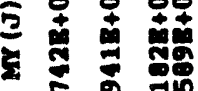

वे क्ष

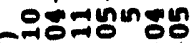

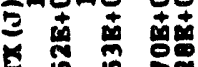

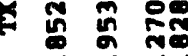

- im min

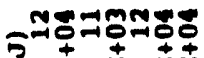

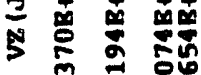

- i $\operatorname{in}$

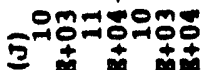

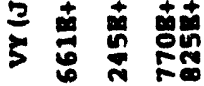

i 6 ن

mgagmgs

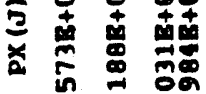

in iे ma

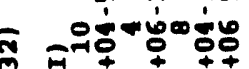

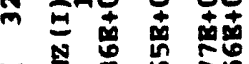

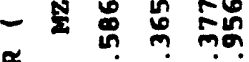

黄

minoinmiñ

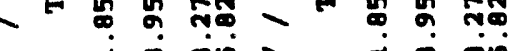

$\rightarrow$ in min

- Emampros:

ร

in - i

- in min

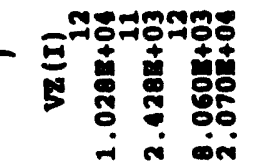

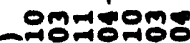

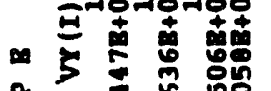

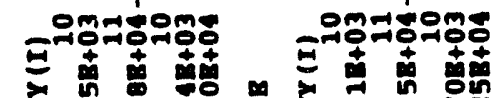

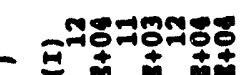

5 훙

- के

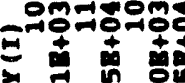

* है 앵으

- $\quad$ i i rim

a m

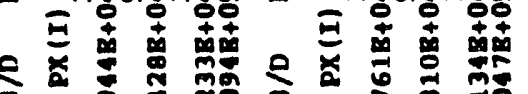

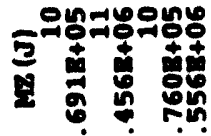

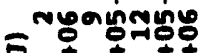

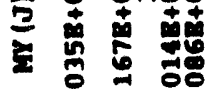

웅ㄱํำํํㅇ

క

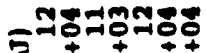

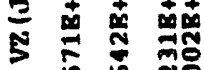

ำำํํํำำ

Е 站

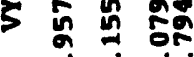

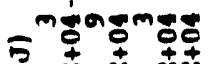

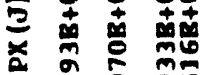

ल

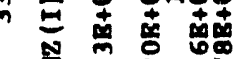

Nî̉ ñ. miñ

三

폴

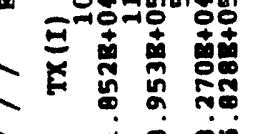

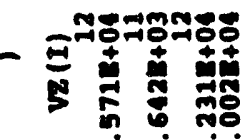

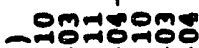

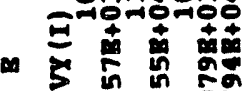

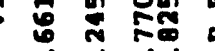

mo no

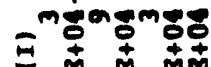

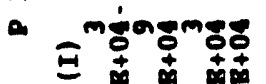

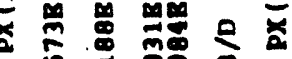

की ने क्व

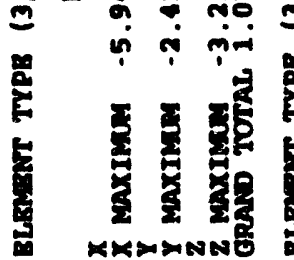

KMMNNE in के न्ष

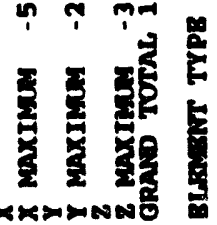

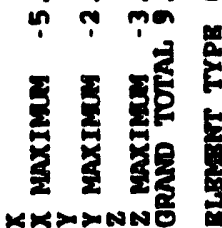

- $\dot{m}-\dot{0}$ - i نं ir ถู ผू సึั - $m$ min - 9 im i $\therefore$ mis

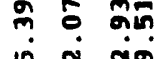

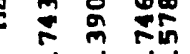
ने

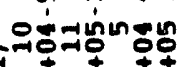

- m min

- $\dot{m}$ im

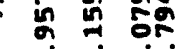

व $\sim$ mir

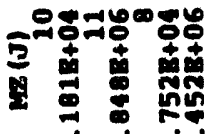
$\therefore-i$

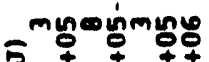

క i $\dot{m} \div-$

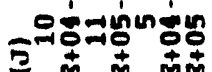

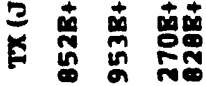

- im min

"canmmmo:

可产

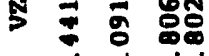

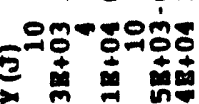

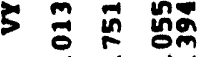

$\rightarrow$ in

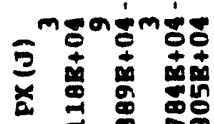
in -

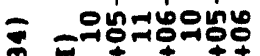

产南南

- है

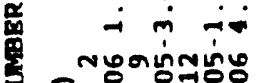

三

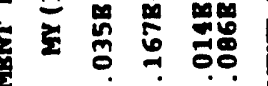

i in

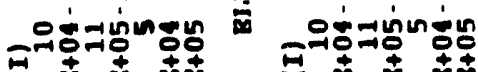

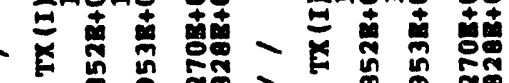

- $-\dot{m}$ min - $-i$ in min

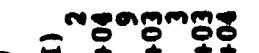

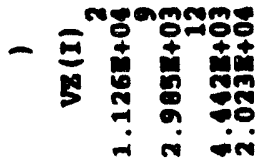

- iे மंi

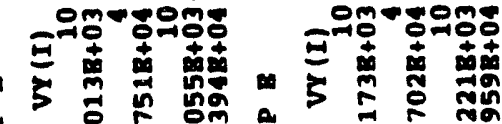

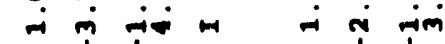

mรaํำ

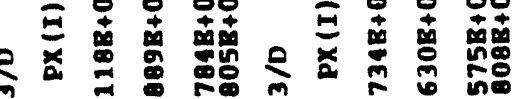

ติ o. ẫn

in iो लंब

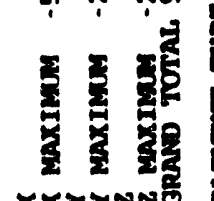



xKMMNNE in $\dot{1}$ iं

$\div \div$

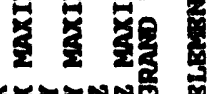

ह ह
3 उह 


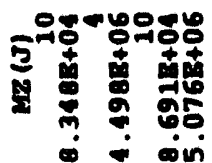

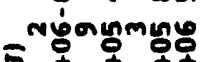

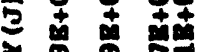

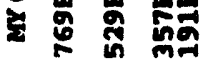

- i in im

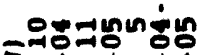

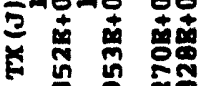

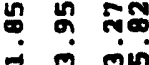

Noñmromo

ร $\therefore$ i mं

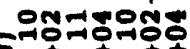

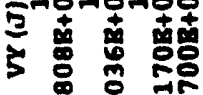
i in ani

5 mongm

ล

- i rir

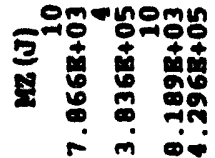

munaํํㅇㅎㅇ용

놀 홍 훙형 . ก . 웜ํํํ영

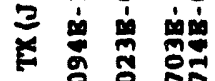
- $\dot{0} \dot{m} \dot{0}$ $\overline{\text { mmañmmo: }}$

క

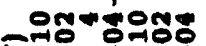

క ก

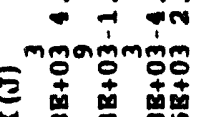

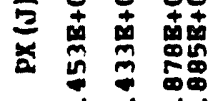
in i $\therefore$ in

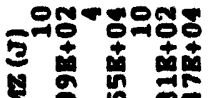

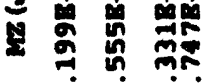

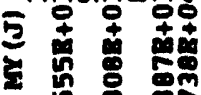

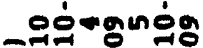

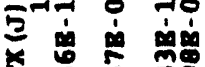

*

- $\dot{1}$ rí

mmoñomom

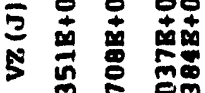
$\dot{m}$ i

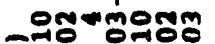

క 5 훙 형 ڤ.

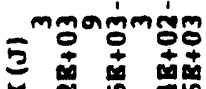

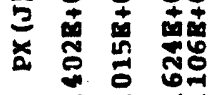

mman்m क் in is ma

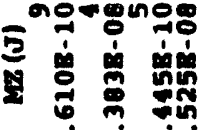

- r vor

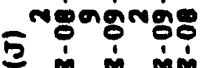

용 홍 in in

우이용웡

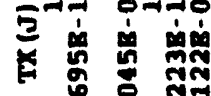

$\dot{0} \dot{0}$ in

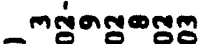

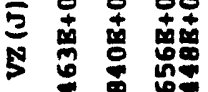

in $\dot{m}$ in

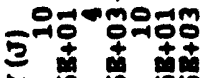

5 ถั: वे लंख

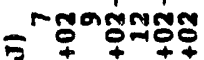

ฮ

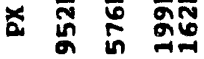

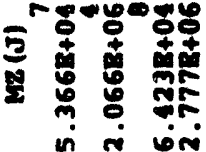

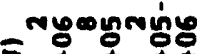

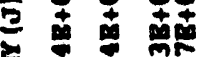

동 ํㅜㅇㅇㅛ - $\dot{m} \dot{m}$

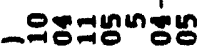

후

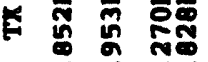
-i m ivi

mormemóg

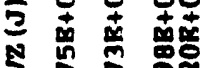
:5 ूัญ - $\div$ vic

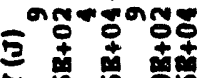

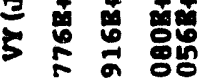
$-i$ in

हmgamm 훙ㅎㅎ

三.

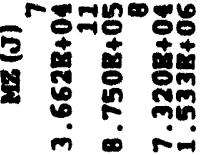

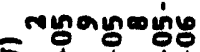

놀 总

$\therefore \therefore$ in

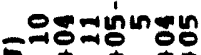

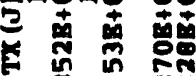

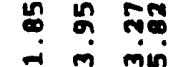

mgammmo

इ

m ₹ ถีต

$5^{0-1}$

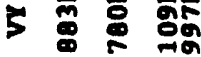
$\dot{m}$ i

$=$ manmmgo

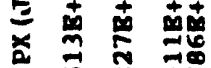

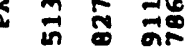
m $\sim-\dot{0}$

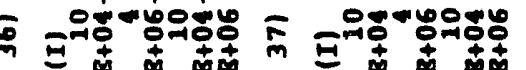

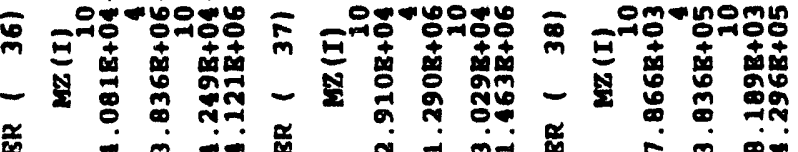

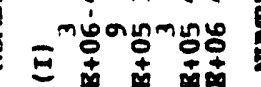

N -1 mi

Em

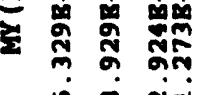

in ले तं

害

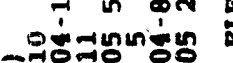

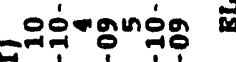

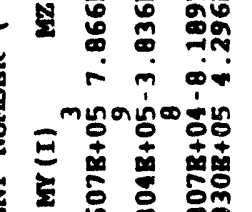

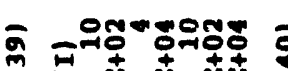

m a Nin

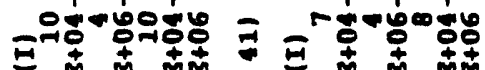

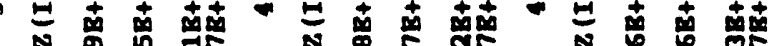

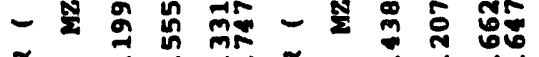

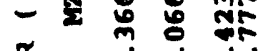

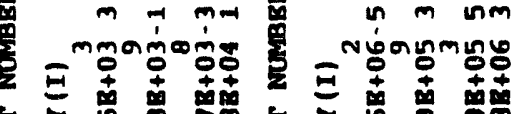

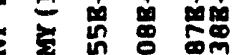
嵌

i vi

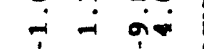

-

\& ó்mó்

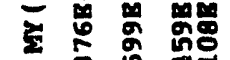

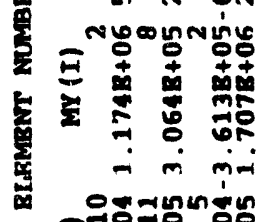

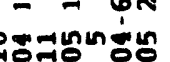

一

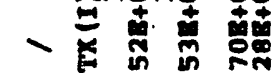

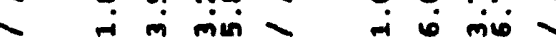

-i i लं $>$

i in in

- E

픈

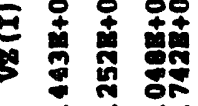

-

$-$

mğagrom

5 Е

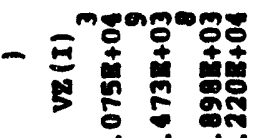

manmmm

in $\dot{m}$ min

- i isi

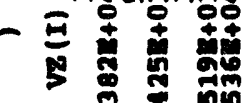

क त

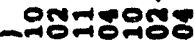

우당요

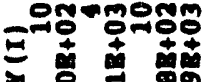

5 \%

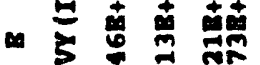

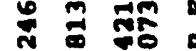

5 훙 영 앰

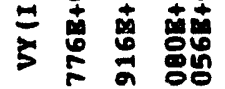

-i $\therefore$ in

i i ivi $m$

-

Emgagimg் a

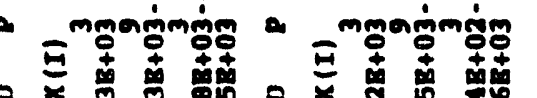

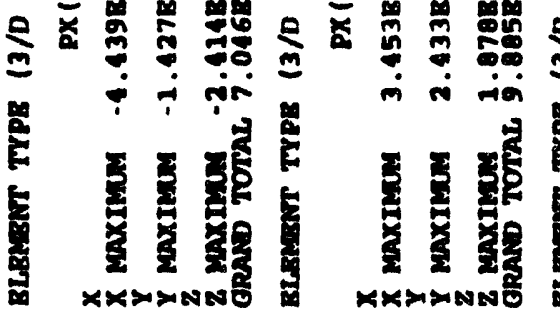

三 xxingivo

mgangm

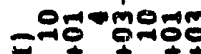

anogang:

a

క ถ้ รู है त में - $\dot{-1}$ ini

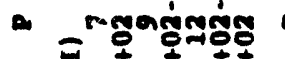

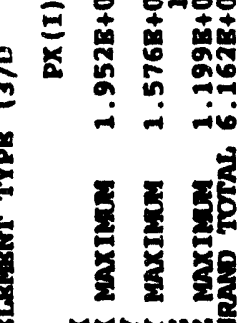

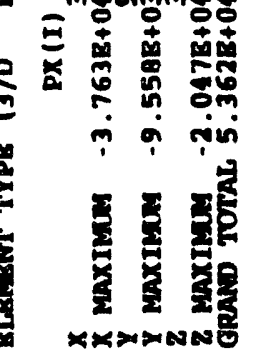

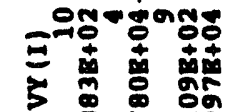

$\dot{m}$ i $\dot{\sim}$ iे

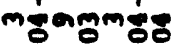

E 离 ติ ส

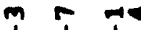

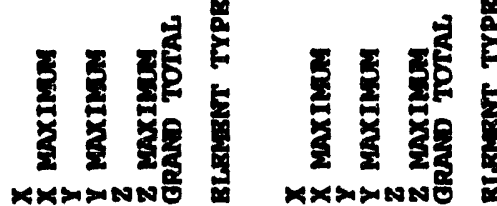




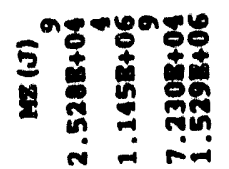

mýăg்

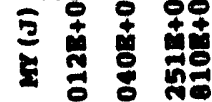

-i i i

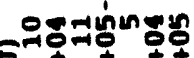

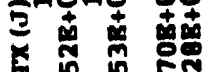

* กู ตू

- m min

mgammmg

इ

รั สิ

- i

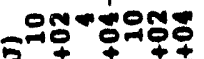

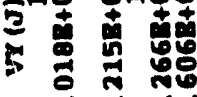

i $\dot{m} \dot{0}$

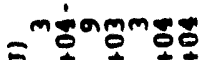

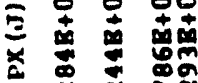

ก

ल 6

₹ E

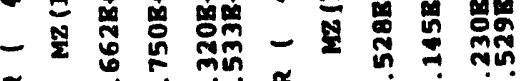

लmang

E E

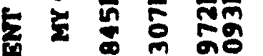

क्षे

- Е

mónogongo

눈

mgammmó

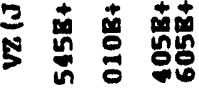

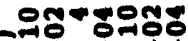

5 兽 影

mgammge

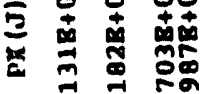

- $\because$ ก

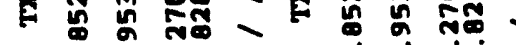

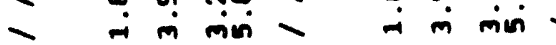

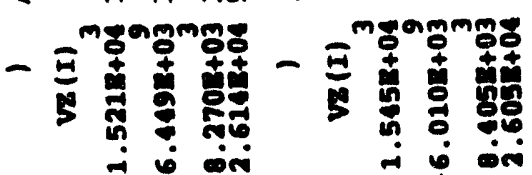

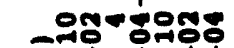

- $5 \stackrel{5}{ \pm}$ 兽

$m$

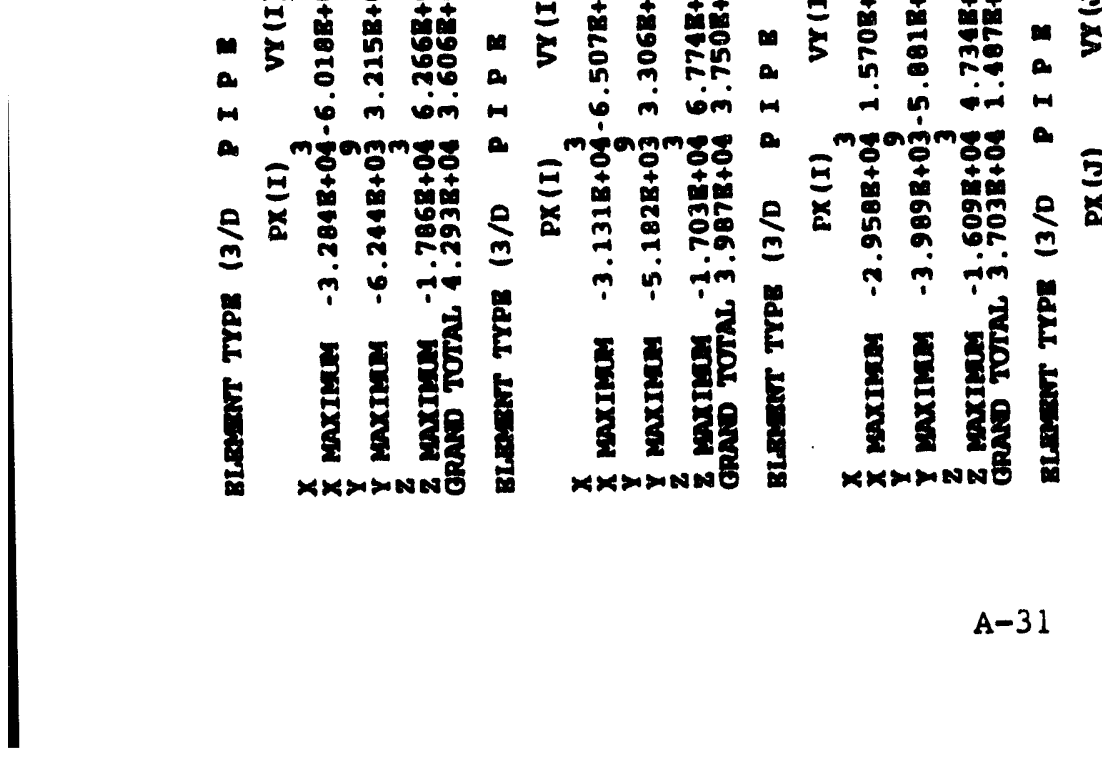

E

E

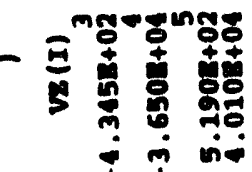

Ј

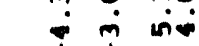

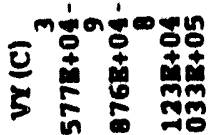

ते in min

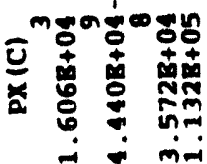

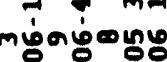

- E声 兽 兽 总

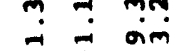

-

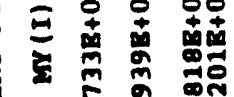

i

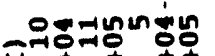

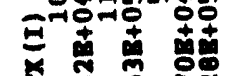

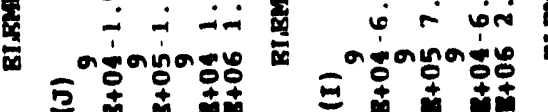

- $\overline{2}^{\text {Nungunongo }} \bar{t}$

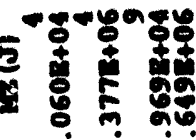

$\dot{m}$ in rim

mǵangmyg

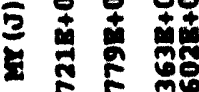

i $\dot{\text { in }}$

a grangağ

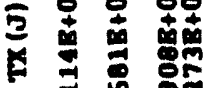

$\dot{m} \dot{0} \dot{0}$

mgromm

इ i - im

ร

- in rim

三

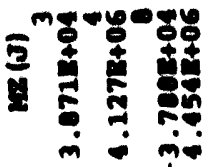

myangmge

농

in

agongage

$\frac{2}{*}$ 苗

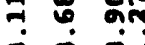

mgromm

ए

i in

mํํ욤ำ

క

? กั

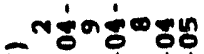

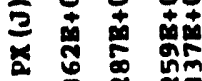

-

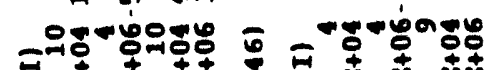

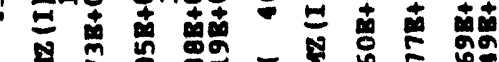

- 2 in

i $\dot{0}$ ir

F

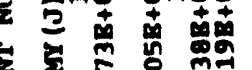

Ning

三

iे $\dot{m}$ rim

-mégngmuge

$\equiv \pm+\infty+\frac{1}{m}$

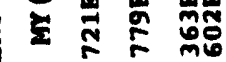

- - on

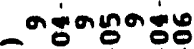

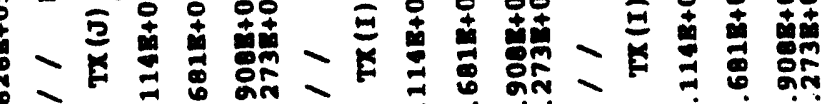

- $\dot{m}$ miv $-\dot{m} \dot{\oplus} \dot{\oplus}$

$\dot{m} \dot{\infty} \dot{\phi}-$

$\dot{m} \dot{0}$

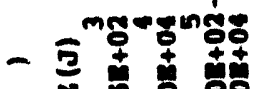

mgomm gं

mgommgo

3 응

5

in $\Rightarrow$

in $\dot{i m}$

플

- m no

mǵnomm

ma்- क्nलि:

if in in

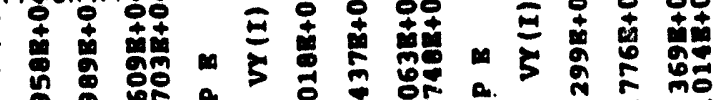

mก-gำ

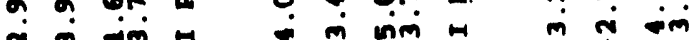

opa in

2 gृage

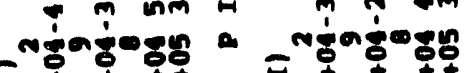

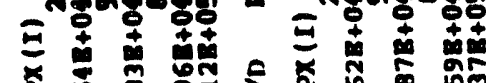

in :

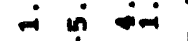

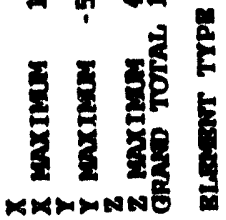

$-\dot{n}-\dot{0}$
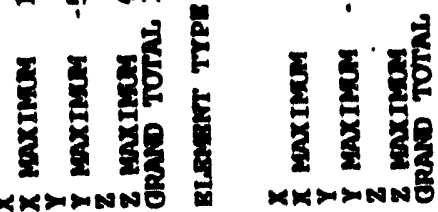


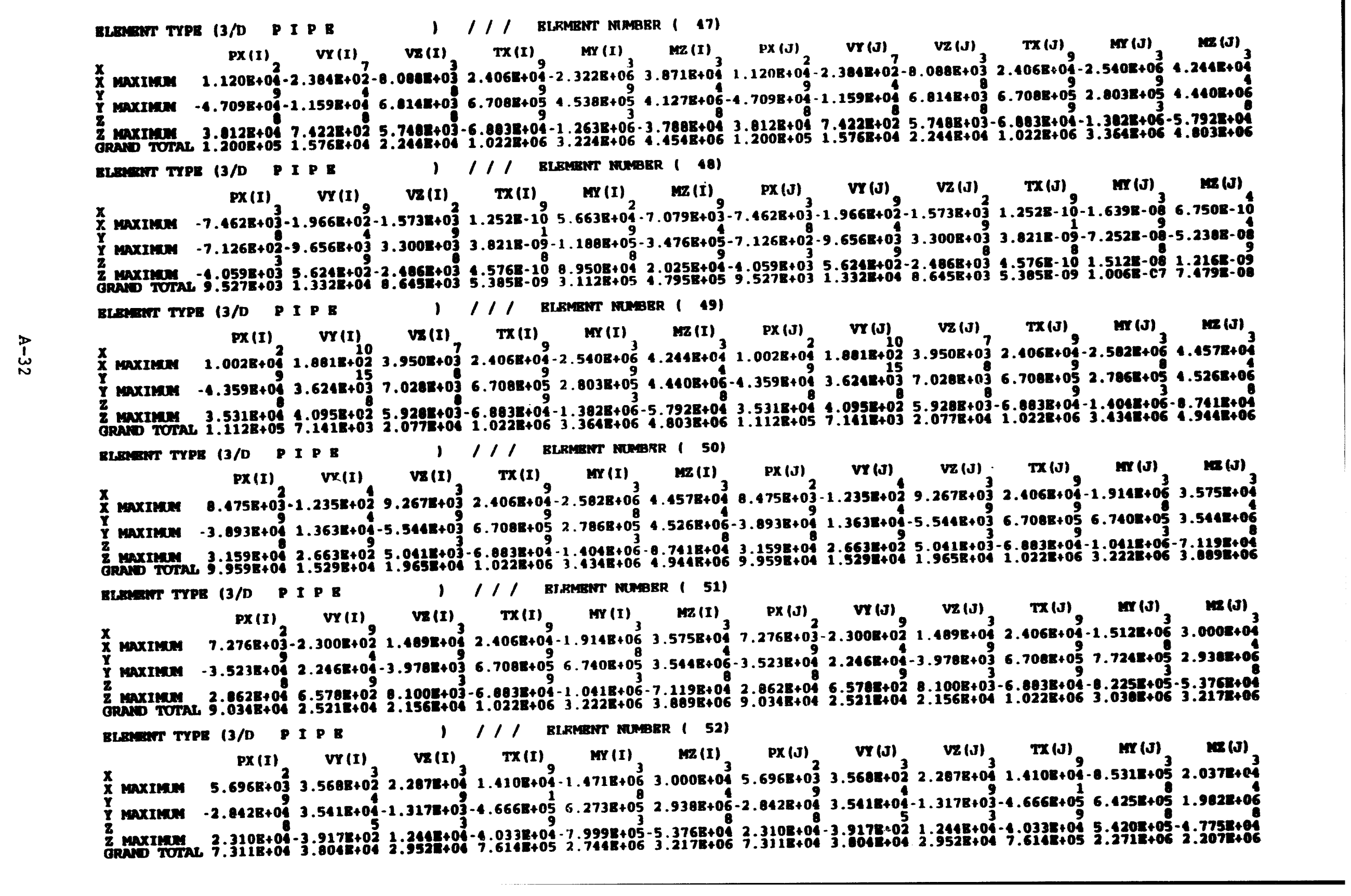




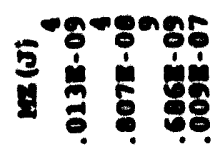
- 1 i

"

놀 i $\dot{0}$ agagnge

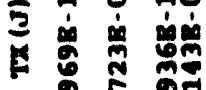
वं :ं نं

풍ํํ용혀

క

$\therefore \dot{-\dot{m}}$

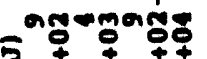

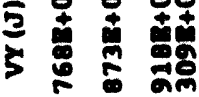
i $r$ i

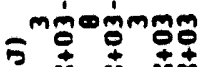

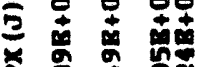

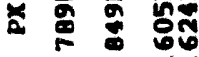

- - iं

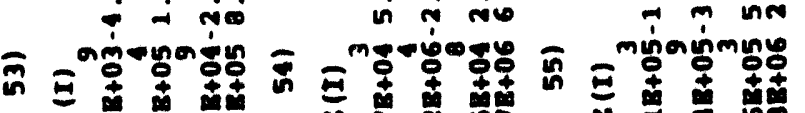

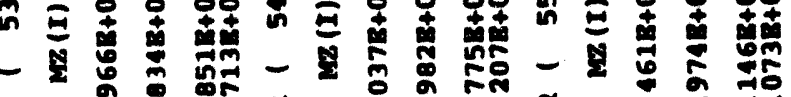

* ดे

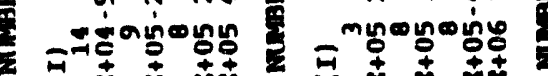

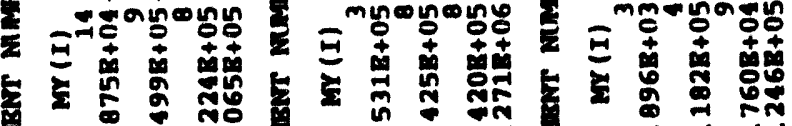

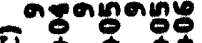

营 总

amagang

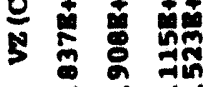

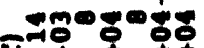

5 ล

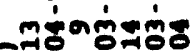

눙

- $<--\infty$

ánghog:

क

$\because$ :

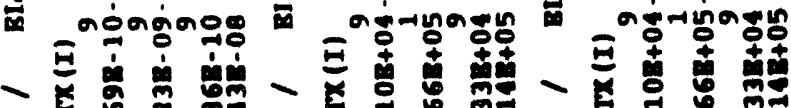

-agagags

동

$\therefore \dot{-i n}$

myogogy

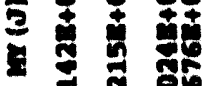

- i

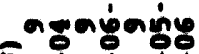

₹ 8 \& i iv im

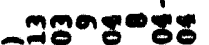

ร

$\therefore$ -

amaga

5 商

त $\therefore \dot{0}$

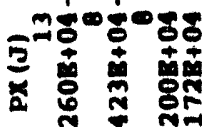

i- in

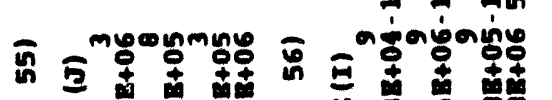

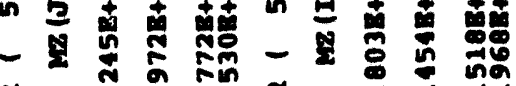

* \.

$\dot{0}$ is îं

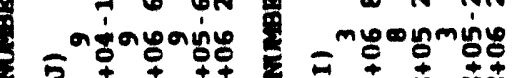

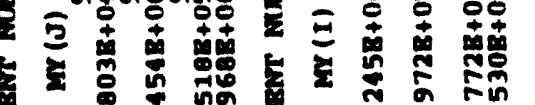
के लि

agngañ in

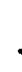

- :

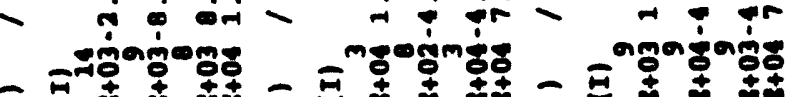

- Е"

$\therefore$ im

i is im

i :

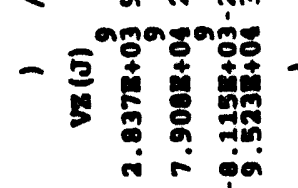

inagis

융 mang

mn-

mơ ํํㅇํํ

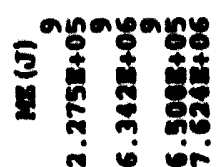

${ }^{m}$ mangóg

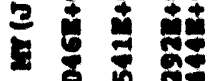

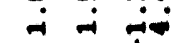

- gुagang.

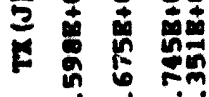

a i

mmagต

$\frac{2}{5} \div$ 훟

-

amagame

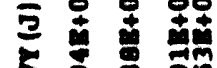

5 \& 8 ริํํㅇ

is in ir

$5^{m}{ }^{\infty} \delta^{\infty}$

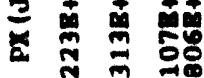

$\therefore-i$

aýnghang.

트 훙 훙 훙

- 일 号

- $-\dot{\text { in }}$

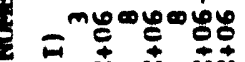

క - พิ

agağang: >E

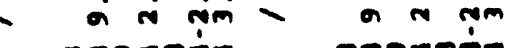

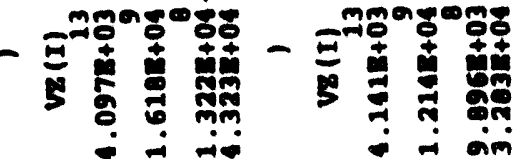

amagamis amagañ.

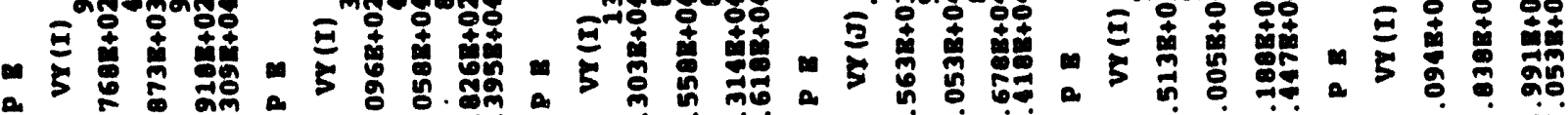

a

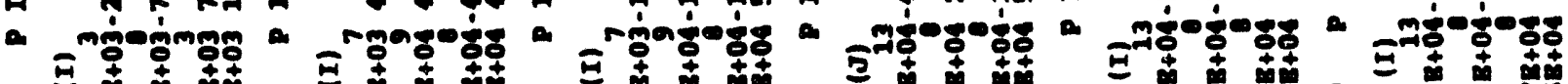

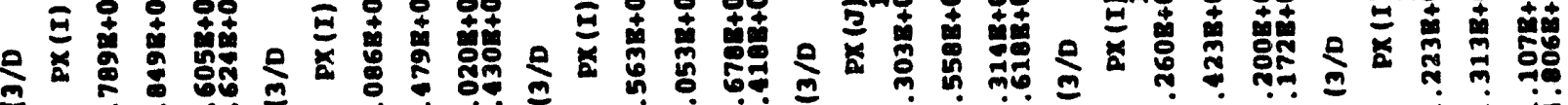

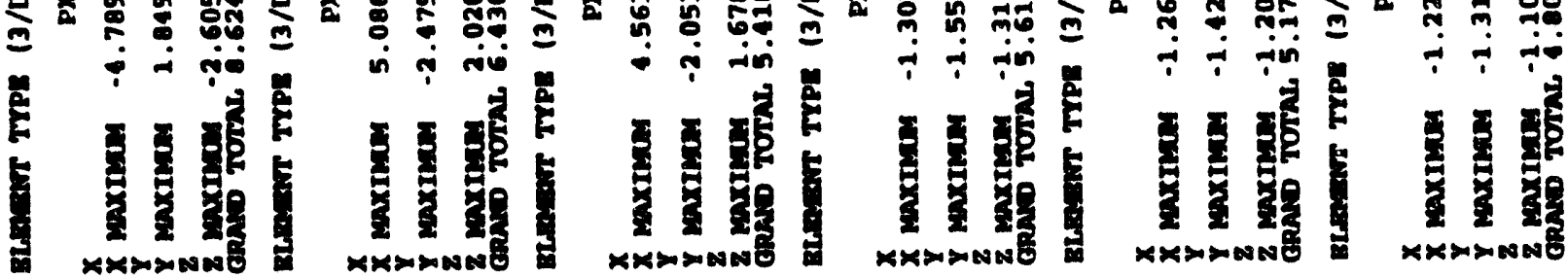




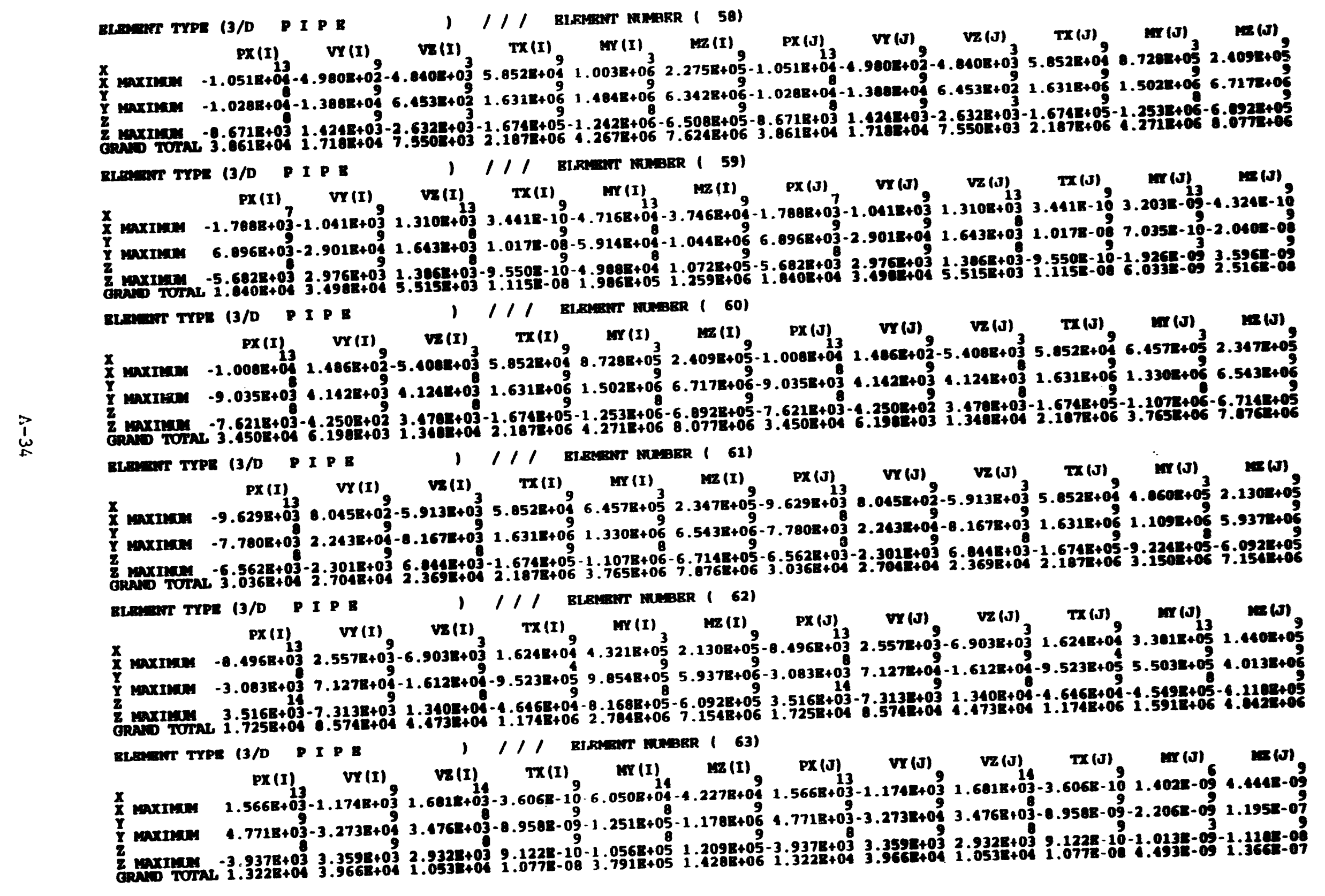




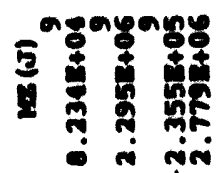

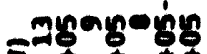

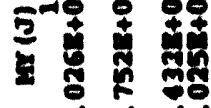

in $\dot{1}$ i

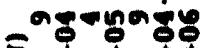

둔

i $\dot{0}:-1$

mmag் हैं

ร

$\therefore$ i i.

amágam

5 5

i $\dot{0 \dot{\theta}}$

궁ำ

ะ

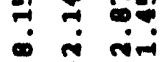

8

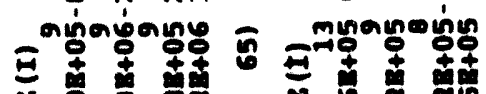

- $\frac{0}{2}$ ำ

量

플

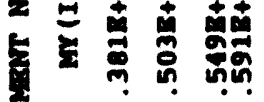

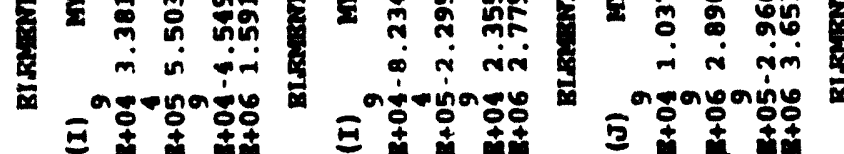

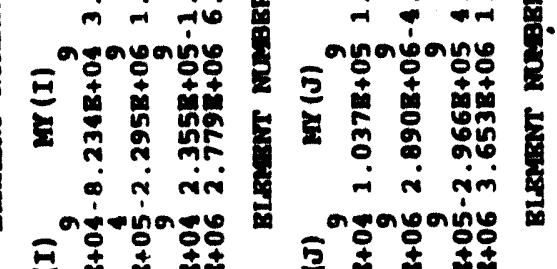

angingatg

군 8 훈

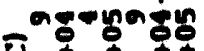

₹

-i i ma

amagames

ร

mmange

5 啇 㩆

mmagom

เ

$m=$ ă

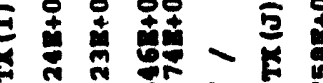

- สู

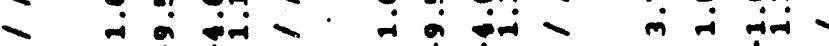

- E

$\therefore$ i

- E 5 i

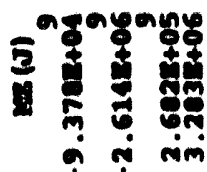

mignag்eng

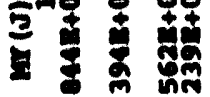
i $\dot{m} \dot{m}$

aǵağange

₹

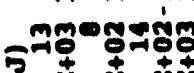

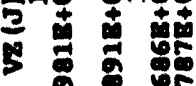

\&

agromang

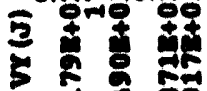

5 ㅇำ

iे ì mir

mต் क्षेळ

₹

$\therefore$ -

angayanǵg

호 홍

- 송 용

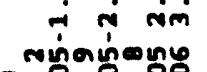

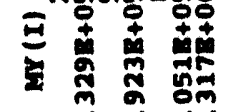

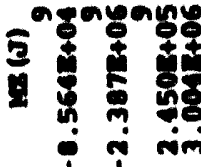

argangeys

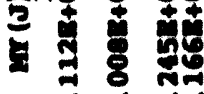

in $\dot{m}$ in

aǵaǵnge

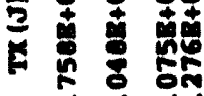

m i

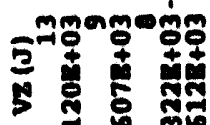

-i

axูomax่

₹ 5 \%

in 9 ตे

ร

$\therefore$ - im

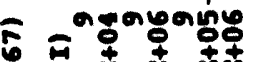

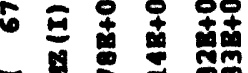

- $4 \sum_{m}^{\circ}$ สำ

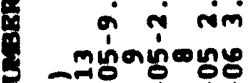

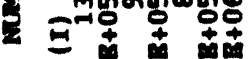

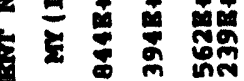

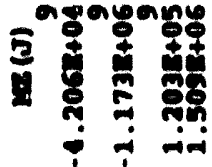

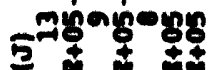

1 영 형

a çaǵange

- 5 웅

in $i$ i-

క 5 क

$\therefore$ iा

agagan்

5 형 홍

- i

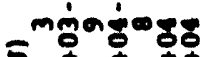

2

$\therefore$ i

- agagañ

6 E -

- के i rim

- mingongongo

5 i. ज्ञ

agağang:

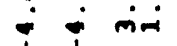

ágab́anc

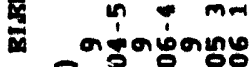

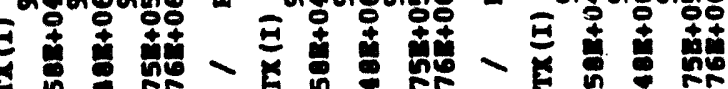
ํำ $m$ i

- $m$ -

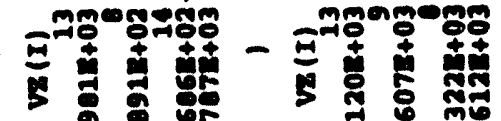
$-\frac{\operatorname{mang}}{5} \frac{+}{5}$ i $\dot{0}$ ic i. ?. ongogong की E + ongagango

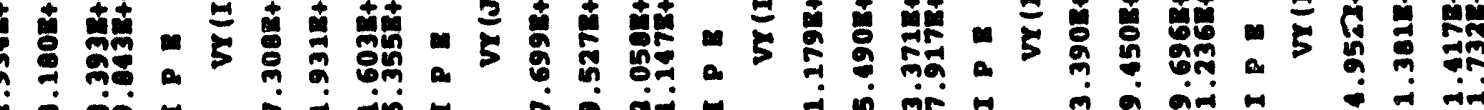

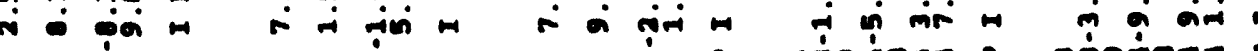

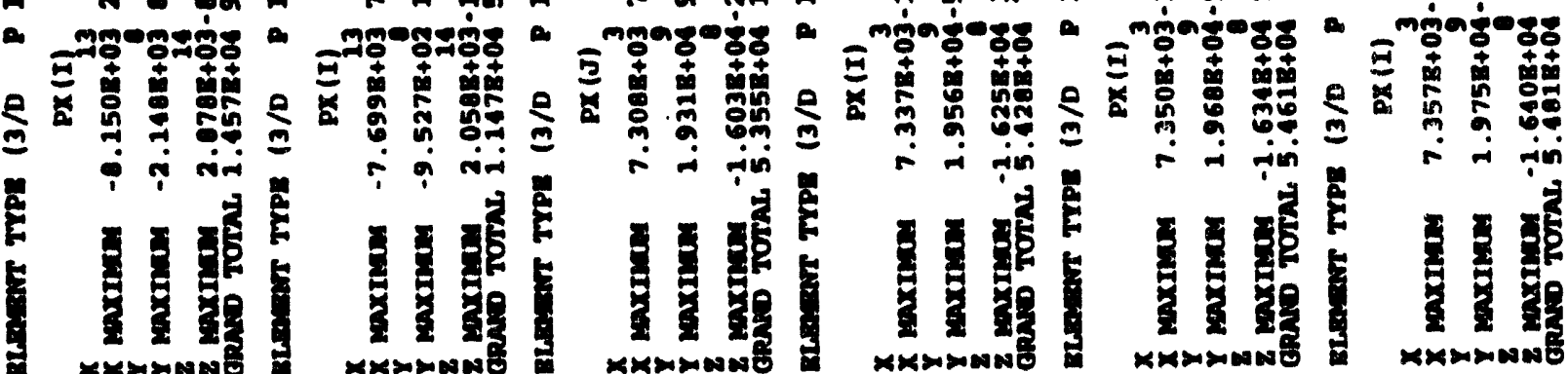




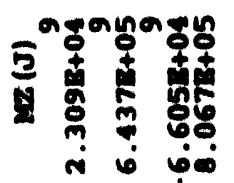

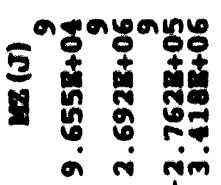

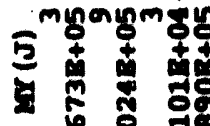

miğangong

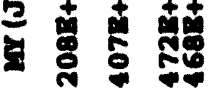

- $\dot{\text { in }}$

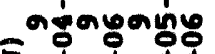

ฐ

iे $\dot{\text { i }}$

mmamem

รั

in के लंन

5

क में in

agayang:

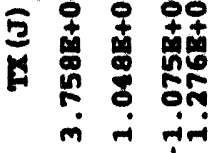

mmañom

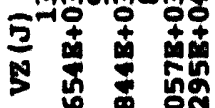

$\sim \dot{m}$ mंन

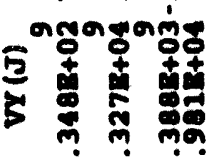

क ते riv

i in ivi

mming

క

mmintor

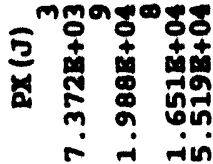

- argagangy

-

-

ก. ๆ พึ.

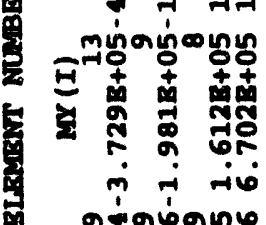

蓄 i

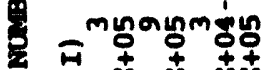

불 i i im

a aramanic

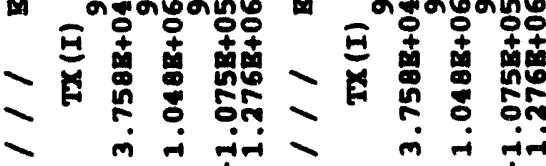

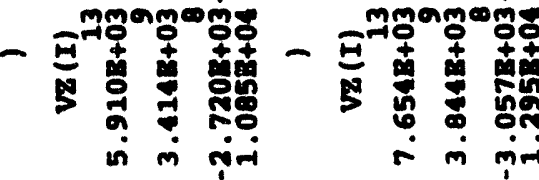

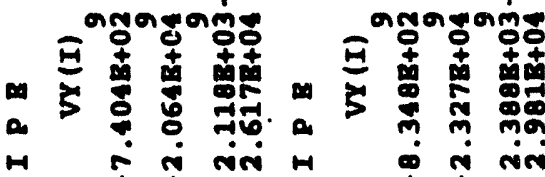

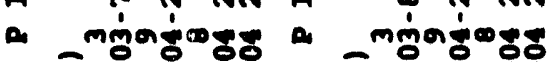

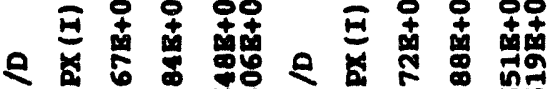

ㄹ.

ค م

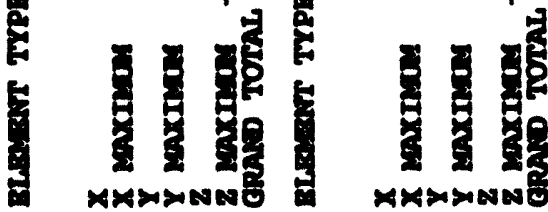




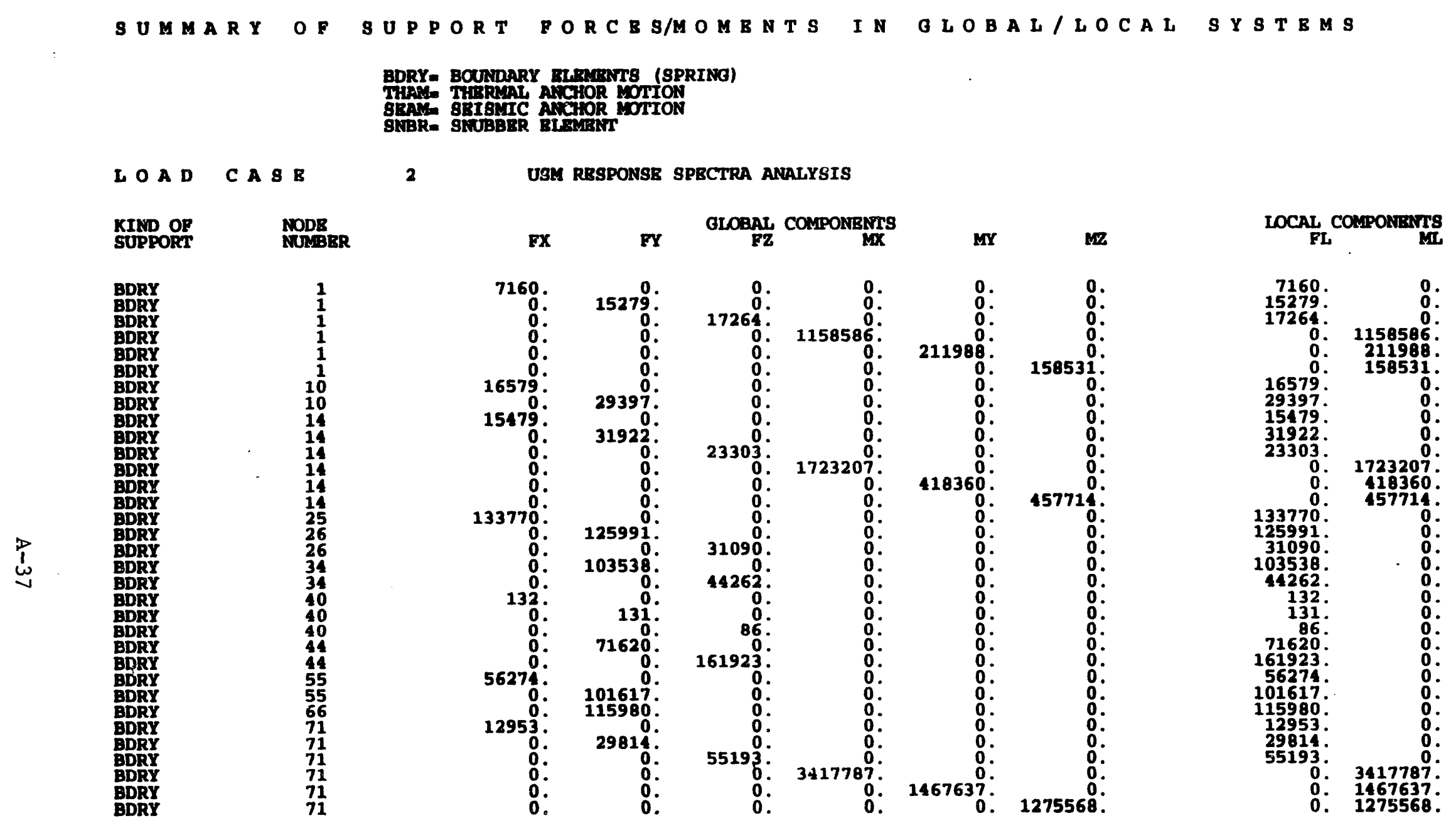

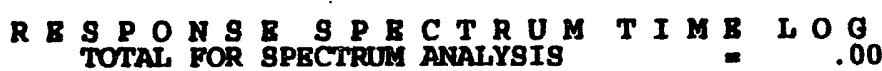


BENCHMARK PROBLEM 1

HIGHER FREQUENCY RESPONSE

(RIGID RESPONSE) 


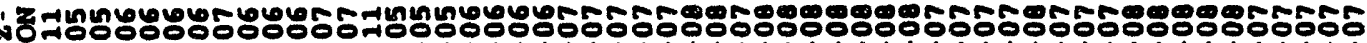

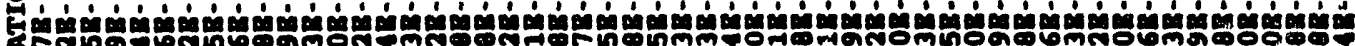
S

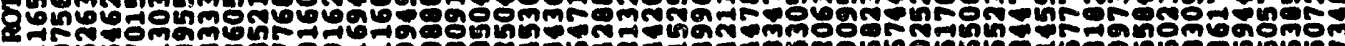
- ont

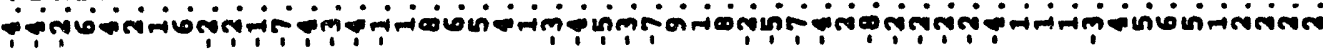

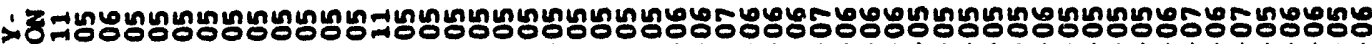

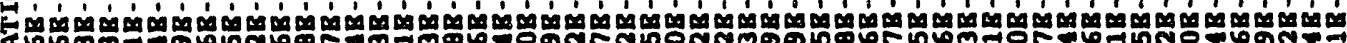

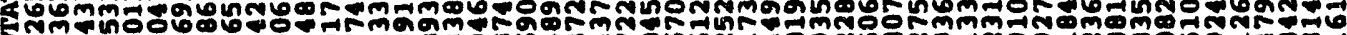
ono-n ๓

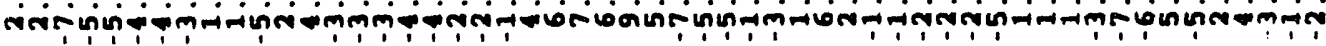

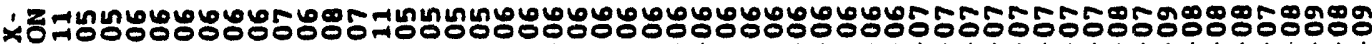

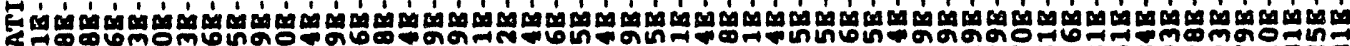

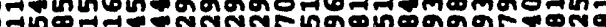
50\%

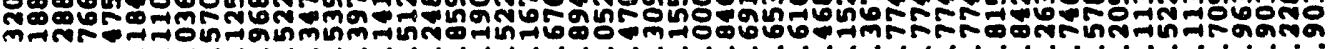

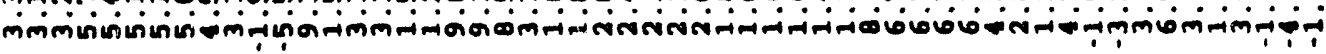

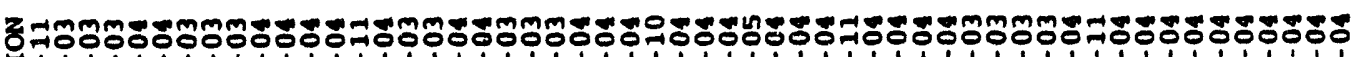

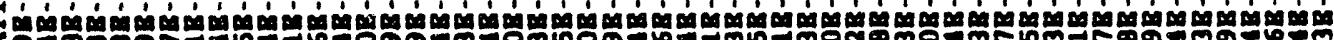
न

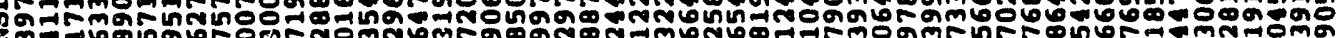
mönbongh

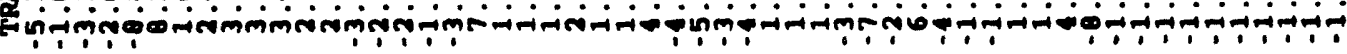

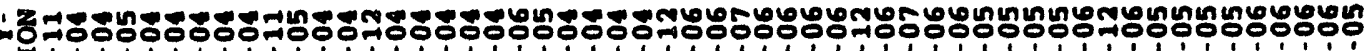
-

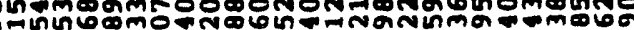

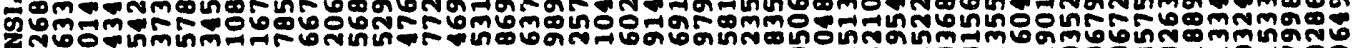

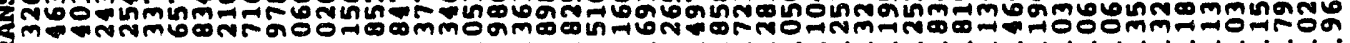

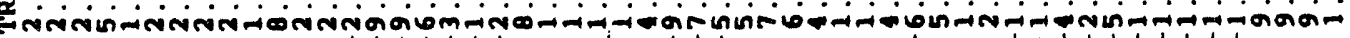

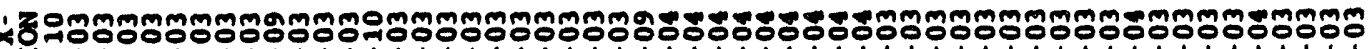
¿

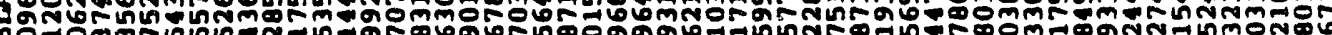

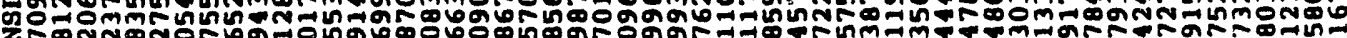
द A 


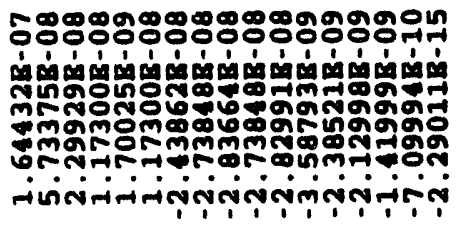

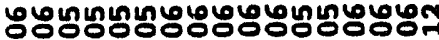

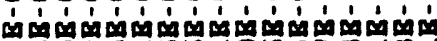

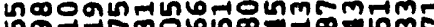
minom 응ำ

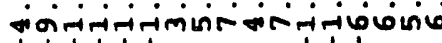

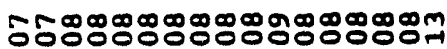
1́1 ที กิตmb. สnต ज้

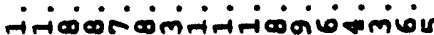

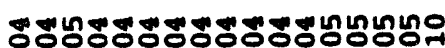
ḿn ทีन-1 Orm

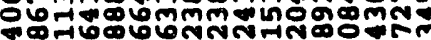

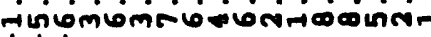

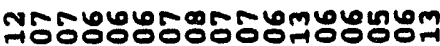

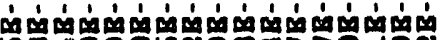
๘ँ 7o

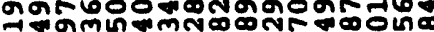
7.m.

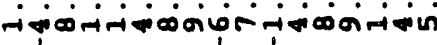

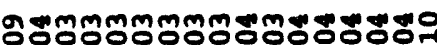

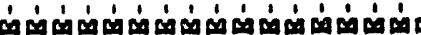
으욤ำ Don

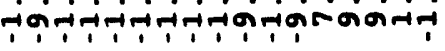

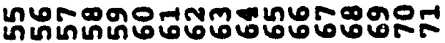




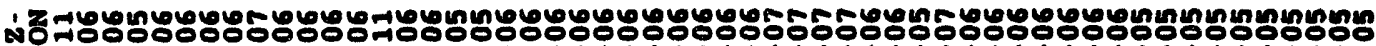

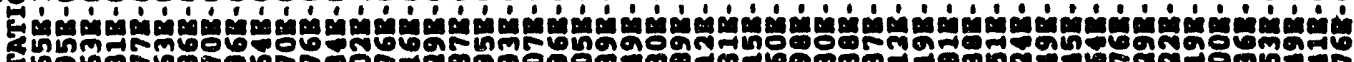
0 ó 6ด̆

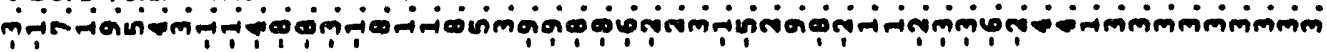

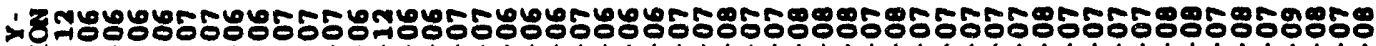

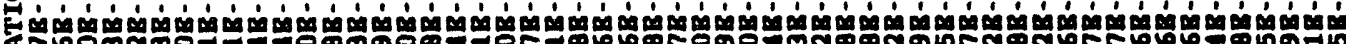
作

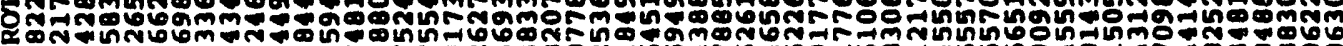

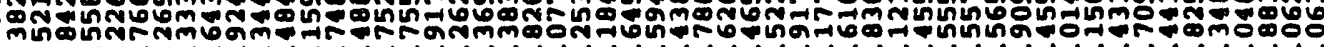

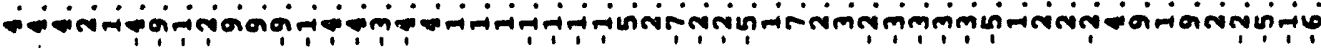

×்

Fُ दm-

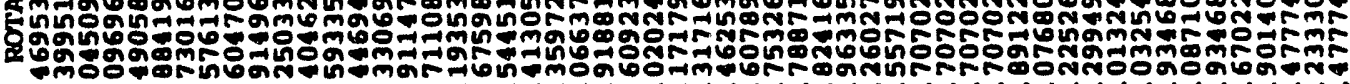

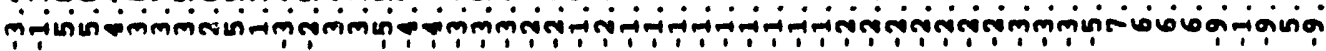

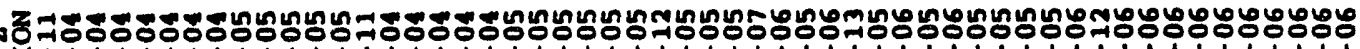

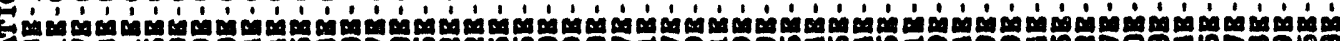
ร 作

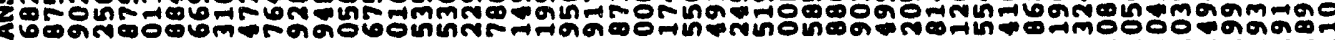

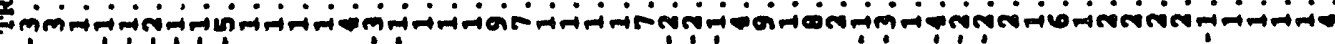
눙

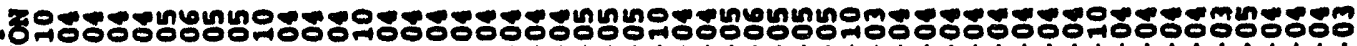

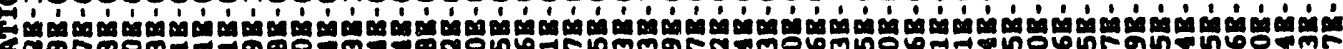

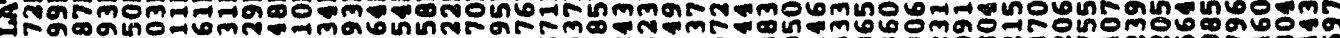
ng.

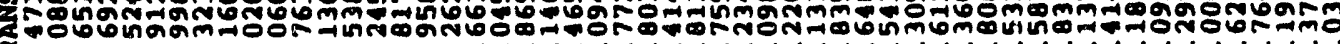
تmámon-

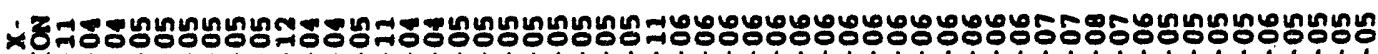
تn்

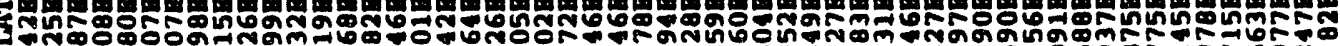
б-ที่ำ द⿱⺈

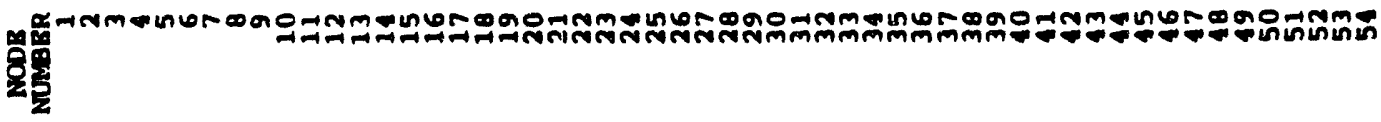




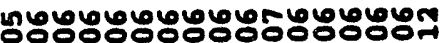

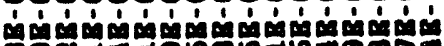

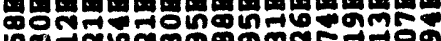

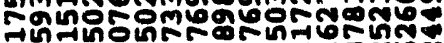

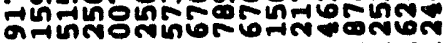
-iतनimnimio

5

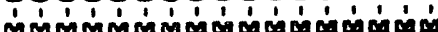

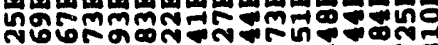

งั0ิ

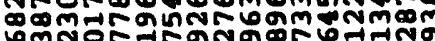

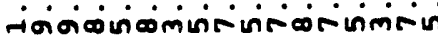

19.

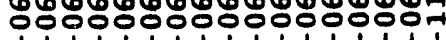

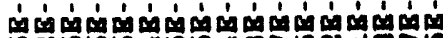

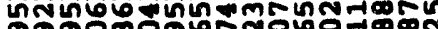
भून

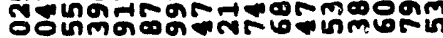

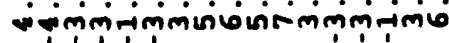

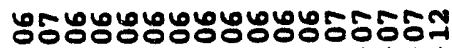

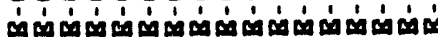
- lo ติก

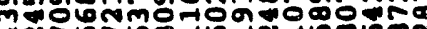

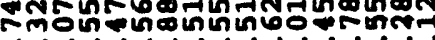
-

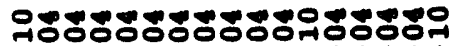
1́一่ ทู워नำ ๕ฐืตี

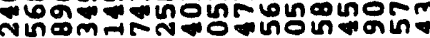

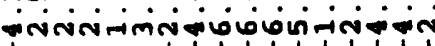

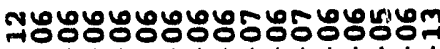

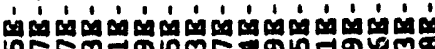
ก도금ำ

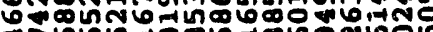

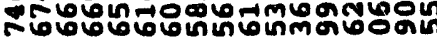

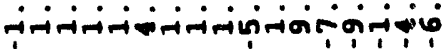

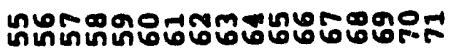




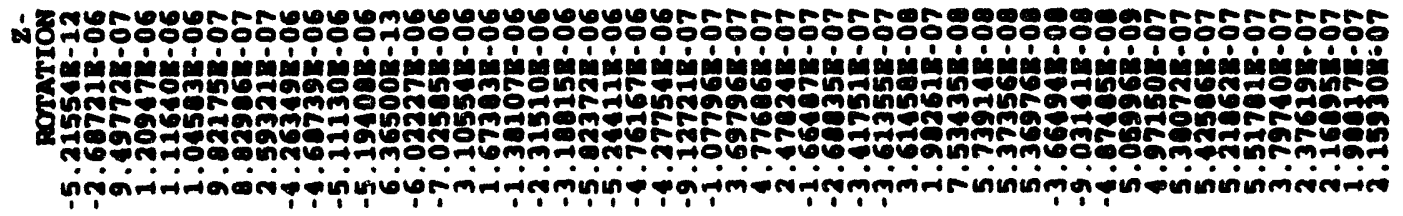

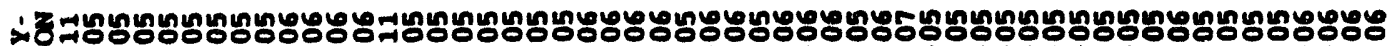

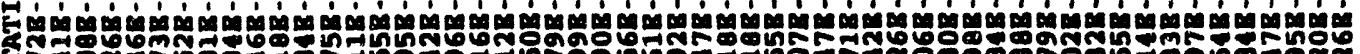

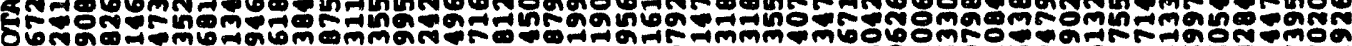
\& - - -imminim

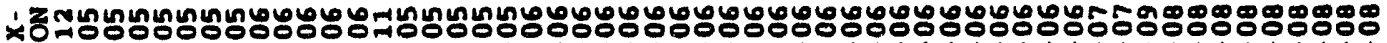

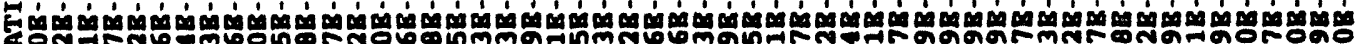
(1)

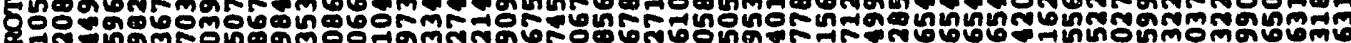
\&

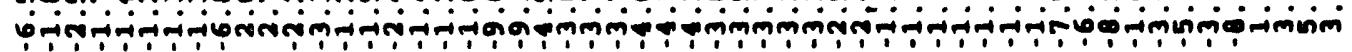

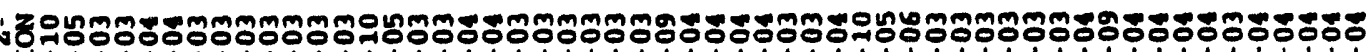

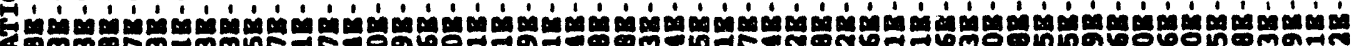

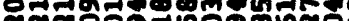

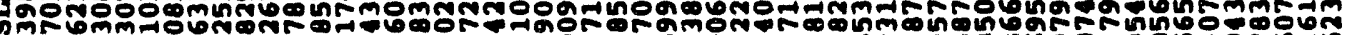

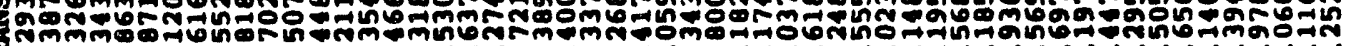

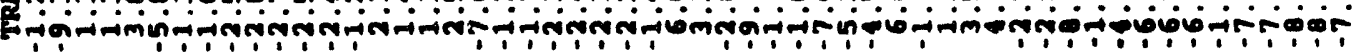

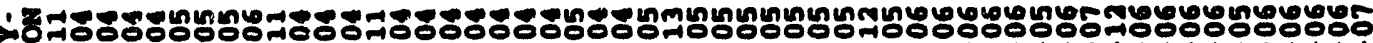

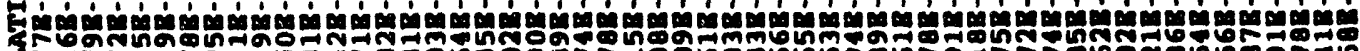

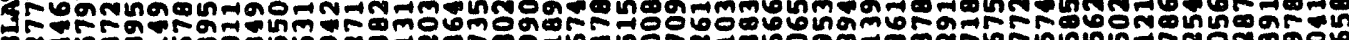

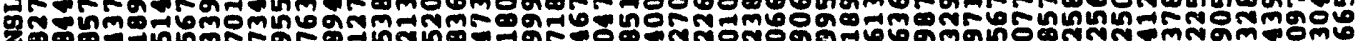
\% E-1,

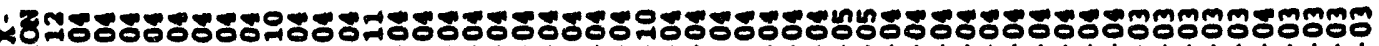
H:

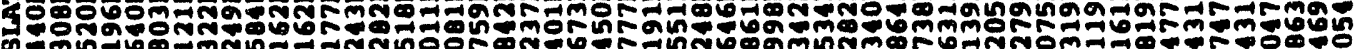
n द 붕

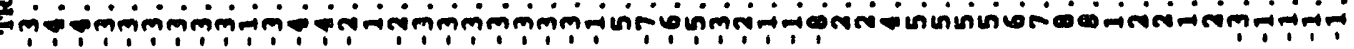

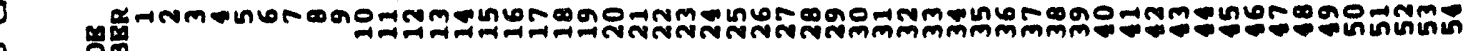




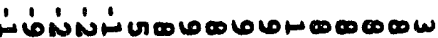
Nivinoniogioojiobiojis O NNaN 6 If जo 3 u พิ

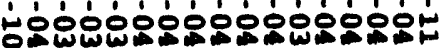

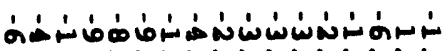

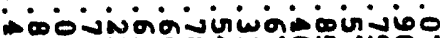
o ๑ - - UUज N พิ

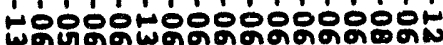

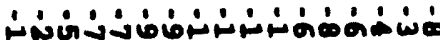
inioivoiroioninin vinivini wh H J

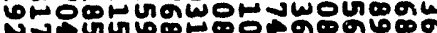
개 ตัต

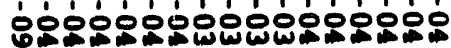

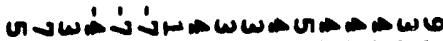
inivinioonioo oniogion (1) ON63

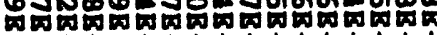

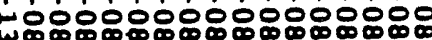

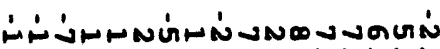

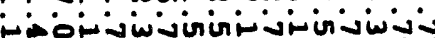
ง⿻日禸 Fัo u涫

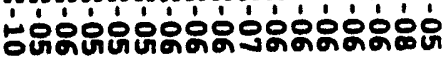

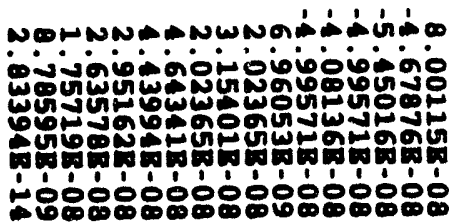




\begin{tabular}{|c|c|c|c|c|c|c|c|c|c|}
\hline 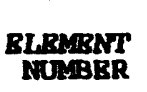 & $\begin{array}{c}\text { BLEMRNT } \\
\text { TYPB }\end{array}$ & $\begin{array}{l}\angle O A D \\
\text { CASB }\end{array}$ & STATION & $\begin{array}{l}\text { AXIAL } \\
\text { BORCR }\end{array}$ & $\begin{array}{l}\text { Y-AXIS } \\
\text { SHRAR }\end{array}$ & $\begin{array}{c}\text { z-AXIS } \\
\text { SHEAR }\end{array}$ & TORSTOMAL & Y-AxIs & 2-axis \\
\hline 1 & BEND & 1 & $\begin{array}{c}\text { END-I } \\
\text { CENTER } \\
\text { END-J }\end{array}$ & $\begin{array}{r}-930.959 \\
-822: 791 \\
-232.566\end{array}$ & $\begin{array}{r}-232.689 \\
4933.806 \\
930.990\end{array}$ & $\begin{array}{l}1686.120 \\
1686.120 \\
1686.120\end{array}$ & $\begin{array}{l}54710.15 \\
37100 \\
27390.45\end{array}$ & $\begin{array}{r}-23192.59 \\
-19316.67 \\
-1123.50\end{array}$ & $\begin{array}{r}7749.64 \\
1504.59 \\
-13202.15\end{array}$ \\
\hline 1 & BEND & 2 & $\begin{array}{c}\text { RSD-I } \\
\text { CENRR } \\
\text { CEND -J }\end{array}$ & $\begin{array}{r}384.945 \\
2225: 171 \\
2828: 178\end{array}$ & $\begin{array}{r}2828.229 \\
1727.513 \\
-385.318\end{array}$ & $\begin{array}{l}135.843 \\
115.843 \\
135.843\end{array}$ & $\begin{array}{r}1450.45 \\
-8979.57 \\
-306.65\end{array}$ & $\begin{array}{r}-\begin{array}{r}382.27 \\
-1242 \\
2625.41\end{array} \\
2625\end{array}$ & $\begin{array}{r}50516.96 \\
-6999.82 \\
-22780.03\end{array}$ \\
\hline 1 & BEND & 3 & 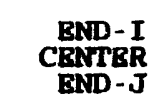 & $\begin{array}{l}466.127 \\
646.581 \\
448.215\end{array}$ & $\begin{array}{r}448.277 \\
-12.665 \\
-466.186\end{array}$ & $\begin{array}{l}517.243 \\
517.243 \\
517.243\end{array}$ & $\begin{array}{r}-915.67 \\
-89798: 12 \\
-2547.79\end{array}$ & $\begin{array}{r}-18067.24 \\
-1154: 01 \\
16635: 34\end{array}$ & $\begin{array}{l}8291.57 \\
2877 \\
8828.92\end{array}$ \\
\hline 2 & TANGRNT & 1 & $\frac{\text { END-I }}{\text { END-J }}$ & $\begin{array}{l}-170.529 \\
-170.529\end{array}$ & $\begin{array}{l}-371.409 \\
-371.109\end{array}$ & 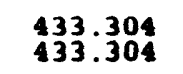 & $\begin{array}{l}27390.99 \\
27390.99\end{array}$ & $\begin{array}{r}6422.13 \\
32095.39\end{array}$ & $\begin{array}{r}-12248.53 \\
9757.46\end{array}$ \\
\hline 2 & TANGENT & 2 & $\frac{\mathrm{END}-\mathrm{I}}{\mathrm{END}-\mathrm{J}}$ & $\begin{array}{l}1551.099 \\
1551: 099\end{array}$ & $\begin{array}{l}-210.643 \\
-210: 643\end{array}$ & $\begin{array}{l}-204.592 \\
-204.592\end{array}$ & $\begin{array}{l}-306.99 \\
-306.99\end{array}$ & $\begin{array}{r}17964.33 \\
5842.25\end{array}$ & $\begin{array}{r}-14251.19 \\
-1770.90\end{array}$ \\
\hline 2 & TANOBNT & 3 & $\begin{array}{l}\text { END-I } \\
\text { END-J }\end{array}$ & $\begin{array}{l}279.227 \\
279.227\end{array}$ & $\begin{array}{l}299.182 \\
299.182\end{array}$ & $\begin{array}{l}-28.936 \\
-28.936\end{array}$ & $\begin{array}{l}-2549.96 \\
-2549.96\end{array}$ & $\begin{array}{l}5378.31 \\
3663.85\end{array}$ & $\begin{array}{r}17864.29 \\
137.74\end{array}$ \\
\hline 3 & BEND & 1 & 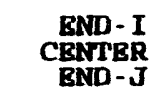 & $\begin{array}{l}-108.279 \\
6684.449 \\
1076.236\end{array}$ & $\begin{array}{r}1076.236 \\
837.578 \\
108.279\end{array}$ & $\begin{array}{l}306.941 \\
306.941 \\
306.941\end{array}$ & $\begin{array}{r}27390.99 \\
15165.82 \\
-549.24\end{array}$ & $\begin{array}{r}-9757.46 \\
-19756.73 \\
-18182.77\end{array}$ & $\begin{array}{r}32095.39 \\
8313.55 \\
-3440.05\end{array}$ \\
\hline 3 & BRND & 2 & $\begin{array}{l}\text { RND-I } \\
\text { CENRBR } \\
\text { END -J }\end{array}$ & $\begin{array}{r}299.359 \\
524: 009 \\
111.702\end{array}$ & $\begin{array}{r}441.702 \\
100 \\
-299.652 \\
-259\end{array}$ & $\begin{array}{l}-97.203 \\
-97.203 \\
-97.203\end{array}$ & $\begin{array}{r}-306.99 \\
191.04 \\
-1145.18\end{array}$ & $\begin{array}{r}\begin{array}{r}1770.90 \\
-592 \\
-2609.69\end{array}\end{array}$ & $\begin{array}{l}5842.25 \\
7997 \\
1571.26\end{array}$ \\
\hline 3 & BEND & 3 & $\begin{array}{c}\text { END-I } \\
\text { CRNTRR } \\
\text { END-J }\end{array}$ & $\begin{array}{l}112.047 \\
256.972 \\
251.366\end{array}$ & $\begin{array}{r}251.366 \\
98.513 \\
-112.047\end{array}$ & $\begin{array}{l}169.182 \\
116.182 \\
169.182\end{array}$ & $\begin{array}{r}-2549.96 \\
-413 \\
-4937.73\end{array}$ & $\begin{array}{l}-137.74 \\
5294: 59 \\
7625.13\end{array}$ & $\begin{array}{r}3663.85 \\
-6033 \\
-515.72\end{array}$ \\
\hline 4 & TANGRNT & 1 & $\begin{array}{l}\text { END-I } \\
\text { END-J }\end{array}$ & $\begin{array}{r}2186.716 \\
2186.716\end{array}$ & $\begin{array}{l}72.599 \\
72.599\end{array}$ & $\begin{array}{l}787.481 \\
787.481\end{array}$ & $\begin{array}{l}-549.24 \\
-549.24\end{array}$ & $\begin{array}{r}-18182.77 \\
5441.66\end{array}$ & $\begin{array}{l}-3440.05 \\
-5618.02\end{array}$ \\
\hline 4 & TANGENT & 2 & $\begin{array}{l}\text { END-I } \\
\text { END-J }\end{array}$ & $\begin{array}{l}373.192 \\
373.192\end{array}$ & $\begin{array}{l}204.201 \\
204.201\end{array}$ & $\begin{array}{l}-44.043 \\
-44.043\end{array}$ & $\begin{array}{l}-1145.18 \\
-1145.18\end{array}$ & $\begin{array}{r}-2609.09 \\
-3930.38\end{array}$ & $\begin{array}{r}-1571.95 \\
-6554.08\end{array}$ \\
\hline 4 & TANGENT & 3 & $\begin{array}{l}\text { END-I } \\
\text { END-J }\end{array}$ & $\begin{array}{l}411.206 \\
111: 206\end{array}$ & $\begin{array}{l}-19.147 \\
-19.147\end{array}$ & $\begin{array}{l}-224.878 \\
-224.878\end{array}$ & $\begin{array}{l}4937.73 \\
4937.73\end{array}$ & $\begin{array}{r}7625.43 \\
879.10\end{array}$ & $\begin{array}{r}-515.72 \\
58.69\end{array}$ \\
\hline 5 & TANGENT & 1 & $\begin{array}{l}\text { RND-I } \\
\text { END -J }\end{array}$ & $\begin{array}{l}3049.966 \\
3049.966\end{array}$ & $\begin{array}{l}46.299 \\
46.299\end{array}$ & $\begin{array}{l}982.571 \\
982.571\end{array}$ & $\begin{array}{l}-549.24 \\
-549.24\end{array}$ & $\begin{array}{r}5441.66 \\
36918.78\end{array}$ & $\begin{array}{l}-5618.02 \\
-7006.98\end{array}$ \\
\hline 5 & TANGENT & 2 & $\frac{\text { END- }}{\text { END-J }}$ & $\begin{array}{l}321.792 \\
321.792\end{array}$ & $\begin{array}{l}266.491 \\
266.491\end{array}$ & $\begin{array}{l}-16.733 \\
-16.733\end{array}$ & $\begin{array}{l}-1145.18 \\
-1145.18\end{array}$ & $\begin{array}{l}-3930.38 \\
-4432.37\end{array}$ & $\begin{array}{r}-4554.08 \\
-12548.81\end{array}$ \\
\hline 5 & TANGENT & 3 & $\begin{array}{l}\text { END-I } \\
\text { END-J }\end{array}$ & $\begin{array}{l}535.386 \\
535.386\end{array}$ & $\begin{array}{l}15.463 \\
15.863\end{array}$ & $\begin{array}{l}-396.968 \\
-396.968\end{array}$ & $\begin{array}{l}4937.73 \\
4937.73\end{array}$ & $\begin{array}{r}879 \\
-11029: 94\end{array}$ & $\begin{array}{r}58.69 \\
-105.20\end{array}$ \\
\hline
\end{tabular}




\begin{tabular}{|c|c|c|c|c|c|c|c|c|c|}
\hline $\begin{array}{l}\text { BLRQRRNT } \\
\text { NUTERRR }\end{array}$ & 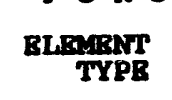 & $\underset{C A B B}{L A A D}$ & STATION & $\begin{array}{l}\text { AXIAY } \\
\text { FORCB }\end{array}$ & $\begin{array}{l}\text { Y-AXXS } \\
\text { SHIBAR }\end{array}$ & $\begin{array}{c}\text { Z-AXIS } \\
\text { SHIRARR }\end{array}$ & $\begin{array}{c}\text { TORSTONAL } \\
\text { MOURATIT }\end{array}$ & 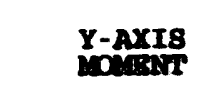 & 2-Axis \\
\hline 6 & TANGENT & 1 & $\frac{\text { RND-I }}{\text { BND-J }}$ & $\begin{array}{l}424.956 \\
1224.956\end{array}$ & $\begin{array}{l}10.609 \\
10.609\end{array}$ & $\begin{array}{l}999.521 \\
999.521\end{array}$ & $\begin{array}{l}-549.24 \\
-549.24\end{array}$ & $\begin{array}{r}34918.78 \\
52910.16\end{array}$ & $\begin{array}{l}-7006.98 \\
-7197.94\end{array}$ \\
\hline 6 & TAMGRNT & 2 & $\frac{\operatorname{RND}-\mathbf{I}}{\mathrm{END}-\mathrm{J}}$ & $\begin{array}{l}254: 402 \\
254: 402\end{array}$ & $\begin{array}{l}106.781 \\
106.781\end{array}$ & $\begin{array}{l}8.837 \\
8.837\end{array}$ & $\begin{array}{l}-1145.18 \\
-1145.18\end{array}$ & $\begin{array}{r}-4432.37 \\
-1273.30\end{array}$ & $\begin{array}{l}-12548.81 \\
-14770: 87\end{array}$ \\
\hline 6 & TANGRNT & 3 & $\frac{\mathrm{END}-\mathrm{I}}{\mathrm{END}-\mathrm{J}}$ & $\begin{array}{l}704.256 \\
704.256\end{array}$ & $\begin{array}{l}23.333 \\
23.333\end{array}$ & $\begin{array}{l}-398.328 \\
-398.328\end{array}$ & $\begin{array}{r}4937.73 \\
1937.73\end{array}$ & $\begin{array}{l}-11029.94 \\
-18199.84\end{array}$ & $\begin{array}{l}-805.20 \\
-825.20\end{array}$ \\
\hline 7 & TANGBNT & 1 & $\frac{\operatorname{RND}-\mathbf{I}}{\operatorname{RND}-J}$ & $\begin{array}{l}5711.956 \\
5711.956\end{array}$ & $\begin{array}{l}-34.941 \\
-34.941\end{array}$ & $\begin{array}{l}842.021 \\
842.021\end{array}$ & $\begin{array}{l}-549.24 \\
-549.24\end{array}$ & $\begin{array}{l}52910.16 \\
68066: 53\end{array}$ & $\begin{array}{l}-71979.94 \\
-6569.00\end{array}$ \\
\hline 7 & TANGENT & 2 & $\frac{\mathrm{END}-\mathrm{I}}{\mathrm{END}-\mathrm{J}}$ & $\begin{array}{l}170.062 \\
170: 062\end{array}$ & $\begin{array}{l}-123.539 \\
-123.539\end{array}$ & $\begin{array}{l}38.117 \\
38.117\end{array}$ & $\begin{array}{l}-1145.18 \\
-115.18\end{array}$ & $\begin{array}{l}-4273.30 \\
-3587.19\end{array}$ & $\begin{array}{l}-14470.87 \\
-12217.17\end{array}$ \\
\hline 7 & TAMGRNT & 3 & $\frac{\operatorname{RND}-\mathbf{I}}{\operatorname{BND}-\mathrm{J}}$ & 9177866 & $\begin{array}{l}14.833 \\
14.833\end{array}$ & $\begin{array}{r}-197.678 \\
-197.678\end{array}$ & $\begin{array}{l}4937.73 \\
4937.73\end{array}$ & $\begin{array}{l}-18199.84 \\
-21758.03\end{array}$ & $\begin{array}{l}-825.20 \\
-1092.19\end{array}$ \\
\hline 8 & TANGBNT & 1 & $\begin{array}{l}\text { RND - I } \\
\operatorname{RND}-J\end{array}$ & $\begin{array}{l}7144.736 \\
7144.736\end{array}$ & $\begin{array}{l}-79.241 \\
-79.241\end{array}$ & $\begin{array}{l}524.891 \\
524.891\end{array}$ & $\begin{array}{l}-549.24 \\
-549.24\end{array}$ & $\begin{array}{r}68066.53 \\
93292.78\end{array}$ & $\begin{array}{l}-6569.00 \\
-2760.66\end{array}$ \\
\hline 8 & TANGRNT & 2 & $\frac{\operatorname{RND}-I}{\operatorname{RND}-J}$ & $\begin{array}{l}89.742 \\
89.742\end{array}$ & $\begin{array}{l}-300.779 \\
-300.779\end{array}$ & $\begin{array}{l}65.747 \\
65.747\end{array}$ & $\begin{array}{l}-1145.18 \\
-1145.18\end{array}$ & $\begin{array}{l}-3587.19 \\
-427.38\end{array}$ & $\begin{array}{r}-12247.17 \\
2208.26\end{array}$ \\
\hline 8 & TANGRAT & 3 & $\frac{\operatorname{RND}-\mathrm{I}}{\mathrm{RND}-\mathrm{J}}$ & $\begin{array}{l}1123.556 \\
1123.556\end{array}$ & $\begin{array}{l}-5.677 \\
-5.677\end{array}$ & $\begin{array}{l}200.432 \\
200.432\end{array}$ & $\begin{array}{r}4937.73 \\
4937.73\end{array}$ & $\begin{array}{l}-21758.03 \\
-12125.26\end{array}$ & $\begin{array}{l}-1092.19 \\
-019.36\end{array}$ \\
\hline 9 & BBND & 1 & $\begin{array}{c}\text { END-I } \\
\text { CENPR } \\
\text { CEND-J }\end{array}$ & $\begin{array}{r}8503.926 \\
5937.241 \\
-107.399\end{array}$ & $\begin{array}{r}-107.399 \\
-6069.126 \\
-8503.926\end{array}$ & $\begin{array}{l}120.851 \\
120.851 \\
120.851\end{array}$ & $\begin{array}{r}-549.24 \\
-1278 \\
864.56\end{array}$ & $\begin{array}{r}-2760.66 \\
999 \\
4179.78\end{array}$ & 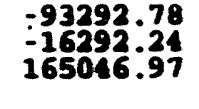 \\
\hline 9 & BEND & 2 & $\begin{array}{c}\text { RND-I } \\
\text { CRNTRR } \\
\text { CRND-J }\end{array}$ & $\begin{array}{l}18.702 \\
80.140 \\
9=057\end{array}$ & $\begin{array}{r}95.057 \\
53.991 \\
-18.702\end{array}$ & $\begin{array}{l}-166.911 \\
-166.911 \\
-166.911\end{array}$ & $\begin{array}{l}-1145.18 \\
-714.900 \\
-2799.07\end{array}$ & $\begin{array}{r}2208.26 \\
-1169 \\
-3062.15 \\
-3062.15\end{array}$ & $\begin{array}{r}-127.38 \\
-1824.75 \\
-1863.27\end{array}$ \\
\hline 9 & BRND & 3 & $\begin{array}{c}\text { END-I } \\
\text { CENTER } \\
\text { BNDD-J }\end{array}$ & $\begin{array}{l}1317.746 \\
1755.273 \\
1150.142\end{array}$ & $\begin{array}{r}1150.442 \\
-118: 302 \\
-1317.746\end{array}$ & $\begin{array}{l}15.947 \\
15: 947 \\
15.947\end{array}$ & $\begin{array}{r}4937.73 \\
3052 \\
-340.95\end{array}$ & $\begin{array}{r}-819.36 \\
-3732 \\
-4159.59 \\
\end{array}$ & $\begin{array}{l}12125.26 \\
17004.54 \\
1710.37\end{array}$ \\
\hline 10 & TANGRAT & 1 & $\begin{array}{l}\text { END-I } \\
\text { END-J }\end{array}$ & $\begin{array}{l}-859.259 \\
-859.259\end{array}$ & $\begin{array}{l}50.825 \\
50.825\end{array}$ & $\begin{array}{l}2891.984 \\
2891.984\end{array}$ & $\begin{array}{l}864.87 \\
864.87\end{array}$ & $\begin{array}{r}-165046.97 \\
38664.41\end{array}$ & $\begin{array}{r}4174.78 \\
594: 69\end{array}$ \\
\hline 10 & TANGERT & 2 & $\begin{array}{l}\text { ENDD-I } \\
\text { RND -J }\end{array}$ & $\begin{array}{l}67.727 \\
67.727\end{array}$ & $\begin{array}{l}-248.141 \\
-248.141\end{array}$ & $\begin{array}{l}-54.625 \\
-54.625\end{array}$ & $\begin{array}{l}-2799.07 \\
-2799.07\end{array}$ & $\begin{array}{r}1863.27 \\
-1964.50\end{array}$ & $\begin{array}{l}-3862.15 \\
\mathbf{1 3 6 1 6 . 9 3}\end{array}$ \\
\hline 10 & TANGRNT & 3 & $\begin{array}{l}\mathrm{END}-\mathbf{I} \\
\mathrm{BNN}-\mathrm{J}\end{array}$ & $\begin{array}{l}2678.9822 \\
2678.982\end{array}$ & $\begin{array}{l}-123.296 \\
-123.296\end{array}$ & $\begin{array}{l}298.093 \\
298.093\end{array}$ & $\begin{array}{l}-340.95 \\
-340.95\end{array}$ & $\begin{array}{r}-17144.37 \\
3853.30\end{array}$ & $\begin{array}{l}-4459.32 \\
425.64\end{array}$ \\
\hline 11 & TANGRNT & 1 & $\begin{array}{l}\mathrm{END}-\mathrm{I} \\
\mathrm{END}-\mathrm{J}\end{array}$ & $\begin{array}{l}-1757.239 \\
-1757: 239\end{array}$ & $\begin{array}{l}73.675 \\
73.675\end{array}$ & $\begin{array}{l}1162.214 \\
1162.214\end{array}$ & $\begin{array}{l}864: 87 \\
864: 87\end{array}$ & $\begin{array}{r}38664.11 \\
120530.79\end{array}$ & $\begin{array}{r}594.69 \\
-1594.95\end{array}$ \\
\hline
\end{tabular}




\begin{tabular}{|c|c|c|c|c|c|c|c|c|c|}
\hline 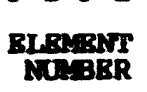 & $\begin{array}{c}\text { BLapers } \\
\text { TYPB }\end{array}$ & LAAD & STATION & MXIAL & $\begin{array}{c}\text { Y-AXIS } \\
\text { SHEAR }\end{array}$ & $\begin{array}{l}\text { 2-AXIS } \\
\text { SHRARR }\end{array}$ & TORSIOMALI & y-Axis & 2.-Axrs \\
\hline 11 & TANGRAT & 2 & $\frac{\operatorname{End}}{\operatorname{ExD}-\mathbf{x}}$ & $\begin{array}{l}38.087 \\
36: 087\end{array}$ & $\begin{array}{l}248.429 \\
248.429\end{array}$ & $\begin{array}{l}.445 \\
.415\end{array}$ & $\begin{array}{l}-2799.07 \\
-2799.07\end{array}$ & $\begin{array}{l}-1984.50 \\
-1953.14\end{array}$ & $\begin{array}{r}13616.93 \\
-3882.38\end{array}$ \\
\hline 11 & TANORNT & 3 & $\frac{R M D}{R M D}-\mathbf{I}$ & $\begin{array}{l}4516.562 \\
4516.562\end{array}$ & $\begin{array}{l}-29.466 \\
-29.166\end{array}$ & $\begin{array}{l}120.973 \\
120.973\end{array}$ & $\begin{array}{l}-340.95 \\
-340.95\end{array}$ & $\begin{array}{r}3853.30 \\
12374.64\end{array}$ & $\begin{array}{l}1225.64 \\
6301.21\end{array}$ \\
\hline 12 & TANGRNT & 1 & $\begin{array}{l}\text { END-I } \\
\text { END-J }\end{array}$ & $\begin{array}{r}-2382.019 \\
-2382.019\end{array}$ & $\begin{array}{l}70.605 \\
70.605\end{array}$ & $\begin{array}{l}-178.736 \\
-178.736\end{array}$ & $\begin{array}{l}864.87 \\
864.87\end{array}$ & $\begin{array}{l}120530.79 \\
116958.22\end{array}$ & $\begin{array}{l}-4594.95 \\
-6006.20\end{array}$ \\
\hline 12 & TANGRAT & 2 & $\begin{array}{l}\text { END-I } \\
\text { END-J }\end{array}$ & $\begin{array}{l}19.777 \\
19: 777\end{array}$ & 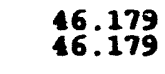 & $\begin{array}{l}82.365 \\
82.365\end{array}$ & $\begin{array}{l}-2799.87 \\
-2799.07\end{array}$ & $\begin{array}{l}-1953.14 \\
-306.82\end{array}$ & $\begin{array}{r}-3862.38 \\
-1805.40\end{array}$ \\
\hline 12 & TANGRMT & 3 & END-I & $\begin{array}{l}5805.722 \\
5805.722\end{array}$ & $\begin{array}{l}55.464 \\
55.164\end{array}$ & $\begin{array}{l}-99.757 \\
-99.757\end{array}$ & $\begin{array}{l}-340.95 \\
-340.95\end{array}$ & 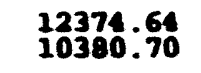 & $\begin{array}{l}6301.21 \\
5192.59\end{array}$ \\
\hline 13 & TANGBRT & 1 & 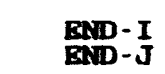 & $\begin{array}{l}-2711.359 \\
-2711.359\end{array}$ & $\begin{array}{l}66.785 \\
66.785\end{array}$ & $\begin{array}{l}-9901.486 \\
-901.486\end{array}$ & $\begin{array}{l}864.87 \\
864: 87\end{array}$ & $\begin{array}{l}116958.22 \\
101622.15\end{array}$ & $\begin{array}{r}-6006.20 \\
-7142.34\end{array}$ \\
\hline 13 & TANGBRMT & 2 & $\begin{array}{l}\operatorname{Ran}-I \\
\operatorname{RND}-J\end{array}$ & $\begin{array}{l}10.127 \\
10.127\end{array}$ & $\begin{array}{l}-16.051 \\
-16.051\end{array}$ & $\begin{array}{l}121.065 \\
121.065\end{array}$ & $\begin{array}{l}-2799.07 \\
-2799.07\end{array}$ & $\begin{array}{l}-306.82 \\
1752.74\end{array}$ & $\begin{array}{l}-4805.40 \\
-4532.33\end{array}$ \\
\hline 13 & TANORNT & 3 & $\begin{array}{l}\mathrm{END}-\mathrm{I} \\
\mathrm{END}-\mathrm{J}\end{array}$ & $\begin{array}{l}6486.812 \\
6486.812\end{array}$ & $\begin{array}{l}91.674 \\
91.674\end{array}$ & $\begin{array}{l}-196.267 \\
-196.267\end{array}$ & $\begin{array}{l}-340.95 \\
-340.95\end{array}$ & $\begin{array}{r}10380.70 \\
7041.81\end{array}$ & $\begin{array}{l}5192.59 \\
3633.03\end{array}$ \\
\hline 14 & BEND & 1 & 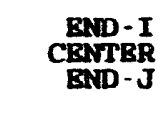 & $\begin{array}{r}-1429.203 \\
-995.280 \\
21.757\end{array}$ & $\begin{array}{r}21.569 \\
1025: 916 \\
1429.200\end{array}$ & $\begin{array}{l}-933.9966 \\
-933.9996 \\
-933.996\end{array}$ & $\begin{array}{r}-11524.85 \\
5902.43 \\
3455.30\end{array}$ & $\begin{array}{r}31477.37 \\
11591: 87 \\
-16499.19\end{array}$ & $\begin{array}{r}33297.48 \\
202799 \\
-10231.33\end{array}$ \\
\hline 14 & BEND & 2 & 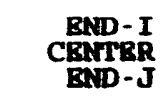 & $\begin{array}{r}391.121 \\
2324 \\
2895.369\end{array}$ & $\begin{array}{l}2895.141 \\
1770.659 \\
-391.502\end{array}$ & $\begin{array}{l}-181.720 \\
-181.720 \\
-181.720\end{array}$ & $\begin{array}{r}-2800.66 \\
-2199 \\
-703.57\end{array}$ & $\begin{array}{r}1740.13 \\
1762 \\
-2651.58 \\
-265.58\end{array}$ & $\begin{array}{r}51547.82 \\
-66460 \\
-23580.24\end{array}$ \\
\hline 14 & BEND & 3 & 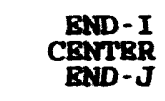 & $\begin{array}{l}-575.832 \\
-860.625 \\
-641.195\end{array}$ & $\begin{array}{r}-641.271 \\
576.216 \\
575.916\end{array}$ & $\begin{array}{l}291.231 \\
291 \\
291.231 \\
\end{array}$ & $\begin{array}{r}-8082.02 \\
-13501.52 \\
-5091.96\end{array}$ & $\begin{array}{r}-14631: 10 \\
1154.51 \\
16820.88\end{array}$ & \\
\hline 15 & TANGRNT & 1 & END-I & $\begin{array}{l}-14.571 \\
-14.571\end{array}$ & $\begin{array}{l}-257.114 \\
-257.114\end{array}$ & $\begin{array}{l}252.024 \\
252.024\end{array}$ & $\begin{array}{l}3457.48 \\
3457.48\end{array}$ & $\begin{array}{l}19991.01 \\
33033.46\end{array}$ & $\begin{array}{r}-4431.73 \\
10802.29\end{array}$ \\
\hline 15 & TANGEMT & 2 & $\frac{\operatorname{END}-\mathbf{I}}{\mathrm{END}-\mathrm{J}}$ & $\begin{array}{l}1575.951 \\
1575.951\end{array}$ & $\begin{array}{l}237.510 \\
237.510\end{array}$ & $\begin{array}{l}-199.548 \\
-199.548\end{array}$ & $\begin{array}{l}-703.48 \\
-703.46\end{array}$ & $\begin{array}{r}18548.76 \\
6725.51\end{array}$ & $\begin{array}{r}14798.73 \\
726.28\end{array}$ \\
\hline 15 & TANGENT & 3 & ENDD-I & $\begin{array}{l}-461.541 \\
-161.541\end{array}$ & $\begin{array}{l}322.764 \\
322.764\end{array}$ & $\begin{array}{l}191.773 \\
191.773\end{array}$ & $\begin{array}{l}-5894.17 \\
-5894.17\end{array}$ & $\begin{array}{r}-6928.822 \\
-1633.75\end{array}$ & $\begin{array}{l}1695.39 \\
-2265.39\end{array}$ \\
\hline 16 & BEND & 1 & 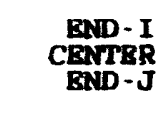 & $\begin{array}{r}-48.821 \\
8266.660 \\
1217.896\end{array}$ & $\begin{array}{r}1217.896 \\
895: 704 \\
48.821\end{array}$ & $\begin{array}{l}194.456 \\
194.156 \\
194.156\end{array}$ & $\begin{array}{r}3457.48 \\
-3484.92 \\
-4968.61\end{array}$ & $\begin{array}{r}-10902.29 \\
-5955: 15 \\
2376.20\end{array}$ & $\begin{array}{l}33833.44 \\
3568: 99 \\
-4168.07\end{array}$ \\
\hline 16 & BRND & 2 & $\begin{array}{l}\text { RND-I } \\
\text { CENTRR } \\
\text { RND -J }\end{array}$ & $\begin{array}{l}273.851 \\
475: 981 \\
399.288\end{array}$ & $\begin{array}{r}399.288 \\
88.698 \\
-273.851\end{array}$ & $\begin{array}{l}56.790 \\
566.790 \\
56.790\end{array}$ & $\begin{array}{r}-703.48 \\
-5111: 99 \\
977.42\end{array}$ & $\begin{array}{l}726.28 \\
1120 \\
2607.58 \\
\end{array}$ & $\begin{array}{l}6725.51 \\
6961.60 \\
2962.39\end{array}$ \\
\hline
\end{tabular}




\begin{tabular}{|c|c|c|c|c|c|c|c|c|c|}
\hline 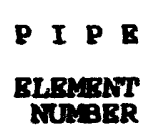 & $\begin{array}{l}\text { BLEGEMT } \\
\text { TYPPE }\end{array}$ & $\begin{array}{l}\text { LOAD } \\
\text { CASB }\end{array}$ & $\begin{array}{l}\text { A N D M M } \\
\text { STATION }\end{array}$ & AXIAL & $\begin{array}{c}\text { Y-AXIS } \\
\text { SkRRAR }\end{array}$ & $\begin{array}{l}\text { 2-AXIS } \\
\text { SHEAR }\end{array}$ & $\begin{array}{l}\text { TORSIONaL } \\
\text { HODeant }\end{array}$ & . & 2-4xis \\
\hline 16 & BEND & 3 & $\begin{array}{c}\text { END-I } \\
\text { CENTRR } \\
\text { END-J }\end{array}$ & $\begin{array}{l}-280.341 \\
-269.397 \\
-100.643\end{array}$ & $\begin{array}{r}-100.643 \\
127.605 \\
280.341\end{array}$ & $\begin{array}{l}54.184 \\
54.184 \\
54.184\end{array}$ & $\begin{array}{r}-5894.17 \\
-2099 \\
3890.91\end{array}$ & $\begin{array}{l}2265.39 \\
6919: 10 \\
7519.70\end{array}$ & $\begin{array}{r}4433.75 \\
4105 \\
4057.19\end{array}$ \\
\hline 17 & TANGEMT & 1 & $\begin{array}{l}\frac{\mathrm{RND}-\mathrm{I}}{\mathrm{RND}-\mathrm{J}} \\
\mathrm{BN}\end{array}$ & $\begin{array}{l}2286.176 \\
2286.176\end{array}$ & $\begin{array}{l}61.091 \\
61.091\end{array}$ & $\begin{array}{l}538.056 \\
538.096\end{array}$ & $\begin{array}{r}-4968.61 \\
-4968.61\end{array}$ & $\begin{array}{r}2376.20 \\
18519: 07\end{array}$ & $\begin{array}{l}-4168.07 \\
-6000.82\end{array}$ \\
\hline 17 & TANGBRT & 2 & $\frac{\text { END- }}{\text { BND-J }}$ & $\begin{array}{l}340.368 \\
340: 368\end{array}$ & $\begin{array}{l}322.559 \\
322.559\end{array}$ & $\begin{array}{l}-2.160 \\
-2.160\end{array}$ & 977.42 & $\begin{array}{l}2407.17 \\
2312.37\end{array}$ & $\begin{array}{r}2962.39 \\
-6714.39\end{array}$ \\
\hline 17 & Tanogars & 3 & RND-I & $\begin{array}{l}-3.803 \\
-3.803\end{array}$ & $\begin{array}{l}170.521 \\
170.521\end{array}$ & $\begin{array}{l}-398.956 \\
-398.956\end{array}$ & $\begin{array}{l}3890.91 \\
3890.91\end{array}$ & $\begin{array}{r}7519: 20 \\
-1418.97\end{array}$ & $\begin{array}{r}-957.19 \\
-6072.82\end{array}$ \\
\hline 18 & TANGRNT & 1 & $\frac{\operatorname{RND}-\mathrm{I}}{\mathrm{END}-\mathrm{J}}$ & $\begin{array}{l}3117.956 \\
3117: 956\end{array}$ & $\begin{array}{l}51.471 \\
51.171\end{array}$ & $\begin{array}{l}686.7866 \\
686: 726\end{array}$ & $\begin{array}{r}-4968.61 \\
-4968.61\end{array}$ & $\begin{array}{l}18519.07 \\
39122.65\end{array}$ & $\begin{array}{l}-6000.82 \\
-7541.96\end{array}$ \\
\hline 18 & TAMGgNT & 2 & $\begin{array}{l}\text { END-I } \\
\text { BND-J }\end{array}$ & $\begin{array}{l}296.1888 \\
296.188\end{array}$ & $\begin{array}{l}462.689 \\
162.689\end{array}$ & $\begin{array}{l}-29.970 \\
-29.970\end{array}$ & $\begin{array}{l}977.12 \\
977.12\end{array}$ & $\begin{array}{l}2342.37 \\
1443.26\end{array}$ & $\begin{array}{r}-6714.39 \\
-20595.06\end{array}$ \\
\hline 18 & TANGRNT & 3 & $\begin{array}{l}\text { ENDI-I } \\
\text { END -J }\end{array}$ & $\begin{array}{l}71.897 \\
71.897\end{array}$ & $\begin{array}{l}95.151 \\
95.151\end{array}$ & $\begin{array}{l}-567.266 \\
-567.266\end{array}$ & $\begin{array}{l}3890.91 \\
3890.91\end{array}$ & $\begin{array}{r}-4448.97 \\
-21466.95\end{array}$ & $\begin{array}{l}-6072.82 \\
-8927.34\end{array}$ \\
\hline 19 & TANGBart & 1 & $\begin{array}{l}\text { RND-I } \\
\text { BND -J }\end{array}$ & $\begin{array}{r}\mathbf{4} 52.706 \\
\mathbf{2 5 2} 2.706\end{array}$ & $\begin{array}{l}20.871 \\
20.871\end{array}$ & $\begin{array}{l}708.186 \\
708.186\end{array}$ & $\begin{array}{r}-4968.61 \\
-4968.61\end{array}$ & $\begin{array}{l}39122.65 \\
51869: 99\end{array}$ & $\begin{array}{r}-7544.96 \\
-7920.65\end{array}$ \\
\hline 19 & TANGBNT & 2 & ENDD-I & $\begin{array}{l}238.348 \\
238.348\end{array}$ & $\begin{array}{l}335.279 \\
335.279\end{array}$ & $\begin{array}{l}-45.890 \\
-45.890\end{array}$ & 9777.12 & $\begin{array}{r}1443.26 \\
617.24\end{array}$ & $\begin{array}{l}-20595.06 \\
-26630.09\end{array}$ \\
\hline 19 & TANGENT & 3 & $\begin{array}{l}\text { END-I } \\
\text { END - J }\end{array}$ & $\begin{array}{l}175.527 \\
175.527\end{array}$ & $\begin{array}{l}11.341 \\
11.141\end{array}$ & $\begin{array}{l}-504.096 \\
-504.096\end{array}$ & $\begin{array}{l}3890.91 \\
3890.91\end{array}$ & $\begin{array}{l}-21466.95 \\
-30540.67\end{array}$ & $\begin{array}{l}-9927.34 \\
-9127.88\end{array}$ \\
\hline 20 & TANGRArT & 1 & $\frac{\text { RND -I }}{\text { END-J }}$ & $\begin{array}{l}5690.106 \\
5690.106\end{array}$ & $\begin{array}{l}-23.719 \\
-23.719\end{array}$ & $\begin{array}{l}592.606 \\
592.606\end{array}$ & $\begin{array}{r}-4968.61 \\
-4968.61\end{array}$ & $\begin{array}{l}51869.99 \\
62536.90\end{array}$ & $\begin{array}{r}-7920.65 \\
-7493.71\end{array}$ \\
\hline 20 & TAMGRNT & 2 & $\frac{\operatorname{RND}-\mathbf{I}}{\operatorname{BND}-\mathbf{J}}$ & $\begin{array}{l}166.008 \\
166: 008\end{array}$ & $\begin{array}{l}33.969 \\
33.969\end{array}$ & $\begin{array}{r}-53.470 \\
-53.470\end{array}$ & 977.42 & $\begin{array}{r}617.24 \\
-345.23\end{array}$ & $\begin{array}{r}-26630.09 \\
-27261.53\end{array}$ \\
\hline 20 & TANGBNT & 3 & $\begin{array}{l}\text { RNDD-I } \\
\text { ENDD-J }\end{array}$ & $\begin{array}{l}306.897 \\
306: 897\end{array}$ & $\begin{array}{r}-76.619 \\
-76.619\end{array}$ & $\begin{array}{l}-189.356 \\
-189.356\end{array}$ & $\begin{array}{l}3890.91 \\
3890.91\end{array}$ & $\begin{array}{l}-30540.67 \\
-33949.07\end{array}$ & $\begin{array}{l}-9127.88 \\
-7746: 74\end{array}$ \\
\hline 21 & TANGRNT & 1 & END-I & $\begin{array}{l}7257.366 \\
7257.366\end{array}$ & $\begin{array}{r}-74.819 \\
-74.819\end{array}$ & $\begin{array}{l}310.856 \\
310.856\end{array}$ & $\begin{array}{r}-4968.61 \\
-4968.61\end{array}$ & $\begin{array}{l}62536.90 \\
81516.51\end{array}$ & $\begin{array}{l}-7493.71 \\
-2925.59\end{array}$ \\
\hline 21 & TANGENT & 2 & RND-I & $\begin{array}{l}89.238 \\
88.238\end{array}$ & $\begin{array}{r}-393.071 \\
-393.071\end{array}$ & $\begin{array}{r}-50.550 \\
-50.550\end{array}$ & 977.42 & $\begin{array}{r}-345.23 \\
-3431.62\end{array}$ & $\begin{array}{r}-27241.53 \\
-3242.19\end{array}$ \\
\hline 21 & TANGEMT & 3 & $\frac{\operatorname{RND}-\mathbf{I}}{\mathrm{END}-\mathrm{J}}$ & $\begin{array}{l}450.217 \\
450.217\end{array}$ & $\begin{array}{r}-149.679 \\
-149.679\end{array}$ & $\begin{array}{l}412.004 \\
412: 004\end{array}$ & $\begin{array}{l}3890.91 \\
3890.91\end{array}$ & $\begin{array}{r}-33949.07 \\
-8793.74\end{array}$ & $\begin{array}{r}-7748.74 \\
1390.06\end{array}$ \\
\hline 22 & TANGENT & 1 & END-I & $\begin{array}{l}8347.116 \\
8367.116\end{array}$ & $\begin{array}{l}-89.839 \\
-89.839\end{array}$ & $\begin{array}{l}-163.634 \\
-163.634\end{array}$ & $\begin{array}{r}-4968.61 \\
-1968.61\end{array}$ & $\begin{array}{l}91516.51 \\
78734.08\end{array}$ & $\begin{array}{l}-2925.59 \\
-1397.98\end{array}$ \\
\hline
\end{tabular}




\begin{tabular}{|c|c|c|c|c|c|c|c|c|c|}
\hline $\begin{array}{l}\text { RLRMESWT } \\
\text { NUMBRR }\end{array}$ & $\begin{array}{l}\text { BLERGMP } \\
\text { TYPE }\end{array}$ & $\underset{C A S Z}{\operatorname{LAAD}}$ & STATION & $\begin{array}{l}\text { AXIALL } \\
\text { PORCE }\end{array}$ & $\begin{array}{c}\mathbf{Y} \text {-AXIS } \\
\text { SHIRAR }\end{array}$ & $\begin{array}{l}\text { z-AXIS } \\
\text { StRARR }\end{array}$ & $\begin{array}{l}\text { TORSIONAL } \\
\text { MOARNT }\end{array}$ & Y-Axrs & 2-Axis \\
\hline 22 & TANGBarT & 2 & $\begin{array}{l}\text { END-I } \\
\text { BND-J }\end{array}$ & $\begin{array}{l}40.038 \\
40: 038\end{array}$ & $\begin{array}{r}-495.931 \\
-495.931\end{array}$ & $\begin{array}{l}-52.760 \\
-52.760\end{array}$ & $977: 12$ & $\begin{array}{r}-3431.62 \\
-4328.75\end{array}$ & $\begin{array}{r}-3242.19 \\
5190.61\end{array}$ \\
\hline 22 & TANGRNT & 3 & $\begin{array}{l}\text { END-I } \\
\text { BND-J }\end{array}$ & $\begin{array}{l}550.037 \\
550.037\end{array}$ & $\begin{array}{l}-131.799 \\
-131.799\end{array}$ & $\begin{array}{l}1351.814 \\
1351.814\end{array}$ & $\begin{array}{l}3890.91 \\
3890.91\end{array}$ & $\begin{array}{r}-8793.74 \\
14192.50\end{array}$ & $\begin{array}{l}\frac{1390.06}{3631.17} \\
\end{array}$ \\
\hline 23 & TANGBNT & 1 & $\frac{\mathrm{END}-\mathbf{I}}{\mathrm{END}-\mathrm{J}}$ & $\begin{array}{l}-3284.8933 \\
-3284: 833\end{array}$ & $\begin{array}{l}-28.884 \\
-28.884\end{array}$ & $\begin{array}{l}-10155.121 \\
-10155.121\end{array}$ & $\begin{array}{l}-533.11 \\
-533.11\end{array}$ & $\begin{array}{r}180356.23 \\
7719.16\end{array}$ & $\begin{array}{l}-2173.73 \\
-1682.70\end{array}$ \\
\hline 23 & TANGBNT & 2 & $\begin{array}{l}\text { END-I } \\
\text { END-.J }\end{array}$ & $\begin{array}{l}-53.683 \\
-53.683\end{array}$ & $\begin{array}{r}-494.292 \\
-494.292\end{array}$ & $\begin{array}{l}119.707 \\
119.707\end{array}$ & $\begin{array}{l}2391.54 \\
2391.54\end{array}$ & $\begin{array}{r}-2576.01 \\
-541.00\end{array}$ & $\begin{array}{r}-5509.75 \\
2893.22\end{array}$ \\
\hline 23 & TANGRNT & 3 & $\frac{\mathrm{END}-\mathrm{I}}{\mathrm{END}-\mathrm{J}}$ & $\begin{array}{l}8687.656 \\
8687.656\end{array}$ & $\begin{array}{l}-8.315 \\
-8.315\end{array}$ & $\begin{array}{l}-829.344 \\
-829.314\end{array}$ & $\begin{array}{l}3290.22 \\
3290.22\end{array}$ & $\begin{array}{r}21234.31 \\
7135.47\end{array}$ & $\begin{array}{l}-257.88 \\
-116.53\end{array}$ \\
\hline 24 & TANGRNT & 1 & $\begin{array}{l}\text { RND-I } \\
\text { END-J }\end{array}$ & $\begin{array}{l}-3587.623 \\
-3587: 623\end{array}$ & $\begin{array}{l}-33.044 \\
-33.044\end{array}$ & $\begin{array}{r}-10818.541 \\
-10818.541\end{array}$ & $\begin{array}{l}-533.11 \\
-533.11\end{array}$ & $\begin{array}{r}7719.16 \\
-176045.15\end{array}$ & $\begin{array}{l}-1682.70 \\
-1118.97\end{array}$ \\
\hline 24 & TANOBNT & 2 & $\begin{array}{l}\text { END-I } \\
\text { END-J }\end{array}$ & $\begin{array}{l}-61.823 \\
-61.823\end{array}$ & $\begin{array}{r}-375.912 \\
-375.912\end{array}$ & $\begin{array}{l}134.577 \\
134.577\end{array}$ & $\begin{array}{l}2391.54 \\
2391.54\end{array}$ & $\begin{array}{r}-541.00 \\
1754.88\end{array}$ & $\begin{array}{r}2893.22 \\
9306.28\end{array}$ \\
\hline 24 & TANGRNT & 3 & $\frac{\operatorname{END} \cdot \mathbf{I}}{\operatorname{RND} \cdot \mathbf{J}}$ & $\begin{array}{l}9315: 706 \\
9315: 706\end{array}$ & $\begin{array}{l}4.785 \\
4.785\end{array}$ & $\begin{array}{l}-859.654 \\
-859.654\end{array}$ & $\begin{array}{l}3290.22 \\
3290.22\end{array}$ & $\begin{array}{r}7135.47 \\
-7530.22\end{array}$ & $\begin{array}{l}-116.53 \\
-198.16\end{array}$ \\
\hline 25 & BEND & 1 & $\begin{array}{l}\text { END-I } \\
\text { CRNTRR } \\
\text { RND-J }\end{array}$ & $\begin{array}{r}-4241.953 \\
-93303.179 \\
-8916.728\end{array}$ & $\begin{array}{r}-8914.728 \\
-3341.151 \\
1241.953\end{array}$ & $\begin{array}{l}-41.674 \\
-11.674 \\
-41.674\end{array}$ & $\begin{array}{l}-533.11 \\
-325 \\
-381.29\end{array}$ & $\begin{array}{r}1118.97 \\
1107.35 \\
-967.15\end{array}$ & $\begin{array}{r}-17645.15 \\
5358 \\
-8625.26\end{array}$ \\
\hline 25 & BEND & 2 & $\begin{array}{l}\text { END-I } \\
\text { CRNRR } \\
\text { CRND-J } \\
\text { BND -J }\end{array}$ & $\begin{array}{l}-78.893 \\
-662.949 \\
-10.131\end{array}$ & $\begin{array}{l}-10.131 \\
48.622 \\
78.893\end{array}$ & $\begin{array}{l}158.898 \\
158.898 \\
158.898\end{array}$ & $\begin{array}{r}2391.54 \\
-3214 \\
-3585.96\end{array}$ & $\begin{array}{r}-9306.28 \\
-42266.73 \\
3328.78\end{array}$ & $\begin{array}{r}1754.88 \\
1180 \\
-720.91 \\
-720\end{array}$ \\
\hline 25 & BEND & 3 & $\begin{array}{c}\text { END-I } \\
\text { CENBR } \\
\text { CRND-J }\end{array}$ & $\begin{array}{r}10674.746 \\
12312: 265 \\
6737.426\end{array}$ & $\begin{array}{r}6737.426 \\
-2784: 106 \\
-10674.746\end{array}$ & $\begin{array}{l}11.985 \\
11: 985 \\
11.985\end{array}$ & $\begin{array}{r}3290.22 \\
25993 \\
629.03 \\
63\end{array}$ & $\begin{array}{r}198 . \cdot 16 \\
-1861 \\
-2858.75 \\
-2858\end{array}$ & $\begin{array}{r}-7530.22 \\
-66500.90 \\
134213.31\end{array}$ \\
\hline 26 & TANGENT & 1 & $\begin{array}{l}\text { END-I } \\
\text { END -J }\end{array}$ & $\begin{array}{l}-8035.568 \\
-8035.568\end{array}$ & $\begin{array}{l}5.241 \\
5.241\end{array}$ & $\begin{array}{l}-229.716 \\
-229.716\end{array}$ & $\begin{array}{l}-381.29 \\
-381.29\end{array}$ & $\begin{array}{r}-8625.26 \\
-19881.34\end{array}$ & $\begin{array}{l}967.15 \\
710.36\end{array}$ \\
\hline 26 & TANGBNT & 2 & END-I & $\begin{array}{l}-5.161 \\
-5.161\end{array}$ & $\begin{array}{l}-617.656 \\
-617.656\end{array}$ & $\begin{array}{l}-8.885 \\
-8.885\end{array}$ & $\begin{array}{l}-3585.96 \\
-3585.96\end{array}$ & $\begin{array}{r}-720.56 \\
-1155.92\end{array}$ & $\begin{array}{l}-3328.78 \\
26936.39\end{array}$ \\
\hline 26 & TANGENT & 3 & $\begin{array}{l}\text { END-I } \\
\text { RND-J }\end{array}$ & $\begin{array}{l}6601.756 \\
6601.756\end{array}$ & $\begin{array}{l}15.625 \\
15.625\end{array}$ & $\begin{array}{l}-1610.117 \\
-1610.117\end{array}$ & $\begin{array}{l}629.63 \\
629.63\end{array}$ & $\begin{array}{r}134213.31 \\
55317.58\end{array}$ & $\begin{array}{l}2858.75 \\
2093.11\end{array}$ \\
\hline 27 & TANGBNT & 1 & $\begin{array}{l}\text { RND-I } \\
\text { BND-J }\end{array}$ & $\begin{array}{r}-7279.468 \\
-7279.168\end{array}$ & $\begin{array}{l}7.641 \\
7.641\end{array}$ & $\begin{array}{l}17.234 \\
17.234\end{array}$ & $\begin{array}{l}-381.29 \\
-381.29\end{array}$ & $\begin{array}{l}-19981.34 \\
-19071.34\end{array}$ & $\begin{array}{l}710.36 \\
351.25\end{array}$ \\
\hline 27 & TANGBNT & 2 & $\frac{\text { END-I }}{\text { END-J }}$ & $\begin{array}{l}-1.101 \\
-1.101\end{array}$ & $\begin{array}{l}349.424 \\
349.424\end{array}$ & $\begin{array}{l}3.625 \\
3.625\end{array}$ & $\begin{array}{l}-3585.96 \\
-3585.96\end{array}$ & $\begin{array}{l}-1155.92 \\
-985.54\end{array}$ & $\begin{array}{l}26936.39 \\
10513.48\end{array}$ \\
\hline
\end{tabular}




\begin{tabular}{|c|c|c|c|c|c|c|c|c|c|}
\hline 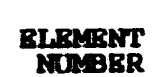 & 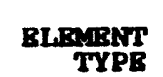 & $\underset{\text { CASB }}{\text { LOAD }}$ & STATION & $\begin{array}{l}\text { AXIAL } \\
\text { BORCE }\end{array}$ & $\begin{array}{l}\mathbf{Y} \text {-AXXS } \\
\text { SHRARR }\end{array}$ & $\begin{array}{l}\text { z-AXIS } \\
\text { SHBRAR }\end{array}$ & TORSIONAI & Y-Axis & 2-4xis \\
\hline 27 & TANGRNT & 3 & END-I & $\begin{array}{l}6471.786 \\
6471.786\end{array}$ & $\begin{array}{l}23.895 \\
23.895\end{array}$ & $\begin{array}{l}-682.357 \\
-682.357\end{array}$ & $\begin{array}{l}629.63 \\
629: 63\end{array}$ & $\begin{array}{r}55317.58 \\
23246: 81\end{array}$ & $\begin{array}{r}2093.11 \\
970.04\end{array}$ \\
\hline 28 & TANGENT & 1 & $\begin{array}{l}\text { END-I } \\
\text { END-J }\end{array}$ & $\begin{array}{l}-6579.168 \\
-6579.168\end{array}$ & $\begin{array}{r}8.381 \\
8.381\end{array}$ & $\begin{array}{l}230.774 \\
230.774\end{array}$ & $\begin{array}{l}-381.29 \\
-381.29\end{array}$ & $\begin{array}{l}-19071.34 \\
-8224: 95\end{array}$ & $\begin{array}{r}351.25 \\
-12.64\end{array}$ \\
\hline 28 & TANGRNT & 2 & $\begin{array}{l}\text { RND-I } \\
\text { BND-J }\end{array}$ & $\begin{array}{l}2.449 \\
2.419\end{array}$ & $\begin{array}{l}439.714 \\
439.714\end{array}$ & $\begin{array}{l}14.035 \\
14.035\end{array}$ & $\begin{array}{l}-3585.96 \\
-3585.96\end{array}$ & $\begin{array}{r}-985.54 \\
-325.89\end{array}$ & $\begin{array}{r}10513.48 \\
-10153.05\end{array}$ \\
\hline 28 & TANGRNT & 3 & $\frac{\text { END -I }}{\text { END-.J }}$ & $\begin{array}{l}6338.616 \\
6338.616\end{array}$ & $\begin{array}{l}26.555 \\
26.555\end{array}$ & $\begin{array}{r}-458.347 \\
-458.347\end{array}$ & $\begin{array}{l}629.63 \\
629.63\end{array}$ & $\begin{array}{r}23246.81 \\
1704.51\end{array}$ & $\begin{array}{r}970.04 \\
-278.06\end{array}$ \\
\hline 29 & TANGBNT & 1 & $\begin{array}{l}\text { LNDD-I } \\
\text { END-J }\end{array}$ & $\begin{array}{l}-5917.998 \\
-5917: 998\end{array}$ & $\begin{array}{l}6.051 \\
6.051\end{array}$ & $\begin{array}{l}302.344 \\
302.344\end{array}$ & $\begin{array}{l}-381.29 \\
-381.29\end{array}$ & $\begin{array}{r}-8224.95 \\
5985.22\end{array}$ & $\begin{array}{r}-327.64 \\
-327.02\end{array}$ \\
\hline 29 & TANGRNT & 2 & $\frac{\mathrm{RND}-\mathrm{I}}{\mathrm{END}-\mathrm{J}}$ & $\begin{array}{l}5.569 \\
5.569\end{array}$ & $\begin{array}{l}8.884 \\
8.884\end{array}$ & $\begin{array}{l}16.085 \\
16.085\end{array}$ & $\begin{array}{l}-3585.96 \\
-3585.96\end{array}$ & $\begin{array}{l}-325.89 \\
\mathbf{1 3 0 . 1 1}\end{array}$ & $\begin{array}{r}-10153.05 \\
-10570.58\end{array}$ \\
\hline 29 & TANGRNT & 3 & $\begin{array}{l}\text { END - I } \\
\text { END-J }\end{array}$ & $\begin{array}{l}6199.826 \\
6199: 826\end{array}$ & $\begin{array}{l}19.535 \\
19.535\end{array}$ & $\begin{array}{l}-590.897 \\
-590.897\end{array}$ & $\begin{array}{l}629.63 \\
629.63\end{array}$ & $\begin{array}{r}1704.51 \\
-26067.64\end{array}$ & $\begin{array}{r}-278.06 \\
-1196.21\end{array}$ \\
\hline 30 & TAMarNT & 1 & $\begin{array}{l}\text { ENDD - I } \\
\text { END - J }\end{array}$ & $\begin{array}{l}-5294.988 \\
-5294: 988\end{array}$ & $\begin{array}{l}1.481 \\
1.481\end{array}$ & $\begin{array}{l}234.834 \\
234.834\end{array}$ & $\begin{array}{l}-381.29 \\
-381.29\end{array}$ & $\begin{array}{r}5985.22 \\
17022.42\end{array}$ & $\begin{array}{r}-327.02 \\
-396.61\end{array}$ \\
\hline 30 & TANGRNT & 2 & $\begin{array}{l}\text { END-I } \\
\text { END-J }\end{array}$ & $\begin{array}{l}8.259 \\
8.259\end{array}$ & $\begin{array}{l}-398.086 \\
-398.086\end{array}$ & $\begin{array}{l}10.075 \\
10.075\end{array}$ & $\begin{array}{l}-3585.96 \\
-3585.96\end{array}$ & $\begin{array}{l}430.11 \\
903.64\end{array}$ & $\begin{array}{r}-10570.58 \\
8139.48\end{array}$ \\
\hline 30 & TANGBNT & 3 & $\begin{array}{l}\text { END - I } \\
\text { RND - J }\end{array}$ & $\begin{array}{l}6055.736 \\
6055: 736\end{array}$ & $\begin{array}{l}5.635 \\
5.635\end{array}$ & $\begin{array}{l}-623.367 \\
-623.367\end{array}$ & $\begin{array}{l}629.63 \\
629.63\end{array}$ & $\begin{array}{l}-26067.64 \\
-55365.86\end{array}$ & $\begin{array}{l}-1196.21 \\
-1461.07\end{array}$ \\
\hline 31 & TANGERT & 1 & $\frac{\mathrm{BND}-\mathrm{I}}{\mathrm{BND}-\mathrm{J}}$ & $\begin{array}{r}-1696.148 \\
-1696.148\end{array}$ & $\begin{array}{l}-3.119 \\
-3.119\end{array}$ & $\begin{array}{l}107.104 \\
107.104\end{array}$ & $\begin{array}{l}-381.29 \\
-381.29\end{array}$ & $\begin{array}{l}17022: 42 \\
19914: 23\end{array}$ & $\begin{array}{r}-396.61 \\
-312.39\end{array}$ \\
\hline 31 & TANGENT & 2 & $\frac{\mathrm{END}-\mathbf{I}}{\mathrm{END}-\mathrm{J}}$ & $\begin{array}{l}10.579 \\
10.579\end{array}$ & $\begin{array}{l}-229.166 \\
-229.165\end{array}$ & $\begin{array}{l}1.135 \\
1.135\end{array}$ & $\begin{array}{l}-3585.96 \\
-3585.96\end{array}$ & $\begin{array}{l}903.64 \\
934.29\end{array}$ & $\begin{array}{r}9139.48 \\
16326.98\end{array}$ \\
\hline 31 & TANGRNT & 3 & $\frac{\text { RND - I }}{\text { END - J }}$ & $\begin{array}{l}5903.386 \\
5903.386\end{array}$ & $\begin{array}{l}-8.535 \\
-8.535\end{array}$ & $\begin{array}{l}-136.457 \\
-136.457\end{array}$ & $\begin{array}{l}629.63 \\
625.63\end{array}$ & $\begin{array}{l}-55365.88 \\
-59050.21\end{array}$ & $\begin{array}{l}-1461.07 \\
-1230.63\end{array}$ \\
\hline 32 & TANGERT & 1 & $\begin{array}{l}\text { END-I } \\
\text { END-J }\end{array}$ & $\begin{array}{r}-4094.598 \\
-4094.598\end{array}$ & $\begin{array}{l}-6.819 \\
-6.819\end{array}$ & $\begin{array}{r}-14.666 \\
-14.666\end{array}$ & $\begin{array}{l}-381.29 \\
-381.29\end{array}$ & $\begin{array}{l}19914.23 \\
19518.25\end{array}$ & $\begin{array}{l}-312.39 \\
-128.27\end{array}$ \\
\hline 32 & TAMGRNT & 2 & $\begin{array}{l}\text { END - I } \\
\text { END -J }\end{array}$ & $\begin{array}{l}12.839 \\
12.839\end{array}$ & $\begin{array}{l}127.314 \\
127.314\end{array}$ & $\begin{array}{l}-6.915 \\
-6.915\end{array}$ & $\begin{array}{l}-3585.96 \\
-3585.96\end{array}$ & $\begin{array}{l}934.29 \\
747.58\end{array}$ & $\begin{array}{r}14326.98 \\
2789.51\end{array}$ \\
\hline 32 & TANGENT & 3 & ${ }_{\text {RND }-J}^{\text {RND }}$ & $\begin{array}{l}5746: 436 \\
5746: 436\end{array}$ & $\begin{array}{l}-20.135 \\
-20.135\end{array}$ & $\begin{array}{l}749.993 \\
749.993\end{array}$ & $\begin{array}{l}629.63 \\
629.63\end{array}$ & $\begin{array}{l}-59050.21 \\
-38800.39\end{array}$ & $\begin{array}{r}-1230.63 \\
-686.99\end{array}$ \\
\hline 33 & TANGERT & $x$ & $\begin{array}{l}\mathrm{END}-\mathrm{I} \\
\mathrm{END}-\mathrm{J}\end{array}$ & $\begin{array}{l}-3527.518 \\
-3527.518\end{array}$ & $\begin{array}{l}-9.119 \\
-9.119\end{array}$ & $\begin{array}{l}-106.356 \\
-106.356\end{array}$ & $\begin{array}{l}-381.29 \\
-381.29\end{array}$ & $\begin{array}{l}19518.25 \\
14732.24\end{array}$ & $\begin{array}{r}-128.27 \\
282.10\end{array}$ \\
\hline
\end{tabular}




\begin{tabular}{|c|c|c|c|c|c|c|c|c|c|}
\hline $\begin{array}{l}\text { P I P B } \\
\text { RLRMEST } \\
\text { WUMBRBR }\end{array}$ & BLEMINS & $\begin{array}{c}\text { B S } \\
\text { LOAD } \\
\text { CASB }\end{array}$ & $\begin{array}{l}\text { A N D } \\
\text { STATION }\end{array}$ & $\begin{array}{l}\text { AXIAL } \\
\text { BORCB }\end{array}$ & $\begin{array}{l}\text { Y-AXIS } \\
\text { SHEAR }\end{array}$ & $\begin{array}{r}\text { z-AXIS } \\
\text { SHRARR }\end{array}$ & $\begin{array}{l}\text { TORSIONAL } \\
\text { MOMBNTIT }\end{array}$ & 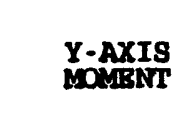 & $\begin{array}{l}\text { 2-AKIS } \\
\text { wotiRNT }\end{array}$ \\
\hline 33 & TANGBNT & 2 & $\begin{array}{l}\text { END-I } \\
\text { END-J }\end{array}$ & 14:889 & $\begin{array}{l}1541.684 \\
1541.684\end{array}$ & $\begin{array}{l}-12.595 \\
-12.595\end{array}$ & $\begin{array}{l}-3585.96 \\
-3585.96\end{array}$ & $\begin{array}{l}747.58 \\
180: 81\end{array}$ & $\begin{array}{r}2789.51 \\
-66586.25\end{array}$ \\
\hline 33 & TANGENT & 3 & END-I & $\begin{array}{l}5594.866 \\
5594: 866\end{array}$ & $\begin{array}{l}-27.615 \\
-27.615\end{array}$ & $\begin{array}{l}1974.253 \\
1974.253\end{array}$ & $\begin{array}{l}629.63 \\
629.63\end{array}$ & $\begin{array}{r}-38800.39 \\
50041.00\end{array}$ & $\begin{array}{r}-686.99 \\
555.67\end{array}$ \\
\hline 34 & TANGENT & 1 & $\begin{array}{l}\text { END-I } \\
\text { END-J }\end{array}$ & $\begin{array}{l}-2725.858 \\
-2725: 858\end{array}$ & $\begin{array}{l}3.394 \\
3.394\end{array}$ & $\begin{array}{l}-424.256 \\
-424.256\end{array}$ & $\begin{array}{l}-381.29 \\
-381.29\end{array}$ & $\begin{array}{r}14732.24 \\
-25996.33\end{array}$ & $\begin{array}{r}282.10 \\
-43: 71\end{array}$ \\
\hline 34 & TANGENT & 2 & $\begin{array}{l}\mathrm{END}-\mathrm{I} \\
\mathrm{END}-\mathrm{J}\end{array}$ & $\begin{array}{l}17.409 \\
17.409\end{array}$ & $\begin{array}{l}-1586.248 \\
-1586.248\end{array}$ & $\begin{array}{l}-9.709 \\
-9.709\end{array}$ & $\begin{array}{l}-3585.96 \\
-3585.96\end{array}$ & $\begin{array}{r}180.81 \\
-751.24\end{array}$ & $\begin{array}{r}-66586.25 \\
85693.56\end{array}$ \\
\hline 34 & TANOBNT & 3 & END-II & $\begin{array}{l}5362.196 \\
5362.196\end{array}$ & $\begin{array}{l}-1.425 \\
-1.425\end{array}$ & $\begin{array}{l}-755.314 \\
-755.314\end{array}$ & $\begin{array}{l}629.63 \\
629.63\end{array}$ & $\begin{array}{r}50041.00 \\
-22469: 16\end{array}$ & $\begin{array}{l}555.67 \\
692.51\end{array}$ \\
\hline 35 & TANGENT & 1 & $\frac{\substack{\mathrm{BNDD}-\mathrm{I} \\
\mathrm{END}-\mathrm{J}}}{}$ & $\begin{array}{l}-1766.768 \\
-1766.768\end{array}$ & $\begin{array}{l}2.804 \\
2.804\end{array}$ & $\begin{array}{l}24.274 \\
24.274\end{array}$ & $\begin{array}{l}-381.29 \\
-381.29\end{array}$ & $\begin{array}{l}-25996.33 \\
-23666.02\end{array}$ & $\begin{array}{r}-43.71 \\
-312.89\end{array}$ \\
\hline 35 & TANGENT & 2 & $\begin{array}{l}\mathrm{END}-\mathrm{I} \\
\mathrm{END}-\mathrm{J}\end{array}$ & $\begin{array}{l}19.269 \\
19.269\end{array}$ & $\begin{array}{l}1161.012 \\
1161.012\end{array}$ & $\begin{array}{l}5.211 \\
5.211\end{array}$ & $\begin{array}{l}-3585.96 \\
-3585.96\end{array}$ & $\begin{array}{l}-751.24 \\
-250.97\end{array}$ & $\begin{array}{r}85693.56 \\
-25763.59\end{array}$ \\
\hline 35 & TANGENT & 3 & $\begin{array}{l}\text { END-I } \\
\text { END-J }\end{array}$ & $\begin{array}{l}5031: 876 \\
5031: 876\end{array}$ & 9.125 & $\begin{array}{r}917.486 \\
917.486\end{array}$ & $\begin{array}{l}629.63 \\
629.63\end{array}$ & $\begin{array}{r}-22469.16 \\
65609.48\end{array}$ & $\begin{array}{r}692.51 \\
-183.46\end{array}$ \\
\hline 36 & TANGBNT & 1 & $\begin{array}{l}\text { ENDD-I } \\
\text { END-J }\end{array}$ & $\begin{array}{l}-1136.188 \\
-1136.188\end{array}$ & $\begin{array}{l}1.954 \\
1.954\end{array}$ & $\begin{array}{l}136.114 \\
136.114\end{array}$ & $\begin{array}{l}-381.29 \\
-381.29\end{array}$ & $\begin{array}{l}-23666.02 \\
-17072.11\end{array}$ & $\begin{array}{r}-312.89 \\
-407.55\end{array}$ \\
\hline 36 & TANGENT & 2 & $\frac{\mathrm{END}-\mathrm{I}}{\mathrm{END}-\mathrm{J}}$ & $\begin{array}{l}19.599 \\
19.599\end{array}$ & $\begin{array}{l}1042.132 \\
1042.132\end{array}$ & $\begin{array}{l}6.621 \\
6.621\end{array}$ & $\begin{array}{l}-3585.96 \\
-3585.96\end{array}$ & $\begin{array}{r}-250.97 \\
69.78\end{array}$ & $\begin{array}{r}-25763.59 \\
-76248.63\end{array}$ \\
\hline 36 & TANGENT & 3 & ENDD- & $\begin{array}{l}4775.946 \\
8775.946\end{array}$ & $\begin{array}{l}8.215 \\
8.215\end{array}$ & $\begin{array}{l}648.716 \\
648.716\end{array}$ & $\begin{array}{l}629.63 \\
629.63\end{array}$ & $\begin{array}{l}65609.48 \\
97035.87\end{array}$ & $\begin{array}{l}-183.46 \\
-581.40\end{array}$ \\
\hline 37 & TANGENT & 1 & $\begin{array}{l}\text { END-I } \\
\text { END-J }\end{array}$ & $\begin{array}{l}692.728 \\
692.728\end{array}$ & 8.424 & $\begin{array}{l}496.748 \\
496.748\end{array}$ & $\begin{array}{l}.00 \\
: 00\end{array}$ & $\begin{array}{l}-11508.42 \\
-16671.02\end{array}$ & $\begin{array}{l}692.95 \\
271.73\end{array}$ \\
\hline 37 & TANGBNT & 2 & ENDD - I & $\begin{array}{r}-.252 \\
\because .252\end{array}$ & $\begin{array}{l}696.823 \\
696.823\end{array}$ & $\begin{array}{l}4.893 \\
4.893\end{array}$ & $\begin{array}{l}.00 \\
.00\end{array}$ & $\begin{array}{l}-449.17 \\
-204.51\end{array}$ & $\begin{array}{l}48619.15 \\
13778.00\end{array}$ \\
\hline 37 & TANGENT & 3 & $\begin{array}{l}\text { END-I } \\
\text { END-J }\end{array}$ & $\begin{array}{l}-307.161 \\
-307.161\end{array}$ & $\begin{array}{l}4.049 \\
4.049\end{array}$ & $\begin{array}{r}-1707.361 \\
-1707.361\end{array}$ & $\begin{array}{l}.00 \\
.00\end{array}$ & $\begin{array}{r}135191.23 \\
49823.18\end{array}$ & $\begin{array}{r}277: 29 \\
74: 83\end{array}$ \\
\hline 38 & TANGRNT & 1 & ENDPII - I & $\begin{array}{l}291.068 \\
291.068\end{array}$ & $\begin{array}{l}5.624 \\
5.624\end{array}$ & $\begin{array}{l}344.928 \\
344.928\end{array}$ & $\begin{array}{l}.00 \\
.00\end{array}$ & $\begin{array}{r}-16671.02 \\
-804.34\end{array}$ & $\begin{array}{r}271.73 \\
13.01\end{array}$ \\
\hline 38 & TANGENT & 2 & $\frac{\text { END-I }}{\mathrm{END}-\mathrm{J}}$ & $\because .112$ & $\begin{array}{l}286.853 \\
286.853\end{array}$ & $\begin{array}{l}4.223 \\
4.223\end{array}$ & $\begin{array}{l}.00 \\
.00\end{array}$ & $\begin{array}{r}-204.51 \\
-10.24\end{array}$ & $\begin{array}{r}13778.00 \\
582.76\end{array}$ \\
\hline 38 & TANGENT & 3 & $\begin{array}{l}\text { END-I } \\
\text { END-J }\end{array}$ & $\begin{array}{l}-129.251 \\
-129.251\end{array}$ & $\begin{array}{l}1.559 \\
1.559\end{array}$ & $\begin{array}{l}-1032.201 \\
-1032.201\end{array}$ & $\begin{array}{l}.00 \\
: 00\end{array}$ & $\begin{array}{r}49823.18 \\
2341.93\end{array}$ & $\begin{array}{r}74.83 \\
3.11\end{array}$ \\
\hline
\end{tabular}




\begin{tabular}{|c|c|c|c|c|c|c|c|c|c|}
\hline $\begin{array}{l}\text { P I P B } \\
\text { BLEMERT } \\
\text { NUMBRR }\end{array}$ & $\begin{array}{c}\text { P O R C } \\
\text { BLROARPT } \\
\text { TYPB }\end{array}$ & $\begin{array}{l}\text { E S } \\
\text { LAAD } \\
\text { CASB }\end{array}$ & $\begin{array}{l}\text { A N D M M } \\
\text { STATION }\end{array}$ & AXIAL & $\begin{array}{c}\text { Y-AXIS } \\
\text { SHIRAR }\end{array}$ & $\begin{array}{l}\text { z-AXIS } \\
\text { SHEAR }\end{array}$ & $\begin{array}{l}\text { TORSIONAL } \\
\text { MOMIENT }\end{array}$ & Y-AXIS & $\begin{array}{l}\text { Z-AxIS } \\
\text { MOABRIS }\end{array}$ \\
\hline 39 & TANGENT & 1 & $\begin{array}{l}\text { END-I } \\
\text { END-J }\end{array}$ & $\begin{array}{l}48.828 \\
48.828\end{array}$ & $\begin{array}{l}1.084 \\
1.084\end{array}$ & $\begin{array}{l}\begin{array}{r}67.028 \\
67.028\end{array}\end{array}$ & $\begin{array}{l}.00 \\
.00\end{array}$ & $\begin{array}{l}-804.34 \\
-00\end{array}$ & 13.01 \\
\hline 39 & TANGENT & 2 & END-I & $\because .022$ & $\begin{array}{l}48.563 \\
48.563\end{array}$ & $\begin{array}{l}.853 \\
.853\end{array}$ & $\begin{array}{l}.00 \\
: 00\end{array}$ & $\begin{array}{r}-10.24 \\
.00\end{array}$ & $\begin{array}{l}582.76 \\
.00\end{array}$ \\
\hline 39 & TANGENT & 3 & END-I & $\begin{array}{l}-21.641 \\
-21.641\end{array}$ & .259 & $\begin{array}{l}-195.161 \\
-195.161\end{array}$ & $\begin{array}{l}.00 \\
.00\end{array}$ & $\begin{array}{r}2341.93 \\
.00\end{array}$ & $\begin{array}{r}3.11 \\
.00\end{array}$ \\
\hline 40 & TANOBNT & 1 & $\begin{array}{l}\text { END-I } \\
\text { END-J }\end{array}$ & $\begin{array}{l}206.581 \\
206.581\end{array}$ & $\begin{array}{r}-3.510 \\
-3.510\end{array}$ & $\begin{array}{l}368.232 \\
368.232\end{array}$ & $\begin{array}{l}-381.29 \\
-381.29\end{array}$ & $\begin{array}{l}24436.31 \\
46366.74\end{array}$ & $\begin{array}{l}285.40 \\
494.46\end{array}$ \\
\hline 40 & TANOBNT & 2 & END-I & $\begin{array}{l}19.167 \\
19.167\end{array}$ & $\begin{array}{l}-356.001 \\
-356.001\end{array}$ & $\begin{array}{l}4.374 \\
4.374\end{array}$ & $\begin{array}{l}-3595.96 \\
-3585.96\end{array}$ & $\begin{array}{l}518.95 \\
779.47\end{array}$ & $\begin{array}{l}-27629.48 \\
-6427.47\end{array}$ \\
\hline 40 & TANGRNT & 3 & $\begin{array}{l}\text { END-I } \\
\text { END-J }\end{array}$ & $\begin{array}{l}4183.065 \\
4183.065\end{array}$ & $\begin{array}{l}-.695 \\
-.695\end{array}$ & $\begin{array}{r}-975.685 \\
-975.685\end{array}$ & $\begin{array}{l}629.63 \\
629.63\end{array}$ & $\begin{array}{l}-38155.36 \\
-96263.26\end{array}$ & $\begin{array}{l}-304.11 \\
-262.75\end{array}$ \\
\hline 41 & TANGENT & 1 & END-I & $\begin{array}{l}659.281 \\
659.281\end{array}$ & $\begin{array}{l}2.830 \\
2.830\end{array}$ & $\begin{array}{l}-152.898 \\
-152.898\end{array}$ & $\begin{array}{l}-381.29 \\
-381.29\end{array}$ & $\begin{array}{l}46366.74 \\
37192.86\end{array}$ & $\begin{array}{l}194.46 \\
324.69\end{array}$ \\
\hline 41 & TANGBNT & 2 & END-I & $\begin{array}{l}18.657 \\
18.657\end{array}$ & $\begin{array}{l}-546.321 \\
-546.321\end{array}$ & $\begin{array}{l}-5.336 \\
-5.336\end{array}$ & $\begin{array}{l}-3585.96 \\
-3585.96\end{array}$ & $\begin{array}{l}779.47 \\
159.34\end{array}$ & $\begin{array}{l}-6427.47 \\
26351.79\end{array}$ \\
\hline 41 & TANGENT & 3 & END-I & $\begin{array}{l}3968.915 \\
3968.915\end{array}$ & $\begin{array}{l}-4.265 \\
-4.265\end{array}$ & $\begin{array}{l}228.505 \\
228.505\end{array}$ & $\begin{array}{l}629.63 \\
629.63\end{array}$ & $\begin{array}{l}-96263.26 \\
-82552.97\end{array}$ & $\begin{array}{r}-262.75 \\
-6.87\end{array}$ \\
\hline 42 & TANGENT & 1 & $\begin{array}{l}\text { END-I } \\
\text { END-J }\end{array}$ & $\begin{array}{l}1040.071 \\
1040.071\end{array}$ & 7.880 & $\begin{array}{l}-604.318 \\
-604.318\end{array}$ & $\begin{array}{l}-381.29 \\
-381.29\end{array}$ & $\begin{array}{r}37192.86 \\
8185.60\end{array}$ & $\begin{array}{r}324.69 \\
-53.53\end{array}$ \\
\hline 42 & TANGBNT & 2 & $\begin{array}{l}\text { END - I } \\
\text { END-J }\end{array}$ & $\begin{array}{l}17.907 \\
17.907\end{array}$ & $\begin{array}{l}210.409 \\
210.409\end{array}$ & $\begin{array}{l}-11.986 \\
-11.986\end{array}$ & $\begin{array}{l}-3585.96 \\
-3585.96\end{array}$ & $\begin{array}{r}459.34 \\
-115.97\end{array}$ & $\begin{array}{l}26351.79 \\
16252.17\end{array}$ \\
\hline 42 & TANGBNT & 3 & $\begin{array}{l}\text { END-I } \\
\text { END-J }\end{array}$ & $\begin{array}{l}3776.215 \\
3776.215\end{array}$ & $\begin{array}{l}-5.725 \\
-5.725\end{array}$ & $\begin{array}{l}2217.395 \\
2217.395\end{array}$ & $\begin{array}{l}629.63 \\
629.63\end{array}$ & $\begin{array}{l}-82552.97 \\
23801.99\end{array}$ & 267.91 \\
\hline 43 & TANGENT & 1 & END-I & $\begin{array}{l}1280.421 \\
1280.421\end{array}$ & $\begin{array}{l}9.120 \\
9.120\end{array}$ & $\begin{array}{l}-731.758 \\
-731.758\end{array}$ & $\begin{array}{l}-381.29 \\
-381.29\end{array}$ & $\begin{array}{l}8185.60 \\
-9376.59\end{array}$ & $\begin{array}{r}-53.53 \\
-272.41\end{array}$ \\
\hline 43 & TANGENT & 2 & END-I & $\begin{array}{l}17.277 \\
17.277\end{array}$ & $\begin{array}{l}1322.849 \\
1322.849\end{array}$ & $\begin{array}{l}-13.446 \\
-13.446\end{array}$ & $\begin{array}{l}-3585.96 \\
-3585.96\end{array}$ & $\begin{array}{l}-115.97 \\
-438.67\end{array}$ & $\begin{array}{r}16252.17 \\
-15496.21\end{array}$ \\
\hline 43 & TANGENT & 3 & $\begin{array}{l}\text { END-I } \\
\text { END-J }\end{array}$ & $\begin{array}{l}3648.555 \\
3648.555\end{array}$ & $\begin{array}{l}-5.835 \\
-5.835\end{array}$ & $\begin{array}{l}3649.905 \\
3649.905\end{array}$ & $\begin{array}{l}629.63 \\
629.63\end{array}$ & $\begin{array}{r}23881.99 \\
111479: 70\end{array}$ & $\begin{array}{l}267.91 \\
107.94\end{array}$ \\
\hline 44 & BEND & 1 & $\begin{array}{l}\text { END-I } \\
\text { CENTER } \\
\text { END-J }\end{array}$ & $\begin{array}{l}1542.331 \\
2234.111 \\
1617.179\end{array}$ & $\begin{array}{r}1617.179 \\
52.926 \\
-1542.331\end{array}$ & $\begin{array}{l}-11.560 \\
-11.560 \\
-11.560\end{array}$ & $\begin{array}{l}-381.29 \\
-198.88 \\
-143.76\end{array}$ & $\begin{array}{l}272.41 \\
167.96 \\
-34.88\end{array}$ & $\begin{array}{r}-9376.59 \\
-34290.68 \\
-12071.14\end{array}$ \\
\hline 44 & BEND & 2 & $\begin{array}{c}\text { END-I } \\
\text { CENTER } \\
\text { END - J }\end{array}$ & $\begin{array}{r}16.497 \\
11.804 \\
.196\end{array}$ & $\begin{array}{r}-196 \\
-11: 527 \\
-16.497\end{array}$ & $\begin{array}{l}-1268.047 \\
-1268.047 \\
-1268.047\end{array}$ & $\begin{array}{r}-3585.96 \\
-49486 \\
-30153.67 \\
-30150\end{array}$ & $\begin{array}{r}15496.21 \\
-18786: 09 \\
-12063: 75\end{array}$ & $\begin{array}{r}-438.67 \\
-269.72 \\
148.17\end{array}$ \\
\hline
\end{tabular}




\begin{tabular}{|c|c|c|c|c|c|c|c|c|c|}
\hline \multirow{2}{*}{$\begin{array}{l}\text { P I P E } \\
\text { BLEMREFT } \\
\text { NUMBER }\end{array}$} & \multicolumn{2}{|c|}{ PORCES } & \multicolumn{2}{|c|}{ AND MOMBNTS } & \multirow[b]{2}{*}{$\begin{array}{l}\text { Y-AXIS } \\
\text { SHEAR }\end{array}$} & \multirow[b]{2}{*}{$\begin{array}{l}\text { Z-AXIS } \\
\text { SHEAR }\end{array}$} & \multirow[b]{2}{*}{$\begin{array}{l}\text { TORSIONAL } \\
\text { MOMRAT }\end{array}$} & \multirow[b]{2}{*}{$\begin{array}{l}\text { Y-AXIS } \\
\text { MOMIRAT }\end{array}$} & \multirow[b]{2}{*}{$\begin{array}{l}\text { Z-AXIS } \\
\text { moxaAnT }\end{array}$} \\
\hline & $\underset{\text { TYPE }}{\text { BLLGART }}$ & $\begin{array}{l}\text { IOAD } \\
\text { CASE }\end{array}$ & STATION & $\underset{\text { FORCB }}{\text { AXIAL }}$ & & & & & \\
\hline 44 & BEND & 3 & $\begin{array}{r}\text { END-I } \\
\text { CRNTER } \\
\text { END-J }\end{array}$ & $\begin{array}{l}3506.345 \\
7348.617 \\
6886.169\end{array}$ & $\begin{array}{r}6886.169 \\
2389.896 \\
-3506.345\end{array}$ & $\begin{array}{l}9.422 \\
9.422 \\
9.422\end{array}$ & $\begin{array}{l}629.63 \\
256.10 \\
-68.76\end{array}$ & $\begin{array}{r}-407.94 \\
-493.84 \\
-290.45\end{array}$ & $\begin{array}{l}111479.70 \\
-26842.08 \\
-10193.93\end{array}$ \\
\hline 45 & TAMGENT & 1 & $\begin{array}{l}\text { END - I } \\
\text { END -J }\end{array}$ & $\begin{array}{l}1379.589 \\
1379.589\end{array}$ & $\begin{array}{l}-8.780 \\
-8.780\end{array}$ & $\begin{array}{l}1803.601 \\
1803.601\end{array}$ & $\begin{array}{l}-143.76 \\
-143.76\end{array}$ & $\begin{array}{r}-12071.14 \\
112377.29\end{array}$ & $\begin{array}{r}34.88 \\
640.71\end{array}$ \\
\hline 45 & TANGERT & 2 & $\begin{array}{l}\text { END-I } \\
\text { END-J }\end{array}$ & $\begin{array}{l}1.046 \\
1.046\end{array}$ & $\begin{array}{l}456.553 \\
456.553\end{array}$ & $\begin{array}{l}12.127 \\
12.127\end{array}$ & $\begin{array}{l}-30153.50 \\
-30153.50\end{array}$ & $\begin{array}{l}148.17 \\
984.96\end{array}$ & $\begin{array}{r}\mathbf{4 2 0 6 3 . 7 5} \\
10561.62\end{array}$ \\
\hline 45 & TANOENT & 3 & $\begin{array}{l}\text { END - I } \\
\text { END - J }\end{array}$ & $\begin{array}{l}6202.489 \\
6202.189\end{array}$ & $\begin{array}{l}8.552 \\
8.552\end{array}$ & $\begin{array}{l}2052.655 \\
2052.655\end{array}$ & $\begin{array}{l}-68.76 \\
-68.76\end{array}$ & $\begin{array}{r}-10193.93 \\
131139.29\end{array}$ & $\begin{array}{r}290.45 \\
-299.61\end{array}$ \\
\hline 46 & TANOBNT & 1 & $\begin{array}{l}\text { END - I } \\
\text { END -J }\end{array}$ & $\begin{array}{l}1118.819 \\
1118.819\end{array}$ & $\begin{array}{l}-2.930 \\
-2.930\end{array}$ & $\begin{array}{l}1117.821 \\
1117.821\end{array}$ & $\begin{array}{l}-143.76 \\
-143.76\end{array}$ & $\begin{array}{l}112377.29 \\
142558.45\end{array}$ & $\begin{array}{l}640.71 \\
719.83\end{array}$ \\
\hline 46 & TANOBNT & 2 & $\begin{array}{l}\text { END - I } \\
\text { END - J }\end{array}$ & $\begin{array}{l}1.806 \\
1.806\end{array}$ & $\begin{array}{l}925.903 \\
925.903\end{array}$ & $\begin{array}{l}4.477 \\
4.477\end{array}$ & $\begin{array}{l}-30153.50 \\
-30153.50\end{array}$ & $\begin{array}{r}984.96 \\
2105.85\end{array}$ & $\begin{array}{r}10561.62 \\
-14437: 75\end{array}$ \\
\hline 46 & TANGENT & 3 & $\begin{array}{l}\text { END-I } \\
\text { END-J }\end{array}$ & $\begin{array}{l}5654.729 \\
5654.729\end{array}$ & $\begin{array}{l}6.742 \\
6.742\end{array}$ & $\begin{array}{l}879.195 \\
879.195\end{array}$ & $\begin{array}{l}-68.76 \\
-68.76\end{array}$ & $\begin{array}{l}131439.29 \\
155177.57\end{array}$ & $\begin{array}{r}-299.61 \\
-481.63\end{array}$ \\
\hline 47 & TANGENT & 1 & $\begin{array}{l}\text { END-I } \\
\text { END-J }\end{array}$ & $\begin{array}{l}703.219 \\
703.219\end{array}$ & $\begin{array}{l}3.310 \\
3.310\end{array}$ & $\begin{array}{l}-581.019 \\
-581.019\end{array}$ & $\begin{array}{l}-202.44 \\
-202.14\end{array}$ & $\begin{array}{r}135527.65 \\
119840.12\end{array}$ & $\begin{array}{l}719.83 \\
630.47\end{array}$ \\
\hline 47 & TANGBNT & 2 & $\begin{array}{l}\text { END-I } \\
\text { END-J }\end{array}$ & $\begin{array}{l}1.086 \\
1.086\end{array}$ & $\begin{array}{l}173.933 \\
173.933\end{array}$ & $\begin{array}{l}-9.323 \\
-9.323\end{array}$ & $\begin{array}{l}1529.02 \\
1529.02\end{array}$ & $\begin{array}{r}1057.61 \\
805.90\end{array}$ & $\begin{array}{l}-11437.75 \\
-19133.92\end{array}$ \\
\hline 47 & TANGBMT & 3 & $\begin{array}{l}\text { END-I } \\
\text { END-J }\end{array}$ & $\begin{array}{l}4056.359 \\
4056.359\end{array}$ & $\begin{array}{l}-5.288 \\
-5.288\end{array}$ & $\begin{array}{l}-959.985 \\
-959.9855\end{array}$ & $\begin{array}{l}315.72 \\
315.72\end{array}$ & $\begin{array}{r}113695.85 \\
87776.26\end{array}$ & $\begin{array}{l}-481.63 \\
-338.84\end{array}$ \\
\hline 48 & TANGEAT & 1 & $\begin{array}{l}\text { END-I } \\
\text { END-J }\end{array}$ & $\begin{array}{l}1019.730 \\
1019.730\end{array}$ & $\begin{array}{l}-1.630 \\
-1.630\end{array}$ & $\begin{array}{l}-195.300 \\
-195.300\end{array}$ & $\begin{array}{l}.00 \\
.00\end{array}$ & $\begin{array}{r}7030.80 \\
.00\end{array}$ & $\begin{array}{r}-58.68 \\
.00\end{array}$ \\
\hline 48 & TAMGENT & 2 & $\begin{array}{l}\text { END-I } \\
\text { END-J }\end{array}$ & $\begin{array}{l}8.280 \\
8.280\end{array}$ & $\begin{array}{l}880.070 \\
880.070\end{array}$ & $\begin{array}{l}-1.340 \\
-1.340\end{array}$ & $\begin{array}{l}.00 \\
.00\end{array}$ & $\begin{array}{r}48.24 \\
.00\end{array}$ & $\begin{array}{r}31682.52 \\
.00\end{array}$ \\
\hline 48 & TANOENT & 3 & $\begin{array}{l}\text { END - I } \\
\text { END-J }\end{array}$ & $\begin{array}{l}1103.590 \\
1103.550\end{array}$ & $\begin{array}{l}10.680 \\
10.680\end{array}$ & $\begin{array}{l}-1152.270 \\
-1152.270\end{array}$ & .00 & $\begin{array}{r}41481.72 \\
.00\end{array}$ & $\begin{array}{r}384.48 \\
.00\end{array}$ \\
\hline 49 & TANGENT & 1 & $\begin{array}{l}\text { END-I } \\
\text { END-J }\end{array}$ & $\begin{array}{l}389.029 \\
389.029\end{array}$ & $\begin{array}{l}9.030 \\
9.030\end{array}$ & $\begin{array}{l}-1591.529 \\
-1591.529\end{array}$ & $\begin{array}{l}-202.44 \\
-202.14\end{array}$ & $\begin{array}{r}119840.12 \\
5250.00\end{array}$ & $\begin{array}{l}630.47 \\
-19.68\end{array}$ \\
\hline 49 & TANGENT & 2 & $\begin{array}{l}\text { END-I } \\
\text { END-J }\end{array}$ & $\begin{array}{l}1.956 \\
1.956\end{array}$ & $\begin{array}{l}34.383 \\
34.383\end{array}$ & $\begin{array}{l}-15.113 \\
-15.113\end{array}$ & $\begin{array}{l}1529.02 \\
1529.02\end{array}$ & $\begin{array}{r}805.90 \\
-282.20\end{array}$ & $\begin{array}{r}-19133.92 \\
-21609.47\end{array}$ \\
\hline 49 & TANGENT & 3 & $\begin{array}{l}\text { END - I } \\
\text { END-J }\end{array}$ & $\begin{array}{l}3439.849 \\
3439.849\end{array}$ & $\begin{array}{l}-6.608 \\
-6.608\end{array}$ & $\begin{array}{l}-1625.715 \\
-1625.715\end{array}$ & $\begin{array}{l}315.72 \\
315.72\end{array}$ & $\begin{array}{r}87776.26 \\
-29275.19\end{array}$ & $\begin{array}{r}-338.84 \\
136.96\end{array}$ \\
\hline 50 & TANGENT & 1 & $\begin{array}{l}\text { END - I } \\
\text { END -J }\end{array}$ & $\begin{array}{r}-42.151 \\
-42.151\end{array}$ & $\begin{array}{l}9.990 \\
9.990\end{array}$ & $\begin{array}{l}-1999.539 \\
-1999.539\end{array}$ & $\begin{array}{l}-202.44 \\
-202.44\end{array}$ & $\begin{array}{r}5250.00 \\
-138716.85\end{array}$ & $\begin{aligned}-19.68 \\
-738.94\end{aligned}$ \\
\hline
\end{tabular}




\begin{tabular}{|c|c|c|c|c|c|c|c|c|c|}
\hline $\begin{array}{l}\text { P I P I } \\
\text { RLBURRT } \\
\text { KUUMBRR }\end{array}$ & $\begin{array}{c}\text { F O R C } \\
\text { BLEMgRrs } \\
\text { TYPPB }\end{array}$ & $\begin{array}{l}\text { E } 9 \\
\text { LOAD } \\
\text { CASE }\end{array}$ & $\begin{array}{l}\text { A N D M } \\
\text { STATION }\end{array}$ & $\begin{array}{c}\text { M N T S } \\
\text { AXIAL } \\
\text { BORCE }\end{array}$ & $\begin{array}{l}\text { Y-AXIS } \\
\text { SHIIAR }\end{array}$ & $\begin{array}{l}\text { Z-AXIS } \\
\text { SHEAR }\end{array}$ & $\begin{array}{l}\text { TORSIONAL } \\
\text { MOMIEAT }\end{array}$ & $\frac{\text { Y-AxIS }}{\text { maxarit }}$ & $\begin{array}{l}\text { Z-AxIS } \\
\text { MOXIBNT }\end{array}$ \\
\hline 50 & TANGENT & 2 & $\begin{array}{l}\text { END-I } \\
\text { END-J }\end{array}$ & $\begin{array}{l}3.046 \\
3.046\end{array}$ & $\begin{array}{l}-525.307 \\
-525.307\end{array}$ & $\begin{array}{l}-11.163 \\
-11.163\end{array}$ & $\begin{array}{l}1529.02 \\
1529.02\end{array}$ & $\begin{array}{r}-282.20 \\
-1085.90\end{array}$ & $\begin{array}{r}-21609.47 \\
16212.67\end{array}$ \\
\hline 50 & TANGENT & 3 & $\begin{array}{l}\text { END -I } \\
\text { END-J }\end{array}$ & $\begin{array}{l}2722.249 \\
2722.249\end{array}$ & $\begin{array}{l}-4.108 \\
-4.108\end{array}$ & $\begin{array}{l}-1151.695 \\
-1151.695\end{array}$ & $\begin{array}{l}315.72 \\
315.72\end{array}$ & $\begin{array}{r}-29275.19 \\
-112197.20\end{array}$ & $\begin{array}{r}136.96 \\
\quad 432.77\end{array}$ \\
\hline 51 & TAMGENT & 1 & $\begin{array}{l}\text { END -I } \\
\text { END-J }\end{array}$ & $\begin{array}{l}-392.221 \\
-392.221\end{array}$ & $\begin{array}{l}6.200 \\
6.200\end{array}$ & $\begin{array}{l}-783.289 \\
-783.289\end{array}$ & $\begin{array}{l}-202.44 \\
-202.44\end{array}$ & $\begin{array}{l}-138716.85 \\
-159865.66\end{array}$ & $\begin{array}{l}-738.94 \\
-906.34\end{array}$ \\
\hline 51 & TANGBNT & 2 & END-I & $\begin{array}{l}3.856 \\
3.856\end{array}$ & $\begin{array}{l}-198.037 \\
-198.037\end{array}$ & $\begin{array}{l}-2.863 \\
-2.863\end{array}$ & $\begin{array}{l}1529.02 \\
1529.02\end{array}$ & $\begin{array}{l}-1085.90 \\
-1163.19\end{array}$ & $\begin{array}{l}16212.67 \\
21559.68\end{array}$ \\
\hline 51 & TANOBNT & 3 & $\begin{array}{l}\text { RND-I } \\
\text { END-J }\end{array}$ & $\begin{array}{l}2217.639 \\
2217.639\end{array}$ & $\begin{array}{l}-.188 \\
-.188\end{array}$ & $\begin{array}{l}-356.805 \\
-356.805\end{array}$ & $\begin{array}{l}315.72 \\
315.72\end{array}$ & $\begin{array}{l}-112197.20 \\
-121830.92\end{array}$ & $\begin{array}{r}132.77 \\
437.86\end{array}$ \\
\hline 52 & TAMGENT & 1 & $\begin{array}{l}\text { END-I } \\
\text { END-J }\end{array}$ & $\begin{array}{l}-1759.451 \\
-1759.151\end{array}$ & $\begin{array}{l}-7.900 \\
-7.900\end{array}$ & $\begin{array}{l}2354.121 \\
2354.121\end{array}$ & 200.04 & $\begin{array}{l}-200079.46 \\
-136518.21\end{array}$ & $\begin{array}{l}-906.34 \\
-693.03\end{array}$ \\
\hline 52 & TANGENT & 2 & $\begin{array}{l}\text { END-I } \\
\text { END-J }\end{array}$ & $\begin{array}{l}6.516 \\
6.516\end{array}$ & $\begin{array}{l}-561.107 \\
-561.107\end{array}$ & $\begin{array}{l}11.067 \\
11.067\end{array}$ & $\begin{array}{l}35508.70 \\
35508.70\end{array}$ & $\begin{array}{r}-1087.95 \\
-789.13\end{array}$ & $\begin{array}{l}21559.68 \\
36709.58\end{array}$ \\
\hline 52 & TANGENT & 3 & $\begin{array}{l}\text { END - I } \\
\text { END-J }\end{array}$ & $\begin{array}{l}1487.659 \\
1487.659\end{array}$ & $\begin{array}{l}3.552 \\
3.552\end{array}$ & $\begin{array}{l}940.165 \\
940.165\end{array}$ & $\begin{array}{l}275.40 \\
275.40\end{array}$ & $\begin{array}{l}-135406.52 \\
-110022.06\end{array}$ & $\begin{array}{l}\mathbf{4 3 7 . 8 6} \\
341.96\end{array}$ \\
\hline 53 & TANGEATT & 1 & $\begin{array}{l}\text { END-I } \\
\text { END-J }\end{array}$ & $\begin{array}{l}-1882.530 \\
-1882.530\end{array}$ & $\begin{array}{l}11.180 \\
11.180\end{array}$ & $\begin{array}{l}-1117.050 \\
-1117.050\end{array}$ & .00 & $\begin{array}{r}40213.80 \\
.00\end{array}$ & $\begin{array}{r}102.48 \\
.00\end{array}$ \\
\hline 53 & TANGENT & 2 & $\begin{array}{l}\text { END-I } \\
\text { END-J }\end{array}$ & $\begin{array}{l}-8.360 \\
-8.360\end{array}$ & $\begin{array}{l}943.880 \\
943.880\end{array}$ & $\begin{array}{l}2.090 \\
2.090\end{array}$ & .00 & $\begin{array}{r}-75.24 \\
.00\end{array}$ & $\begin{array}{r}33979.68 \\
.00\end{array}$ \\
\hline 53 & TANGEIT & 3 & $\begin{array}{l}\text { END -I } \\
\text { END-J }\end{array}$ & $\begin{array}{l}-778.390 \\
-778.390\end{array}$ & $\begin{array}{l}-1.120 \\
-1.120\end{array}$ & $\begin{array}{l}-377.100 \\
-377.100\end{array}$ & $\begin{array}{l}.00 \\
.00\end{array}$ & $\begin{array}{r}13575.60 \\
.00\end{array}$ & $\begin{array}{r}-40.32 \\
.00\end{array}$ \\
\hline 54 & TANGENT & 1 & $\begin{array}{l}\text { END-I } \\
\text { BND-J }\end{array}$ & $\begin{array}{l}-2102.851 \\
-2102.851\end{array}$ & $\begin{array}{l}-11.620 \\
-11.620\end{array}$ & $\begin{array}{r}4553.441 \\
4553.441\end{array}$ & $\begin{array}{l}200.04 \\
200.04\end{array}$ & $\begin{array}{r}-136518.21 \\
177669.19\end{array}$ & $\begin{array}{r}-693.03 \\
108.76\end{array}$ \\
\hline 54 & TANGENT & 2 & $\begin{array}{l}\text { END-I } \\
\text { END-J }\end{array}$ & $\begin{array}{l}7.276 \\
7.276\end{array}$ & $\begin{array}{l}758.103 \\
758.103\end{array}$ & $\begin{array}{l}17.457 \\
17.457\end{array}$ & $\begin{array}{l}35508.70 \\
35508.70\end{array}$ & $\begin{array}{r}-789.13 \\
115.43\end{array}$ & $\begin{array}{r}36709.58 \\
-15599.19\end{array}$ \\
\hline 54 & TANGENT & 3 & $\begin{array}{l}\text { END - I } \\
\text { END-J }\end{array}$ & $\begin{array}{l}1009.679 \\
1009.679\end{array}$ & $\begin{array}{l}6.582 \\
6.582\end{array}$ & $\begin{array}{l}1530.025 \\
1530.025\end{array}$ & $\begin{array}{l}275.10 \\
275.10\end{array}$ & $\begin{array}{r}-110022.06 \\
-4450.31\end{array}$ & $\begin{array}{r}341.96 \\
-112.17\end{array}$ \\
\hline 55 & BEND & 1 & $\begin{array}{c}\text { END-I } \\
\text { CRNTBR } \\
\text { BND-J }\end{array}$ & $\begin{array}{l}-2500.231 \\
-8408.247 \\
-9390.827\end{array}$ & $\begin{array}{r}-9390.827 \\
-4872.387 \\
2500.231\end{array}$ & $\begin{array}{l}-.305 \\
-.305 \\
-.305\end{array}$ & $\begin{array}{r}200.04 \\
215.13 \\
97.77\end{array}$ & $\begin{array}{r}108.76 \\
-72.32 \\
-211.03\end{array}$ & $\begin{array}{r}-177669.19 \\
35019.41 \\
70392.26\end{array}$ \\
\hline 55 & BEND & 2 & $\begin{array}{c}\text { END-I } \\
\text { CENTER } \\
\text { END-J }\end{array}$ & $\begin{array}{l}8.076 \\
5.705 \\
-.009\end{array}$ & $\begin{array}{r}-.009 \\
-5.717 \\
-8.076\end{array}$ & $\begin{array}{l}499.287 \\
499.287 \\
499.287\end{array}$ & $\begin{array}{r}35508.70 \\
19342.49 \\
2374.84\end{array}$ & $\begin{array}{l}-15599.49 \\
-23429: 18 \\
-17534: 37\end{array}$ & $\begin{array}{l}-415.43 \\
-330.05 \\
-124.36\end{array}$ \\
\hline 55 & BEND & 3 & $\begin{array}{c}\text { END-I } \\
\text { CENTER } \\
\text { END -J }\end{array}$ & $\begin{array}{r}485.179 \\
1690: 035 \\
1904.892\end{array}$ & $\begin{array}{l}1904.892 \\
1003.889 \\
-485.179\end{array}$ & $\begin{array}{l}4.153 \\
4.153 \\
4.153\end{array}$ & $\begin{array}{r}275.40 \\
159.22 \\
37.35\end{array}$ & $\begin{array}{l}-112.17 \\
-168: 32 \\
-125.88\end{array}$ & $\begin{array}{r}4150.31 \\
-38924.52 \\
-46659.36\end{array}$ \\
\hline
\end{tabular}




\begin{tabular}{|c|c|c|c|c|c|c|c|c|c|}
\hline $\begin{array}{l}\text { P I P } \\
\text { BLEMRMT } \\
\text { WUMBER }\end{array}$ & $\begin{array}{l}\text { ELEMERT } \\
\text { TYPB }\end{array}$ & $\begin{array}{l}\text { ES } \\
\text { LAAD } \\
\text { CASB }\end{array}$ & $\begin{array}{l}\text { A N D } \\
\text { STATION }\end{array}$ & $\begin{array}{l}\text { AXIALL } \\
\text { PORCE }\end{array}$ & $\begin{array}{c}\text { Y-AXIS } \\
\text { SHEARR }\end{array}$ & $\begin{array}{l}\text { Z.AXIS } \\
\text { SHRRRR }\end{array}$ & $\begin{array}{c}\text { TORSIONAL } \\
\text { MOMBNT }\end{array}$ & Y-AX1S & 2-AXIS \\
\hline 56 & TANGBNT & 1 & $\begin{array}{l}\text { END }-I \\
\text { END -J }\end{array}$ & $\begin{array}{l}-8257.607 \\
-8257.607\end{array}$ & $\begin{array}{r}.325 \\
.325\end{array}$ & $\begin{array}{l}1995.941 \\
1995.941\end{array}$ & 97.77 & $\begin{array}{r}-70392.26 \\
-4526.21\end{array}$ & $\begin{array}{l}-211.03 \\
-221.77\end{array}$ \\
\hline 56 & TANGRMT & 2 & $\frac{\substack{\mathrm{END}-\mathrm{I} \\
\mathrm{END}-\mathrm{J}}}{4}$ & $\begin{array}{l}-.549 \\
-.549\end{array}$ & $\begin{array}{l}340.473 \\
340.473\end{array}$ & $\begin{array}{l}-8.136 \\
-8.136\end{array}$ & $\begin{array}{l}2374.84 \\
2374: 84\end{array}$ & $\begin{array}{r}124.36 \\
-144: 14\end{array}$ & $\begin{array}{l}-17534.37 \\
-28769.98\end{array}$ \\
\hline 56 & TANGBMT & 3 & $\frac{\text { END-I }}{\text { END-J }}$ & $\begin{array}{l}1604.672 \\
1604.672\end{array}$ & $\begin{array}{l}-4.683 \\
-4.683\end{array}$ & $\begin{array}{r}-413.389 \\
-413.389\end{array}$ & $\begin{array}{l}37.35 \\
37.35\end{array}$ & $\begin{array}{l}\begin{array}{l}46659.36 \\
33017.54\end{array} \\
\end{array}$ & $\begin{array}{r}-125.89 \\
28.67\end{array}$ \\
\hline 57 & TANGBNT & 1 & $\begin{array}{l}\text { END-I } \\
\text { END-J }\end{array}$ & $\begin{array}{r}-7365.117 \\
-7365.117\end{array}$ & $\begin{array}{l}.095 \\
.095\end{array}$ & $\begin{array}{l}1434.221 \\
1434.221\end{array}$ & 97.77 & $\begin{array}{r}-4526.21 \\
34197.75\end{array}$ & $\begin{array}{l}-221.77 \\
-224.35\end{array}$ \\
\hline 57 & TANGENT & 2 & END-I & $\begin{array}{r}-.979 \\
-979\end{array}$ & $\begin{array}{l}414.283 \\
414.283\end{array}$ & $\begin{array}{l}-7.116 \\
-7.116\end{array}$ & $\begin{array}{l}2374.84 \\
2374: 84\end{array}$ & $\begin{array}{l}-144.14 \\
-336.29\end{array}$ & $\begin{array}{l}-28769.98 \\
-39955.62\end{array}$ \\
\hline 57 & TANGBNT & 3 & $\begin{array}{l}\mathrm{END}-\mathrm{I} \\
\mathrm{END}-\mathrm{J}\end{array}$ & $\begin{array}{l}\begin{array}{l}1362.372 \\
1362.372\end{array} \\
1372\end{array}$ & $\begin{array}{l}-4.543 \\
-4.543\end{array}$ & $\begin{array}{l}-457.699 \\
-457.699\end{array}$ & $\begin{array}{l}37.35 \\
37.35\end{array}$ & $\begin{array}{l}33017.54 \\
20659.68\end{array}$ & $\begin{array}{r}28.67 \\
151: 34\end{array}$ \\
\hline 58 & TANGBNT & 1 & $\frac{\substack{\text { BND-I } \\
\text { END-J }}}{4}$ & $\begin{array}{l}-5017.927 \\
-5017.927\end{array}$ & $\begin{array}{l}-1.905 \\
-1.905\end{array}$ & $\begin{array}{l}-113.099 \\
-113.099\end{array}$ & $\begin{array}{l}160.41 \\
160.41\end{array}$ & $\begin{array}{l}84002.31 \\
80948.63\end{array}$ & $\begin{array}{l}-224.35 \\
-172.92\end{array}$ \\
\hline 58 & TANGENT & 2 & $\begin{array}{l}\text { END - I } \\
\text { END-J }\end{array}$ & $\begin{array}{l}-3.529 \\
-3.529\end{array}$ & $\begin{array}{l}-381.517 \\
-381.517\end{array}$ & $\begin{array}{l}-2.386 \\
-2.386\end{array}$ & $\begin{array}{l}22526.92 \\
22526.92\end{array}$ & $\begin{array}{l}-411.17 \\
-475.60\end{array}$ & $\begin{array}{r}-39955.62 \\
-29654.66\end{array}$ \\
\hline 58 & TANGBNT & 3 & ENDD-I & $\begin{array}{l}649.632 \\
649.632\end{array}$ & $\begin{array}{l}-2.203 \\
-2.203\end{array}$ & $\begin{array}{l}-589.339 \\
-589.339\end{array}$ & $\begin{array}{l}-25.29 \\
-25.29\end{array}$ & $\begin{array}{r}4504.32 \\
-11407.82\end{array}$ & $\begin{array}{l}\frac{151}{210.34} \\
2103\end{array}$ \\
\hline 59 & TANGBNT & 1 & $\begin{array}{l}\text { END -I } \\
\text { BND-J }\end{array}$ & $\begin{array}{l}928.620 \\
928.620\end{array}$ & $\begin{array}{l}1.740 \\
1.740\end{array}$ & $\begin{array}{l}1383.460 \\
1383.460\end{array}$ & :00 & -49804.56 & $\begin{array}{l}62.64 \\
.00\end{array}$ \\
\hline 59 & TAMGRNT & 2 & $\begin{array}{l}\text { END-I } \\
\text { END-J }\end{array}$ & $\begin{array}{l}-2.840 \\
-2.840\end{array}$ & $\begin{array}{l}559.780 \\
559.780\end{array}$ & $\begin{array}{l}-2.080 \\
-2.080\end{array}$ & $\begin{array}{l}.00 \\
.00\end{array}$ & $\begin{array}{l}74.88 \\
.00\end{array}$ & 20152.08 \\
\hline 59 & TANGENT & 3 & $\begin{array}{l}\text { END - I } \\
\text { END -J }\end{array}$ & $\begin{array}{l}79.260 \\
79.260\end{array}$ & $\begin{array}{l}-1.740 \\
-1.740\end{array}$ & $\begin{array}{r}-448.760 \\
-448.760\end{array}$ & .00 & $\begin{array}{r}16155.36 \\
: 00\end{array}$ & $\begin{array}{r}-62.64 \\
.00\end{array}$ \\
\hline 60 & TANGRNT & 1 & $\frac{\mathrm{END}-\mathrm{I}}{\mathrm{END}-\mathrm{J}}$ & $\begin{array}{l}-4034.897 \\
-1034: 897\end{array}$ & $\begin{array}{l}-2.085 \\
-2.085\end{array}$ & $\begin{array}{l}-696.259 \\
-696.259\end{array}$ & $\begin{array}{l}160.41 \\
160.41\end{array}$ & $\begin{array}{l}80948.63 \\
51705.75\end{array}$ & $\begin{array}{r}-172.92 \\
-85.37\end{array}$ \\
\hline 60 & TANGBNT & 2 & $\frac{\mathrm{END}-\mathrm{I}}{\mathrm{END}-\mathrm{J}}$ & $\begin{array}{l}-4.009 \\
-4.009\end{array}$ & $\begin{array}{l}-807.277 \\
-807.277\end{array}$ & $\begin{array}{l}.174 \\
: 174\end{array}$ & $\begin{array}{l}22526.92 \\
22526.92\end{array}$ & $\begin{array}{r}-475.60 \\
-468.31\end{array}$ & $\begin{array}{r}-29654.66 \\
4250.97\end{array}$ \\
\hline 60 & TANGENT & 3 & $\begin{array}{l}\text { END - I } \\
\text { END -J }\end{array}$ & $\begin{array}{l}378.432 \\
378.432\end{array}$ & $\begin{array}{l}-1.223 \\
-1.223\end{array}$ & $\begin{array}{l}-590.199 \\
-590.199\end{array}$ & $\begin{array}{l}-25.29 \\
-25.29\end{array}$ & $\begin{array}{l}-11407.82 \\
-36196.16\end{array}$ & $\begin{array}{l}210.83 \\
262.21\end{array}$ \\
\hline 61 & TANGRAT & 1 & $\begin{array}{l}\text { ENDP-I } \\
\text { END-J }\end{array}$ & $\begin{array}{l}-3080.347 \\
-3080: 347\end{array}$ & $\begin{array}{l}-1.925 \\
-1.925\end{array}$ & $\begin{array}{r}-1044.769 \\
-1044.769\end{array}$ & $\begin{array}{l}160.41 \\
160.41\end{array}$ & $\begin{array}{l}51705.75 \\
23496.98\end{array}$ & $\begin{array}{l}-85.37 \\
-33.41\end{array}$ \\
\hline 61 & TANGENT & 2 & END-I & $\begin{array}{l}-1.159 \\
-1.469\end{array}$ & $\begin{array}{l}-1000.077 \\
-1000.077\end{array}$ & $\begin{array}{l}2.824 \\
2.624\end{array}$ & $\begin{array}{l}22526.92 \\
22526.92\end{array}$ & $\begin{array}{r}-468.31 \\
-392.08\end{array}$ & $\begin{array}{r}4250.97 \\
31253.05\end{array}$ \\
\hline
\end{tabular}




\begin{tabular}{|c|c|c|c|c|c|c|c|c|c|}
\hline$\frac{\text { RLRMRNT }}{\text { NUNBRR }}$ & $\begin{array}{c}\text { BLRMRNT } \\
\text { TYPE }\end{array}$ & $\begin{array}{l}\text { LOAD } \\
\text { CASB }\end{array}$ & STATION & $\underset{\text { BORCB }}{\text { BXIAL }}$ & $\begin{array}{c}\text { Y-AXIS } \\
\text { SHRAR }\end{array}$ & $\begin{array}{l}\text { z-AXIS } \\
\text { SHRARR }\end{array}$ & $\underset{\text { TORSIONAL }}{\text { MOMENT }}$ & $\underset{\text { MDNBRIS }}{\text { Y-AXIS }}$ & $\begin{array}{l}\text { Z-AXIS } \\
\text { MONIRNT }\end{array}$ \\
\hline 61 & TANGENT & 3 & $\begin{array}{l}\text { END-I } \\
\text { END-J }\end{array}$ & $\begin{array}{l}109.112 \\
109.112\end{array}$ & $\begin{array}{r}-.053 \\
-.053\end{array}$ & $\begin{array}{r}-303.599 \\
-303.599\end{array}$ & $\begin{array}{l}-25.29 \\
-25.29\end{array}$ & $\begin{array}{l}-36196.16 \\
-44393.32\end{array}$ & $\begin{array}{l}262.21 \\
263.64\end{array}$ \\
\hline 62 & TANGENT & 1 & $\begin{array}{l}\mathrm{END}-\mathrm{I} \\
\mathrm{END}-\mathrm{J}\end{array}$ & $\begin{array}{l}-1184.117 \\
-1184.117\end{array}$ & $\begin{array}{l}-1.035 \\
-1.035\end{array}$ & $\begin{array}{l}-1399.199 \\
-1399.199\end{array}$ & $\begin{array}{l}142.41 \\
142.41\end{array}$ & $\begin{array}{l}58653.86 \\
20875.48\end{array}$ & $\begin{array}{r}-33.41 \\
-5.47\end{array}$ \\
\hline 62 & TANGRNT & 2 & END-I & $\begin{array}{r}-1.479 \\
-1.479\end{array}$ & $\begin{array}{r}-453.307 \\
-453.307\end{array}$ & $\begin{array}{l}8.094 \\
8.094\end{array}$ & $\begin{array}{l}11213.92 \\
11213.92\end{array}$ & $\begin{array}{l}-376.60 \\
-158.07\end{array}$ & $\begin{array}{l}31253.05 \\
13492.33\end{array}$ \\
\hline 62 & TAMGENT & 3 & $\frac{\text { END - I }}{\text { END-J }}$ & $\begin{array}{l}-1161.488 \\
-1161.488\end{array}$ & $\begin{array}{l}2.947 \\
2.947\end{array}$ & $\begin{array}{l}1123.521 \\
1123.521\end{array}$ & $\begin{array}{r}-98.01 \\
-98.01\end{array}$ & $\begin{array}{l}-80736.04 \\
-50600.96\end{array}$ & $\begin{array}{l}263.64 \\
184.08\end{array}$ \\
\hline 63 & TANGENT & 1 & $\begin{array}{l}\text { END-I } \\
\text { END-J }\end{array}$ & $\begin{array}{l}212,700 \\
212: 700\end{array}$ & $\begin{array}{l}-.500 \\
-.500\end{array}$ & $\begin{array}{l}976.580 \\
976.580\end{array}$ & $\begin{array}{l}.00 \\
.00\end{array}$ & -35156.88 & $\begin{array}{l}-18.00 \\
-00\end{array}$ \\
\hline 63 & TANGERT & 2 & ENDD-I & $\begin{array}{l}-3.160 \\
-3.160\end{array}$ & $\begin{array}{l}-314.250 \\
-314.250\end{array}$ & $\begin{array}{l}.430 \\
.430\end{array}$ & :00 & $\begin{array}{l}-15.48 \\
.00\end{array}$ & -11313.000 \\
\hline 63 & TAMGENT & 3 & $\underset{\mathrm{END}-\mathrm{J}}{\mathrm{END}-\mathrm{I}}$ & $\begin{array}{l}-856.140 \\
-856.140\end{array}$ & $\begin{array}{l}-2.020 \\
-2.020\end{array}$ & $\begin{array}{r}-1009.520 \\
-1009.520\end{array}$ & .000 & $\begin{array}{r}36342.72 \\
.00\end{array}$ & $\begin{array}{r}-72.72 \\
.00\end{array}$ \\
\hline 64 & TANGENT & 1 & $\frac{\text { END- }-\mathbf{Y}}{\text { END-J }}$ & $\begin{array}{l}-485.077 \\
-685 ; 077\end{array}$ & $\begin{array}{l}: .585 \\
: .585\end{array}$ & $\begin{array}{r}-1360.169 \\
-1360.169\end{array}$ & $\begin{array}{l}142.41 \\
142.41\end{array}$ & $\begin{array}{r}20875.49 \\
-7688.07\end{array}$ & $\begin{array}{r}-5.47 \\
6: 80\end{array}$ \\
\hline 64 & TANGENT & 2 & $\begin{array}{l}\text { RND-I } \\
\text { END-J }\end{array}$ & $\begin{array}{l}-4.809 \\
-4: 809\end{array}$ & $\begin{array}{l}110.983 \\
110.983\end{array}$ & $\begin{array}{l}9.204 \\
9.204\end{array}$ & $\begin{array}{l}11213.92 \\
11213.92\end{array}$ & $\begin{array}{r}-158.07 \\
35.20\end{array}$ & $\begin{array}{l}43492.33 \\
11161.69\end{array}$ \\
\hline 64 & TANGENT & 3 & $\frac{\text { END-I }}{\mathrm{END}-\mathrm{J}}$ & $\begin{array}{l}-1360.698 \\
-1360.698\end{array}$ & $\begin{array}{l}3.487 \\
3.487\end{array}$ & $\begin{array}{l}1813.231 \\
1813.231\end{array}$ & $\begin{array}{l}-98.01 \\
-98.01\end{array}$ & $\begin{array}{l}-50400.96 \\
-12323.10\end{array}$ & $\begin{array}{l}184.08 \\
110.86\end{array}$ \\
\hline 65 & BEND & 1 & $\begin{array}{l}\text { RND-I } \\
\text { CRNTRR } \\
\text { CRND-J }\end{array}$ & $\begin{array}{r}400.303 \\
1125 \\
1190.909\end{array}$ & $\begin{array}{r}1190.909 \\
555: 004 \\
-160.303\end{array}$ & $\begin{array}{l}.125 \\
.125 \\
.125\end{array}$ & $\begin{array}{r}142.41 \\
97.21 \\
-2.29\end{array}$ & $\begin{array}{r}-66.80 \\
-102: 32 \\
-137: 89\end{array}$ & $\begin{array}{r}-7688.07 \\
-337782: 81 \\
-36169: 88\end{array}$ \\
\hline 65 & BEND & 2 & $\begin{array}{l}\text { END-I } \\
\text { CENTER } \\
\text { CEND-J }\end{array}$ & $\begin{array}{r}-5.219 \\
-10.771 \\
-10.014\end{array}$ & $\begin{array}{r}-10.014 \\
-3.390 \\
5.219\end{array}$ & $\begin{array}{l}1289.073 \\
11289.073 \\
1289.073\end{array}$ & $\begin{array}{r}11213.92 \\
-7584.09 \\
5244.94\end{array}$ & $\begin{array}{r}-41161.69 \\
-4220.771 \\
35192.71\end{array}$ & $\begin{array}{r}\begin{array}{r}35.20 \\
235: 08 \\
207: 81\end{array}\end{array}$ \\
\hline 65 & BEND & 3 & $\begin{array}{c}\text { END-I } \\
\text { CENTER } \\
\text { CEND-J }\end{array}$ & $\begin{array}{l}-1614.428 \\
-32477.055 \\
-2977.601\end{array}$ & $\begin{array}{r}-2977.601 \\
-966.909 \\
1614.428\end{array}$ & $\begin{array}{l}\begin{array}{l}3.867 \\
3 \\
3.867 \\
3.867\end{array}\end{array}$ & $\begin{array}{r}-98.01 \\
-106.92 \\
28.34\end{array}$ & $\begin{array}{r}-110.86 \\
89.34 \\
237.21\end{array}$ & $\begin{array}{r}-12323.10 \\
4651.47 \\
36751.12\end{array}$ \\
\hline 66 & TANGENT & 1 & $\begin{array}{l}\text { ENDD-I } \\
\text { END-J }\end{array}$ & $\begin{array}{l}1259.819 \\
1259.819\end{array}$ & $\begin{array}{l}4.831 \\
4.831\end{array}$ & $\begin{array}{l}1161.443 \\
1161.443\end{array}$ & $\begin{array}{l}-2.29 \\
-2.29\end{array}$ & $\begin{array}{r}-36149.88 \\
61411.37\end{array}$ & $\begin{array}{r}137.89 \\
-267.89\end{array}$ \\
\hline 66 & TANGENT & 2 & $\frac{\text { END -I }}{\text { END-J }}$ & $\begin{array}{l}-10.584 \\
-10.584\end{array}$ & $\begin{array}{l}-425.028 \\
-425.028\end{array}$ & $\begin{array}{l}-5.589 \\
-5.589\end{array}$ & $\begin{array}{l}5244.94 \\
5244: 94\end{array}$ & $\begin{array}{r}207.81 \\
-261.67\end{array}$ & $\begin{array}{r}-35192.71 \\
509.66\end{array}$ \\
\hline 66 & TANGENT & 3 & ENDD-I & $\begin{array}{l}-6011.281 \\
-6011.281\end{array}$ & $\begin{array}{l}-5.751 \\
-5.751\end{array}$ & $\begin{array}{l}-1246.798 \\
-1246.798\end{array}$ & $\begin{array}{l}28.34 \\
28: 34\end{array}$ & $\begin{array}{r}36751.12 \\
.67979 .94\end{array}$ & $\begin{array}{r}-237.21 \\
245.83\end{array}$ \\
\hline
\end{tabular}




\begin{tabular}{|c|c|c|c|c|c|c|c|c|c|}
\hline 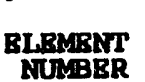 & $\underset{\text { BLEMENT }}{\text { TYPB }}$ & LASB & Station & $\begin{array}{l}\text { AXIALL } \\
\text { BORCB }\end{array}$ & 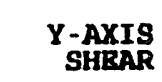 & $\begin{array}{l}\text { 2-AXIS } \\
\text { SHEARR }\end{array}$ & $\begin{array}{l}\text { TORSIONAL } \\
\text { MONENTI }\end{array}$ & $\begin{array}{l}\text { Y-AXIS } \\
\text { MOEIENTI }\end{array}$ & $\begin{array}{l}\text { Z.AXIS } \\
\text { WOMRENT }\end{array}$ \\
\hline 67 & TANGBNT & 1 & $\begin{array}{l}\text { END-I } \\
\text { END-J }\end{array}$ & $\begin{array}{l}1290.659 \\
1290.659\end{array}$ & $\begin{array}{l}2.121 \\
2.121\end{array}$ & $\begin{array}{l}296.893 \\
296.893\end{array}$ & $\begin{array}{l}-2.29 \\
-2.29\end{array}$ & $\begin{array}{l}61411.37 \\
68536.82\end{array}$ & $\begin{array}{l}-267.89 \\
-318.79\end{array}$ \\
\hline 67 & TANGENT & 2 & $\begin{array}{l}\text { END-I } \\
\text { END-J }\end{array}$ & $\begin{array}{l}-10.834 \\
-10.834\end{array}$ & $\begin{array}{l}-107.138 \\
-107.138\end{array}$ & $\begin{array}{l}-2.719 \\
-2.719\end{array}$ & $\begin{array}{l}5244.94 \\
5244: 94\end{array}$ & $\begin{array}{l}-261.67 \\
-326: 93\end{array}$ & $\begin{array}{r}509.66 \\
3080.98\end{array}$ \\
\hline 67 & TANGENT & 3 & $\begin{array}{l}\text { END-I } \\
\text { END-J }\end{array}$ & $\begin{array}{l}-7874.901 \\
-7874.901\end{array}$ & $\begin{array}{l}-2.821 \\
-2.821\end{array}$ & $\begin{array}{l}-469.348 \\
-469.348\end{array}$ & $\begin{array}{l}28.34 \\
28.34\end{array}$ & $\begin{array}{r}-67979.94 \\
-79244.31\end{array}$ & $\begin{array}{l}245.83 \\
313.53\end{array}$ \\
\hline 68 & TANGBNT & 1 & $\begin{array}{l}\text { END-I } \\
\text { END-J }\end{array}$ & $\begin{array}{l}1309.299 \\
1309: 299\end{array}$ & $\begin{array}{l}.041 \\
.041\end{array}$ & $\begin{array}{l}-346.777 \\
-346.777\end{array}$ & $\begin{array}{l}-2.29 \\
-2.29\end{array}$ & $\begin{array}{l}68536.82 \\
38020.48\end{array}$ & $\begin{array}{l}-318.79 \\
-322.38\end{array}$ \\
\hline 68 & TANGRNT & 2 & $\begin{array}{l}\text { END-I } \\
\text { END-J }\end{array}$ & $\begin{array}{l}-10.984 \\
-10.984\end{array}$ & $\begin{array}{l}-99.348 \\
-99.348\end{array}$ & $\begin{array}{r}-.439 \\
-.439\end{array}$ & $\begin{array}{l}5244.94 \\
5244.94\end{array}$ & $\begin{array}{l}-326.93 \\
-365.56\end{array}$ & $\begin{array}{r}3000.98 \\
11823.63\end{array}$ \\
\hline 68 & TANGENT & 3 & $\begin{array}{l}\operatorname{END}-\mathrm{I} \\
\operatorname{END}-\mathrm{J}\end{array}$ & $\begin{array}{l}-9117.181 \\
-9117: 181\end{array}$ & $=. .511$ & $\begin{array}{l}71.112 \\
71.112\end{array}$ & $\begin{array}{l}28.34 \\
28.34\end{array}$ & $\begin{array}{r}-79244.31 \\
-72986.49\end{array}$ & $\begin{array}{l}313.53 \\
358.45\end{array}$ \\
\hline 69 & TANGERT & 1 & $\begin{array}{l}\text { END-I } \\
\text { END-J }\end{array}$ & $\begin{array}{l}1333.469 \\
1333.469\end{array}$ & $\begin{array}{l}-4.039 \\
-4.039\end{array}$ & $\begin{array}{l}-902.907 \\
-902.907\end{array}$ & $\begin{array}{l}-2.29 \\
-2.29\end{array}$ & $\begin{array}{r}38020.48 \\
-41435.29\end{array}$ & $\begin{array}{r}-322.38 \\
33.07\end{array}$ \\
\hline 69 & TAMGRNT & 2 & $\frac{\text { END-I }}{\text { END-J }}$ & $\begin{array}{l}-11.184 \\
-11: 184\end{array}$ & $\begin{array}{r}-245.878 \\
-245.878\end{array}$ & $\begin{array}{l}4.351 \\
4.351\end{array}$ & $\begin{array}{l}5244.94 \\
5244.94\end{array}$ & $\begin{array}{r}-365.56 \\
17.32\end{array}$ & $\begin{array}{l}11823.63 \\
33460.92\end{array}$ \\
\hline 69 & TANGBNT & 3 & $\frac{\mathrm{END}-\mathrm{I}}{\mathrm{END}-\mathrm{J}}$ & $\begin{array}{l}-11537.011 \\
-11537.011\end{array}$ & $\begin{array}{l}4.289 \\
4.289\end{array}$ & $\begin{array}{l}915.392 \\
915.392\end{array}$ & $\begin{array}{l}28.34 \\
28.34\end{array}$ & $\begin{array}{r}-72986.49 \\
7567.97\end{array}$ & $\begin{array}{r}358.45 \\
-19.02\end{array}$ \\
\hline 70 & TANGENT & 1 & END-I & $\begin{array}{l}1345.579 \\
1345.579\end{array}$ & $\begin{array}{l}-5.849 \\
-5.849\end{array}$ & $\begin{array}{l}396.563 \\
396.563\end{array}$ & $\begin{array}{l}-2.29 \\
-2.29\end{array}$ & $\begin{array}{r}-41435.29 \\
-6537.71\end{array}$ & $\begin{array}{r}33.07 \\
547.80\end{array}$ \\
\hline 70 & TANGENT & 2 & $\frac{\text { END-I }}{\text { END-J }}$ & $\begin{array}{l}-11.284 \\
-11.284\end{array}$ & $\begin{array}{l}1122.312 \\
1122.312\end{array}$ & $\begin{array}{l}6.551 \\
6.551\end{array}$ & $\begin{array}{l}5244: 94 \\
5244: 94\end{array}$ & $\begin{array}{r}17.32 \\
593.81\end{array}$ & $\begin{array}{r}33460.92 \\
-65302.52\end{array}$ \\
\hline 70 & TANOENT & 3 & $\frac{\mathrm{END}-\mathrm{I}}{\mathrm{END}-\mathrm{J}}$ & $\begin{array}{l}-13969.521 \\
-13969: 521\end{array}$ & $\begin{array}{l}6.489 \\
6.489\end{array}$ & $\begin{array}{l}1244.972 \\
1244: 972\end{array}$ & $\begin{array}{l}28.34 \\
28.34\end{array}$ & $\begin{array}{r}7567.97 \\
117125.47\end{array}$ & $\begin{array}{l}-199.02 \\
-590.10\end{array}$ \\
\hline
\end{tabular}




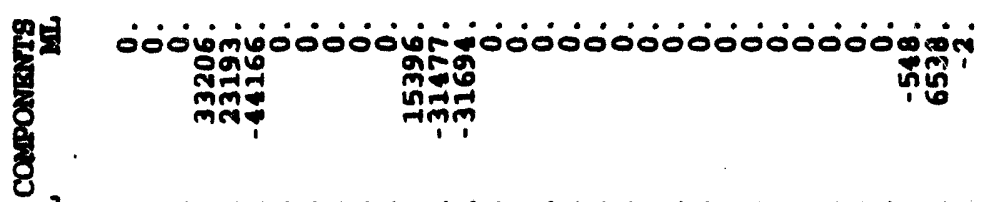

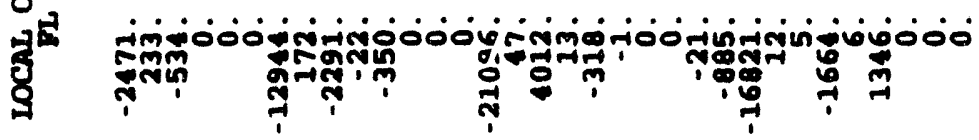

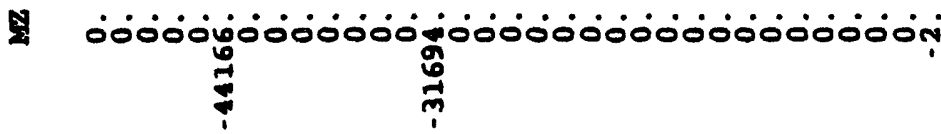

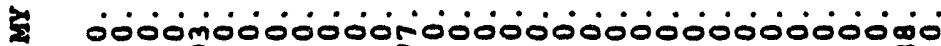
ते

勒

零

จ ०

*

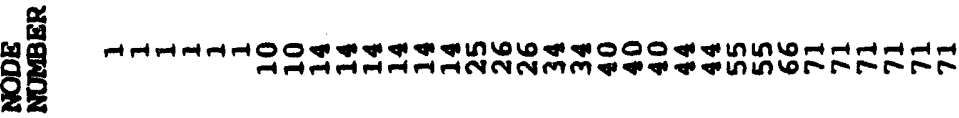




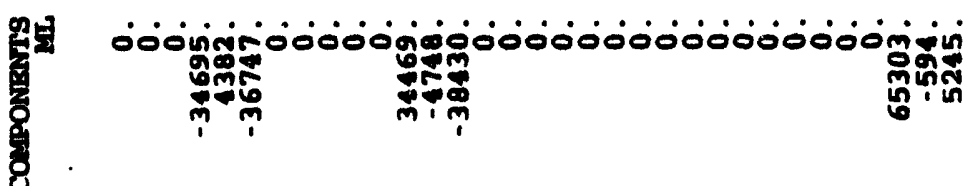

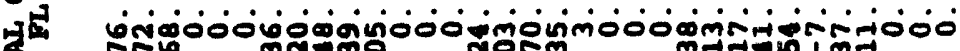
§ิ

*

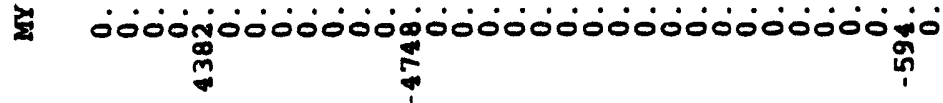
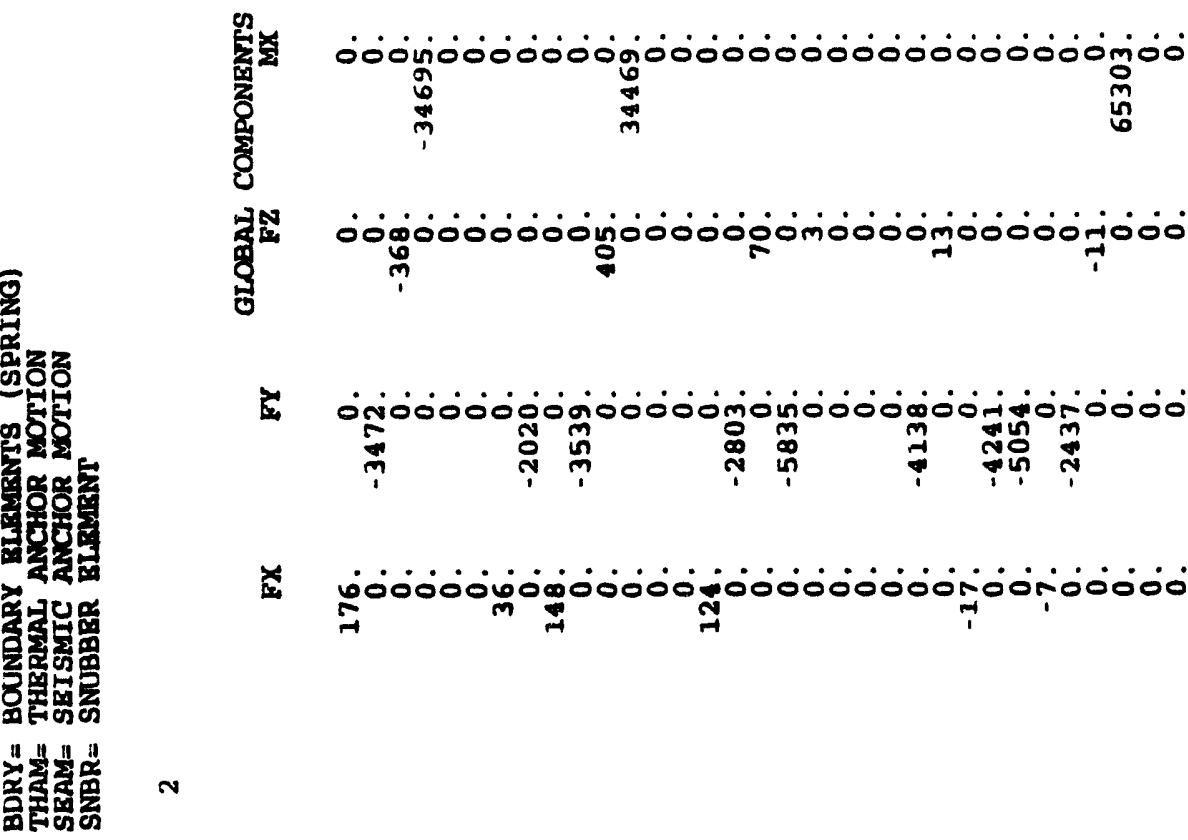

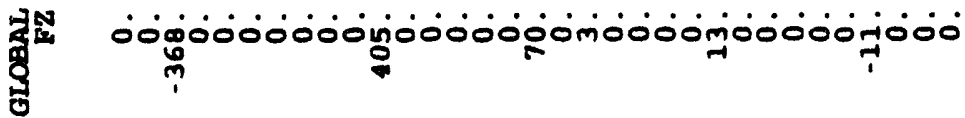

* o

*

$D$

是
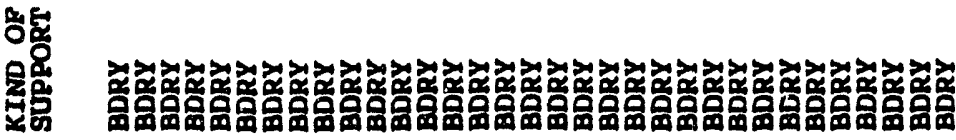


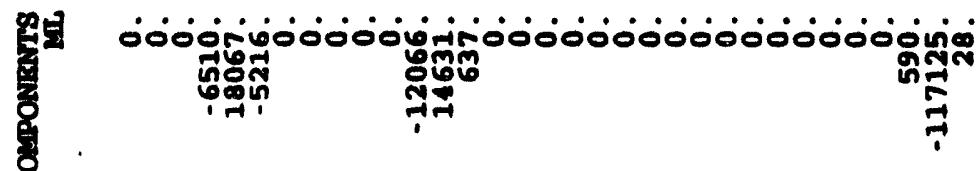
马ु

U 000000 तิ

2. 0000
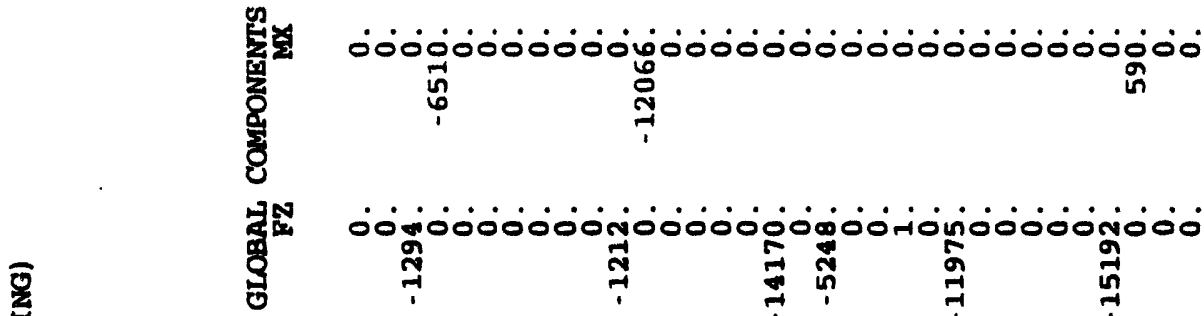

ใิ้

敦

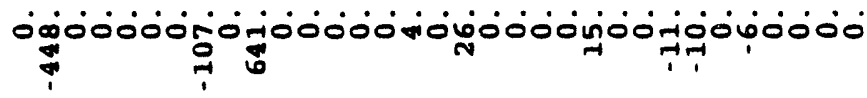

$\stackrel{m}{1}$

ஸें
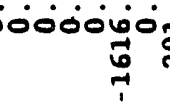

$\overbrace{0}^{1}$
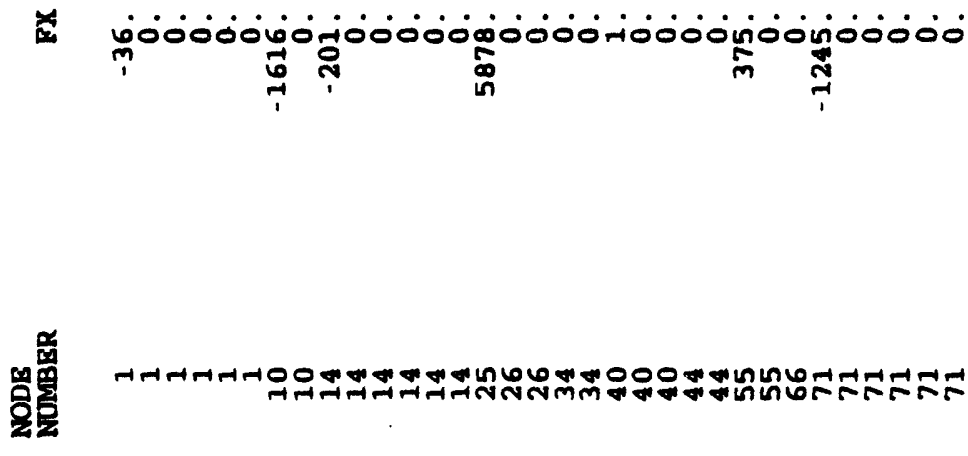

hathm-thos

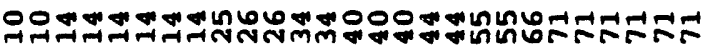




\section{BENCHMARK PROBLEM 1}

TOTAL RESPONSE 


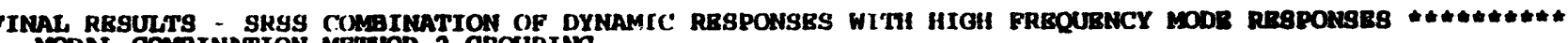

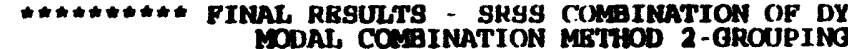

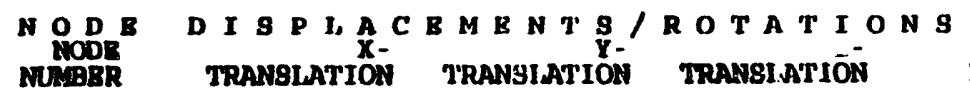
ROTATION

ROTATró

Rorarrö̀i

DrRectr

nCCBLBRATIOng If 0 's

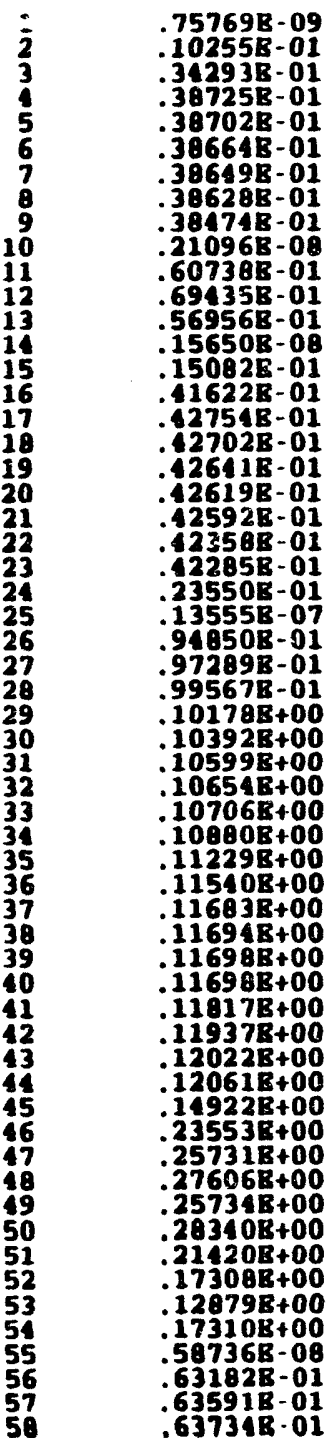

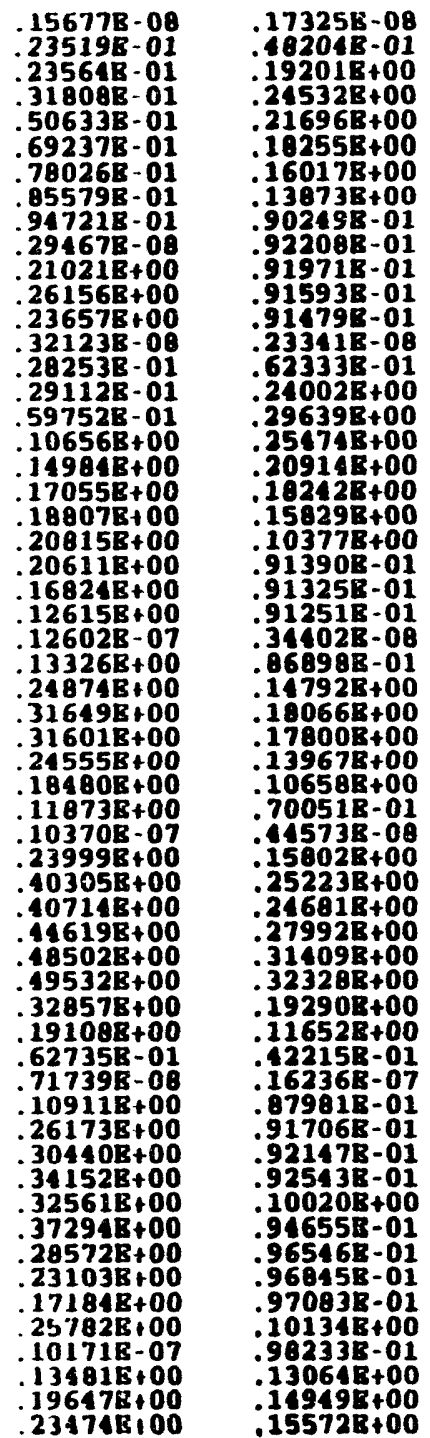

$115968-08$
$.223188-02$ $.223988-02$ $.196478-02$ $.200268-02$ $.210608-02$ $.214178-02$ $.25060 \mathrm{~B}-02$

$.31993 \mathrm{~B}-02$

$.20228-02$

166768-02

$.172378-08$ .300068-02 $.203828-02$ $.19073 \mathrm{~B}-02$

$.103888-02$

$.183304 \mathrm{E}-02$

$.19771 \mathrm{~B}-02$

.244068-02

$.271798-02$

$.314828-02$

$.303128-02$

.292441 - 02

$.274658-02$

$.272838-02$

$.27110 \mathrm{~B}-02$

$.25006 \mathrm{~B}-02$

$.25846 \mathrm{~B}-02$

$.26132 \mathrm{~B}-02$

$.261328-02$

$.26717 \mathrm{~B}-02$

$.276468-02$

$.29143 \mathrm{~B}-02$

$.20084 \mathrm{~B}-02$

$17466-02$
$.15472 B-02$

$.13194 \mathrm{R}-02$

$.15072 \mathrm{~B}-03$

$.19173 \mathrm{~B}-02$

$.209468-02$

$.209465-02$

$.245158-02$

$.21630 \mathrm{~B}-02$

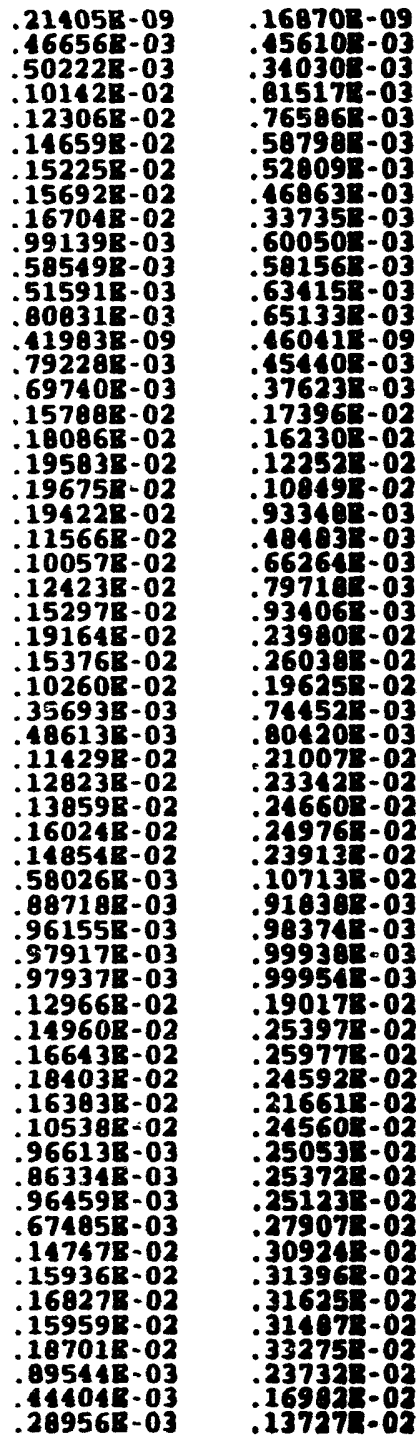

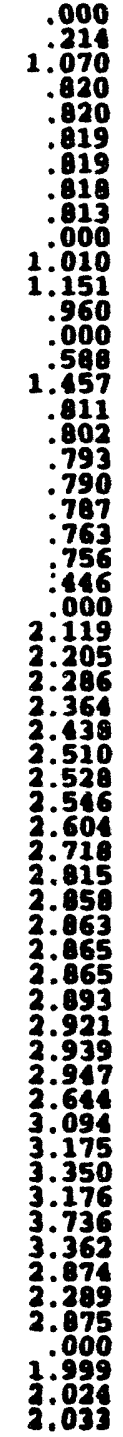

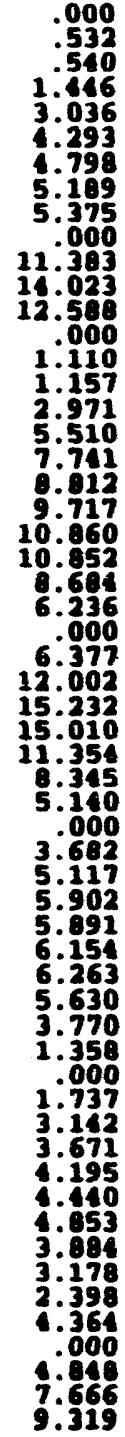

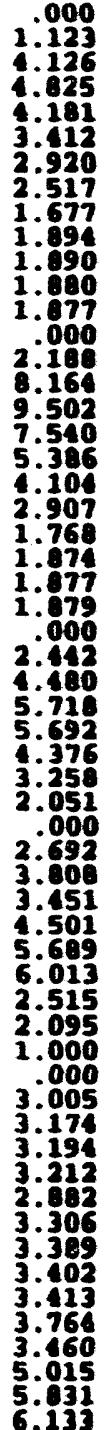


m-19oramoarang

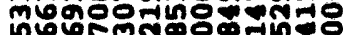

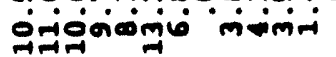

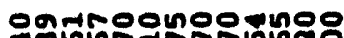

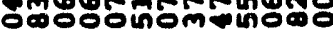
inirinciminiminicin

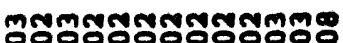
í

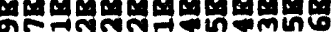

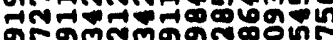
ḡmom

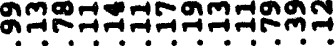

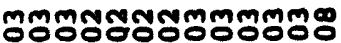

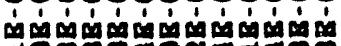
7оm 눅ำ

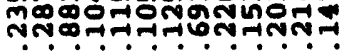

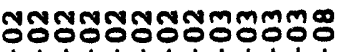

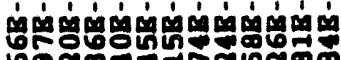

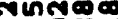

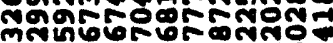

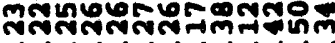

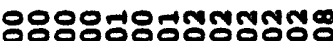

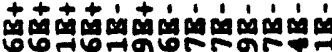
-7م นทำ

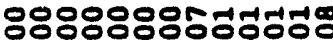

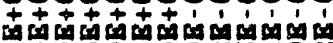
ตूनी ติธ

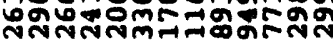

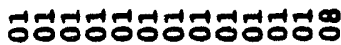

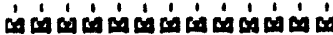

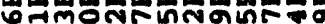
-6or-tin

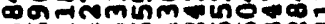

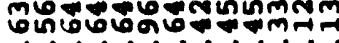

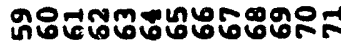




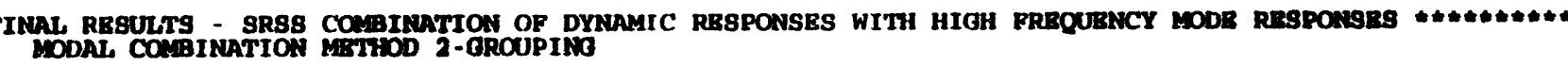

\begin{tabular}{|c|c|c|c|c|c|c|c|c|}
\hline 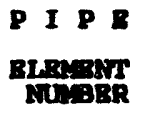 & $\begin{array}{c}\text { POR C } \\
\text { RLEager } \\
\text { TYPE }\end{array}$ & $\begin{array}{lr}\text { B 9 A } \\
\text { STATION }\end{array}$ & $\begin{array}{c}\text { MO M } \\
\text { AXIAI } \\
\text { BORCE }\end{array}$ & $\begin{array}{l}\text { Y-AXIS } \\
\text { ShRRAR }\end{array}$ & $\begin{array}{l}\text { Z-AXIS } \\
\text { SHEAR }\end{array}$ & $\begin{array}{l}\text { TORSIOMAi } \\
\text { Movisit }\end{array}$ & y-Axrs & $\frac{2-4 \times 18}{20109}$ \\
\hline 1 & BRED & $\begin{array}{c}\text { RND-I } \\
\text { CRMPRR } \\
\text { CRND-J }\end{array}$ & $\begin{array}{l}14965.024 \\
19023 \\
15545.6592\end{array}$ & $\begin{array}{l}15546.861 \\
10088.888 \\
14966.135\end{array}$ & $\begin{array}{l}11302.380 \\
11302.380 \\
11302.380\end{array}$ & $\begin{array}{l}897084.89 \\
668852.29 \\
300536.37\end{array}$ & $\begin{array}{l}214061.66 \\
160105: 42 \\
569535.08\end{array}$ & $\begin{array}{l}753075.19 \\
625900 \\
360067.05\end{array}$ \\
\hline 2 & TALGBRT & $\begin{array}{l}\text { RND }-I \\
\text { RND }-\mathbf{J}\end{array}$ & $\begin{array}{l}15316.157 \\
15316.157\end{array}$ & $\begin{array}{l}15587.622 \\
15587.622\end{array}$ & $\begin{array}{l}7076.302 \\
7076.302\end{array}$ & $\begin{array}{l}300600.57 \\
300600.57\end{array}$ & $\begin{array}{l}156975.16 \\
361623: 53\end{array}$ & $\begin{array}{r}667957.21 \\
691906.09\end{array}$ \\
\hline 3 & BESD & 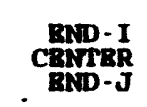 & $\begin{array}{r}15161.856 \\
11773.272 \\
6046.100\end{array}$ & $\begin{array}{r}6046.400 \\
6906: 309 \\
15161.856\end{array}$ & $\begin{array}{r}9488.173 \\
9488.173 \\
9488.173\end{array}$ & $\begin{array}{l}300600.57 \\
\$ 72286.008 \\
722916.88\end{array}$ & $\begin{array}{r}191906.09 \\
501630: 71 \\
514753.77\end{array}$ & $\begin{array}{l}361623.53 \\
3661656.90 \\
151333.05\end{array}$ \\
\hline 4 & TAROBRT & END - I & $\begin{array}{l}6343.655 \\
6343.655\end{array}$ & $\begin{array}{l}13698.928 \\
13698.928\end{array}$ & $\begin{array}{r}4930.293 \\
1930.293\end{array}$ & $\begin{array}{l}722916.89 \\
722916.88\end{array}$ & $\begin{array}{l}514753.77 \\
620507.03\end{array}$ & $\begin{array}{r}151333.05 \\
305360.67\end{array}$ \\
\hline 5 & TARBGATT & RARD-I & $\begin{array}{l}6855.222 \\
6855.222\end{array}$ & $\begin{array}{l}11141.894 \\
11141.894\end{array}$ & $\begin{array}{l}2739.709 \\
2739.709\end{array}$ & $\begin{array}{l}722916.88 \\
722916.88\end{array}$ & $\begin{array}{l}620507.03 \\
634562.11\end{array}$ & $\begin{array}{r}385360.67 \\
705022.64\end{array}$ \\
\hline 6 & TAligBar & END - I & $\begin{array}{l}7787.849 \\
7787.849\end{array}$ & $\begin{array}{l}6205.572 \\
6205.572\end{array}$ & $\begin{array}{r}1134.374 \\
1134.374\end{array}$ & $\begin{array}{l}722916.88 \\
722916: 88\end{array}$ & $\begin{array}{l}634562.11 \\
578010: 83\end{array}$ & $\begin{array}{l}704022.64 \\
809597.36\end{array}$ \\
\hline 7 & TANGBNT & BND-I & $\begin{array}{l}9146.800 \\
9146.800\end{array}$ & $\begin{array}{r}1982.617 \\
1982.617\end{array}$ & $\begin{array}{l}8104.673 \\
8104.673\end{array}$ & $\begin{array}{l}722915.88 \\
722916.88\end{array}$ & $\begin{array}{l}579010.93 \\
152035.35\end{array}$ & $\begin{array}{l}809597.36 \\
707454: 21\end{array}$ \\
\hline 8 & TAMOBMT & $\begin{array}{l}\text { RNDD-I } \\
\text { BND-J }\end{array}$ & $\begin{array}{l}10625.177 \\
10625.177\end{array}$ & $\begin{array}{l}9787.608 \\
8787.608\end{array}$ & $\begin{array}{l}11414.426 \\
11414.426\end{array}$ & $\begin{array}{l}722916.88 \\
722916.88\end{array}$ & $\begin{array}{r}152035.35 \\
208204: 00\end{array}$ & $\begin{array}{l}787454.21 \\
380121: 72\end{array}$ \\
\hline 9 & BBND & $\begin{array}{c}\text { END-I } \\
\text { CENPRR } \\
\text { BNID-J }\end{array}$ & $\begin{array}{l}12122.761 \\
11056.800 \\
12742.450\end{array}$ & $\begin{array}{l}12742.450 \\
13831: 076 \\
12122.761\end{array}$ & $\begin{array}{l}15993.344 \\
15893.344 \\
15993.344\end{array}$ & $\begin{array}{r}722916.88 \\
154186.62 \\
170602.80\end{array}$ & $\begin{array}{l}380121.72 \\
149832: 12 \\
196199.73\end{array}$ & $\begin{array}{r}209204.00 \\
116799.55 \\
666141.97\end{array}$ \\
\hline 10 & TANOBEN & ERD - I & $\begin{array}{l}14200.158 \\
14200.158\end{array}$ & $\begin{array}{l}39997.992 \\
39997.992\end{array}$ & $\begin{array}{l}13607.714 \\
13607.714\end{array}$ & $\begin{array}{l}170602.80 \\
170602.80\end{array}$ & $\begin{array}{l}666441.97 \\
812549.40\end{array}$ & $\begin{array}{r}196199.73 \\
2570050.30\end{array}$ \\
\hline 11 & TANOBNT & $\begin{array}{l}\text { RND -I } \\
\text { END-J }\end{array}$ & $\begin{array}{l}16610.169 \\
16610.169\end{array}$ & $\begin{array}{l}17212.076 \\
17212.076\end{array}$ & $\begin{array}{l}12072.780 \\
12072.780\end{array}$ & $\begin{array}{l}170602: 80 \\
170602.80\end{array}$ & $\begin{array}{r}812549.40 \\
1517272.89\end{array}$ & $\begin{array}{l}2570055.30 \\
3741951.24\end{array}$ \\
\hline 12 & TANOENT & BND-I & $\begin{array}{l}18578.253 \\
18578.253\end{array}$ & $\begin{array}{l}5126.194 \\
5126.194\end{array}$ & $\begin{array}{l}11040.405 \\
11040.405\end{array}$ & $\begin{array}{l}170602.80 \\
170602.80\end{array}$ & $\begin{array}{l}1517272.89 \\
1711351.68\end{array}$ & $\begin{array}{l}3741951.24 \\
3601159.29\end{array}$ \\
\hline 13 & TANOENT & $\begin{array}{l}\text { RERD-I } \\
\text { BEDD-J }\end{array}$ & $\begin{array}{l}19685.980 \\
19685.980\end{array}$ & $\begin{array}{l}13461.087 \\
13461.007\end{array}$ & $\begin{array}{l}10723.947 \\
10723.947\end{array}$ & $\begin{array}{l}170602.80 \\
170602.80\end{array}$ & $\begin{array}{l}2712351.60 \\
1069700.73\end{array}$ & $\begin{array}{l}3694159.29 \\
3677477.29\end{array}$ \\
\hline 14 & BEND & $\begin{array}{c}\text { RND-I } \\
\text { CRNTRR } \\
\text { BND-J }\end{array}$ & $\begin{array}{l}17066.401 \\
27957: 155 \\
32058.663\end{array}$ & $\begin{array}{l}32059.068 \\
23738.616 \\
17068.105\end{array}$ & $\begin{array}{l}24201.065 \\
24201.066 \\
24201.066\end{array}$ & $\begin{array}{r}1387902.81 \\
1051158: 81 \\
163022.82\end{array}$ & $\begin{array}{l}419824: 20 \\
701299: 71 \\
839242: 91\end{array}$ & $\begin{array}{r}1085397.62 \\
701628: 76 \\
153862.66\end{array}$ \\
\hline 15 & TANORNT & $\begin{array}{l}\text { END-I } \\
\text { BND-. }\end{array}$ & $\begin{array}{l}30836.659 \\
30836.659\end{array}$ & $\begin{array}{l}20211.909 \\
20211.909\end{array}$ & $\begin{array}{l}14772.143 \\
18772.113\end{array}$ & $\begin{array}{l}163122.95 \\
163122.95\end{array}$ & $\begin{array}{l}359989.66 \\
678069.23\end{array}$ & $\begin{array}{l}898593.37 \\
550023.13\end{array}$ \\
\hline 16 & BEND & $\begin{array}{c}\text { BND-I } \\
\text { CEIFTRR } \\
\text { BND-J }\end{array}$ & $\begin{array}{l}29850.813 \\
29948: 308 \\
13260: 370\end{array}$ & $\begin{array}{l}13260.370 \\
13091.197 \\
29850.813\end{array}$ & $\begin{array}{l}9307.263 \\
9307.263 \\
9307.263\end{array}$ & $\begin{array}{r}163122.95 \\
297148.43 \\
734555.76\end{array}$ & $\begin{array}{l}550023.13 \\
767797.61 \\
603714: 12\end{array}$ & $\begin{array}{l}676069.23 \\
676293.04 \\
197623.69\end{array}$ \\
\hline 17 & TANOBNI & RND - I & $\begin{array}{l}13827.255 \\
13827.255\end{array}$ & $\begin{array}{l}26925.042 \\
26925.042\end{array}$ & $\begin{array}{l}7524.377 \\
7524.377\end{array}$ & $\begin{array}{l}734555.76 \\
734555.76\end{array}$ & $\begin{array}{l}683714: 12 \\
652147.39\end{array}$ & $\begin{array}{l}197623.69 \\
708670.86\end{array}$ \\
\hline 18 & TANOEMI & $\begin{array}{l}\text { END. I } \\
\text { BNDD.J J }\end{array}$ & $\begin{array}{l}14336.205 \\
11336.205\end{array}$ & $\begin{array}{r}22319.459 \\
22319.459\end{array}$ & $\begin{array}{l}12034.74 y \\
12034.749\end{array}$ & $\begin{array}{r}734555.76 \\
731555.76\end{array}$ & $\begin{array}{l}652147.39 \\
599375.09\end{array}$ & $\begin{array}{r}709470.86 \\
2354129: 30\end{array}$ \\
\hline
\end{tabular}



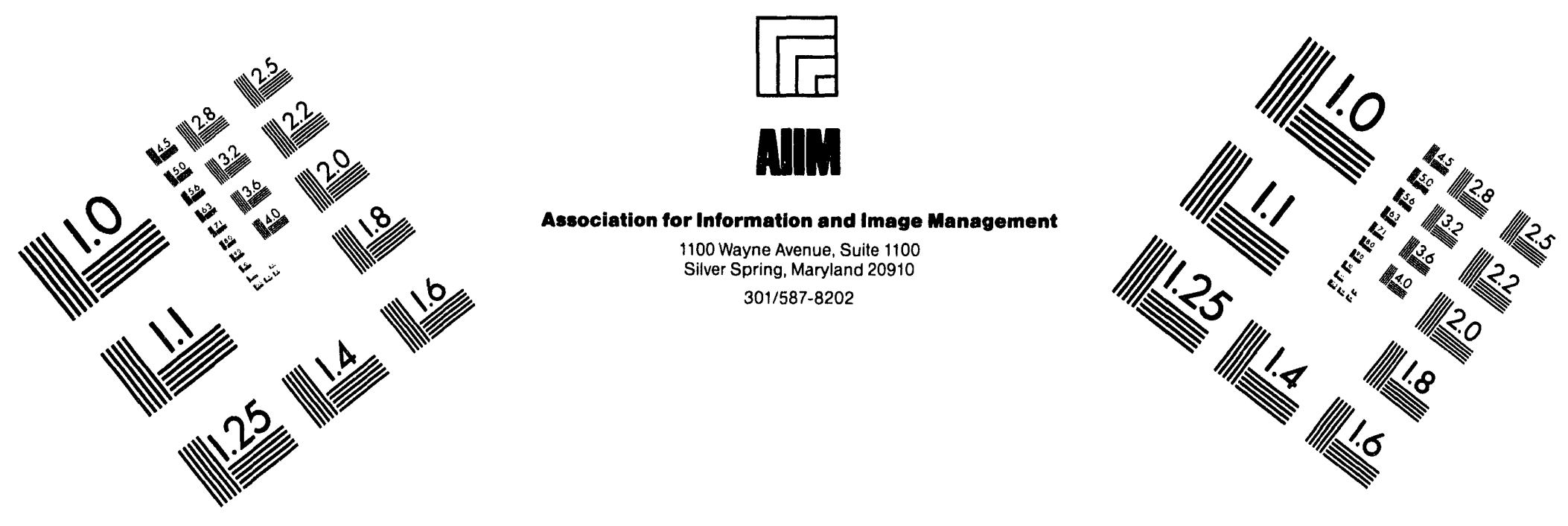

\section{Centimeter}

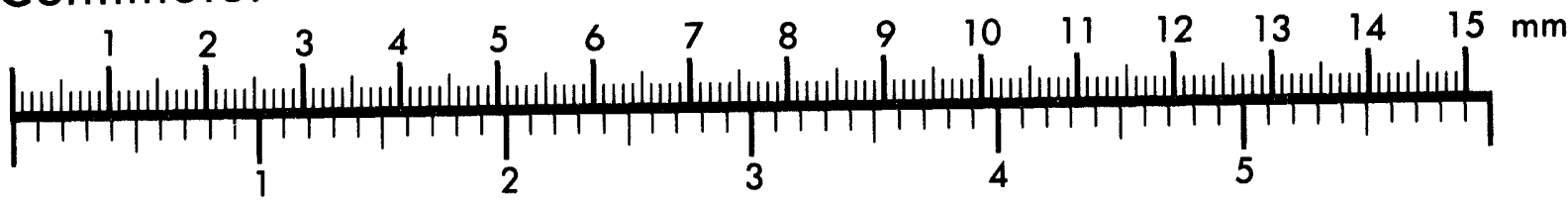
Inches

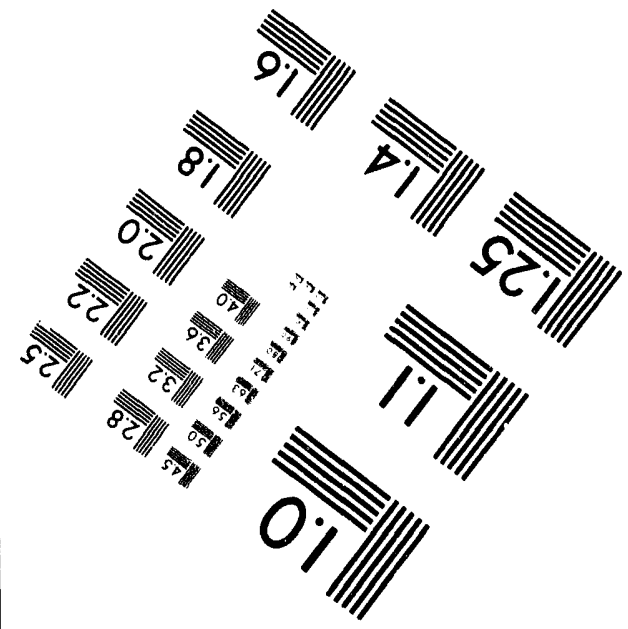

MANUFACTURED TO AIIM STANDARDS BY APPLIED IMAGE. INC.

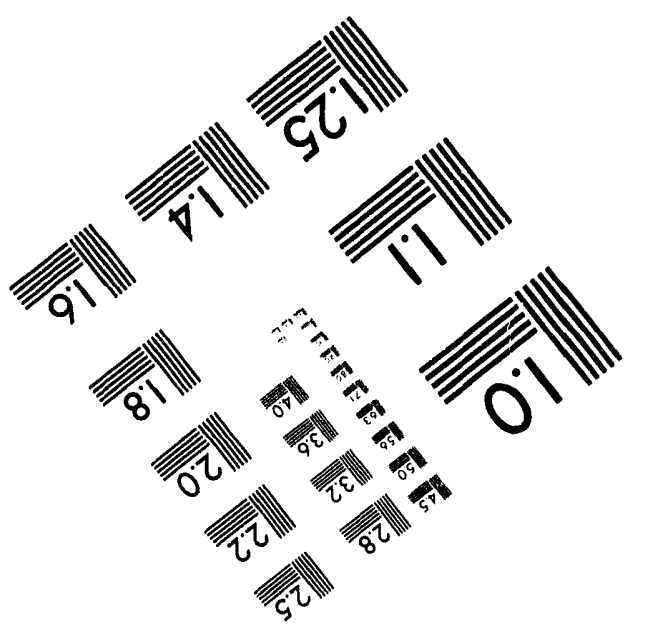



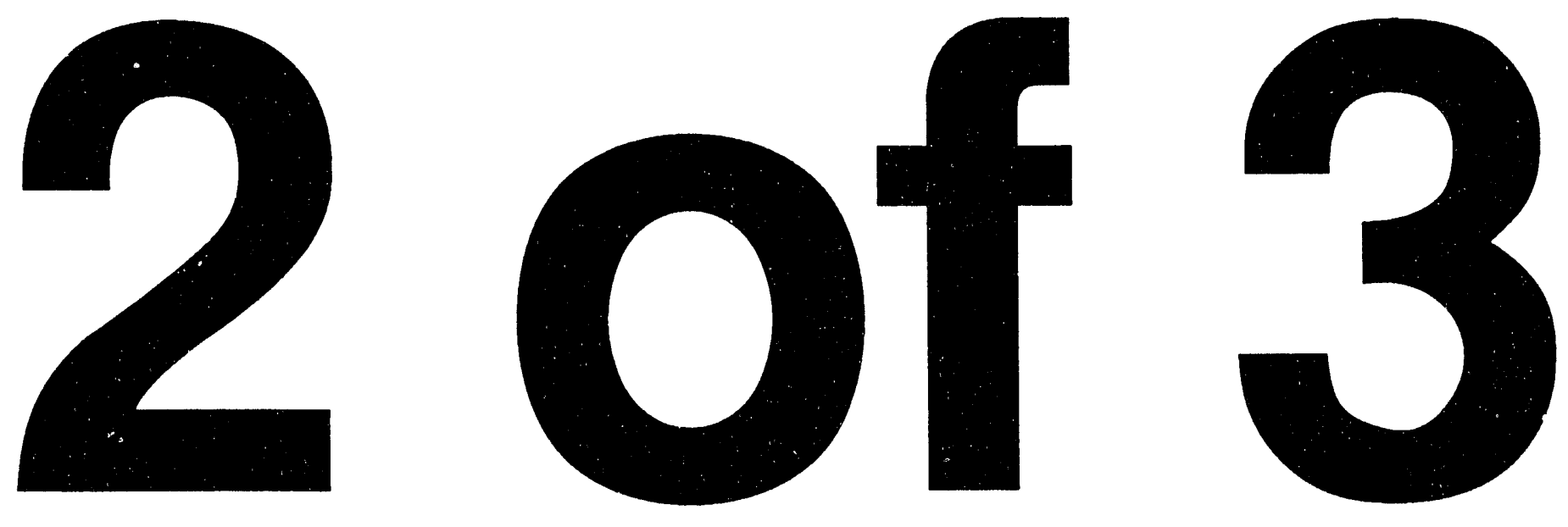


\begin{tabular}{|c|c|c|c|c|c|c|c|c|}
\hline 19 & TanoBart & $\frac{\operatorname{END}-I}{\operatorname{BNDD}-J}$ & $\begin{array}{l}15112: 428 \\
15112: 828\end{array}$ & $\begin{array}{l}13566.364 \\
13566.364\end{array}$ & $\begin{array}{l}17455.519 \\
17455.519\end{array}$ & $\begin{array}{l}734555.76 \\
734555.76\end{array}$ & $\begin{array}{l}599375.09 \\
646626.43\end{array}$ & $\begin{array}{l}1354129.38 \\
1591932.93\end{array}$ \\
\hline 20 & TANGERT & 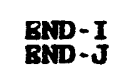 & $\begin{array}{l}16225.035 \\
16225: 035\end{array}$ & $\begin{array}{l}5674.269 \\
5674: 269\end{array}$ & $\begin{array}{l}22960.192 \\
22960: 192\end{array}$ & $\begin{array}{l}734555.76 \\
734555: 76\end{array}$ & $\begin{array}{l}646626.43 \\
843097: 20\end{array}$ & $\begin{array}{l}1591932.93 \\
1601016.54\end{array}$ \\
\hline 21 & TANOBNT & $\frac{\operatorname{RSD}-\mathbf{I}}{\mathrm{BNSD}-\mathrm{J}}$ & $17574: 979$ & $\begin{array}{l}17948.385 \\
17948.385\end{array}$ & $\begin{array}{l}27050.271 \\
27050.271\end{array}$ & $\begin{array}{l}734555.76 \\
736555.76\end{array}$ & $\begin{array}{r}843097.20 \\
2260410.53\end{array}$ & $\begin{array}{r}1601816.54 \\
921681.59\end{array}$ \\
\hline 22 & TAMORAMT & $\frac{\operatorname{Rg} D-I}{\operatorname{RNSD}-\mathbf{J}}$ & $\begin{array}{l}18593.401 \\
18593.401\end{array}$ & $\begin{array}{l}29532.395 \\
29532: 395\end{array}$ & $\begin{array}{l}26053.162 \\
26053: 162\end{array}$ & $\begin{array}{l}734555.76 \\
736555: 76\end{array}$ & $\begin{array}{l}2260440.53 \\
2676918.16\end{array}$ & $\begin{array}{l}921681.59 \\
679640.72\end{array}$ \\
\hline 23 & TAMOGNT & 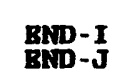 & $\begin{array}{l}27013.943 \\
27013: 943\end{array}$ & $\begin{array}{l}52476.737 \\
52676: 737\end{array}$ & $\begin{array}{l}502.647 \\
502.647\end{array}$ & 70887.87 & $\begin{array}{l}3200175.72 \\
3160407.53\end{array}$ & $\begin{array}{l}3914926.69 \\
303169: 55\end{array}$ \\
\hline 24 & t tangarat & $\frac{\text { RND-I }}{\text { BNAD-J }}$ & $\begin{array}{l}27196.528 \\
27196.528\end{array}$ & $\begin{array}{l}9414.918 \\
8444: 918\end{array}$ & $\begin{array}{l}902.962 \\
902.962\end{array}$ & 70887.87 & 60497.53 52523.79 & $\begin{array}{l}303148 \\
205035\end{array}$ \\
\hline 25 & BRND & 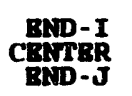 & $\begin{array}{r}27948.735 \\
93832 \\
131000.1157\end{array}$ & $\begin{array}{r}131890.147 \\
96277.115 \\
27848.735\end{array}$ & $\begin{array}{l}67596.901 \\
67596: 901 \\
67596: 901\end{array}$ & $\begin{array}{l}970897.87 \\
970578: 81 \\
562625.10\end{array}$ & $\begin{array}{r}2050355.53 \\
02456506 \\
2617676.26\end{array}$ & $\begin{array}{r}3152523.79 \\
169055: 80 \\
1970589.84\end{array}$ \\
\hline 26 & 5 TAMNORBTT & 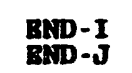 & $\begin{array}{l}127281.970 \\
127281.970\end{array}$ & $\begin{array}{l}65356.321 \\
65356.321\end{array}$ & 457.808 & $\begin{array}{l}582825.10 \\
582825.10\end{array}$ & $\begin{array}{l}1070589.84 \\
1604401.45\end{array}$ & $\begin{array}{l}261797 \\
107659\end{array}$ \\
\hline 27 & TANOBST & 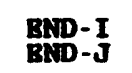 & $\begin{array}{l}123022.107 \\
123022.107\end{array}$ & $\begin{array}{l}53134.455 \\
53134.455\end{array}$ & 9956.120 & $\begin{array}{r}582825 \cdot 10 \\
582825 ; 10\end{array}$ & $\begin{array}{l}1904491.45 \\
2125173.36\end{array}$ & $\begin{array}{l}10765: \\
331222\end{array}$ \\
\hline 28 & TANOBAT & $\begin{array}{l}\text { ENDD-I } \\
\text { BND-J }\end{array}$ & $\begin{array}{l}118718.341 \\
118718.341\end{array}$ & $\begin{array}{l}31032.028 \\
31032: 028\end{array}$ & $\begin{array}{l}12949.879 \\
12949.879\end{array}$ & $\begin{array}{l}582825.10 \\
582825.10\end{array}$ & $\begin{array}{r}2125173.36 \\
2455907: 09\end{array}$ & $\begin{array}{r}3312260.95 \\
7716032.64\end{array}$ \\
\hline 29 & TANGERT & 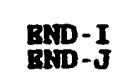 & $\begin{array}{l}114286.741 \\
114286.741\end{array}$ & $\begin{array}{l}10791.523 \\
10791.523\end{array}$ & $\begin{array}{l}9144.835 \\
9144: 835\end{array}$ & $\begin{array}{l}582825.10 \\
582825: 10\end{array}$ & $\begin{array}{l}2455997.09 \\
2473532: 14\end{array}$ & 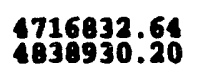 \\
\hline 30 & t tangrart & 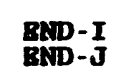 & $\begin{array}{l}109735.139 \\
109735.139\end{array}$ & $\begin{array}{l}3058 \mathrm{C} \\
3058 \mathrm{C}\end{array}$ & $\begin{array}{l}14271.758 \\
14271.758\end{array}$ & $\begin{array}{l}582825.10 \\
582825.10\end{array}$ & $\begin{array}{r}2473532.44 \\
2096272.91\end{array}$ & $\begin{array}{r}4838930.20 \\
3616719.18\end{array}$ \\
\hline 31 & I TANGERT & 良积D-I & $\begin{array}{l}1045 \\
1045\end{array}$ & & 27 & 22825.10 & $\begin{array}{l}2096 \\
1753\end{array}$ & 3816 \\
\hline 32 & TANORRT & $\frac{\operatorname{sND}-\mathbf{I}}{\mathrm{END}-\mathrm{J}}$ & $\begin{array}{l}10000 \\
10000\end{array}$ & $\begin{array}{l}682 \\
6824\end{array}$ & $\begin{array}{l}548.101 \\
548.101\end{array}$ & :10 & & $\begin{array}{l}29 \\
25\end{array}$ \\
\hline 33 & 3 TANGRAT & 胥D-I & 474 & 7795 & $\begin{array}{l}30082.544 \\
30082.544\end{array}$ & $\begin{array}{l}582825.10 \\
582825.10\end{array}$ & 25896 & $\begin{array}{l}2577 \\
6556\end{array}$ \\
\hline 34 & - TANGERT & 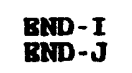 & $\begin{array}{l}88254.236 \\
88254.236\end{array}$ & $\begin{array}{r}\mathbf{4} 3968.523 \\
\mathbf{3} 3968.523\end{array}$ & $\begin{array}{l}28031.491 \\
28031.191\end{array}$ & $\begin{array}{r}582825.10 \\
582825.10\end{array}$ & $\begin{array}{l}2086784.00 \\
1534329.08\end{array}$ & 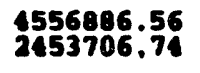 \\
\hline 35 & 5 TANORET & 茈D-I & $\begin{array}{l}78263.616 \\
78263.616\end{array}$ & $\begin{array}{l}39609.121 \\
39608.121\end{array}$ & $\begin{array}{l}20253.008 \\
20253.008\end{array}$ & $\begin{array}{l}582825.10 \\
582825.10\end{array}$ & $\begin{array}{l}1534 \\
2793 t\end{array}$ & $\begin{array}{r}245370 \\
812102\end{array}$ \\
\hline 36 & 6 TAMOBNT & $\begin{array}{l}\text { END-I } \\
\text { END-J }\end{array}$ & $\begin{array}{l}7062 \\
7062\end{array}$ & $\begin{array}{l}27023.603 \\
27023.603\end{array}$ & $\begin{array}{l}11302.954 \\
11302.954\end{array}$ & $\begin{array}{l}582825.10 \\
582825.10\end{array}$ & $\begin{array}{l}27938 \\
31923\end{array}$ & $\begin{array}{l}\$ 12102 \\
307653\end{array}$ \\
\hline 37 & TANGRAT & $\begin{array}{l}\mathrm{RND}-1 \\
\mathrm{BND}-\mathrm{s}\end{array}$ & 9913.803 & $\begin{array}{l}20740.811 \\
20740.811\end{array}$ & $\begin{array}{l}17506.042 \\
17506.042\end{array}$ & 00 & $\begin{array}{l}128065 \\
10643\end{array}$ & $\begin{array}{r}1463722.68 \\
429773.00\end{array}$ \\
\hline 38 & TANOBNT & $\begin{array}{l}\text { RNDD-I } \\
\text { BNDD-J }\end{array}$ & $\begin{array}{l}4118.632 \\
1118.632\end{array}$ & $\begin{array}{l}8963.393 \\
8963.393\end{array}$ & $\begin{array}{l}8454.440 \\
8454.440\end{array}$ & :00 & $\begin{array}{l}40643 \\
1755\end{array}$ & $\begin{array}{c}429733.00 \\
17476.42\end{array}$ \\
\hline 39 & TANGB & SWD-I & & 310 & $\begin{array}{l}1463.026 \\
1463.026\end{array}$ & .00 & & \\
\hline 10 & O TANGRMT & ESOD-1 & & & 86 & 5.10 & . & . \\
\hline
\end{tabular}




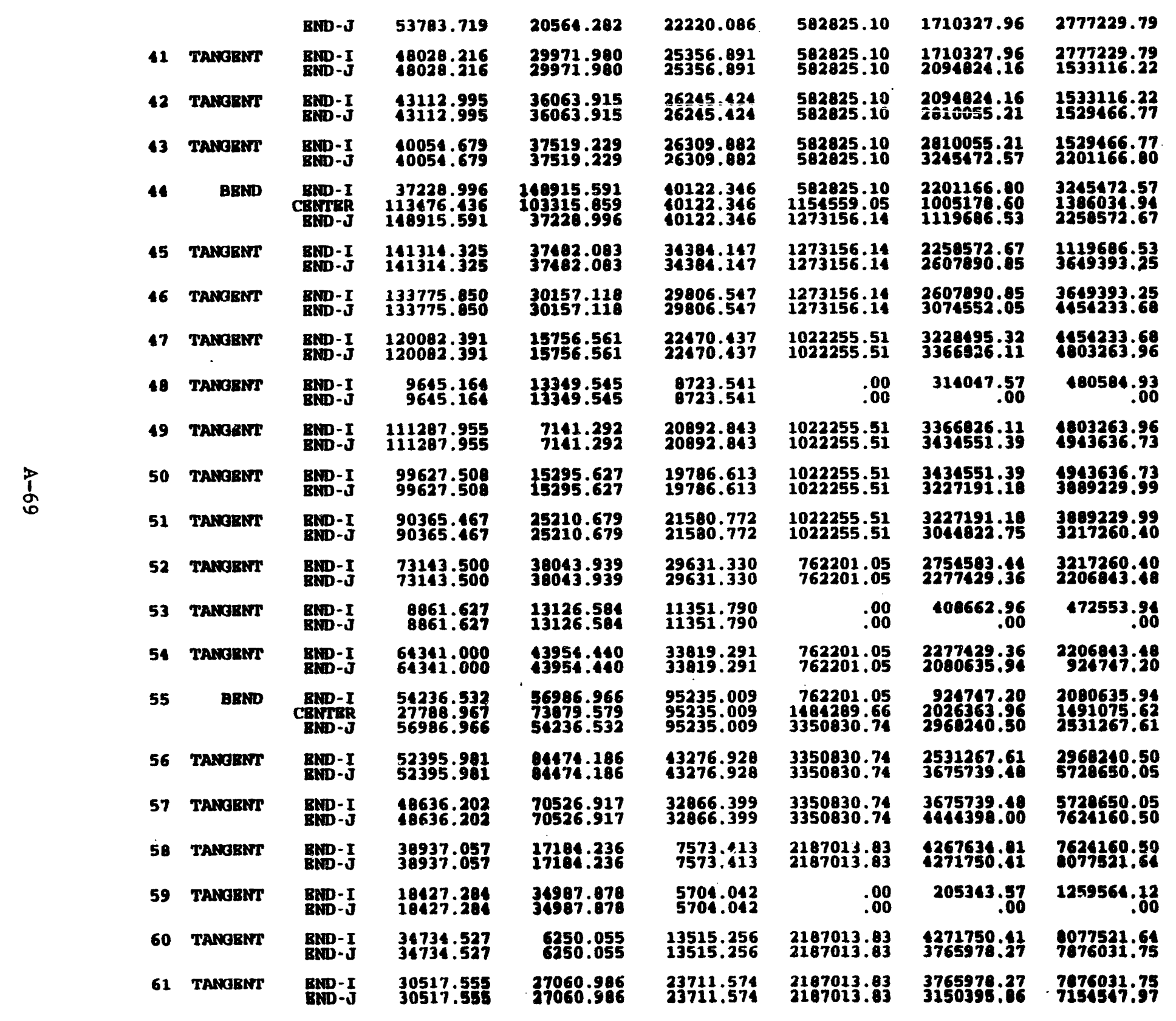




\begin{tabular}{|c|c|c|c|c|c|c|c|c|}
\hline 62 & TANGBNT & $\begin{array}{l}\text { END-1 } \\
\text { END-J }\end{array}$ & $\begin{array}{l}17329.960 \\
17329.960\end{array}$ & $\begin{array}{l}85744.398 \\
85744.398\end{array}$ & $\begin{array}{r}14767.479 \\
44767.479\end{array}$ & $\begin{array}{l}1174330.16 \\
1174330.16\end{array}$ & $\begin{array}{l}2785782.49 \\
1591456.69\end{array}$ & 715 \\
\hline 63 & TANGRRTY & $\frac{\operatorname{RND}-I}{\operatorname{RND}-\mathrm{J}}$ & $\begin{array}{l}13253.492 \\
13253: .92\end{array}$ & $\begin{array}{l}39657.445 \\
39657.445\end{array}$ & $\begin{array}{l}10624: 255 \\
10624: 255\end{array}$ & :00 & $\begin{array}{r}382474.78 \\
.00\end{array}$ & \\
\hline 64 & TAMOEANT & $\begin{array}{l}\text { RND }-I \\
\text { BND }-J\end{array}$ & $\begin{array}{l}14639.250 \\
14639.250\end{array}$ & $\begin{array}{l}98433.863 \\
98433.863\end{array}$ & $\begin{array}{l}99569.553 \\
19569.553\end{array}$ & $\begin{array}{l}1174330.16 \\
117430.16\end{array}$ & $\begin{array}{r}1591456.69 \\
602677.95\end{array}$ & \\
\hline 65 & BRND & 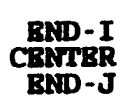 & $\begin{array}{l}11588.987 \\
11329.916 \\
53642.816\end{array}$ & $\begin{array}{l}53642.846 \\
36167: 2700 \\
11588.987\end{array}$ & $\begin{array}{l}11803.832 \\
111803 \\
111803.832 \\
111832\end{array}$ & $\begin{array}{l}1174330.16 \\
985556.50 \\
1275578.48\end{array}$ & $\begin{array}{r}2779419.41 \\
7759939.19 \\
3653317.32\end{array}$ & \\
\hline 66 & TAMGERNE & - & $\begin{array}{l}54628.266 \\
54628.266\end{array}$ & $\begin{array}{l}7928.604 \\
7928.604\end{array}$ & $\begin{array}{l}6998.020 \\
6998.020\end{array}$ & $\begin{array}{l}1275578.48 \\
1275578.48\end{array}$ & $\begin{array}{l}1317985.95 \\
1212299.39\end{array}$ & \\
\hline 67 & TANGERT & $\underset{\text { RND }}{\text { RND - I }}$ & $\begin{array}{l}55189.865 \\
55189.865\end{array}$ & $\begin{array}{l}12361.365 \\
12361.365\end{array}$ & 45.219 & $\begin{array}{l}1275578.48 \\
1275578.48\end{array}$ & & \\
\hline 68 & TANGRATP & END-I & $\begin{array}{l}55576.562 \\
55576.562\end{array}$ & $\begin{array}{l}17324.485 \\
17324.485\end{array}$ & $\begin{array}{l}6116.353 \\
6116.353\end{array}$ & $\begin{array}{l}1275578.48 \\
1275578.48\end{array}$ & $\begin{array}{r}117 \\
67\end{array}$ & \\
\hline 69 & TANOERT & $\begin{array}{l}\text { RND - I } \\
\text { BND-J }\end{array}$ & $\begin{array}{l}56276.025 \\
56276.025\end{array}$ & $\begin{array}{l}26168.756 \\
26168.756\end{array}$ & $\begin{array}{l}10922.046 \\
10922: 046\end{array}$ & $\begin{array}{l}1275578.48 \\
1275578.48\end{array}$ & & \\
\hline 70 & TANOBNT & $\begin{array}{l}\operatorname{RED}-\mathbf{I} \\
\text { BND -J }\end{array}$ & $\begin{array}{l}56949.324 \\
56949.324\end{array}$ & $\begin{array}{l}29834.718 \\
29834: 718\end{array}$ & $\begin{array}{l}\begin{array}{l}13018.238 \\
13018.238\end{array} \\
\end{array}$ & $\begin{array}{r}1275578.48 \\
1275578.48\end{array}$ & & \\
\hline
\end{tabular}




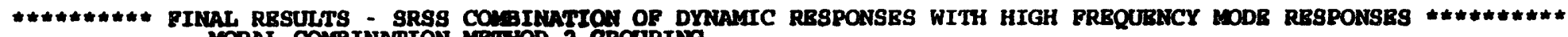
MODAL COABILATION MIFTHOD 2-GROOPINO

\begin{tabular}{|c|c|c|c|}
\hline 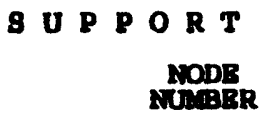 & $\begin{array}{l}\text { P O R C B S } \\
\text { COMPONBAT } \\
\text { DIRECTION }\end{array}$ & A N D & 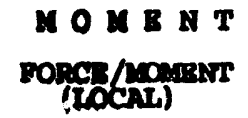 \\
\hline $\begin{array}{l}1 \\
1 \\
1 \\
1 \\
1 \\
1 \\
10 \\
10 \\
14 \\
14 \\
14 \\
14 \\
14 \\
14 \\
25 \\
26 \\
26 \\
34 \\
34 \\
40 \\
40 \\
40 \\
44 \\
14 \\
55 \\
55 \\
66 \\
71 \\
71 \\
71 \\
71 \\
71 \\
71\end{array}$ & 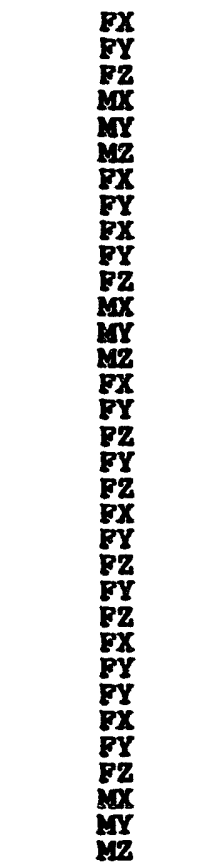 & & 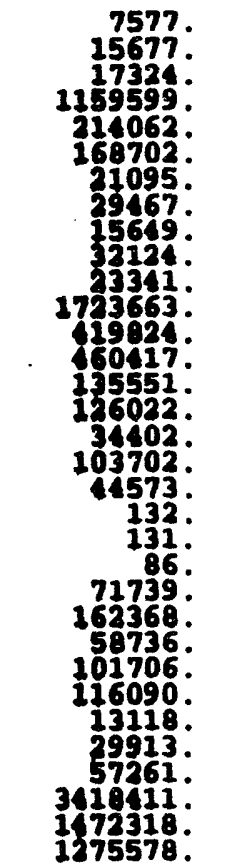 \\
\hline
\end{tabular}




\section{BENCHMARK PROBLEM 2}

\section{DIRECT INTEGRATION TIME HISTORY ANALYSIS}


SYSTEM 80+ FEEDWATER PIPING, BENCHMARK PROBLBM 2,DIRECT INIBGRATION TIME HISTORY ANALYSIS

CONTROI IN FOR MATION

NUMBER OF NODAL POINTS

NUMBER OF RIEMIENT TYPES

NUMBER OF STATIC LOAD CASES

NUMBER OP DYMAMIC IOAD CASES
NUMBER OF ANCHOR MVMT CASES

SOIBr ô

EO.0, EXECUTION

STRERS'S' CAICULATION FLAG

$\mathrm{EO} .0 \mathrm{NO}$

ASMI CODE EVALUATION FLAG

EO. 2 CLASS2 OR CIASS 3 PIPING

ACCELERATION DUR TO GRAVITY

BO. 0 NO

ARBITRARY NODB NUMBERING FLAG =

BO. 0 NO

LIST OF ANALYSIS TO BB PERFORMIED
LOAD CASE JISK FILE ANALYSIS TYPE

1

TIME HISTORY ANALYSIS 


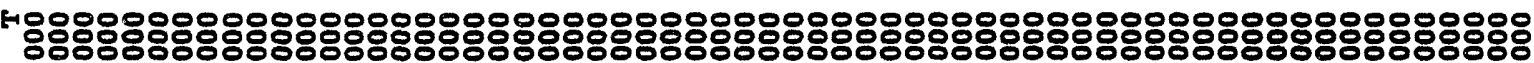
000000000000000000000000000000000000000000000000000000000000000

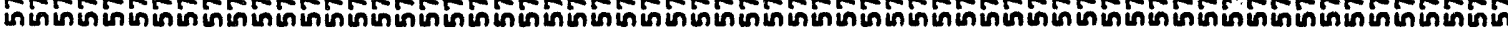

추웅위

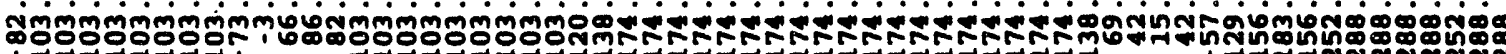

禺

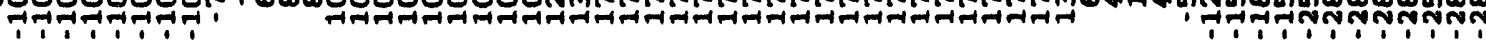

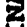

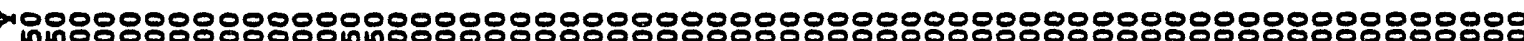

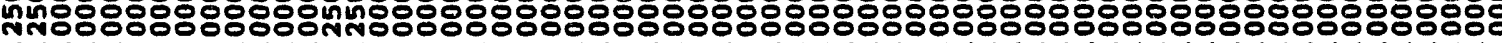
Nمmpq

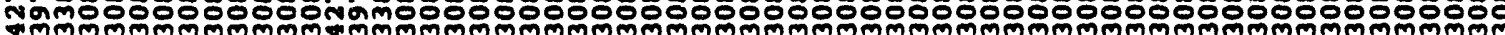

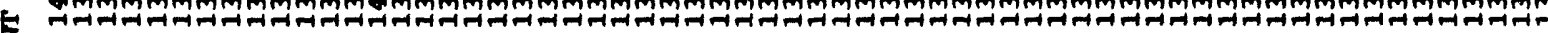

8

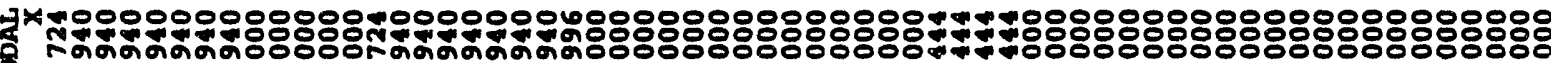
rmmmm

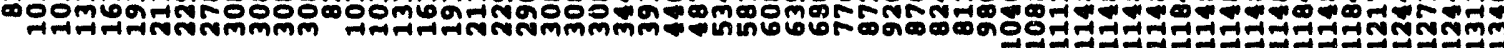

N00000000000000000000000000000000000000000000000000000000000000

on \%, 00000000000000000000000000000000000000000000000000000000000000 8

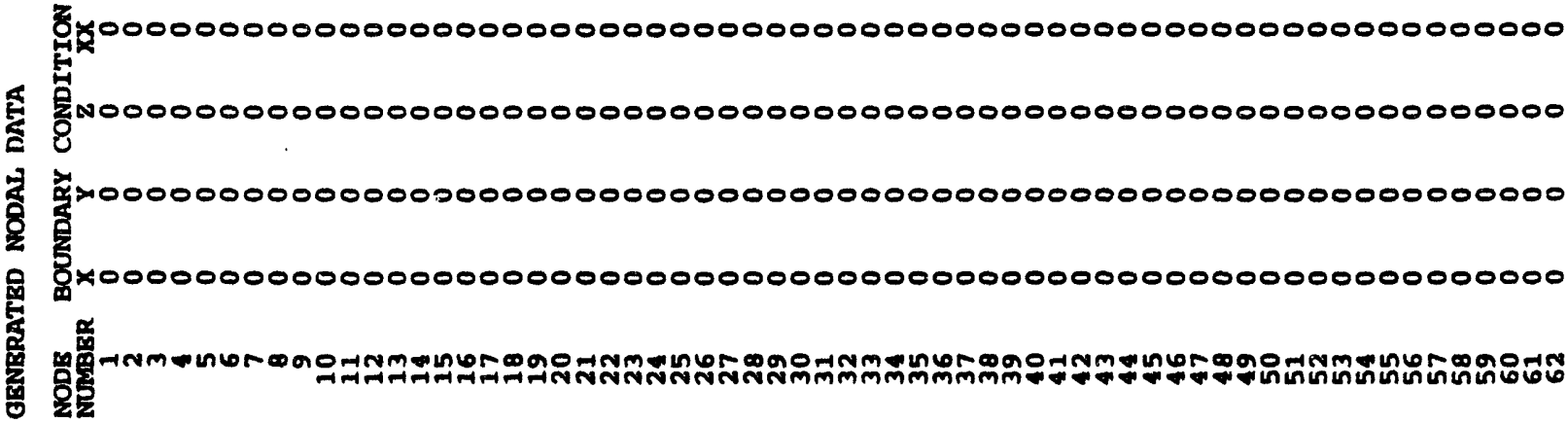


양응양응

000000000

iñninininininininín

양ํํㅇํㅇ요요

inimini

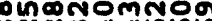

Nतกल

양용ㅇㅇㅇㅛ

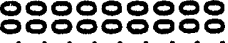

\%०00000000

mpm

Trif

요용ㅇำ

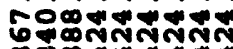

जिलन

000000000

000000000

000000000

000000000

000000000

000000000

ูํำแัธธ์ณำำ 


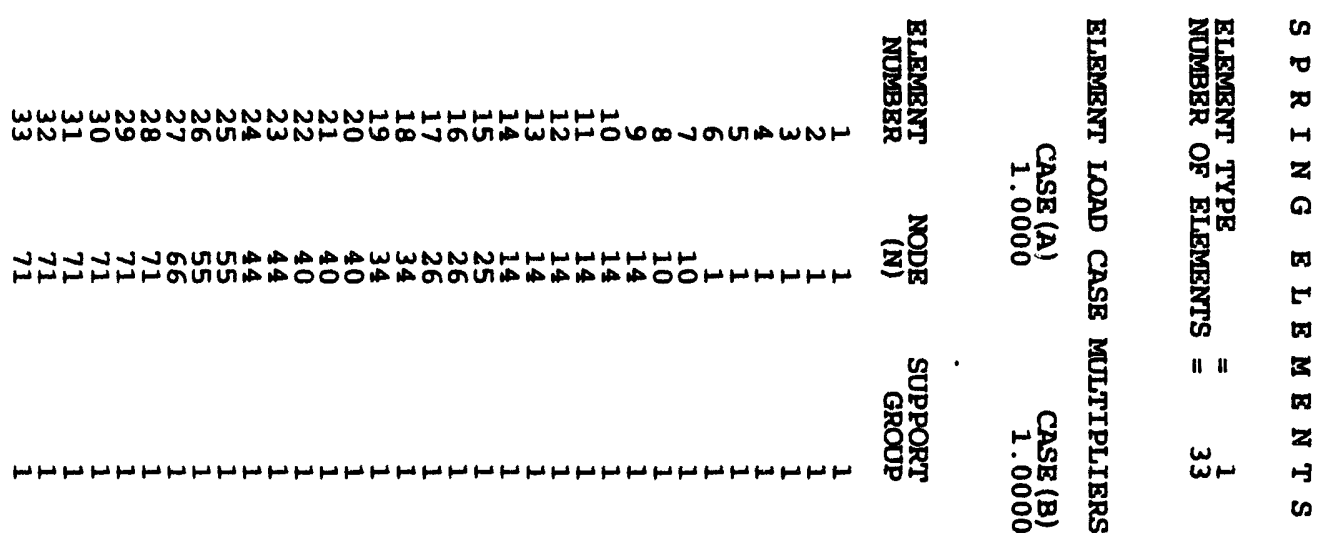

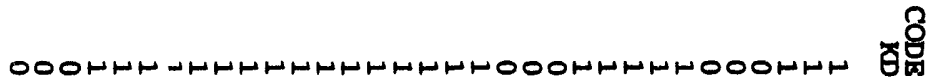

ผ

ค.

品量

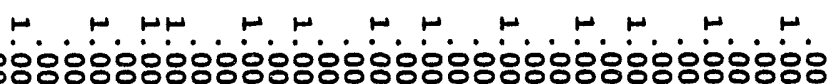

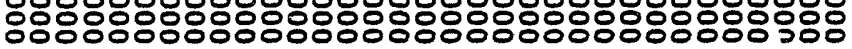

药

เั

里

蛋

哭

is ర $\infty$ $H$ 内 以 a 
P I P E E L B M E N T I N P U T D A T A

CONTROL INFORMATION

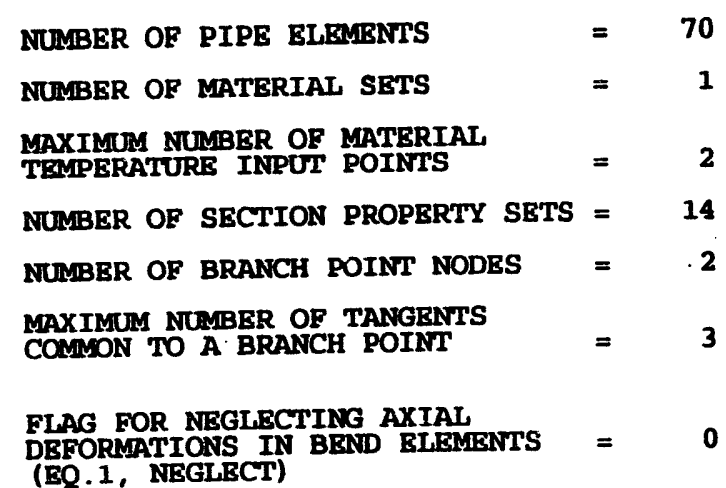


MATERIALTPOPERTY TABLES

\begin{tabular}{|c|c|c|c|c|}
\hline $\begin{array}{l}\text { MATERIA } \\
\text { NUMBER } \\
\text { TEMPERA } \\
\text { IDENTIF }\end{array}$ & $\begin{array}{l}\text { NUMBER } \\
\text { JRB POINTS } \\
\text { CATION } \\
\text { C. }\end{array}$ & $\begin{array}{l}\text { 1) } \\
\text { 2) }\end{array}$ & & \\
\hline $\begin{array}{l}\text { POINT } \\
\text { NUUBER }\end{array}$ & TEMPERATURE & $\begin{array}{l}\text { YOUNG'S } \\
\text { MODULUS }\end{array}$ & $\begin{array}{l}\text { POISSON'S } \\
\text { RATIO }\end{array}$ & $\begin{array}{l}\text { THERMAL } \\
\text { EXPANSION }\end{array}$ \\
\hline$\frac{1}{2}$ & $\begin{array}{r}70.00 \\
651.00\end{array}$ & $\begin{array}{l}2.950 \mathrm{E}+07 \\
2.610 \mathrm{E}+07\end{array}$ & $\begin{array}{l}.300 \\
.300\end{array}$ & $\begin{array}{l}5.600 \mathrm{~B}-06 \\
8.700 \mathrm{~B}-06\end{array}$ \\
\hline
\end{tabular}


SECTION PROPBRTY TABLE

\begin{tabular}{|c|c|c|c|c|c|c|}
\hline $\begin{array}{l}\text { SECTIION } \\
\text { NURBERR }\end{array}$ & $\begin{array}{c}\text { OUTSIDE } \\
\text { DIAMETER }\end{array}$ & $\begin{array}{l}\text { WALL } \\
\text { THICKNESS }\end{array}$ & $\begin{array}{l}\text { SHAPE FACTOR } \\
\text { FOR SHEAR }\end{array}$ & $\begin{array}{l}\text { WEIGHT/ } \\
\text { UNIT LBNGTH }\end{array}$ & UNIT IBASS/ & DESCRIPTION \\
\hline $\begin{array}{r}1 \\
2 \\
3 \\
4 \\
5 \\
6 \\
7 \\
8 \\
9 \\
10 \\
11\end{array}$ & $\begin{array}{l}20.000 \\
24.000 \\
24.000 \\
28.000 \\
33.000 \\
22.062 \\
26.436 \\
27.624 \\
27.600 \\
22.000 \\
26.000\end{array}$ & $\begin{array}{r}1.0310 \\
1.2180 \\
1.8120 \\
2.0000 \\
1.5000 \\
2.0620 \\
2.4360 \\
3.6240 \\
10.0000 \\
1.1240 \\
1.9060\end{array}$ & $\begin{array}{l}.0000 \\
.0000 \\
.0000 \\
.0000 \\
.0000 \\
.0000 \\
.0000 \\
.0000 \\
.0000 \\
.0000 \\
.0000\end{array}$ & $\begin{array}{l}.2898 \mathrm{~B}+02 \\
.4075 \mathrm{~B}+02 \\
.5043 \mathrm{~B}+02 \\
.3169 \mathrm{~B}+02 \\
.4205 \mathrm{~B}+02 \\
.8333 \mathrm{~B}+02 \\
.7407 \mathrm{~B}+02 \\
.7407 \mathrm{~B}+02 \\
.0000 \mathrm{~B}+00 \\
.4075 \mathrm{~B}+02 \\
.3737 \mathrm{~B}+01\end{array}$ & $\begin{array}{l}.7499 \mathrm{~B}-01 \\
.1055 \mathrm{~B}+00 \\
.1305 \mathrm{E}+00 \\
.8202 \mathrm{~B}-01 \\
.1088 \mathrm{~B}+00 \\
.2157 \mathrm{~B}+00 \\
.1917 \mathrm{~B}+00 \\
.1917 \mathrm{~B}+00 \\
.0000 \mathrm{~B}+00 \\
.1055 \mathrm{~B}+00 \\
.9671 \mathrm{E}-02\end{array}$ & $\begin{array}{l}20 S 80 \\
24 S 80 \\
24 S 120 \\
28 S P B C \\
30 I D \\
20 S 80 \text { VLVB } \\
24 S 80 \text { vive } \\
24 S 120 \text { VLVE } \\
\text { VIOP } \\
\text { BRED } 24 \star 20 \\
\text { BRED } 28 \star 24\end{array}$ \\
\hline
\end{tabular}


BRANCH POINT NODE I I S T

\section{BRANCH
POINT NODE
NUMBER \\ 123}

BLEMENT LOAD CASB MULTIPLIIRS

$\begin{array}{lrrrr} & \text { CASE A } & \text { CASE B } & \text { CASB C } & \text { CASB D } \\ \text { X-DIRECTION GRAVITY } & .000 & .000 & .000 & .000 \\ \text { Y-DIRECTION GRAVITY } & -1.000 & .000 & .000 & .000 \\ \text { Z-DIRECTION GRAVITY } & .000 & .000 & .000 & .000 \\ \text { THERRAL DISTORTION } & .000 & .000 & 1.000 & .000 \\ \text { PRESSURB DISTORTION } & .000 & 1.000 & .000 & .000\end{array}$




\begin{tabular}{|c|c|c|c|c|c|c|c|c|c|c|c|c|c|c|}
\hline $\begin{array}{c}\text { ELEMENT } \\
\text { NUMBER }\end{array}$ & $\frac{\text { ELEMPR }}{T Y}$ & & $\underset{-I}{\text { NODE }}$ & $\begin{array}{r}\text { NODE } \\
-J\end{array}$ & $\begin{array}{l}\text { MATL. } \\
\text { NUABER }\end{array}$ & $\begin{array}{l}\text { SECTION } \\
\text { NUIBER }\end{array}$ & $\begin{array}{c}\text { REFERENCE } \\
\text { TBITPERATURB } \\
\text { (BEND } \\
\text { RADIUS) }\end{array}$ & $\begin{array}{c}\text { INTERNAL } \\
\text { PRRSSURE } \\
\text { (THIRD } \\
\text { POINT) }\end{array}$ & $\begin{array}{l}\text { D I } \underset{A}{R} \text { (YX) } \\
\text { (X3- } \\
\text { ORDINATE) }\end{array}$ & $\begin{array}{l}\text { I O }{ }_{A}^{N} \text { C } 0 \\
\text { (Y3- } \\
\text { ORDINATE) }\end{array}$ & $\begin{array}{l}\text { O S I N N }{ }_{\text {A (YZ) }}^{\text {S }} \\
\text { (Z3 - } \\
\text { ORDINATE) }\end{array}$ & $\begin{array}{r}\text { NODE } \\
\text { INCREMENT } \\
\text { (WAII, } \\
\text { ERACTION) }\end{array}$ & $\begin{array}{l}\text { INPUT } \\
\text { TAG } \\
\text { BAND }\end{array}$ & \\
\hline 1 & BEND & & 1 & 2 & 1 & 1 & $\begin{array}{l}70.00 \\
30.0001\end{array}$ & $\left(j^{00}(\right.$ & $103.940)($ & $1427.250)($ & -103.9401 & $.1000)$ & IC & \\
\hline $\begin{array}{l}2 \\
3\end{array}$ & $\begin{array}{l}\text { TANG } \\
\text { BEND }\end{array}$ & $\mathbf{T}$ & $\begin{array}{l}2 \\
3\end{array}$ & $\begin{array}{l}3 \\
4\end{array}$ & $\frac{1}{1}$ & $\frac{1}{1}$ & $\begin{array}{l}70.00 \\
70.00 \\
30.0001\end{array}$ & $\left(j^{.00}\right.$ & $\begin{array}{c}.0000 \\
103.940)(\end{array}$ & $\begin{array}{c}.0000 \\
1308.000)(\end{array}$ & $\begin{array}{r}.0000 \\
-103.940)\end{array}$ & $\begin{array}{l}1 \\
.1000)\end{array}$ & II & 2 \\
\hline $\begin{array}{l}4 \\
5 \\
6 \\
7 \\
8 \\
9\end{array}$ & $\begin{array}{l}\text { TANG } \\
\text { TANG } \\
\text { TANG } \\
\text { TANG } \\
\text { TANG } \\
\text { BEND }\end{array}$ & $\begin{array}{l}\mathbf{T} \\
\mathbf{T} \\
\mathbf{T} \\
\mathbf{T} \\
\mathbf{T}\end{array}$ & $\begin{array}{l}4 \\
5 \\
6 \\
7 \\
8 \\
9\end{array}$ & $\begin{array}{r}5 \\
6 \\
7 \\
8 \\
9 \\
10\end{array}$ & $\begin{array}{l}1 \\
1 \\
1 \\
1 \\
1 \\
1\end{array}$ & $\begin{array}{l}1 \\
1 \\
6 \\
6 \\
1 \\
1\end{array}$ & $\begin{array}{l}70.00 \\
70.00 \\
70.00 \\
70.00 \\
70.00 \\
70.00 \\
30.0001\end{array}$ & $\begin{array}{l}.00 \\
.00 \\
.00 \\
.00 \\
.00 \\
1.00\end{array}$ & $\begin{array}{c}.0000 \\
.0000 \\
.0000 \\
.0000 \\
.0000 \\
308.000)(\end{array}$ & $\begin{array}{c}.0000 \\
.0000 \\
.0000 \\
.0000 \\
.0000 \\
1308.000)(\end{array}$ & $\begin{array}{r}.0000 \\
.0000 \\
.0000 \\
.0000 \\
.0000 \\
-103.940)\end{array}$ & $\begin{array}{l}1 \\
1 \\
1 \\
1 \\
1 \\
.1000)\end{array}$ & $\begin{array}{l}\text { II } \\
\text { II } \\
\text { II } \\
\text { II } \\
\text { II } \\
\text { IC }\end{array}$ & $\begin{array}{l}12 \\
12 \\
12 \\
12 \\
12\end{array}$ \\
\hline $\begin{array}{l}10 \\
11 \\
12 \\
13 \\
14\end{array}$ & $\begin{array}{l}\text { TANG } \\
\text { TANG } \\
\text { TANG } \\
\text { TANG } \\
\text { BEND }\end{array}$ & $\begin{array}{l}T \\
T \\
T \\
T\end{array}$ & $\begin{array}{l}10 \\
11 \\
12 \\
13 \\
14\end{array}$ & $\begin{array}{l}11 \\
12 \\
13 \\
23 \\
15\end{array}$ & $\begin{array}{l}1 \\
1 \\
1 \\
1 \\
1\end{array}$ & $\begin{array}{r}1 \\
1 \\
10 \\
2 \\
1\end{array}$ & $\begin{array}{l}70.00 \\
70.00 \\
70.00 \\
70.00 \\
70.00 \\
30.000)\end{array}$ & $\begin{array}{l}.00 \\
.00 \\
.00 \\
.00 \\
1.00\end{array}$ & $\begin{array}{r}.0000 \\
.0000 \\
.0000 \\
.0000 \\
103.940)(\end{array}$ & $\begin{array}{r}.0000 \\
.0000 \\
.0000 \\
.0000 \\
1427.250)(\end{array}$ & $\begin{array}{r}.0000 \\
.0000 \\
.0000 \\
.0000 \\
103.940)\end{array}$ & $\begin{array}{l}1 \\
i \\
3 \\
j \\
.10001\end{array}$ & $\begin{array}{l}\text { II } \\
\text { II } \\
\text { II } \\
\text { II } \\
\text { IC }\end{array}$ & $\begin{array}{l}12 \\
12 \\
12 \\
66\end{array}$ \\
\hline $\begin{array}{l}15 \\
16\end{array}$ & $\begin{array}{l}\text { TANG } \\
\text { BEND }\end{array}$ & $\mathbf{T}$ & $\begin{array}{l}15 \\
16\end{array}$ & $\begin{array}{l}16 \\
17\end{array}$ & $\begin{array}{l}1 \\
1\end{array}$ & $\frac{1}{1}$ & $\begin{array}{l}70.00 \\
70.00 \\
(\quad 30.000)\end{array}$ & $\left(i^{.00}\right.$ & $\begin{array}{c}.0000 \\
103.940)(\end{array}$ & $\begin{array}{c}.0000 \\
1308.000)(\end{array}$ & $\begin{array}{r}.0000 \\
103.940)\end{array}$ & $.1070)$ & II & 12 \\
\hline $\begin{array}{l}17 \\
18 \\
19 \\
20 \\
21 \\
22 \\
23 \\
24 \\
25\end{array}$ & $\begin{array}{l}\text { TANG } \\
\text { TANG } \\
\text { TANG } \\
\text { TANG } \\
\text { TANG } \\
\text { TANG } \\
\text { TANG } \\
\text { TANG } \\
\text { BEND }\end{array}$ & $\begin{array}{l}\mathbf{T} \\
\mathbf{T} \\
\mathbf{T} \\
\mathbf{T} \\
\mathbf{T} \\
\mathbf{T} \\
\mathbf{T} \\
\mathbf{T}\end{array}$ & $\begin{array}{l}17 \\
18 \\
19 \\
20 \\
21 \\
22 \\
23 \\
24 \\
25\end{array}$ & $\begin{array}{l}18 \\
19 \\
20 \\
21 \\
22 \\
23 \\
24 \\
25 \\
26\end{array}$ & $\begin{array}{l}1 \\
1 \\
1 \\
1 \\
1 \\
1 \\
1 \\
1 \\
1\end{array}$ & $\begin{array}{l}1 \\
1 \\
6 \\
6 \\
1 \\
1 \\
2 \\
2 \\
2\end{array}$ & $\begin{array}{l}70.00 \\
70.00 \\
70.00 \\
70.00 \\
70.00 \\
70.00 \\
70.00 \\
70.00 \\
70.00 \\
36.000)\end{array}$ & $\begin{array}{l}.00 \\
.00 \\
.00 \\
.00 \\
.00 \\
.00 \\
.00 \\
.00 \\
1.00\end{array}$ & $\begin{array}{r}.0000 \\
.0000 \\
.0000 \\
.0000 \\
.0000 \\
.0000 \\
.0000 \\
.0000 \\
308.000)(\end{array}$ & $\begin{array}{r}.0000 \\
.0000 \\
.0000 \\
.0000 \\
.0000 \\
.0000 \\
.0000 \\
.0000 \\
1308.000)(\end{array}$ & $\begin{array}{r}.0000 \\
.0000 \\
.0000 \\
.0000 \\
.0000 \\
.0000 \\
.0000 \\
.0000 \\
174.000)\end{array}$ & $\begin{array}{l}1 \\
1 \\
1 \\
1 \\
1 \\
1 \\
1 \\
1 \\
.1000)\end{array}$ & $\begin{array}{l}\text { III } \\
\text { III } \\
\text { III } \\
\text { II } \\
\text { II } \\
\text { II } \\
\text { II } \\
\text { II } \\
\text { IC }\end{array}$ & $\begin{array}{l}12 \\
12 \\
12 \\
12 \\
12 \\
12 \\
12 \\
12\end{array}$ \\
\hline $\begin{array}{l}26 \\
27 \\
28 \\
29 \\
30 \\
31 \\
32 \\
33 \\
34 \\
35 \\
36 \\
37 \\
38\end{array}$ & $\begin{array}{l}\text { TANG } \\
\text { TANG } \\
\text { TANG } \\
\text { TANG } \\
\text { TANG } \\
\text { TANG } \\
\text { TANG } \\
\text { TANG } \\
\text { TANG } \\
\text { TANG } \\
\text { TANG } \\
\text { TANG } \\
\text { TANG }\end{array}$ & $\begin{array}{l}\mathbf{T} \\
\mathbf{T} \\
\mathbf{T} \\
\mathbf{T} \\
\mathbf{T} \\
\mathbf{T} \\
\mathbf{T} \\
\mathbf{T} \\
\mathbf{T} \\
\mathbf{T} \\
\mathbf{T} \\
\mathbf{T} \\
\mathbf{T}\end{array}$ & $\begin{array}{l}26 \\
27 \\
28 \\
29 \\
30 \\
31 \\
32 \\
33 \\
34 \\
35 \\
36 \\
37 \\
38\end{array}$ & $\begin{array}{l}27 \\
28 \\
29 \\
30 \\
31 \\
32 \\
33 \\
34 \\
35 \\
36 \\
37 \\
38 \\
39\end{array}$ & $\begin{array}{l}1 \\
1 \\
1 \\
1 \\
1 \\
1 \\
1 \\
1 \\
1 \\
1 \\
1 \\
1 \\
1\end{array}$ & $\begin{array}{l}2 \\
2 \\
2 \\
2 \\
2 \\
7 \\
7 \\
2 \\
2 \\
2 \\
2 \\
5 \\
5\end{array}$ & $\begin{array}{l}70.00 \\
70.00 \\
70.00 \\
70.00 \\
70.00 \\
70.00 \\
70.00 \\
70.00 \\
70.00 \\
70.00 \\
70.00 \\
70.00 \\
70.00\end{array}$ & $\begin{array}{l}.00 \\
.00 \\
.00 \\
.00 \\
.00 \\
.00 \\
.00 \\
.00 \\
.00 \\
.00 \\
.00 \\
.00 \\
.00\end{array}$ & $\begin{array}{l}.0000 \\
.0000 \\
.0000 \\
.0000 \\
.0000 \\
.0000 \\
.0000 \\
.0000 \\
.0000 \\
.0000 \\
.0000 \\
.0000 \\
.0000\end{array}$ & $\begin{array}{l}.0000 \\
.0000 \\
.0000 \\
.0000 \\
.0000 \\
.0000 \\
.0000 \\
.0000 \\
.0000 \\
.0000 \\
.0000 \\
.0000 \\
.0000\end{array}$ & $\begin{array}{l}.0000 \\
.0000 \\
.0000 \\
.0000 \\
.0000 \\
.0000 \\
.0000 \\
.0000 \\
.0000 \\
.0000 \\
.0000 \\
.0000 \\
.0000\end{array}$ & $\begin{array}{l}1 \\
1 \\
1 \\
1 \\
1 \\
1 \\
1 \\
1 \\
1 \\
1 \\
1 \\
1 \\
1\end{array}$ & $\begin{array}{l}\text { II } \\
\text { III } \\
\text { II } \\
\text { II } \\
I I \\
I I \\
I I \\
I I \\
I I \\
I I \\
I I \\
I I \\
\text { II }\end{array}$ & $\begin{array}{l}12 \\
12 \\
12 \\
12 \\
12 \\
12 \\
12 \\
12 \\
12 \\
12 \\
12 \\
12 \\
12\end{array}$ \\
\hline
\end{tabular}




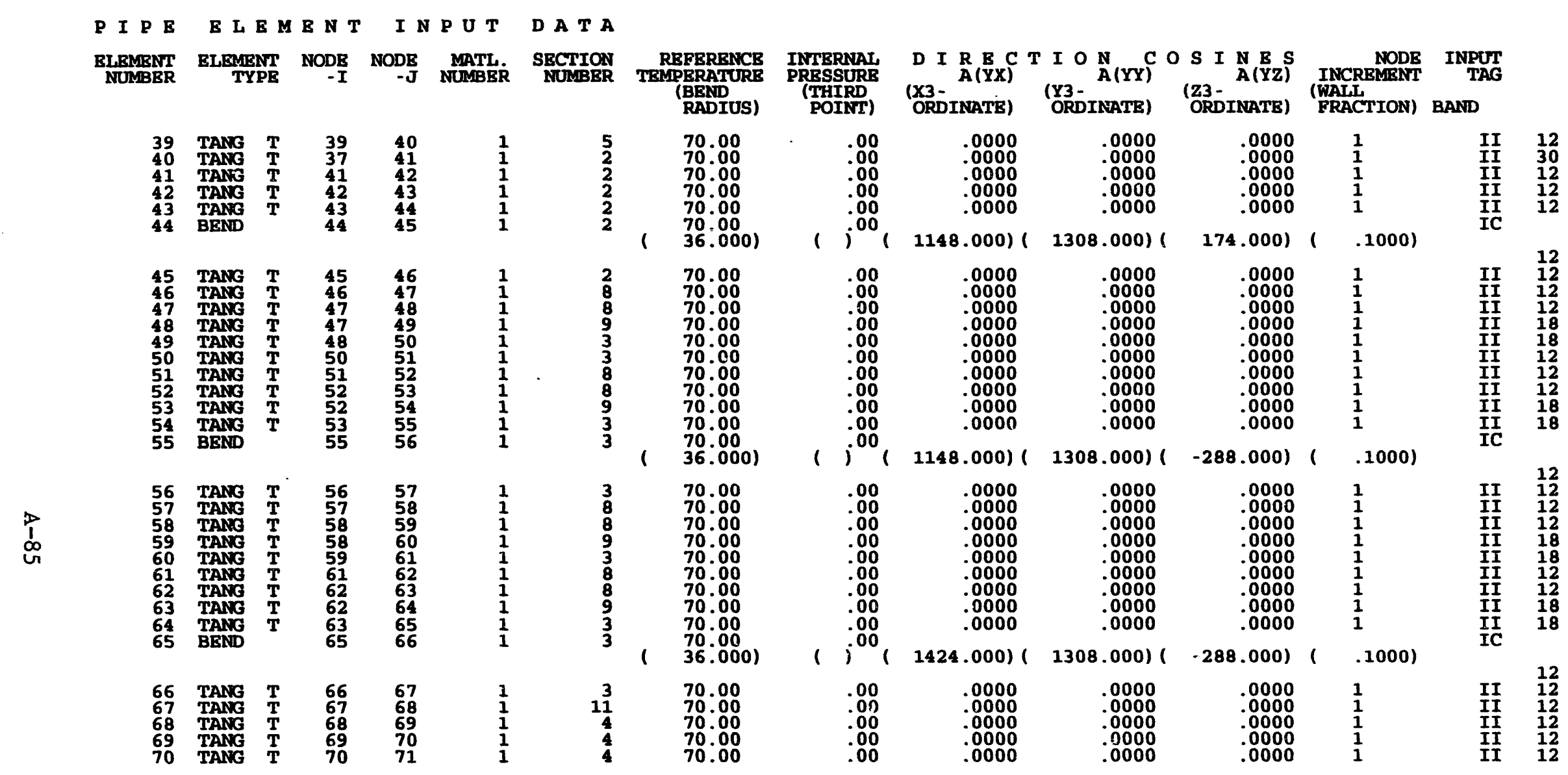




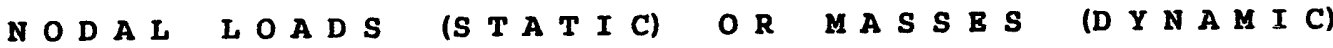

\begin{tabular}{|c|c|c|c|c|c|c|c|}
\hline $\begin{array}{r}\text { NODE } \\
\text { NUMBER }\end{array}$ & $\begin{array}{l}\text { LOAD } \\
\text { CASE }\end{array}$ & $\begin{array}{c}\text { X-AXIS } \\
\text { FORCE }\end{array}$ & $\begin{array}{r}\text { Y-AXIS } \\
\text { BORCB }\end{array}$ & $\begin{array}{c}\text { z-AXIS } \\
\text { BORCE }\end{array}$ & $\begin{array}{l}\text { X-AXIS } \\
\text { MORIENT }\end{array}$ & $\begin{array}{l}\text { Y-AXIS } \\
\text { MONIENT }\end{array}$ & $\begin{array}{l}\text { Z-AXIS } \\
\text { MOMIINT }\end{array}$ \\
\hline $\begin{array}{l}49 \\
49 \\
54 \\
54 \\
60 \\
60 \\
64 \\
64\end{array}$ & $\begin{array}{l}0 \\
1 \\
0 \\
1 \\
0 \\
1 \\
0 \\
1\end{array}$ & $\begin{array}{l}7.76398 \mathrm{E}+00 \\
0.00000 \mathrm{E}+00 \\
7.76398 \mathrm{~B}+00 \\
0.00000 \mathrm{E}+00 \\
7.76398 \mathrm{E}+00 \\
0.00000 \mathrm{E}+00 \\
7.76398 \mathrm{E}+00 \\
0.00000 \mathrm{E}+00\end{array}$ & $\begin{array}{r}7.76398 \mathrm{E}+00 \\
-3.00000 \mathrm{~B}+03 \\
7.76398 \mathrm{E}+00 \\
-3.00000 \mathrm{E}+03 \\
7.76398 \mathrm{E}+00 \\
-3.00000 \mathrm{E}+03 \\
7.76398 \mathrm{E}+00 \\
-3.00000 \mathrm{E}+03\end{array}$ & $\begin{array}{l}7.76398 \mathrm{~B}+00 \\
0.00000 \mathrm{E}+00 \\
7.76398 \mathrm{~B}+00 \\
0.00000 \mathrm{~B}+00 \\
7.76398 \mathrm{E}+00 \\
0.00000 \mathrm{E}+00 \\
7.76398 \mathrm{~B}+00 \\
0.00000 \mathrm{~B}+00\end{array}$ & $\begin{array}{l}0.00000 \mathrm{E}+00 \\
0.00000 \mathrm{~B}+00 \\
0.00000 \mathrm{E}+00 \\
0.00000 \mathrm{~B}+00 \\
0.00000 \mathrm{~B}+00 \\
0.00000 \mathrm{~B}+00 \\
0.00000 \mathrm{~B}+00 \\
0.00000 \mathrm{~B}+00\end{array}$ & $\begin{array}{l}0.00000 \mathrm{~B}+00 \\
0.00000 \mathrm{~B}+00 \\
0.00000 \mathrm{~B}+00 \\
0.00000 \mathrm{~B}+00 \\
0.00000 \mathrm{~B}+00 \\
0.00000 \mathrm{~B}+00 \\
\mathrm{C} .00000 \mathrm{~B}+00 \\
0.00000 \mathrm{~B}+00\end{array}$ & $\begin{array}{l}0.00000 \mathrm{E}+00 \\
0.00000 \mathrm{E}+00 \\
0.00000 \mathrm{E}+00 \\
0.00000 \mathrm{E}+00 \\
0.00000 \mathrm{E}+00 \\
0.00 \mathrm{C} 00 \mathrm{~B}+00 \\
0.00000 \mathrm{~B}+00 \\
0.00000 \mathrm{E}+00\end{array}$ \\
\hline
\end{tabular}

ALL EQUATIONS HAVE STIFENESS ATTACHED

\section{STRUCTURE
LOAD CASE}

1

\begin{tabular}{rrrrr} 
& BLEMIENT & LOAD & \multicolumn{2}{c}{ MULTIPLIERS } \\
.000 & .000 & .000 & .000
\end{tabular}


STEP-BY-STEP SOLUTION CONTROL INEORMATION

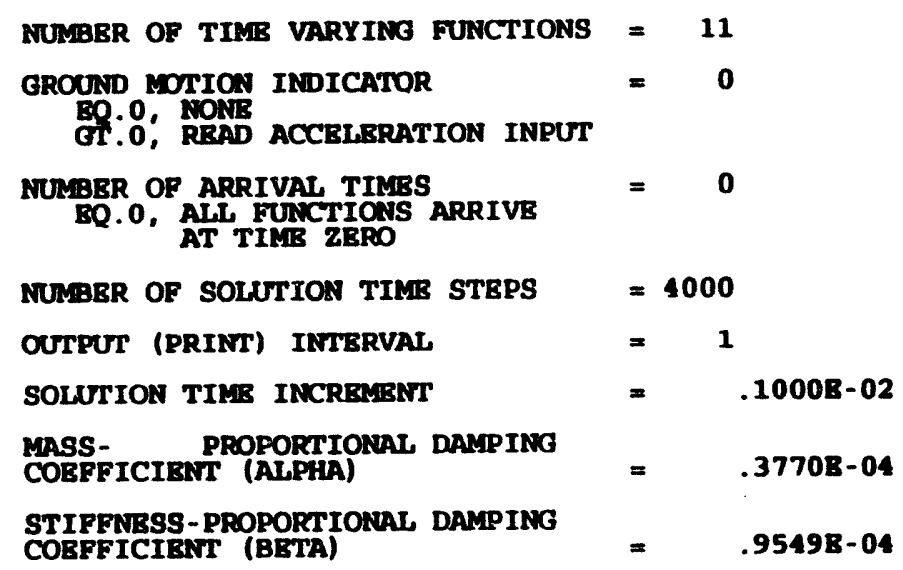

STIFFNESS MATRIX PARAMIBTRS

MINIYMY NON-28RO DIAGONAL BLEMIRT $=2.873 \mathrm{~B}+06$

MAXIMUA DIAGONAL BLEMRNT $=\frac{1.0008+15}{3.481 \mathrm{~B}+08}$

$\begin{array}{ll}\text { MAXIMUN/MINIMUM } & =3.4818+08 \\ \text { AVBRAGE DIAGONAL ELEMIENT } & =2.163 \mathrm{~B}+13 \\ \text { DENSITY OF THE MATRIX } & =5.3 \mathrm{PCT} .\end{array}$

$\underset{1}{\infty}$

DENSITY OF THB MATRIX 
D Y A M I C L O A D I N P U T

\begin{tabular}{|c|c|c|c|c|}
\hline $\begin{array}{r}\text { NODE } \\
\text { NUMBER }\end{array}$ & $\begin{array}{c}\text { DEGREE OF } \\
\text { FREEDOM }\end{array}$ & $\begin{array}{l}\text { FUNCTION } \\
\text { RBEBRENCE }\end{array}$ & $\begin{array}{r}\text { ARRIVAL TIMB } \\
\text { NUMBER }\end{array}$ & $\begin{array}{r}\text { FUNCTION } \\
\text { MLTIPLIER }\end{array}$ \\
\hline $\begin{array}{r}1 \\
1 \\
3 \\
9 \\
14 \\
14 \\
16 \\
17 \\
25 \\
44 \\
55 \\
65 \\
66\end{array}$ & $\begin{array}{l}1 \\
3 \\
2 \\
1 \\
1 \\
3 \\
2 \\
1 \\
3 \\
3 \\
1 \\
3 \\
1 \\
3\end{array}$ & $\begin{array}{r}1 \\
1 \\
2 \\
3 \\
9 \\
9 \\
10 \\
11 \\
4 \\
5 \\
6 \\
7 \\
8\end{array}$ & $\begin{array}{l}1 \\
1 \\
1 \\
1 \\
1 \\
1 \\
1 \\
1 \\
1 \\
1 \\
1 \\
1 \\
1\end{array}$ & 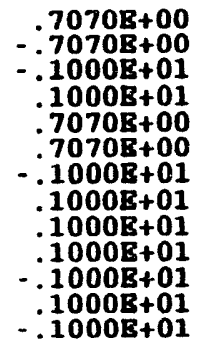 \\
\hline
\end{tabular}

REQUIRED BLANK COMMON FOR THIS STEP = 9374

A RRI VAL T IME VALUES

INPUT ARDER

1
1
$\infty$

T I B F U N C T I ON D A T A

801

1.0
0.000
0.015
0.030
0.045
0.060
0.075
0.090
0.105
0.120
0.135
0.150
0.165
0.180
0.195
0.210
0.225

FUNCTION 1

0.000

0.045
0.044

0.044

0.042
0.039

0.035

0.032
0.028

0.028
0.024

0.024
0.020

0.020
0.015

0.011

0.006

0.001
-0.004
-0.010

-0.004
-0.010
0.005
0.020

0.020
0.035
0.050

0.050

0.080

0.095

0.110

0.140

0.155

0.185

0.215
0.230

0.045
0.045
0.043
0.041
0.038
0.034
0.031
0.027
0.023
0.018
0.014
0.009
0.004
-0.001
-0.006
-0.012

0.010

0.025

0.055

0.070

0.085

0.100
0.115

0.130

0.145

0.160

0.175
0.190

0.1905

0.220

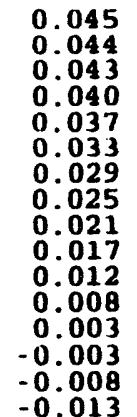




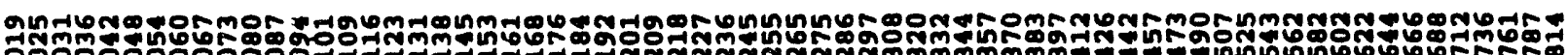

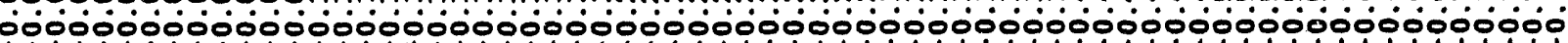

애은

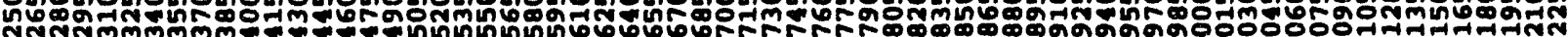

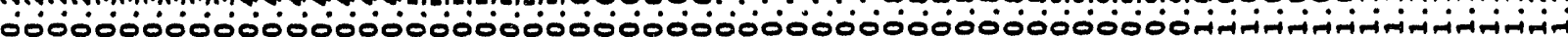

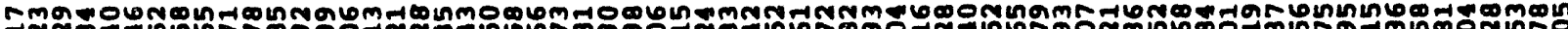

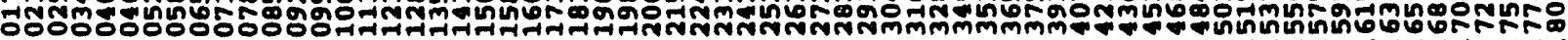

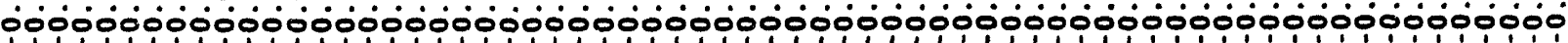

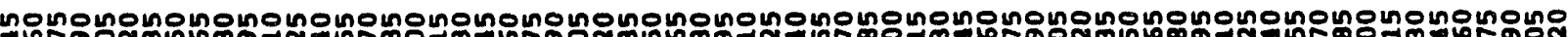
సีస్ㄱำ 0000000000000000000000000000000000000000000000000 -

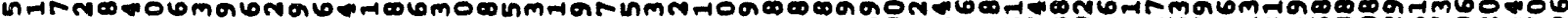

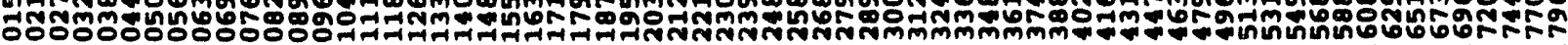

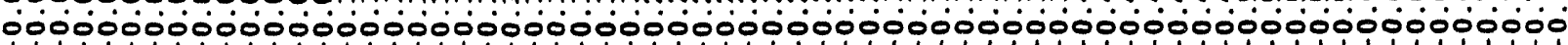

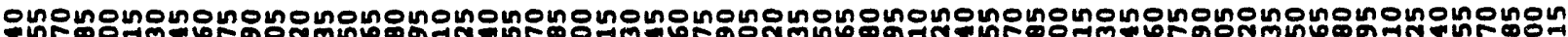
ผสnกำmmmm

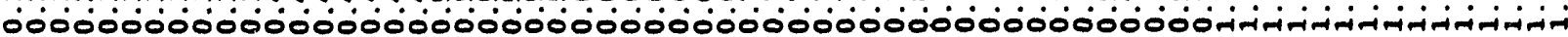




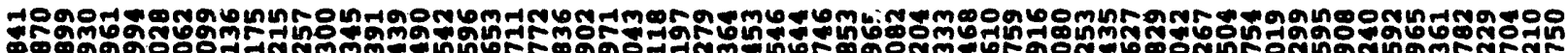

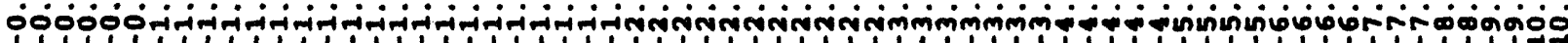

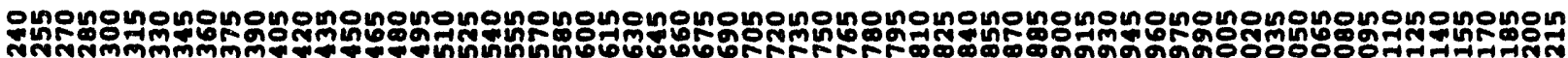

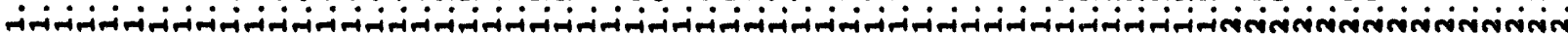

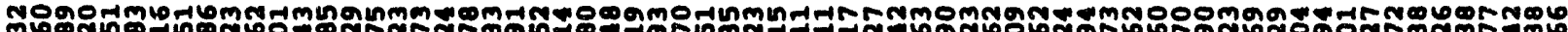

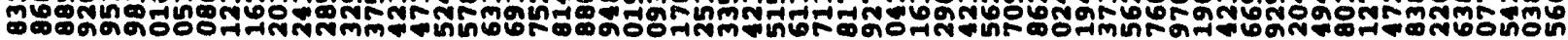

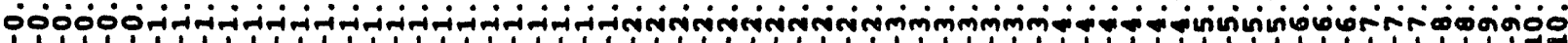

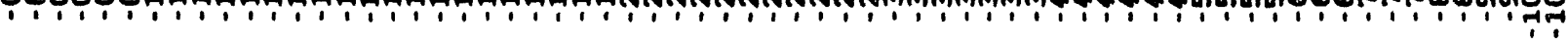

n으애은

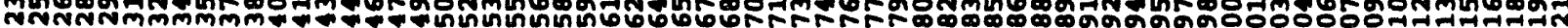
-

m-10\%ㅇำ

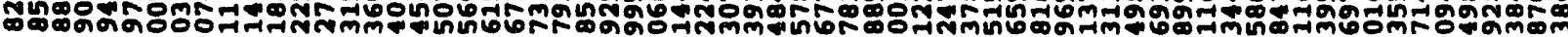

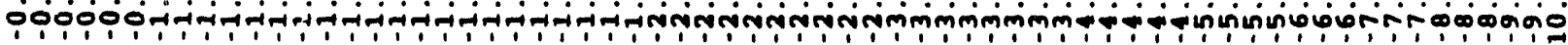

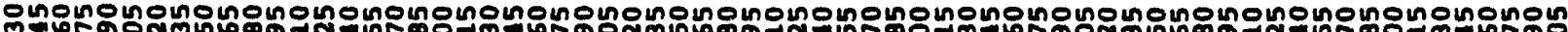

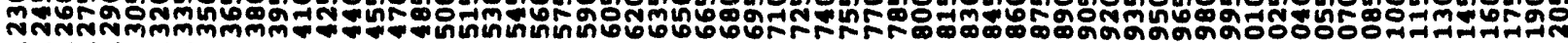

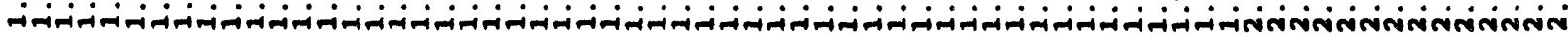




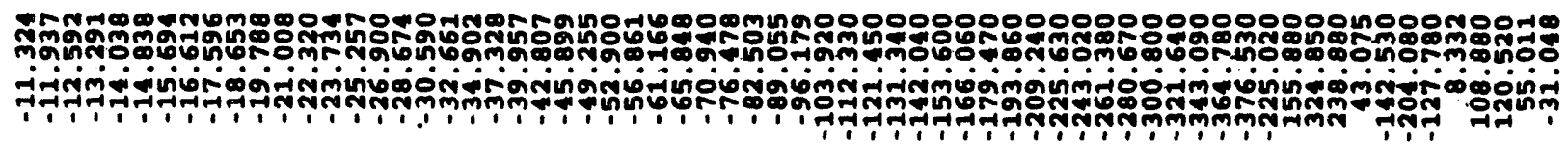

Ongnononongnononongnononononononononononononononononononononononon

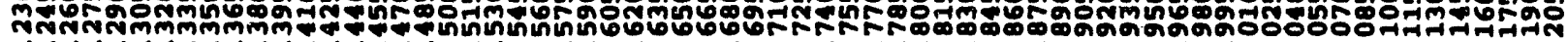

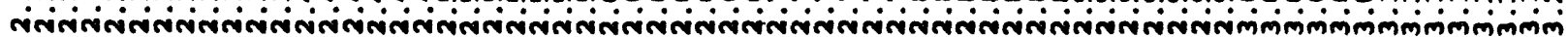

ㄱํํํำ Aำกํำ

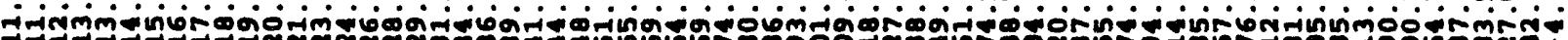
†ศન

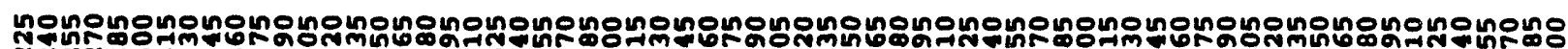

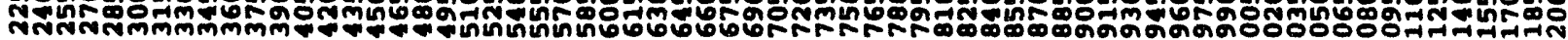

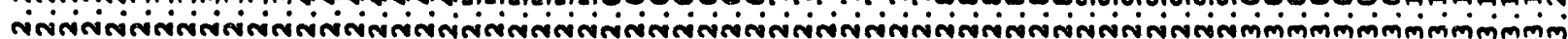

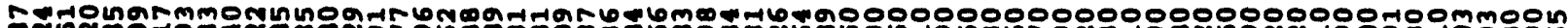

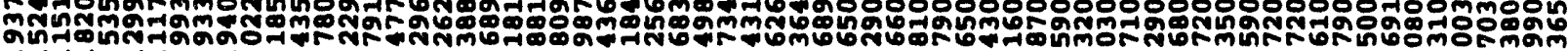
- - ${ }^{2}$ n

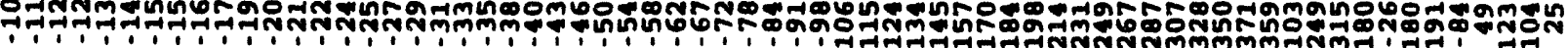

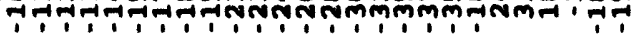

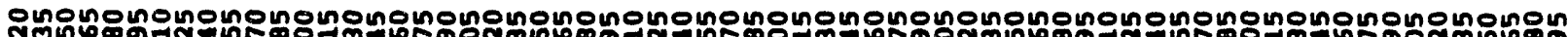

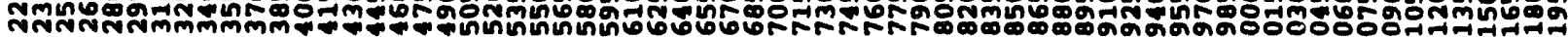

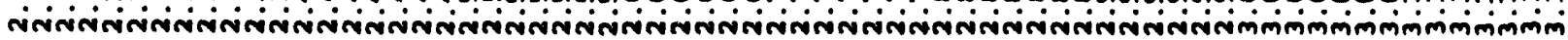




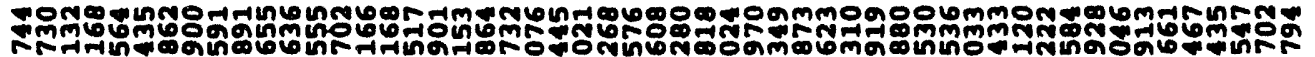

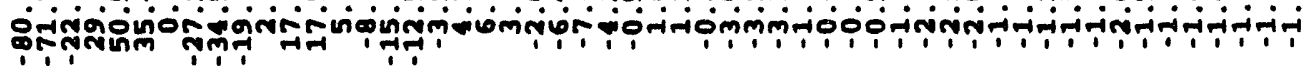

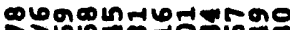

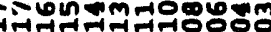
000000000000

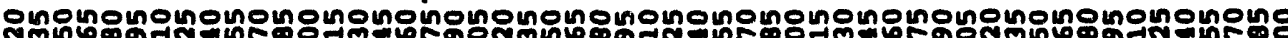

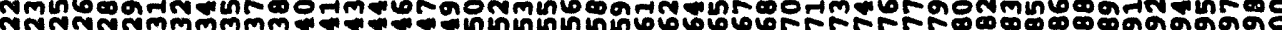

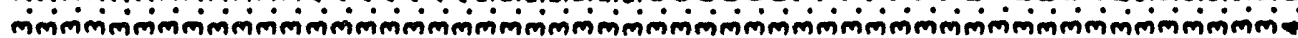

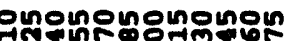

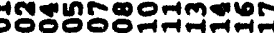
000000000000

욱근

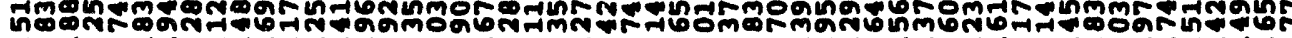

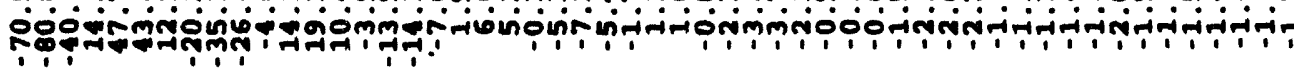

armorombomint

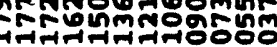
000000000000

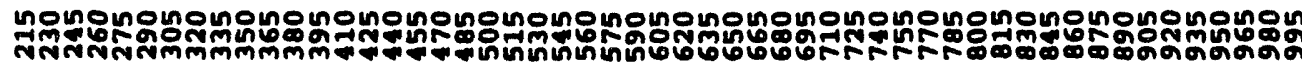

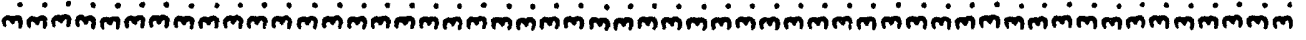
(2) nonono ớco 000000000000

(1)

후

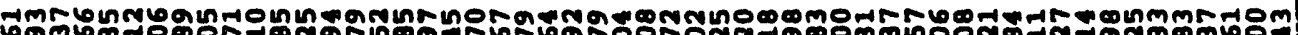
চดmbm mino

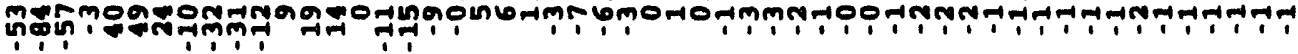

?

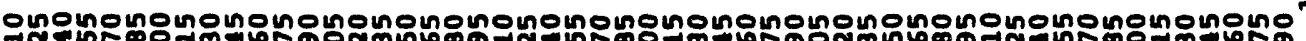

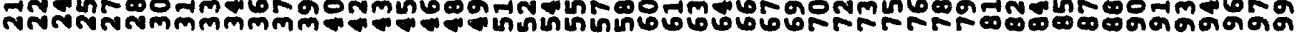

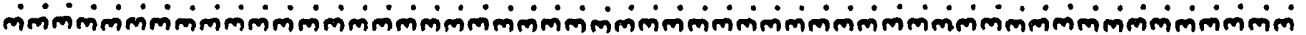

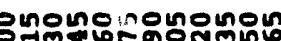

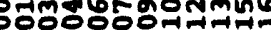
-00000000000 
70

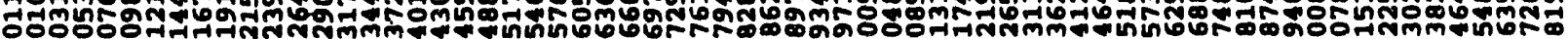

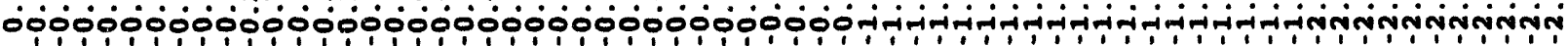

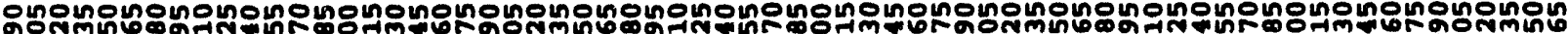

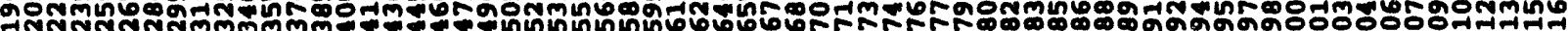
00000000000000000000000000000000000000000000000 -

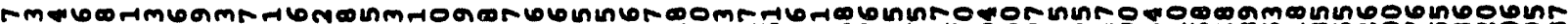

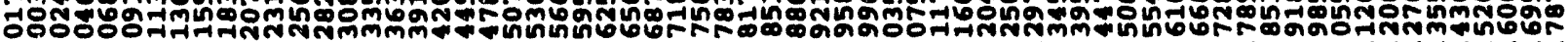

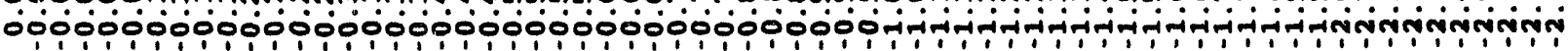

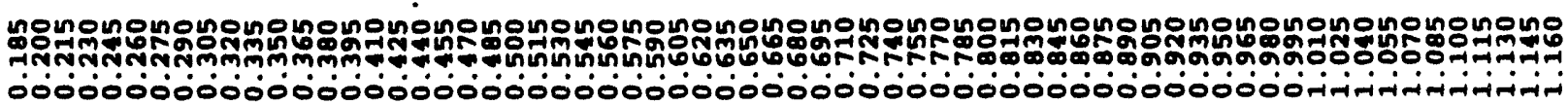

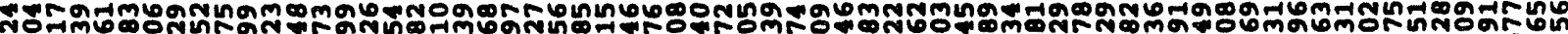

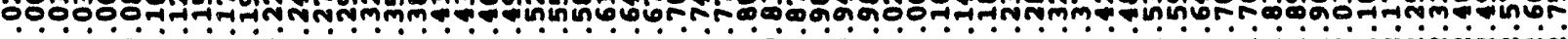

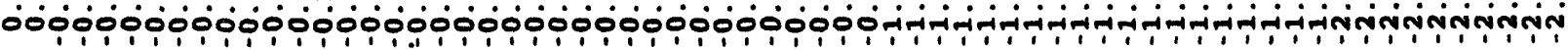

onononononongnononongnononononnonononononononononononongnongnononon

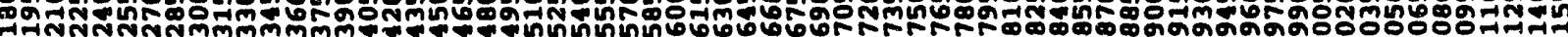
000000000000000000000000000000000000000000000000000 - 


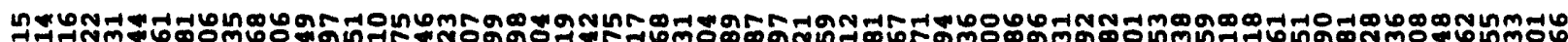

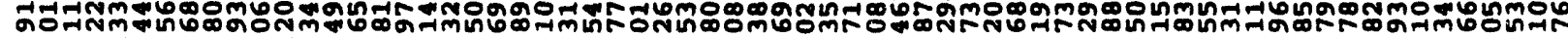

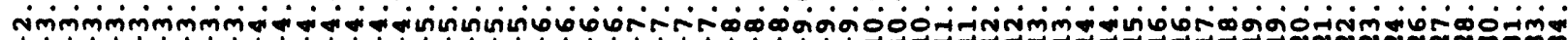

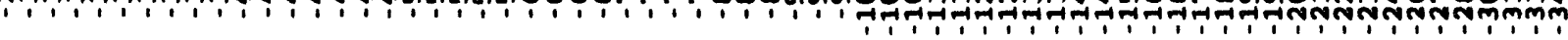

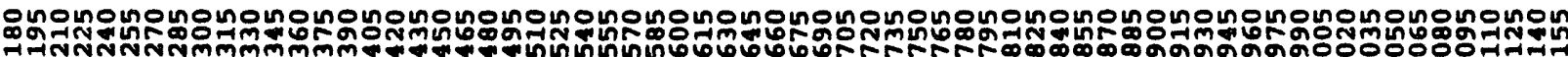

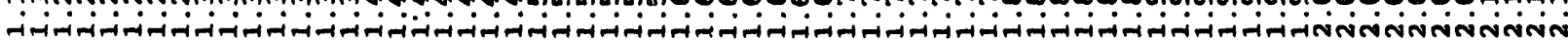

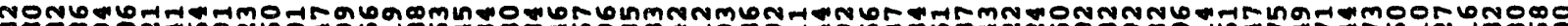

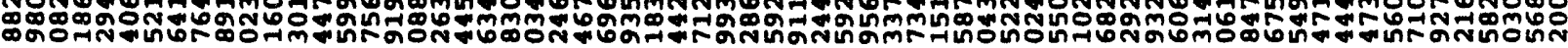

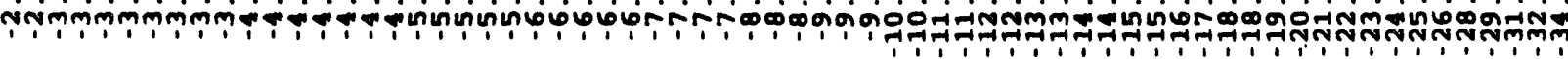

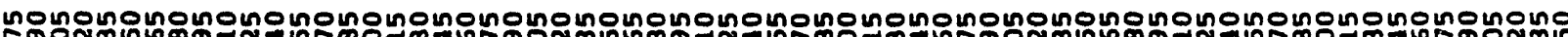

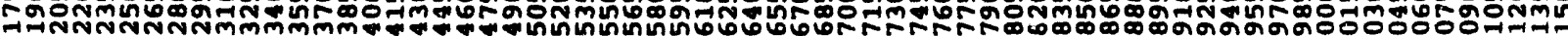
-

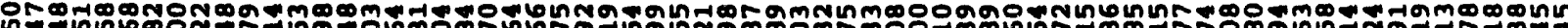

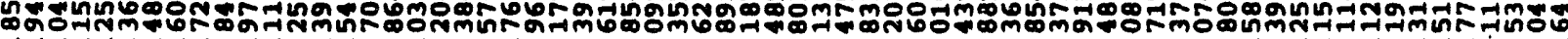

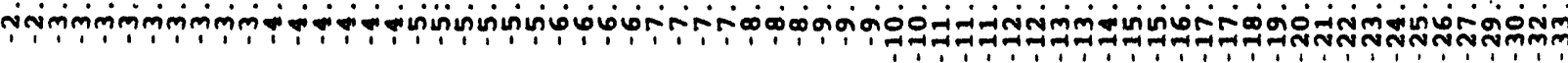

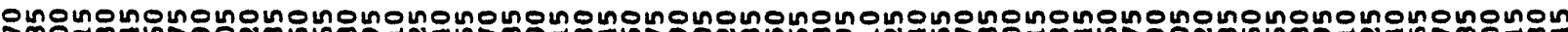

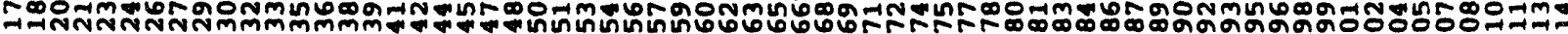

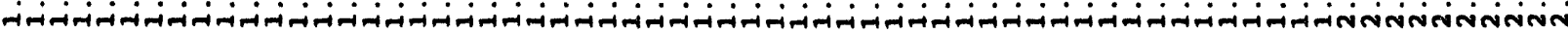




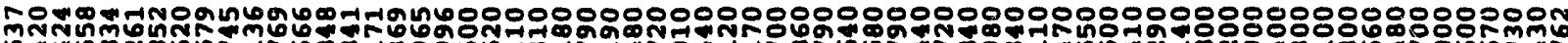

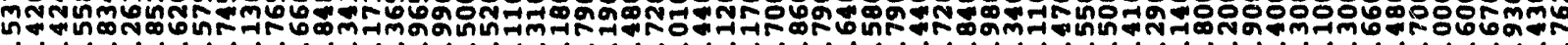

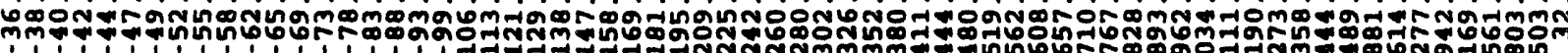

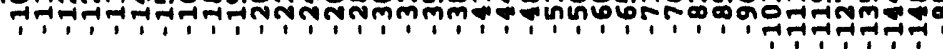

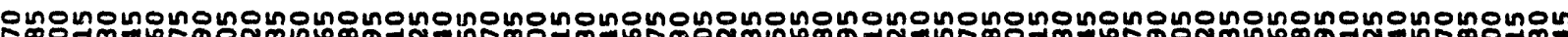

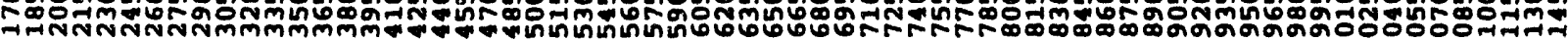

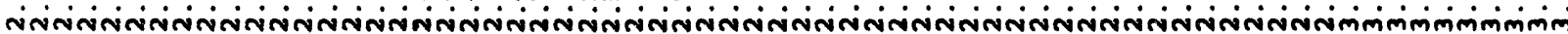

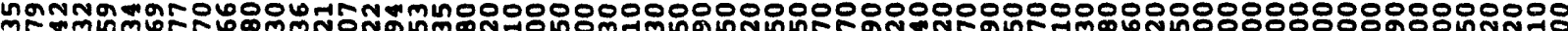

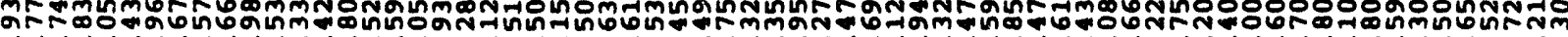

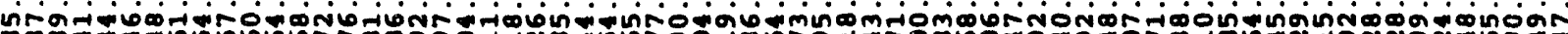

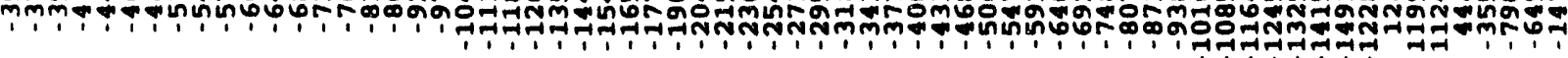

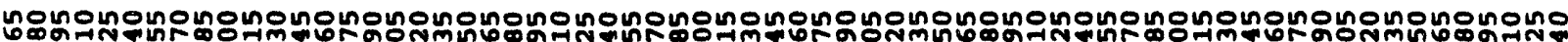

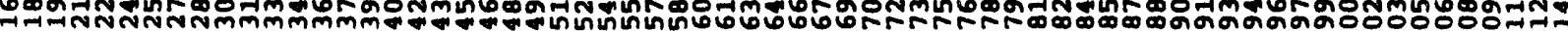

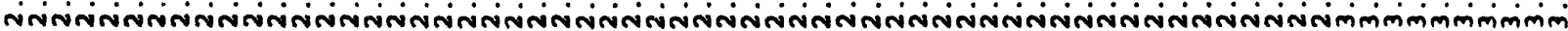

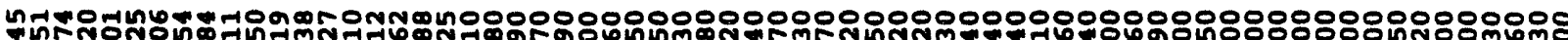
লñ nंm̄ं

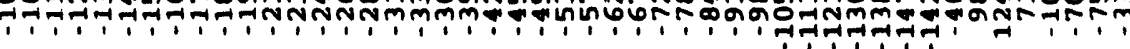

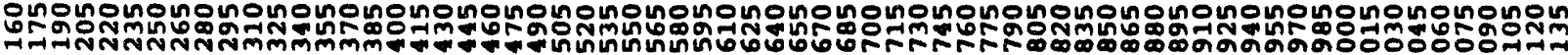

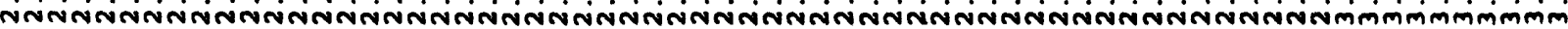




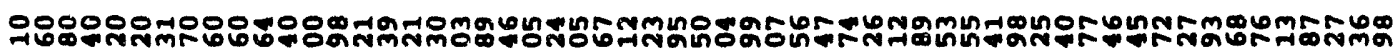

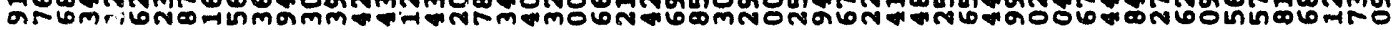

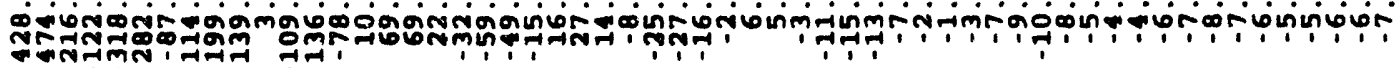

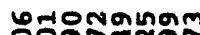

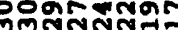
000000000

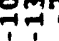
i.

1,1

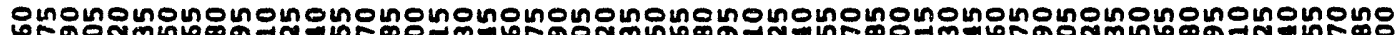
ఈ

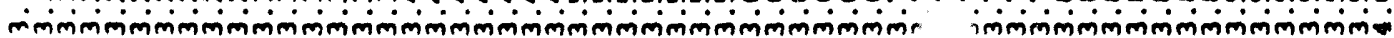

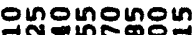
ใำำกำำ D00000000

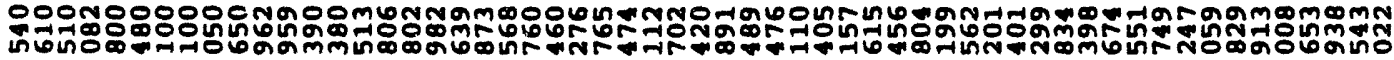
por rmugrman

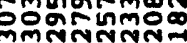
00000000

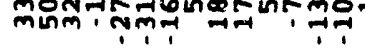

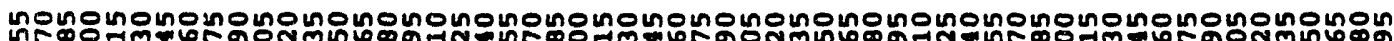

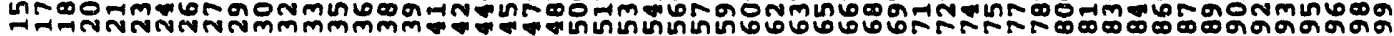

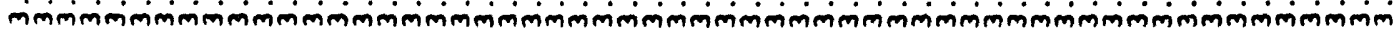

noinonono

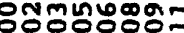

z

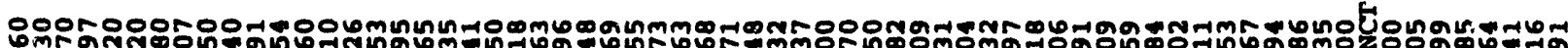
ำตำ viomo- -

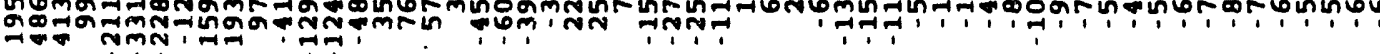

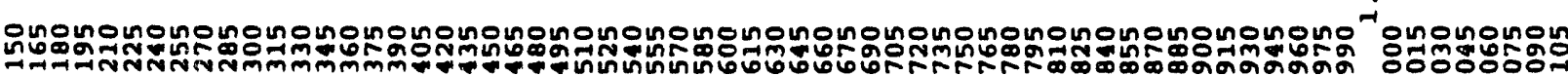

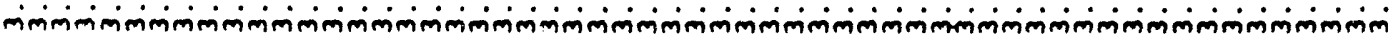
00000000 


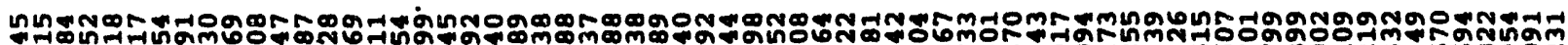

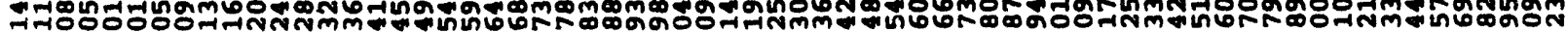
000000 1

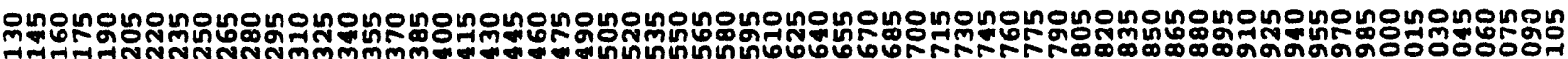

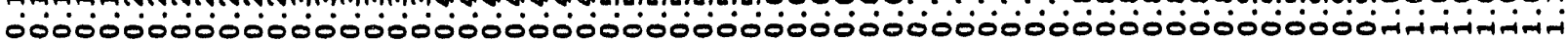

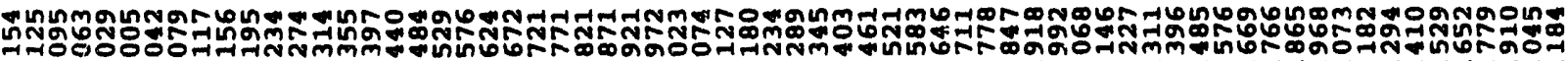
$000000000000000000000000-1-1-1 \mu_{1}$

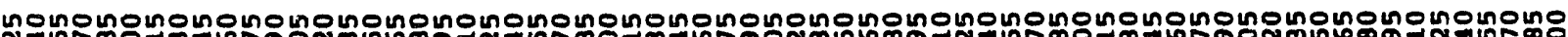

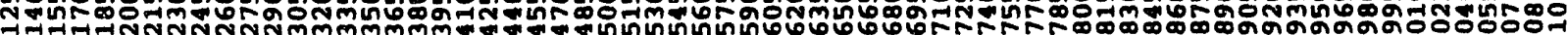
00000000000000000000000000000000000000000000000000000000 -

ซีnนา -

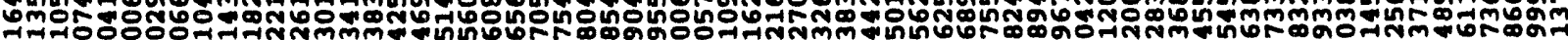

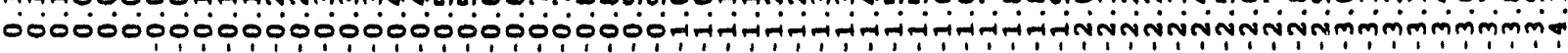

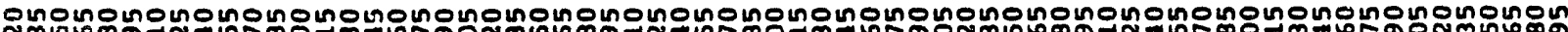

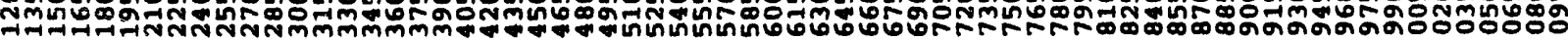
000000000000000000000000000000000000000000000000000000 - 
下oீ-1 4

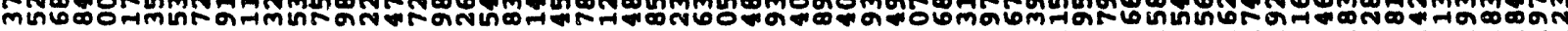

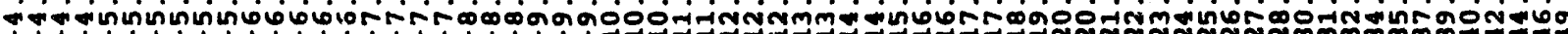
1.7.

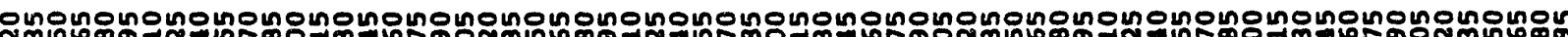

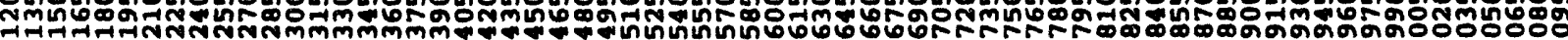
-

๓6ồ

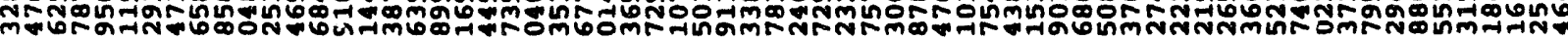
П.十,

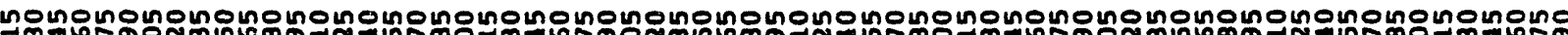
-

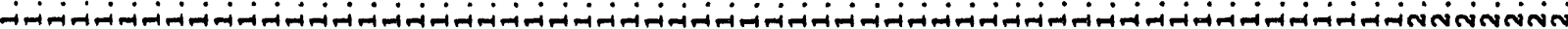

ด้อ

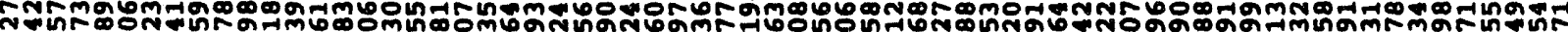

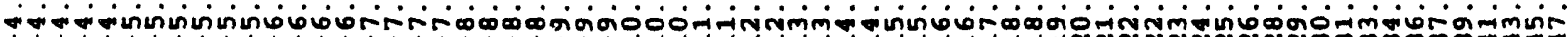

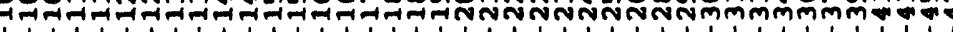

on은

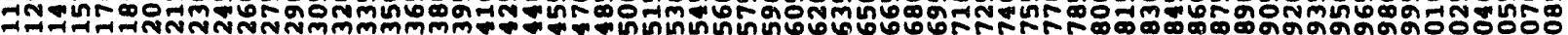

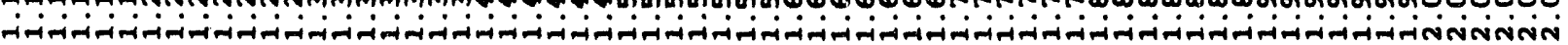




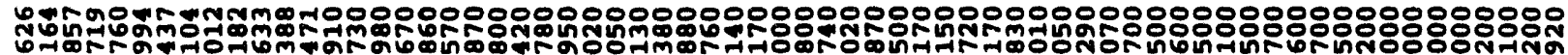
-

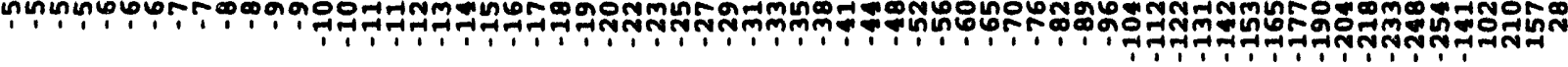

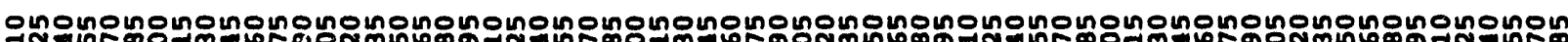
T패

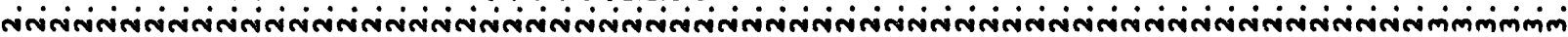

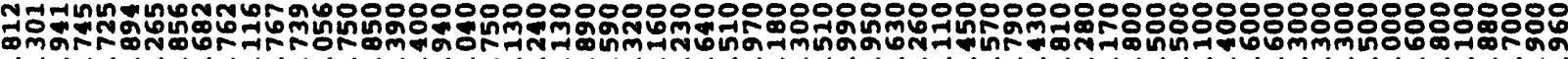

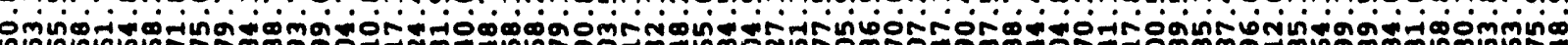

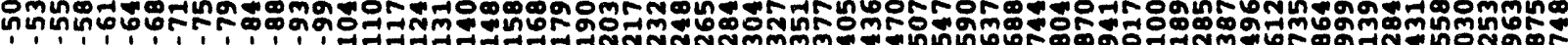

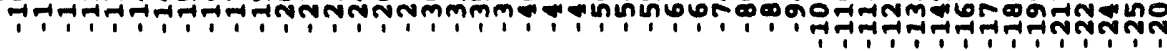

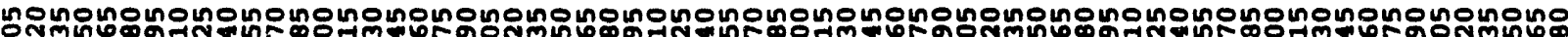

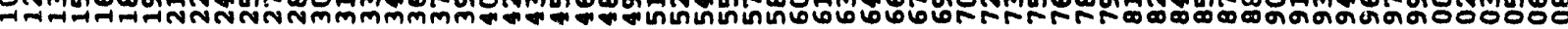

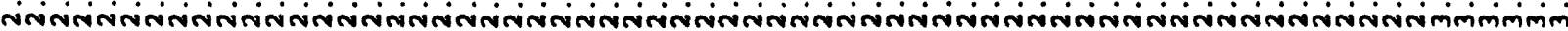

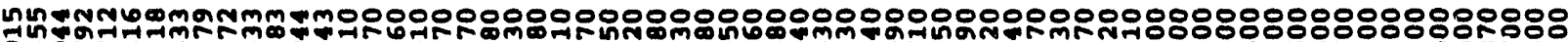
పี

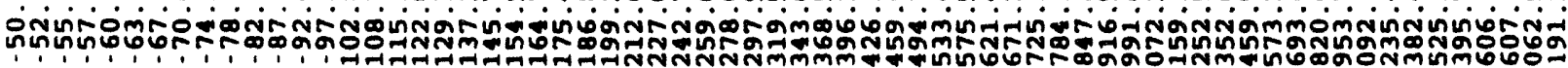

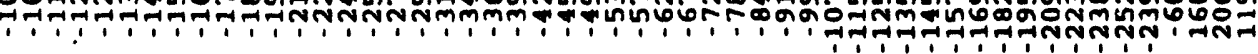

ononononononononononononononononononononononononononononononononon

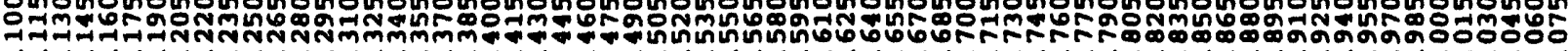

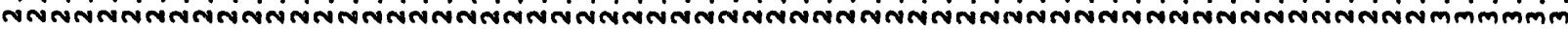




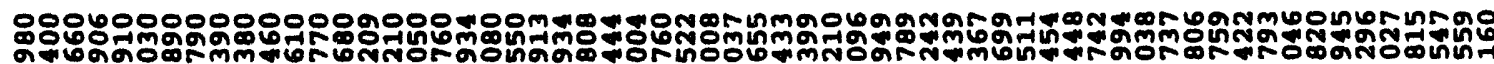
nе०

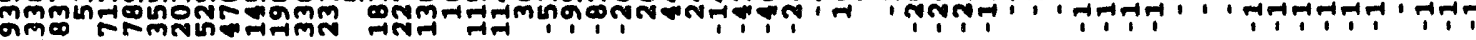
$0000^{\circ}$ Tึ: $\because$.

Onononononononononononononononononononononononononononononono 구튼

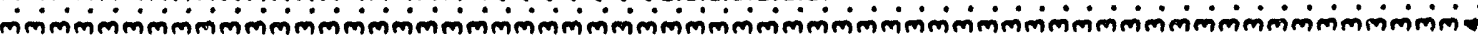

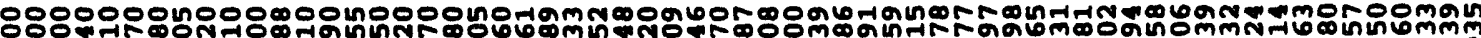
लrरำ Sू

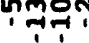

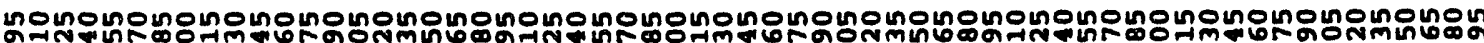
ดี-

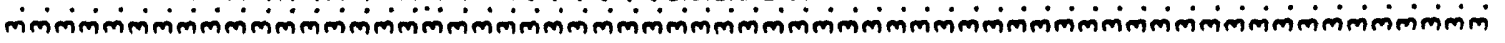

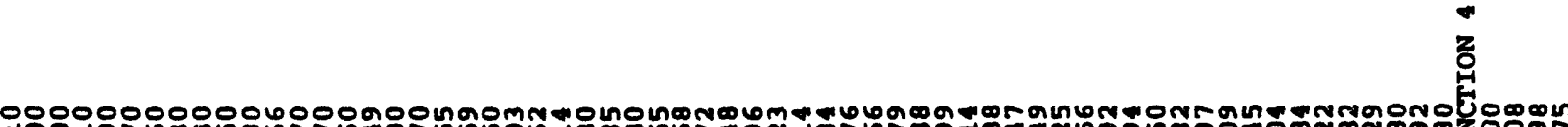

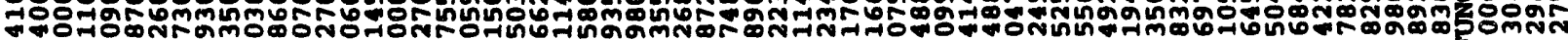
कomminin- -

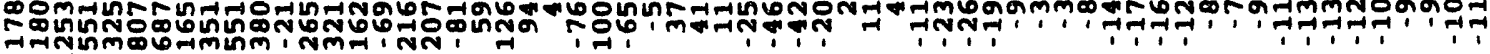

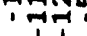

0

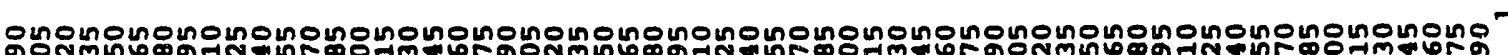

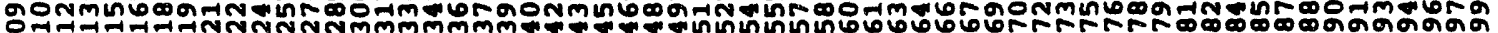

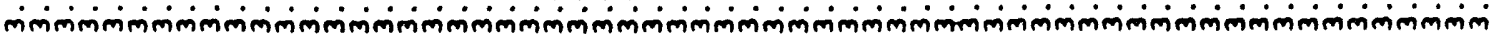


иำ-176-

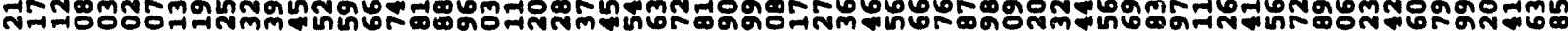
- 0000 o

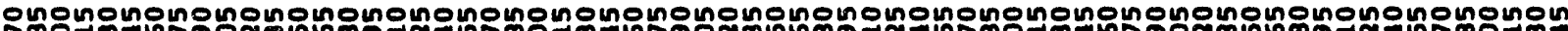

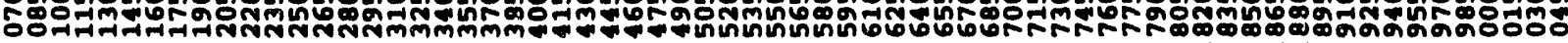

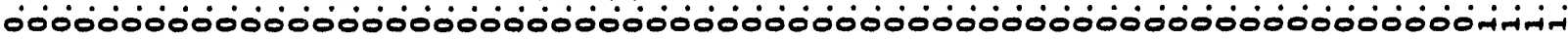

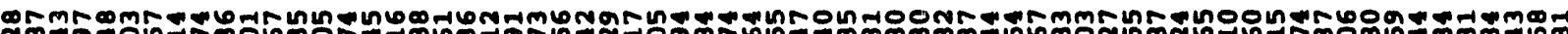
N0000000000000000000- - - - - -

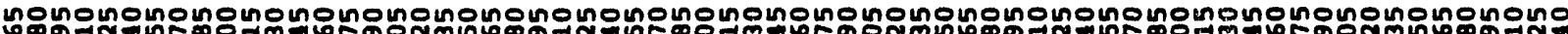

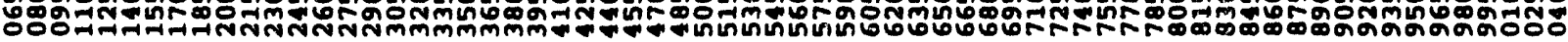
0000000000000000000000000000000000000000000000000000000

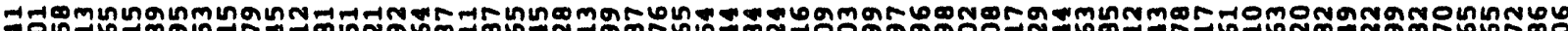

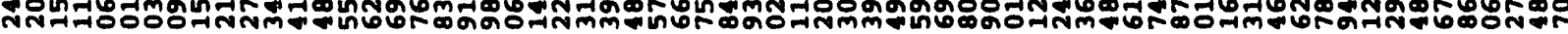

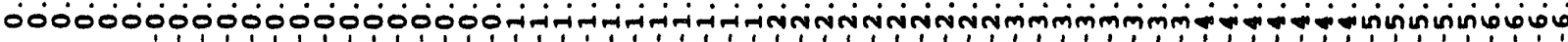

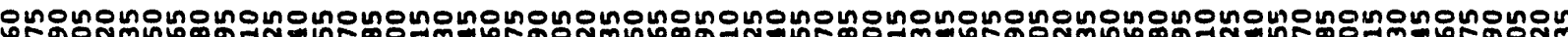

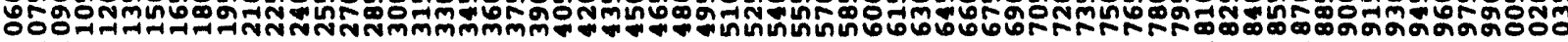

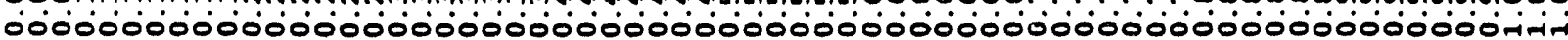




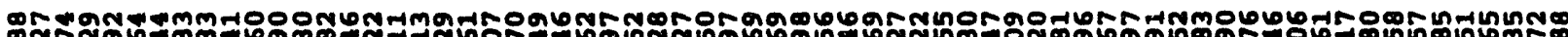

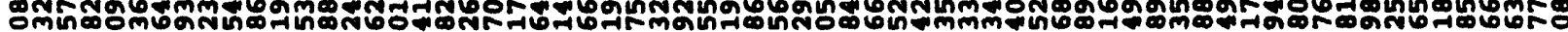

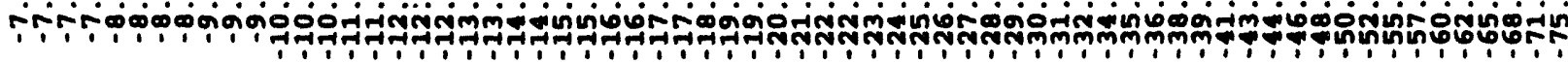

으은

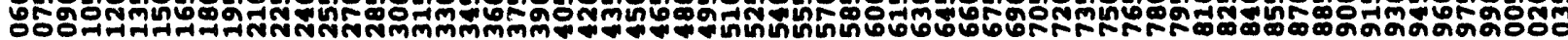

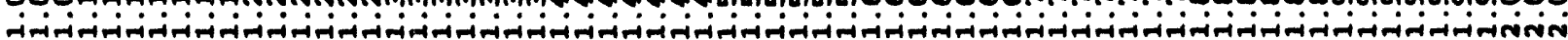

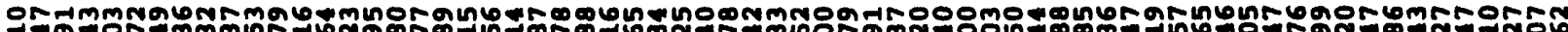
oี ำ - r

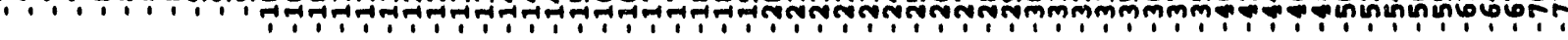

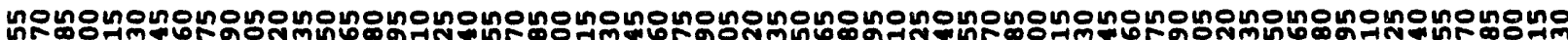
o०o-

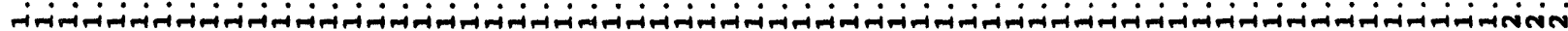

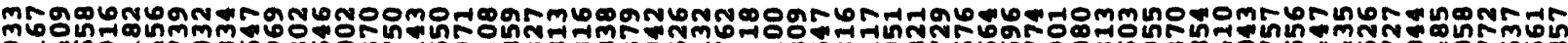

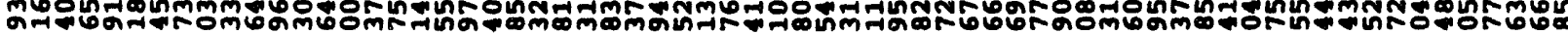

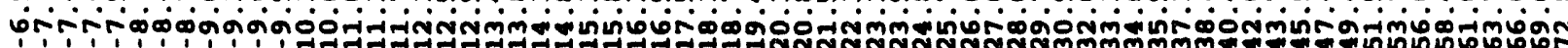
7न-

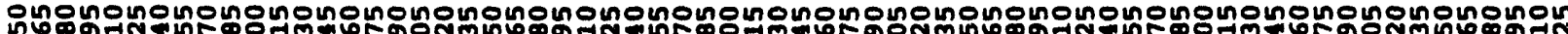

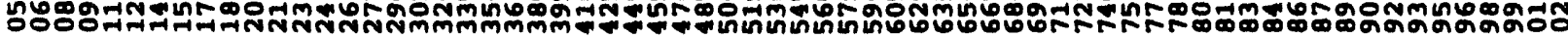

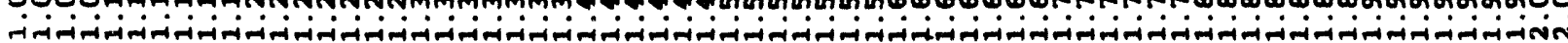




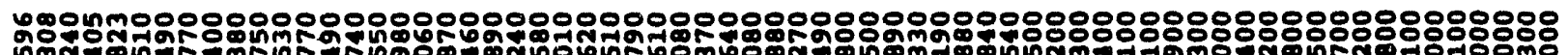
कno Фळono 1,

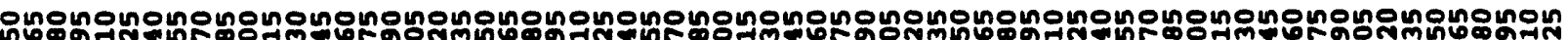

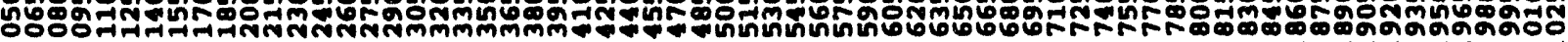

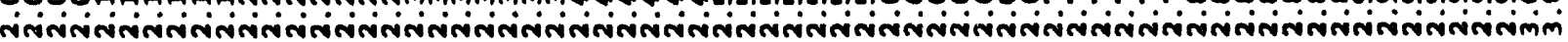

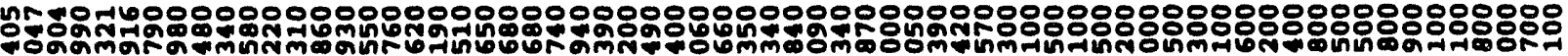
-j-jmingminno ค.

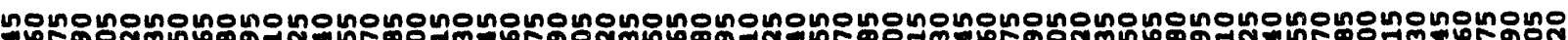

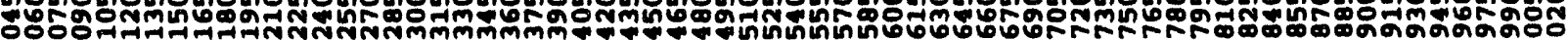

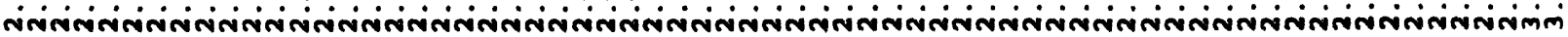

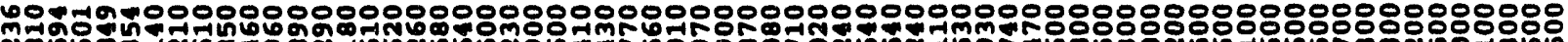

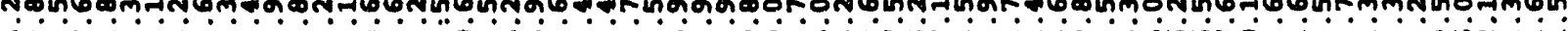

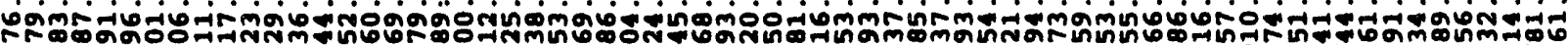
구

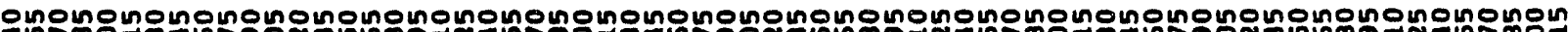

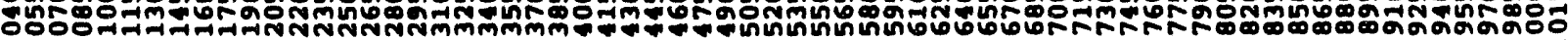

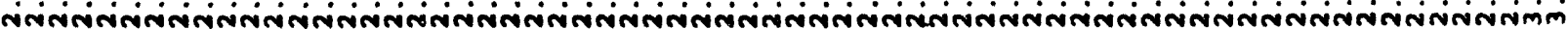




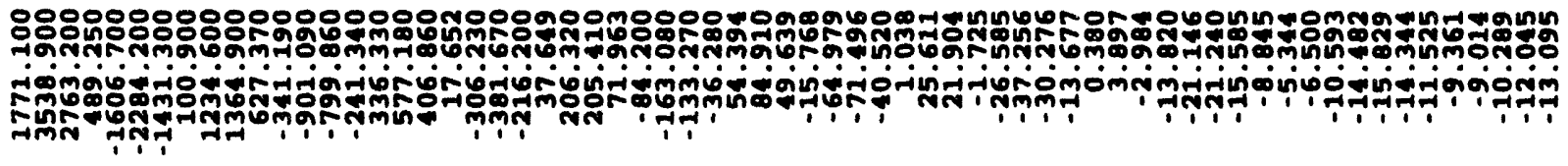

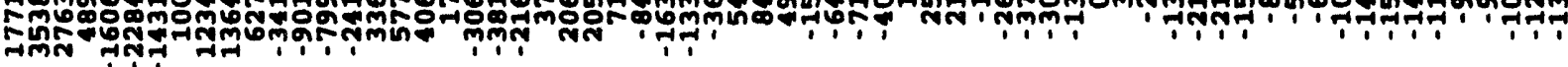

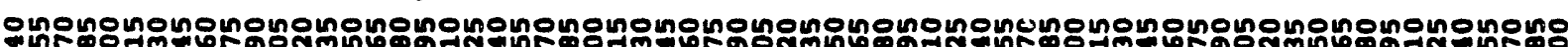

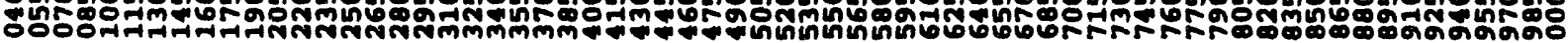

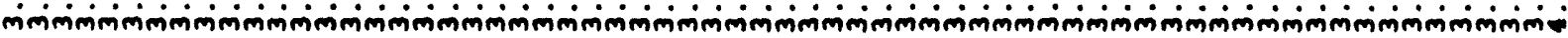

ํำㅇำㅇำ

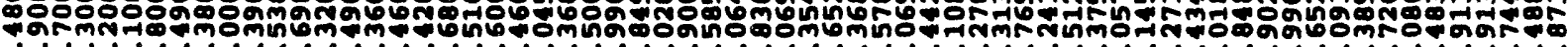
नैं 11

nonononounononongnononongnoungnonononononounongnongnongnongnonononon

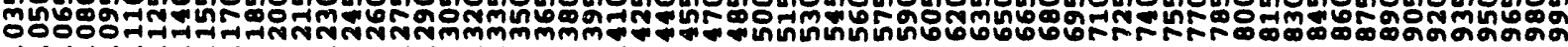

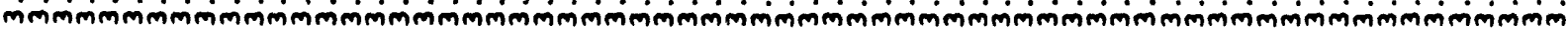

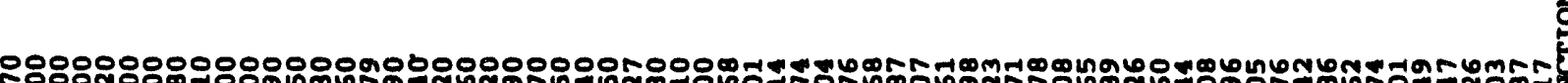

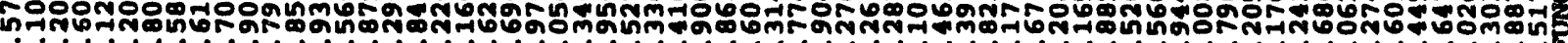
rin-ia-jon

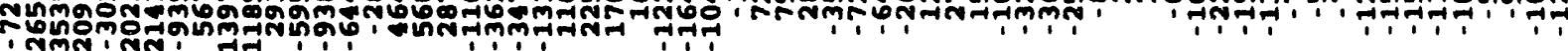

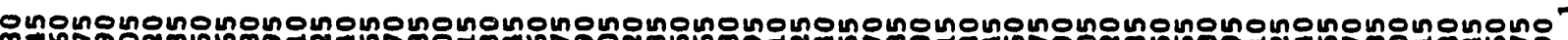

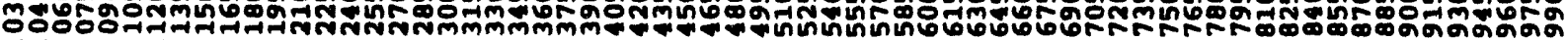

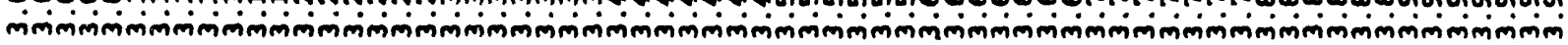




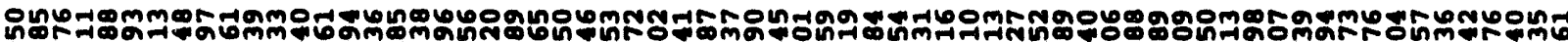

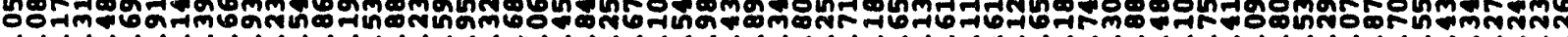

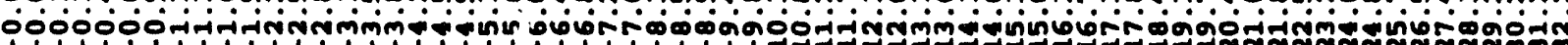
(1)

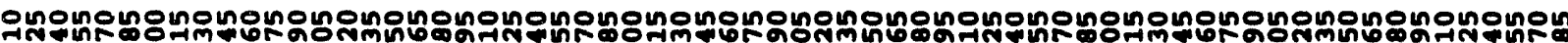

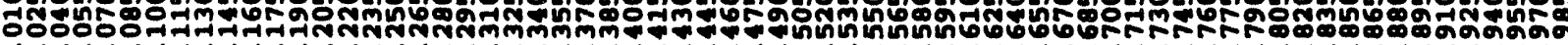

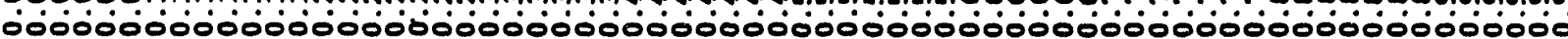

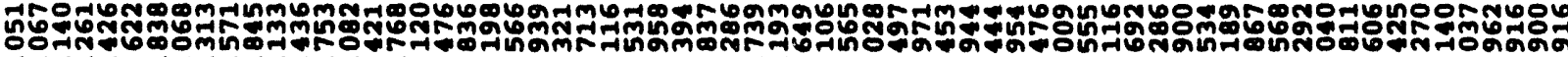
o

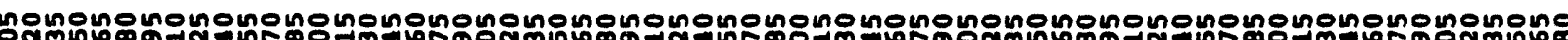

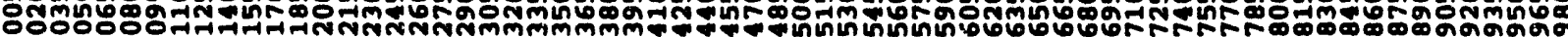

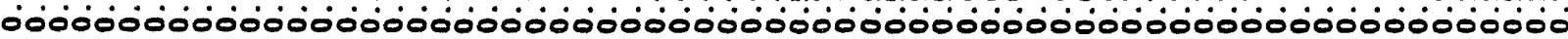

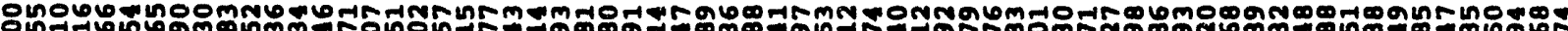

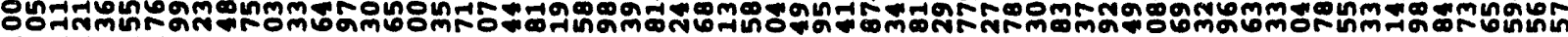

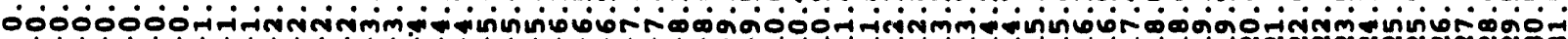
П.

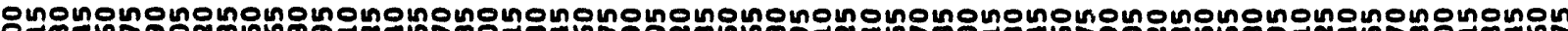

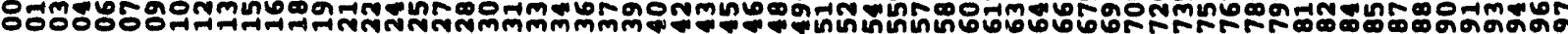
-000000000000000000000000000000000000000000000000000000000000 


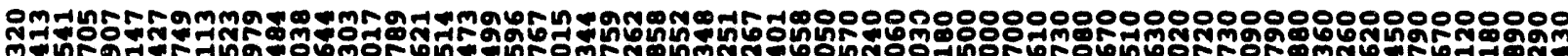
mंniono -

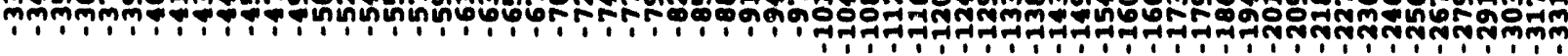

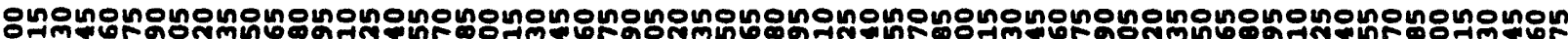

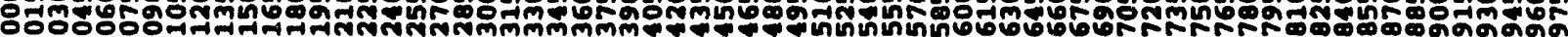

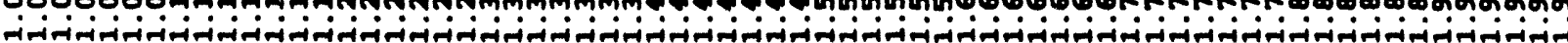

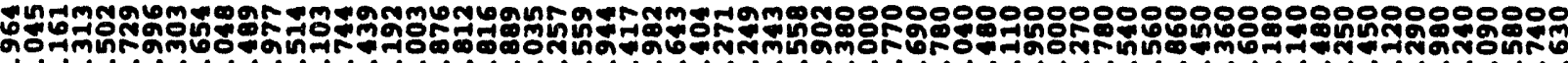

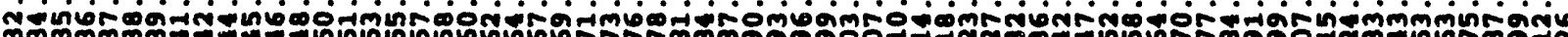
๑.

unononononongnonononononononononongnonononononononononononounonomono

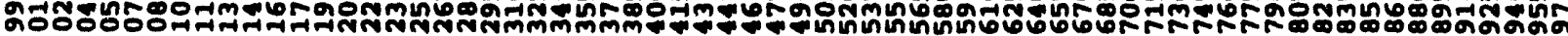

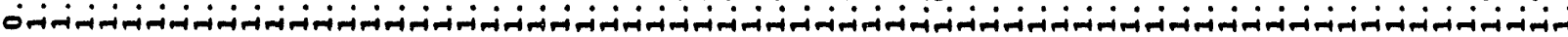

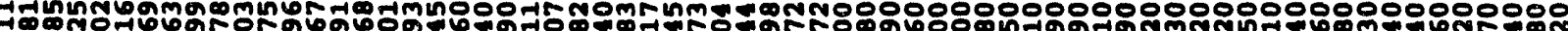
6ซึूm

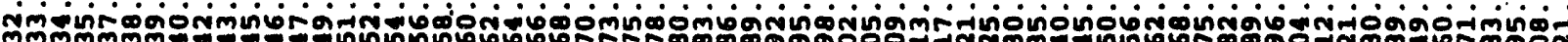
mmmmmp ? :

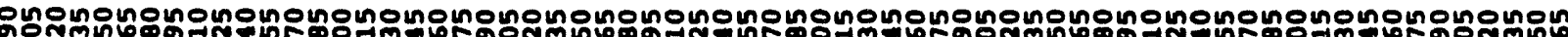

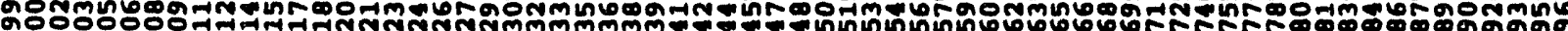

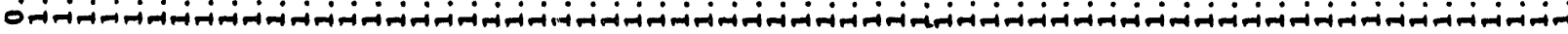




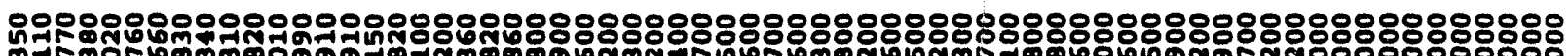
Hrim mmmm -

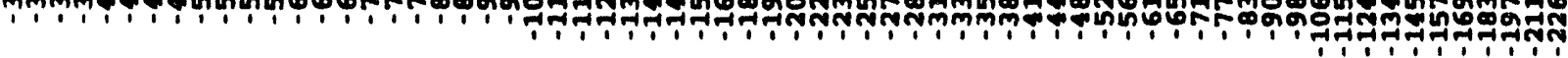

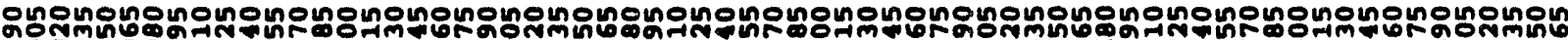

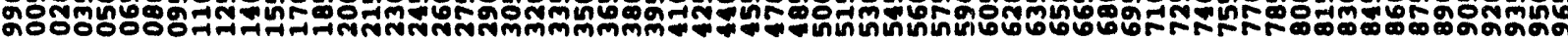
- in

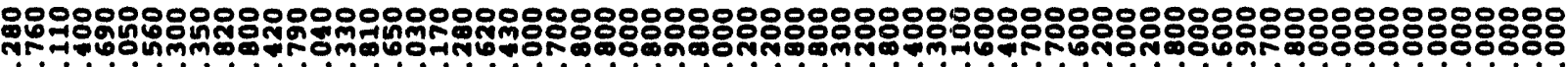
- jmomom

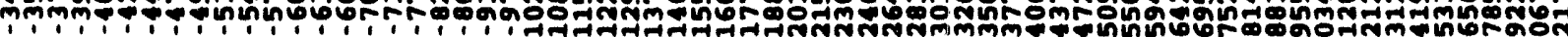
1. ?

uonongunonononononononononononononononongnonongnononononononononono

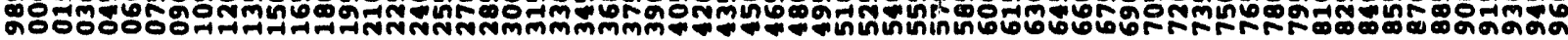

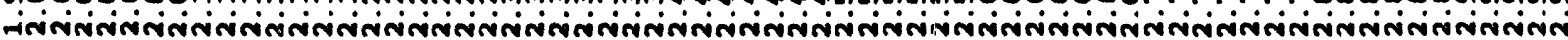

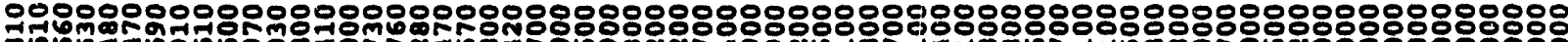

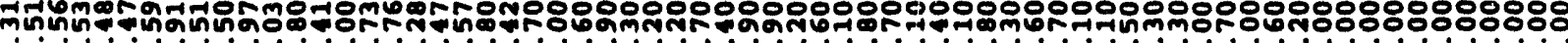
- -

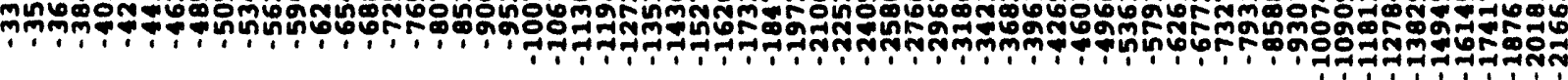

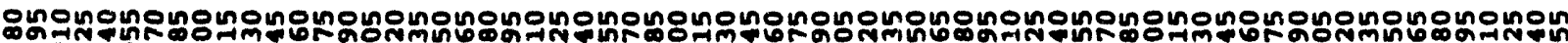

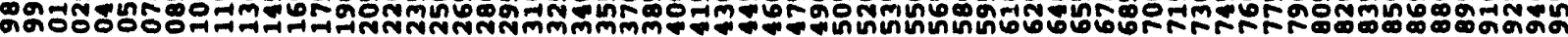

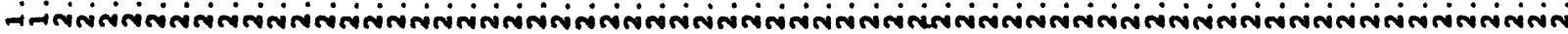




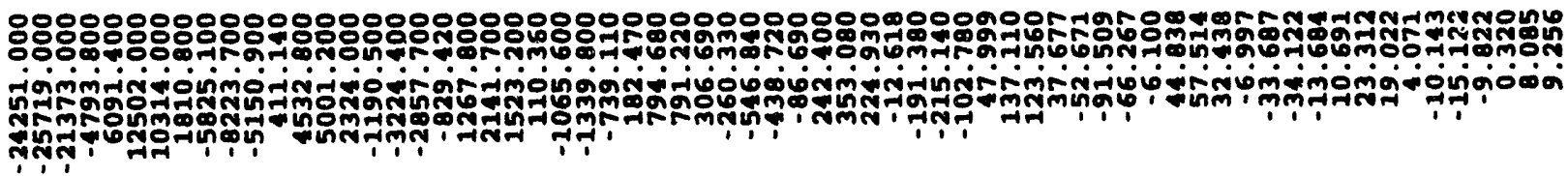

onononononongnononononononongnongnononononononguphongnonononghongn

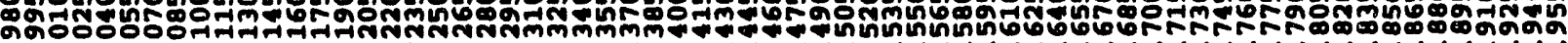

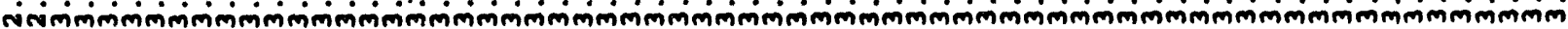

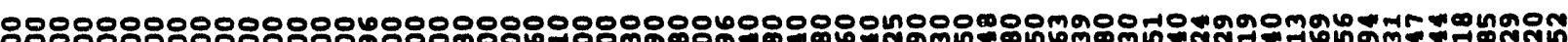

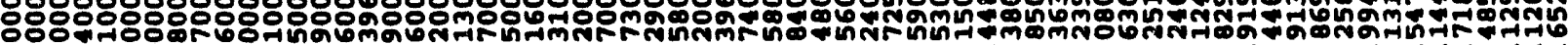
คै०0\%

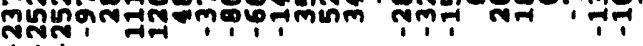

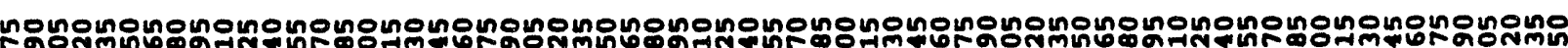

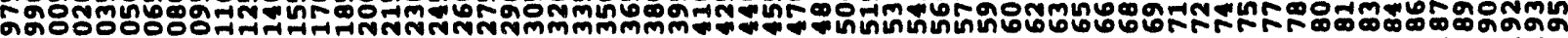

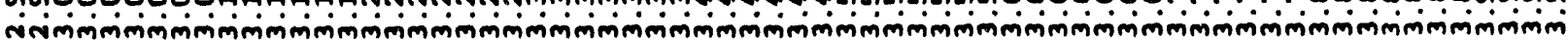

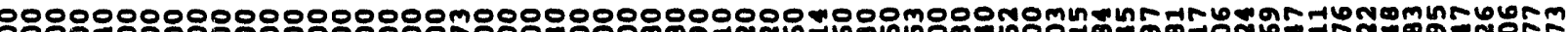

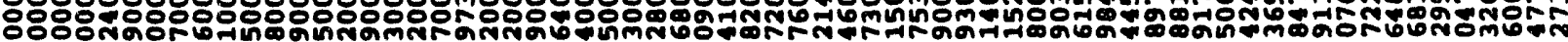

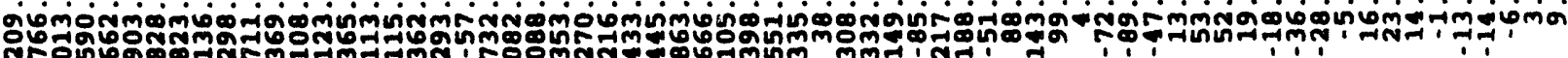

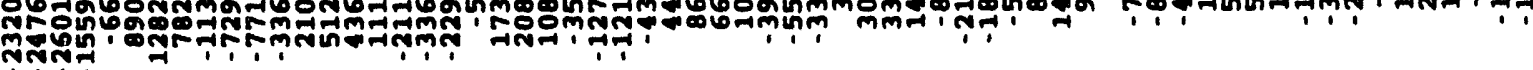

ononononononononononononononononononononounonongnononononononononon

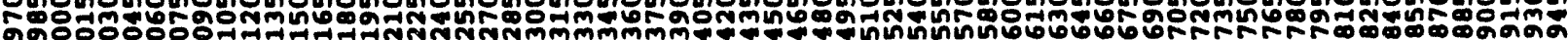

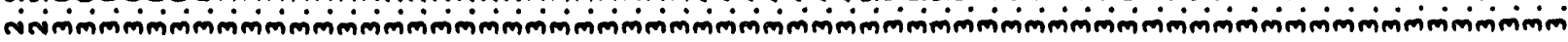


omf

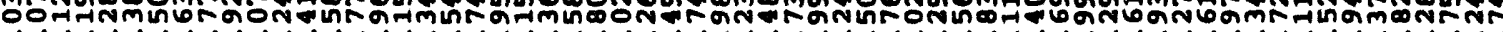

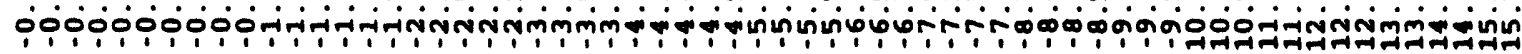

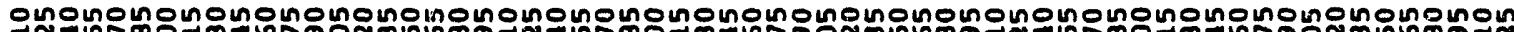

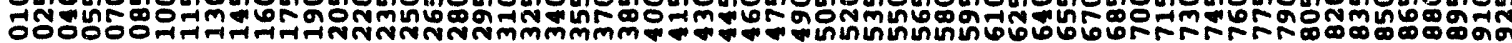
0000000000000000000000000000000000000000000000000000000000000

Ф000906 สํํ구 -0000000009

non nononononononononononononononononononononononongnonononononono Кू̆ minm 00000000000000000000000000000000000000000000000000000000000000 6

z

007 7m650 or nis o0000000000-

o

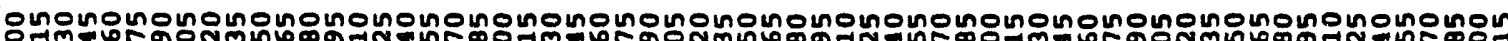

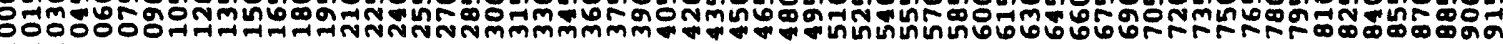
00000000000000000000000000000000000000000000000000000000000000 


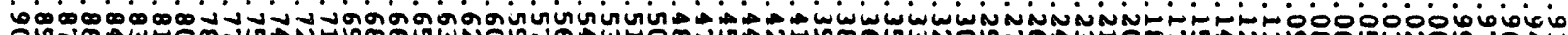

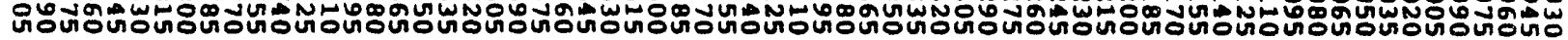

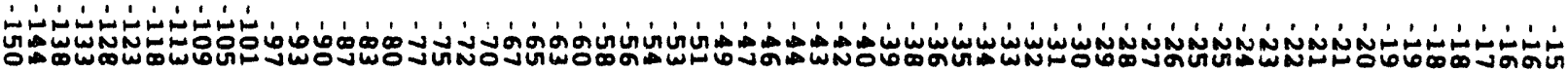

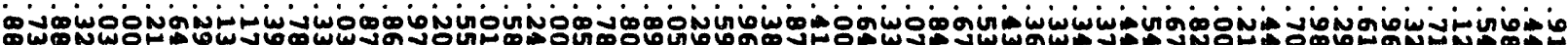

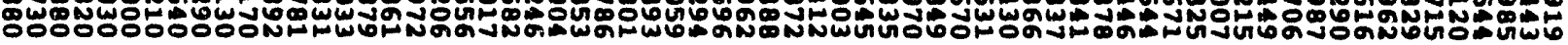

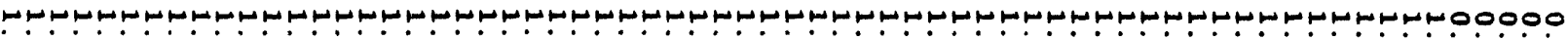

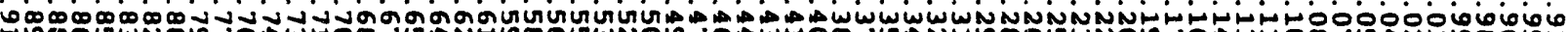
ด ดัดง

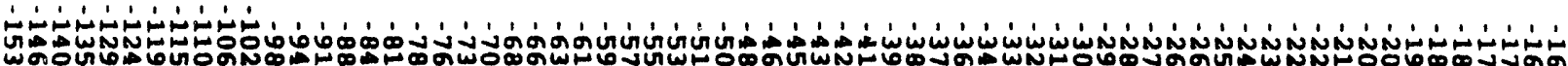
oे

60

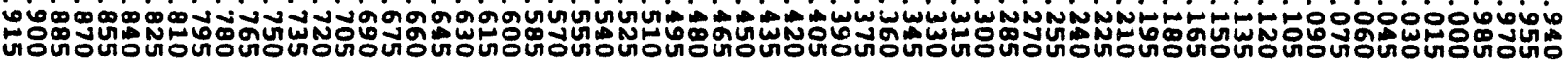

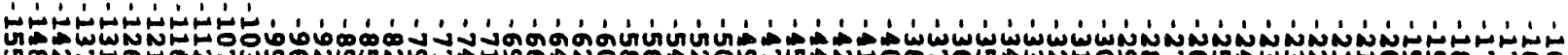

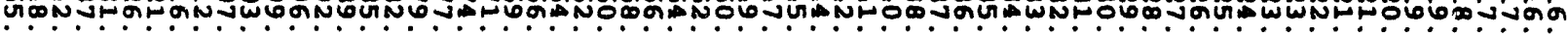
ğ 


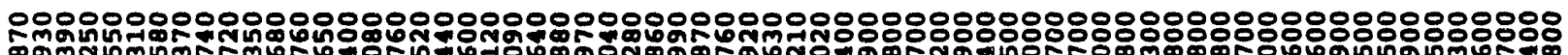
- Bo - v-jo - min

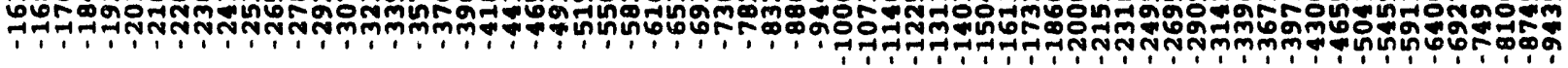

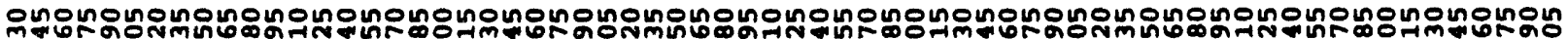

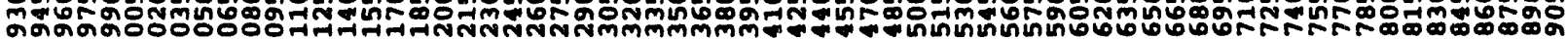

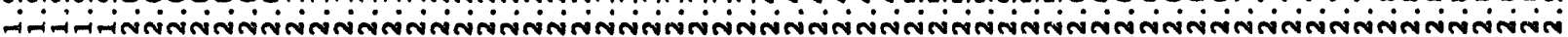

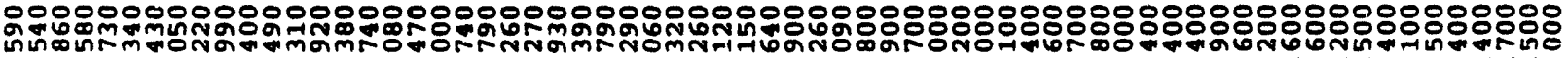

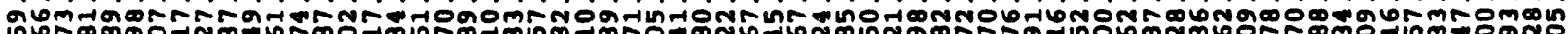

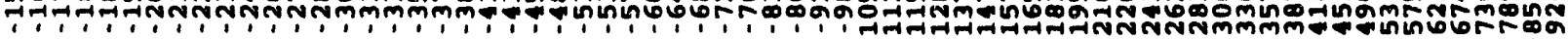

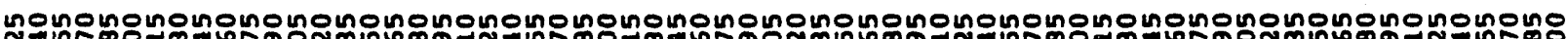
สু

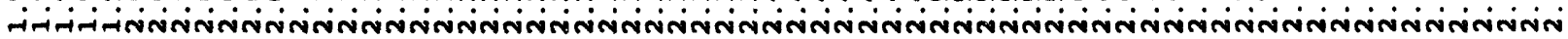

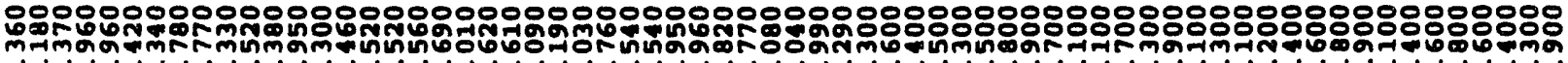

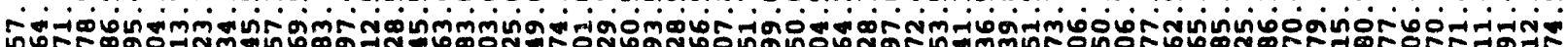

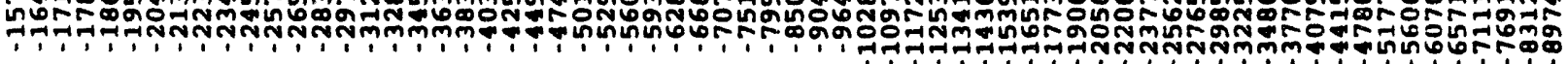

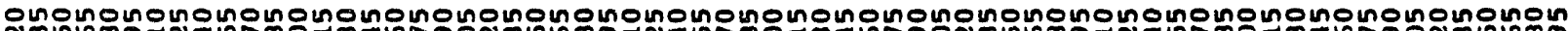

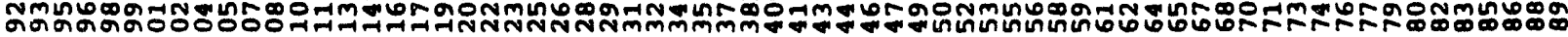

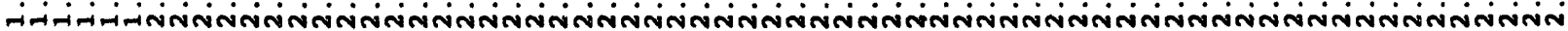




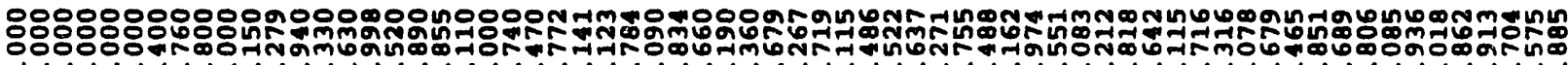

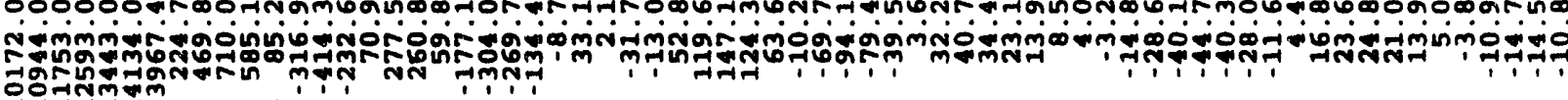

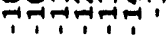

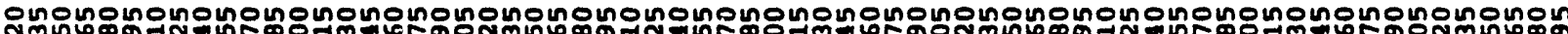

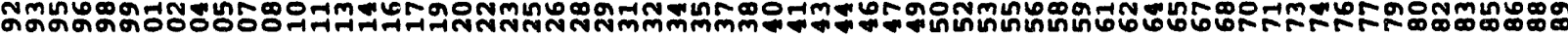

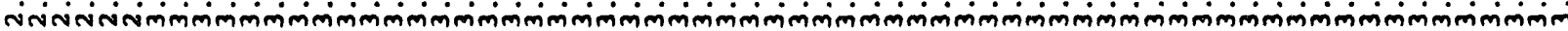

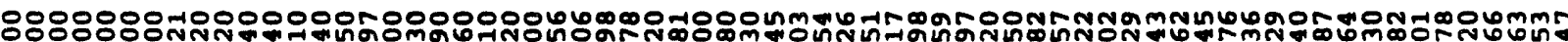
ํํㅇํㅇำ mivo - vomom ลูळ

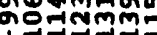
17.7.

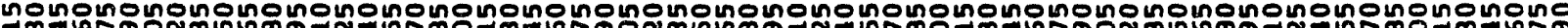

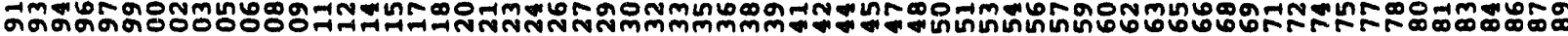

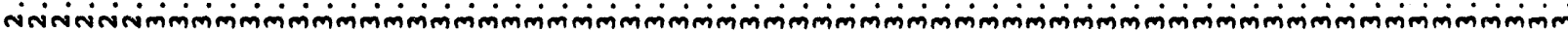

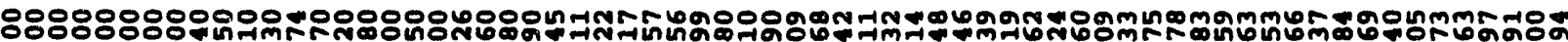

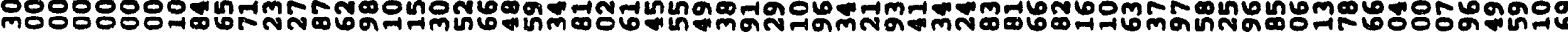 ningon

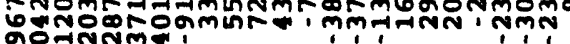

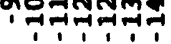

onononononononononononongnononongnonononononononongnononononononon

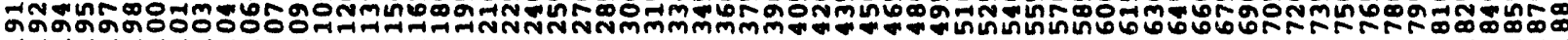

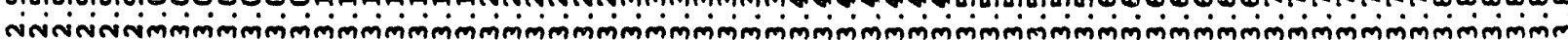


ononononononononononononononononononononononononononononon

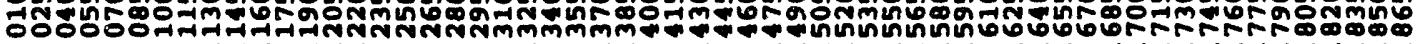

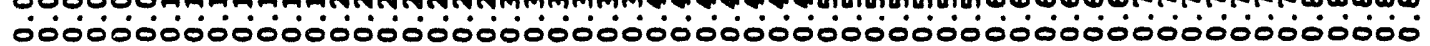

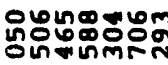
ripinägo

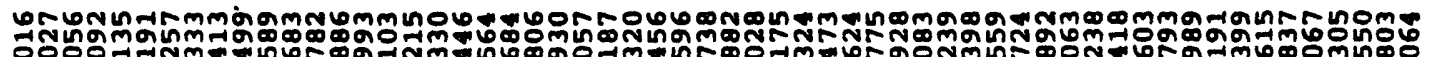

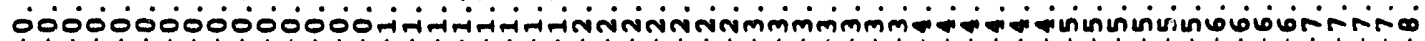

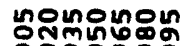 ดूनूनूनू} mimiminiminim

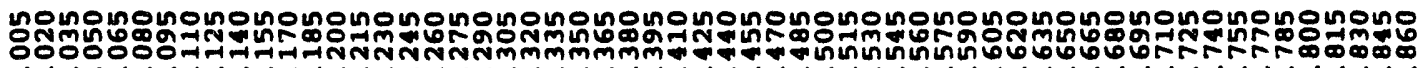
0000000000000000000000000000000000000000000000000000000000

争

క

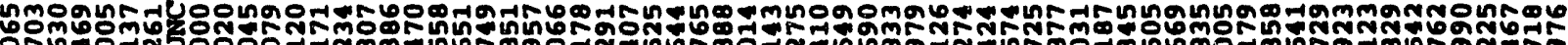

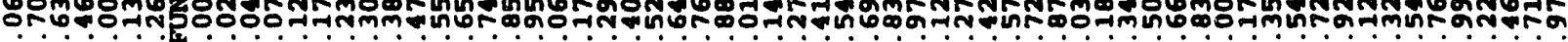

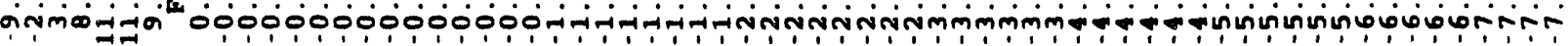

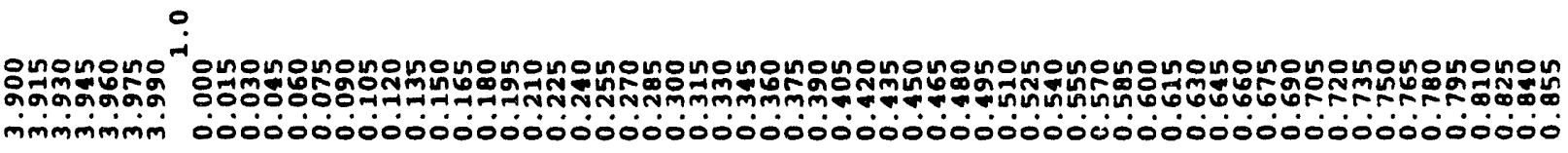




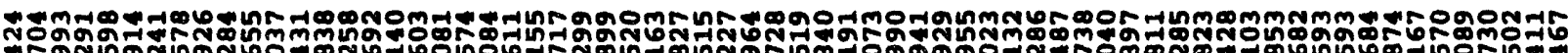
1.

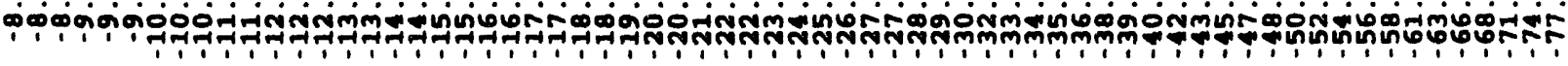

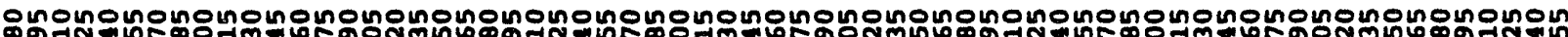

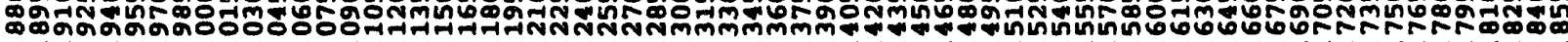
த0000000-

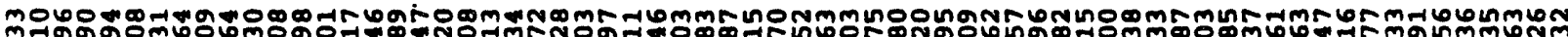
mైథ్య

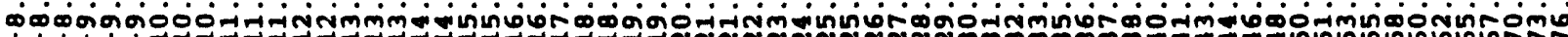
1.

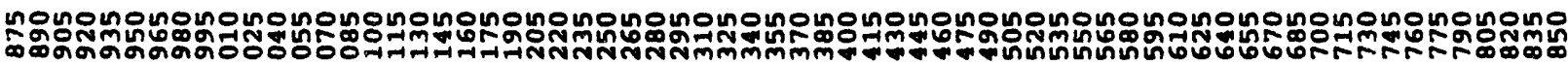
000000000- -

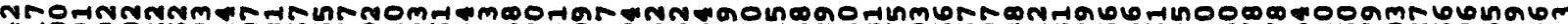

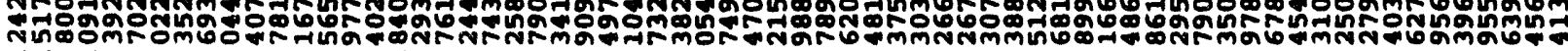

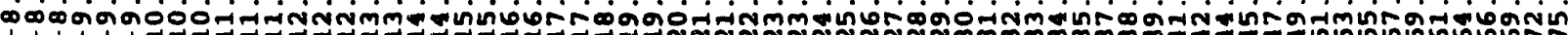
-

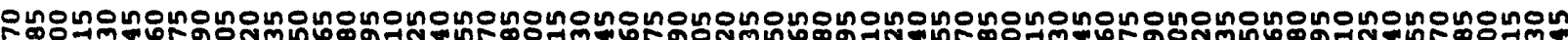

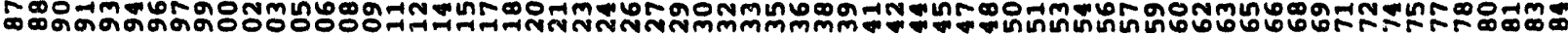

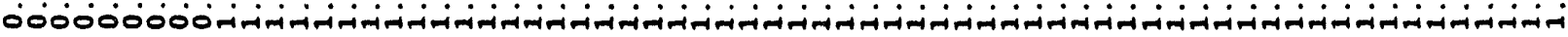



นิํํำ ذं宀 Ф,

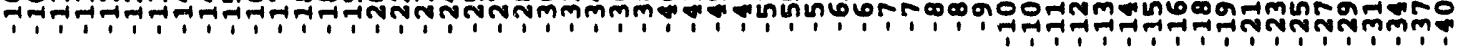

으은

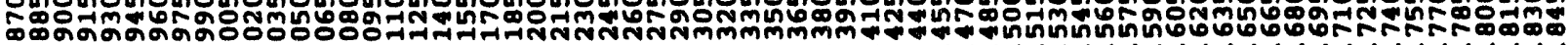

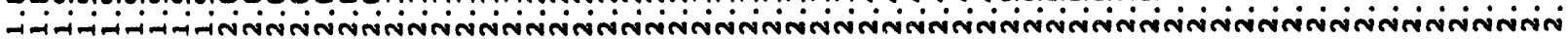

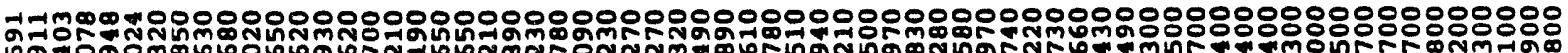

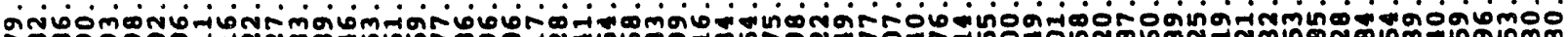

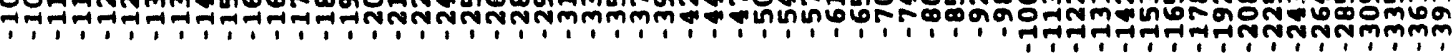

개쓴

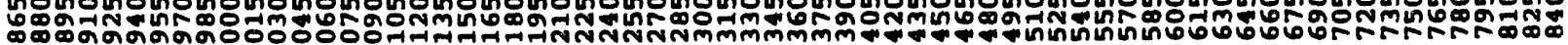

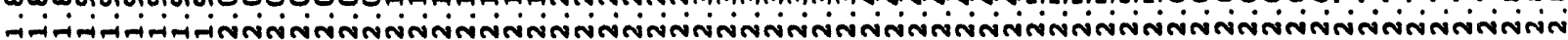

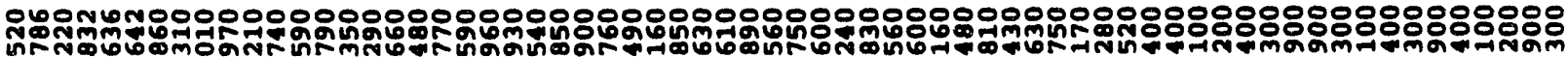

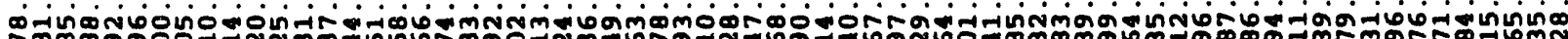
-

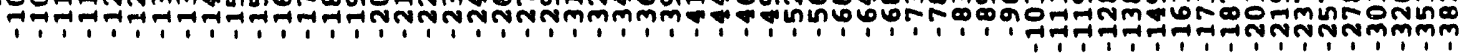

ongunonononononononononononongnononononononononononounounonounonounonon

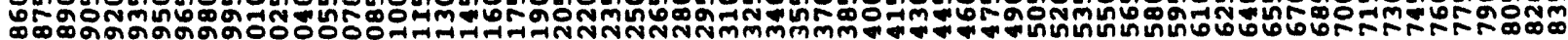

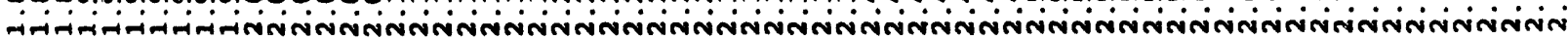


W

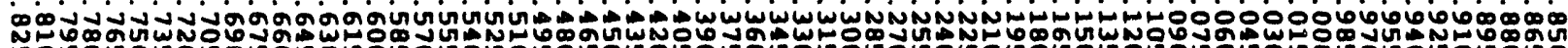

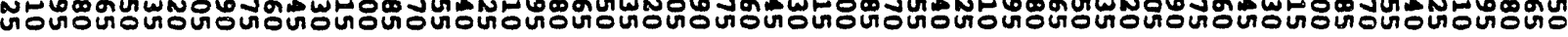

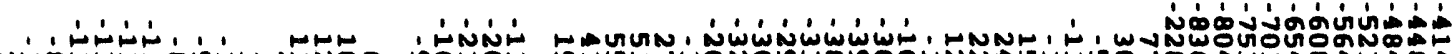
w

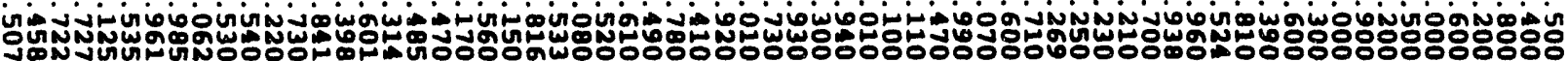

WWWW

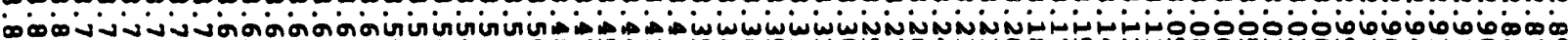

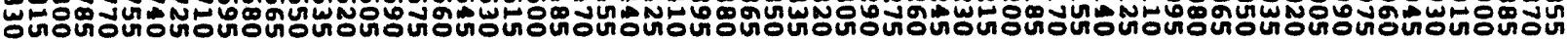

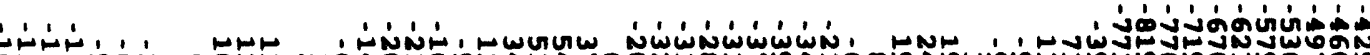

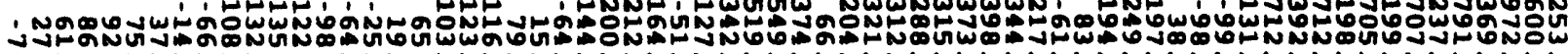

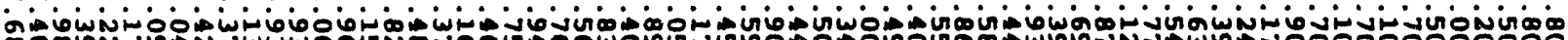

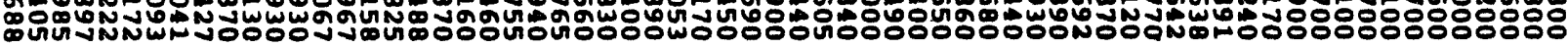

שแ" wo

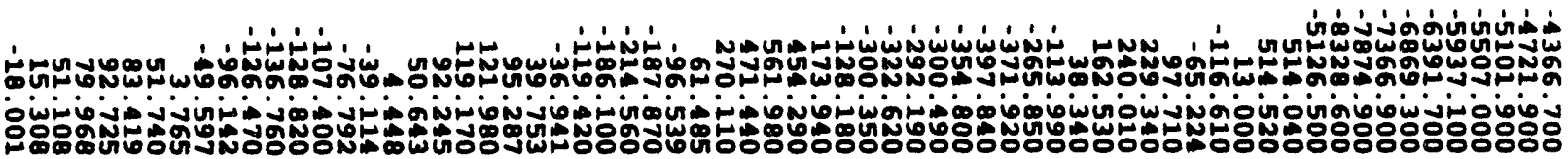




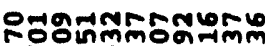

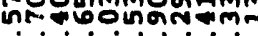

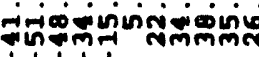

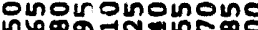

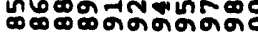
miniminiminiminimi-

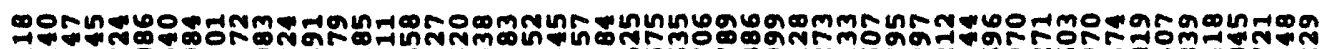

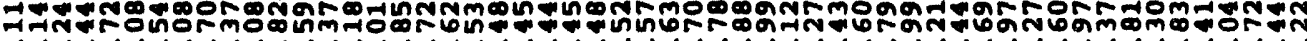

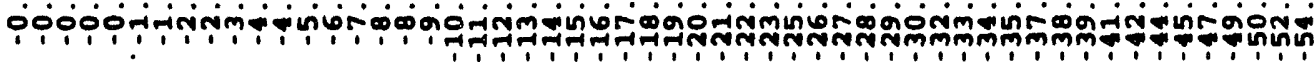

ononononononononononononononononononononnononononononon

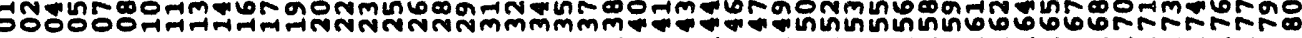
000000000000000000000000000000000000000000000

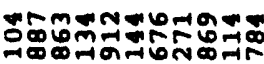

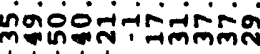

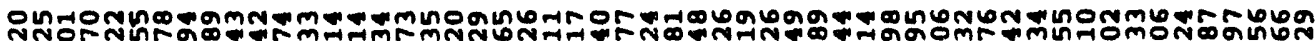
-1

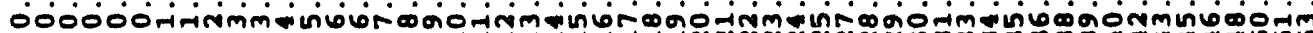

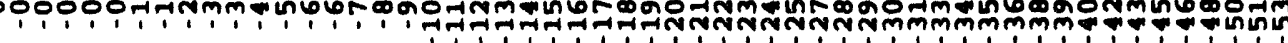

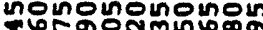
iminiminiminimiminim

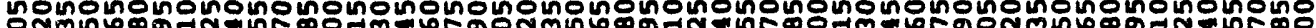

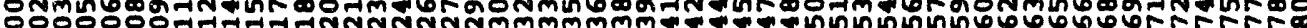
000000000000000000000000000000000000000000000000000000

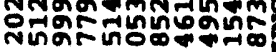

Dogn-anr

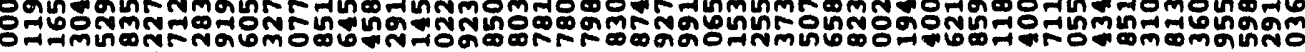
กษึี

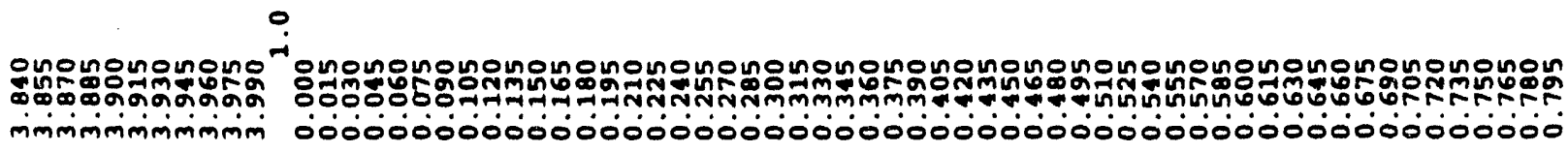

$\overline{\mathbf{D}}$ 


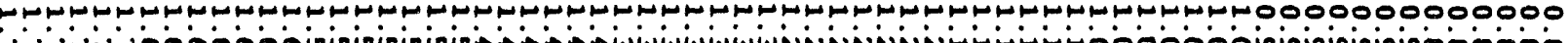

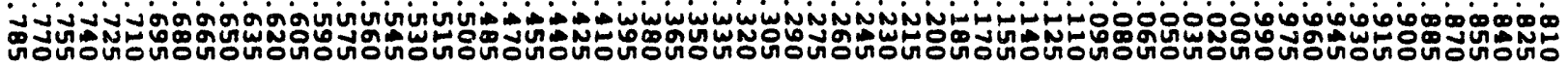

每

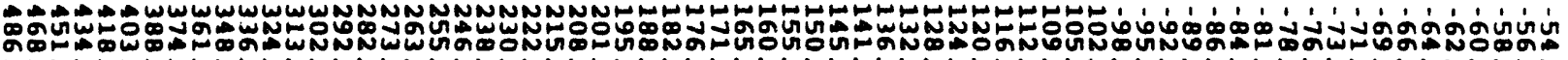

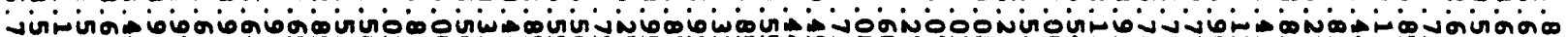
\$.

M

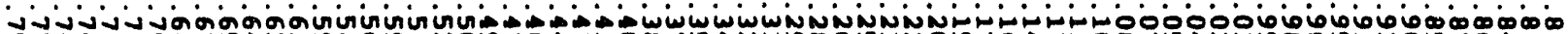
๑ง

ด̈

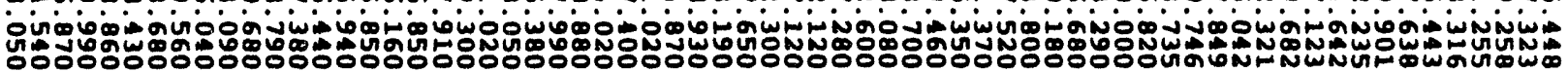

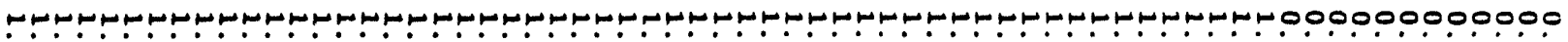

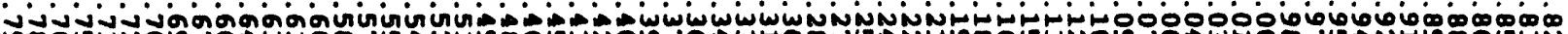

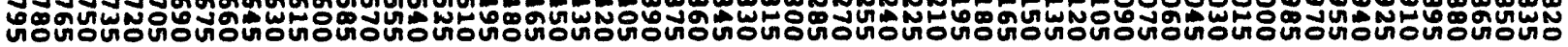

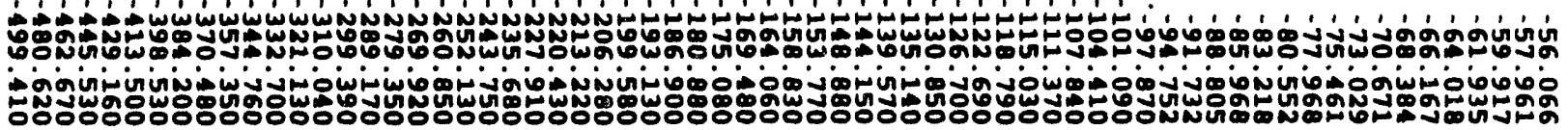




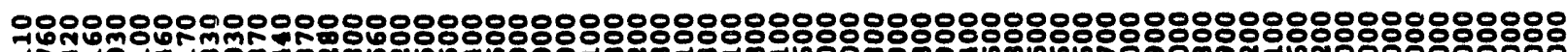
7 r. - - mom m m n Go 6 im

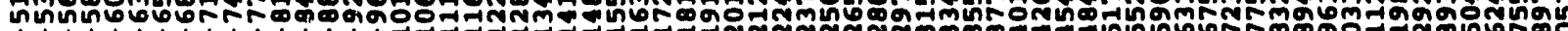

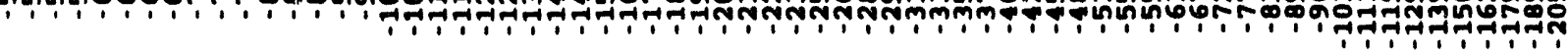

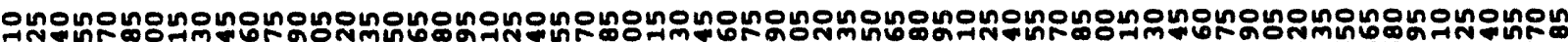

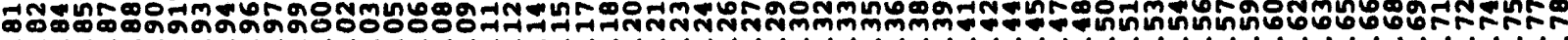

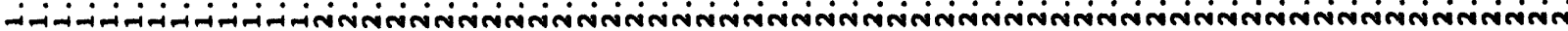

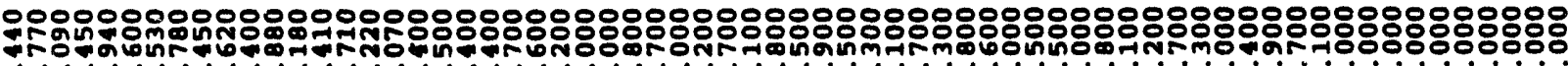
7nูn

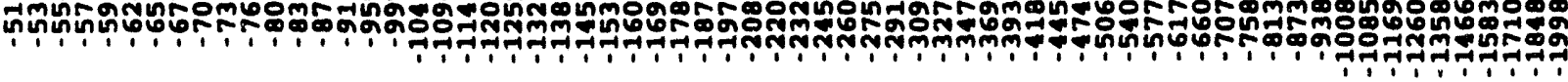

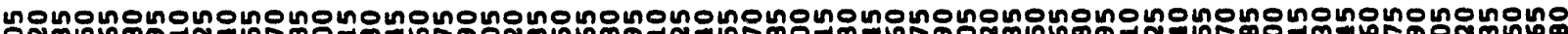

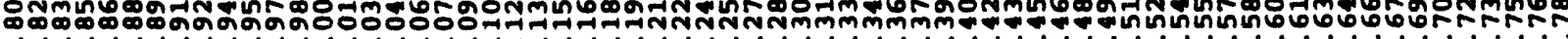

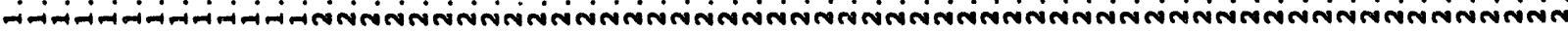

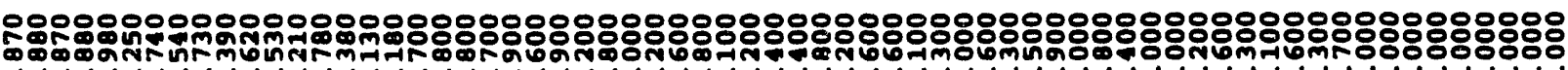

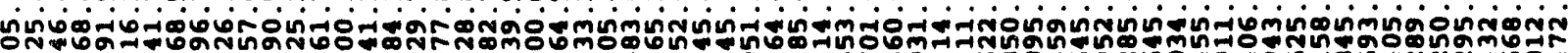

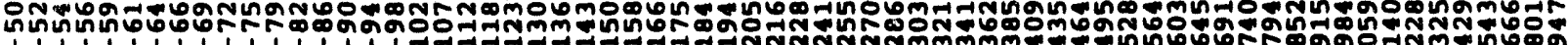

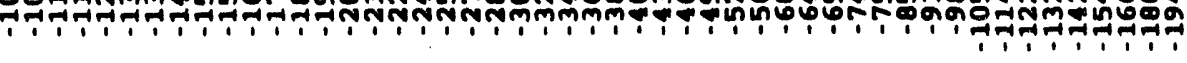

onononononononononongnongnononongnonononongnonononongnonononononon

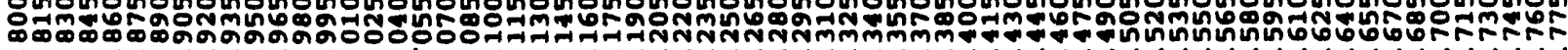

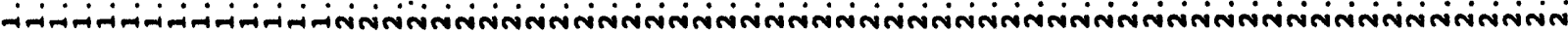




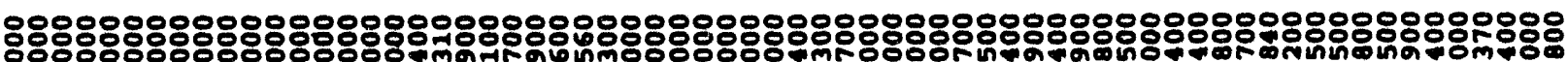
ॠrsंmm

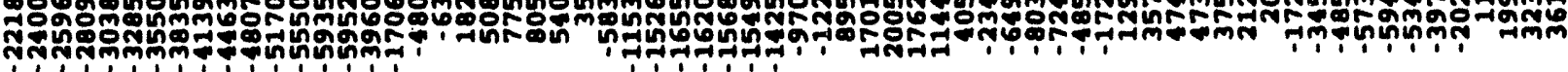

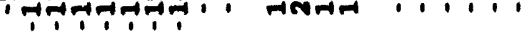

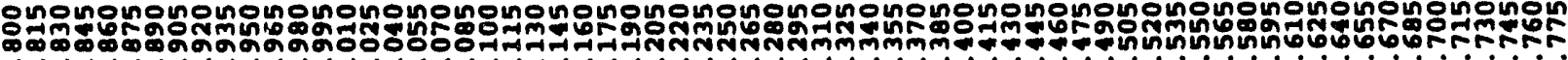

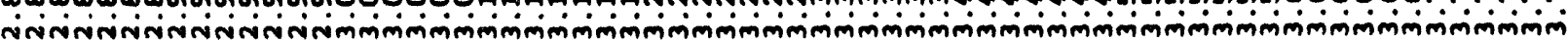

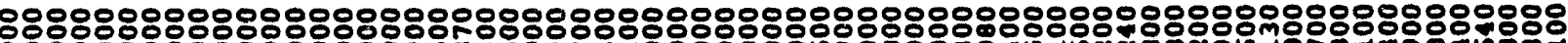

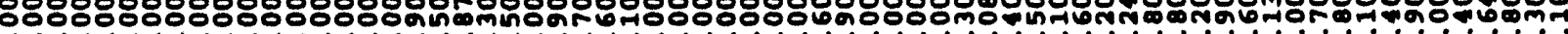
nंnison 6m

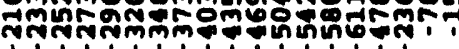

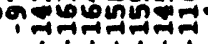

uononononononononononononononononononongnonononononononononononono

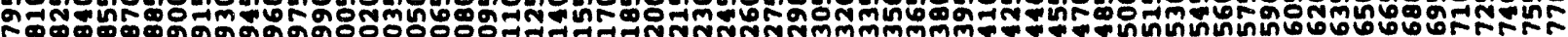

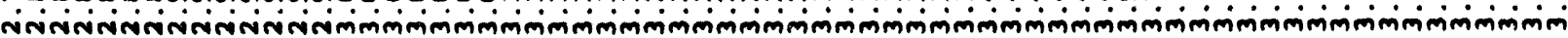

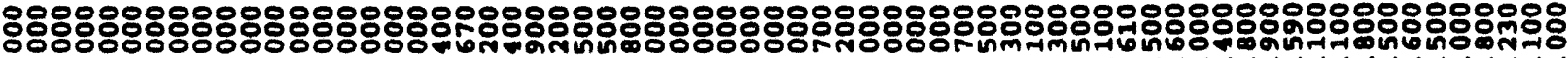
rionornin

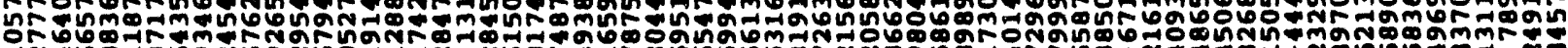

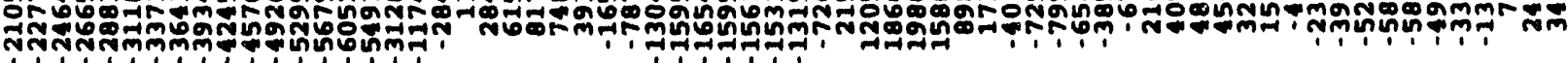

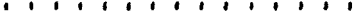

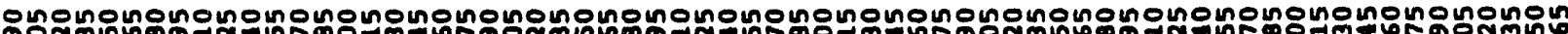

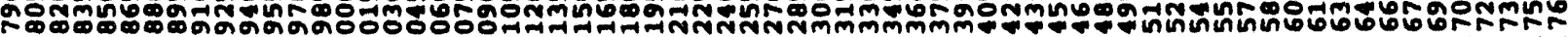

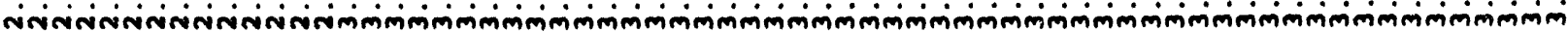




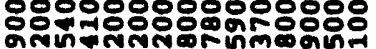 ชnc 80ำ

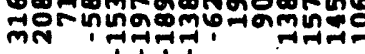

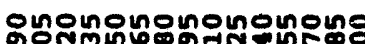

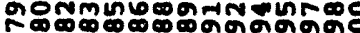

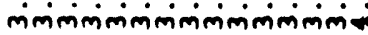

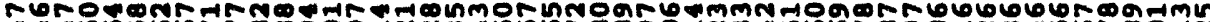

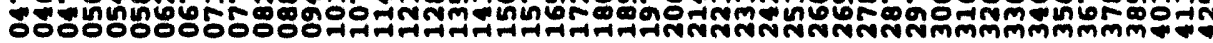
o00000000000000000000000000000000000000

ononononongnononononononongnongnongnongnononononon

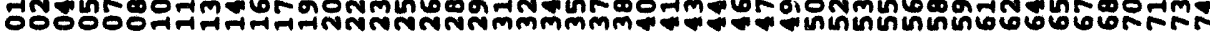
000000000000000000000000000000000000000000

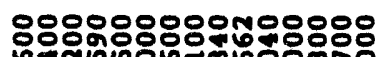
- بnต̣

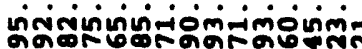

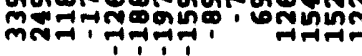

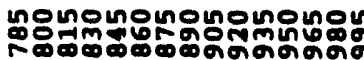

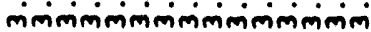

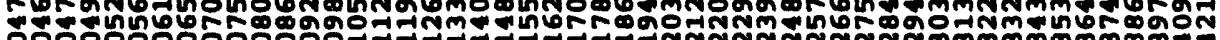
0000 iopopopo

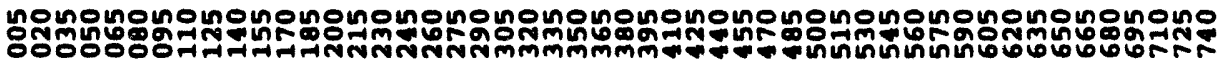
00000000000000000000000000000000000000000000000000

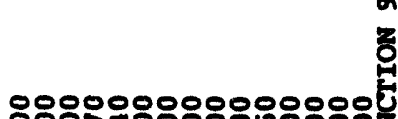

井

แดด̆

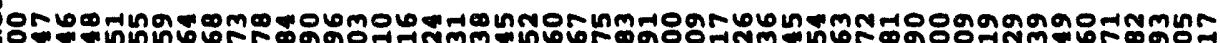

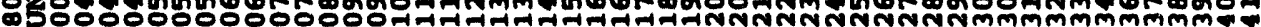
on - r r o

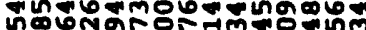
ज्ञलत T.1.

0

으onononongnono

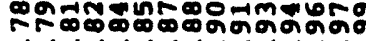
miniminiminiminiminiming

ononononononononononononononononononononononononon

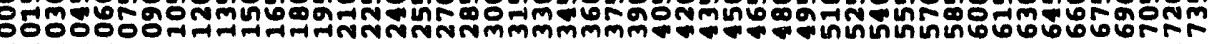
000000000000000000000000000000000000000000 


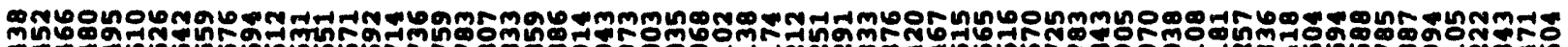

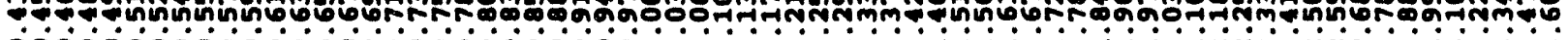

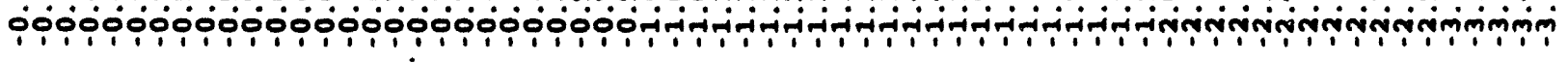

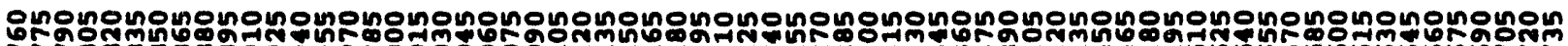

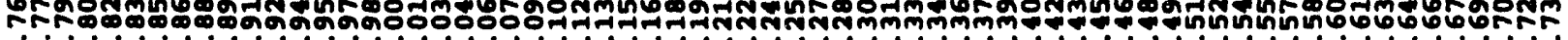

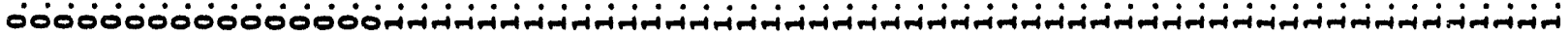

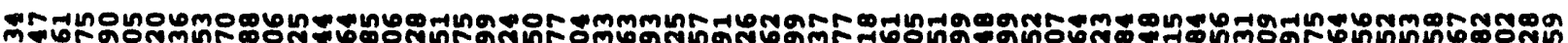

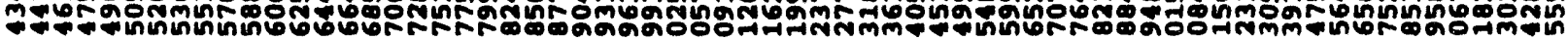
io po

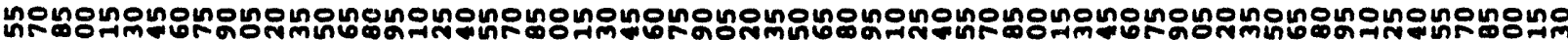
ก 00000000000000000 -

웁ํํํํำ

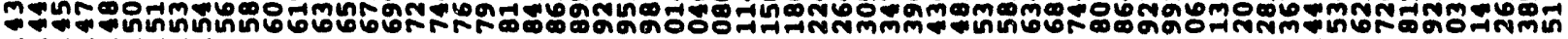
o

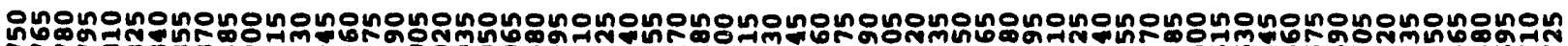

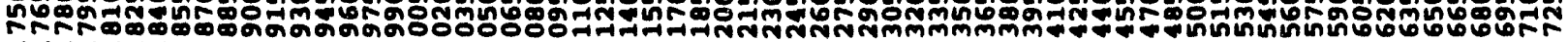
00000000000000000 - 
-ommm

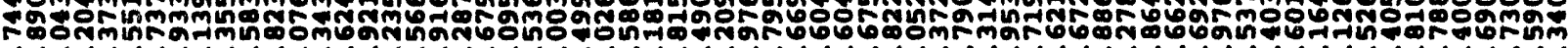
mm-

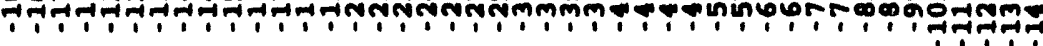

Ononononononononoinonononononononononononononononongnongnononononon

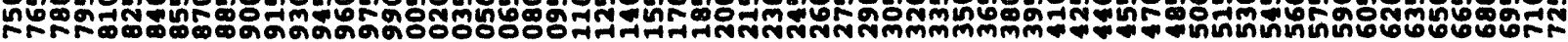

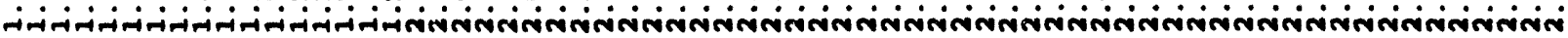

மono6- ดøgn

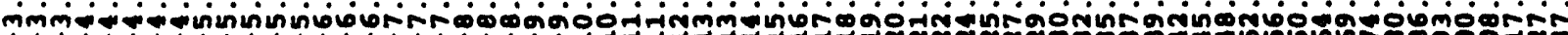

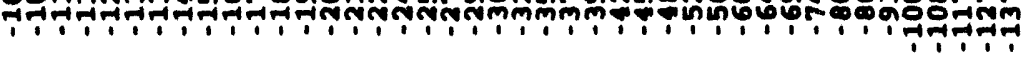

nongnononounonongnonongnononononononononongnononongnononononononono

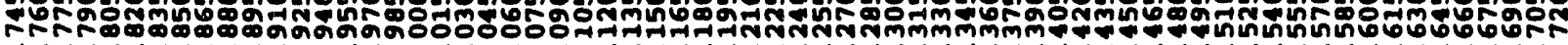
-

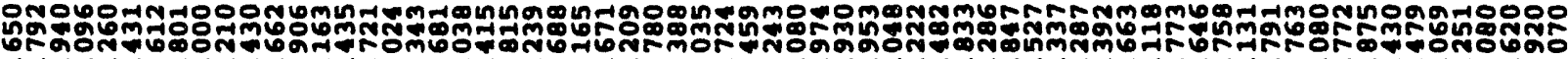
mmm - -

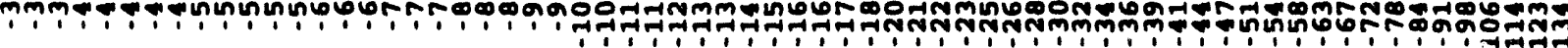

$$
\text { 7., }
$$

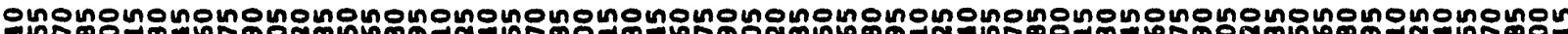

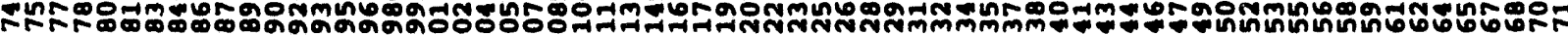

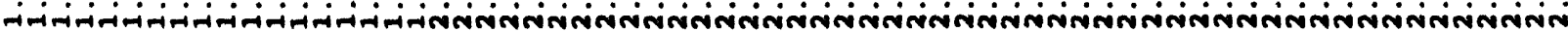




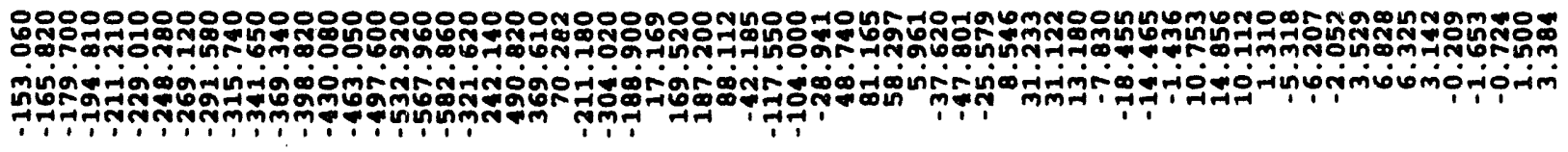

onononononononononononononononononononononononononononononononongn

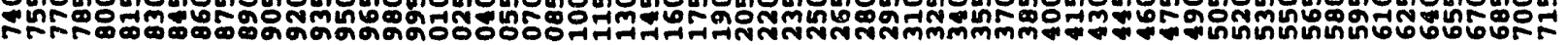

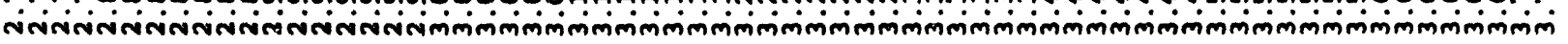

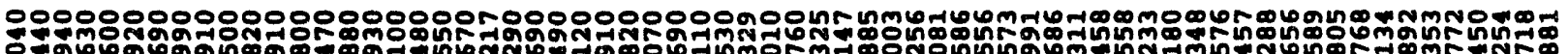
on ق̆

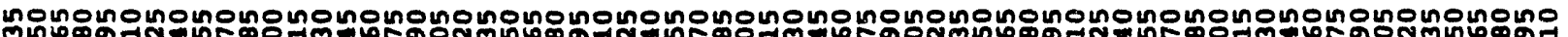

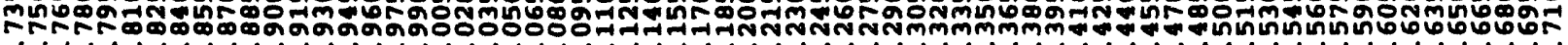

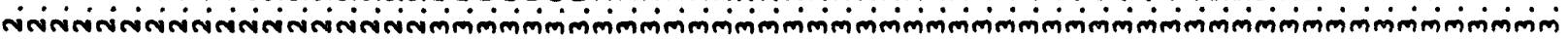

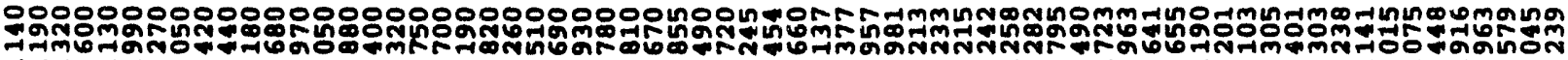
viñ

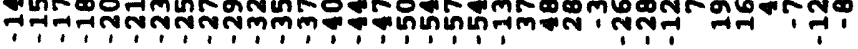

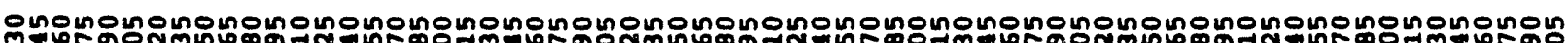

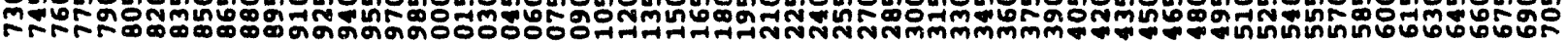

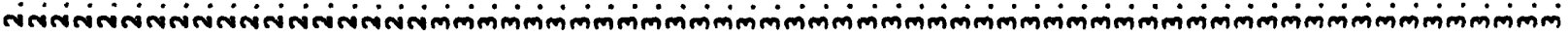




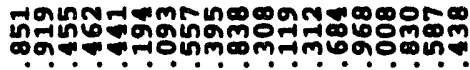
minio

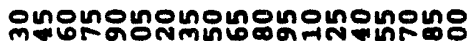

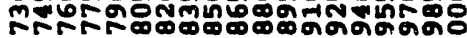

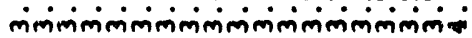

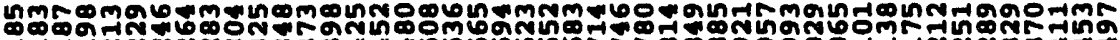
1

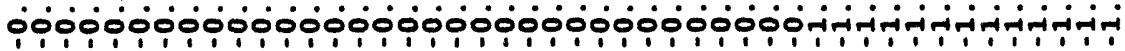

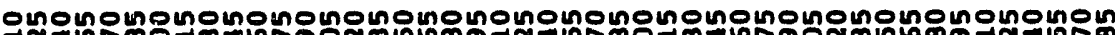

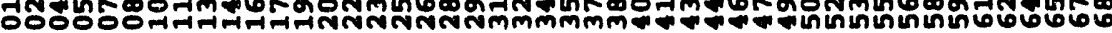
0000000000000000000000000000000000000000000000

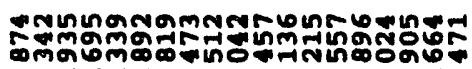

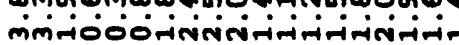

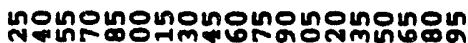

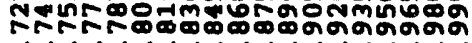
iniminiminiminiminiminiminiminimin

a-conmmngomoneos

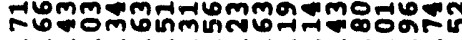

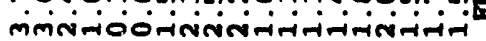

๑

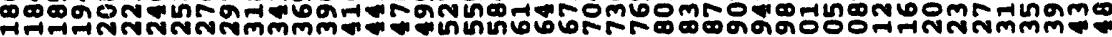
iojo

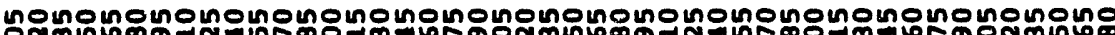

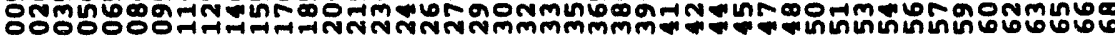
- 00000000000000000000000000000000000000000000

0 - $0 m \infty$ wo

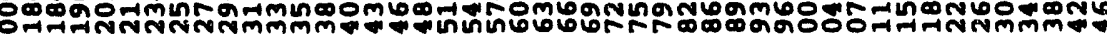

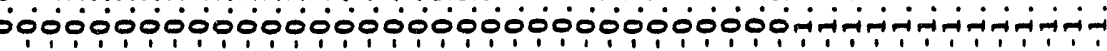

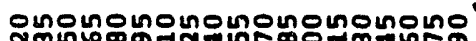

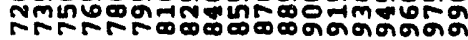

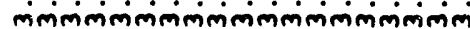

ononononononononononononononononononononononon

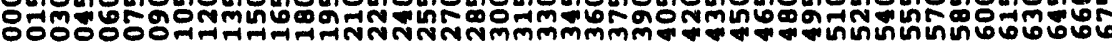
- 0000000000000000000000000000000000000

8. 


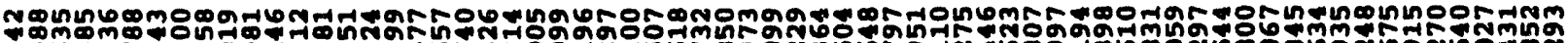

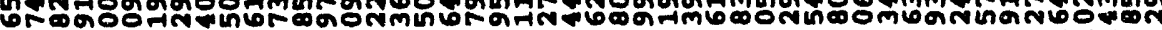

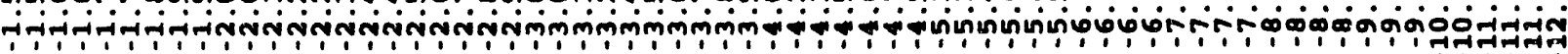

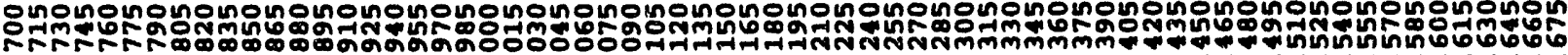
00000000000000000000 -iन

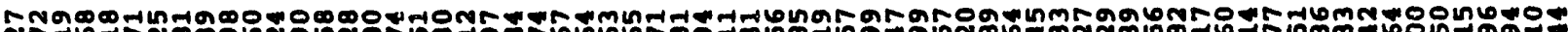

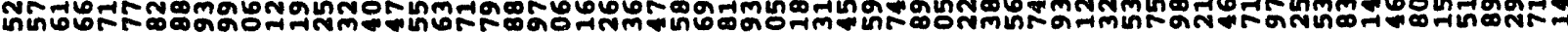

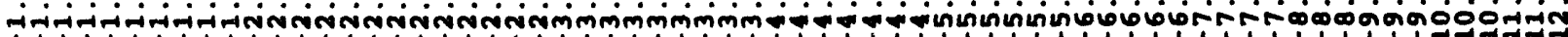

nonononononononononononononononononononononononononononononononono

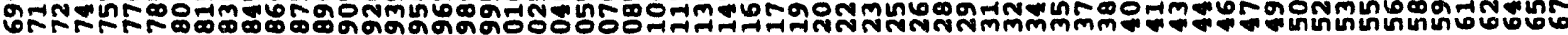
0000000000000000000000 -

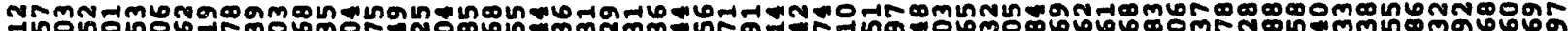

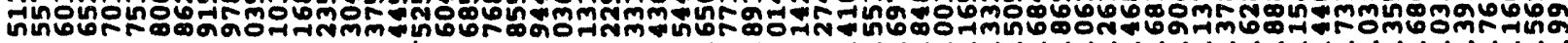

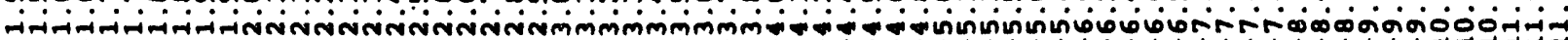

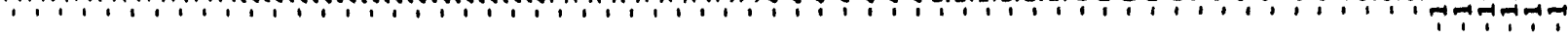

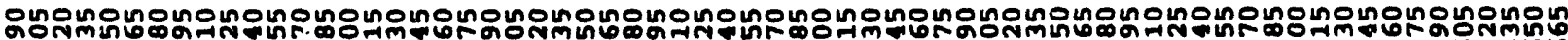

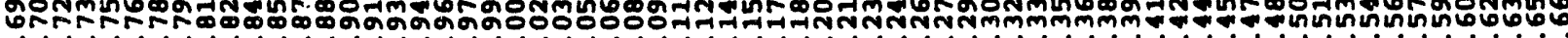

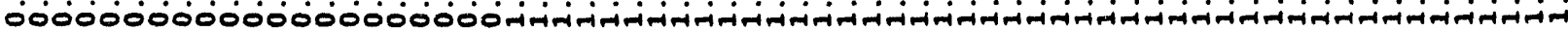




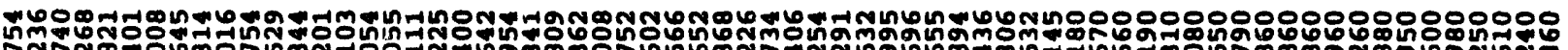
TNN न नНन

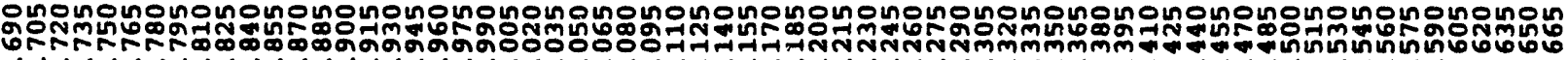

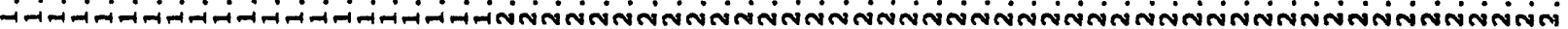

mตด

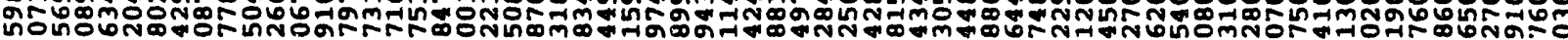
Nmm viniono

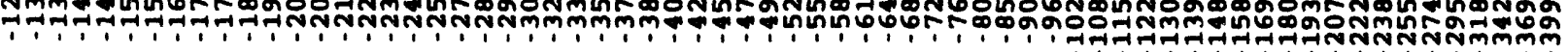

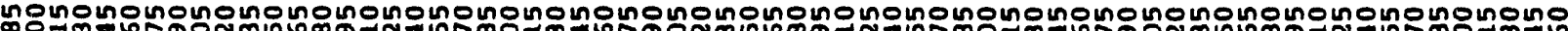

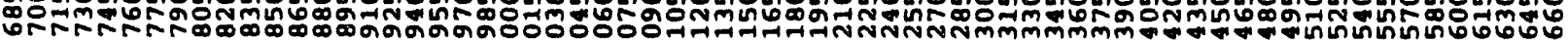

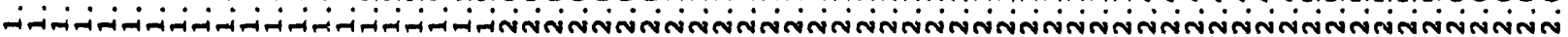

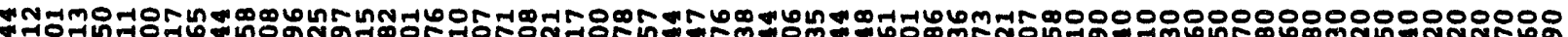

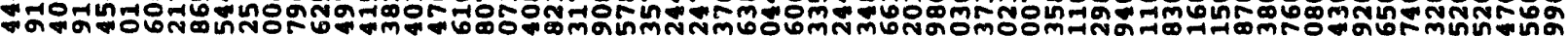

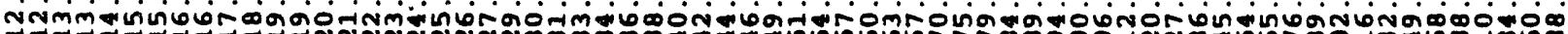

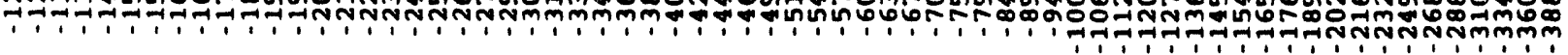

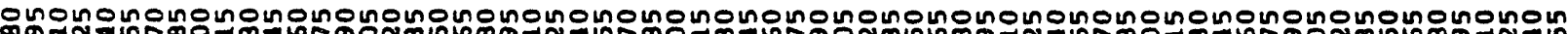

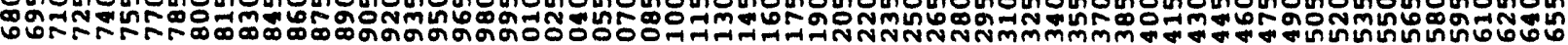

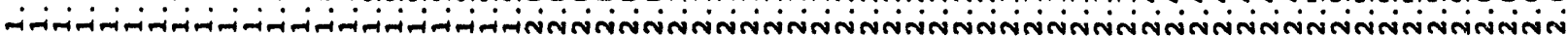




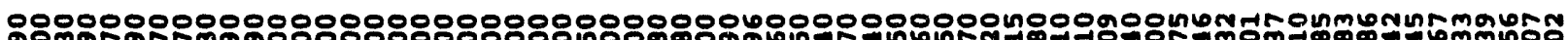

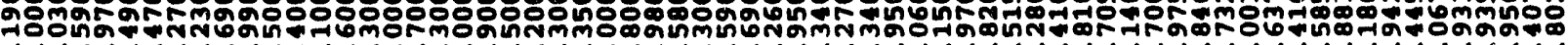

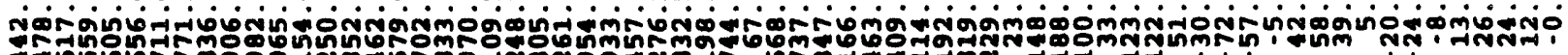

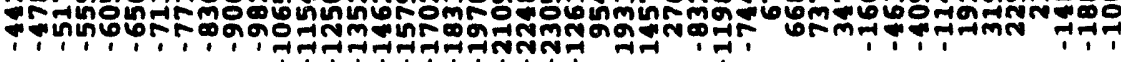

onononongnononononononononononononononononononongnonononononononon

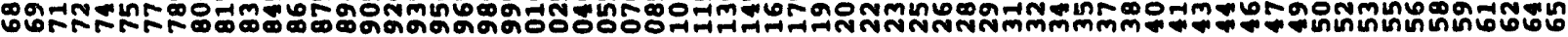
نN

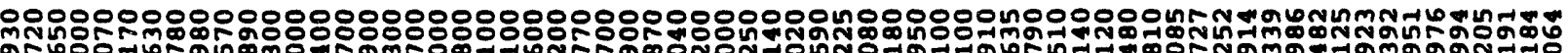
o mio Punvo

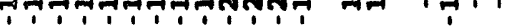

$$
1 \text {. }
$$

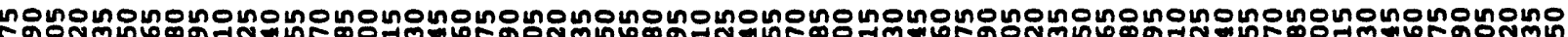

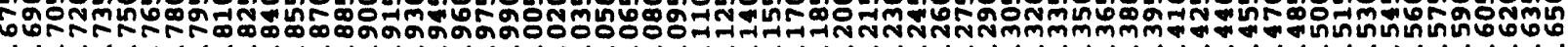

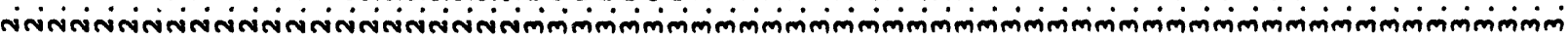

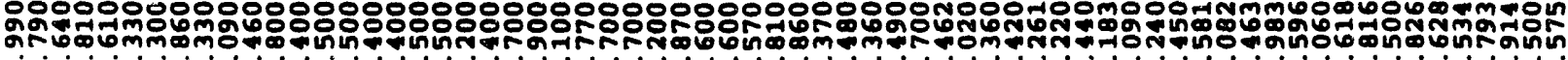
Gต่อ - Tñ

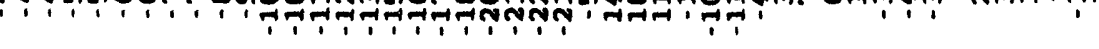

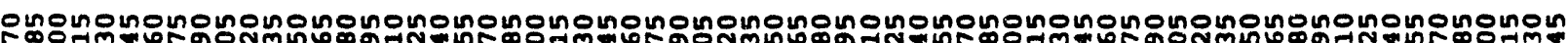

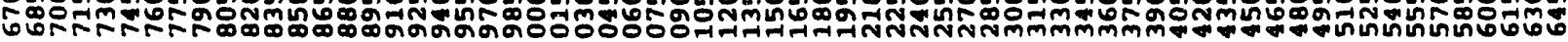

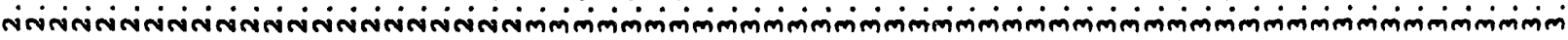




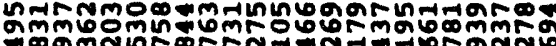

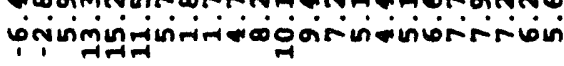

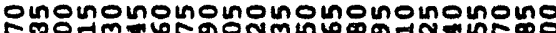

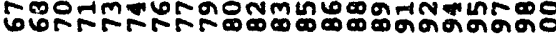

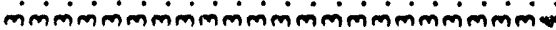

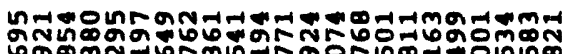

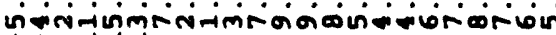
i.

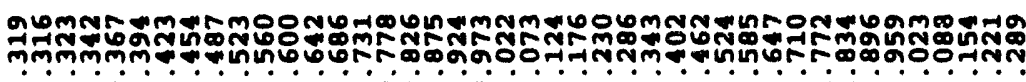

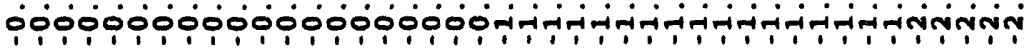

onononononononomononononononononononononon

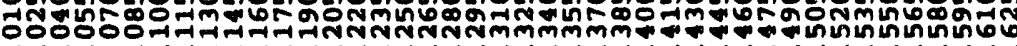
00000000000000000000000000000000000000

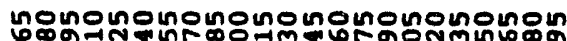

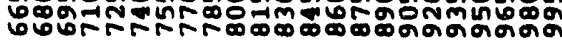

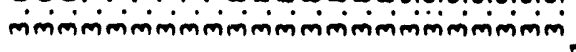

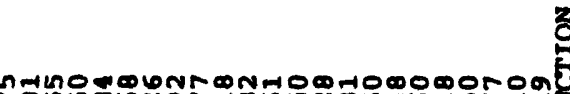

-69nmum -6 mmmmm

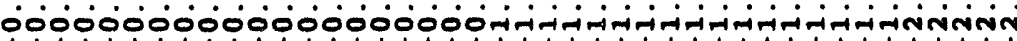

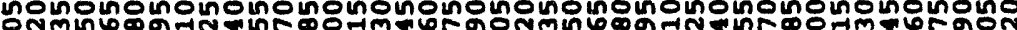
จำ 00000000000000000000000000000000000

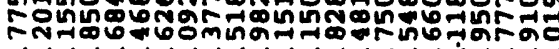

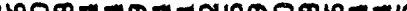

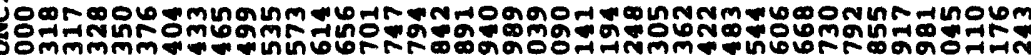

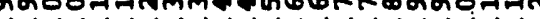
o.

○

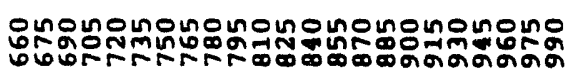

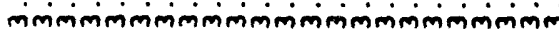

io

onongnononongnonononono

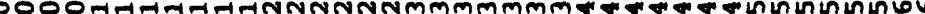
000000000000000000000000000000000000000000 
作

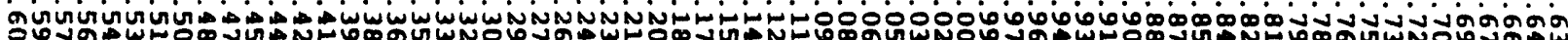

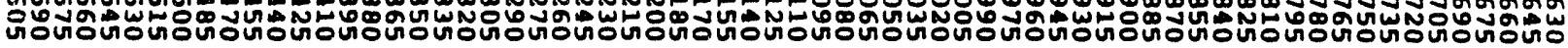

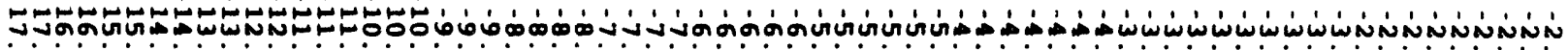

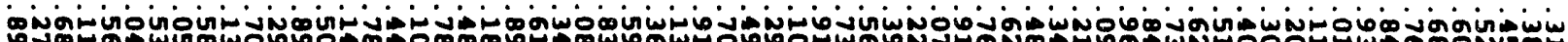
:

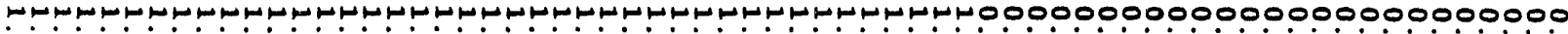
gininivinini

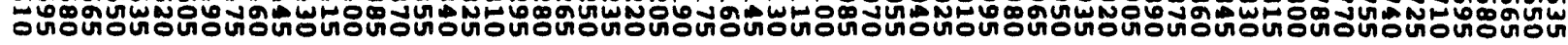

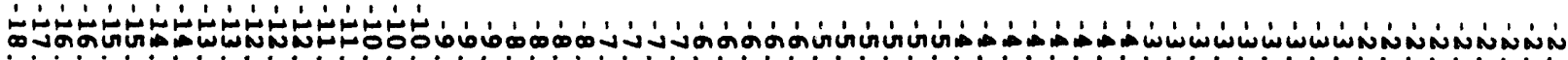

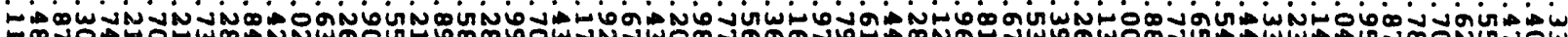

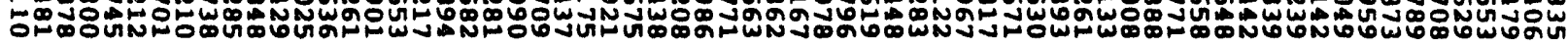

ПHトル grinuninun

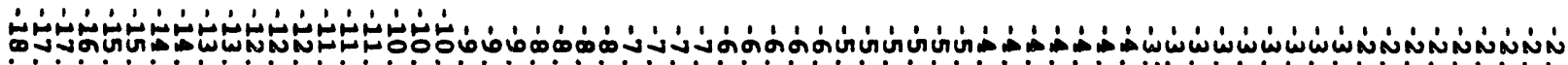
wojo

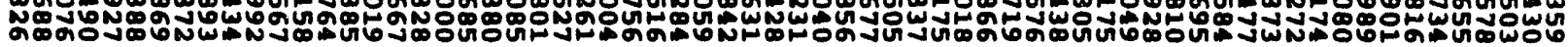




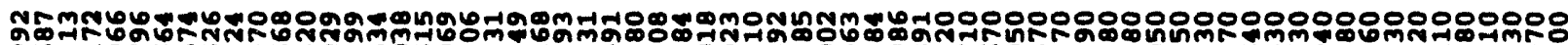

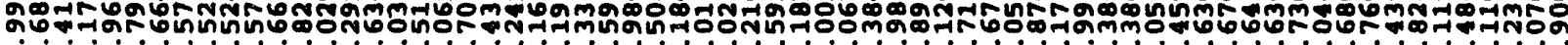

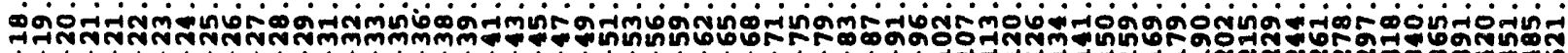
年

ononononononononononongnononononononongnonononononnonononononononon

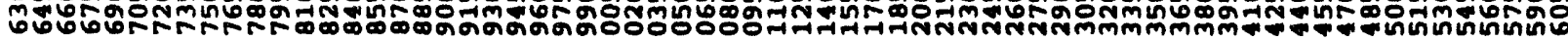

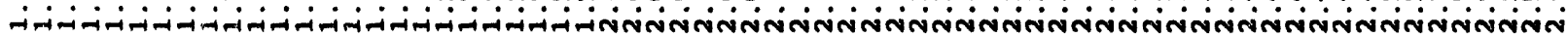

분ำ

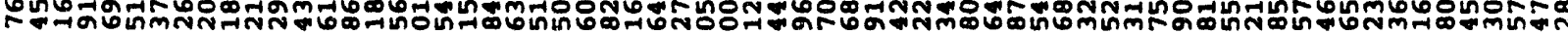
mojo- Nm

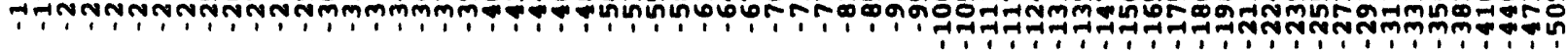

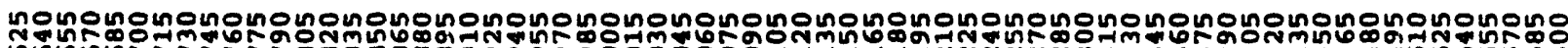

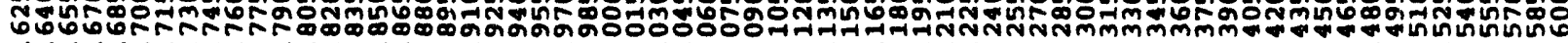

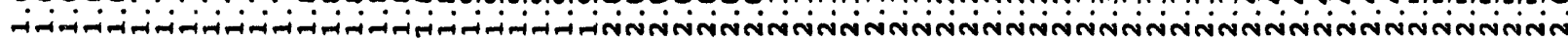

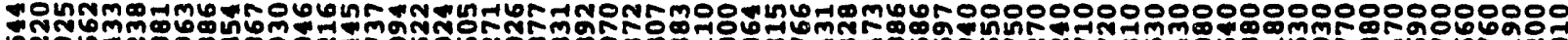

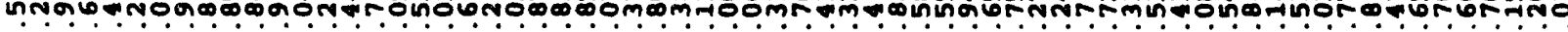

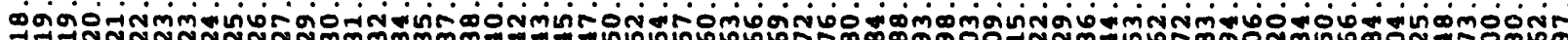

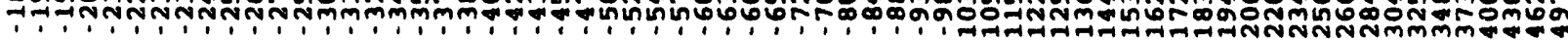

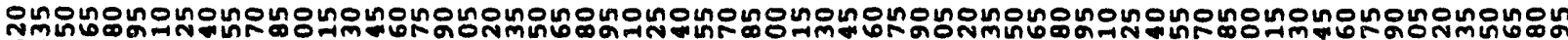

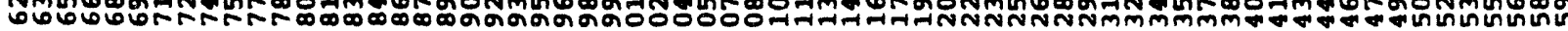

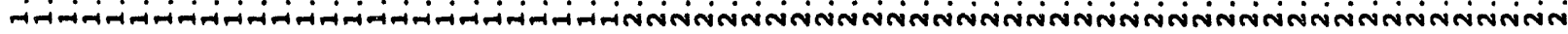




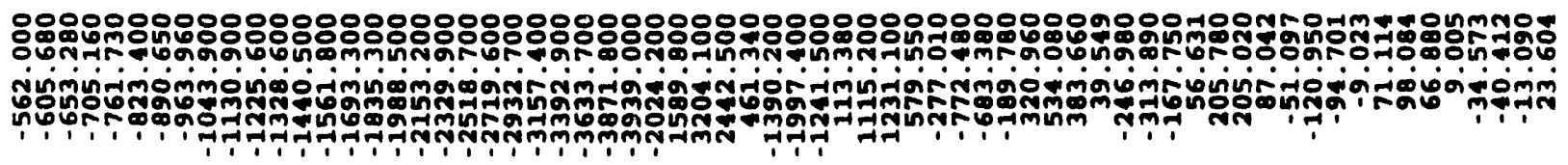

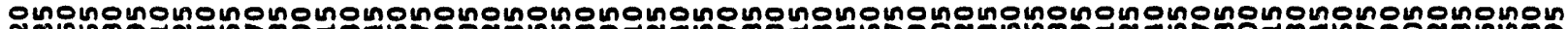

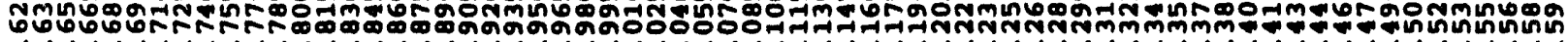

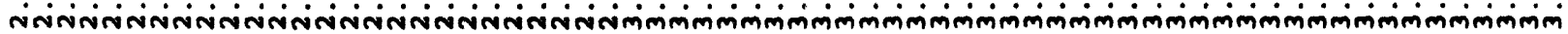

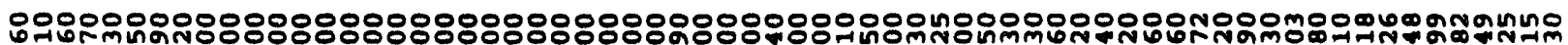
Nกศmm

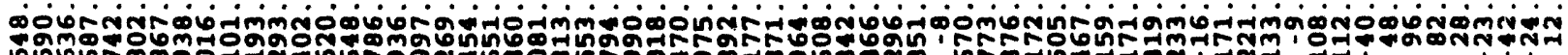

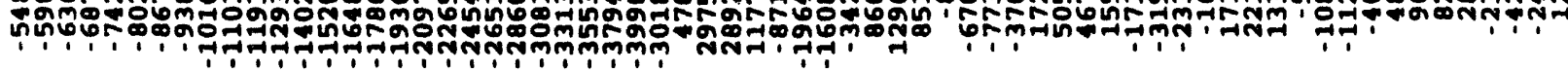

nongnononononononononoungnounonononononongunongnonononounonononounongno

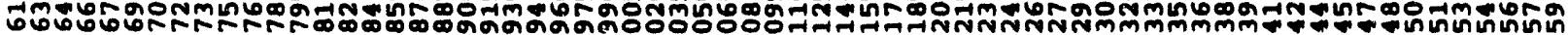

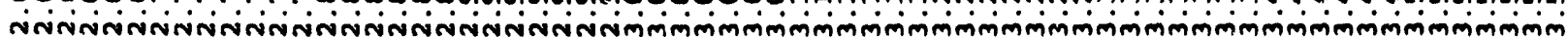

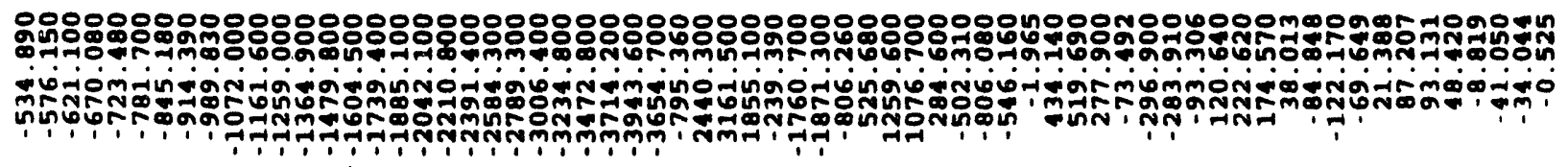

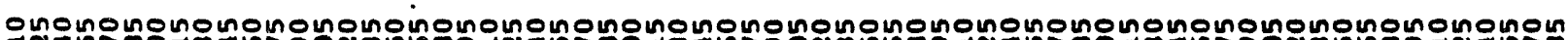

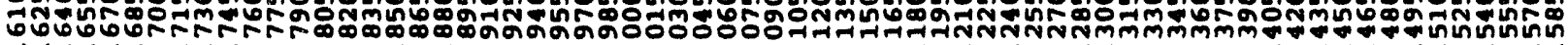

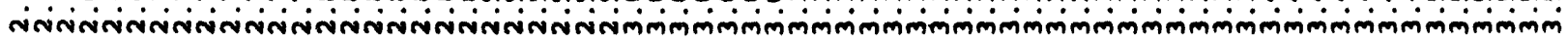


ผแผ Vioioioio o o o o

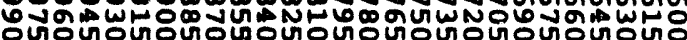

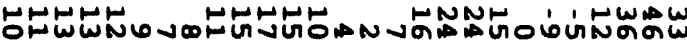
woow оำ

Wแผ ioioioioiocón O.

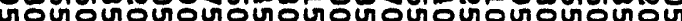

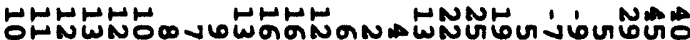

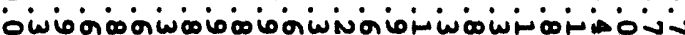

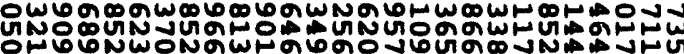

-ioivioio ó ó कo

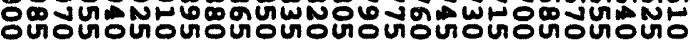

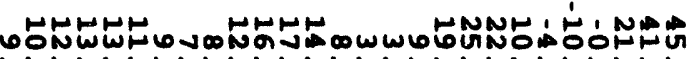

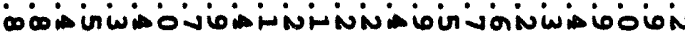
mon 
DYnaurc RRSPONSES 
D I S P LACEMENTMAXIMA,

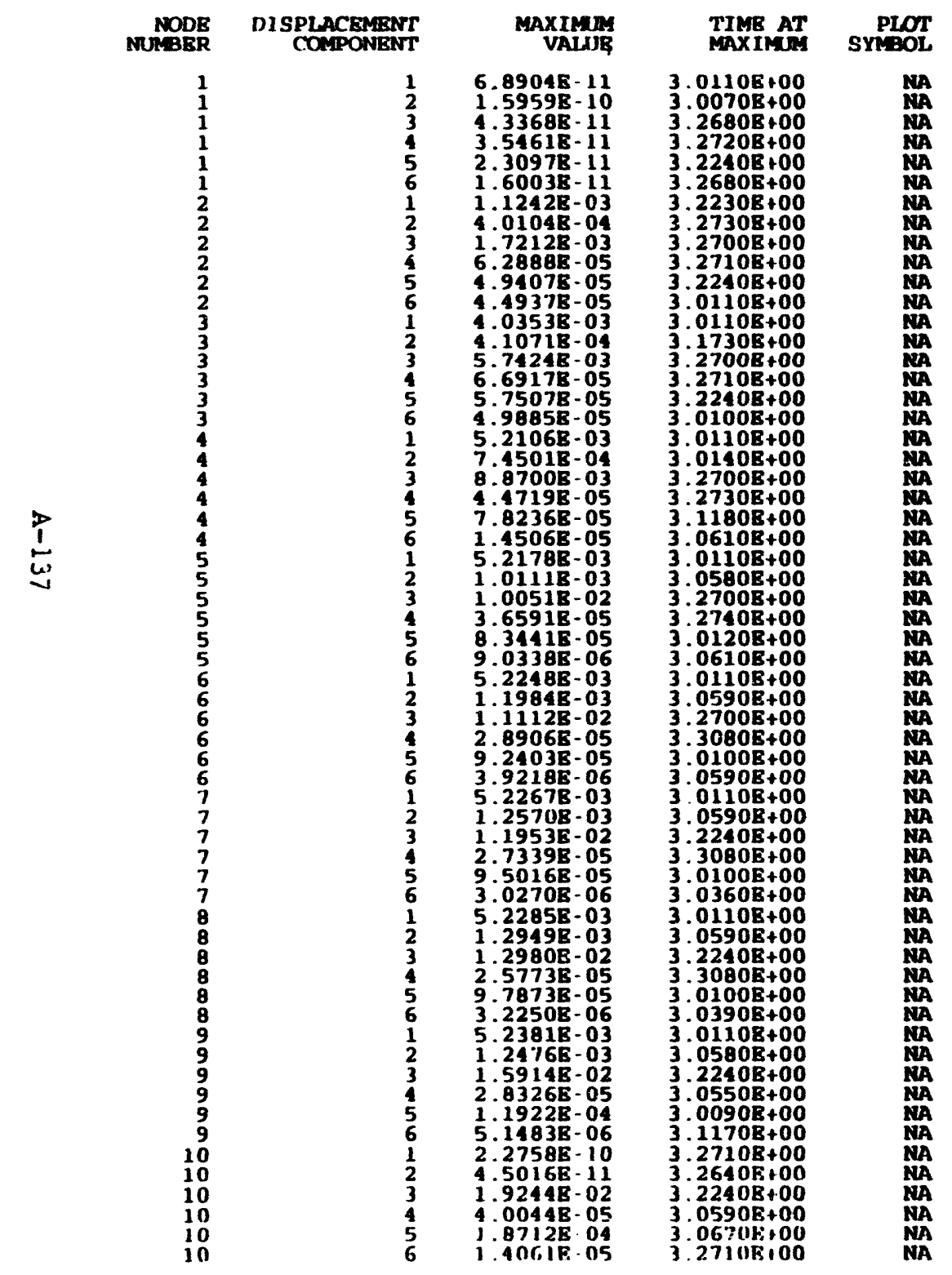




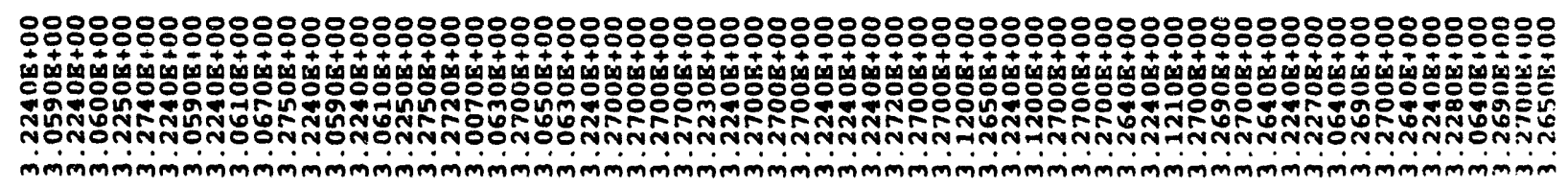

ว

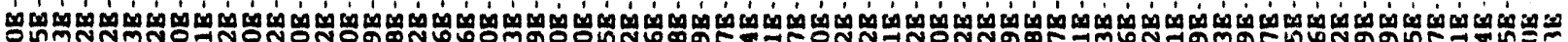

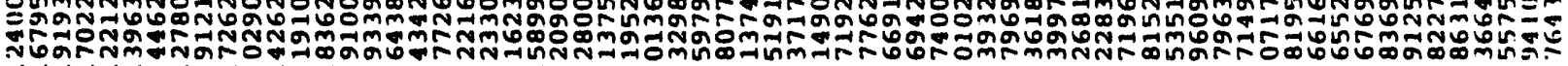

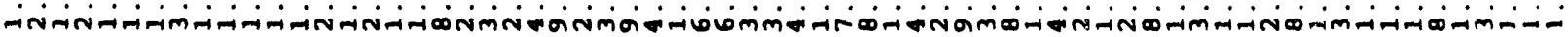



Q

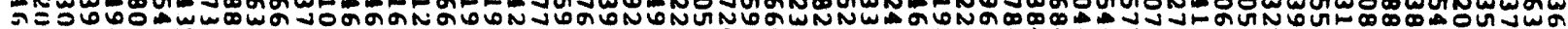

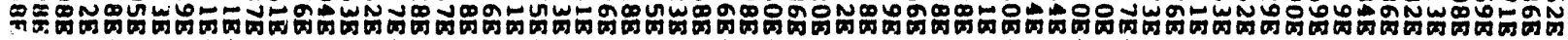

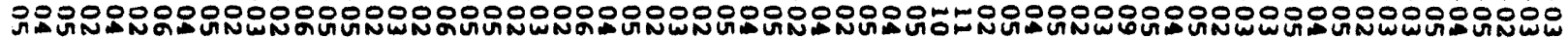

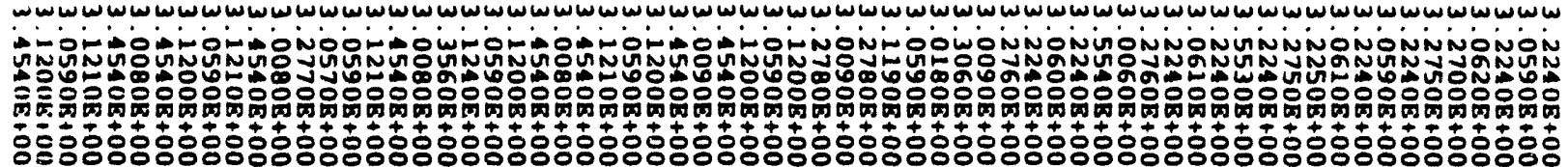

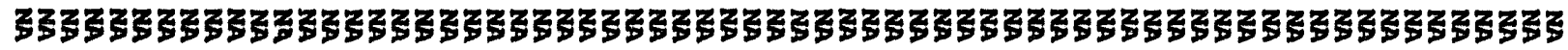




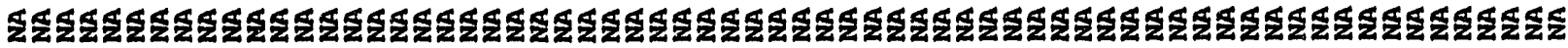

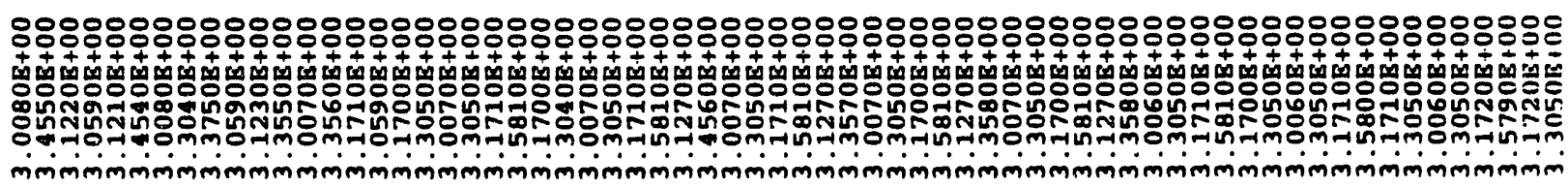

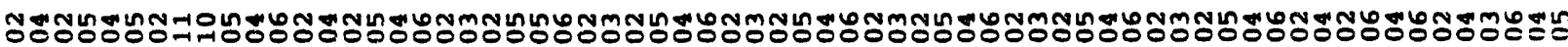

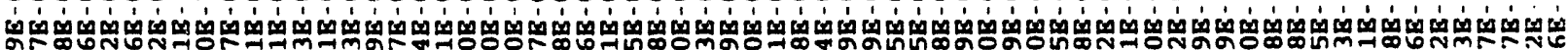
ת-

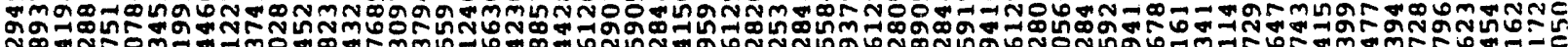

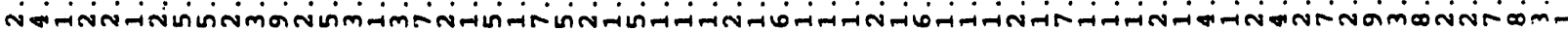

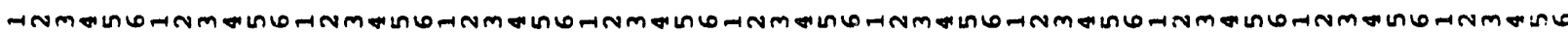




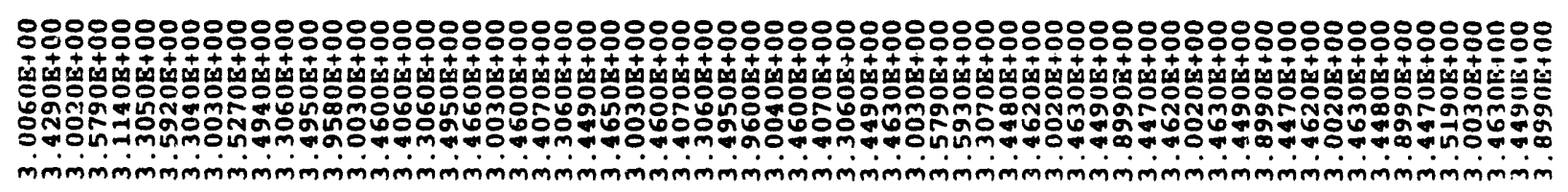

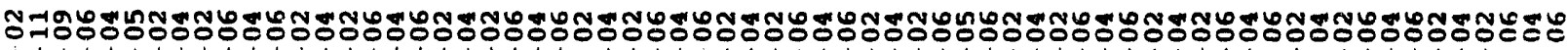

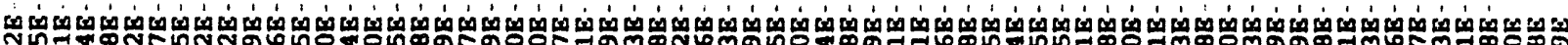

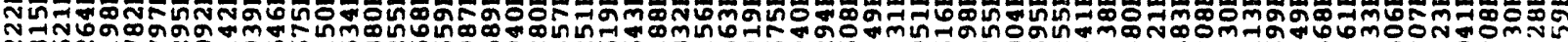

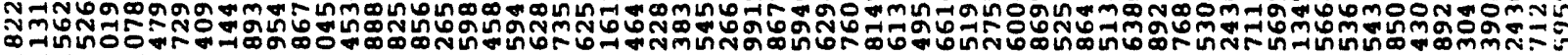

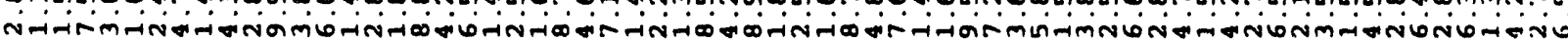

HNm 

owgi-n in 3-NG

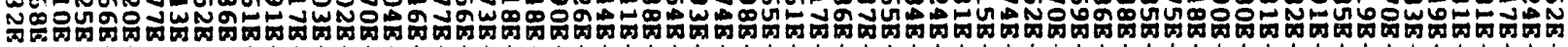

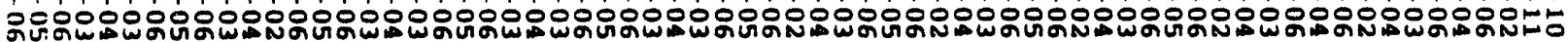

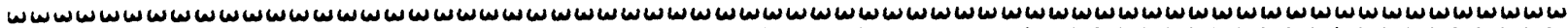

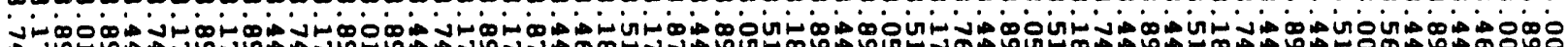
¿ ป็

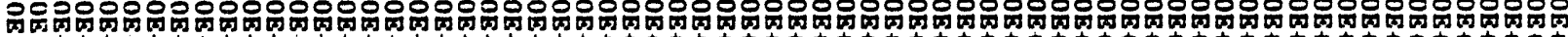

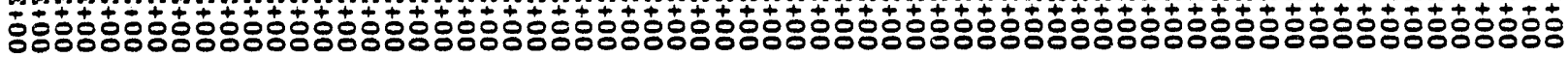

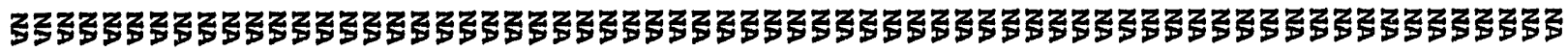




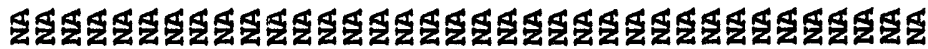

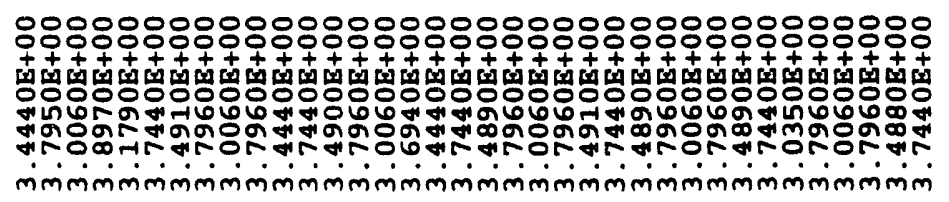

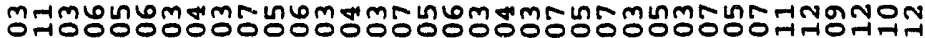

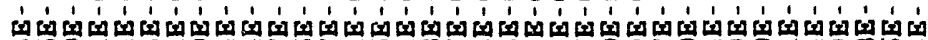

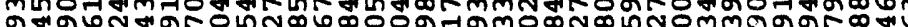
○ी "mun

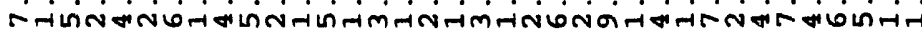

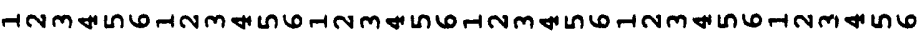

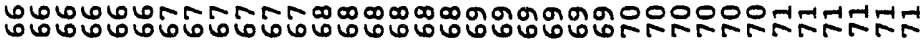


S TRESS C OM P ONEN T MAX IMA ELEMENT TYPE NUMBER

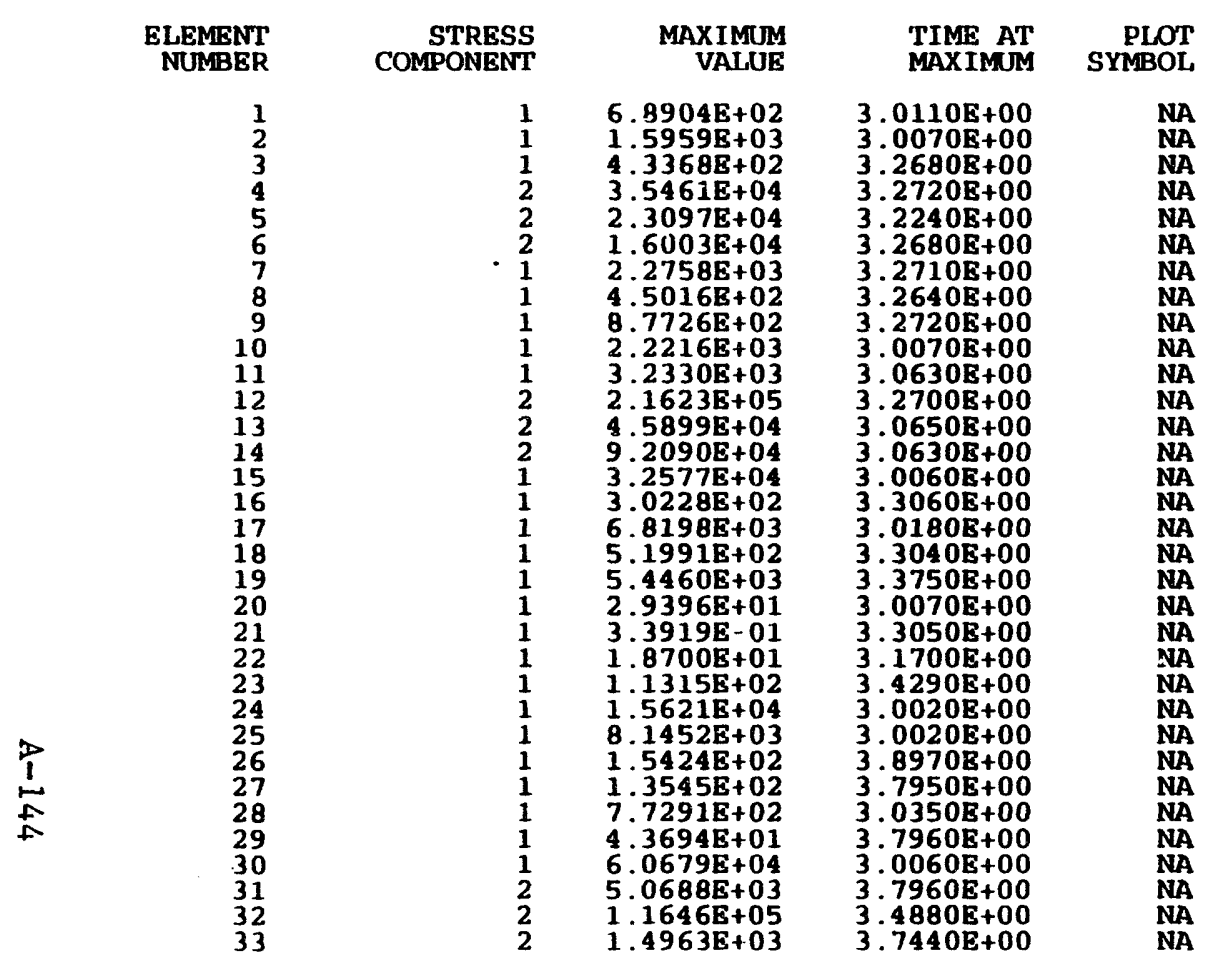


STRESS COM UNENT M XXIMA

FI,RMENT TYPE NUMBER $=2$

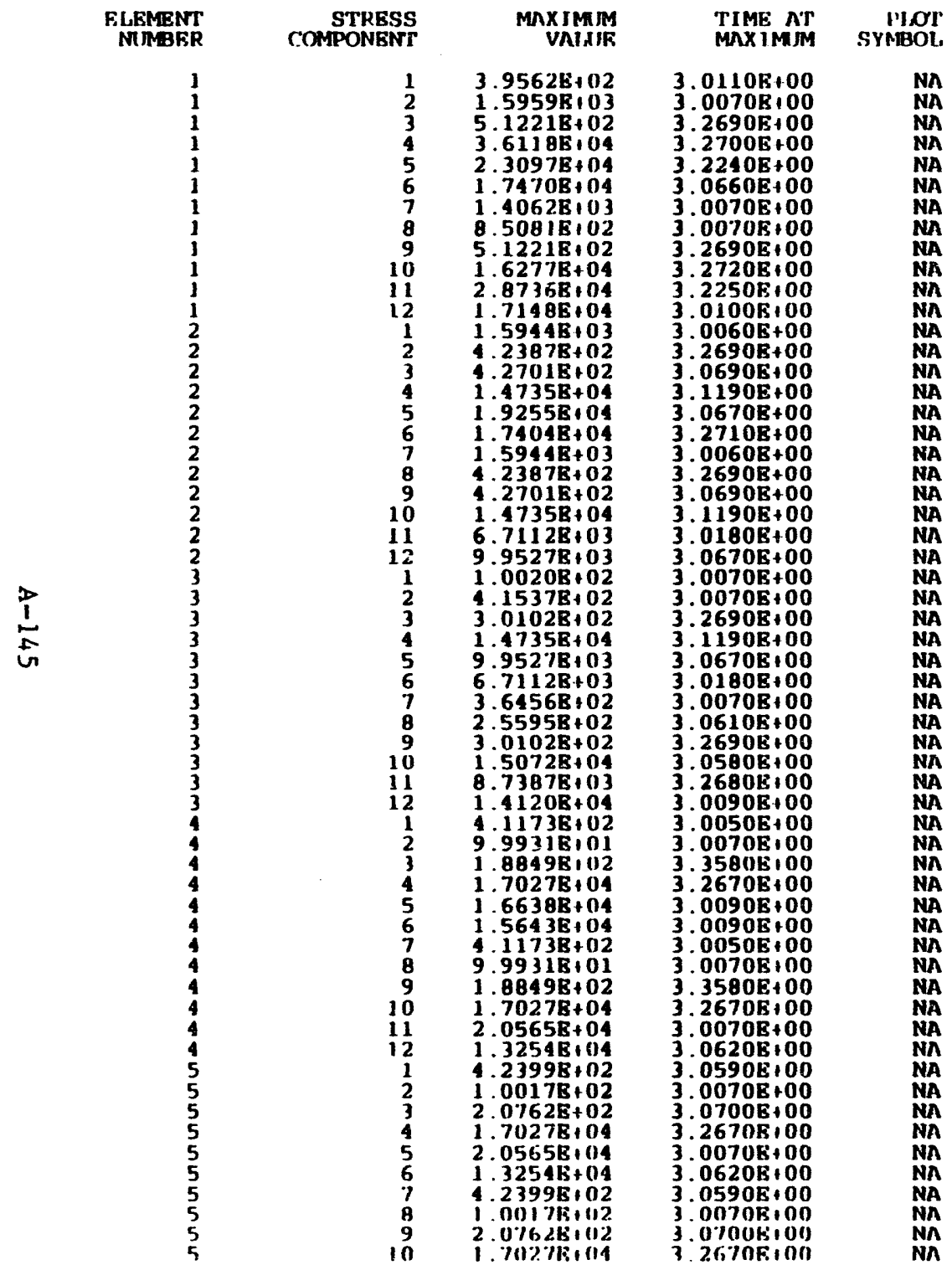




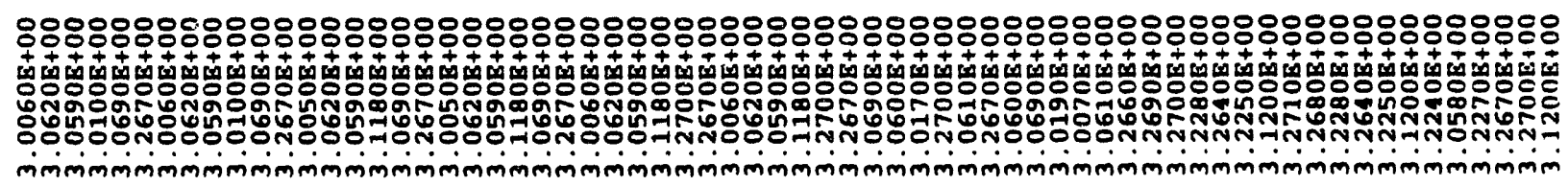

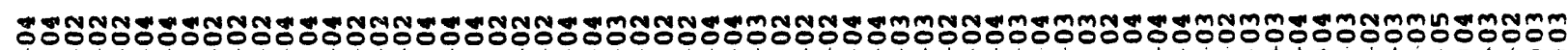

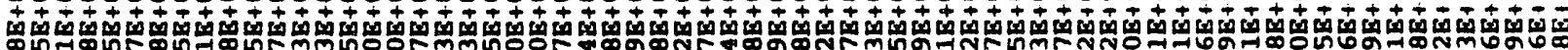

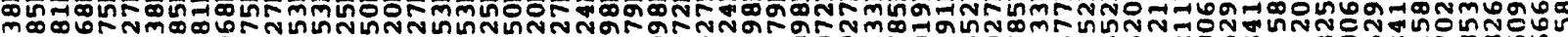

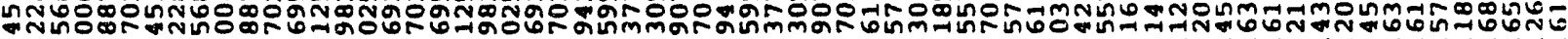

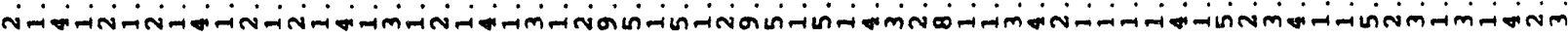

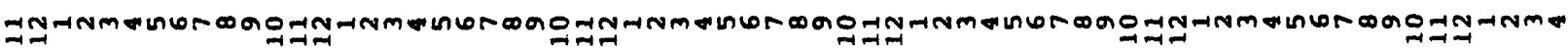




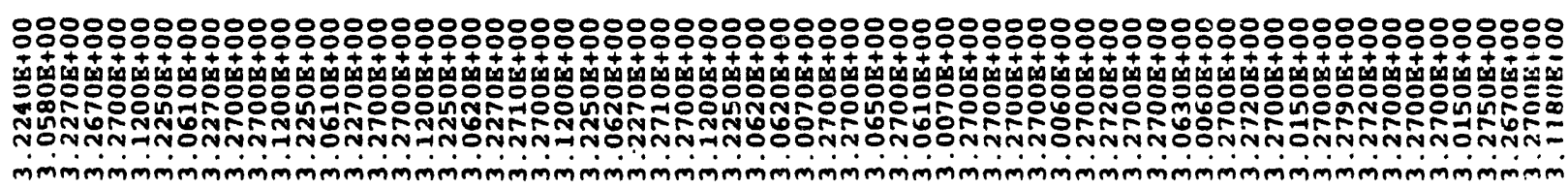

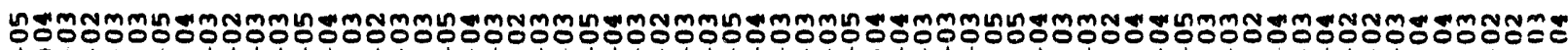

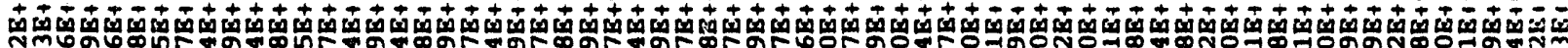

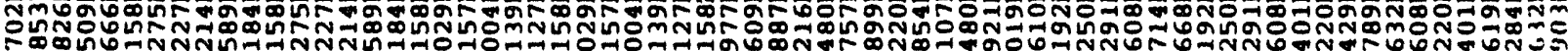

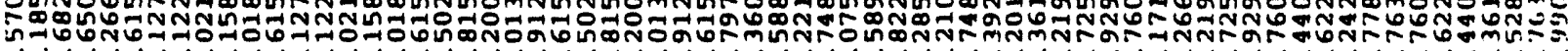
-im-inmmoñ

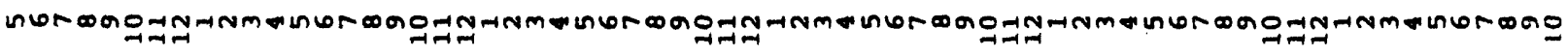
然 


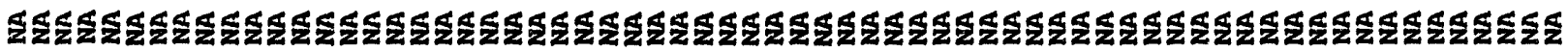
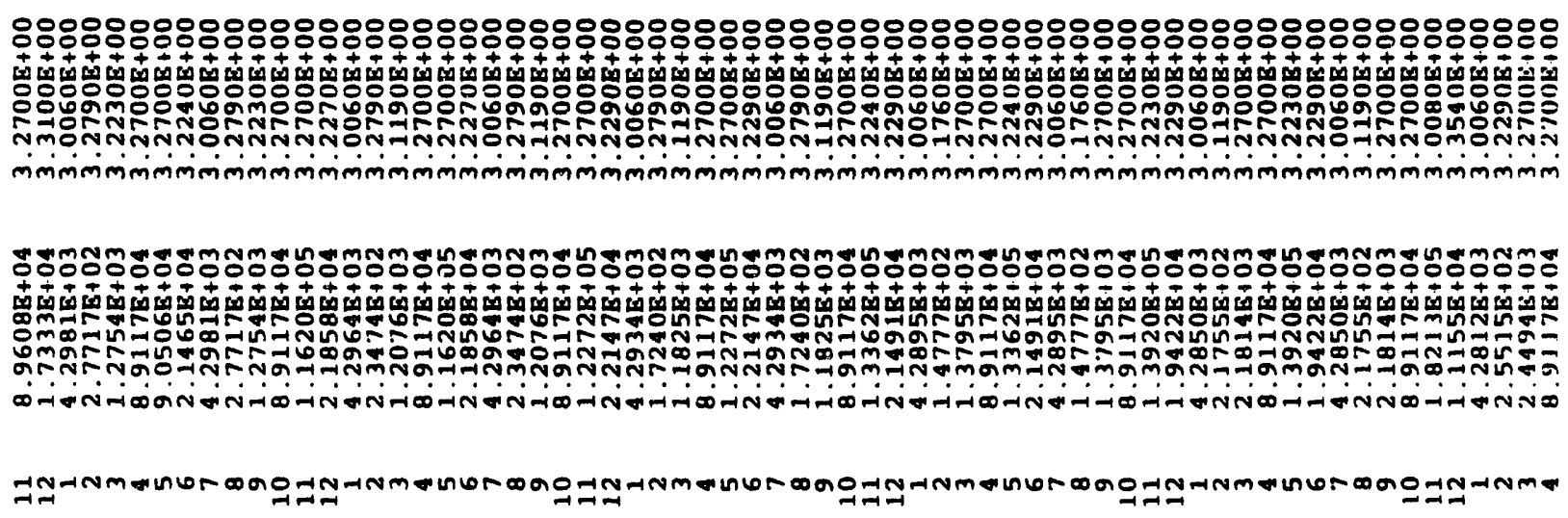

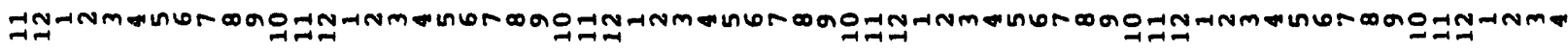




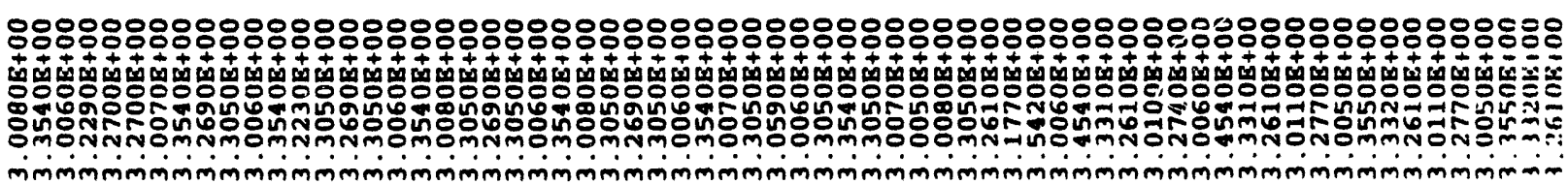

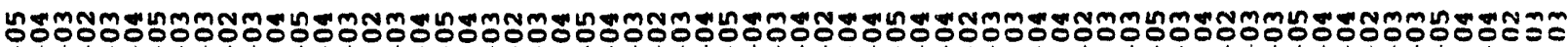

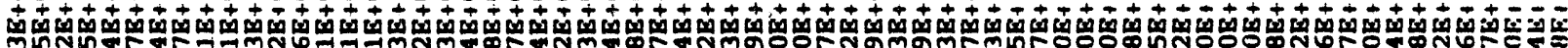

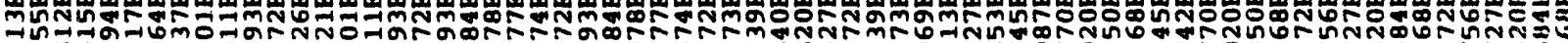

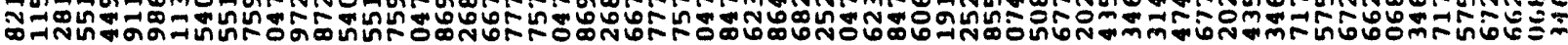

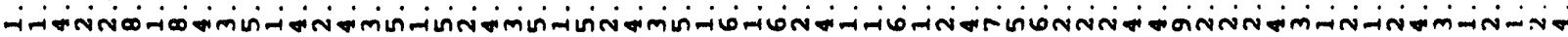

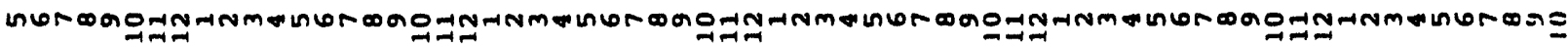

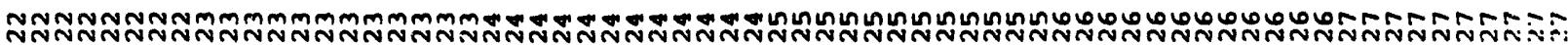




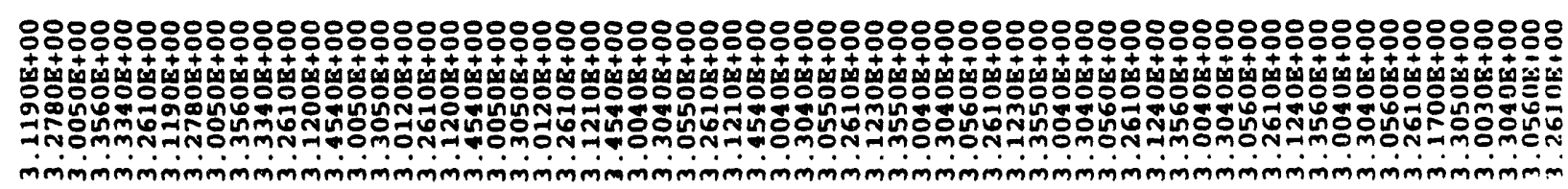

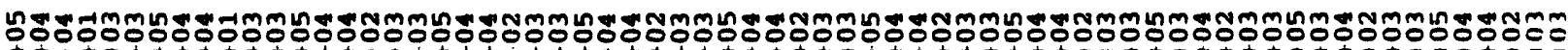

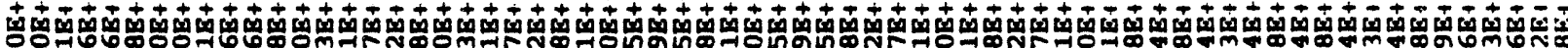

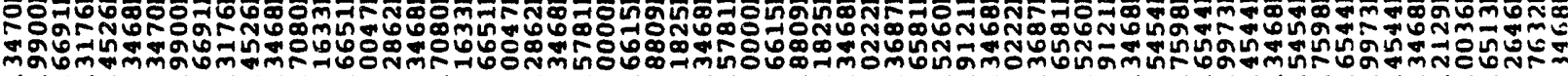

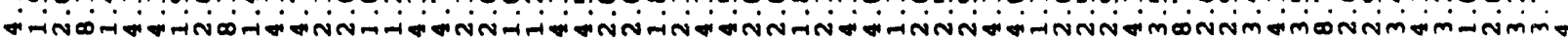

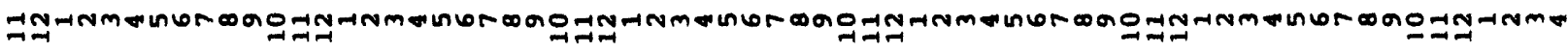

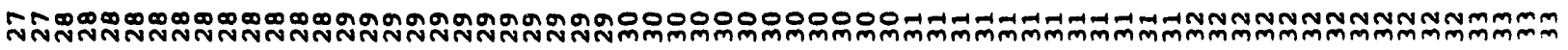




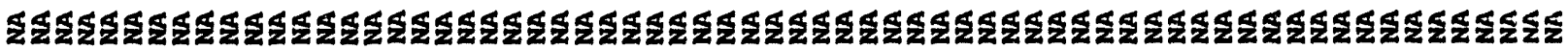

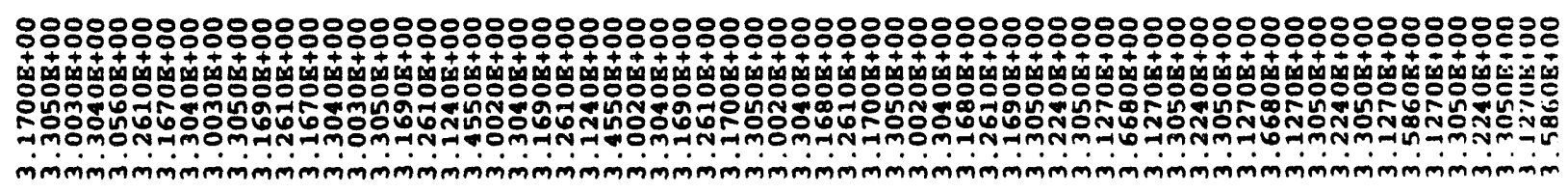

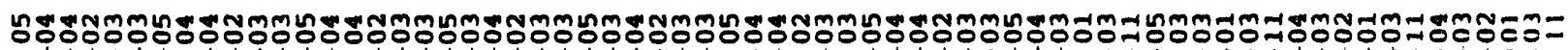

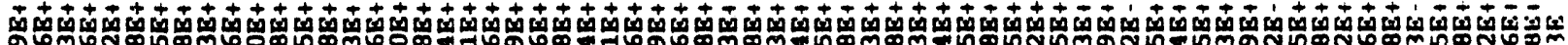

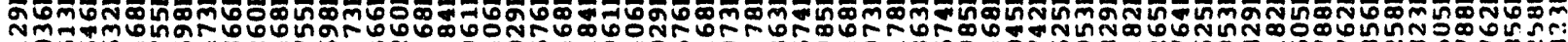

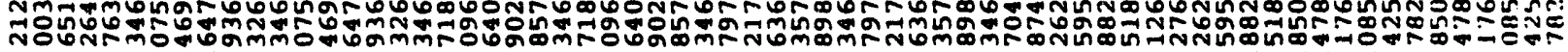

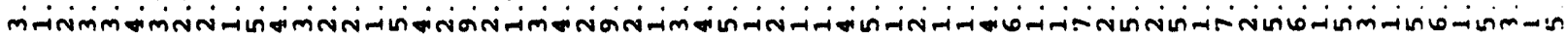

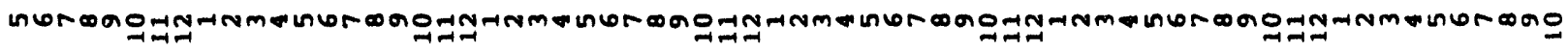

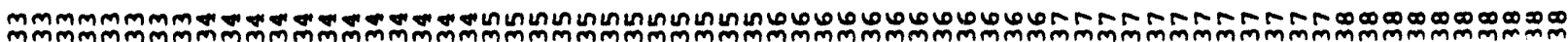




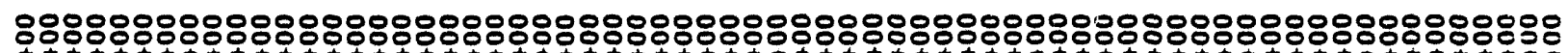

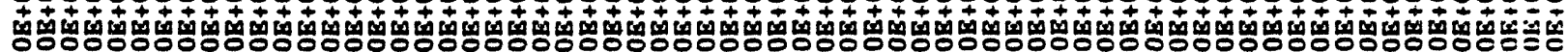
Noñ

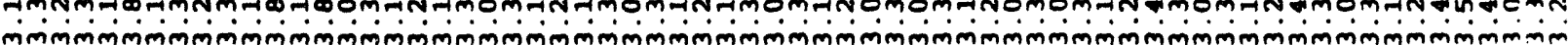

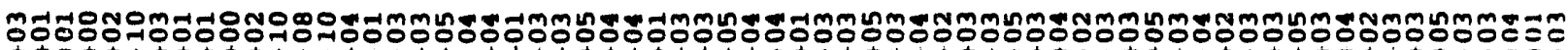

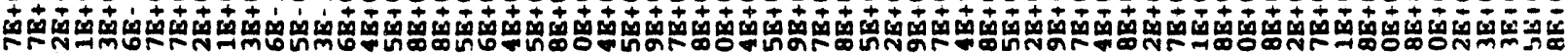

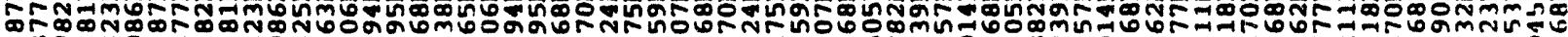

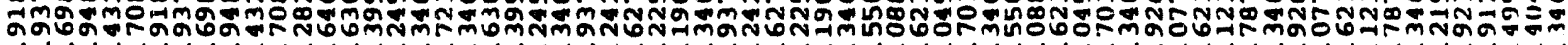
Ninioñ

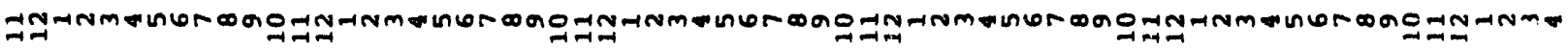

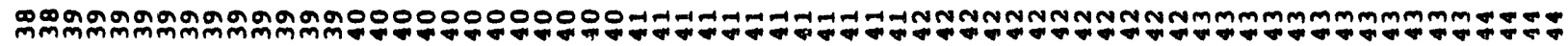




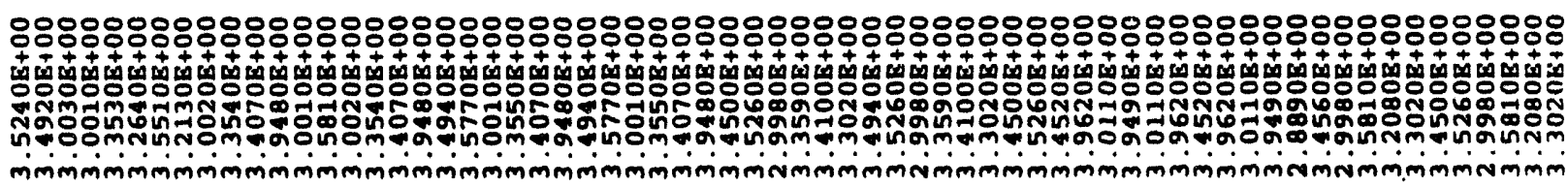

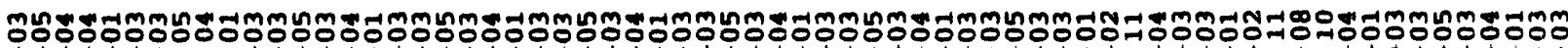

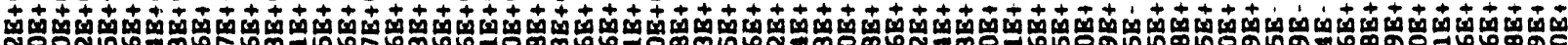

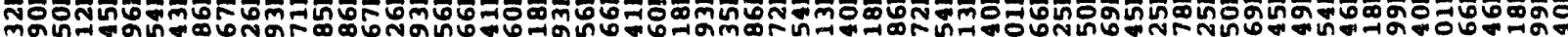

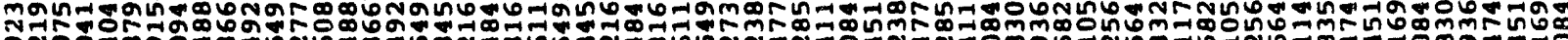

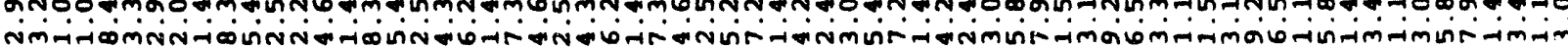

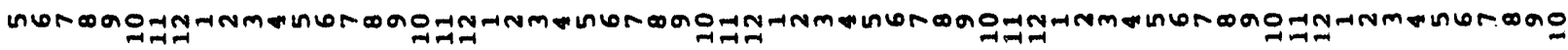

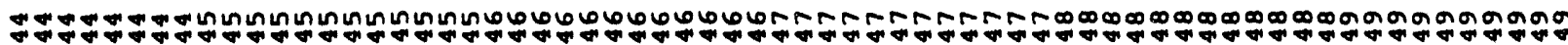



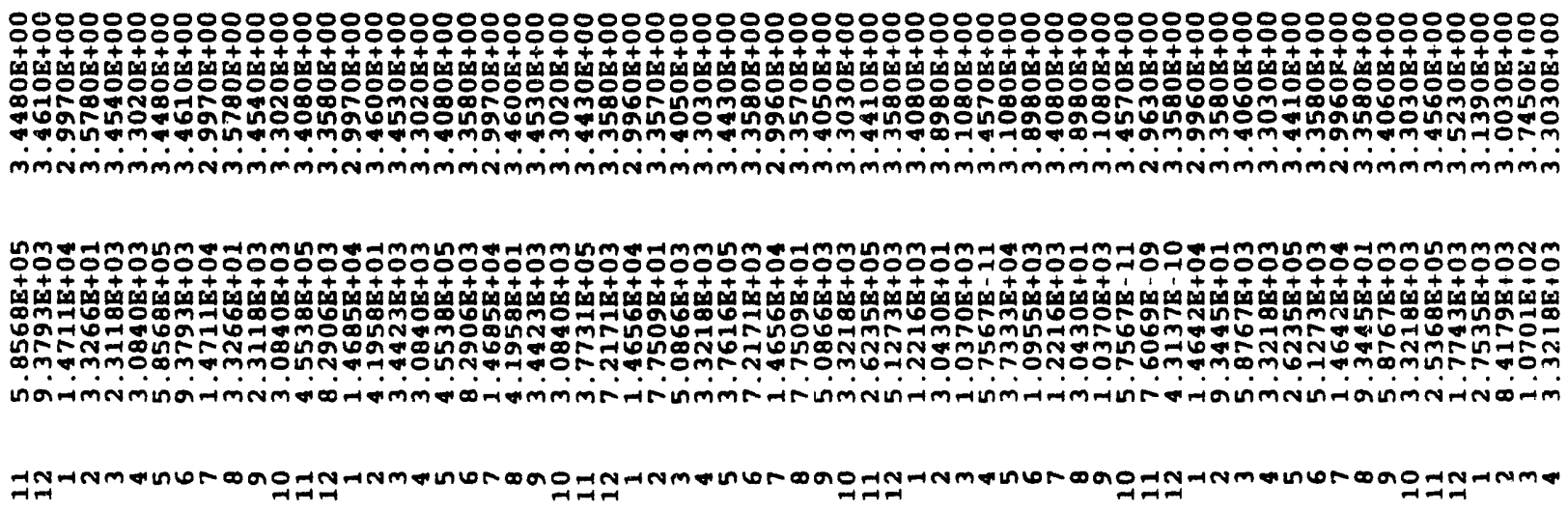

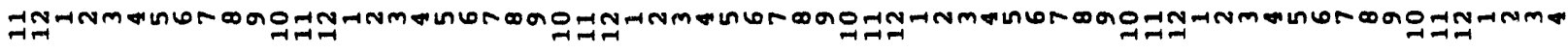




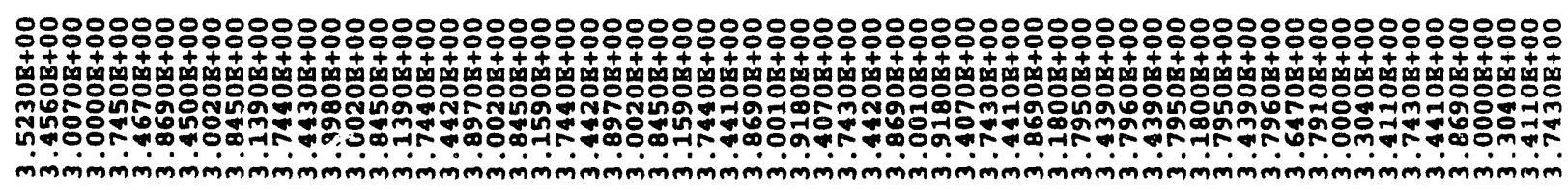

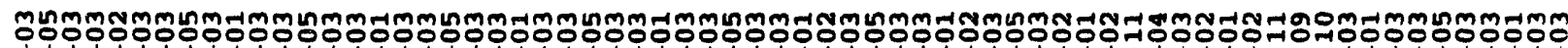

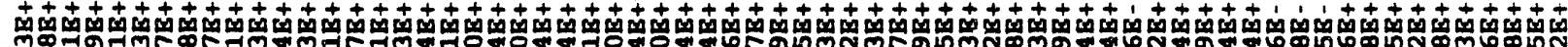

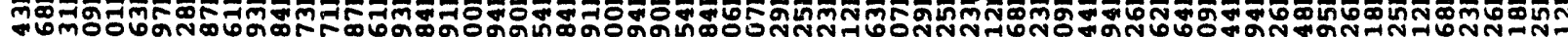
F⿻ำ

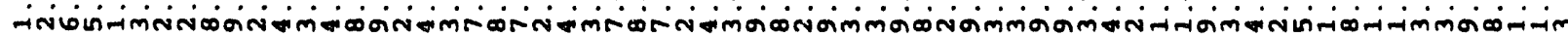

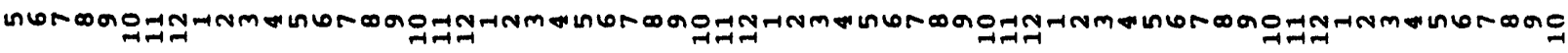

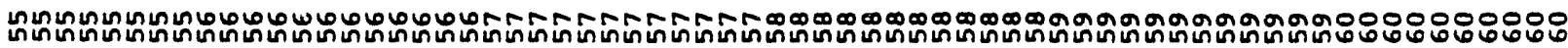




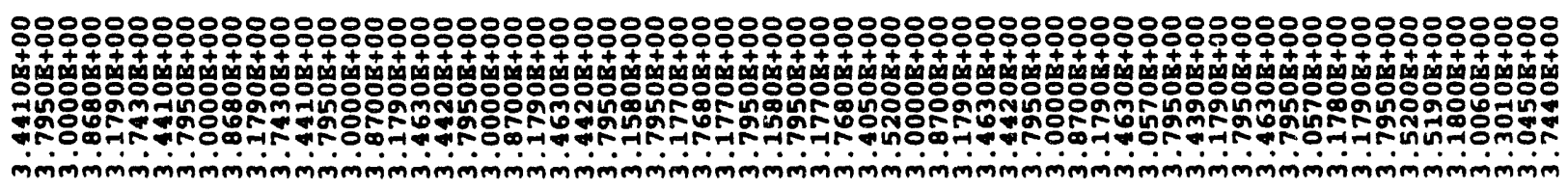

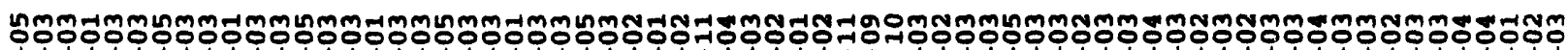

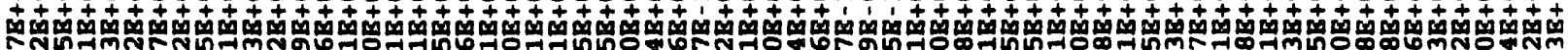

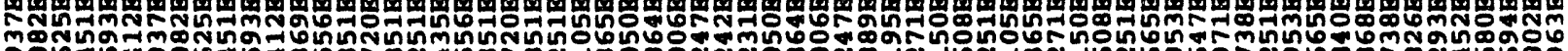

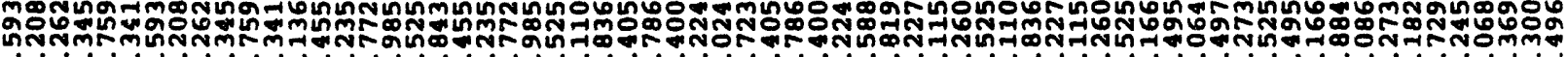

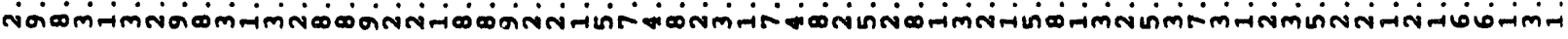

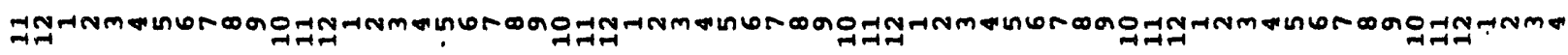
8ำดี 


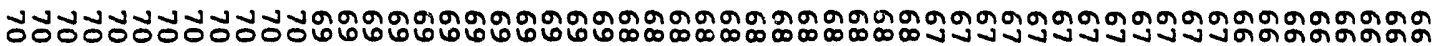

NF⿱

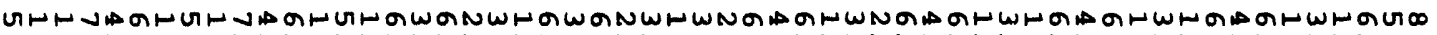

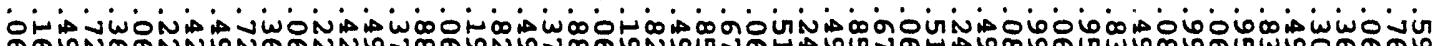
Ğफ

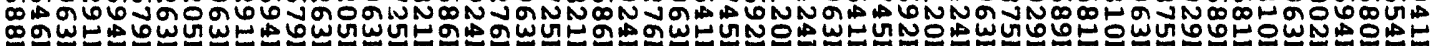

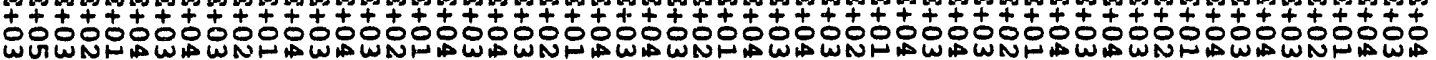

W

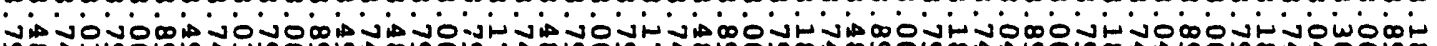
ร้๊ผ

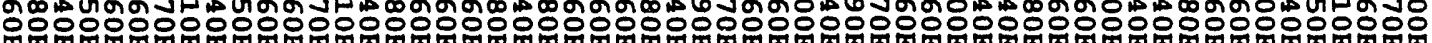

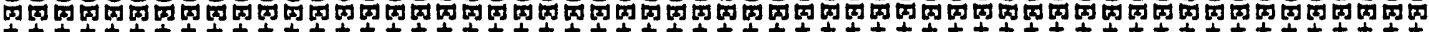

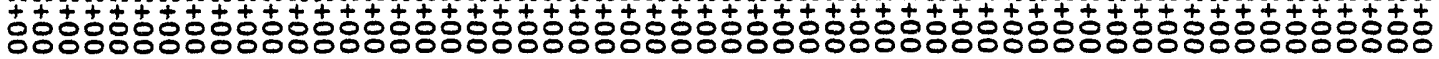

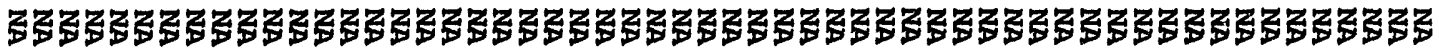


BENCHMARK PROBLEM 3

MODAL SUPERPOSITION TIME HISTORY ANALYSIS 
SYSTRM BO+ PRESSURIZER SUHGK L.INE - MODAI, SUPERPOSITION TIME HISTORY RUN

CONTROL INBORMATION

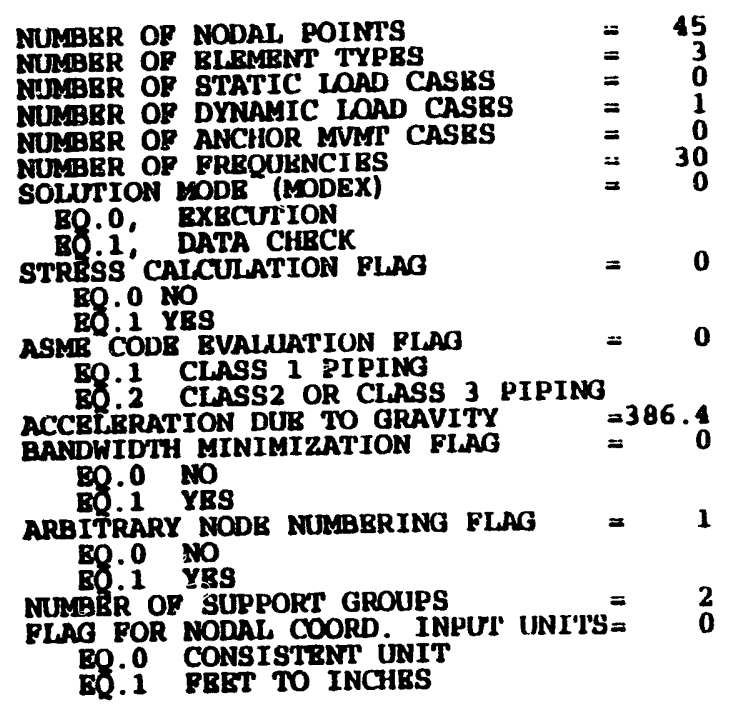

LIST OP ANALYSIS 'TO BE PBRFORMED LOAD CASB DISK FILE ANALYSIS TYPB 


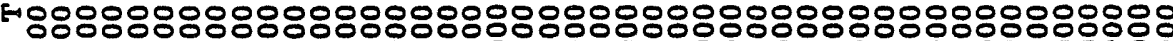

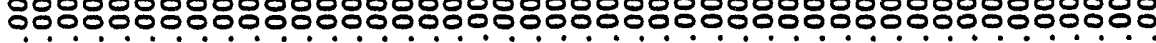

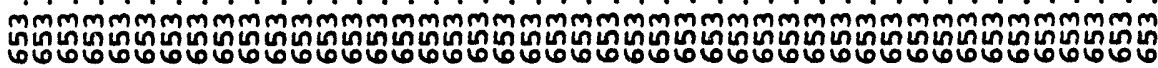

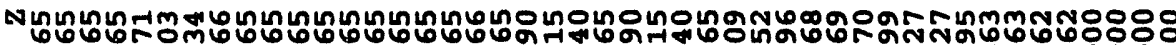

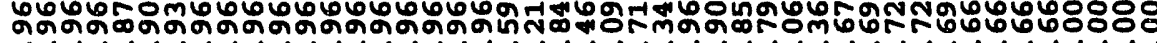 Firiving

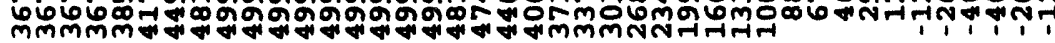 \\ Hทn

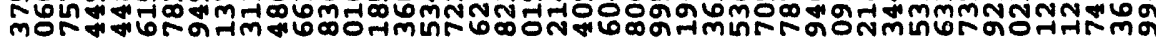 - ตึฒదం Mind

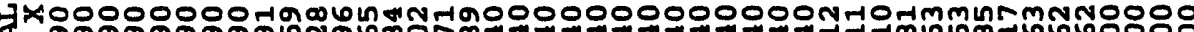

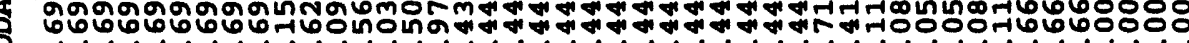 riñ

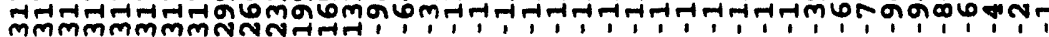

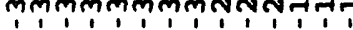

惫

N000000000000000000000000000000000000000000000 里 8

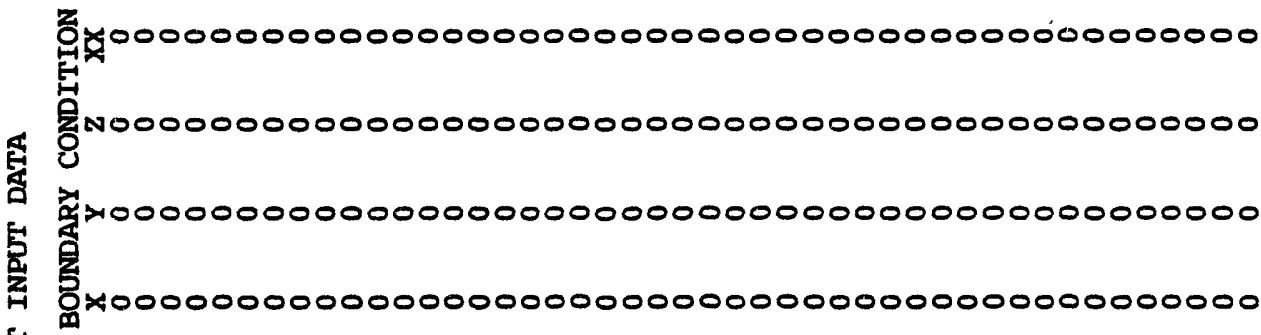

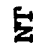

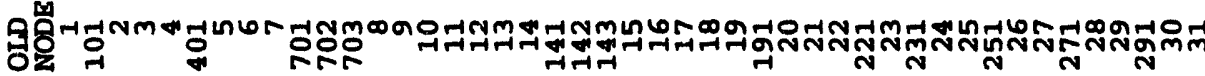

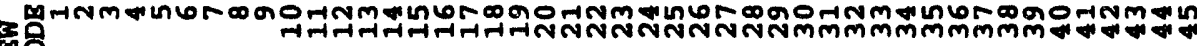

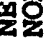


S P R I N G B L E M B N T S

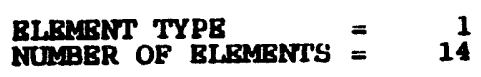

BLRMENT LOAD CASE MULTIPLIERS

$\begin{array}{rr}\text { CASE (A) } & \text { CASE (B) } \\ 1.0000 & 1.0000\end{array}$

$\begin{array}{rr}\text { CASE (C) } & \text { CASE(D) } \\ 1.0000 & 1.0000\end{array}$

BLRARET
NULBER
1
1
3
3
4
5
6
7
8
9
10
11
12
13
14

NODB
$(\mathrm{N})$
1
1
1
1
1
1
1
19
23
31
31
31
31
31
31

SUPPORT
GROUP

$\underset{\mathbf{K D}}{\mathrm{CODB}}$

DIREC

$\mathrm{X}-$ CONIN

Y- WRT GLOBA

AXA

1.000

.000

-

SPECIFIED
DISPIACEMENT

.000

$\begin{array}{r}.000 \\ .000 \\ \hline .000\end{array}$

.000
1.000

1.000

1.000

1.000

1.000

1.000

1.000

000

1.000

.000

.000

1.000
.000

.000
.000

.000

.000
.000

$1.000 \quad 000 \quad 1.000$

$\begin{array}{rrr}.000 & 1.000 & .000 \\ .000 & .000 & 1.000\end{array}$

$\stackrel{p}{\stackrel{1}{a}}$

1.000

.000
.000
.000
.000
.000
.000
.000
.000
.000
.000
.000
.000
.000
.000

SPECIFIBD
ROTATION

$\begin{array}{ll}.000 & 1.0000 \mathrm{~B}+13 \\ .000 & 1.0000 \mathrm{~B}+13\end{array}$

$.000 \quad 1.0000 \mathrm{E}+13$

$.0001 .0000 \mathrm{~B}+15$

.000

.000

.000

$1.0000 \mathrm{~B}+15$

$1.0000 \mathrm{~B}+15$
$.0000 \mathrm{~B}+05$

$5.00008+05$

$.0000 \mathrm{~B}+13$

$1.0000 \mathrm{~B}+13$

$.0000 \mathrm{~B}+15$

$1.0000 \mathrm{~B}+15$

S N U B B E R E L E M E N T S

$\begin{array}{ll}\text { RLBMERT TYPE } & =4 \\ \text { NUYBER OF BLBMIBNTS }= & 1\end{array}$

BLBLENT LOAD CASE MULTIPLIERS

$$
\text { CASE (A) CASE (B) }
$$

$\begin{array}{rr}\text { CASE (C) } & \text { CASE (D) } \\ 1.0000 & 1.0000\end{array}$

$\begin{array}{rrrr}\text { BLERYRNT } & \text { NODE } & \begin{array}{r}\text { SUPPORT } \\ \text { GROUP }\end{array} & \text { COD } \\ \text { NULBBR } & \text { (N) } & 2 & \end{array}$

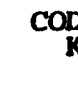
DIRECTION COSINES WRT GLOBAL AXES
000
.000
1.000

SPECIFIED
DISPLACEYBNT

.000

$\begin{array}{rr}\text { SPECIPIBD } & \text { SPRING } \\ \text { ROTATION } & \text { RATE }\end{array}$

$000 \quad 2.0000 \mathrm{~B}+05$ 
P I P E B LEMENT IN P UT DATA

CONTROL INFORMATION

NUMBER OF PIPE ELEMIENTS

NUEBER OF MATERIAL SETS

MAXIMUM NUMBER OF MATERIAL
TEMPBRATURB INPUT POINTS

$=44$

$=1$

PROPERTY SETS

MAXIMUM NUMBER OF TANGENTS

COMNON TO A BRANCH POINT

$=3$

FLAG FOR NEGLECTING AXIAL DEFORMATIONS IN BEND ELBMENTS

(EQ.1, NEGLECT) 


\begin{tabular}{|c|c|c|c|c|}
\hline $\begin{array}{l}\text { MATBRI } \\
\text { NUMBRR } \\
\text { TRMPER } \\
\text { IDEMIII }\end{array}$ & $\begin{array}{l}\text { NUMBER } \\
\text { RE POINTS } \\
\text { ATION }\end{array}$ & $\begin{array}{c}\text { 9) } \\
\text { SA358 TYPE }\end{array}$ & 316 STAINLES & S STBRL PIPE) \\
\hline $\begin{array}{c}\text { POINT } \\
\text { NUUBBR }\end{array}$ & TEMPERATURE & $\begin{array}{l}\text { YOUAG'S } \\
\text { MODULUS }\end{array}$ & $\begin{array}{r}\text { POISSON'S } \\
\text { RATIO }\end{array}$ & $\begin{array}{l}\text { THERYAL } \\
\text { EXPANSIOA }\end{array}$ \\
\hline $\begin{array}{l}1 \\
2 \\
3 \\
4 \\
5 \\
6 \\
7 \\
8 \\
9\end{array}$ & $\begin{array}{r}70.00 \\
100.00 \\
200.00 \\
300.00 \\
400.00 \\
500.00 \\
600.00 \\
650.00 \\
700.00\end{array}$ & $\begin{array}{l}2.830 \mathrm{~B}+07 \\
2.820 \mathrm{~B}+07 \\
2.770 \mathrm{~B}+07 \\
2.710 \mathrm{~B}+07 \\
2.660 \mathrm{~B}+07 \\
2.610 \mathrm{~B}+07 \\
2.540 \mathrm{~B}+07 \\
2.510 \mathrm{~B}+07 \\
2.480 \mathrm{~B}+07\end{array}$ & $\begin{array}{l}.300 \\
.300 \\
.300 \\
.300 \\
.300 \\
.300 \\
.300 \\
.300 \\
.300\end{array}$ & $\begin{array}{l}8.420 \mathrm{~B}-06 \\
8.590 \mathrm{~B}-06 \\
9.090 \mathrm{~B}-06 \\
9.560 \mathrm{~B}-06 \\
9.950 \mathrm{~B}-06 \\
1.030 \mathrm{~B}-05 \\
1.050 \mathrm{~B}-05 \\
1.060 \mathrm{~B}-05 \\
1.080 \mathrm{~B}-05\end{array}$ \\
\hline
\end{tabular}

$\frac{p}{5}$

S B C T I ON PRO PERT Y TABLE

\begin{tabular}{|c|c|c|c|c|c|c|}
\hline $\begin{array}{l}\text { MECTION } \\
\text { NUEAER }\end{array}$ & $\begin{array}{c}\text { OUTSIDE } \\
\text { DIAMETER }\end{array}$ & $\begin{array}{r}\text { WALL } \\
\text { THICKNESS }\end{array}$ & $\begin{array}{c}\text { SHAPE FACTOR } \\
\text { FOR SHBAR }\end{array}$ & $\begin{array}{l}\text { WEIGHT/ } \\
\text { UNIT LELGTH }\end{array}$ & NIT IENGS/ & $\mathbf{N}$ \\
\hline & 12 & 1.3120 & .0000 & $1.9200 \mathrm{~B}+01$ & $4.9689 \mathrm{~B}-02$ & CH SCH 160 \\
\hline
\end{tabular}

B L B M B N T LOA D

CASEM U L T I P L I B R S

X-DIRECTION GRAVITY

-DIRRCTION GRAVITY

TrPMA DISTORTIO

PRBSSURB DISTORTION

CASE A
.000
-1.000
.000
.000
.000

CASB B CASB C

$.000 \quad .000$

.000

1.000

.000
.000
.000

CASE D

.000

.000
.000

.00 


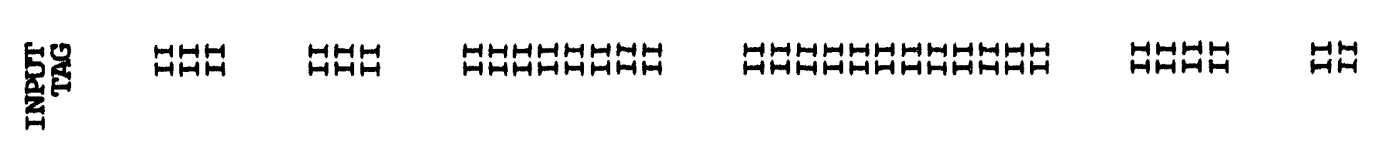

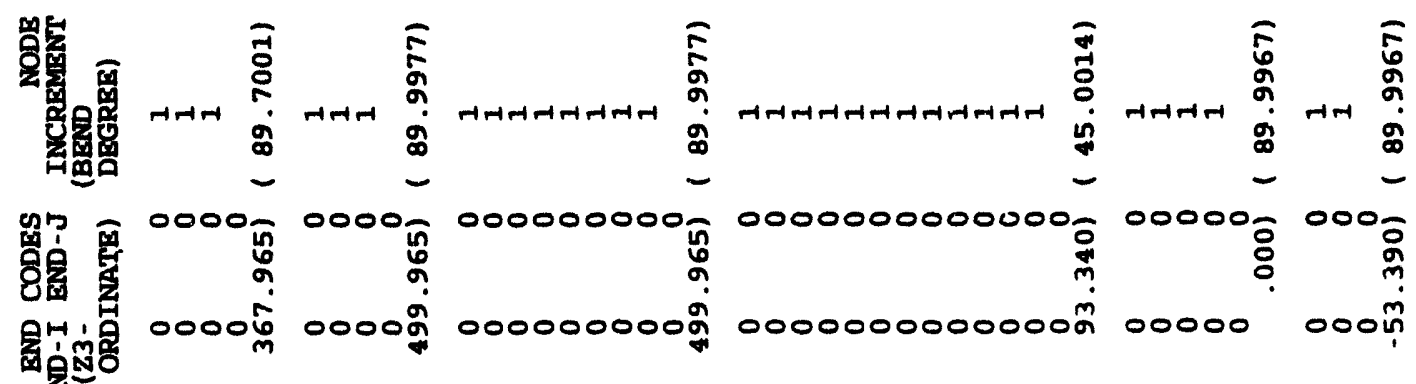

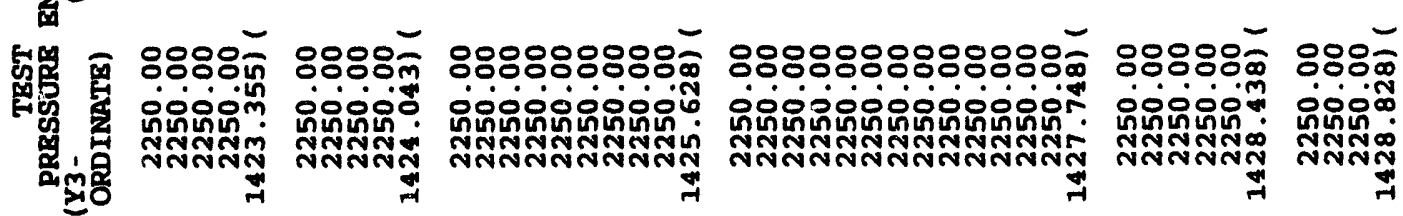
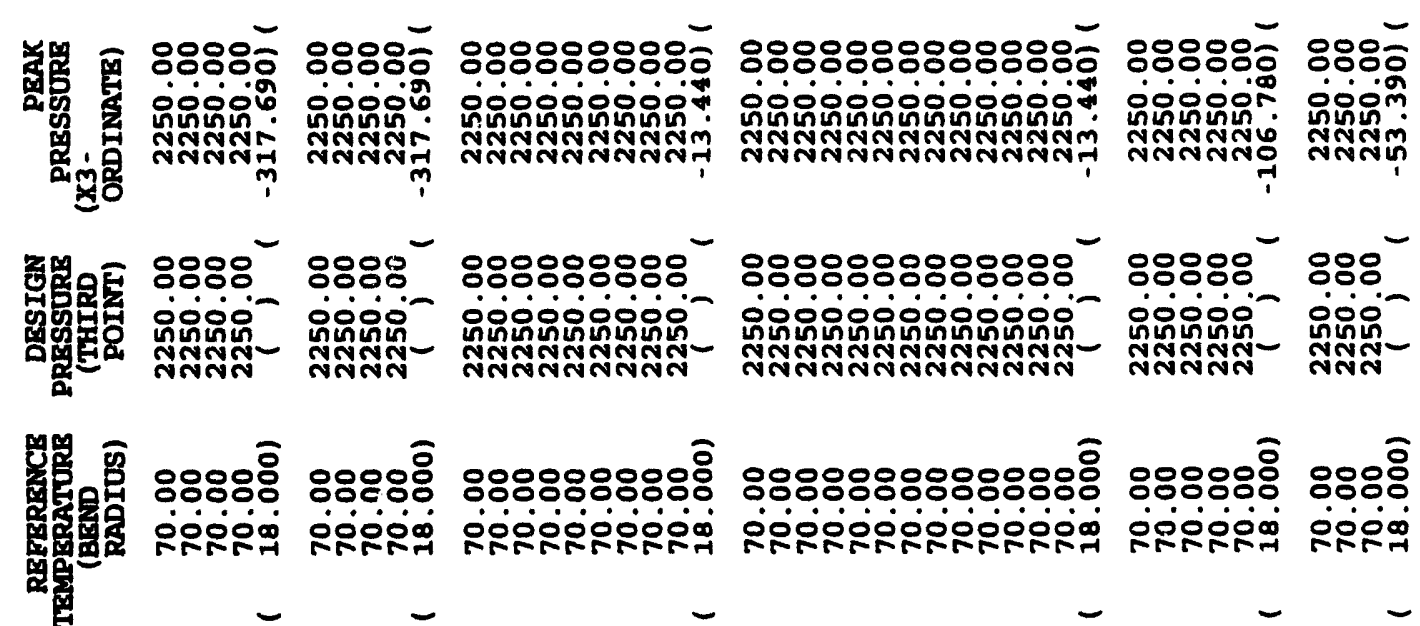

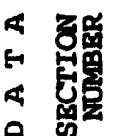

HनलA HनलA

hHAm-AhHA

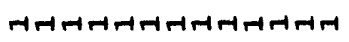

hHAm hHA

是量

$z$

郭

कृतmo

$\sin ^{\text {gin }}$

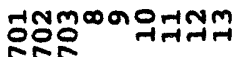

สี

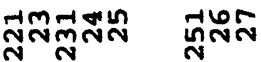

郭

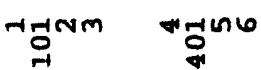

ํํำ

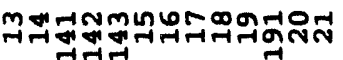

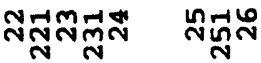

2

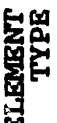
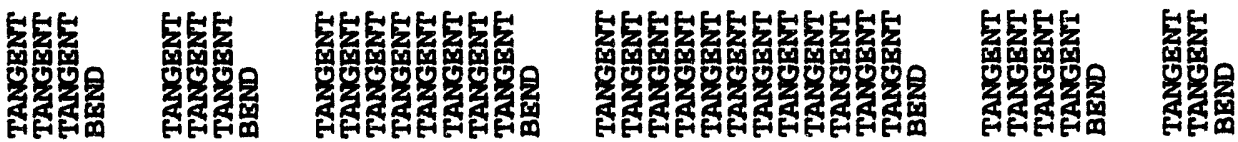


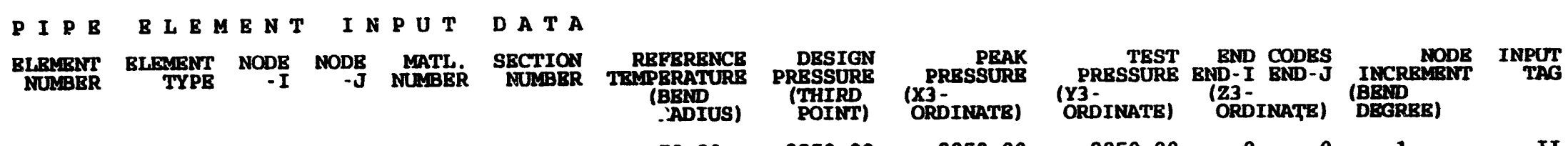
$\begin{array}{llrrrrrrrrrrrr}39 & \text { TANGENT } & 27 & 271 & 1 & 1 & 70.00 & 2250.00 & 2250.00 & 2250.00 & 0 & 0 & 1 & 1 \\ 1 & \text { TANGENT } & 271 & 28 & 1 & 1 & 70.00 & 2250.00 & 2250.00 & 2250.00 & 0 & 0 & 1 & 1\end{array}$

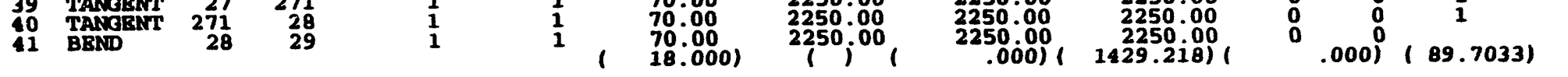

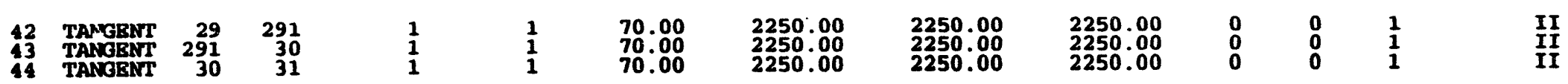

$\underset{1}{\stackrel{p}{a}}$

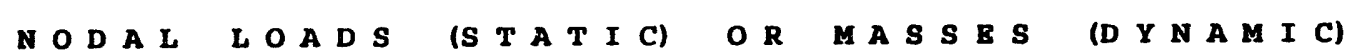

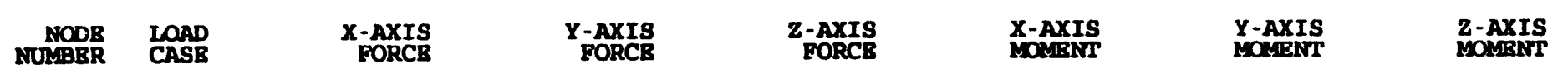


DYUAMIC ANALYSIS

\section{STRUCTURR}

1 WODAL BUP. TIG HISTORY ANALYSIS

\begin{tabular}{|c|c|c|}
\hline & $\mathrm{B}^{\text {LOAI }}$ & My \\
\hline .000 & .000 & .000 \\
\hline
\end{tabular}

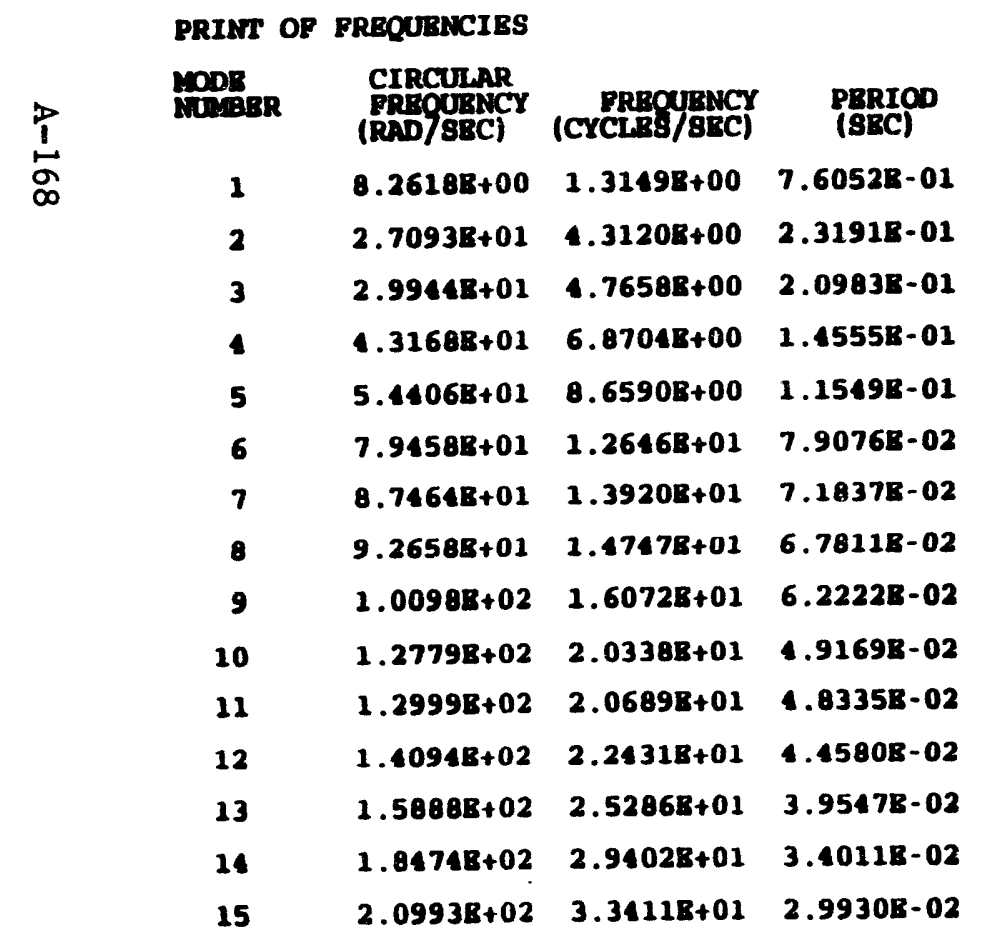


$2.1821 B+02 \quad 3.47298+01 \quad 2.8795 B-02$

$2.6913 B+02 \quad 4.2834 B+01 \quad 2.3346 B-02$

$2.7654 B+02 \quad 4.4013 B+01 \quad 2.2721 B-02$

$3.2874 \mathrm{~B}+02 \quad 5.2321 \mathrm{~B}+01 \quad 1.9113 \mathrm{~B}-02$

$3.3603 \mathrm{~B}+02 \quad 5.3481 \mathrm{~B}+01 \quad 1.8698 \mathrm{~B}-02$

$3.5468 \mathrm{~B}+02 \quad 5.64498+01 \quad 1.7715 \mathrm{~B}-02$

$4.0643 \mathrm{~B}+02 \quad 6.4686 \mathrm{~B}+01 \quad 1.5459 \mathrm{~B}-02$

$4.3682 \mathrm{~B}+02 \quad 6.9521 \mathrm{~B}+01 \quad 1.4384 \mathrm{~B}-02$

$5.2406 \mathrm{~B}+02 \quad 8.3406 \mathrm{~B}+01 \quad 1.1990 \mathrm{~B}-02$

$5.6313 \mathrm{E}+02 \quad 8.9625 \mathrm{~B}+01 \quad 1.1158 \mathrm{E}-02$

6.0389B+02 $9.6112 \mathrm{~B}+01 \quad 1.0404 \mathrm{~B}-02$

$6.2127 \mathrm{~B}+02 \quad 9.8878 \mathrm{~B}+01 \quad 1.0114 \mathrm{~B}-02$ 

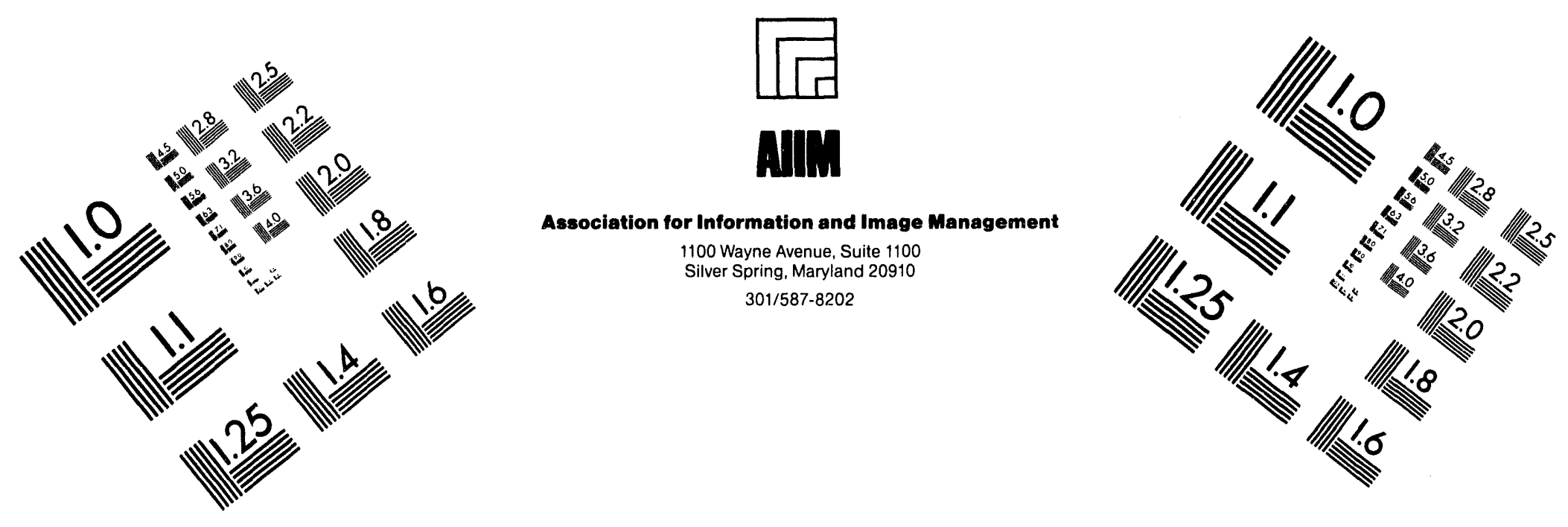

\section{Centimeter}

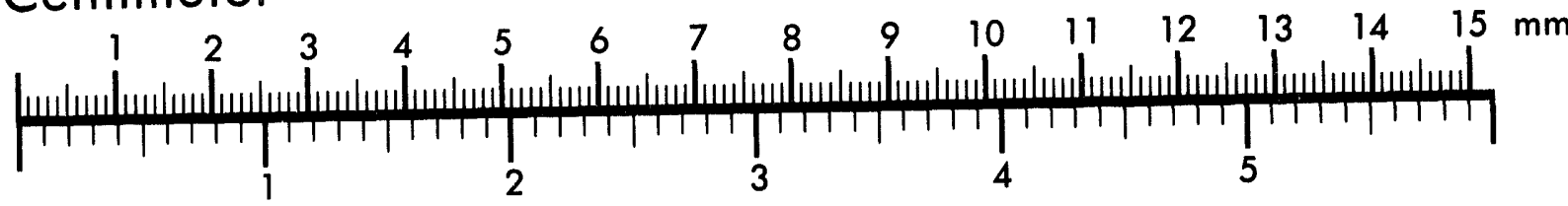
Inches
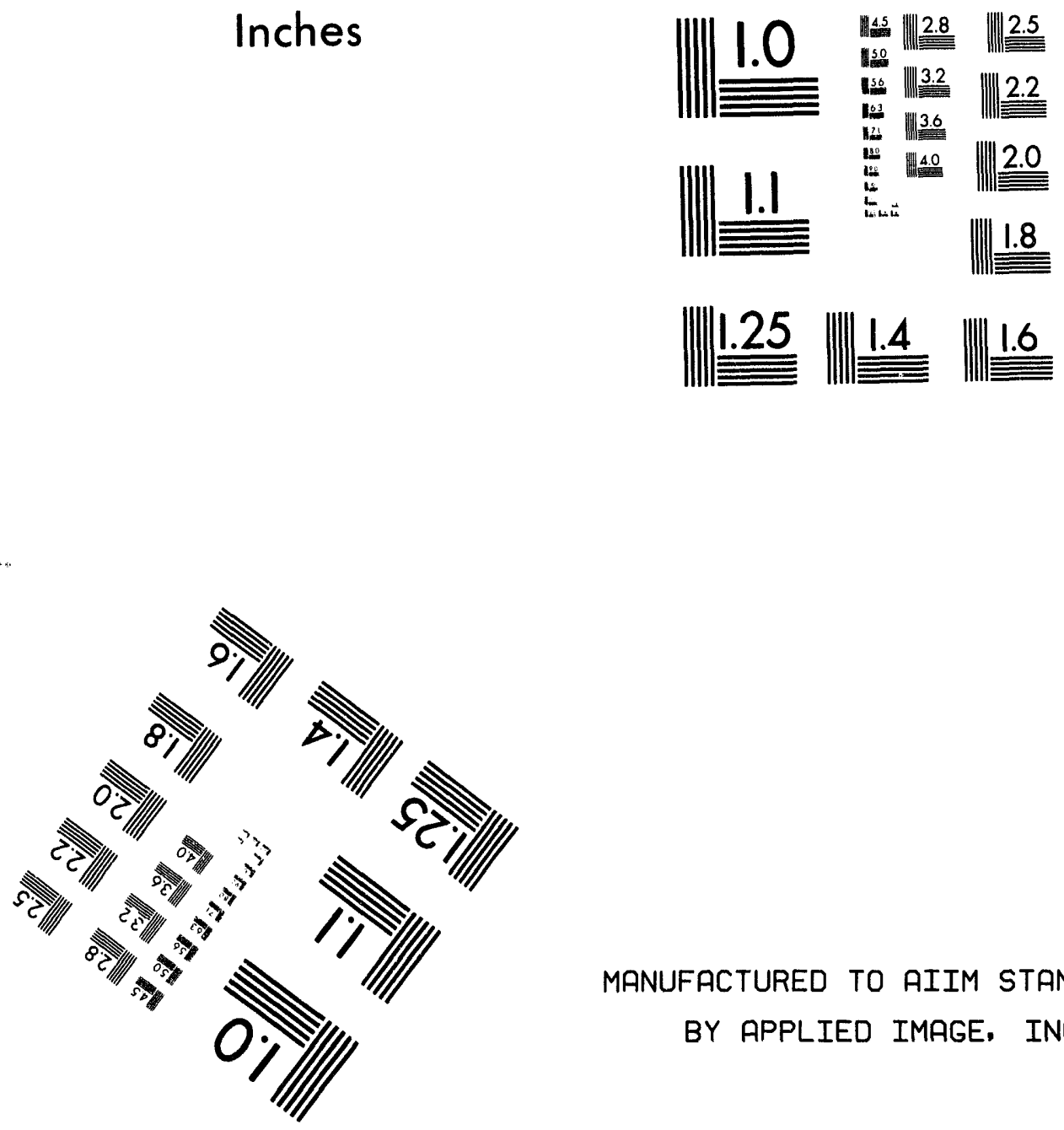

MANUFACTURED TO AIIM STANDARDS BY APPLIED IMAGE, INC.

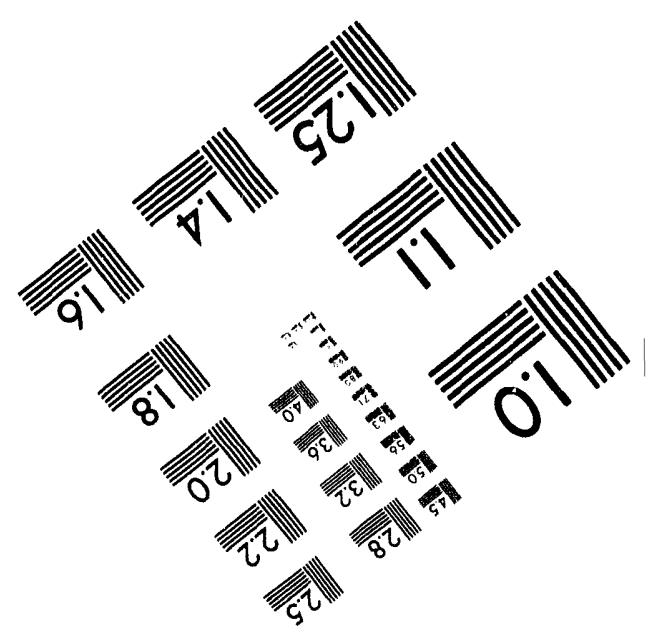



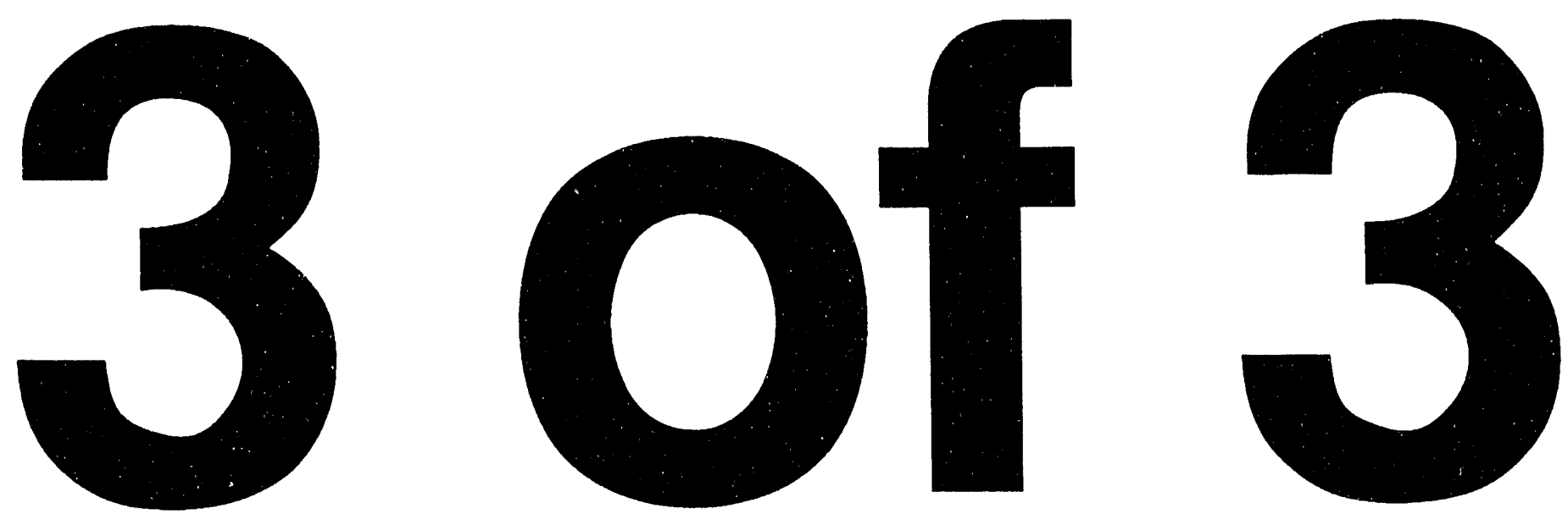
בODAL PARTICIPATION FACTORS

MODE FREO (CPS) $X$-DIRECTION

\begin{tabular}{|c|c|c|c|c|}
\hline & (א. & 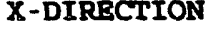 & Y-DIRECTION & $\infty$ \\
\hline 1 & 1.315 & $-3.13428-01$ & $5.5930 \mathrm{~B}+00$ & $3.6742 E-02$ \\
\hline 2 & 4.312 & $-3.44438+00$ & $-8.3543 E-01$ & $1.3840 \mathrm{E}-01$ \\
\hline 3 & 4.766 & $3.7270 \mathrm{~B}+00$ & $-2.2035 B-01$ & $-2.6006 \mathrm{~B}-01$ \\
\hline 4 & 6.870 & $-1.8266 \mathrm{~B}+00$ & $3.7298 \mathrm{E}+00$ & $-1.2499 \mathrm{~B}+00$ \\
\hline 5 & 8.659 & $-2.0107 \mathrm{~B}+00$ & $-1.0364 \mathrm{~B}+00$ & $-8.7982 E-01$ \\
\hline 6 & 12.646 & $-1.1180 \mathrm{~B}+00$ & $-9.1453 B-01$ & $-4.5747 \mathrm{E}+00$ \\
\hline 7 & 13.920 & $1.61818+00$ & $2.6979 \mathrm{~B}-01$ & $-3.5310 \mathrm{E}+00$ \\
\hline 8 & 14.747 & $1.84918+00$ & $5.82838-02$ & $-5.91348-01$ \\
\hline 9 & 16.072 & $-2.3210 \mathrm{~B}+00$ & $-9.8060 \mathrm{~B}-01$ & $1.30068-01$ \\
\hline 10 & 20.338 & $1.2198 \mathrm{~B}+00$ & $-3.8252 \mathrm{E}-01$ & $2.6970 \mathrm{~B}-01$ \\
\hline 11 & 20.689 & $-2.1071 B+00$ & $-5.8416 \mathrm{~B}-01$ & $1.1530 \mathrm{~g}+00$ \\
\hline 12 & 22.431 & $-5.0618 \mathrm{E}-01$ & $2.18478-01$ & $-2.3183 \mathrm{~B}+00$ \\
\hline 13 & 25.286 & $-4.5807 \mathrm{E}-01$ & $4.4204 \mathrm{~B}-01$ & $-2.1787 E+00$ \\
\hline 14 & 29.402 & $1.24428+00$ & $-3.0068 \mathrm{~B}-01$ & $-2.5390 \mathrm{~B}+00$ \\
\hline 15 & 33.411 & $9.1065 \mathrm{E}-01$ & $1.5253 \mathrm{E}-01$ & $1.7502 \mathrm{E}+00$ \\
\hline 16 & 34.729 & $4.3527 \mathrm{E}-01$ & $-2.39578-01$ & $-2.9287 \mathrm{~B}-01$ \\
\hline 17 & 42.834 & $7.3281 \mathrm{E}-01$ & 9.08848-01 & $1.0715 \mathrm{~B}+00$ \\
\hline 18 & 44.013 & $-1.4236 \mathrm{~B}+00$ & $9.3622 \mathrm{~B}-02$ & $-2.01258-01$ \\
\hline 19 & 52.321 & $-d .6640 \mathrm{~B}-01$ & $-2.04778-01$ & $-1.2027 \mathrm{~B}-01$ \\
\hline 20 & 53.481 & $3.41728-01$ & $-6.51478-01$ & $-4.7860 \mathrm{E}-01$ \\
\hline 21 & 56.449 & $5.35818-03$ & $-2.3499 B-02$ & $7.9029 \mathrm{~B}-02$ \\
\hline 22 & 64.686 & $6.8516 \mathrm{~B}-02$ & $-2.2147 \mathrm{~B}-01$ & $-1.0776 \mathrm{~B}-01$ \\
\hline 23 & 69.521 & $-7.49288-01$ & $1.8389 \mathrm{~B}-02$ & $-2.50368-01$ \\
\hline 24 & 83.406 & $1.0429 \mathrm{E}-02$ & $9.2250 \mathrm{~B}-01$ & $4.39518-01$ \\
\hline 25 & 89.625 & 4.53298-01 & $2.95118-03$ & $-1.58648-01$ \\
\hline 26 & 96.112 & $2.63188-02$ & $-3.97038-02$ & $2.08478-02$ \\
\hline 27 & 98.878 & $1.5594 \mathrm{~B}-02$ & $2.8390 \mathrm{~B}-03$ & $2.5659 \mathrm{~B}-01$ \\
\hline
\end{tabular}


F ORCED RE S P ONSE A NALY S I S

SUPPORT GROUP NUMBER $=1$

CONTROL INFORYATION NURARR OP TINR EUNCTIONS BO.0, NONB

BO.1, GROOND INPUT NURBBR OR ARRIVAI TIMES

TULBBR OR TIME STEPS

OUTPUT PRINT INIERVAL

TIME STEP

$\begin{array}{ll}= & 3 \\ = & 1\end{array}$

$=9980$

$=10.00010$

.02000

$\stackrel{P}{2}$

GROUND ACCELERATION INPUT

X-DIRECTION Y-DIRBCTION Z-DIRBCTION

TIMR FUNCTION NUARER (S)

$\begin{array}{lll}1 & 2 & 3\end{array}$

ARRIVAY TIME VALUBS

$\underset{\text { EMTRY }}{\text { ARRIVAL TIMB }}$

1.000000 


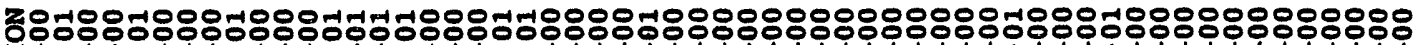

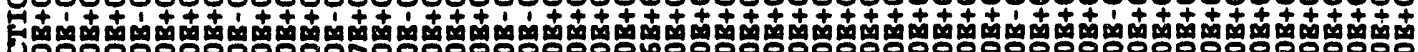

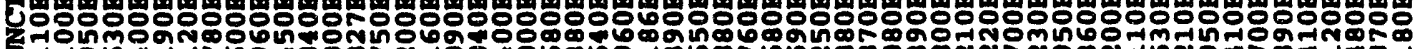
కmo

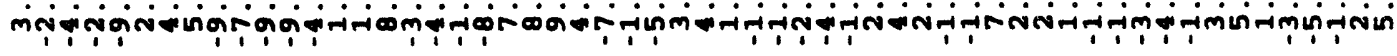

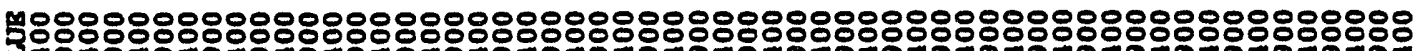

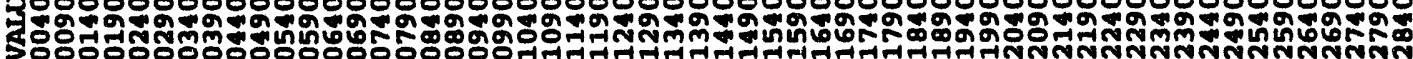

善

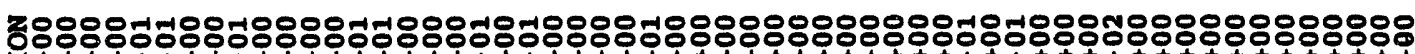

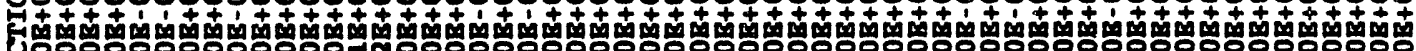

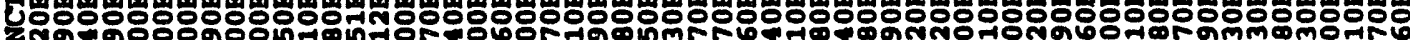

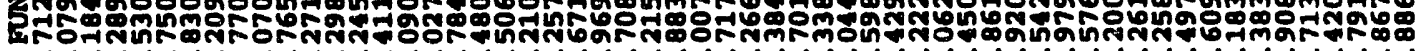

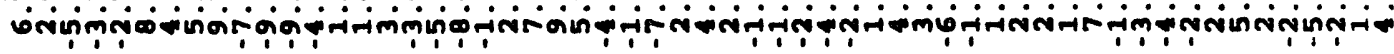

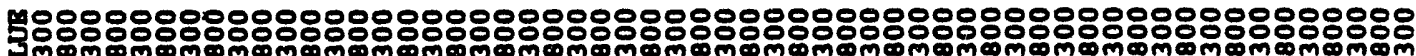

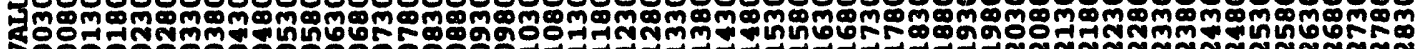
思 E

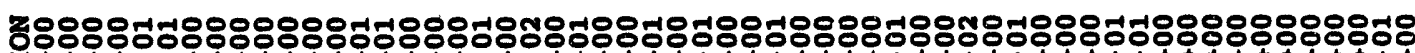

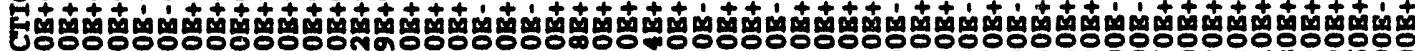
 50

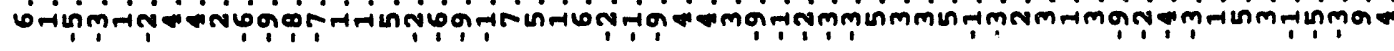
ল

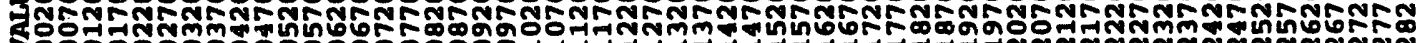
ת.. 兽

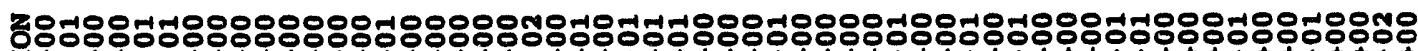

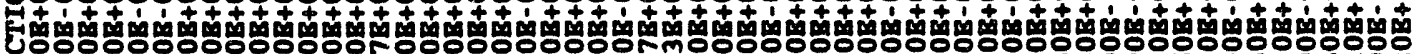

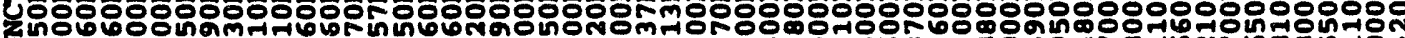
Eस ن pุin ল สైะี 난

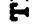

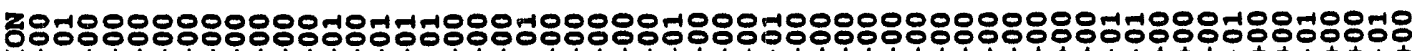

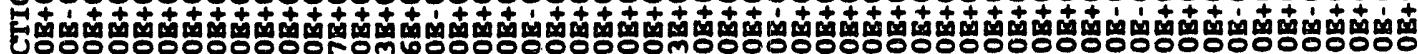

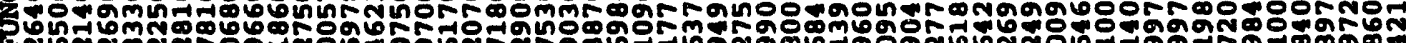

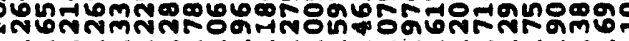
mp̣mpi 

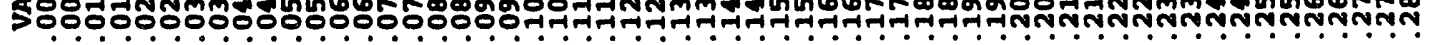
畄 


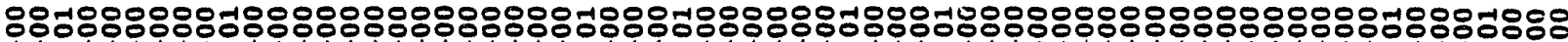

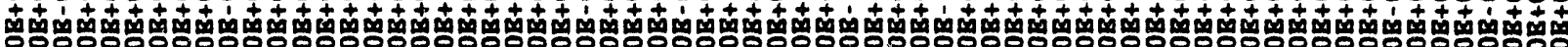

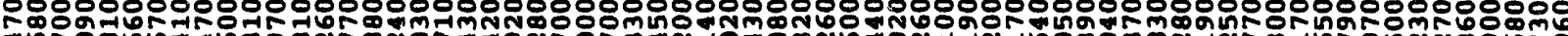
ợำ

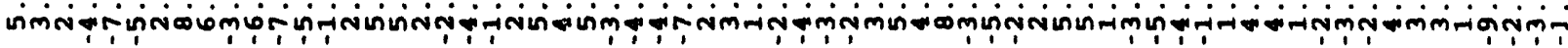

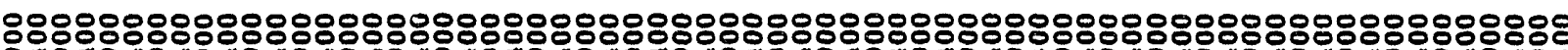

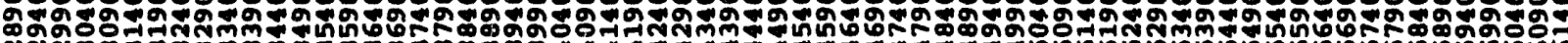

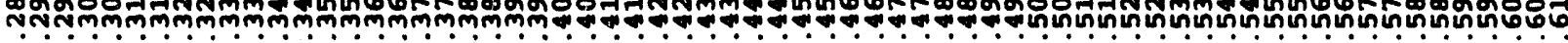

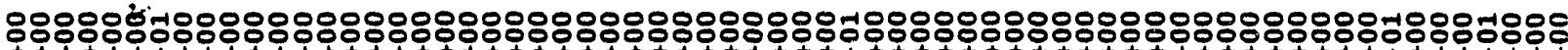

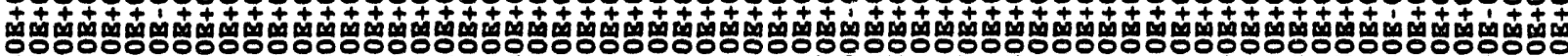
గ్p ๑ొळ

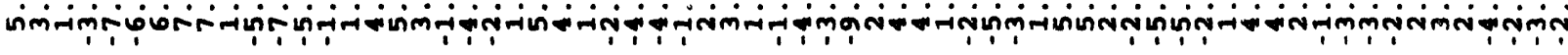

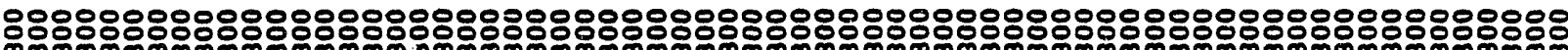

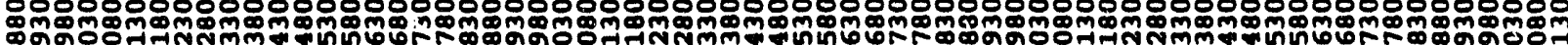

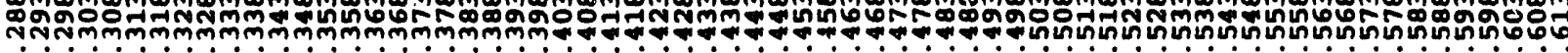

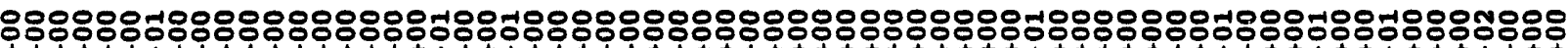

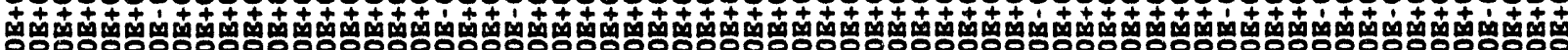

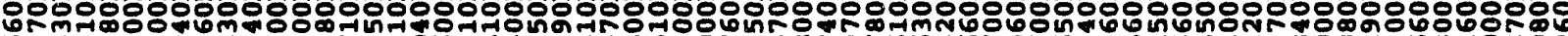

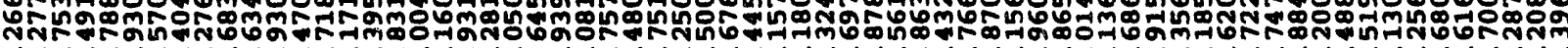
vं- -

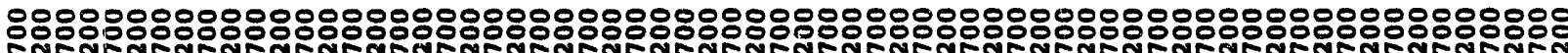

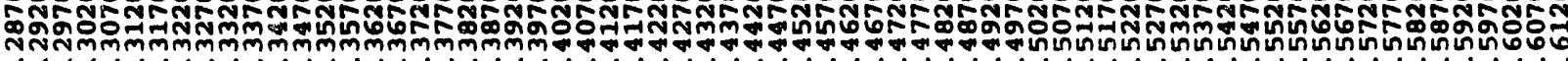

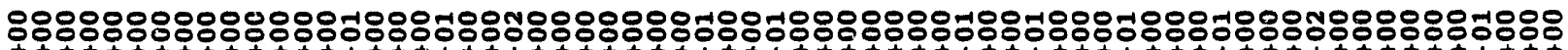

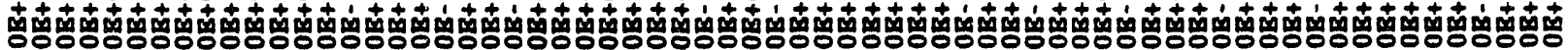
ôn̄ ñ

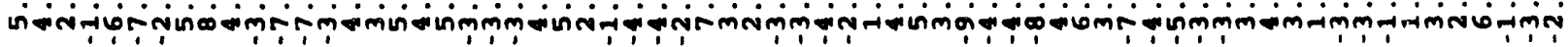

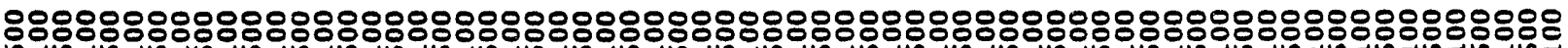
๓⿴囗大ํำ

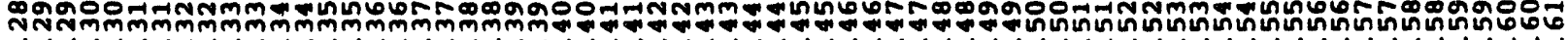

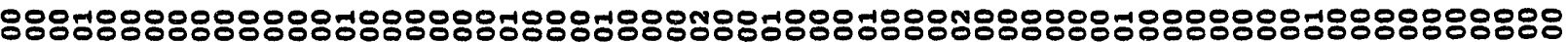

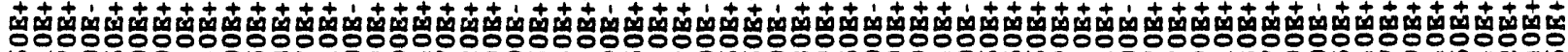

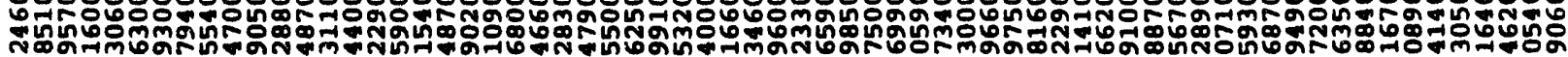
n-

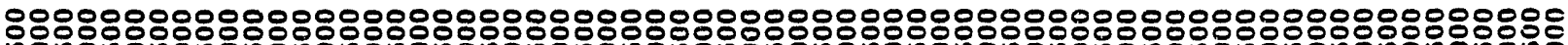
ที่大

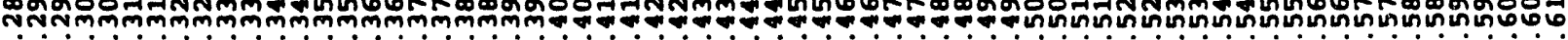




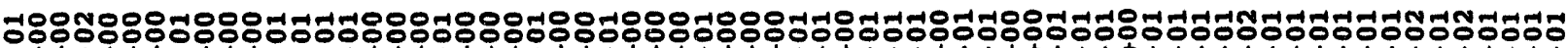

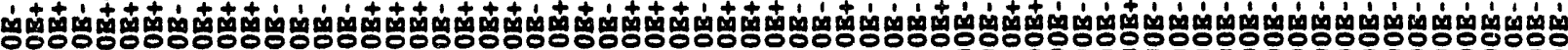

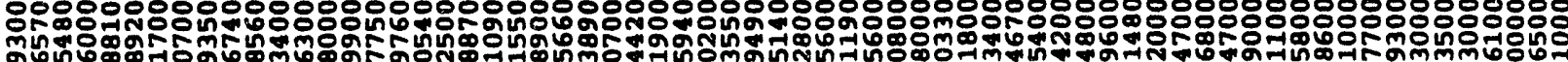

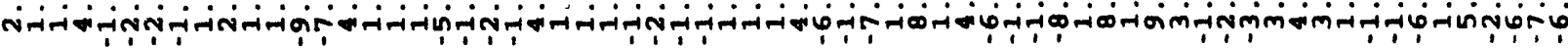

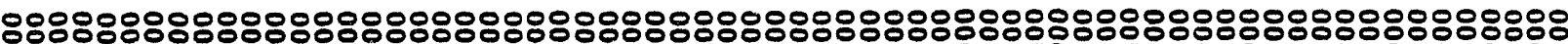
สิสนตำ

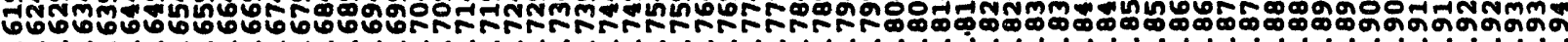

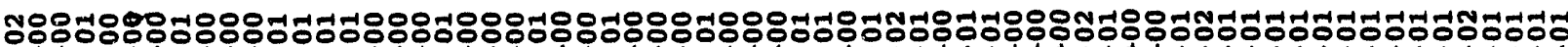

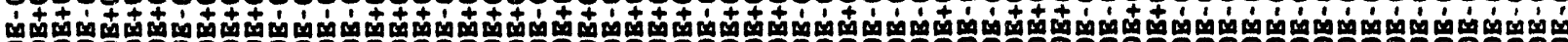

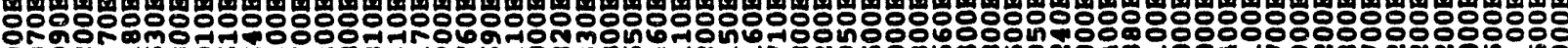

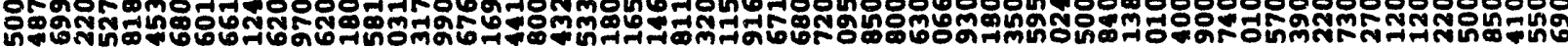

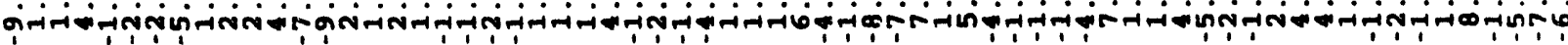

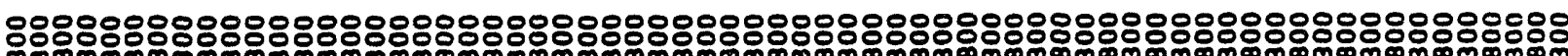
Фmథmळm

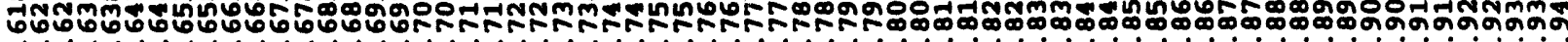

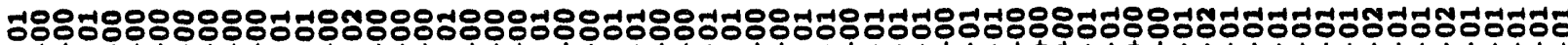

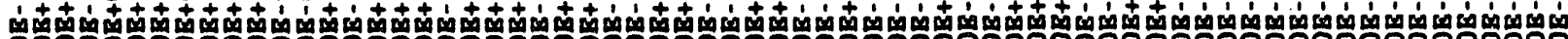

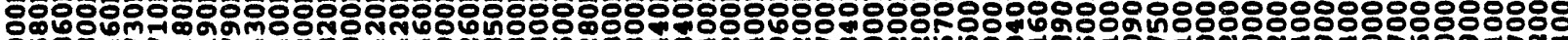

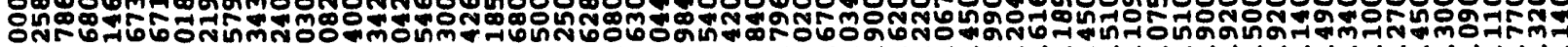

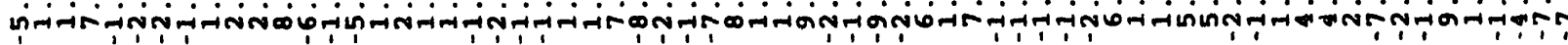

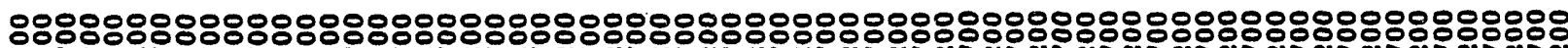

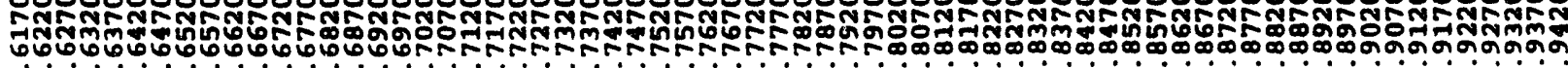

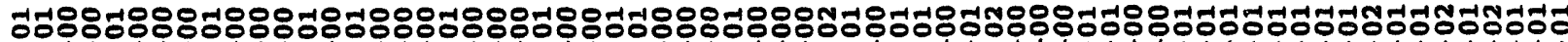

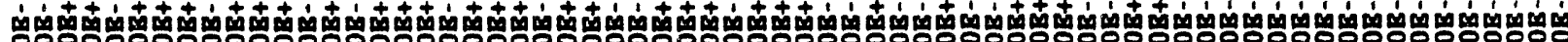

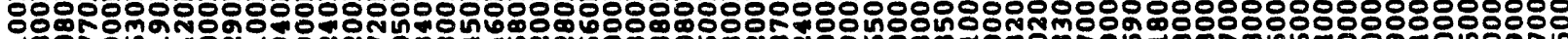

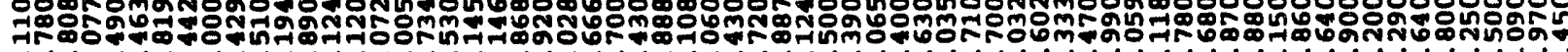

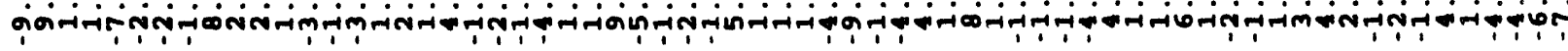

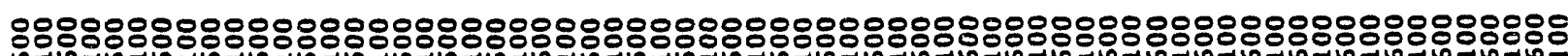

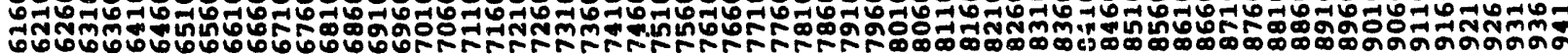

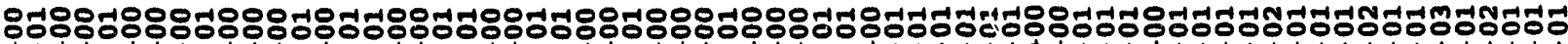

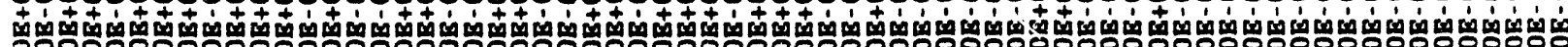

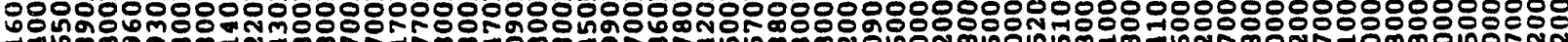
mưำ

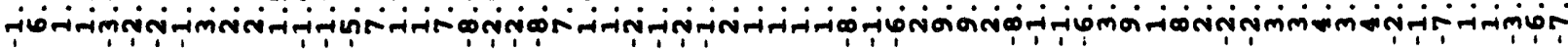

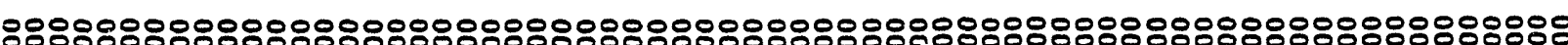

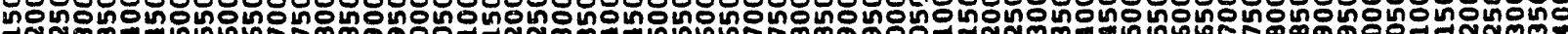

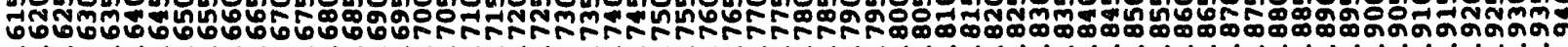




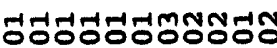

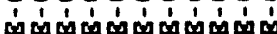

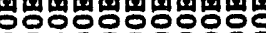

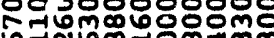

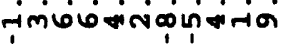

야용용 ต

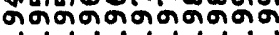

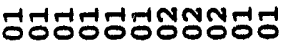

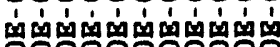

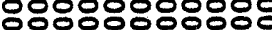

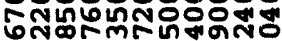

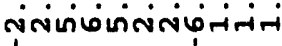

응응ㅇㅇ응용요 . "nที่

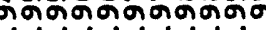

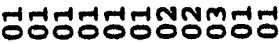

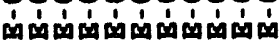

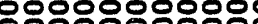

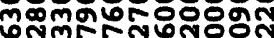
mंनirivimiónirim 응ㅇㅇㅇㅇㅇㅇㅇㅇㅇㅇㅇㅇㅛ กสกสร

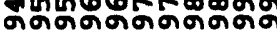

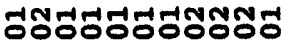

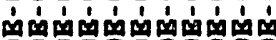

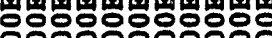

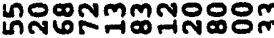

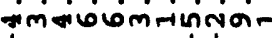
응ㅇㅇㅇㅇㅇㅇㅇㅇㅇㅛ

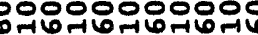
in नूनूनूनकनून

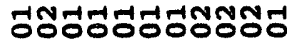

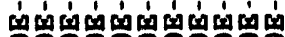

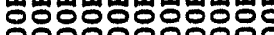

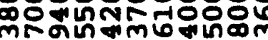

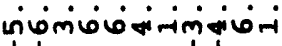

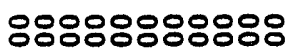

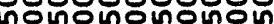

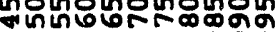

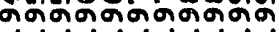




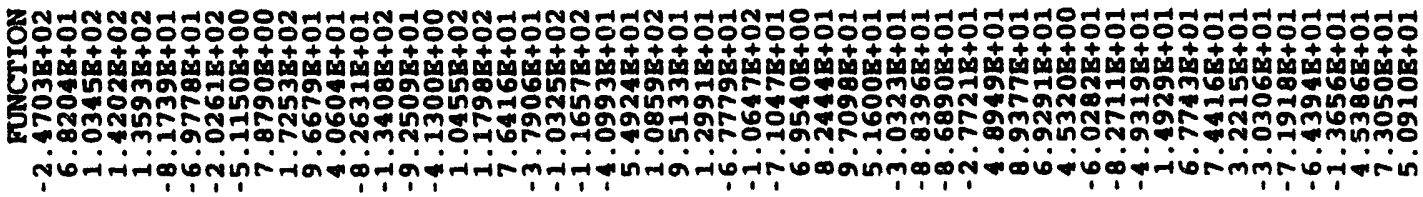

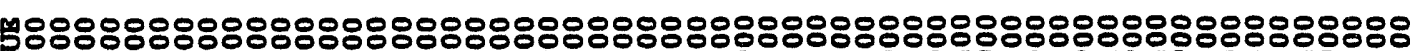
3

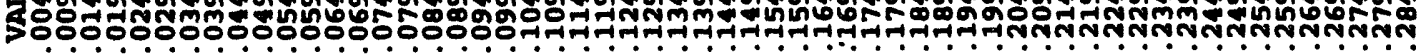
幽

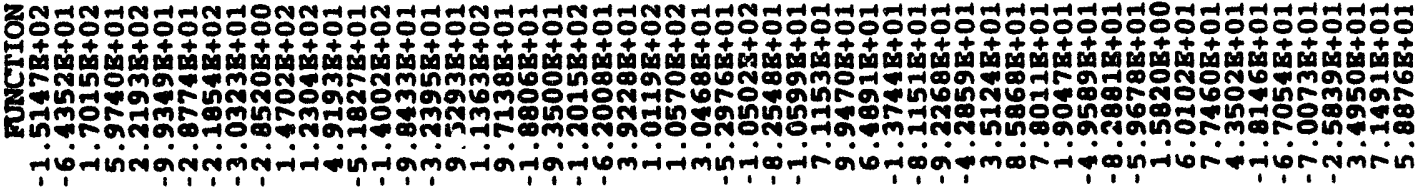
 द⿱乛龰:

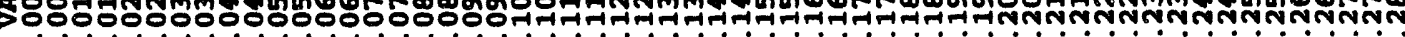
爽

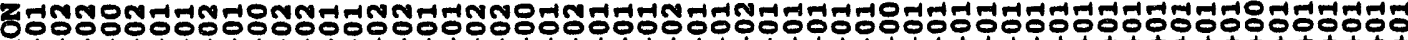

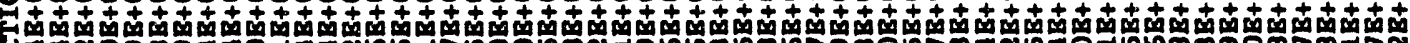
हnู

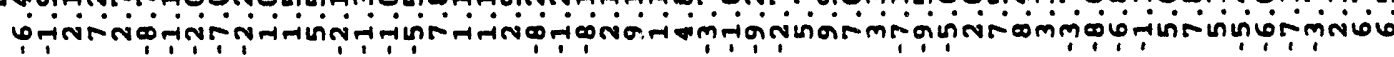

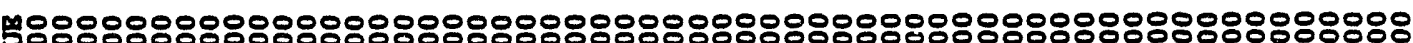
ไม่ 究

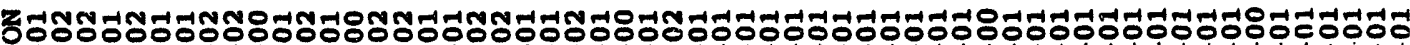

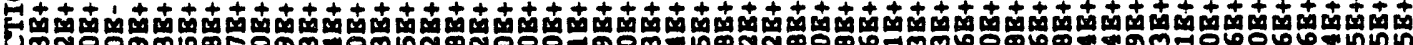

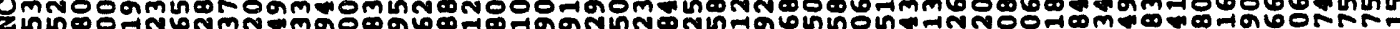

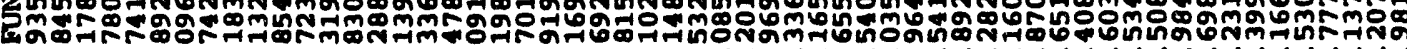

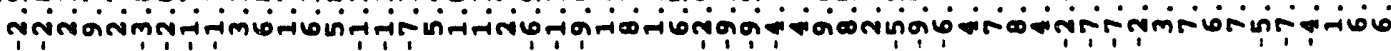

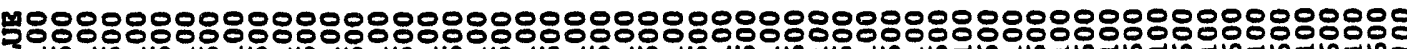

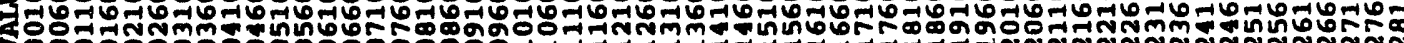
(1)000000000000000000 急

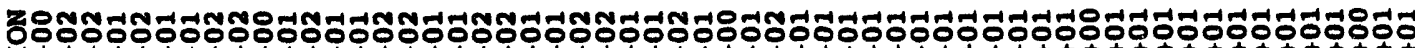

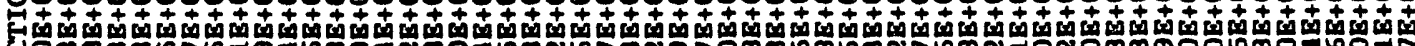
Uำ๊

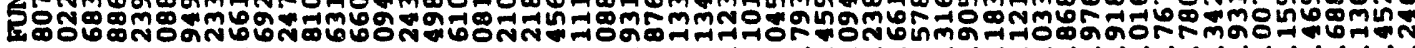

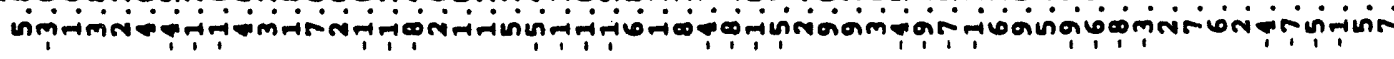

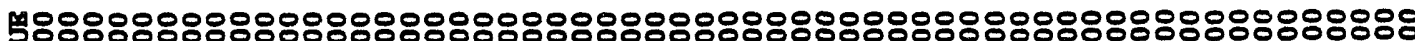

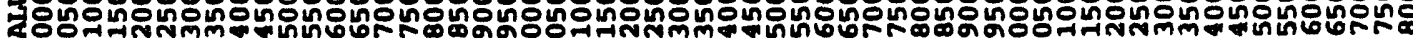

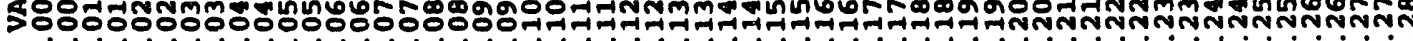
晃 


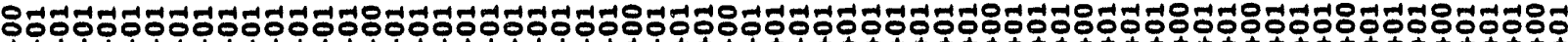

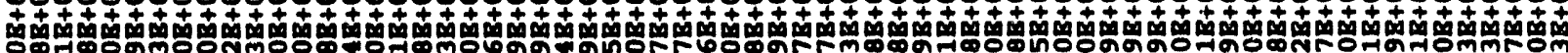
go-m.

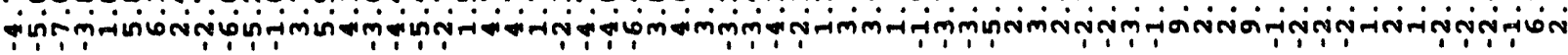

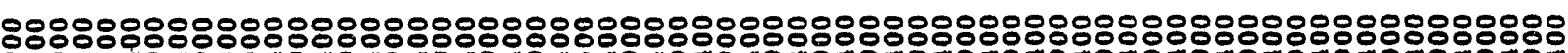

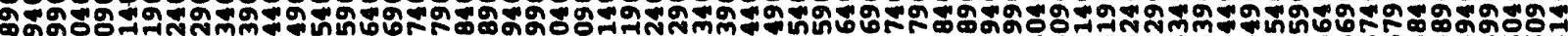

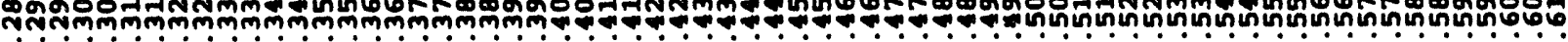

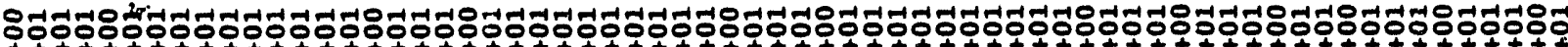

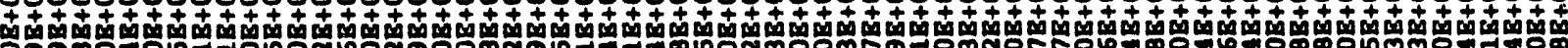
o슬

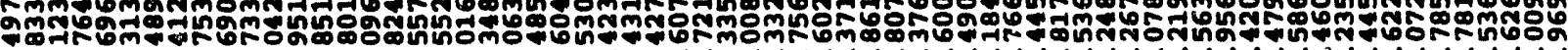

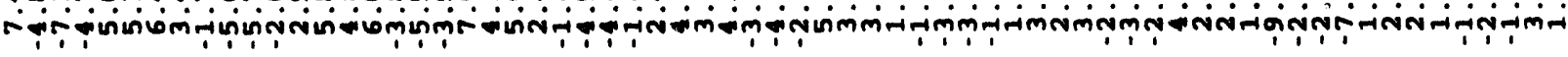

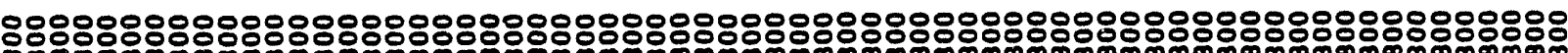

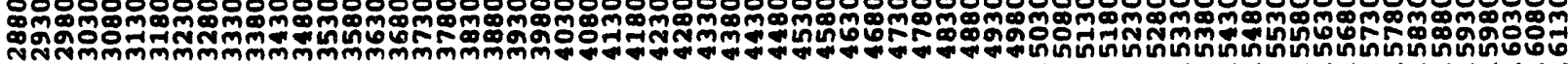

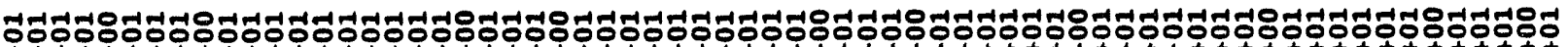

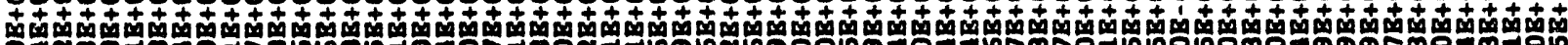

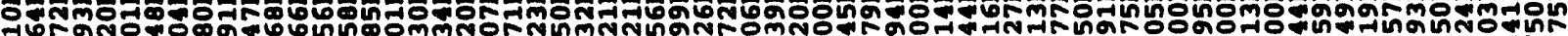

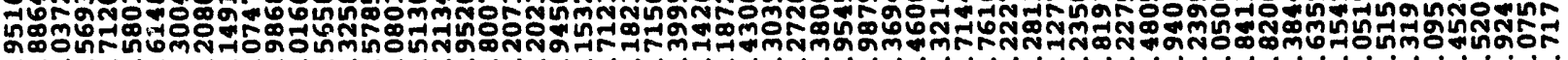

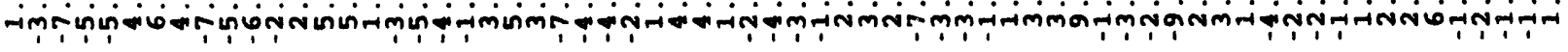

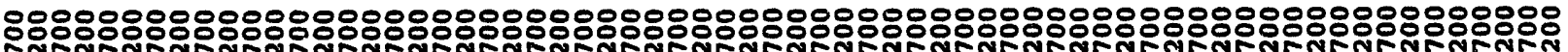

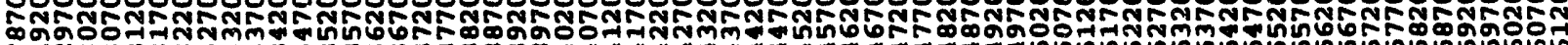
ง

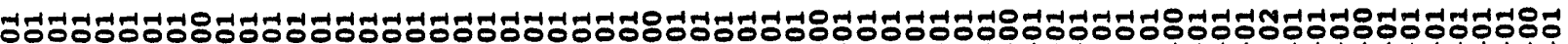

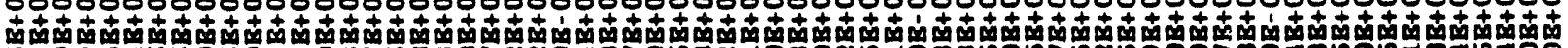

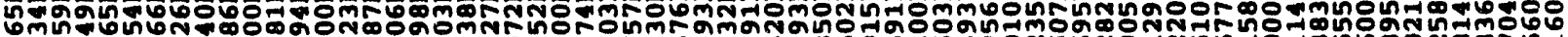
ŏm

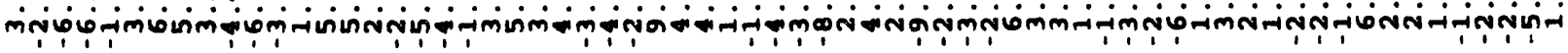

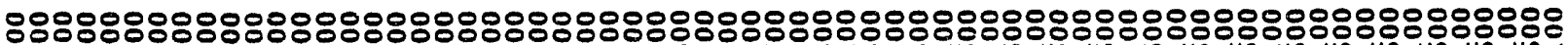
oño-

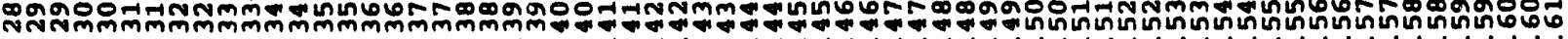

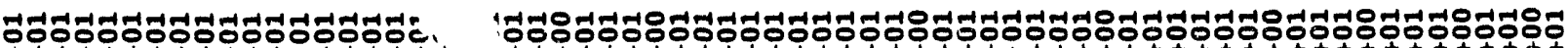

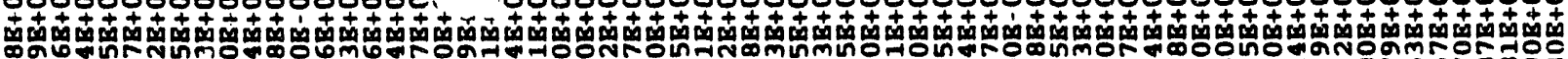

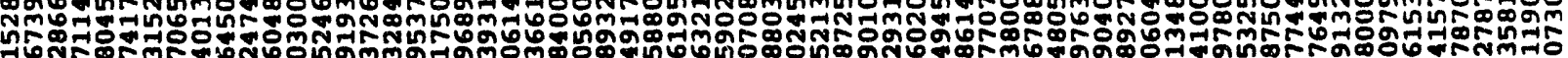
- ib

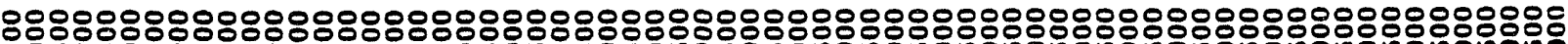
ผ 


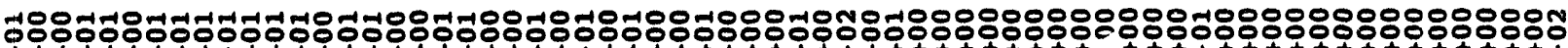

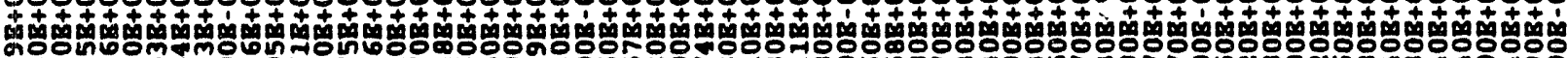

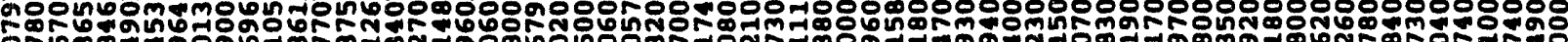

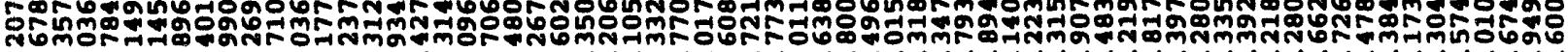
rळộ,

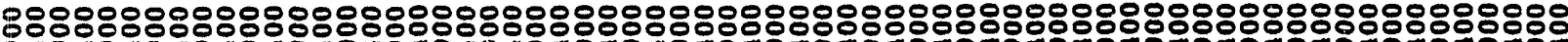

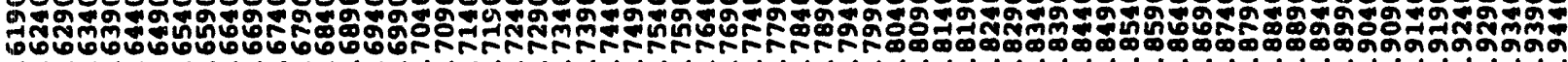

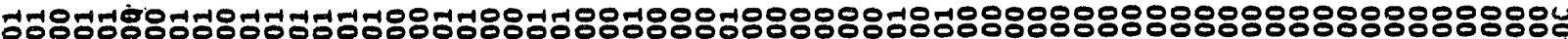

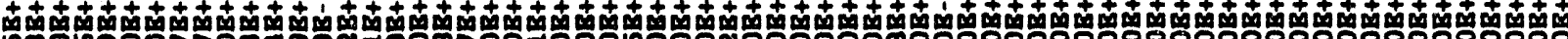
น̂m

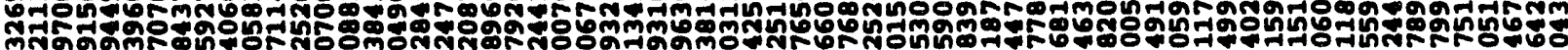

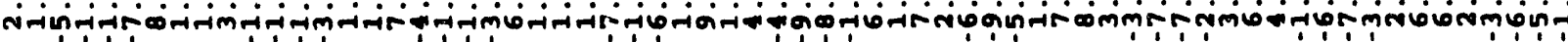

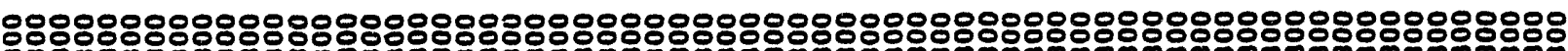

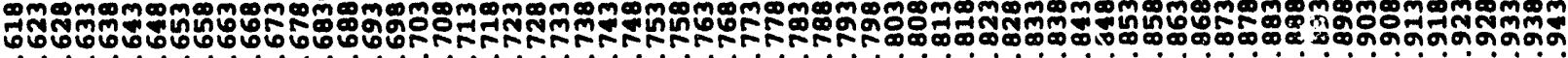

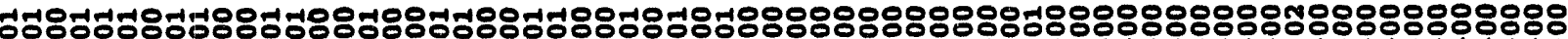

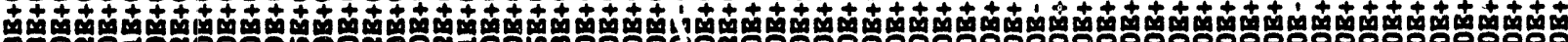

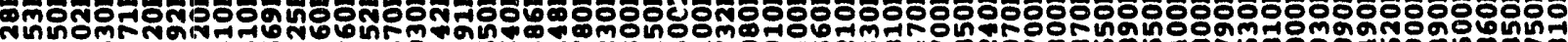

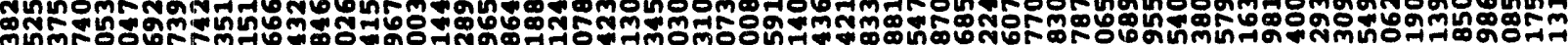

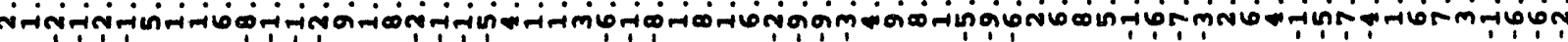

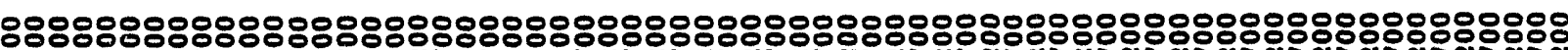
స எสชูตแ

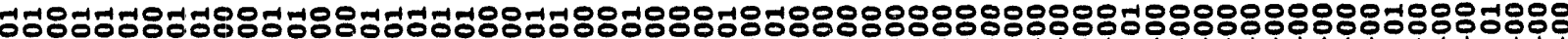

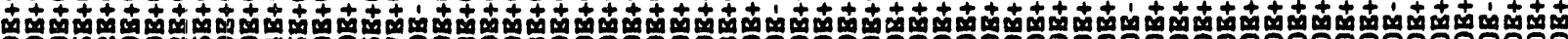

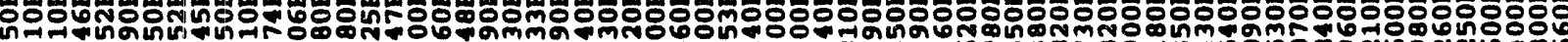

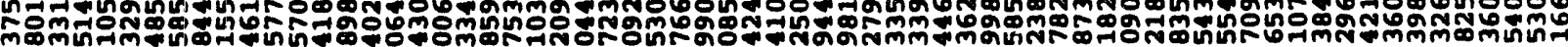
N-ifr, ৪৪৪৪৪৪ 울

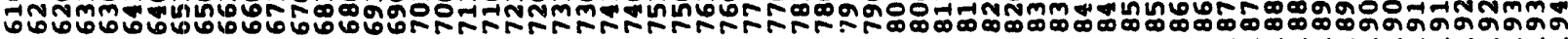

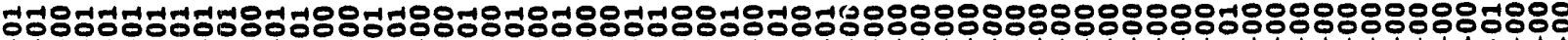

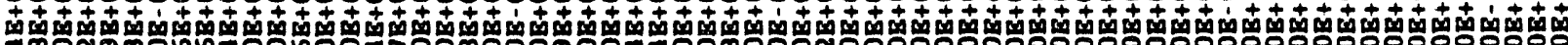

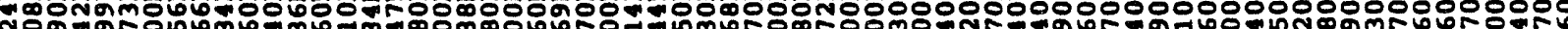

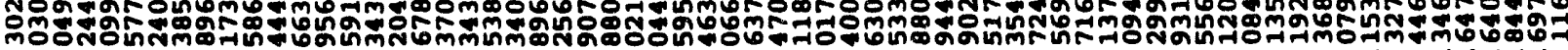

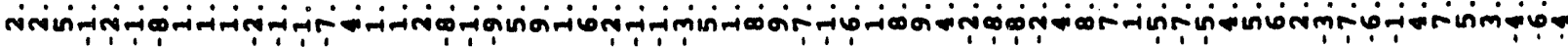

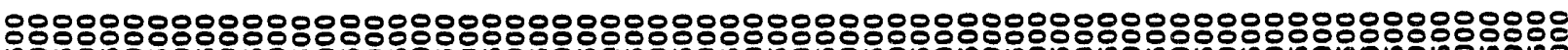

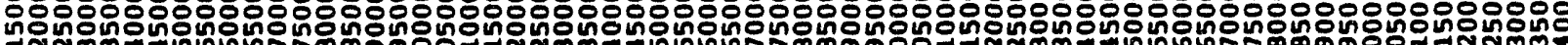

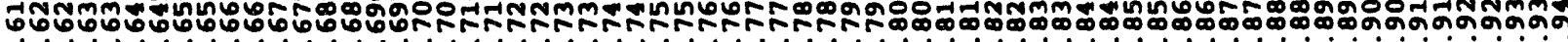




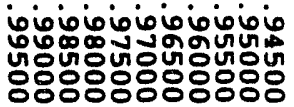

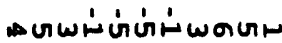
inivinisi

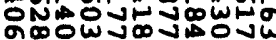

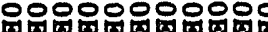
$+t++t+t+t+t$ 양ㅎㅇㅇㅇㅇㅇㅇㅇㅇㅇㅇㅇㅇㅇㅇㅇㅇ

joioioioioioioio 60\%

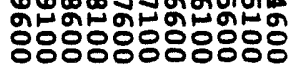
wruviśññNan vorois ongoñoin

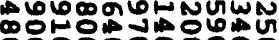

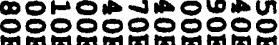

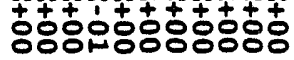

joioioioioioioioio ด0\%ั

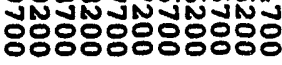

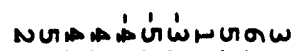
Girivingosinginis N.

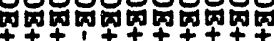
ธะ8ำㅇํㅇำ

ioioioioioioioio 60\%

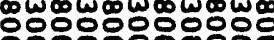
.

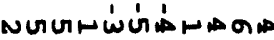

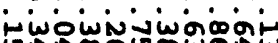
जู

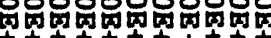
ㅇํำ

ioioioioioioioioio

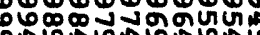
양응양ㅇㅇㅇㅇㅇㅇㅇㅇㅇㅇㅇ

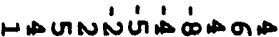
viois iniojogio

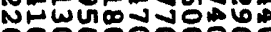

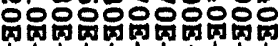
tㅎㅇㅇㅇㅇㅇㅇㅇㅇㅇㅇㅇㅇㅇㅇㅇㅇㅇㅇㅇㅇ 


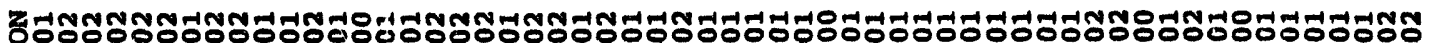

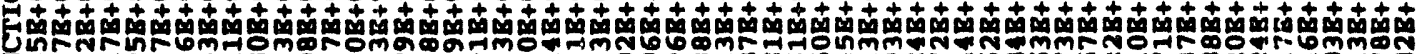

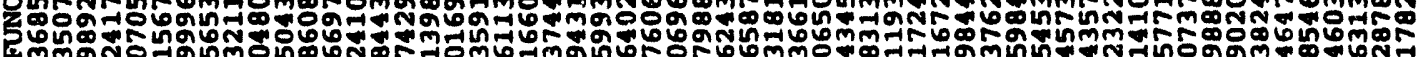

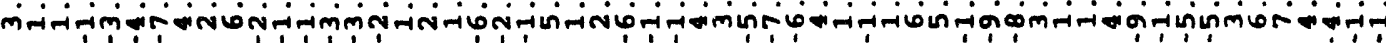

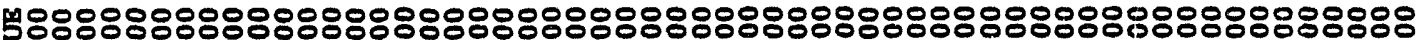

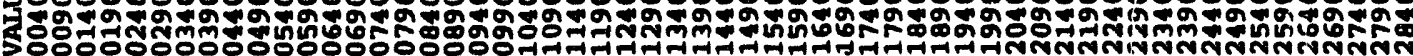
举

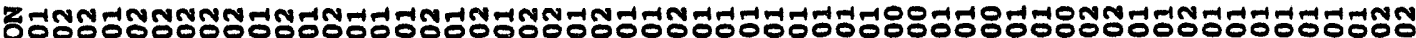

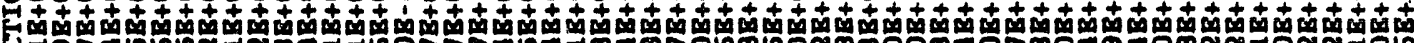
거으

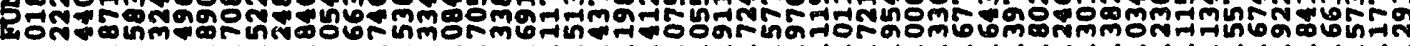

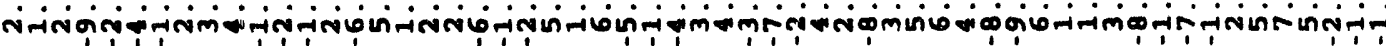

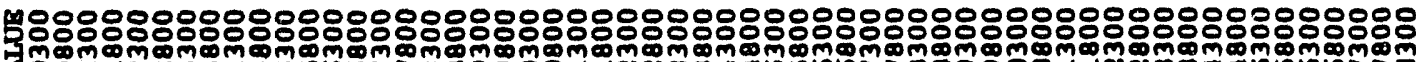
కöㅇำ 罩

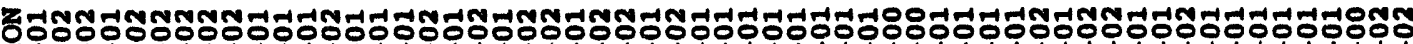

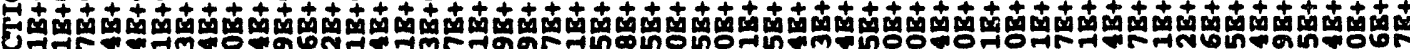
N-1 Do 0 - hom

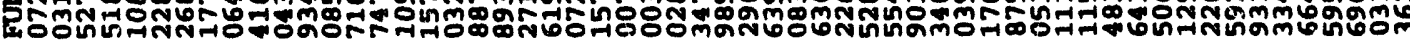

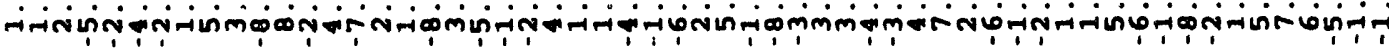

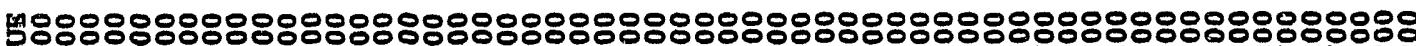
उสิ

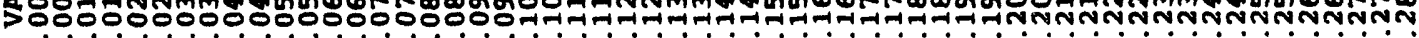
里

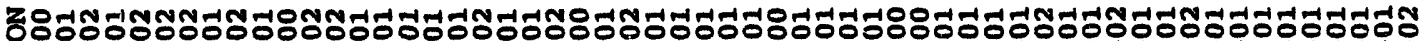

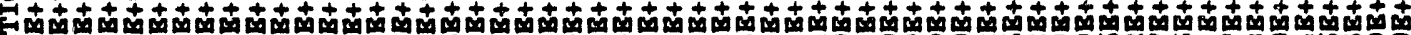
우순

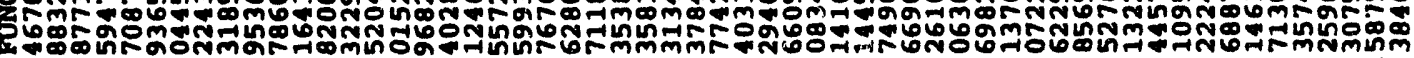
மr-iñmmon sn

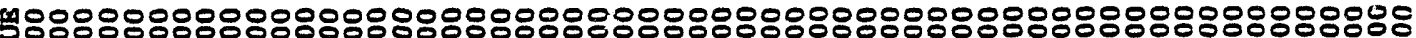
दై 兴

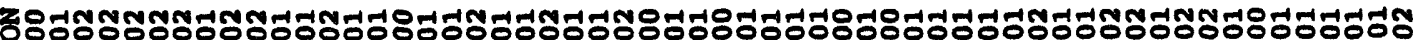

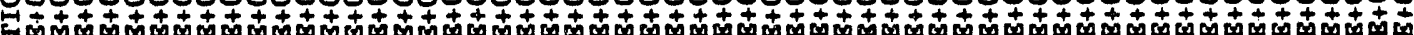
bono

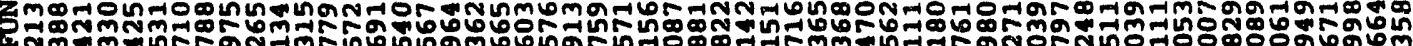

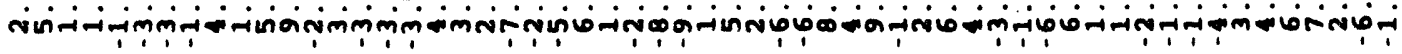

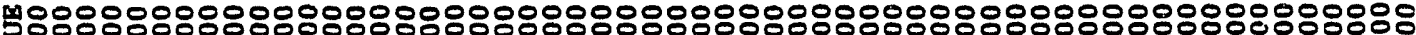

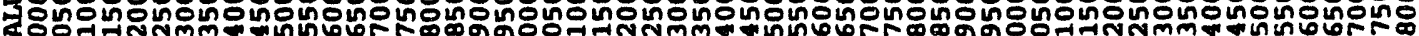

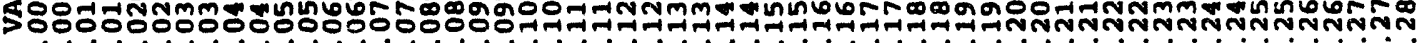
空 


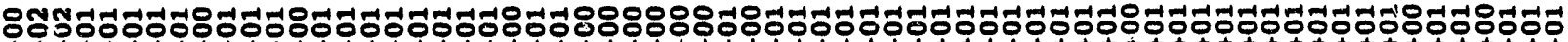
+.

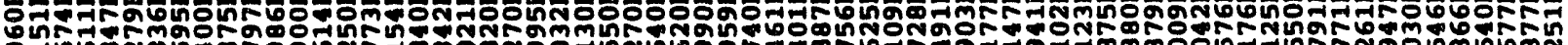

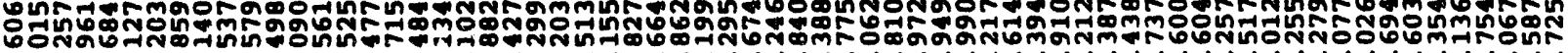

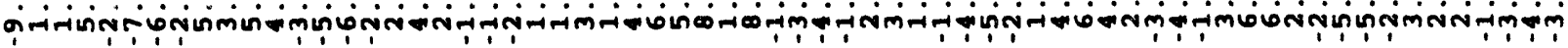

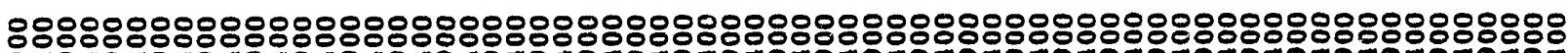

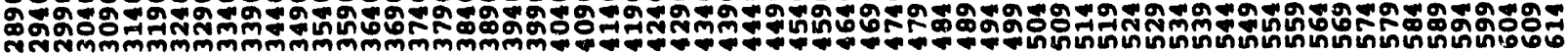

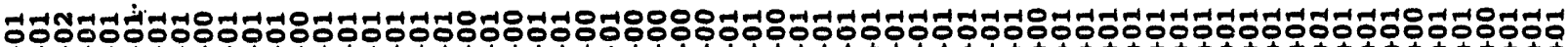

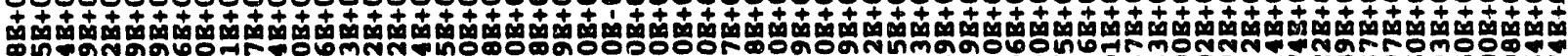
๓๐น

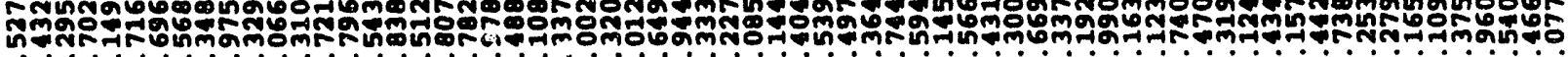

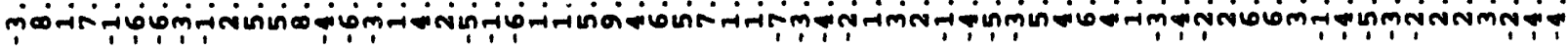

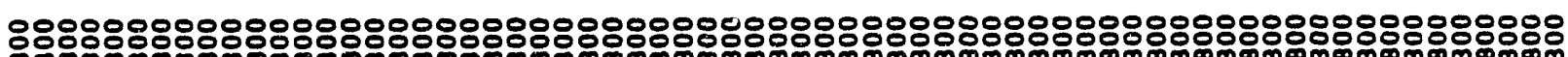

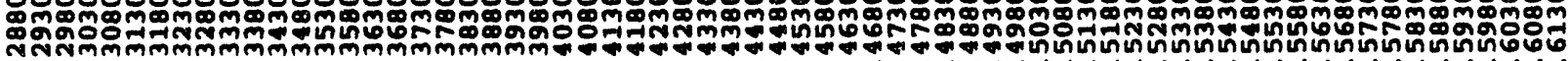

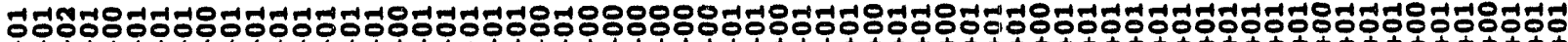

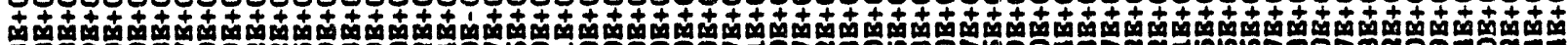

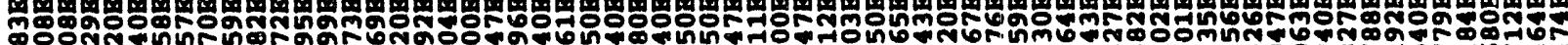

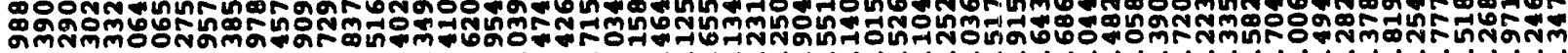
นึம -

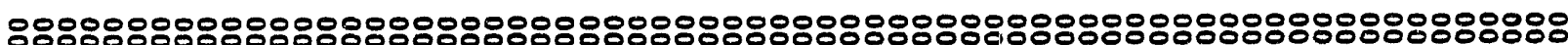

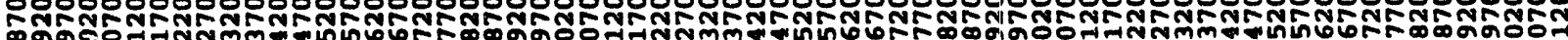

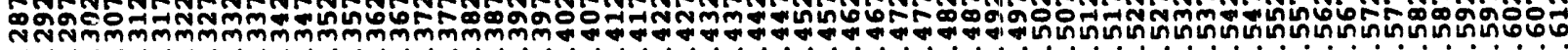

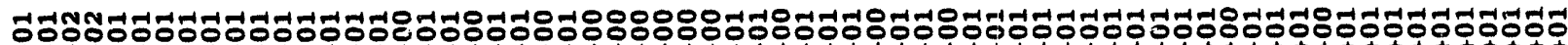

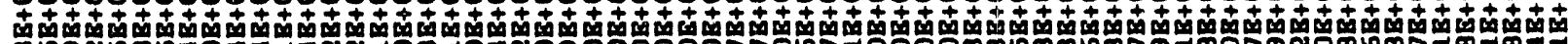
mn

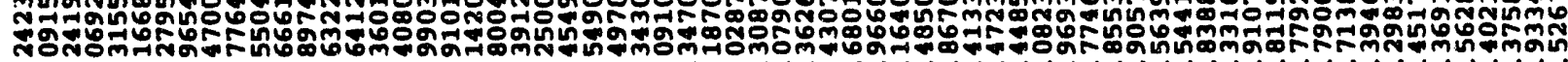

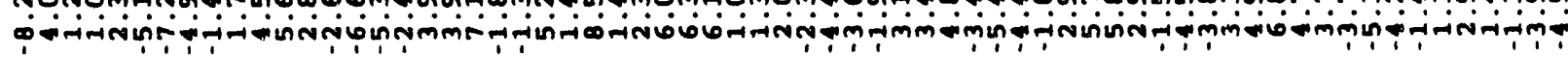

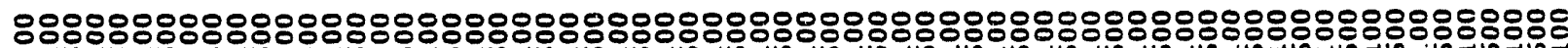

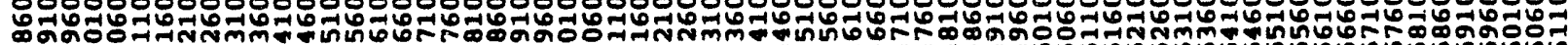
ง Nommmmmmmmmmmmmmmmmm

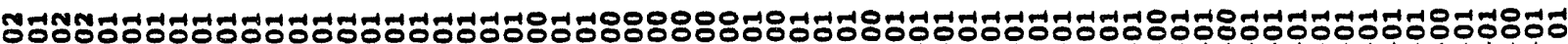

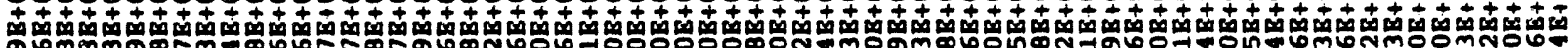

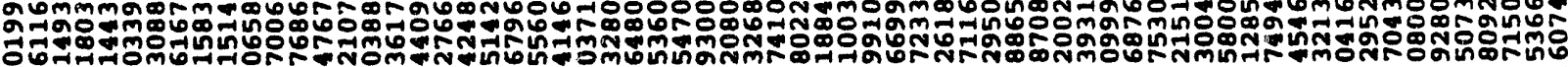
-

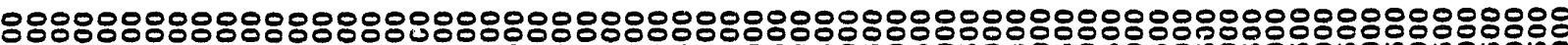

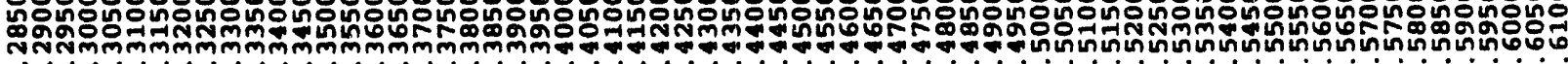




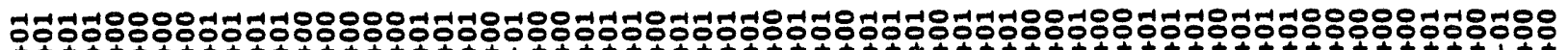

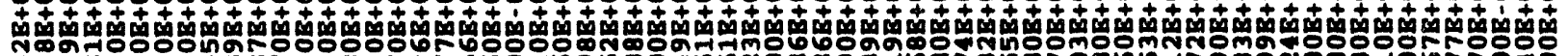

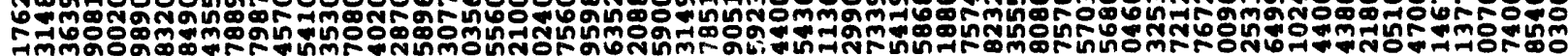

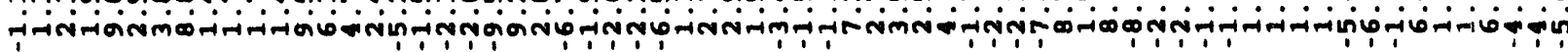

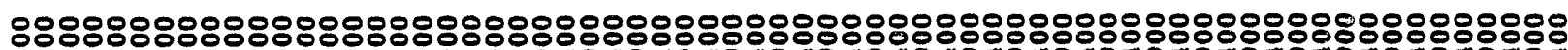

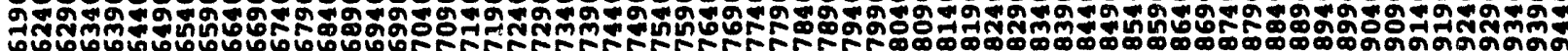

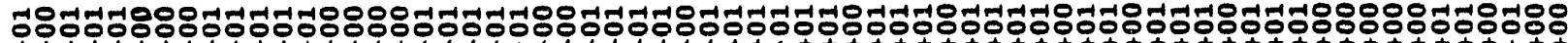

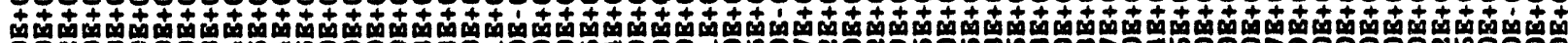

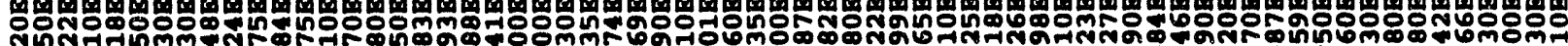

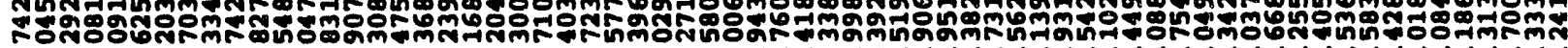

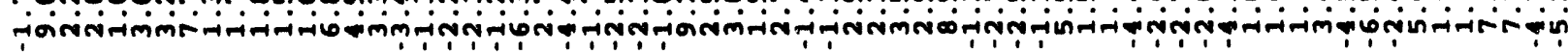
:

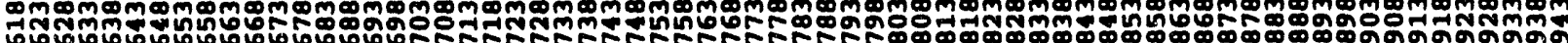

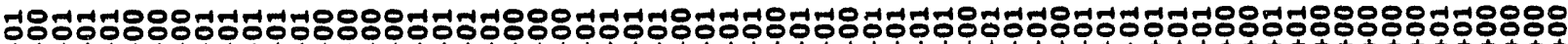

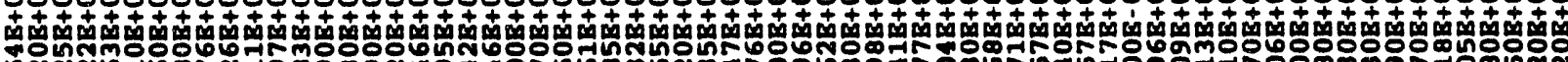

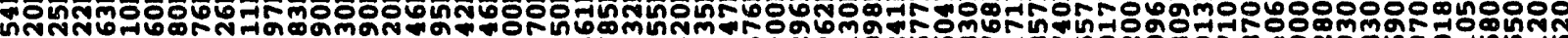

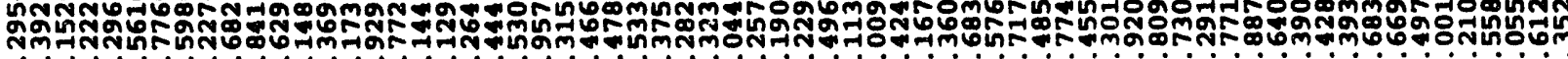

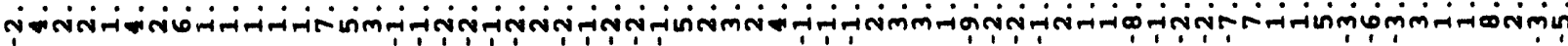

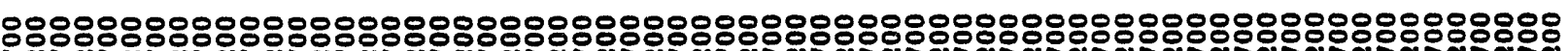
సస ำ

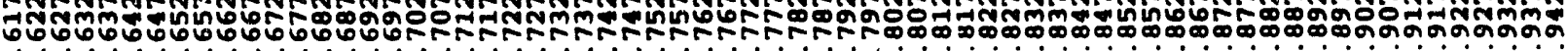

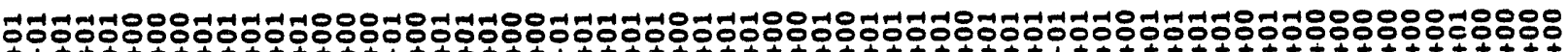

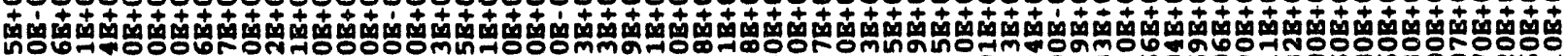

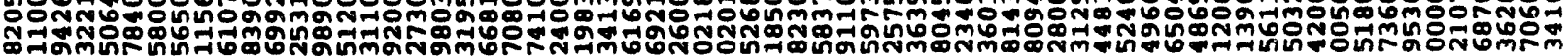

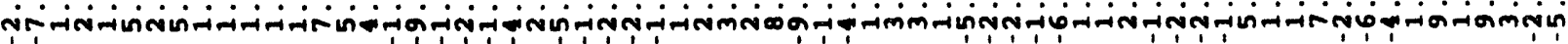

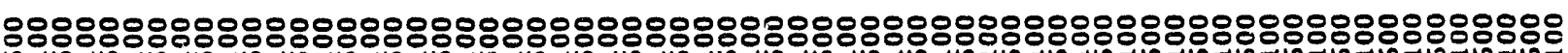
สํํํํำ

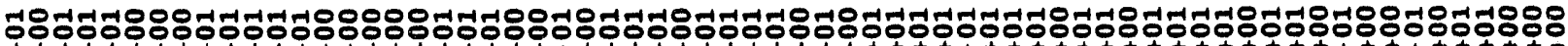

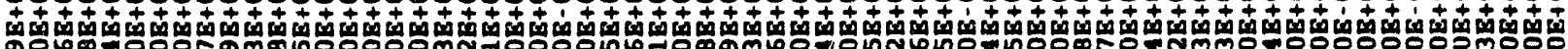

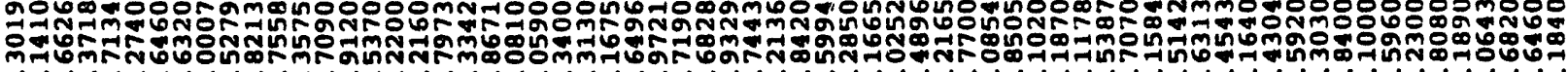

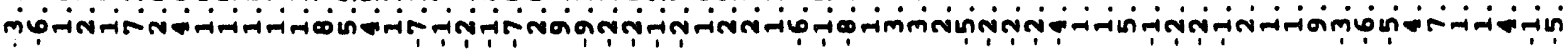

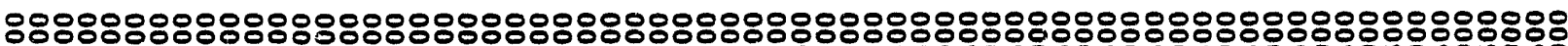

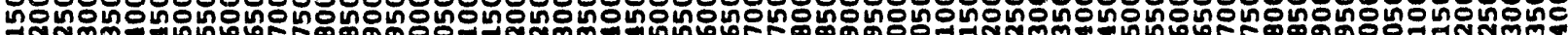

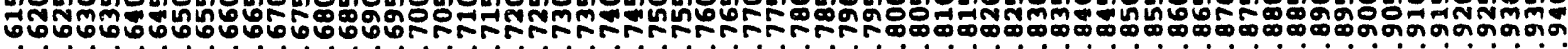


ioiobioibioiviojo

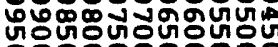

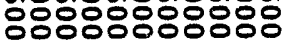

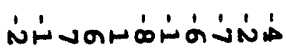
संnivio

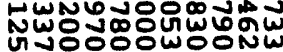
이임이잉 t+ometat

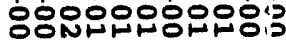

ioivioiviviojio 6.5 양용용ㅇㅇㅇㅇㅇㅇㅇㅇㅇㅇㅇㅇㅇ

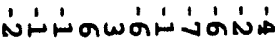
oirquisonini

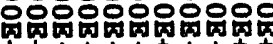

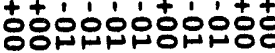

ioioioioioioioioio

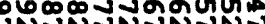

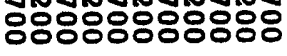

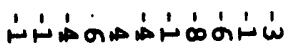
ดัن

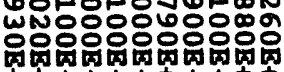

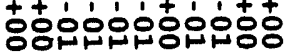

¡ioioioioioioioio ००0

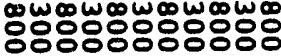

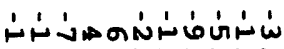
oigoninonivi

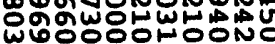

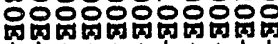

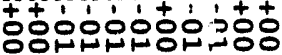

ioioioioioioioioio 붕ํำ 양융ㅇㅇㅇㅇㅇㅇㅇㅇㅇ융요

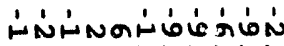
óogivinivivio ง

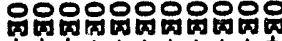

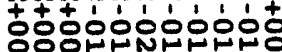


SUPPORT GROUP NUMBER $=2$

CONTROL INEORYATION

NURABR OP TIMI EUNCTIONS $=1$
GROUND MOTION INDICATOR $=1$

BO.0, NONR INDICATOR

MOE. $\frac{1}{1}$ GROUID INPUT

TIME STEPS

$\begin{array}{lll} & = & \\ \text { TIIST STRP } & .00010 \\ \text { DALYPING BACTOR } & = & .02000\end{array}$

$\underset{\substack{p \\ ⿱ 亠 乂}}{\stackrel{p}{+}}$

GROUND ACCELBRATION INPUT

X-DIRECTION Y-DIRECTION Z-DIRBCTION

TIMR FUNCTION NUMBR $(S)=$
ARRIVAL TILIS NULBER $(\mathbf{S})=$

1

1

1

ARRIVAL TIMB VALUES

RINTRY ARRIVAL TIME
VUIURBR

$1 \quad .000000$ 
TIMR FUNCTION NUEBRR

EUNCTION DESCRIPTION

MUgBar OF ABgCISgAR
FUICTION SCAIB BACTOR

TILB VALUB FUNCTION TIMB VALUE

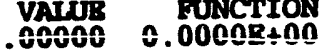

$=1 \quad 1)$

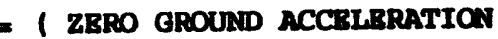

$=(2) .1000 \mathrm{~B}+01)$ 99900 $0.00003+00$
FUNCTION TIMB VALUE FUNCTION TIMB VALUE

FUNCTION 
DYNAMMIC RESPONSES 
D I S P I ACEMENT MAXIMA,

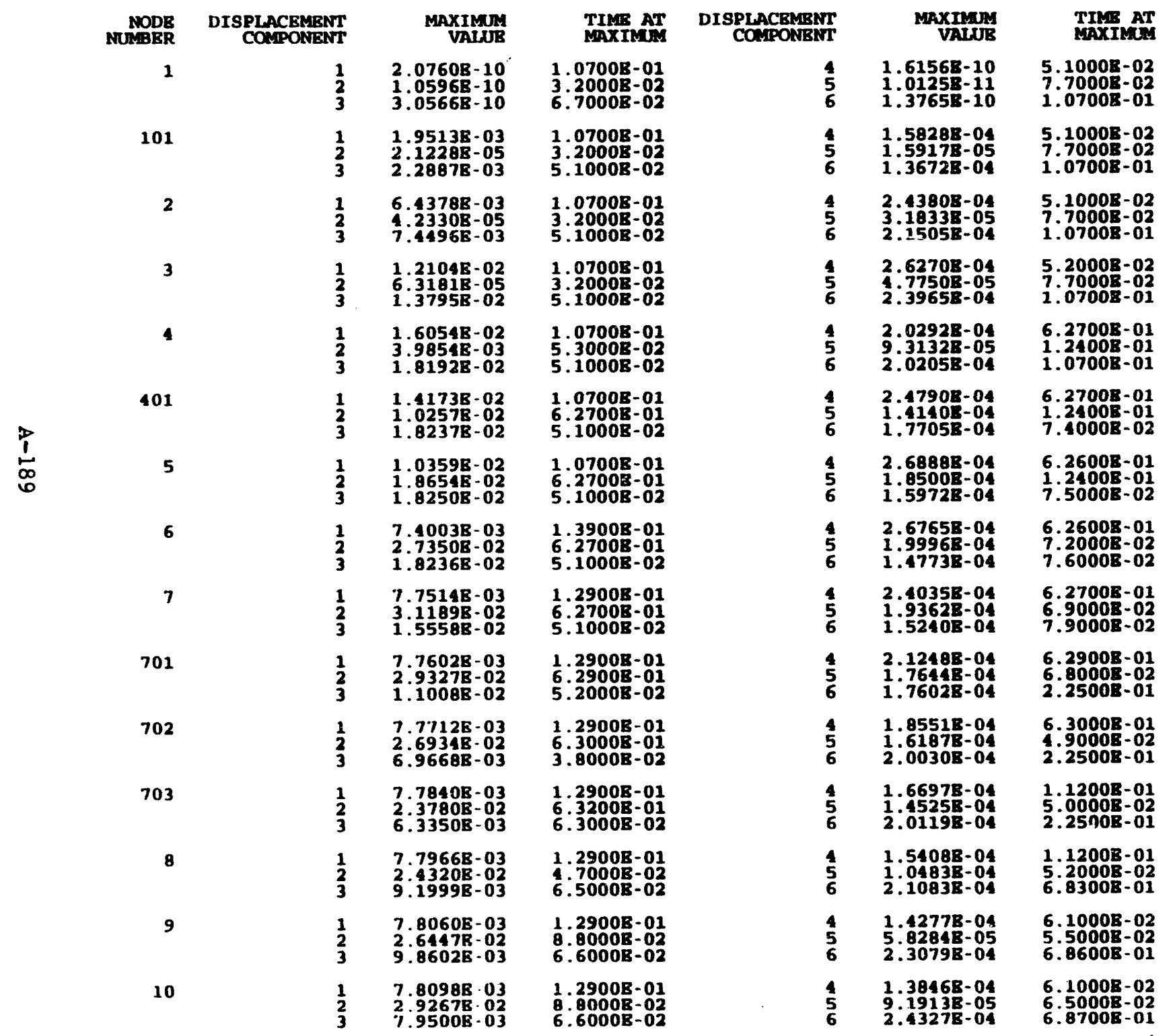



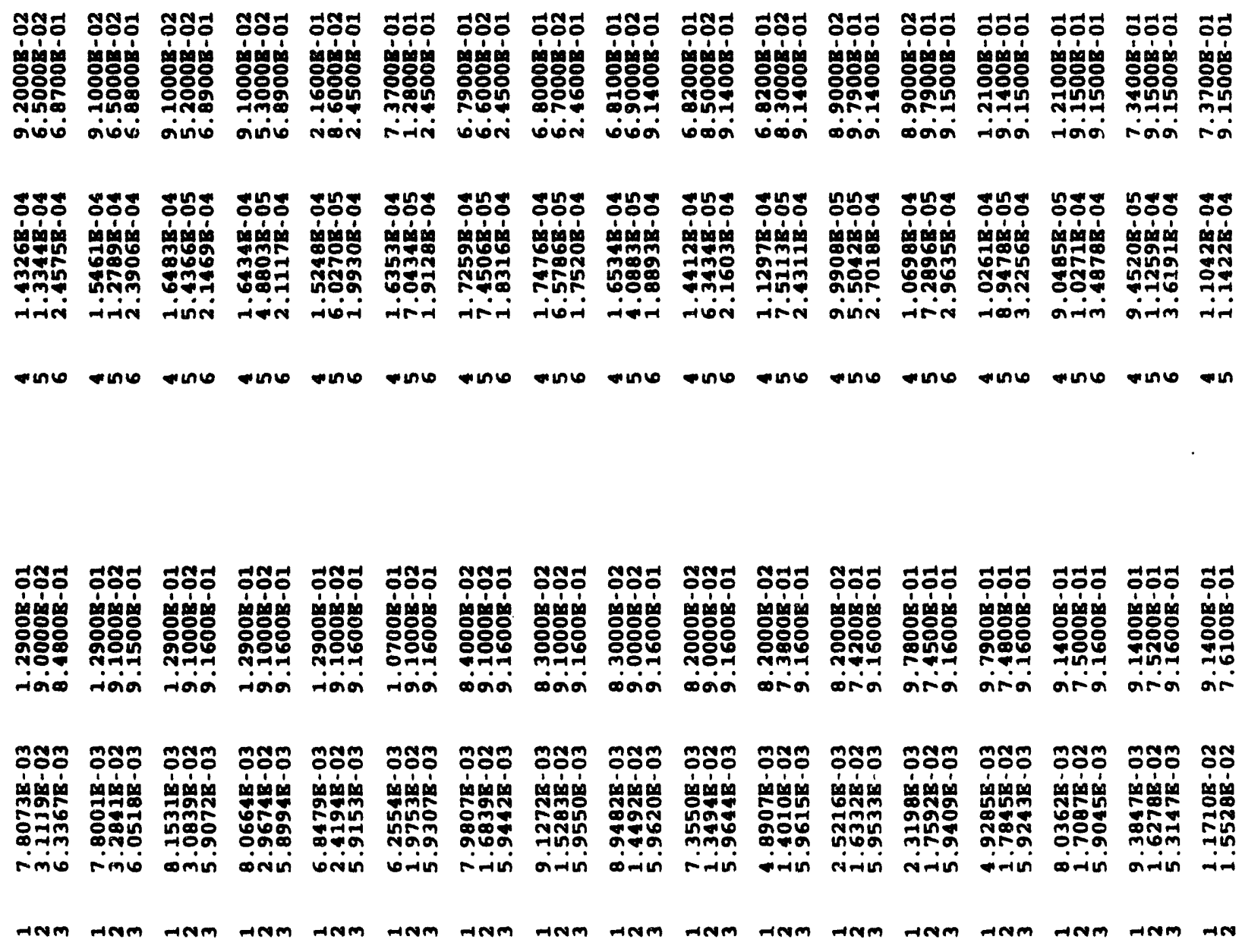

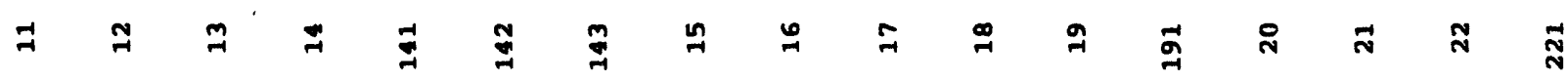




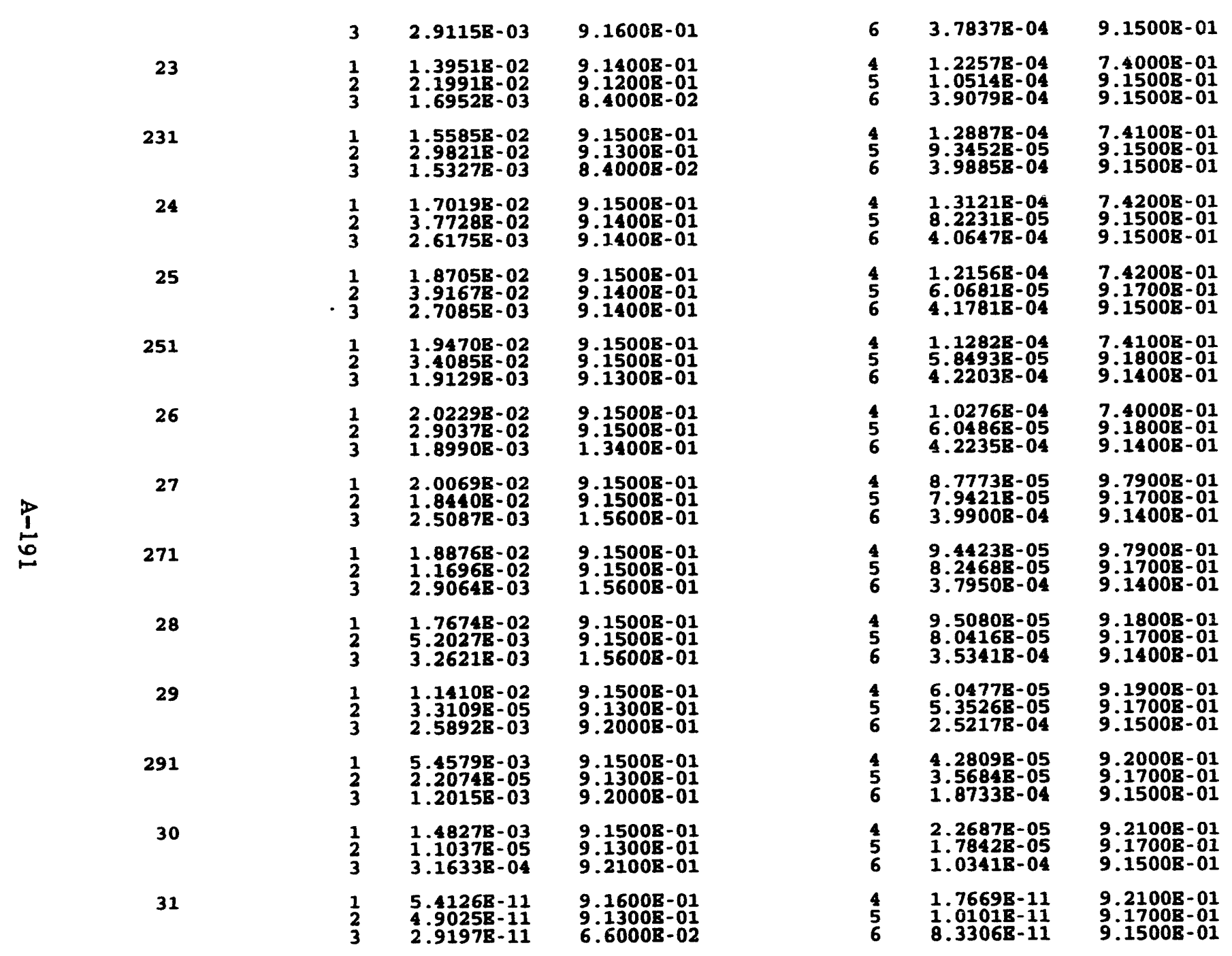


ACCELERATION MAXIMA

\begin{tabular}{|c|c|c|c|c|c|c|}
\hline \multirow{2}{*}{$\begin{array}{r}\text { MODR } \\
\text { NUBRR } \\
1\end{array}$} & $\begin{array}{l}\text { ACCRLERATION } \\
\text { COMPONENTI }\end{array}$ & $\underset{\text { VALIUB }}{\operatorname{MaxIMUM}}$ & $\begin{array}{l}\text { TIMR AT } \\
\text { MaxIMTM }\end{array}$ & $\begin{array}{l}\text { ACCRLBRATION } \\
\text { COMPONBNANT }\end{array}$ & $\begin{array}{c}\text { MAXIMUM } \\
\text { VALUE }\end{array}$ & 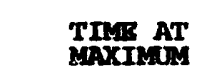 \\
\hline & $\frac{1}{2}$ & $\begin{array}{l}1.8016 \mathrm{~B}-08 \\
6.1112 \mathrm{~B} \\
4.1103 \mathrm{~B}-08 \\
4.08\end{array}$ & $\begin{array}{l}1.0600 \mathrm{~B}-01 \\
3.1000 \mathrm{~B} \\
6.6000 \mathrm{~B}-02 \\
6.602\end{array}$ & $\begin{array}{l}4 \\
5 \\
6\end{array}$ & $\begin{array}{l}1.85718-08 \\
7.37908-10 \\
1.17968-08\end{array}$ & $\begin{array}{l}6.60008-02 \\
4.40008-02 \\
9.00008-02 \\
9002\end{array}$ \\
\hline 101 & $\frac{1}{3}$ & $\begin{array}{l}1.67218-01 \\
1.23048-02 \\
2.61968-01\end{array}$ & $\begin{array}{l}9.00008-02 \\
3.10008 \\
6.60008-02 \\
6.002\end{array}$ & $\begin{array}{l}4 \\
5 \\
6\end{array}$ & $\begin{array}{l}1.66908-02 \\
1: 16008003 \\
1.17158-02\end{array}$ & 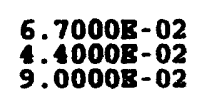 \\
\hline 2 & $\frac{1}{2}$ & $\begin{array}{l}5.51678-01 \\
2.45248-02 \\
7.83198-01 \\
4.83198\end{array}$ & $\begin{array}{l}9.0000-02 \\
3: 010008-02 \\
6: 7000 \mathrm{~B}-02\end{array}$ & $\begin{array}{l}4 \\
5 \\
6\end{array}$ & 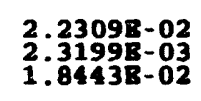 & $\begin{array}{l}6.70008-02 \\
4: 40008-02 \\
9.00008-02 \\
9000\end{array}$ \\
\hline 3 & $\frac{1}{2}$ & 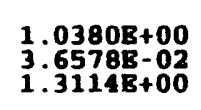 & $\begin{array}{l}9.0000 \mathrm{~B}-02 \\
3.01000 \mathrm{~B} \\
6.7000 \mathrm{~B}-02 \\
6.702\end{array}$ & $\begin{array}{l}4 \\
5 \\
6\end{array}$ & $\begin{array}{l}2.1846 \mathrm{~B}-02 \\
3: 4799 \mathrm{~B} \\
2.0652 \mathrm{0}-02 \\
2.062\end{array}$ & $\begin{array}{l}5.3000 \mathrm{~B}-02 \\
\mathbf{4}: 4000 \mathrm{R}-02 \\
9.0000 \mathrm{~B}-02\end{array}$ \\
\hline 4 & $\frac{1}{3}$ & $\begin{array}{l}1.3458 \mathrm{~B}+00 \\
5.2893 \mathrm{~B} \\
1.5097 \mathrm{~B}+00\end{array}$ & $\begin{array}{l}9.0000 \mathrm{~B}-02 \\
3.7000 \mathrm{~B} \\
5.2000 \mathrm{~B}-02\end{array}$ & $\begin{array}{l}4 \\
5 \\
6\end{array}$ & $\begin{array}{l}2.5898 \mathrm{~B}-02 \\
6.245458 \\
1.8022 \mathrm{~B}-02 \\
1.02\end{array}$ & $\begin{array}{l}2.600 \mathrm{~B}-02 \\
1: 22020 \mathrm{z} \\
9.0000 \mathrm{E}-02\end{array}$ \\
\hline 401 & $\frac{1}{3}$ & $\begin{array}{l}1.1235 \mathrm{~B}+00 \\
1.24298 \mathrm{~B} \\
1.513 \mathrm{~B}+00 \\
15+00\end{array}$ & $\begin{array}{l}1.070 \mathrm{~B}-01 \\
2.6000 \mathrm{~B} \\
5.2000 \mathrm{~B}-02\end{array}$ & $\begin{array}{l}4 \\
5 \\
6\end{array}$ & 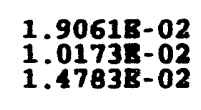 & $\begin{array}{l}2.7008-02 \\
1.23000 \mathrm{~B} \\
8.9000 \mathrm{E}-02\end{array}$ \\
\hline i & $\frac{1}{2}$ & $\begin{array}{l}7.7709 \mathrm{~B}-01 \\
1.53546 \mathrm{~B} \\
1.5129 \mathrm{~B}+00\end{array}$ & $\begin{array}{l}\frac{1}{2} .0600 \mathrm{~B}-01 \\
2.6000 \mathrm{~B} \\
5.2000 \mathrm{~B}-02\end{array}$ & $\begin{array}{l}4 \\
5 \\
6\end{array}$ & 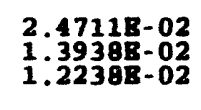 & 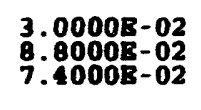 \\
\hline 6 & $\begin{array}{l}\frac{1}{2} \\
\frac{1}{3}\end{array}$ & $\begin{array}{l}3.61198-01 \\
1.5964 \mathrm{~B}+00 \\
1.50918+00\end{array}$ & $\begin{array}{l}9.10008-02 \\
2.1000-02 \\
5.20008-02 \\
502\end{array}$ & $\begin{array}{l}4 \\
5 \\
6\end{array}$ & $\begin{array}{l}3.88918-02 \\
1.58928-02 \\
1.04758-02\end{array}$ & $\begin{array}{l}3.10008-02 \\
8.10008-02 \\
5.50008-02\end{array}$ \\
\hline 7 & $\frac{1}{2}$ & $\begin{array}{l}4.6037 \mathrm{~B}-01 \\
1.9653 \mathrm{~B}+00 \\
1.3022 \mathrm{~B}+00\end{array}$ & $\begin{array}{r}4.1000 \mathrm{~B}-02 \\
2: 9000 \mathrm{~B}-02 \\
5: 2000 \mathrm{~B}-02\end{array}$ & $\begin{array}{l}4 \\
5 \\
6\end{array}$ & $\begin{array}{l}3.9000-02 \\
1: 44768-02 \\
1: 09018-02 \\
1.02\end{array}$ & $\begin{array}{r}3.10008-02 \\
6.80008 \\
1.80008-02 \\
1.8000\end{array}$ \\
\hline 701 & $\frac{1}{2}$ & $\begin{array}{l}4.6015 \mathrm{E}-01 \\
1: 8715 \mathrm{~B}+00 \\
9.7189 \mathrm{E}-01\end{array}$ & $\begin{array}{l}4.10008-02 \\
2: 90008-02 \\
5.20008-02\end{array}$ & $\begin{array}{l}4 \\
5 \\
6\end{array}$ & $\begin{array}{l}3.44878-02 \\
11.41818-02 \\
1.36448-02\end{array}$ & $\begin{array}{l}3.10008-02 \\
6.70008-02 \\
2.50008-02\end{array}$ \\
\hline 702 & $\frac{1}{2}$ & $\begin{array}{l}\mathbf{4} .5997 \mathrm{E}-01 \\
1.968628+00 \\
6.1064 \mathrm{E}-01\end{array}$ & 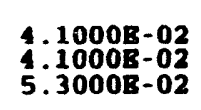 & $\begin{array}{l}4 \\
5 \\
6\end{array}$ & $\begin{array}{l}2.99798-02 \\
1: 40858-02 \\
1.6300 \mathrm{~B}-02\end{array}$ & $\begin{array}{l}3.10008-02 \\
6.770008 \\
3.70008-02 \\
3.702\end{array}$ \\
\hline 703 & $\frac{1}{2}$ & $\begin{array}{l}4.60758-01 \\
2.17798+00 \\
4.0714 E-01\end{array}$ & $\begin{array}{l}4.10008-02 \\
2.70008 \\
4.2000-02 \\
4.02\end{array}$ & $\begin{array}{l}4 \\
5 \\
6\end{array}$ & $\begin{array}{l}2.64538-02 \\
1: 310988-02 \\
1.21458-02 \\
1.2145\end{array}$ & $\begin{array}{l}3.8000 \mathrm{R}-02 \\
5.1000 \mathrm{~B} \\
6.1000 \mathrm{~B}-02 \\
6.1002\end{array}$ \\
\hline 8 & $\frac{1}{3}$ & $\begin{array}{l}4.6258 \mathrm{~B}-01 \\
2.0719 \mathrm{~B} \\
5.1390 \mathrm{~B}-01\end{array}$ & $\begin{array}{l}4.1000 \mathrm{~B}-02 \\
2.7000 \mathrm{R} \\
6.5000 \mathrm{~B}-02\end{array}$ & $\begin{array}{l}4 \\
5 \\
6\end{array}$ & $\begin{array}{l}2.34998-02 \\
1: 05438-02 \\
1.18208-02\end{array}$ & 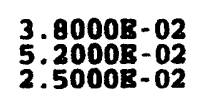 \\
\hline 9 & $\frac{1}{2}$ & $\begin{array}{r}4.6511 E-01 \\
1.90848+00 \\
6.7155-01\end{array}$ & $\begin{array}{l}4.1000-02 \\
9.10008 \\
6.4000-02 \\
6.02\end{array}$ & $\begin{array}{l}4 \\
5 \\
6\end{array}$ & $\begin{array}{r}2.0538 \mathrm{R}-02 \\
5.5508 \mathrm{~B} \\
1.3518 \mathrm{0}-02 \\
1.02\end{array}$ & $\begin{array}{l}3.80008-02 \\
5.40008-02 \\
2.00008-02\end{array}$ \\
\hline 10 & $\frac{1}{2}$ & $\begin{array}{l}4.6655 \mathrm{E}-01 \\
1.8734 \mathrm{E}+00 \\
6.1484 \mathrm{E}-01\end{array}$ & $\begin{array}{l}4.100 \mathrm{~B}-02 \\
9.11000 \mathrm{~B} \\
6.4000 \mathrm{~B}-02\end{array}$ & $\begin{array}{l}4 \\
5 \\
6\end{array}$ & $\begin{array}{l}1.8634 \mathrm{~B}-02 \\
5: 0112 \mathrm{~B} \\
1.1301 \mathrm{~B}-02 \\
1.02\end{array}$ & $\begin{array}{l}9.3008-02 \\
4: 770008-02 \\
6.10008-02\end{array}$ \\
\hline
\end{tabular}



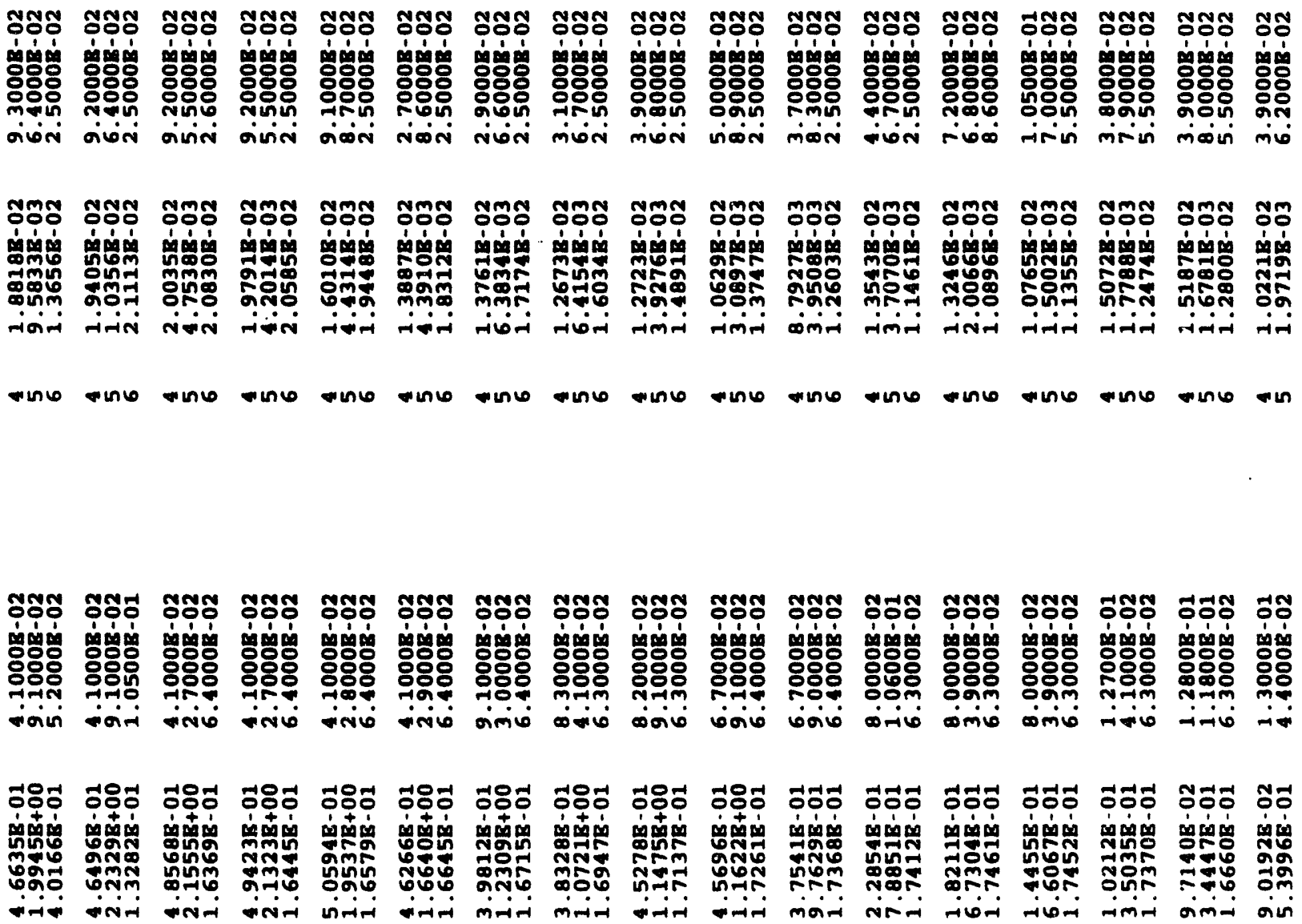

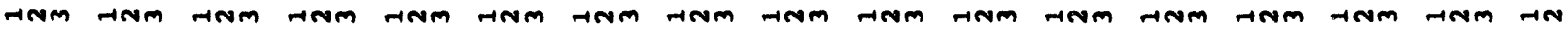

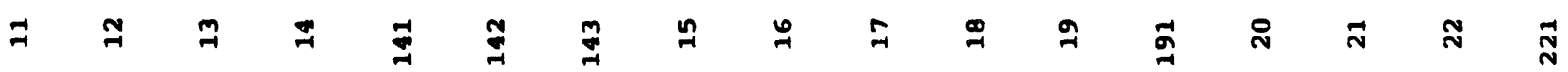




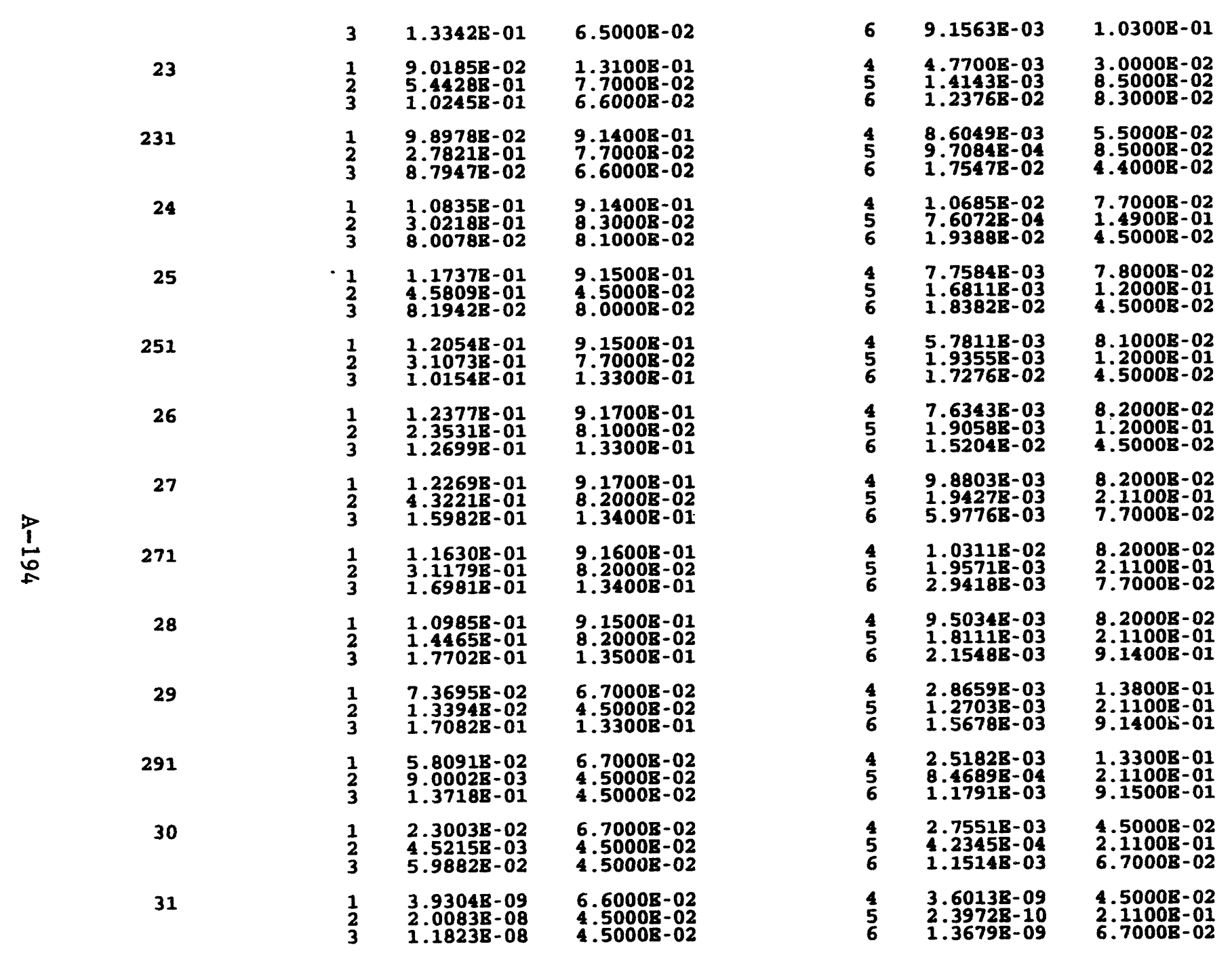




\begin{tabular}{|c|c|c|c|}
\hline $\begin{array}{c}\text { ELEMANT } \\
\text { NOABRR } \\
1\end{array}$ & $\begin{array}{l}\text { FORCE } \\
\text { COMPONENT } \\
\text { FOR. } \\
\text { MOM. }\end{array}$ & $\begin{array}{r}\text { MAXIMUM } \\
\text { VALUB } \\
2.07608+03 \\
0.00008+00\end{array}$ & $\begin{array}{r}\text { TIMRAT } \\
\text { MAXIMTM } \\
1.0700 \mathrm{~B}-01 \\
0.0000 \mathrm{~B}+00\end{array}$ \\
\hline 2 & FOR: & $\begin{array}{l}1.05968+03 \\
0.0000 \mathrm{~B}+00\end{array}$ & $\begin{array}{l}3.20008-02 \\
0.00008+00\end{array}$ \\
\hline 3 & FOR: & $\begin{array}{l}3.0566 \mathrm{~B}+03 \\
0.0000 \mathrm{~B}+00\end{array}$ & $\begin{array}{l}6.7000-02 \\
0.00008+00\end{array}$ \\
\hline 4 & gor. & $\begin{array}{l}0.0000 \mathrm{~B}+00 \\
1.6156 \mathrm{E}+05\end{array}$ & $\begin{array}{l}0.0000 \mathrm{~B}+00 \\
5.1000 \mathrm{~B}-02\end{array}$ \\
\hline 5 & FOR: & $\begin{array}{l}0.0000 \mathrm{~B}+00 \\
1.0125 \mathrm{~B}+04\end{array}$ & $\begin{array}{l}0.0000+00 \\
7.70008-02\end{array}$ \\
\hline 6 & FOR: & $\begin{array}{l}0.00008+00 \\
1.3765 \mathrm{~B}+05\end{array}$ & $\begin{array}{l}0.0000 \mathrm{~B}+00 \\
1.0700 \mathrm{~B}-01\end{array}$ \\
\hline 7 & FOR. & $\begin{array}{l}1.2608 \mathrm{~B}+03 \\
0.0000 \mathrm{E}+00\end{array}$ & $\begin{array}{l}8.2000 \mathrm{E}-02 \\
0.0000 \mathrm{~B}+00\end{array}$ \\
\hline 8 & FoR: & $\begin{array}{l}8.4760 \mathrm{~B}+02 \\
0.0000 \mathrm{~B}+00\end{array}$ & $\begin{array}{l}8.4000 \mathrm{~B}-02 \\
0.0000 \mathrm{~B}+00\end{array}$ \\
\hline 9 & FOR: & $\begin{array}{l}5.4126 \mathrm{E}+02 \\
0.0000 \mathrm{E}+00\end{array}$ & $\begin{array}{l}9.1600 \mathrm{E}-01 \\
0.0000 \mathrm{~B}+00\end{array}$ \\
\hline 10 & FOR: & $\begin{array}{l}4.90258+02 \\
0.0000 \mathrm{E}+00\end{array}$ & $\begin{array}{l}9.1300 \mathrm{E}-01 \\
0.0000 \mathrm{~B}+00\end{array}$ \\
\hline 11 & FOR: & $\begin{array}{l}2.9197 \mathrm{~B}+02 \\
0.0000 \mathrm{~B}+00\end{array}$ & $\begin{array}{l}6.6000 \mathrm{~B}-02 \\
0.0000 \mathrm{E}+00 \mathrm{C}\end{array}$ \\
\hline 12 & FOR: & $\begin{array}{l}0.0000 \mathrm{E}+00 \\
1.7669 \mathrm{E}+04\end{array}$ & $\begin{array}{l}0.0000 \mathrm{~B}+00 \\
9.2100 \mathrm{~B}-01\end{array}$ \\
\hline 13 & FOR: & $\begin{array}{l}0.0000 \mathrm{~B}+00 \\
1.0101 \mathrm{E}+04\end{array}$ & $\begin{array}{l}0.0000 \mathrm{E}+00 \\
9.1700 \mathrm{E}-01\end{array}$ \\
\hline 14 & FOR: & $\begin{array}{l}0.0000 \mathrm{~B}+00 \\
8.3306 \mathrm{~B}+04\end{array}$ & $\begin{array}{l}0.0000 \mathrm{~B}+00 \\
9.1500 \mathrm{~B}-01\end{array}$ \\
\hline
\end{tabular}


STRESS COM O OENT MAXIMA BLEMIRNT TYPE NUMBER $=4$

\begin{tabular}{|c|c|c|c|c|}
\hline $\begin{array}{l}\text { ELELENT } \\
\text { NULBER }\end{array}$ & LOCATION & $\begin{array}{c}\text { FORCE } \\
\text { COMPONENT }\end{array}$ & $\begin{array}{l}\text { MAXIMUM } \\
\text { VAIUE }\end{array}$ & $\underset{\text { MAXIMU AT }}{\text { TIME }}$ \\
\hline 1 & & $\begin{array}{l}\text { FOR. } \\
\text { MOM. }\end{array}$ & $\begin{array}{l}3.1115 \mathrm{E}+03 \\
0.0000 \mathrm{~B}+00\end{array}$ & $\begin{array}{l}5.1000 \mathrm{E}-02 \\
0.0000 \mathrm{E}+00\end{array}$ \\
\hline
\end{tabular}




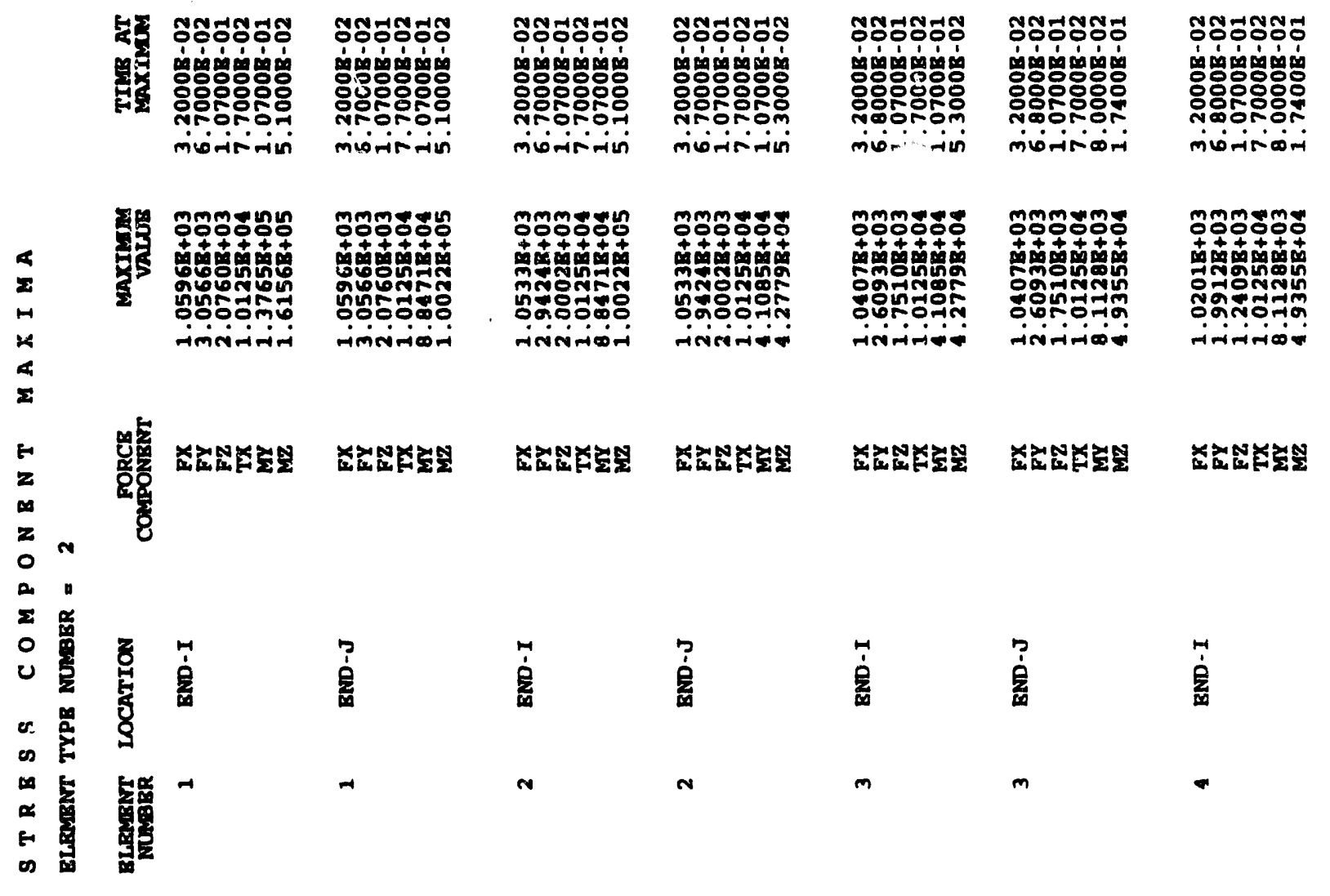




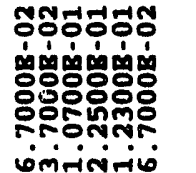

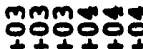

$+{ }^{+}+{ }^{+}+$

어에

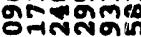

तiनiनiनiं

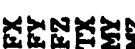

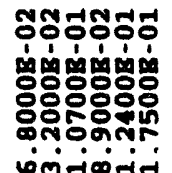

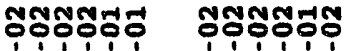
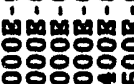

웅응에

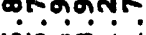

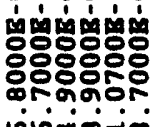

மंin-im

꽁꽁ㅎํ유

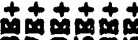

फิ-

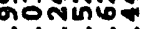

नirinivio

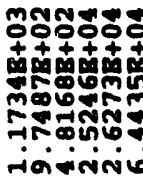

๓ํํㅇํํㅇํํำ

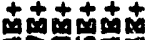
mo 둥 ज下नiovinim

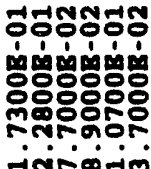

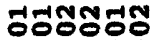

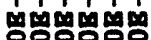

mळoㅇㅇㅛ

-ivirionim

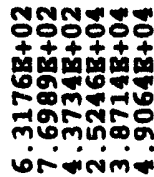

กำํㅇㅇㅇㅇํํำ

$t+t+t$

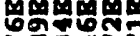

†술.

mominñ

orisinim

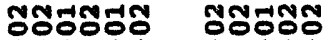

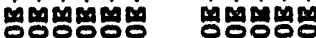

ㅇํ유ํ요

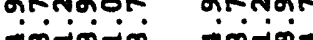

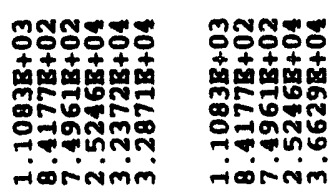

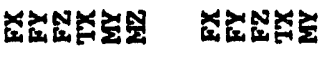

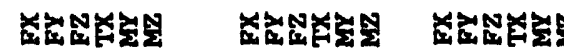

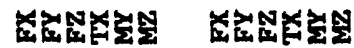

息

?̇

点

量

竞

空

in

$\omega$

$\omega$ 
8 END-I

$\begin{array}{lll}\text { FX } & 1.94078+03 & 5.0000 \mathrm{~B}-02 \\ \text { FY } & 8.06098+02 & 1.23000 \mathrm{~B}-01 \\ \text { FZ } & 6.49358+02 & 3.8000 \mathrm{~B}-02 \\ \text { TX } & 2.5246 \mathrm{~B}+04 & 8.9000 \mathrm{~B}-02 \\ \text { MY } & 9.87808+03 & 6.8000 \mathrm{~B}-01 \\ \mathrm{MZ} & 3.6628 \mathrm{~B}+04 & 1.7000 \mathrm{~B}-02\end{array}$

8 END-J

$\begin{array}{lll}\text { FX } & 1.4187 \mathrm{~B}+03 & 4.8000 \mathrm{~B}-02 \\ \text { FY } & 1.1489 \mathrm{~B}+03 & 5.2000 \mathrm{~B}-02 \\ \text { FZ } & 6.1935 \mathrm{~B}+02 & 3.8000 \mathrm{~B}-02 \\ \text { TX } & 1.7610 \mathrm{~B}+04 & 1.0700 \mathrm{~B}-01 \\ \text { MI } & 2.3212 \mathrm{~B}+04 & 2.2700 \mathrm{~B}-01 \\ \text { WZ } & 3.1455 \mathrm{~B}+04 & 1.6000 \mathrm{~B}-02\end{array}$

8 BND-J

$\begin{array}{lll}F X & 8.06088+02 & 1.2300 \mathrm{~B}-01 \\ F Y & 1.9407 \mathrm{~B}+03 & 5.0000 \mathrm{~B}-02 \\ \text { FZ } & 6.4935+02 & 3.8000 \mathrm{~B}-02 \\ \text { TX } & 1.4227 \mathrm{~B}+04 & 6.8500 \mathrm{~B}-01 \\ \text { MY } & 2.9561 \mathrm{~B}+04 & 8.9000 \mathrm{~B}-02 \\ \text { WZ } & 2.8949 \mathrm{~B}+04 & 1.5700 \mathrm{~B}-01\end{array}$

$\begin{array}{lll}\text { FX } & 7.32868+02 & 7.4000 \mathrm{~B}-02 \\ \text { FY } & 3.8760 \mathrm{~B}+02 & 1.0700 \mathrm{~B}-01 \\ \text { FZ } & 6.70268+02 & 1.0000 \mathrm{~B}-02 \\ \text { TX } & 1.12878+04 & 6.8500 \mathrm{~B}-01 \\ \text { WY } & 2.9007 \mathrm{~B}+04 & 1.5700 \mathrm{~B}-01 \\ \mathrm{WZ} & 2.94428+04 & 8.9000 \mathrm{~B}-02\end{array}$

9 BKD-J

$\begin{array}{lll}\text { FX } & 7.3286 \mathrm{~B}+02 & 7.4000 \mathrm{~B}-02 \\ \text { FY } & 3.8760 \mathrm{~B}+02 & 1.0700 \mathrm{~B}-01 \\ \text { FZ } & 6.7026 \mathrm{~B}+02 & 1.0000 \mathrm{~B}-02 \\ \text { TX } & 1.4287 \mathrm{~B}+04 & 6.8500 \mathrm{~B}-01 \\ \text { MY } & 2.7235 \mathrm{~B}+0 \mathrm{~L} & 1.0700 \mathrm{~B}-01 \\ \text { WZ } & 2.7587 \mathrm{~B}+04 & 1.7200 \mathrm{~B}-01\end{array}$

10 BND-I

$\begin{array}{lll}\text { FX } & 6.60318+02 & 7.4000 \mathrm{~B}-02 \\ \text { FY } & 5.4079 \mathrm{~B}+02 & 1.0700 \mathrm{~B}-01 \\ \text { FZ } & 1.8144 \mathrm{~B}+02 & 4.5000 \mathrm{~B}-02 \\ \text { TX } & 1.4287 \mathrm{~B}+04 & 6.8500 \mathrm{~B}-01 \\ \text { MY } & 2.7235 \mathrm{~B}+04 & 1.0700 \mathrm{~B}-01 \\ \mathrm{MZ} & 2.7587 \mathrm{~B}+04 & 1.7200 \mathrm{~B}-01\end{array}$

10 BND-J

$\begin{array}{ll}\text { FX } & 6.60318+02 \\ \text { FY } & 5.40798+02 \\ \text { FZ } & 4.81488+02 \\ \text { TX } & 1.42678+04 \\ M Y & 3.1598+04 \\ M Z & 2.52828+04\end{array}$

7.40608-02 57008-01 $8500 \mathrm{~B}-01$ $.0600 \mathrm{~B}-01$
$7300 \mathrm{~B}-01$

$7.40008-02$
$5.1000 \mathrm{E}-02$ 

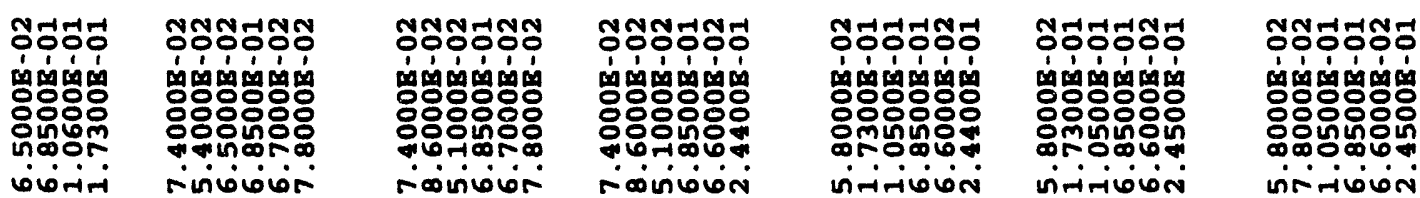

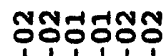

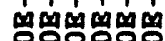
응응응응 이앵ㅆㄷำ

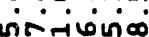
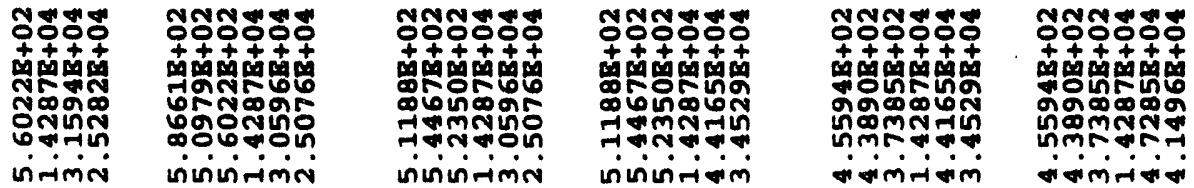

ํํㅇ주유ํํํำ

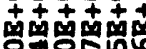
Tตिएक.

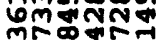
अiिmiनi-

สํํㅇํํํำ $+++++$

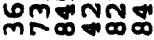
निंminimin

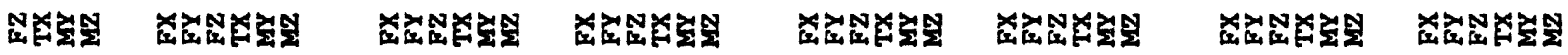

?

量

占

点

是

量

是

I

กั

N

$m$

$m$

$\nexists$

$\pm$ 

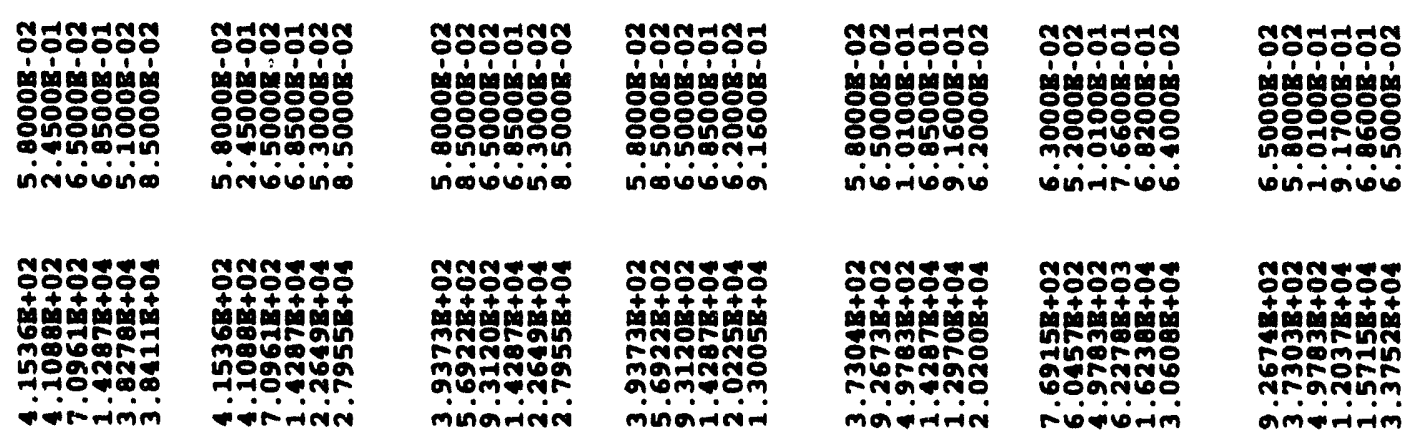

ริํㅇㅇㅇํํㅇㅇㅇำ

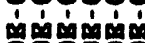

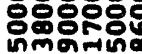

ธringivio

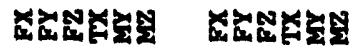
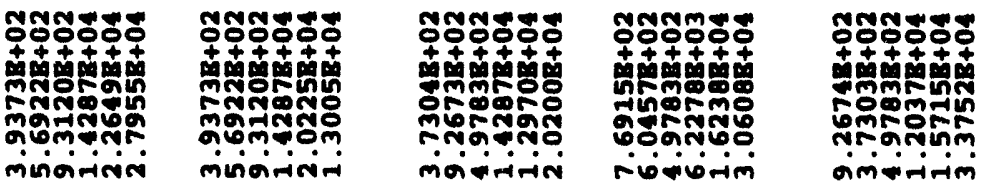

ำํํㅇํㅇㅇํㅇํำ

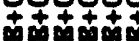

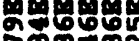

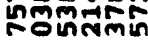

örimiñi

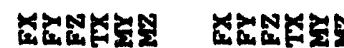

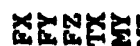

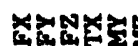

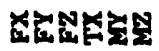

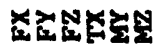

$\dot{9}$

官

官

搌

$\mp$

テ

7

$\stackrel{\infty}{-}$ 

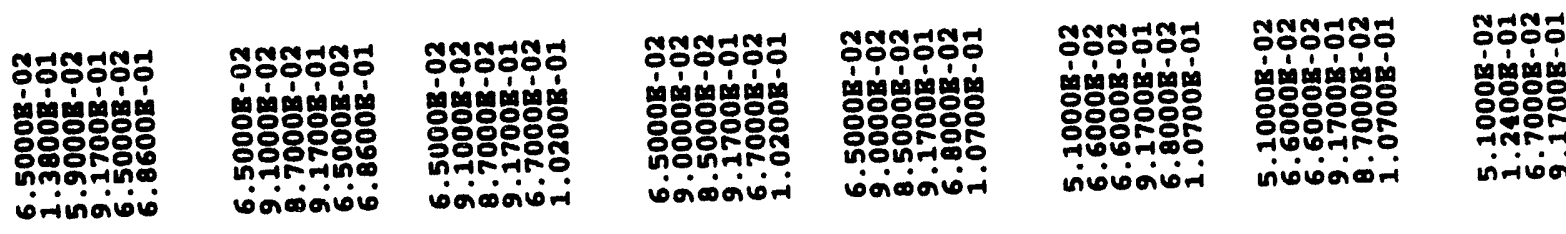
м่́m

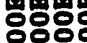
유뮤 जिनंषต
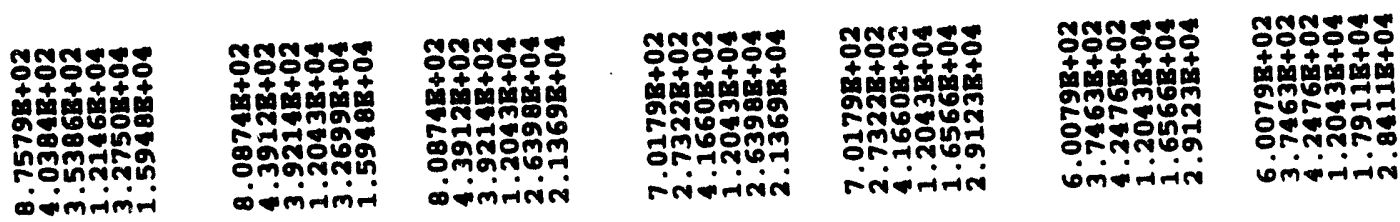

충종여

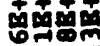
ติ600 ทं宀

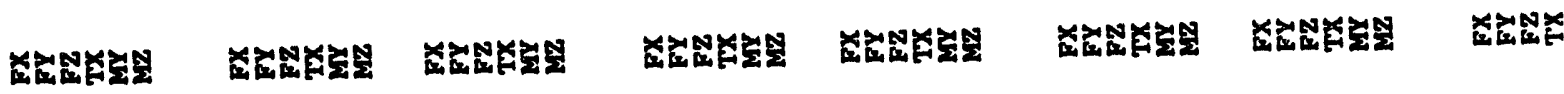

总

葛

是

皇

完

息

?

管

$\underset{-1}{\infty}$

g

a

옹

กำ

$\pi$

ה

ส 

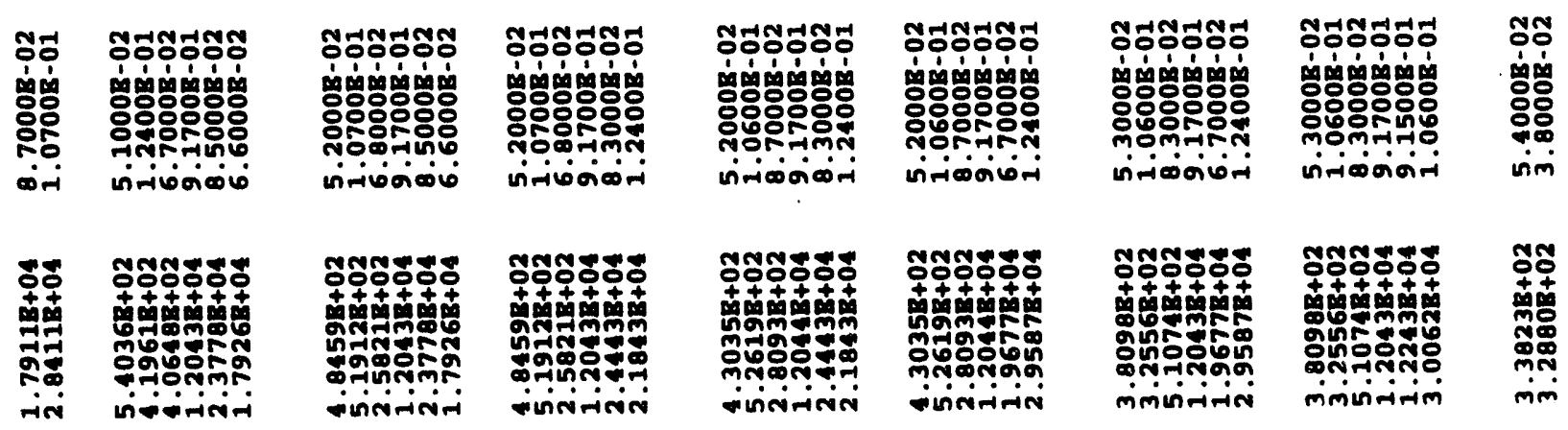

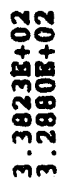

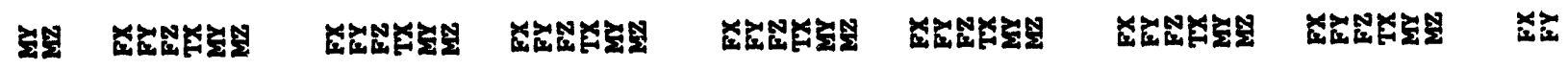

是

*
总 -

完

m

*

量

是

总

竞

竞

הั

$\bar{n}$

*

$\dddot{n}$

$\stackrel{\sim}{*}$ 

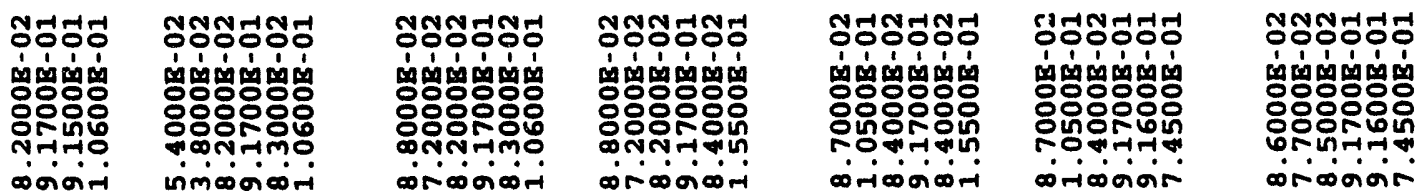

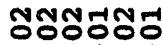

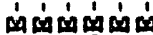
응융용요 ठํำ oricior
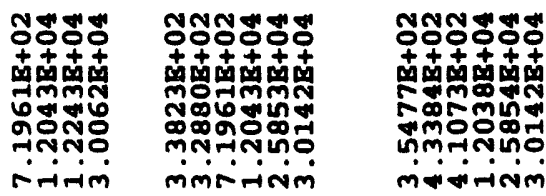

ํㅝㅇ웡ํㅇํำ

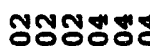
cono criming

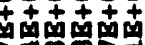
i⿻上丨⿶凵人

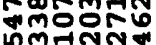

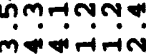

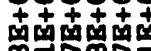
लिन्र UnRतOR

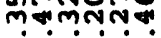

ํํㅇํํํํํํำ

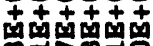

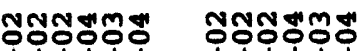
$++t++t+ \pm+++$

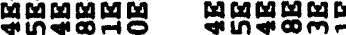
Ominga

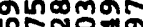

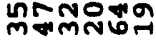

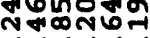
जित्रांक

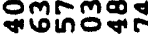
तึक्तmळ जित्रनिन

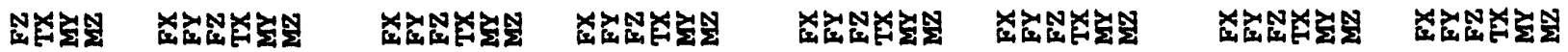

倠

$\stackrel{\text { ก }}{2}$
年

ล
息

ล
望

$\stackrel{\infty}{\sim}$
富

$\stackrel{\infty}{\sim}$
罢

ลิ
量

ลิ 

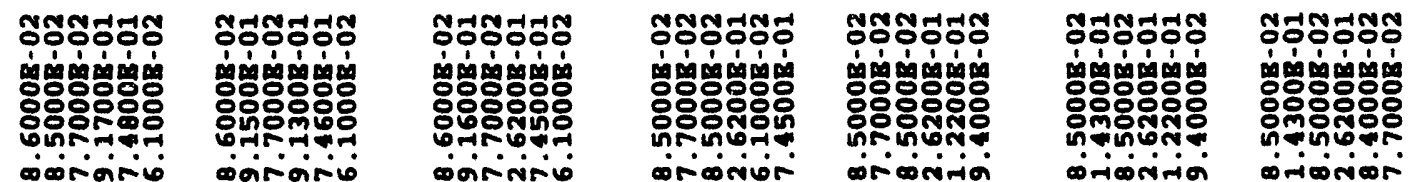

ํํำำำ

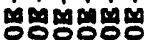
애아융요 mmunt

ธิธุำํํำำ

ริํํํํํำำำ

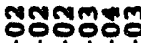

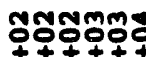

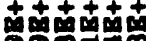

중중ำㅇํㅇ

$t+t+t$

नैन

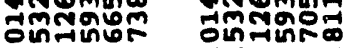

óminaior

omingiñ-
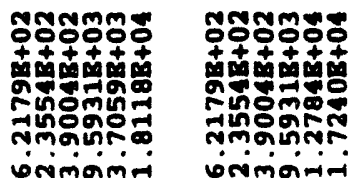

รีำำํํํํำ

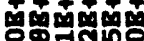

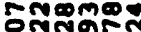
ㅇำสูกิก inc

orimaima

iminairiti

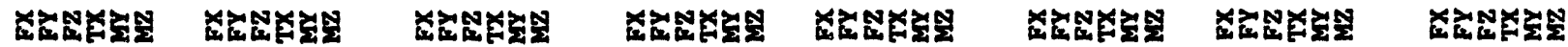

\begin{tabular}{|c|c|c|c|c|c|c|}
\hline & 总 & 总 & 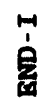 & 桌 & 䈱 & 官 \\
\hline & 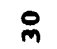 & in & $\ddot{m}$ & $\vec{m}$ & n & m \\
\hline
\end{tabular}



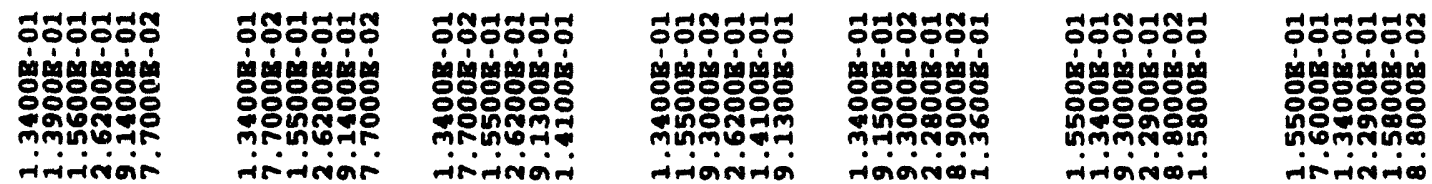

ธีธิธีธีธีดี

नलmor

iniviain

irionitio

नaवरrion
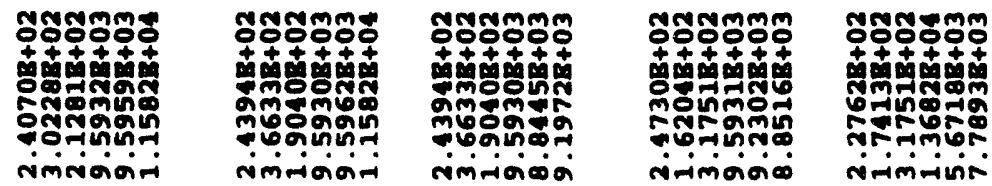

กิ두ํำ

iniminis
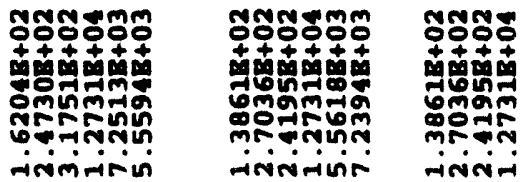

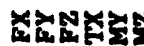

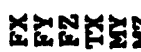

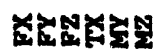

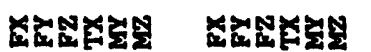

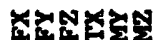

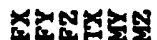

조존든

量

窝

?:

量

?

官

葛

愠

m

$\bar{m}$

m

m

卢

照

$\stackrel{\circ}{m}$

吕 

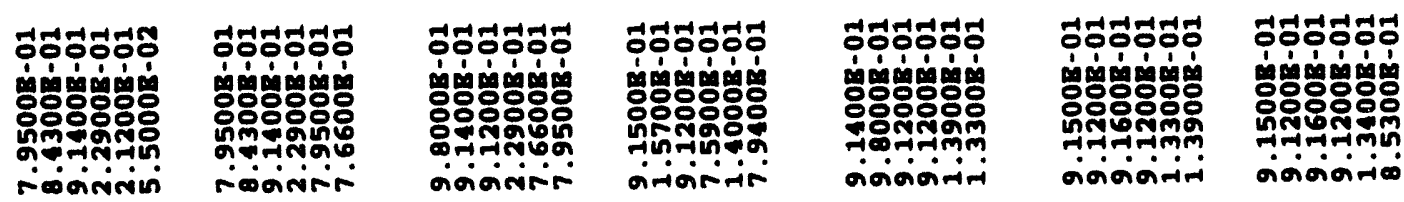

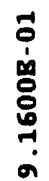
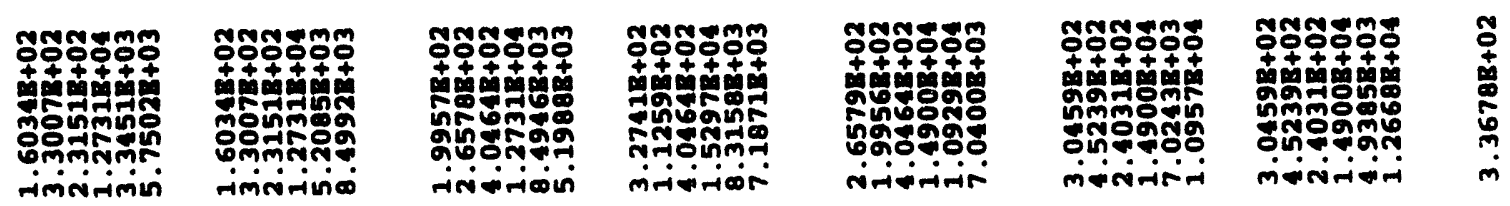

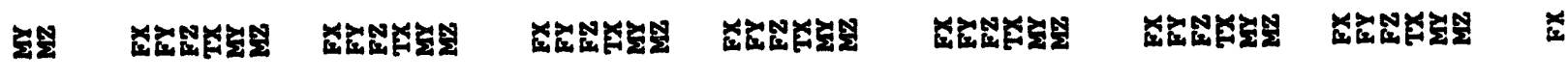

\begin{tabular}{|c|c|c|c|c|c|c|}
\hline 芒 & 章 & 息 & 总 & 葟 & 总 & 葛 \\
\hline$\frac{m}{m}$ & $\tilde{m}$ & $\bar{m}$ & $\underset{m}{\infty}$ & $\underset{m}{\infty}$ & gे & g \\
\hline
\end{tabular}



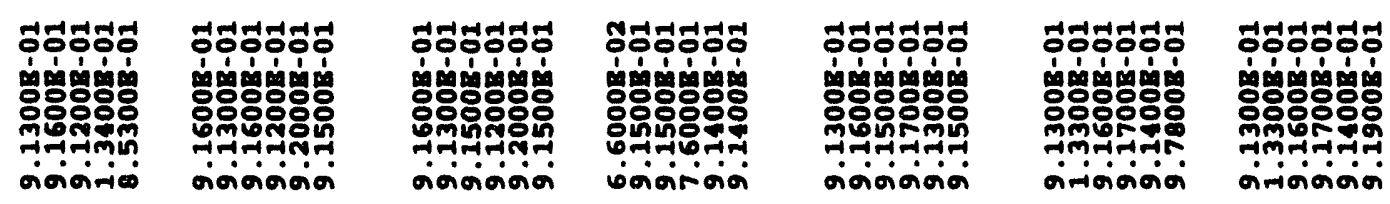

멍해엉멍영

뿌ำํํำํำ

앖으용

निन-Aन-

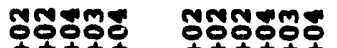

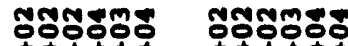

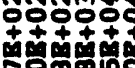

똥종ํํํํํำ

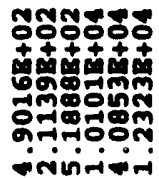

ธํํㅇㅈํㅇํํำ

+

-1900 응

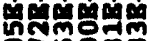

oxn.ำ

ărọ:

웡ำ

किiviniñ

ํํㅇ졍ㅎํㅇํํำ

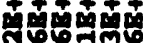

얗ํㅇํำ

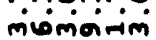

inivinini-

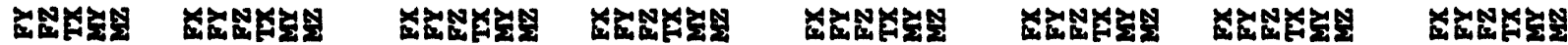

量

是

是

是

$\ddot{\theta}$

?

最

?

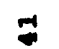

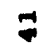

$\exists$

Y

พ

$\boldsymbol{F}$ 

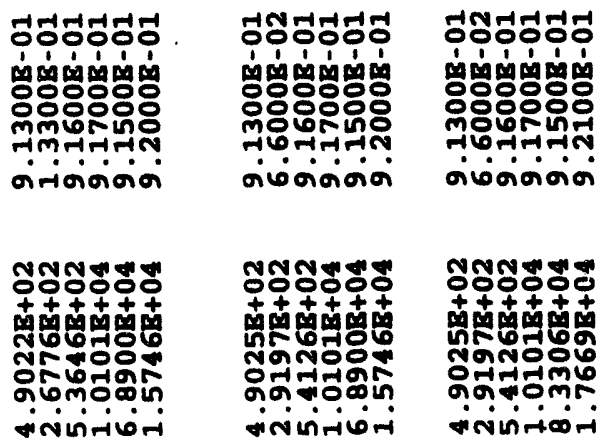

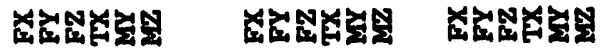

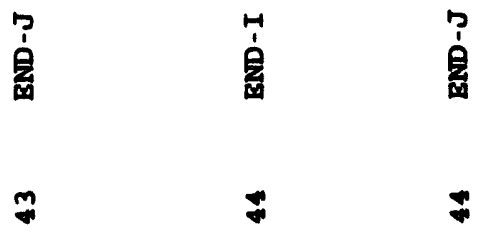



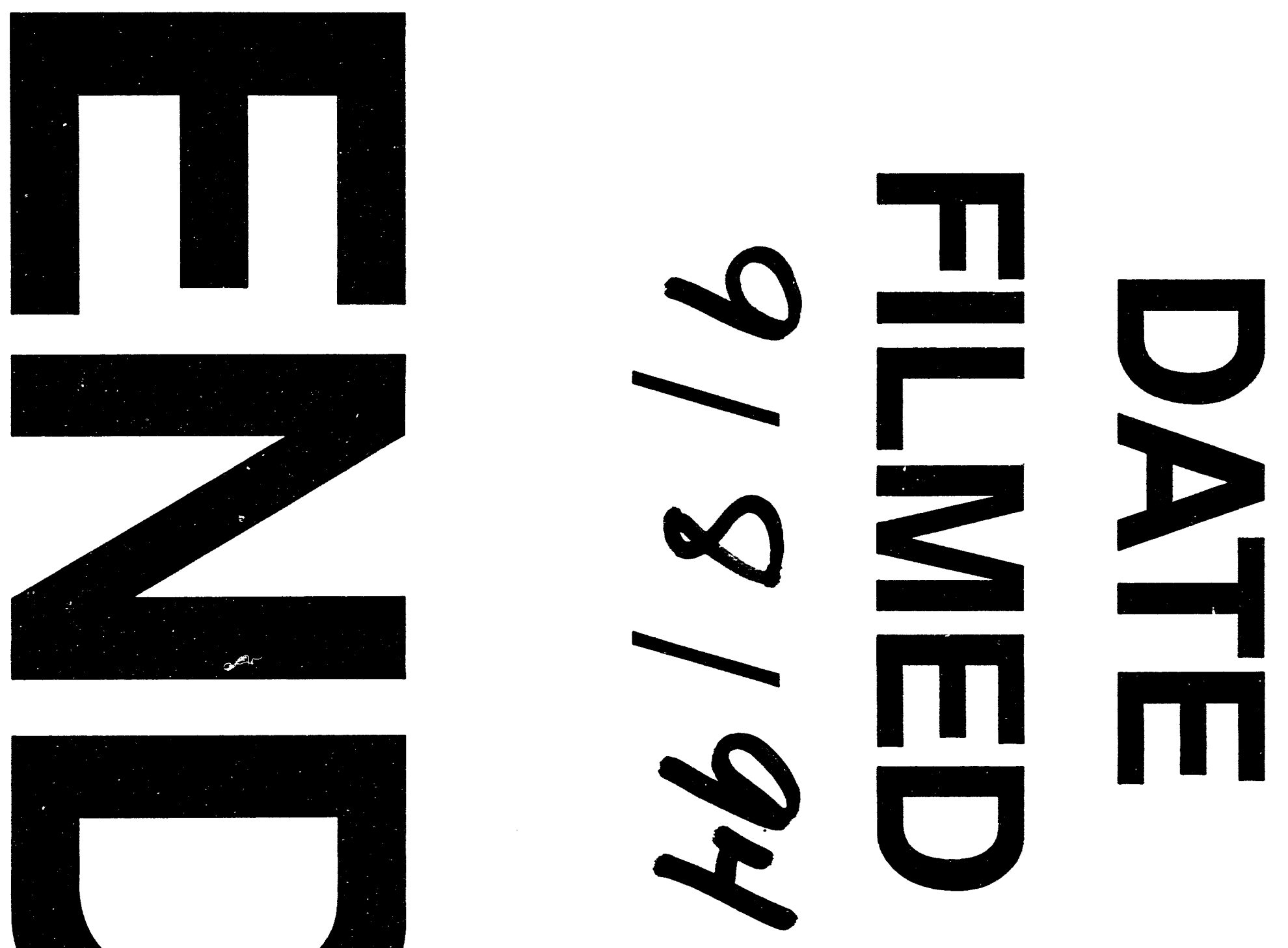